\title{
Dreidimensionale thermische Evolutionsmodelle für das Innere von Mars und Merkur
}

\author{
Dissertation \\ zur Erlangung des Doktorgrades \\ der Mathematisch-Naturwissenschaftlichen Fakultäten \\ der Georg-August-Universität zu Göttingen
}

vorgelegt von

Monika Buske

aus Stadtlohn

Göttingen 2006 
D7

Referent: Prof. Dr. Ulrich Christensen

Korreferent: Prof. Dr. Andreas Tilgner

Tag der mündlichen Prüfung: 25. April 2006 


\section{Inhaltsverzeichnis}

Zusammenfassung $\quad 5$

$\begin{array}{ll}\text { Summary } & 7\end{array}$

1 Einleitung 9

2 Einführung: Mars $\quad 11$

2.1 Topographie und Vulkanismus des Mars . . . . . . . . . . . . . . . . . 12

2.2 Ursachen des Vulkanismus . . . . . . . . . . . . . . . . . . . . . 14

2.3 Innerer Aufbau des Mars . . . . . . . . . . . . . . . . . 15

2.3.1 Bestimmung der Kerngröße . . . . . . . . . . . . . . . 15

2.3.2 Mineralstruktur des Mantels . . . . . . . . . . . . . . . . . . . 17

2.3.3 Parameter der Phasengrenzkurven . . . . . . . . . . . . . . . 19

2.3.4 Modelle der inneren Struktur des Mars _ . . . . . . . . . . . . 20

2.4 Einfluss von Phasengrenzen auf die Manteldynamik . . . . . . . . . . 23

2.5 Magnetfeld und Dynamo . . . . . . . . . . . . . . . . . 26

2.6 Thermische Evolutionsmodelle und Zielsetzung . . . . . . . . . . . . . 30

3 Theoretische Grundlagen $\quad 37$

3.1 Grundgleichungen der Strömungsmechanik . . . . . . . . . . . . . . . 37

3.2 Boussinesq- und erweiterte Boussinesq-Näherung . . . . . . . . . . . . 39

3.3 Zustandsgleichung . . . . . . . . . . . . . . . . . . 39

3.4 Entdimensionalisieren der Gleichungen . . . . . . . . . . . . . . . . . . . 41

3.5 Viskosität . . . . . . . . . . . . . . . . . . . . . . . 44

3.6 Thermischer Ausdehnungskoeffizient _. . . . . . . . . . . . . 48

3.7 Thermische Leitfähigkeit . . . . . . . . . . . . . . . . . . . . . . . . . . . . . . . . . . . . . 59

3.8 Interne Wärmequellen . . . . . . . . . . . . . . . . . . . 51

3.9 Rand- und Startbedingungen . . . . . . . . . . . . . . . . . 52

4 Numerische Methoden $\quad \mathbf{5 5}$

4.1 Grundlagen . . . . . . . . . . . . . . . . 55

4.2 Gitterparameter: räumliche Auflösung . . . . . . . . . . . . . . 58

4.3 Tiefenabhängiges $\lambda \ldots \ldots \ldots \ldots \ldots \ldots$

4.4 Erweiterte Boussinesq-Näherung . . . . . . . . . . . . . . . . . . . 59

4.5 Bestimmung der Tiefe der Phasengrenze . . . . . . . . . . . . . . . . 60 
5 Ergebnisse der Evolutionsmodelle für Mars

5.1 Einfluss des Aktivierungsvolumens . . . . . . . . . . . . . . . 62

5.2 Einfluss verschiedener Profile von $\alpha(r) \ldots \ldots \ldots$. . . . . . 77

5.3 Einfluss verschiedener Profile von $\lambda(r) \ldots \ldots \ldots$. . . . . . 87

5.4 Einfluss der Kombination von $\alpha(r)$ und $\lambda(r) \ldots \ldots \ldots$

5.4.1 Modelle für Mars mit $\alpha(r), \lambda(r)$ und zusätzlichen exothermen Phasengrenzen .................... 96

5.4.2 Einfluss der Kerntemperatur im Startprofil bei Modellen mit $\alpha(r)$ und $\lambda(r) \ldots \ldots \ldots \ldots \ldots$

5.5 Einfluss der Nicht-Boussinesq-Effekte . . . . . . . . . . . . . . . 113

5.5.1 Konstante thermische Parameter . . . . . . . . . . . . . . . . 113

5.5.2 Druck- und temperaturabhängige thermische Parameter . . . . . . 126

5.6 Der Aufbau des Marskerns . . . . . . . . . . . . . . . . . . . . . . 133

6 Thermische Evolutionsmodelle für Merkur 137

6.1 Innerer Aufbau und Magnetfeld des Merkur . . . . . . . . . . . . . . . 137

6.2 Modellierung des Kerns . . . . . . . . . . . . . . . . . . . . . . . 140

6.3 Ergebnisse der Evolutionsmodelle für Merkur . . . . . . . . . . . . . . . 144

6.3.1 Einfluss des Temperaturstartprofils . . . . . . . . . . . . . . . . . 144

6.3.2 Einfluss des Schwefelgehalts im Kern . . . . . . . . . . . . 155

6.3.3 Dreidimensionale Evolutionsmodelle für das Merkurinnere . . . . 161

6.3.4 Zusammenstellung weiterer Simulationsergebnisse . . . . . . . 171

6.3.4.1 Variation des Aktivierungsvolumens . . . . . . . . 171

7 Zusammenfassung und Diskussion $\quad 173$

7.1 Evolutionsmodelle für Mars . . . . . . . . . . . . . . . . . . 173

7.2 Evolutionsmodelle für Merkur . . . . . . . . . . . . . . . 178

$\begin{array}{ll}\text { A Anhang } & 181\end{array}$

A.1 Abbildungsfunktion: radiales Gitter . . . . . . . . . . . . . . 181

A.2 Bestimmung der Lithosphärendicke . . . . . . . . . . . . . . 183

A.3 Modellparameter für Mars . . . . . . . . . . . . . . . . . . . . . 185

A.4 Modellparameter für Merkur . . . . . . . . . . . . . . . . . . 186

A.5 Änderung des Planetenradius . . . . . . . . . . . . . . . . . . . . 187

$\begin{array}{ll}\text { Literaturverzeichnis } & 189\end{array}$

$\begin{array}{ll}\text { Danksagung } & 199\end{array}$

$\begin{array}{ll}\text { Lebenslauf } & 201\end{array}$ 


\section{Zusammenfassung}

Die innere Struktur und Entwicklung der terrestrischen Planeten ist eng mit Vorgängen im tiefen Inneren verbunden. Diese endogenen Vorgänge sind für viele geologische und tektonische Strukturen an der Oberfläche sowie die Erzeugung des Magnetfeldes durch einen Dynamoprozess verantwortlich. Thermische Evolutionsmodelle können zum Verständnis dieser Vorgänge und der Entwicklung der Planeten beitragen.

Auf dem Mars ist der junge Vulkanismus auf zwei Zentren konzentriert: die TharsisRegion und die Elysium-Region. Anomalien im Schwerefeld über den Vulkanregionen verstärken die Annahme, dass sich das Konvektionsmuster im Marsmantel von dem der Erde unterscheidet und von zwei starken Aufströmen geprägt ist. Eine mögliche Ursache für die starke Konzentration der Aufströme könnte der endotherme Phasenübergang von $\gamma$-Spinell zu Perowskit und Magnesiowüstit sein, der möglicherweise im Mars in der Nähe der Kern-Mantel-Grenze (KMG) auftritt.

Das Magnetfeld des Mars ist durch eine starke Krustenmagnetisierung dominiert. Diese Krustenmagnetisierung, insbesondere auf der alten Hemisphäre legt nahe, dass im Mars nur in den ersten 0.5 Ga seiner Entwicklung ein Dynamoprozess aktiv war, der dann ausgestorben ist.

Ein bestehendes, sphärisches, dreidimensionales Konvektionsprogramm wurde auf die Besonderheiten des Mars angepasst und so erweitert, dass es möglichst realistische Simulationen der thermischen Entwicklung des Planeten erlaubt. Die Viskosität ist tiefenabhängig und variiert, einem Arrheniusterm folgend, mit dem Druck und der horizontal gemittelten Temperatur. Die Temperatur an der KMG nimmt proportional zum Wärmefluss in den Mantel ab, so dass das Abkühlen des Kerns in die Simulationen eingeht. In erweiterten Modellen wird der Einfluss eines tiefenabhängigen thermischen Ausdehnungskoeffizienten $\alpha$ und einer tiefenabhängigen thermischen Leitfähigkeit $\lambda$ auf die thermische Entwicklung und das Konvektionsmuster untersucht. Die thermische Leitfähigkeit hängt dabei vom Druck und der horizontal gemittelten Temperatur ab. Das Modell berücksichtigt die verschiedenen Phasengrenzen des Olivins, insbesondere den endothermen Phasenübergang von $\gamma$-Spinell zu Perowskit und Magnesiowüstit. Die Simulationen werden sowohl in der Boussinesq- als auch in der erweiterten Boussinesq-Näherung durchgeführt. Es zeigt sich, dass der Einfluss der einzelnen Tiefenabhängigkeiten von $\alpha$ und $\lambda$, wie sie im Marsmantel vorkommen, sowohl auf die thermische Entwicklung als auch auf das Konvektionsmuster gering ist. Die kernnahe, endotherme Phasengrenze führt auch in diesen erweiterten Modellen zu einer deutlichen Reduktion der Anzahl der Aufströme. Bei Kombination der tiefenabhängigen Parameter nimmt jedoch der Einfluss dieser Phasengrenze deutlich ab, und auch ohne endotherme Phasengrenze entsteht ein großskaliges

Konvektionsmuster mit nur drei Aufströmen. In vollständigen Modellen, die zudem die exothermen Phasengrenzen des Olivins in der Mantelmitte berücksichtigen, stellt sich 
ein Konvektionsmuster mit zwei Aufströmen ein, das den konzentrierten, langlebigen Vulkanismus auf dem Mars erklären könnte. Die Tiefenabhängigkeit der beiden thermischen Parameter erhöht den Wärmefluss aus dem Kern in der Frühphase der Entwicklung und macht, insbesondere im Zusammenwirken mit den exothermen Phasengrenzen, einen thermisch getriebenen Dynamo zu Beginn der Evolution wahrscheinlich. Dieser Dynamo könnte die Krustenmagnetisierung erklären. Heute ist der Wärmefluss aus dem Kern zu gering, um durch thermische Konvektion einen Dynamo anzutreiben. Der Vergleich der Kerntemperaturen, die von der KMG entlang einer Adiabate in den Kern fortgesetzt werden, mit den Schmelztemperaturen von Eisenlegierungen zeigt, dass der Marskern bei einem Schwefelgehalt von 10 - 14 Gew.-\% flüssig ist und bislang keinen festen inneren Kern ausgebildet hat. Chemische Konvektion entfällt somit als Antriebsmechanismus für den Dynamoprozess, so dass zusammen mit dem geringen Wärmefluss das Fehlen eines heutigen globalen Magnetfelds erklärbar ist.

Die Erweiterung der Boussinesq-Modelle durch Berücksichtigung von Temperatureffekten durch adiabatische Kompression, latente Wärme und viskose Reibung verändern das Konvektionsmuster nicht signifikant. Der Temperatureinfluss wird durch den Beitrag der adiabatischen Kompression und der latenten Wärme der exothermen Phasenübergänge bestimmt. Insbesondere zeigt sich, dass der Beitrag der viskosen Reibung im Marsmantel vernachlässigbar ist.

Im zweiten Teil der Arbeit werden Evolutionsmodelle für Merkur vorgestellt. Die innere Struktur des Merkur ist nur sehr unzureichend bekannt. Die hohe Dichte legt einen großen Eisenkern nahe. Allerdings ist nicht klar, ob dieser Eisenkern fest, flüssig oder zumindest teilweise fest ist. Auch für Merkur stellt sich die Frage nach dem Mechanismus, der das heutige globale, aber sehr schwache Magnetfeld erzeugt. Hier können thermische Evolutionsmodelle, die das Ausbilden eines inneren, festen Kerns mitsimulieren, einen Beitrag zum Verständnis der inneren Struktur liefern.

Achsensymmetrische und erste dreidimensionale Simulationen zeigen, dass Merkur einen festen inneren Kern hat. Die Größe dieses inneren Kerns hängt stark von den Schmelzbedingungen von Eisen mit einigen Prozent Schwefel (0.5 - 5 Gew.-\%) ab, die bisher nicht durch Messungen genau bestimmt sind. Die angenommene Konvektionsstärke und die Temperaturstartbedingungen beeinflussen das Kernwachstum und die Größe zusätzlich. Nach 4.5 Ga liegt der Radius des inneren Kerns zwischen 610 und 1505 km bzw. 32 and $79 \%$ des Kernradius. Das Ausbilden eines inneren Kerns ermöglicht chemische Konvektion und damit den Antrieb eines Dynamos, der eventuell das heute beobachtete Magnetfeld erklären kann. Ein thermisch getriebener Dynamo ist heute nicht mehr möglich, da der Wärmefluss aus dem Kern zu gering ist. Die Konvektion im Merkurmantel ist auf eine dünne Schicht begrenzt, und der Mantel wird von einer dicken Lithosphäre dominiert. 


\section{Summary}

The interior structure and evolution of the terrestrial planets are closely related to the dynamics in their interior. These endogenic processes are responsible for many geological and tectonic structures on the surface and for the production of the magnetic field by a dynamo process. Thermal evolution models can help us to understand the processes in the interior and the thermal evolution of the planet.

On Mars young volcanism is concentrated in only two regions: in the Elysium region and, much more prominently, in the Tharsis region. Strong anomalies in the gravity field in the volcanic regions support the assumption that thermal convection in the martian mantle differs from that in the Earth's mantle and that the convection pattern is dominated by two strong plumes. A possible reason for this strong reduction to a few plumes could be the endothermic phase transition in the mineral structure from $\gamma$-spinel to perovskite and magnesiowüstite, which may occur in Mars close to the core-mantle boundary (CMB). The magnetic field of Mars is today dominated by a strong crust magnetization. The crust magnetization - especially in the old southern hemisphere - suggests that Mars had an active dynamo in the first $0.5 \mathrm{Ga}$ of its evolution but which subsequently died out.

An existing 3-D numerical convection code has been adapted to the specific features on Mars and extended to allow for more realistic simulations of the thermal evolution of the planet. The viscosity is depth-dependent and varies with pressure and the horizontally averaged temperature following an Arrhenius law. The temperature at the CMB decreases proportionally with the heat flux into the mantle. In this way, the cooling of the core is taken into account. In extended models, the influence of a depth-dependent thermal expansivity $\alpha$ and of the depth-dependence of the thermal conductivity $\lambda$ on the convection pattern and the thermal evolution are investigated. The thermal conductivity varies with pressure and the horizontally averaged temperature. The model takes the different phase transitions of olivine -especially the endothermic transition between $\gamma$-spinel and perovskite and magnesiowüstite- into account. The simulations were carried out in both the Boussinesq- and the extended Boussinesq-approximations.

The influence of the depth-dependence of only $\alpha$ or only $\lambda$ in the martian mantle on the convection pattern and the thermal evolution is small. The endothermic phase transition close to the $\mathrm{CMB}$ also reduces the number of upwellings in the extended models. The combination of the depth-dependencies of both parameters weakens the influence of the phase transition. Even in the absence of the endothermic phase transition, a strong reduction in the number of plumes to only three plumes can be observed. A complete model which also includes the exothermic phase transitions leads to a convection pattern with two upwellings which could explain the concentrated volcanism on Mars today. Both depth-dependent parameters $\alpha$ and $\lambda$ increase the heat flux at the CMB at the beginning of the evolution. In combination and especially together with the exothermic phase tran- 
sitions, the heat flux out of the core in the early stage of the planets evolution is probably high enough to allow for a thermally driven dynamo. This early dynamo could explain the crust magnetization. Today, the heat flux is too low to allow for a thermally driven dynamo. The comparison of the interpolated temperature structure in the core with the melting conditions of iron with 10 to $14 \%$ sulfur demonstrates that Mars has not yet frozen out an inner core. The martian core is completely liquid and does not allow for chemical convection as a driving mechanism for a dynamo today. The failure of both a thermally and a chemically driven dynamo in Mars can explain the lack of a global martian magnetic field today. The extension of the Boussinesq-Approximation by including the thermal effects of adiabatic compression, latent heat and viscous heating does not change the convection pattern dramatically. The influence on the temperature is dominated by the adiabatic heating and the latent heat of the exothermic phase transitions. It can be shown that viscous heating plays a negligible role in Mars.

In the second part of the thesis, evolution models for Mercury are shown. The internal structure of Mercury is not well-known at present. The extremely high density of the planet suggests a big iron core. The available data does not provide insight as to whether this big iron core is completely liquid or solid or at least partially solid. Mercury has a weak magnetic field and the mechanism which can produce this field is not yet well constrained. Thermal evolution models for Mercury which can simulate the effects of freezing out a solid inner core can provide important information about the interior structure and give some hints for the magnetic field.

Axisymmetric and first fully 3-D results show that Mercury has a solid inner core. The size of this inner core strongly depends on the exact melting conditions of iron with a small sulfur content $(0.5-5 \%)$, which are not yet well known. The assumed convection strength and the initial temperature conditions additionally influence the evolution and size of the inner core. After $4.5 \mathrm{Ga}$, the radius of the inner solid core is between 610 and $1505 \mathrm{~km}$ (corresponding to $32-79 \%$ of the core radius). Freezing out of an inner core allows for chemical convection, which could explain the present-day magnetic field. A thermally driven dynamo can be ruled out in all simulations because the heat flux out of the core which is transported by the mantle is too low to allow for thermal convection in the core. Mantle convection in the mercurian mantle is restricted to a thin layer. The mantle is dominated by a very thick lithosphere. 


\section{Einleitung}

Die innere Struktur und Entwicklung der terrestrischen Planeten ist eng mit Vorgängen im tiefen Inneren verbunden. Diese endogenen Vorgänge sind für viele geologische und tektonische Strukturen an der Oberfläche sowie die Erzeugung des Magnetfeldes durch einen Dynamoprozess verantwortlich. Beobachtungen der Planeten von der Erde aus oder mit Hilfe von Raumsonden können z.B. durch Kartierung der Oberfläche und durch Vermessung des Schwere- und Magnetfeldes Informationen über diese Prozesse und den möglichen inneren Aufbau der Planeten liefern. Obwohl sich die terrestrischen Planeten hinsichtlich ihrer Entstehung, ihrer chemischen Zusammensetzung und ihres prinzipiellen Aufbaus aus eisenreichem Kern, silikatischem Mantel und fester Kruste ähnlich sind, können sich die Prozesse im Inneren deutlich unterscheiden und zu sehr unterschiedlichen Merkmalen der Planeten führen. Thermische Evolutionsmodelle können dazu beitragen, die unterschiedliche Entwicklung der Planeten, insbesondere im Vergleich zur Erde, und die damit verbundenen Vorgänge im Inneren zu verstehen. In dieser Arbeit werden Konvektionsmodelle für die thermische Entwicklung von Mars und Merkur vorgestellt.

Kartierungen der Marsoberfläche zeigen, das der junge Vulkanismus auf zwei Zentren -die Tharsis-Region und die Elysium-Region- konzentriert ist. Anomalien im Schwerefeld über den Vulkanregionen verstärken die Annahme, dass sich das Konvektionsmuster im Marsmantel von dem der Erde unterscheidet und von zwei starken Aufströmen geprägt ist. Eine mögliche Ursache für die starke Konzentration der Aufströme könnte der endotherme Phasenübergang von $\gamma$-Spinell zu Perowskit und Magnesiowüstit sein, der möglicherweise im Mars in der Nähe der Kern-Mantel-Grenze (KMG) auftritt.

Das Magnetfeld des Mars ist durch eine starke Krustenmagnetisierung dominiert. Diese Krustenmagnetisierung, insbesondere auf der alten Hemisphäre legt nahe, dass im Mars nur in den ersten 0.5 Ga seiner Entwicklung ein Dynamoprozess aktiv war, der dann ausgestorben ist.

Im ersten Teil der Arbeit werden möglichst realitätsgetreue thermische Evolutionsmodelle für Mars vorgestellt, die versuchen, Ursachen für die Konzentration des langlebigen Vulkanismus und die Geschichte des Magnetfeldes zu finden. Kapitel 2 gibt eine Einführung und beschreibt den Vulkanismus auf dem Mars und die Entwicklung des Magnetfeldes. Die innere Struktur des Mars wird diskutiert und die besondere Bedeutung der Phasengrenzen für die Mantelkonvektion im Mars erläutert. Kapitel 3 beschreibt das mathematische Modell und Kapitel 4 gibt einen kurzen Einblick in die numerische Umsetzung. Die Ergebnisse der Simulationen werden in Kapitel 5 vorgestellt.

Der zweite Teil der Arbeit, Kapitel 6, präsentiert Evolutionsmodelle für Merkur. Die innere Struktur des Merkur ist nur sehr unzureichend bekannt. Die hohe Dichte legt einen großen Eisenkern nahe. Allerdings ist nicht klar, ob dieser Eisenkern fest, flüssig oder zumindest teilweise fest ist. Auch für Merkur stellt sich die Frage nach dem Mechanismus, 
der das heutige globale, aber sehr schwache Magnetfeld erzeugt. Hier können thermische Evolutionsmodelle, die das Ausbilden eines inneren, festen Kerns mitsimulieren, einen Beitrag zum Verständnis der inneren Struktur liefern.

In Kapitel 7 werden die Ergebnisse der Evolutionsmodelle für Mars und Merkur zusammengefasst und diskutiert. 


\section{Einführung: Mars}

Mars ist als vierter Planet unseres Sonnensystems der äußere Nachbar der Erde und gehört zu den erdähnlichen Planeten. Mit einem mittleren Radius von $3389.92 \mathrm{~km}$ ist er nur etwa halb so groß wie die Erde. Seine Dichte von $3933.5 \mathrm{~kg} / \mathrm{m}^{3}$ legt aber nahe, dass er der Erde in seiner Zusammensetzung ähnlich ist. Die Schwerebeschleunigung an der Oberfläche entspricht mit $3.711 \mathrm{~m} / \mathrm{s}^{2}$ am Äquator $\left(3.758 \mathrm{~m} / \mathrm{s}^{2}\right.$ am Pol) etwa einem Drittel des Wertes für die Erde. Ein Marsjahr dauert 686.98 Erdtage, wobei die Bahn um die Sonne eine Exzentrizität von 0.0934 hat. Für die Rotation um die eigene Achse benötigt Mars $24.62 \mathrm{~h}$ (Sternentag). Die Rotationsachse ist dabei um $25.19^{\circ}$ gegen die Ekliptik geneigt, so dass es auch auf dem Mars Jahreszeiten gibt, die aber wegen der Exzentrizität der Bahn unterschiedliche Längen auf der Nord- und Südhalbkugel haben. Die Oberflächentemperatur schwankt zwischen 140 und $300 \mathrm{~K}$, die mittlere Temperatur beträgt $210 \mathrm{~K}$. Die Marsatmosphäre besteht im Wesentlichen aus Kohlendioxid (95.32 \%) mit einigen Spurengasen wie Stickstoff $(2.7 \%)$, Argon (1.6\%), Sauerstoff (0.13\%), Kohlenmonoxid (0.07\%), Wasserdampf (0.03 \%), Ozon und Edelgasen. Der Druck an der Oberfläche beträgt nur $560 \mathrm{~Pa}$ (Kieffer et al. 1992).

Die Erforschung des Mars mit Hilfe von Weltraumraumsonden begann 1964 mit der Mariner 4 Mission, die in einem Minimalabstand von $9825 \mathrm{~km}$ an der Marsoberfläche vorbeiflog und die ersten 21 Aufnahmen der Marsoberfläche lieferte. Es folgten 1969 die Mariner 6 und Mariner 7 Missionen, die Bilder machten, auf denen Details von einer GröBe von 300 m erkennbar waren. Noch genauere Aufnahmen wurden 1971 durch Mariner 9 gewonnen. 1971 setzten die sowjetischen Sonden Mars 2 und 3 die erste Landekapseln auf dem Mars ab. Die NASA-Missionen Viking 1 und 2 flogen 1975 zum Mars und setzten ebenfalls Landestufen auf der Marsoberfläche ab. Diese Missionen lieferten Daten bis in die 80er Jahre. Neben der Kartierung der Marsoberfläche mit Hilfe der Orbiter dienten die Landestufen auch der Suche nach Leben auf dem Mars. Nach verschiedenen fehlgeschlagenen Missionen (Mars Observer Mission (1993), Mars 96 (1996)) erreichte im Dezember 1997 die Mars Global Surveyor Mission den Marsorbit. An Bord des Mars Global Surveyor befindet sich u.a. das Mars Orbiter Laser Altimeter (MOLA), das Höhenmessungen auf der Marsoberfläche mit einer Genauigkeit von bis zu $37.5 \mathrm{~cm}$ ermöglicht, und Experimente (MAG/ER), die das Magnetfeld des Mars vermessen haben. 1997 erreicht auch die Mars Pathfinder Mission den Mars und setzt einen Lander mit einem mobilen Rover auf der Marsoberfläche ab. Die NASA-Mission Mars-Odyssey ist im April 2001 gestartet worden und analysiert mit Hilfe eines Orbiters die chemischen Elemente und Minerale auf der Marsoberfläche und sucht nach Hinweisen auf Wasser in den oberflächennahen Schichten. Zudem analysiert die Mission die Strahlung auf dem Mars, um die gesundheitlichen Risiken für den Menschen abzuschätzen. Der Pathfinder Mission folgten im Januar 2004 die im Juni 2003 gestarteten Mars Exploration Rover, 
Spirit and Opportunity, die nach Hinweisen auf Wasser und seinen Einfluss auf die Umwelt des Mars und deren Entwicklung in früheren Zeiten suchen. Zur gleichen Zeit wurde die ESA-Mission MarsExpress gestartet, die einerseits mit Instrumenten ausgestattet ist, die Wasser in der Atmosphäre und oberflächennahen Schichten nachweisen können, und anderseitets über ein hochauflösendes Stereokamerasystem verfügt, dass die Marsoberfläche mit hoher Auflösung kartiert.

Diese Missionen haben zu einer guten Kenntnis der Marsoberfläche und ihren geologischen Erscheinungsformen geführt. Zudem sind Daten ermittelt worden, die Hinweise auf die innere Struktur geben.

\subsection{Topographie und Vulkanismus des Mars}

Mit den Daten des Laseraltimeters (MOLA) der Mars Global Surveyer Mission ist eine hochaufgelöste Karte der Oberflächentopographie des Mars erstellt worden (Abbildung (2.1)). Die Topographiekarte zeigt eine deutliche Zweiteilung der Oberfläche, die als Di-

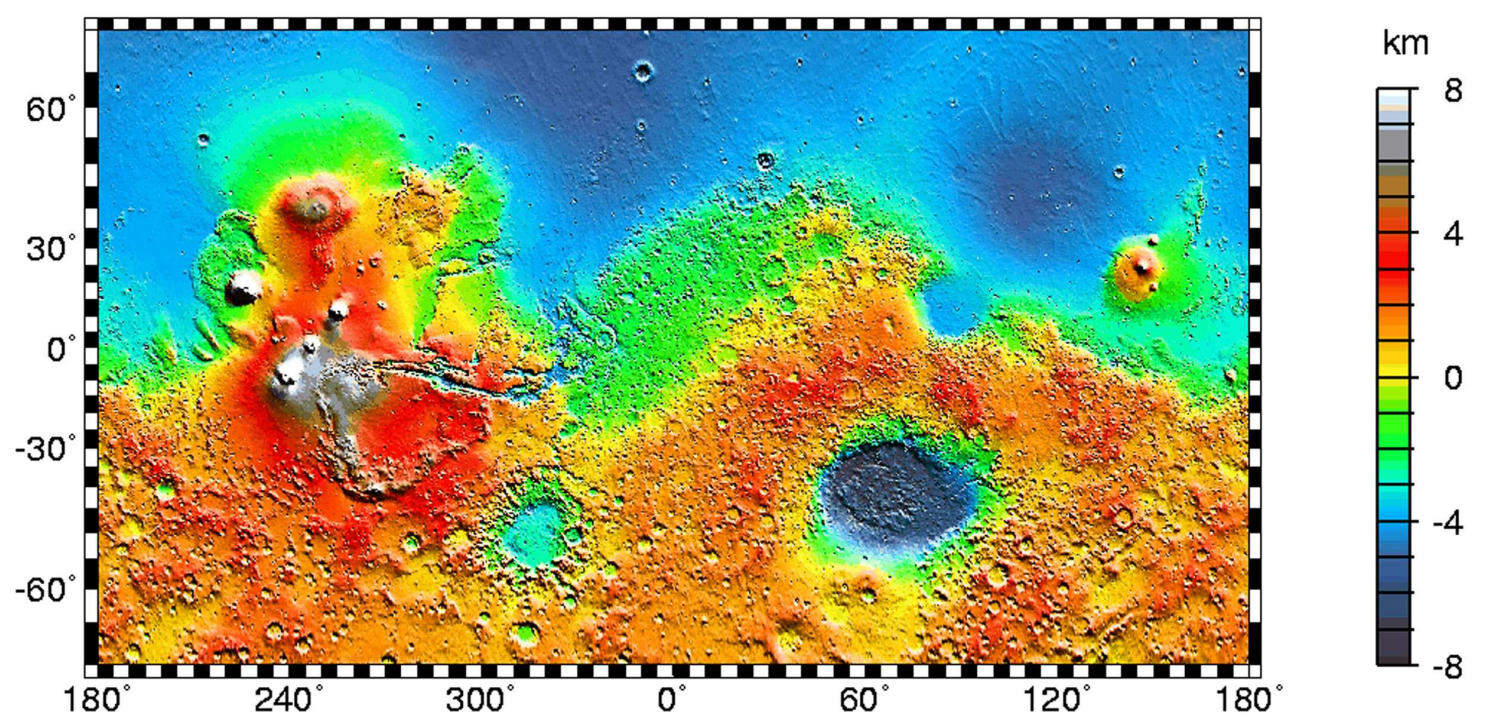

Abbildung 2.1: Topographie der Marsoberfläche (MOLA) (Smith et al. 1999)

chotomie bezeichnet wird. Dabei liegt die Oberfläche der südlichen Hemisphäre einige $\mathrm{km}$ höher als die der Nordhalbkugel und ist dicht mit vielen Kratern übersät, was auf ein hohes Alter $(\sim 3.8 \mathrm{Ga})$ schließen lässt. Dahingegen finden sich auf der nördliche $\mathrm{He}-$ misphäre nur vereinzelte Krater, die anzeigen, dass dieser Krustenteil wesentlich jünger ist. Die Ursache der Dichotomie der Oberfläche ist noch nicht endgültig verstanden. Es werden einerseits große Einschläge verantwortlich gemacht und andererseits Vorgänge im Inneren vermutet. Zhong und Zuber (2001) z.B. beobachten in Konvektionsmodellen für den Marsmantel, die eine geschichtete Viskosität mit einer rheologisch weichen Asthenosphäre annehmen, ein Konvektionsmuster mit der Kugelflächenfunktionen von Grad $l=1$, das dazu beitragen kann, die unterschiedliche Krustenstruktur in der Nord- und Südhalbkugel zu verstehen. Auch Plattentektonik in der frühen Geschichte des Mars wird 
als Ursache diskutiert (Sleep 1994).

Neben der Dichotomie fallen einige besonders große Krater auf der Südhalbkugel auf. Das Hellas Basin $\left(45^{\circ} \mathrm{S}, 70^{\circ} \mathrm{O}\right)$ ist einer dieser großen Einschlagkrater (Radius: 2100 $\mathrm{km})$ und markiert den tiefsten Punkt der Marsoberfläche. Ein weiterer auffallender Krater ist das Agyre Basin $\left(50^{\circ} \mathrm{S}, 320^{\circ} \mathrm{O}\right)$. Von gewaltigem Ausmaß ist auch das Canyon-System Valles-Marineris, das sich zwischen $\left(10^{\circ} \mathrm{S}, 250^{\circ} \mathrm{O}\right)$ und $\left(10^{\circ} \mathrm{S}, 305^{\circ} \mathrm{O}\right)$ über eine Länge von $4000 \mathrm{~km}$ erstreckt und Tiefen von bis zu $8 \mathrm{~km}$ erreicht.

Eine weitere topographische Besonderheit ist die Aufwölbung der Tharsis-Region, die ihr Zentrum etwa am Äquator hat und sich in der Länge zwischen $220^{\circ}$ Ost bis $300^{\circ}$ Ost erstreckt. Die Tharsis-Region ist ein riesiges Vulkangebiet zwischen den nördlichen Tiefebenen und den südlichen höher gelegenen Gebieten. Sie besteht aus zwei breiten Erhebungen, von denen die südliche im Wesentlichen durch die Vulkane Ascaraeus Mons $\left(12^{\circ} \mathrm{N}, 248^{\circ} \mathrm{O}\right)$, Pavonis Mons $\left(0^{\circ}, 247^{\circ} \mathrm{O}\right)$ und Arsia Mons $\left(9^{\circ} \mathrm{S}, 239^{\circ} \mathrm{O}\right)$ geformt wird und die nördliche durch den großen Schildvulkan Alba Patera $\left(42^{\circ} \mathrm{N}, 252^{\circ} \mathrm{O}\right)$. Der größte Vulkan unseres Sonnnensystem Olympus Mons ( $\left.18^{\circ} \mathrm{N}, 225^{\circ} \mathrm{O}\right)$ mit einer Höhe von $24 \mathrm{~km}$ befindet sich am westlichen Rand der Tharsis-Region. Trotz der räumlichen Trennung von Olympus Mons und Alba Patera ist es wahrscheinlich, dass alle Vulkane mit dem Ursprung der Tharsis-Region verbunden sind (Smith et al. 1999). Außerhalb der Tharsis-Region gibt es in der nördlichen Hemisphäre eine zweite Erhöhung die vulkanischen Ursprungs ist: die Elysium-Region $\left(15^{\circ} \mathrm{N}-35^{\circ} \mathrm{N}, 140^{\circ} \mathrm{O}-155^{\circ} \mathrm{O}\right)$ mit den Vulkanen Elysium Mons, Hecates Tholus und Albor Tholus.

Neukum und Hiller (1981) haben Vikingbilder analysiert und mit Hilfe von Kraterstatistiken die Alter der Vulkanregionen und deren Aktivitätszeiträume bestimmt. Neben den zwei schon erwähnten Vulkanregionen fassen sie verschiedene Vulkane (Tyrrhena Patera, Hadriaca Patera und Tempe Patera) auf der stark gekraterten alten Hemisphäre zu einer dritten Gruppe zusammen. Für die Vulkanen dieser letzten Gruppe ermitteln sie ein Alter zwischen 3.8 und 4.0 Ga. Die Lavaflüsse, die diese Vulkane umgeben, sind 3.8 Ga alt und stellen das jüngste Material in den südlichen Hochländern dar. Der Vulkanismus in diesen Regionen ist also ungefähr in dieser Zeit ausgestorben.

Für die Elysium-Region liefert die Kraterdichte ein Alter für die Schildvulkane zwischen 3.2 und 3.9 Ga. Die jüngsten Lavaflüsse haben ein Alter von etwa 1 bis $1.5 \mathrm{Ga}$ (Neukum und Hiller 1981). Die hochaufgelösten Bilder der High Resolution Stereo Camera (HRSC) auf MarsExpress tragen dazu bei, diese Angaben zu präzisieren und geben Hinweise auf einen aktiven Vulkanismus bis in die jüngste geologische Vergangenheit. Neukum et al. (2004) untersuchen mit diesen Daten und verbesserten Statistiken das Alter von verschiedenen Laven auf den Flanken des Vulkans Hecates Tholus und kommen zu Altern zwischen 3.8 und 0.1 Ga. Zudem finden Neukum et al. (2004) und Hauber et al. (2005) hier Calderen mit Altern zwischen 1 und 0.11 Ga. Die neuen Daten bestätigen, dass der Vulkanismus in der Elysium-Region einen sehr langen geologischen Zeitraum überspannt hat und sich bis in die jüngste Vergangenheit fortsetzt.

In der Tharsis-Region begann der Vulkanismus etwa zur selben Zeit wie in der ElysiumRegion (vor 3.3 - 3.8 Ga). Lavaflüsse um Olympus Mons und die drei großen Tharsisvulkane haben Alter zwischen 3.3 und $0.3 \mathrm{Ga}$. Ähnliche Angaben ergeben sich für Alba Patera (3.8 - $0.5 \mathrm{Ga})$. Hier setzt sich die Aktivität bis in die jüngste geologische Vergangenheit fort (Neukum und Hiller 1981). Die Altersbestimmungen mit neueren Daten bestätigen den langlebigen Vulkanismus in der Tharsis-Region. Neukum et al. (2004) be- 
stimmen z.B. das Alter der Caldera von Ascraeus Mons zu 0.1 Ga bzw. 0.1 - 0.2 Ga für die Caldera von Olympus Mons. An der Flanke finden sich Lavaflüsse mit einem Alter von $0.24 \mathrm{Ga}$.

Der junge Vulkanismus auf dem Mars ist somit in nur zwei Zentren (Tharsis und Elysium) konzentriert. Damit unterscheidet sich die Verteilung des Vulkanismus auf dem Mars stark von der Situation auf der Erde, wo Vulkanismus an vielen Orten zu finden ist. Ein Ziel der vorliegenden Arbeit ist es, die Konzentration des Vulkanismus auf wenige Zentren im Mars zu verstehen.

\subsection{Ursachen des Vulkanismus}

Auf der Erde befinden sich Vulkane in der Nähe von Plattenrändern, an denen ozeanische Platten subduziert werden, oder innerhalb der tektonischen Platten an sogenannten 'hot-spots'. Sowohl die Plattenbewegung als auch die Bildung der 'hot-spots' haben ihre Ursache in der Dynamik des Mantels bzw. der Mantelkonvektion. Das feste Gestein im Silikatmantel der terrestrischen Planeten lässt sich unter Druck, hohen mechanischen Spannungen und bei den Temperaturbedingungen im Inneren des Planeten plastisch verformen und zeigt eine Kriechbewegung. Unter den Bedingungen im Planetenmantel verhält sich das Gestein wie eine äußerst zähe Flüssigkeit. Angetrieben durch den Temperaturkontrast zwischen der kalten Oberfläche und dem warmen Kern und zusätzlichen internen Wärmequellen durch den radioaktiven Zerfall von Spurenelementen $\left({ }^{235} \mathrm{U},{ }^{238} \mathrm{U},{ }^{232} \mathrm{Th}\right.$, ${ }^{40} \mathrm{~K}$ ) wird eine thermische Konvektionsbewegung angetrieben. Die tektonischen Platten bilden dabei die kalte thermische Grenzschicht und sind Bestandteil der Konvektionsbewegung. Die Subduktion einer tektonischen Platte stellt den kalten, abtauchenden Zweig der Konvektionsbewegung dar. In diesem subduzierten -wasserhaltigen- Material wird die Solidustemperatur schon in geringer Tiefe erreicht, so dass sich partielle Schmelzen bilden, die zu Vulkanismus führen.

Für den 'hot-spot'-Vulkanismus ist eine besondere Form der Aufströme innerhalb der Konvektionsbewegung verantwortlich, sogenannte Mantelplumes. Diese Mantelplumes sind quasizylindrische, stationäre Aufströme von warmem Mantelmaterial, die aus Instabilitäten in der heißen thermischen Grenzschicht an der Kern-Mantel-Grenze (KMG) entstehen. Erreicht das warme Material in den Plumes oberflächennahe Regionen, entstehen durch die Druckentlastung Schmelzen, die an der Oberfläche Vulkanismus hervorrufen. Auf der Erde gibt es bis zu 100 dieser Plumes (Turcotte und Schubert 2002).

Im Gegensatz zur Erde hat es auf dem Mars wahrscheinlich nur für einen geologisch kurzen Zeitraum Plattentektonik gegeben. Hinweise auf Plattenbewegung finden Connerney et al. (1999) mit Hilfe der MAG/ER-Daten in einem Streifenmuster abwechselnder Magnetisierungsrichtungen auf der alten südlichen Hemisphäre. Das Muster von nahezu parallelen Streifen (in Ost-West-Richtung) abwechselnder Polarität erinnert an das Streifenmuster auf den Ozeanböden. Eventuell ist hier ähnlich wie bei 'seafloorspreading' auf der Erde an einem mittelozeanischen Rücken neue Kruste erzeugt worden, die dann während ihrer Bewegung weg vom Rücken abgekühlt ist und die Magnetfeldrichtung eingefroren hat. Seit dieser kurzen Periode mit möglicher Plattentektonik besteht die Marsoberfläche aus nur einer zusammenhängenden Platte, die keine Subduktion und den damit verbunde- 
nen Vulkanismus zulässt.

Eine mögliche Erklärung für den heute auf wenige Zentren konzentrierten Vulkanismus auf dem Mars könnte in einem Konvektionsmuster im Mantelinneren liegen, das im Gegensatz zur Erde durch nur wenige warme Aufströme -eventuell je einen unter Tharsis und Elysium- geprägt ist. Die lange Aktivität der Marsvulkane, die die Lebensdauer der großen Vulkane auf der Erde um mehrere Größenordnungen übertrifft, lässt zusätzlich auf eine lange Lebensdauer der 'hot-spots' und große Stabilität der Plumes im Mars schließen.

Die Analyse des Schwerefeldes durch 'Doppler-tracking' während der Kartierung durch Mars Global Surveyor zeigt sehr starke, positive Freiluftanomalien über den jungen Vulkanregionen Tharsis und Elysium (Zuber et al. 2000). Diese starken Anomalien beobachten auch Kiefer et al. (1996) bei der Analyse der 'Doppler-tracking'-Daten von Mariner 9, Viking 1 und Viking 2. Sie berücksichtigen bei ihrer Inversion der Topographie und Schwerefelddaten den Einfluss von viskosen Strömungen im Mantel und finden thermische Anomalien, die auf warme Aufströme unter Tharsis und Elysium deuten. Konvektionsvorgänge im Mantel wurden auch von Espostio et al. (1992) bei ihrer Analyse der Schwerefelddaten als Ursache vermutet. Die Schweredaten unterstützen damit einen Zusammenhang der Tharsis-Region mit manteldynamischen Vorgängen und Plumes.

Ein möglicher Mechanismus für die starke Reduzierung der Anzahl der Aufströme im Mars im Vergleich zur Erde kann in der Wechselwirkung zwischen Phasenübergängen in den Mantelmineralen und der Konvektionsströmung liegen (Weinstein 1995, Harder und Christensen 1996, Breuer et al. 1998, Harder 2000). Diese Phasenübergänge finden im Mars in anderen Tiefen statt als in der Erde, so dass ihnen eine besondere Bedeutung zukommen kann. Die innere Struktur des Mars und seine Mineralstruktur wird im nächsten Abschnitt beschrieben.

\subsection{Innerer Aufbau des Mars}

Wie einleitend erwähnt, ist Mars ein terrestrischer Planet, dessen Radius etwa halb so groß ist wie der der Erde und dessen Dichte eine erdähnliche Zusammensetzung vermuten lässt. Der innere Aufbau des Mars ist bis heute nicht genau geklärt, da seismologische Daten, die für die Erde genauen Aufschluss über die innere Struktur geben, für Mars bisher nicht vorliegen und andere Messdaten bislang keine eindeutigen Aussagen zulassen.

\subsubsection{Bestimmung der Kerngröße}

Während der Pathfindermission ist die Präzessionsrate des Mars durch 'radio-Dopplertracking' sehr genau vermessen worden. Die Präzessionsrate eines Planeten ist proportional zu $(C-(A+B) / 2) / C$, wobei $A, B$ und $C$ die Hauptträgheitsmomente des Planeten sind. $A$ und $B$ bezeichnen dabei die Momente um Äquatordurchmesser und $C$ das Moment um die Rotationsachse. Aus der Kenntnis des Schwerefeldes, das schon mit dem Viking Orbiter aber auch während der Mars Global Surveyer Mission genau vermessen worden ist, lässt sich die Größe $C-(A+B) / 2=C_{2}^{0} M R^{2}$ bestimmen. $C_{2}^{0}$ ist der Entwicklungskoeffizient des Schwerefeldes, der durch den Äquatorwulst dominiert wird, $M$ 
und $R$ sind Masse und Radius des Planeten (Radius der Kugel mit gleichem Volumen). Aus der Präzessionsrate zusammen mit den Daten aus dem Schwerefeld kann das dimensionslose Trägheitsmoment um die Rotationsachse $C / M R^{2}=0.3662 \pm 0.0017$ berechnet werden (Folkner et al. 1997). Das Trägheitsmoment stellt eine zusätzliche Bedingung an mögliche Dichte- und Aufbaumodelle für das Innere des Mars.

Das dimensionslose Trägheitsmoment einer homogenen Kugel beträgt 0.4. Ein kleinerer Wert $(<0.4)$ deutet auf eine Massenkonzentration im Inneren z.B. in Form eines dichteren (metallischen) Kerns hin. Mars hat vermutlich ähnlich wie die Erde einen Differentiationsprozess durchlaufen und besteht aus einem Mantel aus Silikatgestein und einem metallischen Kern. Das Trägheitsmoment wird durch die Dichteverteilung bestimmt und hängt von der Kerngröße, der chemischen Zusammensetzung von Kern und Mantel sowie dem Temperaturprofil ab. Da die chemische Zusammensetzung des Kerns nicht genau bekannt ist, kann die Kerngröße nicht eindeutig bestimmt werden. Dabei konkurrieren kleine, relativ dichte Kerne (Fe/Ni) mit vergleichsweise großen, weniger dichten Kernen (FeS). Ohne chemische Beschränkungen sind Kernradien zwischen 1200 und 2400 km mit dem dimensionslosen Trägheitmoment vereinbar (Folkner et al. 1997). Mit Anlehnung an die chemischen Zusammensetzung von Meteoriten, die vom Mars stammen, und unter der Annahme, dass der Schwefelanteil im Marskern kleiner als $50 \%$ ist, geben Folkner et al. (1997) Radien zwischen 1450 und $1700 \mathrm{~km}$ für ein Temperaturprofil mit vergleichsweise hohen Temperaturen und zwischen 1300 und $1450 \mathrm{~km}$ bei einem kalten Temperaturprofil und etwas veränderter Mantelzusammensetzung an.

Die chemische Analyse der SNC-Meteoriten, die sehr wahrscheinlich vom Mars stammen und nach ihren Fundorten auf der Erde Shergotty, Nakhla, Chassigny benannt sind, gibt weitere Informationen über die Zusammensetzung des Mars. Dreibus und Wänke (1987) leiten aus der Untersuchung dieser Meteorite und mit Hilfe von kosmochemischen Argumenten die Zusammensetzung des Marsmantels und Kerns ab. In ihren Modellen hat der Marskern im Vergleich zur Erde einen deutlich höheren Schwefelanteil von 14 \%. Dieser hohe Schwefelanteil lässt eine relativ geringe Kerndichte vermuten, die, um die Anforderung an Masse und Trägheitsmoment zu erfüllen, einen relativ großen Kern im Mars erforderlich macht. Anderseits haben Fei et al. (1995) in Hochdruckexperimenten im Druck- und Temperaturbereich des Marskerns eine sehr kompressible Hochdruckphase von FeS (FeS IV) gefunden, die sowohl einen hohen Schwefelanteil (13.9\%) als auch ein hohe Dichte im Marskern zulässt und somit auch kleinere Kerne, die bisher vorliegenden Daten erklären können. Fei et al. (1995) ermitteln mit der Dichte von FeS IV eine Kerngröße von 1400 km bzw. ein Radienverhältnis zwischen dem Kernradius $r_{i}$ und dem Planetenradius $r_{a}$ von $r_{i} / r_{a}=0.41$.

Aufgrund der fehlenden seismischen Daten ist zudem unklar, ob der Kern fest oder flüssig ist. Yoder et al. (2003) bestimmen mit 'radio-tracking'-Daten der Mars Global Surveyer Mission (MGS) aus der Gezeitendeformation, die die Sonne auf dem Mars bewirkt, die Love-Zahl $k_{2}=0.153 \pm 0.017$. Aus der Größe des Wertes schließen sie, dass der Marskern nicht komplett fest sein kann sondern zumindest einen äußeren flüssigen Anteil hat. Sie berechnen Strukturmodelle für das Innere des Mars mit unterschiedlichen chemischen Zusammensetzungen für Kern und Mantel sowie verschiedenen Annahmen über das Temperaturprofil bzw. die Krustendicke. In ihren Modellen geben Kernradien zwischen 1520 und $1840 \mathrm{~km}$ sowohl das Trägheitsmoment als auch die Love-Zahl richtig wieder. 


\subsubsection{Mineralstruktur des Mantels}

Die Kerngröße beeinflusst die Druckbedingungen im Mantel und ist damit bedeutend für mögliche Phasenübergänge im Mantelmineral. Die mineralische Zusammensetzung des Marsmantels wird in diesem Abschnitt beschrieben.

In der Erde besteht der obere Mantel im Wesentlichen aus den Mineralen Olivin (63\%), Orthopyroxen ( $8 \%)$, Klinopyroxen (16\%) und Garnet (13\%). Mit zunehmenden Druck in zunehmender Tiefe durchlaufen die Minerale dieses Gemisches verschiedene strukturelle Phasenübergänge, die Änderungen in der Kristallstruktur des Minerals darstellen und mit Änderungen der Dichte und anderen physikalischen Eigenschaften einhergehen. In der Erde werden die Tiefen dieser Phasenübergänge des Hauptminerals, Olivin, mit den starken seismischen Diskontinuitäten identifiziert. In einer Tiefe von $410 \mathrm{~km}$ (ca. 14.5 GPa) wandelt sich $\alpha$-Olivin in eine $\beta$-Phase ( $\beta$-Wadsleyit) um, die ihrerseits in einer Tiefe von etwa $500 \mathrm{~km}$ (ca. 17.7 GPa) in $\gamma$-Spinell übergeht. Diese beiden Phasenübergänge sind exotherm und zeichnen sich im Phasendiagramm durch eine positive Steigung $\gamma$ der Phasengrenzkurve (Clausius-Clapeyron-Kurve) aus. Der Übergang zum unteren Mantel in einer Tiefe von $660 \mathrm{~km}$ (ca. 23.3 GPa) wird durch die Phasengrenze vom $\gamma$-Spinell zu einer Mischung aus Perowskit und Magnesiowüstit markiert. Diese Phasenumwandlung ist endotherm und durch eine negative Clausius-Clapeyron-Steigung gekennzeichnet. Die Phasenübergänge der Pyroxene bzw. des Garnets sind gradueller und erzeugen weniger scharfe Diskontinuitäten (Ita und Stixrude 1992, Christensen 1995).

Die Mineralstruktur und die möglichen Phasenübergänge im Marsmantel haben Bertka

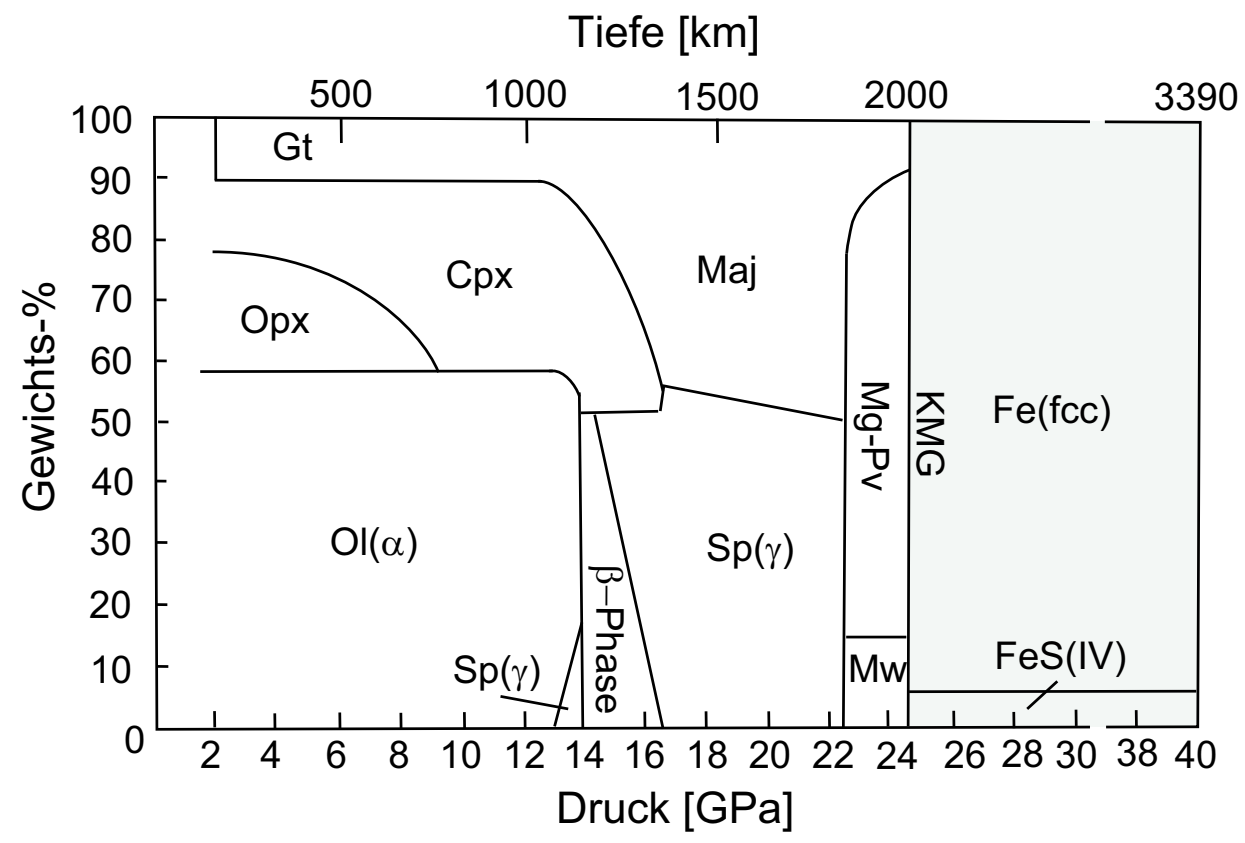

Abbildung 2.2: Zusammensetzung des Marsmantels als Funktion des Drucks (Bertka und Fei 1997). Die Abkürzungen stehen für: Ol: Olivin, Opx: Orthopyroxen, Cpx: Klinopyroxen, Gt: Garnet, Maj: Majorit, Sp: Spinell, Mw: Magnesiowüstit, Mg-Pv: Mg-FePerowskit

und Fei (1997) mit Hilfe von Hochdruckexperimenten im Druck- und Temperaturbereich, 
wie er für Mars angenommen wird, untersucht. Das Temperaturprofil im Marsmantel ist dabei so hoch gewählt, dass der Marskern flüssig ist. Die angenommene chemische $\mathrm{Zu}$ sammensetzung des Mantels entspricht etwa den Daten, die Dreibus und Wänke (1985) aus der Analyse der Marsmeteoriten gewonnen haben und berücksichtigt insbesondere den höheren Eisengehalt des Marsmantels. Abbildung (2.2) zeigt die Zusammensetzung des Marsmantels als Funktion des Drucks. Der obere Marsmantel besteht bei niedrigen Drücken ähnlich wie der Erdmantel aus Olivin (58 \%), Orthopyroxen (20\%), Klinopyroxen (12\%) und Garnet (10\%). Übersteigt der Druck 9 GPa (ca. 750 km), fehlt Orthopyroxen zu Gunsten eines höheren Anteils von Klinopyroxen. Bei $13.5 \mathrm{GPa}$ (ca. $1125 \mathrm{~km}$ ) zeigt ein geringer Anteil von $\gamma$-Spinell den Beginn der Übergangszone an, in der sich bei 14.0 GPa (ca. $1170 \mathrm{~km}$ ) $\alpha$-Olivin und das vorhandene $\gamma$-Spinell in die $\beta$-Phase umwandeln. Das Auftreten von $\gamma$-Spinell vor der Umwandlung in die $\beta$-Phase wird bei der Mineralzusammensetzung des Erdmantels nicht beobachtet. In der Erde wandelt sich $\alpha$-Olivin in einem engen Druckintervall von weniger als 0.5 GPa direkt in die $\beta$-Phase um. Dieser Unterschied ist auf den höheren Eisengehalt im Marsmantel zurückzuführen (Bertka und Fei 1997). Die $\beta$-Phase ist im Mars nur etwa über einen Druckbereich von $1.5 \mathrm{GPa}$ stabil (in der Erde etwa $4 \mathrm{GPa}$ ). Ab $15 \mathrm{GPa}$ (ca. $1250 \mathrm{~km}$ ) wandelt sich ein Teil der $\beta$-Phase in $\gamma$-Spinell um. Bis $17 \mathrm{GPa}$ (ca. $1415 \mathrm{~km}$ ) wächst der Anteil des $\gamma$-Spinells, während der Beitrag der $\beta$-Phase abnimmt. Klinopyroxen existiert im Druckbereich oberhalb von $17 \mathrm{GPa}$ nicht mehr und ist in Majorit umgewandelt. Die genaue Zusammensetzung der verschiedenen Phasen des Olivins in der Übergangsregion hängt stark vom Eisengehalt ab. Der höhere Eisenanteil im Marsmantel verschiebt die Übergangszone um etwa 0.5 $1 \mathrm{GPa} z u$ niedrigeren Drücken. Der Übergang zum unteren Mantel mit dem Phasenübergang zu einer Mischung aus Perowskit (75\%), Magnesiowüstit (15\%) und einem kleinen Anteil von Majorit (10 \%) liegt zwischen 22.8 und 23.5 GPa (ca. 1900 - 1960 km). Fei et al. (1997) weisen jedoch darauf hin, dass diese Angaben stark von der angenommenen Temperaturverteilung im Mars abhängig sind. Das Perowskit im unteren Mantel besteht im Wesentlichen aus Mg-Fe-Perowskit, Ca-Perowskit fehlt im Gegensatz zur Erde bis zu einem Druck von 23.5 GPa, was einerseits auf eine geringeren Calciumgehalt des Marsmantels im Vergleich zur Erde zurückzuführen ist und anderseits wird der Übergang durch den höheren Eisengehalt in einen höheren Druckbereich verschoben (Fei et al. 1997). Für den unteren Mantel finden Bertka und Fei (1996) neben Perowskit, Magnesiowüstit und Majorit auch Stishovit, das in der neueren Arbeit nicht mehr nachgewiesen wird. Dieser Unterschied kann auf etwas unterschiedliche Temperaturbedingungen der Experimente zurückgeführt werden, so dass bei Temperaturen unterhalb von 1973 K Stishovit im unteren Mantel eines Fe-reicheren Mantels eventuell stabil ist, wohingegen es in der Erde fehlt.

In Hochdruckexperimenten mit der Mineralzusammensetzung des Erdmantels beobachtet Hirose (2002) einen Phasenübergang zwischen Majorit und Al-haltigen Mg-reichen Perowskit. Dieser Phasenübergang ist exotherm $(\gamma=+1.3 \mathrm{MPa} / \mathrm{K})$ und findet bei ähnlichen Drücken wie der endotherme Übergang von $\gamma$-Spinell zu Perowskit und Magnesiowüstit statt. Die Phasengrenzkurven schneiden sich zwischen 1973 und 2073 K. Bei Temperaturen oberhalb von 2073 K erfolgt der Phasenübergang zwischen Majorit und Perowskit bei höheren Drücken als die Postspinelltransformation. Der Phasenübergang könnte insbesondere in warmen Aufströmen eine Rolle spielen, in denen dann der Übergang vom Perowskit zum Majorit mit einer positiven Clausius-Clapeyron-Steigung auf- 
tritt. Bertka und Fei (1997) finden allerdings für den unteren Marsmantel kein Al-haltiges Perowskit, so dass nicht klar ist, wie dieser Phasenübergang durch den höheren Eisengehalt im Mars beeinflusst wird. Zudem sind die Temperaturen im unteren Mantel im Mars, die z.B. Bertka und Fei (1997) annehmen, an der unteren Grenze des Bereichs, in dem dieser exotherme Phasenübergang bedeutsam ist, so dass er hier vernachlässigt wird.

Die Unterschiede in den Phasenübergängen zusammen mit dem geringeren Druckgradienten im Mars aufgrund der geringeren Größe des Planeten führen dazu, dass die Phasenübergänge im Mars in größerer Tiefe stattfinden, die Übergangszone verbreitert wird und Diskontinuitäten weniger scharf sind. Die Existenz der endothermen Phasengrenze hängt stark von der Kerngröße und den Druckbedingungen an der KMG ab.

\subsubsection{Parameter der Phasengrenzkurven}

Der Einfluss, den die einzelnen Phasenübergänge auf die Konvektionsströmung haben, hängt einerseits von der Steigung $\gamma=d p / d T$ der Phasengrenzkurve (Clausius-ClapeyronKurve) und anderseits vom Dichtekontrast $\Delta \rho$ durch die Phasenänderung ab. Der prinzipielle Einfluss der Phasengrenzen auf die Manteldynamik wird in Abschnitt (2.4) beschrieben und die mathematische Umsetzung in Kapitel (3.3) erläutert.

Die Steigung der Phasengrenzkurve kann durch Hochdruckexperimente z.B. in einer Diamantstempelpresse direkt gemessen werden, indem Druck- und Temperaturbedingung des Übergangs bestimmt werden. Eine andere Möglichkeit besteht in der Messung von thermodynamischen Größen des Übergangs wie z.B. der Enthalpie, aus denen die Steigung berechnet werden kann. Katsura und Ito (1989) haben experimentell die Steigung der Phasengrenzkurve zwischen $\alpha$-Spinell und der $\beta$-Phase untersucht und finden einen Wert von $\gamma_{\alpha \beta}=+2.5 \pm 1 \mathrm{MPa} / \mathrm{K}$. Diesen Wert bestätigen die Rechnungen von Bina und Helffrich (1994), die aus thermodynamischen Größen (Akaogi et al. 1989) mit Hilfe einer verbesserter Zustandsgleichung $\gamma_{\alpha \beta}=+2.8 \pm 0.1 \mathrm{MPa} / \mathrm{K}$ erhalten. Auch modernere Messverfahren in einer lasergeheizten Diamantstempelpresse (Boehler und Chopelas 1991) unterstützen die Daten von Katsura und Ito (1989). Für den Übergang zwischen der $\beta$-Phase und $\gamma$-Spinell liefern die Experimente eine Steigung $\gamma_{\beta \gamma}=+5.0 \pm 1 \mathrm{MPa} / \mathrm{K}$ (Katsura und Ito 1989). Die Phasengrenzkurve des endothermen Übergangs zwischen $\gamma$-Spinell und Perowskit und Magnesiowüstit ist von Ito und Takahashi (1989) experimentell analysiert worden. Sie bestimmen eine Steigung von $\gamma_{\gamma p v}=-2.8 \mathrm{MPa} / \mathrm{K}$. Dieser Wert liegt im Bereich der von Akaogi und Ito (1993) aus Enthalpiemessungen für diesen Übergang gewonnenen Daten von $\gamma_{\gamma p v}=-3 \pm 1 \mathrm{MPa} / \mathrm{K}$. Die Rechnungen von Bina und Helffrich (1994) liefern Werte zwischen $\gamma_{\gamma p v}=-1.9 \mathrm{MPa} / \mathrm{K}$ und $\gamma_{\gamma p v}=-2.7 \mathrm{MPa} / \mathrm{K}$ und thermodynamische Daten aus Raman-Spektroskopischen Messungen führen zu $\gamma_{\gamma p v}=-2.5 \mathrm{MPa} / \mathrm{K}$ (Chopelas et al. 1994). Dieser Wert wird auch durch Experimente in der Diamantstempelpresse bestätigt.

In den numerischen Simulationen werden für die Steigungen der einzelnen Phasengrenzkurven in Übereinstimmung mit den Messungen folgenden Werte angenommen: $\gamma_{\alpha \beta}=$ $+2.5 \mathrm{MPa} / \mathrm{K}, \gamma_{\beta \gamma}=+5.0 \mathrm{MPa} / \mathrm{K}$ und $\gamma_{\gamma p v}=-2.5 \mathrm{MPa} / \mathrm{K}$.

Die Dichtezunahme an den einzelnen Phasengrenzen lässt sich einem Dichteprofil für den Marsmantel (Bertka und Fei 1998) entnehmen, das mit Hilfe der Daten der Hochdruckexperimente (Bertka und Fei 1997) gewonnen worden ist: $(\Delta \rho)_{\alpha \beta}=252 \mathrm{~kg} / \mathrm{m}^{3}$, 
$(\Delta \rho)_{\beta \gamma}=150 \mathrm{~kg} / \mathrm{m}^{3}$ und $(\Delta \rho)_{\gamma p v}=400 \mathrm{~kg} / \mathrm{m}^{3}$. Diese Werte werden auch von Breuer et al. (1998) für die Konvektionsmodelle angenommen. In den numerischen Simulationen in dieser Arbeit werden ebenfalls diese Dichtekontraste verwendet. Lediglich beim Übergang zum Perowskit wird mit $(\Delta \rho)_{\gamma p v}=252 \mathrm{~kg} / \mathrm{m}^{3}$ ein deutlich kleinerer Wert verwendet, um eine bessere Vergleichbarkeit mit früheren Arbeiten (Harder und Christensen 1996, Harder 2000) zu ermöglichen. Das Produkt $(\gamma \Delta \rho)$ für diesen Übergang stimmt mit früheren Arbeiten überein.

Neben der Steigung der Phasengrenzkurve lässt sich in den Hochdruckexperimenten auch die genaue Lage der Phasengrenzkurven bestimmen, so dass für jede Temperatur $T$ der entsprechende Phasenumwandlungsdruck $p_{\operatorname{Tr}}$ bekannt ist. In den Modellen im Rahmen dieser Arbeit werden die folgenden Gleichungen zur Beschreibung der Phasengrenzkurve angenommen. Diese Kurven stimmen weitgehend mit denen überein, die Sohl und Spohn (1997) bei der Berechnung ihrer Strukturmodelle des Marsinneren verwenden.

Für die Phasengrenzkurve [GPa, K] zwischen $\gamma$-Spinell und Perowskit gilt in Übereinstimmung mit den Daten von Chopelas et al. (1994):

$$
p_{\operatorname{Tr}}(T)=22.9-2.5 \times 10^{-3}(T-2260)
$$

Der Übergang zwischen der $\beta$-Phase und $\gamma$-Spinell wird durch die folgende Beziehung [GPa] beschrieben (Sohl und Spohn 1997):

$$
p_{\operatorname{Tr}}(T)=22.9+5.0 \times 10^{-3}(T-2260)-16.7(1-\mathrm{Mg} \#)
$$

Dabei ist $\mathrm{Mg} \#=\mathrm{Mg}^{2+} /\left(\mathrm{Mg}^{2+}+\mathrm{Fe}^{2+}\right)=0.75$ die Magnesiumzahl, die im Mars durch den höheren Eisenanteil kleiner als in der Erde $(\mathrm{Mg} \#=0.90)$ ist. Dieser Term berücksichtigt den Einfluss des höheren Eisenanteils im Marsmantel auf die Lage der Phasengrenze.

Die Beschreibung der Phasengrenzkurve zwischen $\alpha$-Spinell und der $\beta$-Phase wird gegenüber Sohl und Spohn (1997), die ein Polynom dritter Ordnung verwenden, vereinfacht und wie die anderen Kurven als lineare Funktion beschrieben. Mit den Daten von Katsura und Ito (1989) und der bei Sohl und Spohn (1997) vorgeschlagenen Korrektur für den höheren Eisenanteil im Mars ergibt sich:

$$
p_{\operatorname{Tr}}(T)=10.32+2.5 \times 10^{-3} T-5.4(1-\mathrm{Mg} \#)
$$

\subsubsection{Modelle der inneren Struktur des Mars}

Die genaue Tiefe der Phasenübergänge sowie die Existenz des Phasenübergangs zum Perowskit hängt entscheidend von der Kerngröße und der damit verbundenen Druckverteilung in der Nähe der KMG ab. Harder (1998) hat Dichteprofile für Mars berechnet und versucht die Kerngröße und damit die Druckbedingungen in der Nähe der KMG für einen vorgegebenen Schwefelgehalt des Kerns und das bekannte Trägheitsmoment des Planeten einzuschränken. Die einzelnen Eingabeparameter des Dichtemodells werden dabei mit Hilfe eines Monte-Carlo-Verfahrens zufällig in einem großen Bereich variiert. Auch die Parameter eines flüssigen Kerns werden berücksichtigt. Der hydrostatische Druck im Marsmantel in den Modellen ist weitgehend unabhängig von den verschiedenen Annahmen. Die Erhöhung der Dichte in einer bestimmten Tiefe ist mit einer Abnahme der Schwerebeschleunigung in dieser Tiefe verbunden, da weniger Masse unterhalb 
dieses Radius zur Verfügung steht. Dies führt zu einem nahezu konstanten Druckgradienten und nur kleinen Änderungen im Druck. In den Modellen, die das Trägheitsmoment richtig wiedergeben, findet Harder (1998) bei einem Schwefelgehalt des Kerns zwischen 10 und $20 \%$ Kernradien zwischen 1280 und $1560 \mathrm{~km}$. Für die Umwandlung von $\gamma$ Spinell zu Perowskit nimmt er einen Druck von $23 \mathrm{GPa}$ an und bestimmt den Radius zu $r_{\gamma p v}=1480 \pm 90 \mathrm{~km}$. Die Übergangszone betrachtet er vereinfacht mit der Umwandlung von Olivin zu Spinell bei $13 \mathrm{GPa}$ und findet einen Radius von $r_{\alpha \beta}=2310 \pm 85 \mathrm{~km}$. Die Fehlergrenzen beziehen neben der Schwankungsbreite aus den Simulationen mit ein, dass seine Druckangaben auf Experimenten von Katsura und Ito (1989) bzw. Ito und Takahashi (1989) bei $1873 \mathrm{~K}$ beruhen und einerseits die Temperatur im Marsmantel eventuell davon abweicht und anderseits diese Experimente den höheren Eisenanteil im Marsmantel und die daraus folgenden Effekte nicht berücksichtigen. Der Vergleich der Kernradien mit den Radien der endothermen Phasengrenze zeigt, dass der Phasenübergang zum Perowskit im Mars in der Nähe der KMG möglich ist. Die obere Grenze der Dicke der Perowskitschicht liegt in den Modellen bei $300 \mathrm{~km}$ und der Mittelwert bei $100 \mathrm{~km}$.

Sohl und Spohn (1997) berechnen ausgehend von der Mineralzusammensetzung, die durch die Analyse der SNC-Meteoriten und kosmochemischen Argumenten nahegelegt wird, mit geeigneten Zustandsgleichungen und Annahmen über die Temperatur Dichteund Strukturmodelle für Mars. Sie stellen zwei Modelle für die innere Struktur des Mars vor, von denen das eine optimiert ist, das Trägheitsmoment zu erklären (Modell A), und das andere versucht, die geochemischen Daten aus den SNC Meteoriten, insbesondere das Verhältnis $\mathrm{Fe} / \mathrm{Si}=1.71$ (Dreibus und Wänke 1985) im Mars wiederzugeben (Modell B). In beiden Modellen ist der Kern zu groß bzw. reicht der Druck in der Nähe der KMG nicht aus, um die endotherme Phasengrenze zum Perowskit zu ermöglichen. Die Kernradien liegen bei 1468 km für Modell A und 1667 km für Modell B. Die Übergangszone wird vereinfacht, indem zwei univariante Phasengrenzen zwischen $\alpha$-Olivin und $\beta$-Spinell und zwischen $\beta$ - und $\gamma$-Spinell angenommen werden. Die Reduzierung der Übergangsdrücke durch den höheren Eisengehalt wird berücksichtigt. Die Phasengrenzen liegen in Modell A bei $r_{\alpha \beta}=2360 \mathrm{~km}$ und $r_{\beta \gamma}=2033 \mathrm{~km}$ und in Modell B bei $r_{\alpha \beta}=2332 \mathrm{~km}$ und $r_{\beta \gamma}=1974 \mathrm{~km}$. Der Kern ist in diesen Modellen flüssig. Sohl und Spohn (1997) merken an, dass mit den Dichtewerten für die Hochdruckphase von Eisensulfid (Fei et al. 1995) in ihrem Modell A ein kleinerer Kern und eine sehr dünne Perowskitschicht möglich sind. Die Problematik, nicht gleichzeitig die Anforderungen an die chemische Zusammensetzung und das Trägheitsmoment erfüllen zu können, zeigt sich ebenfalls in den Dichtemodellen von Bertka und Fei (1998). Ihre erste Modellreihe berücksichtigt die vorher experimentell bestimmte Mineralzusammensetzung (Bertka und Fei 1997) und den von Dreibus und Wänke (1987) vorgeschlagenen Schwefelgehalt von 14 \% im Kern, ist aber nicht an die chondritische Zusammensetzung des Planeten gebunden, die Dreibus und Wänke (1985) nahelegen. Die Modelle nehmen im Kern die Hochdruckphase von Eisensulfid an. Damit ergeben sich in Abhängigkeit von der angenommenen Krustendicke und Krustendichte Trägheitsmomente zwischen 0.367 und 0.357, die die Daten von Folkner et al. (1997) zumindest teilweise erfüllen. In diesen Modellen ist im Gegensatz zu Sohl und Spohn (1997) der Druck nahe der KMG ausreichend hoch, um eine Perowskitschicht zu erzeugen. Die Dicke der Perowskitschicht kann $100 \mathrm{~km}$ erreichen. Allerdings ist der Eisengehalt des Planeten im Vergleich zur chondritischen Zusammensetzung zu gering. Wird jedoch das chondritischen Elementvorkommen $(\mathrm{Fe} / \mathrm{Si}=1.71)$ erzwungen, müssen 
die Modelle eine unrealistisch dicke Kruste für den Mars annehmen und das Trägheitsmoment von 0.354 ist zu klein. Hier fehlt die Perowskitschicht.

Zharkov und Gudkova (2000) lösen das Problem des Eisen-Silizium-Verhältnisses, indem sie geringe Mengen Wasserstoff ( 0 - $50 \mathrm{~mol} \%$ ) im Marskern zulassen. In Analogie zur Erde, wo der Eisengehalt in der Kruste im Vergleich zum Mantel erhöht ist, reduzieren sie den Eisengehalt im Marsmantel gegenüber den Daten von Dreibus und Wänke (1985). Die durch den Wasserstoff niedrigere Dichte des Kerns sowie die geringere Manteldichte durch den reduzierten Eisenbeitrag machen einen großen Kern erforderlich und ermöglichen damit ein höheres Eisen-Silizium-Verhältnis. Mit diesen Annahmen finden sie für das Eisen-Silizium Verhältnis Werte zwischen $1.525 \leq \mathrm{Fe} / \mathrm{Si} \leq 1.695$, die relativ gut mit dem chondritischen Wert von 1.71 (Dreibus und Wänke 1985) übereinstimmen und auch das Trägheitsmoment erklären können. Allerdings sind die berechneten Kernradien zwischen 1564 und 1702 km zu groß, um eine Perowskitschicht zuzulassen. Gudkova und Zharkov (2004) erweitern diese Dichtemodelle, indem sie auch eine poröse, etwa $10 \mathrm{~km}$ dicke Regolithschicht in das Dichtemodell einbeziehen. Sie bestimmen die Lage der exothermen Phasengrenzen in Abhängigkeit des Eisengehalts des Mantels. Für $r_{\alpha \beta}$ ermitteln sie Werte zwischen 2260 und $2318 \mathrm{~km}$ und für $r_{\beta \gamma}$ zwischen 1961 und $2026 \mathrm{~km}$. Die Autoren bemerken, dass ohne Wasserstoff im Kern bei einem Schwefelgehalt von $14 \%$ eine bis zu $150 \mathrm{~km}$ dicke Perowskitschicht möglich ist.

Die Richtigkeit der Modelle kann mit den vorliegenden Daten nur unzureichend beurteilt werden. Eine genaue Vermessung des Kernradius könnte eine weitere wichtige Bedingung für die Dichtemodelle liefern.

In den numerischen Modellen wird ein Modell mit einem relativ kleinem Kern $r_{\mathrm{i}}=$

\begin{tabular}{|l|c|c|c|c|c|}
\hline & Harder (1998) & \multicolumn{2}{|c|}{ Sohl und Spohn (1997) } & Gudkova und & Konvektions- \\
& & Modell A & Modell B & Zharkov (2004) & modell \\
\hline$r_{i}[\mathrm{~km}]$ & $1280-1560$ & 1468 & 1667 & $\sim 1600$ & 1360 \\
\hline$r_{\alpha \beta}[\mathrm{km}]$ & - & 2360 & 2332 & $2260-2318$ & 2338 \\
\hline$r_{\beta \gamma}[\mathrm{km}]$ & $2310 \pm 85$ & 2033 & 1974 & $1961-2026$ & 2053 \\
\hline$r_{\gamma p v}[\mathrm{~km}]$ & $1480 \pm 90$ & - & - & - & 1497 \\
\hline $\mathrm{Fe} / \mathrm{Si}$ & - & 1.35 & 1.71 & 1.7 & - \\
\hline $\mathrm{C}$ & $0.3645-0.3679$ & 0.366 & 0.357 & $0.3642-0.3678$ & - \\
\hline $\mathrm{S}$ im Kern & $10 \%-20 \%$ & $14.2 \%$ & $14.2 \%$ & $\leq 20 \%$ & - \\
\hline
\end{tabular}

Tabelle 2.1: Zusammenstellung der Ergebnisse verschiedener Strukturmodelle. Zum Vergleich sind die Daten für die numerischen Konvektionsmodelle angegeben.

$1360 \mathrm{~km}$ bzw. einem Radienverhältnis von $r_{\mathrm{i}} / r_{\mathrm{a}}=0.4$ angenommen, so dass der Druck in der Nähe der KMG hoch genug ist, um die endotherme Phasenumwandlung zum Perowskit und eine $137 \mathrm{~km}$ dicke Perowskitschicht zuzulassen. Bei der Beschreibung der mineralischen Zusammensetzung und Übergänge wird nur das Hauptmineral Olivin betrachtet und die Übergangszone wird, soweit die einzelnen Modelle sie berücksichtigen, ähnlich wie bei Sohl und Spohn (1997) vereinfacht durch zwei univariante, exotherme Übergänge zwischen $\alpha$-Olivin - $\beta$-Spinell und $\beta$-Spinell - $\gamma$-Spinell simuliert, die bei einem Radius von $r_{\alpha \beta}=2338 \mathrm{~km}$ bzw. $r_{\beta \gamma}=2053 \mathrm{~km}$ liegen. Diese Werte weichen nur leicht von den 


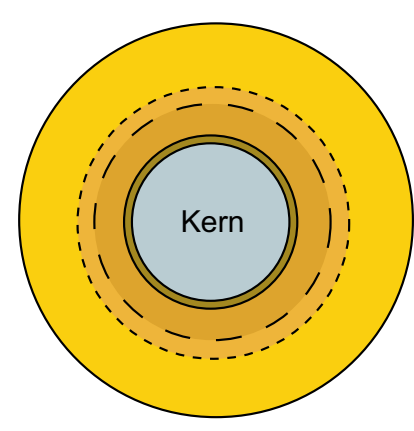

a)

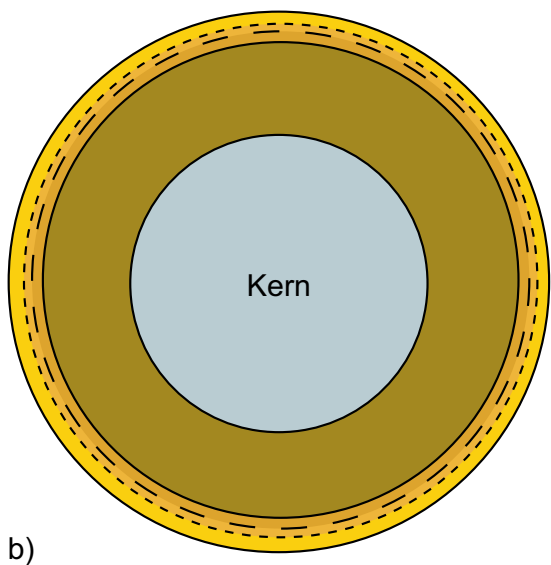

b)

Abbildung 2.3: Lage der Phasengrenzen im verwendeten Modell für den Mars (a) und in der Erde (b). Die Abbildung gibt das Größenverhältnis der beiden Planeten nicht maßstäblich wieder.

Werten $\left(r_{\alpha \beta}=2380 \mathrm{~km}, r_{\beta \gamma}=2040 \mathrm{~km} r_{\gamma \mathrm{pv}}=1530 \mathrm{~km}\right) \mathrm{ab}$, die Breuer et al. (1998) für ihre Konvektionsmodelle verwenden.

\subsection{Einfluss von Phasengrenzen auf die Manteldynamik}

Die Modelle für die innere Struktur des Mars zeigen, dass die Phasengrenzen durch den geringen Druckgradienten in größerer Tiefe als in der Erde liegen und die Übergangszone deutlich breiter als in der Erde ist. Besonders bedeutsam für die Konvektionsmodelle ist die Phasengrenze zum Perowskit, die im Mars nur im Falle eines relativ kleinen Kerns auftritt und nur eine dünne Perowskitschicht zulässt. Wegen der unmittelbaren Nähe zur KMG und insbesondere zur thermischen Grenzschicht ist aber der Einfluss nicht vernachlässigbar.

Phasenübergänge treten im Wesentlichen durch zwei Prozesse mit der Konvektion in Wechselwirkung: Einerseits wird in warmen Aufströmen bzw. kalten Abströmen die Tiefe der Phasenumwandlung verändert und die Phasengrenze gewölbt und andererseits werden durch das Freiwerden oder die Aufnahme von latenter Wärme die Temperaturverhältnisse in der Nähe der Phasengrenze beeinflusst. Beide Effekte wirken sich auf die Auftriebskräfte aus, wobei dem ersten die größere Bedeutung zukommt (Christensen 1995).

Abbildung (2.4) veranschaulicht die Wechselwirkung zwischen einer endothermen Phasengrenze und einem warmen Aufstrom (Plume), in dem die Temperatur höher als im umgebenden Material ist. Die negative Clausius-Clayperon-Steigung $(\gamma=d p / d T)$ der endothermen Phasenumwandlung führt dazu, dass im Bereich der höheren Temperatur der erforderliche Umwandlungsdruck niedriger ist und der Übergang somit in geringerer Tiefe stattfindet, so dass sich die Phasengrenze aufwölbt. Dadurch steigt im Plume die dichtere Phase (Perowskit) in einen Bereich, in dem sie von der weniger dichten Phase ( $\gamma$-Spinell) umgeben ist, so dass die Auftriebskräfte verringert werden und die Aufwärtsbewegung gehemmt wird. Analog findet die Umwandlung in einem kalten Abstrom bei 


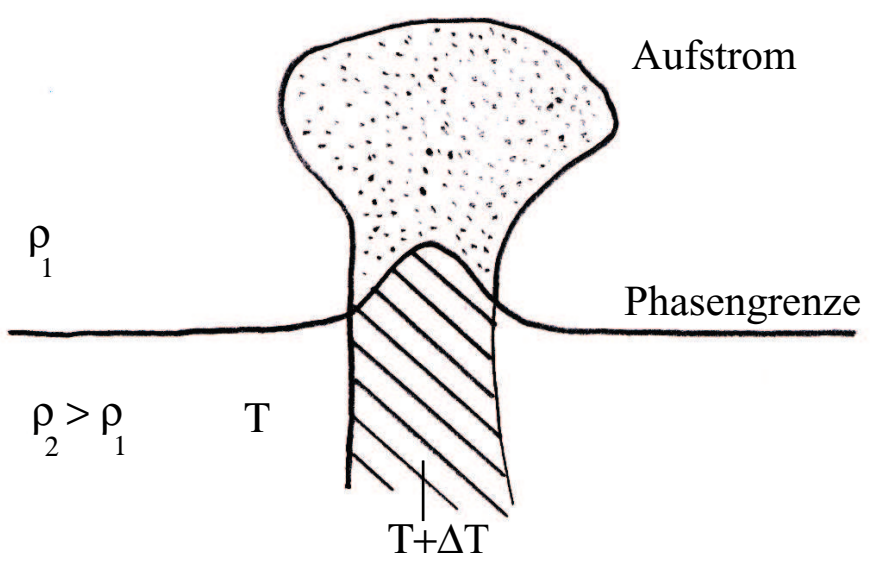

Abbildung 2.4: Aufwölbung einer endothermen Phasengrenze innerhalb eines warmen Aufstromes

einer endothermen Phasengrenze in größerer Tiefe statt, so dass die leichtere Phase tiefer in den dichteren Bereich eintaucht und durch die vergrößerten Auftriebskräfte in ihrer Abwärtsbewegung gebremst wird. Eine endotherme Phasengrenze behindert somit die Konvektionsbewegung. Im Fall der Erde wird dieser Effekt z.B. im Zusammenhang mit der Frage, ob abtauchende, kalte Lithosphärenplatten in den unteren Mantel eindringen können, diskutiert.

Die Situation im Fall einer exothermen Phasengrenze mit $\gamma=d p / d T>0$ ist genau umgekehrt. Hier wölbt sich die Phasengrenze in den Temperaturanomalien gerade so, dass die zusätzlichen Auftriebseffekte deren Bewegungsrichtung unterstützen.

Im Mars liegt die endotherme Phasengrenze in der Nähe der thermischen Grenzschicht, in der Aufströme bzw. Plumes aus thermischen Instabilitäten entstehen. Die endotherme Phasengrenze in der Nähe der KMG behindert in einem sehr frühen Stadium die Aufwärtsbewegung der Instabilitäten und der zunächst noch vielen auch schwächeren Plumes. Dies führt dazu, dass viele der schwächeren Aufströme die Phasengrenze nicht durchdringen können, Instabilitäten unterdrückt werden und ein Konvektionsmuster mit wenigen starken Aufströmen entstehen kann. Dieser Einfluss einer endothermen Phasengrenze in der Nähe der kernnahen thermischen Grenzschicht ist eine mögliche Erklärung für die starke Reduktion der Anzahl der Aufströme, die den konzentrierten Vulkanismus auf dem Mars erklären kann. Harder und Christensen (1996) haben in numerischen Modellen der Mantelkonvektion im Mars zeigen können, dass die Berücksichtigung der kernnahen endothermen Phasengrenze zu einem Konvektionsmuster mit nur einem Aufstrom führt.

Neben dem Aufwölben der Phasengrenze ist die latente Wärme des Phasenübergangs eine zweite Möglichkeit der Wechselwirkung zwischen Phasengrenze und Manteldynamik. Die latente Wärme vergrößert oder verringert die Temperaturanomalie von Auf- und Abströmen. Damit hat sie einen direkten Einfluss auf die thermischen Auftriebskräfte und verändert gleichzeitig auch die Wölbung der Phasengrenze. Durchdringt ein kalter Abstrom eine exotherme Phasengrenze, so wird durch die Kristallumstrukturierung latente 
Wärme frei und erwärmt den Abstrom. Dadurch sinkt der Temperatur- und Dichtekontrast zum Umgebungsmaterial, so dass die negativen Auftriebskräfte reduziert werden und die Abwärtsbewegung verlangsamt wird. Zusätzlich wird in dem nun wärmeren Abstrom die Phasengrenze weniger deformiert, so dass der beschleunigende Effekt der Deformation der Phasengrenze verringert wird. Hier führt die latente Wärme zu einem Abbremsen der Bewegung. Stößt ein Plume durch eine exotherme Phasengrenze, so muss latente Wärme aufgenommen werden und der Plume kühlt etwas ab. Analog zum kalten Abstrom wird dadurch der thermisch bedingte Dichteunterschied zum Umgebungsmaterial geringer und die Deformation der Phasengrenze reduziert, so dass die Aufwärtsbewegung gehemmt wird.

Im Fall der endothermen Phasengrenze führt die latente Wärme zu konkurrierenden Effekten. Ein kalter Abstrom wird beim Durchgang durch die endotherme Phasengrenze durch die aufzubringende latente Wärme abgekühlt, so dass der Dichtekontrast verstärkt wird und das Absinken beschleunigt wird. Diesem Effekt wirkt aber der in kälteren Abstrom verstärkte bremsende Beitrag der Deformation der Phasengrenze entgegen. Die gleiche Konkurrenzsituation entsteht in einem warmen Aufstrom, der sich durch die freiwerdende latente Wärme beim Durchgang durch die endotherme Phasengrenze aufheizt, dadurch einerseits zusätzlichen Auftrieb gewinnt anderseits aber stärker als zuvor durch die Aufwölbung der Phasengrenze behindert wird.

In allen Fällen schwächt sich der Einfluss der latenten Wärme ab, wenn in einer realistischen Betrachtung berücksichtigt wird, dass neben dem Material in den konzentrierten Auf- bzw. Abströmen immer Umgebungsmaterial mit auf- bzw. absteigt und seinerseits latente Wärme aufnimmt oder abgibt. Der Temperaturunterschied durch latente Wärme zwischen Auf- bzw. Abstrom und dem umgebenden Material reduziert sich auf den Anteil, der durch die Temperaturabhängigkeit der latenten Wärme entsteht.

In diesen Betrachtungen sowie in der numerischen Simulation werden die Phasengrenzen als univariante Übergänge angenommen, bei denen die Umwandlung in Abhängigkeit von der Temperatur bei einem exakt definierten Wert für den Druck erfolgt. Divariante Übergänge mit Regionen, in denen beide Phasen koexistieren, werden nicht betrachtet. Außerdem wird vorrausgesetzt, dass die Übergänge im thermodynamischen Gleichgewicht stattfinden. Die Umwandlung der Kristallstruktur bei Fest-Fest-Phasenübergängen geschieht nicht instantan, sondern in endlichen Raten, so dass insbesondere in kalten Abströmen, wo diese Vorgänge langsamer sind, eine Phase im Stabilitätsbereich der anderen Phase existiert und die Phasengrenzen verschoben werden. Diese Metastabilitäten werden nicht berücksichtigt.

Breuer et al. (1998) betrachten Konvektionsmodelle im Mars, in denen der Kern zu groß ist, eine Perowskitschicht zuzulassen. Sie berücksichtigen in ihren Modellen die Effekte von latenter Wärme und erzielen durch die beiden exothermen Phasengrenzen eine starke Reduzierung in der Anzahl der Aufströme. Harder (2000) zeigt allerdings, dass dieses großskalige Konvektionsmuster nicht alleine durch die exothermen Phasengrenzen hervorgerufen wird, sondern darüber hinaus die verwendete weichere Lithosphäre ein Konvektionsmuster mit wenigen Aufströmen wahrscheinlicher macht. 


\subsection{Magnetfeld und Dynamo}

Das Magnetfeld des Mars ist mit Hilfe der Magnetfeldexperimente (MAG/ER) der Mars Global Surveyor Mission vermessen worden. Die Messungen zeigen, dass Mars im Gegensatz zur Erde kein globales Magnetfeld hat, die oberere Grenze für das Dipolmoment liegt bei $\sim 2 \times 10^{18} \mathrm{Am}^{2}$ (Acuña et al. 1999). In einer Messhöhe von etwa $200 \mathrm{~km}$ bzw. bei der größten Annäherung von etwa $110 \mathrm{~km}$ konnte jedoch ein räumlich variierendes, kleinskaliges, relativ starkes Magnetfeld der Krustenmagnetisierung nachgewiesen werden. Die Magnetisierung des Gesteins ist dabei bis zu einer Größenordung stärker als typische Krustenmagnetisierungen auf der Erde (Connerney et al. 1999). Die großen Mengen an magnetisierter Kruste legen nahe, dass der Großteil der Magnetisierung entstanden ist, als das Material in einem zu der Zeit vorhandenen Magnetfeld unter die Curie-Temperatur abgekühlt ist und die vorhandene Magnetisierung thermisch remanent eingefroren hat. Mars hatte somit in seiner frühen Geschichte ein globales Magnetfeld, das wahrscheinlich durch einen Dynamoprozess im Eisenkern des Planeten erzeugt worden ist. Durch die besonders starke Magnetisierung ist es zudem möglich, dass später abgekühltes Material im Krustenfeld des Umgebungsmaterials magnetisiert worden ist. Magnetisierungen durch Einschläge wie sie z.B. für den Mond diskutiert werden, spielen auf dem Mars wegen der großen Menge des magnetisierten Materials und der Stärke der Magnetisierung eine untergeordnete Rolle (Stevenson 2001).

Der Großteil, insbesondere der stark magnetisierten Kruste liegt südlich der Dichotomie-

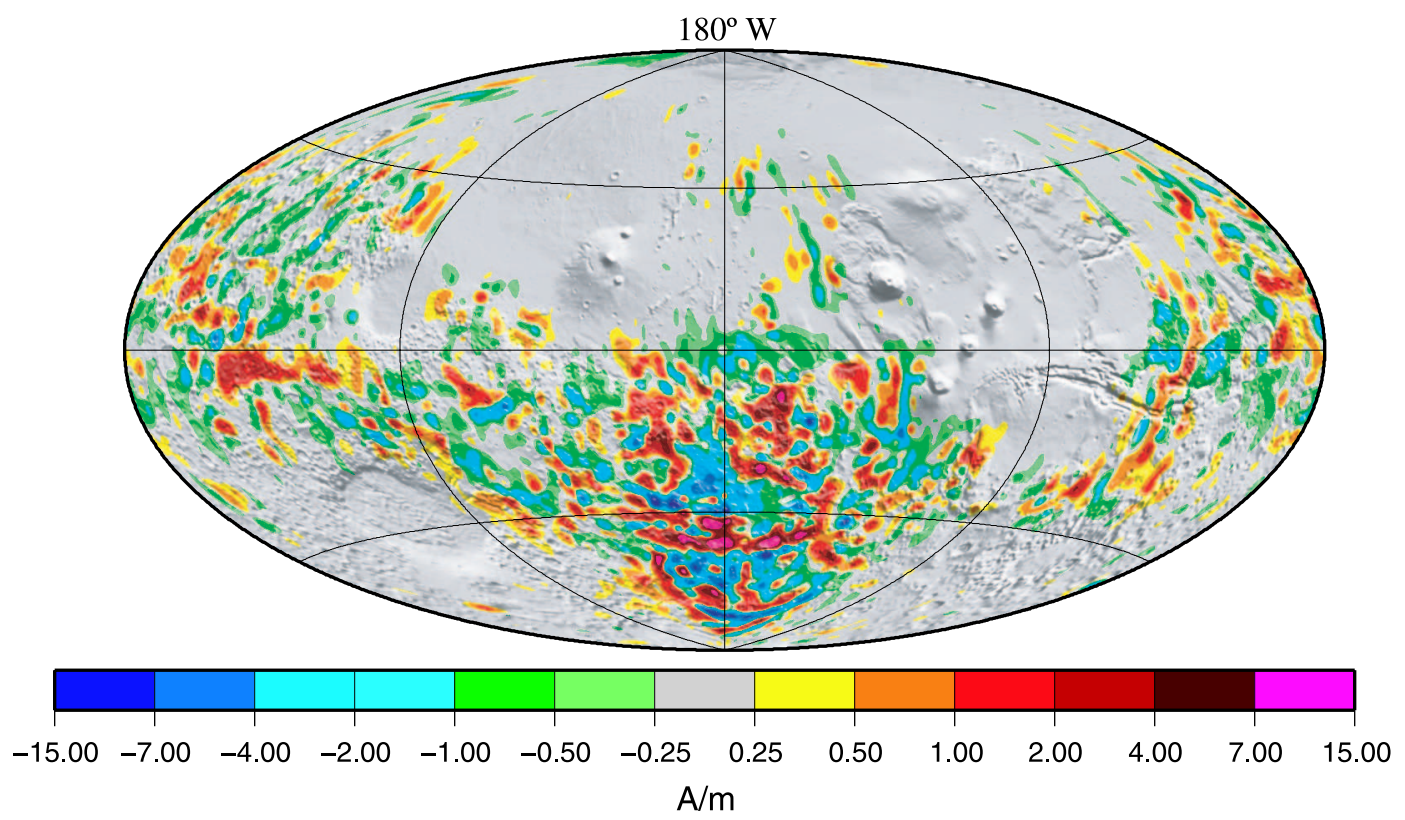

Abbildung 2.5: Radialkomponente der Magnetisierung der Marskruste: Modell von Whaler und Purucker (2005).

Grenze in den relativ alten südlichen Hochländern (Abbildung (2.5)). Die jüngere nördliche Kruste ist nahezu magnetisierungsfrei und die dort auftretende Magnetisierungen sind deutlich schwächer. In den Vulkanregionen Elysium und Tharsis sowie im Valles Marineris befindet sich kein magnetisiertes Gestein. Die Gesteine in den großen Einschlagkrater 
im Süden, insbesondere das Hellas Becken und das Agyre Becken, die vor $\sim 4.0 \mathrm{Ga}$ entstanden sind, sind magnetisierungsfrei. Außerhalb dieser Strukturen ist die Magnetisierung deutlich schwächer. Diese fehlende Magnetisierung in den großen Kratern wird vielfach genutzt, um die Lebensdauer eines möglichen Dynamos im Mars zu bestimmen (Acuña et al. 1999). Eventuell hatte Mars nur für eine sehr kurze Zeit ( $\sim 0.5 \mathrm{Ga}$ ) zu Beginn der Evolution einen Dynamo und damit ein globales Magnetfeld, das schon zur Zeit der Einschläge, die das Hellas und Agyre Becken formten, äußerst schwach oder gänzlich verschwunden war. In diesem Fall wird das vorher magnetisierte Gestein durch die Energie des Einschlags über die Curie-Temperatur erhitzt, verliert seine Magnetisierung und kühlt dann ohne globales Feld wieder ab, so dass keine ausgezeichnete Magnetisierung im Krater entstehen kann. Mohit und Arkani-Hamed (2004) untersuchen den Druckstoß und die Wärme, die beim Hellas Einschlag erzeugt worden ist, und berechnen, dass die Kruste $\sim 100$ Ma benötigt, um wieder unter die Curie-Temperatur abzukühlen. Die Entstehung der Dichotomie-Grenze bzw. die jungen Lavaflüsse im Norden fallen ebenso in die Zeit, als Mars kein globales Magnetfeld mehr hatte. Bei den in der nördlichen Hemisphäre auftreteden Krustenmagnetisierungen überdeckt eventuell eine dünne Schicht von jungem Krustenmaterial altes, magnetisiertes Gestein (Purucker et al. 2000).

Die Bestimmung der Lebensdauer eines Dynamos im Mars durch die fehlende Magnetisierung in den großen Einschlagkratern ist nicht unstrittig. Carpozen et al. (2005) beobachten im Vredefort Krater in Südafrika, der zu einer Zeit entstanden ist, als die Erde ein Magnetfeld hatte, deutlich schwächere Magnetfeldintensitäten. Die Magnetisierung des Gesteins ist zwar sehr stark, aber so zufällig angeordnet, dass bei Messungen in größerer Höhe, wie sie für die Marskrater vorgenommen worden sind, nur sehr geringe Intensitäten gemessen werden. Auch Schubert et al. (2000) argumentieren gegen die Datierung der Dynamoaktivität mit Hilfe der Krater, da sie erwarten, dass ein Einschlag in die magnetisierte Kruste um den Krater herum charakteristische magnetische Anomalien erzeugen sollte, die aber nicht beobachtet worden sind. In ihrer Argumentation ist die Kruste zur Zeit der Einschläge nicht magnetisiert. Der Dynamo wird zu einem späteren Zeitpunkt aktiv und lokale Aufheizprozesse ermöglichen die Magnetisierung der Kruste. Gegen ein spätes Einsetzen des Dynamoprozesses spricht allerdings die stabile remanente Magnetisierung des Marsmeteoriten ALH84001, die 3.9 bis 4.1 Ga alt ist und darauf hindeutet, dass Mars zu dieser Zeit ein Magnetfeld hatte (Weiss et al. 2002). Magnetfelddaten mit einer höheren räumlichen Auflösung können dazu beitragen, die Art der Magnetisierung und die zeitliche Abfolge besser festzulegen.

Beim Dynamoprozess wird mechanische Bewegungsenergie in magnetische Energie umgewandelt. Durch die elektromagnetische Induktion werden bei der Bewegung eines Leiters durch ein Magnetfeld Ströme induziert, die ihrerseits wieder ein Magnetfeld erzeugen. Verstärkt das induzierte Magnetfeld das ursprüngliche Feld, entsteht ein selbsterhaltender Dynamo. Im flüssigen, elektrisch leitenden Eisenkern der terrestrischen Planeten wird die Bewegungsenergie im Wesentlichen durch eine Konvektionsströmung aufgebracht, die thermisch oder chemisch angetrieben werden kann.

Damit in einer Flüssigkeitsschicht eine thermische Konvektionsströmung einsetzt ist u.a. ein ausreichend großer Temperaturgradient innerhalb der Schicht erforderlich. Dazu muss der Kern effizient genug abkühlen können bzw. der Mantel genug Wärme abführen. Als Kriterium der thermischen Konvektion im Kern muss der Wärmefluss aus dem Kern grö- 
Ber sein als der Wärmefluss durch Wärmeleitung entlang der Adiabiate:

$$
F_{\text {Kern }}>F_{\text {Kern,ad }}=\frac{\lambda \alpha T g}{C_{p}}
$$

darin ist $\lambda$ die thermische Leitfähigkeit des Eisens, $\alpha$ der thermische Ausdehnungskoeffizient im Kern, $T$ die Temperatur, $g$ die Schwerebeschleunigung und $C_{p}$ die spezifische Wärmekapazität im Kern bei konstantem Druck. Nimmo und Stevenson (2000) stellen die auftretenden Materialparameter zusammen: Die Schwerebeschleunigung an der KMG beträgt $g=3 \pm 0.3 \mathrm{~ms}^{-2}$ (Longhi et al. 1992). Die Wärmekapazität von Eisen hängt nicht stark vom Druck ab, so dass sich die Werte für den Erdkern von $C_{p}=820-860 \mathrm{~J} / \mathrm{kgK}$ (Anderson 1995) verwenden lassen. Der thermische Ausdehnungskoeffizient von festem Eisen bei $25 \mathrm{GPa}$ (Druck an der KMG im Mars) liegt bei $\alpha=2-3 \cdot 10^{-5} \mathrm{~K}^{-1}$ (Boehler et al. 1990). Urakawa et al. (2004) untersuchen die Zustandsgleichung für FeS(IV) und FeS(V), die als Hochdruckphasen von FeS auftreten und eventuell im Marskern eine Rolle spielen könnten. Für den thermischen Ausdehnungskoeffizient von FeS bei $25 \mathrm{GPa}$ finden sie $\alpha=3-4 \cdot 10^{-5} \mathrm{~K}^{-1}$. Für flüssiges Eisen im Erdkern gibt Anderson (1995) Werte von $\alpha=1.5-2 \cdot 10^{-5} \mathrm{~K}^{-1}$ an. Die größte Unsicherheit besteht bei der Bestimmung der thermischen Leitfähigkeit. Anderson (1998) bestimmt die thermische Leitfähigkeit aus Messungen der elektrischen Leitfähigkeit für Eisen - Siliziumverbindungen. Für Eisen mit $14.5 \% \mathrm{~S}$ bei Bedingungen an der KMG im Mars liefert seine Methode $\lambda=43-88 \mathrm{~W} / \mathrm{mK}$ (Nimmo und Stevenson 2000). (Für die Bedingungen an der KMG in der Erde ergibt sich $\lambda=28.6-60 \mathrm{~W} / \mathrm{mK}$ (Anderson 1998).) Zusammenfassend schätzen Nimmo und Stevenson (2000) für den maximalen Wärmefluss entlang der Adiabate im Kern einen relativ großen von Bereich von $F_{\text {Kern,ad }}=5-19 \mathrm{~mW} \mathrm{~m}^{-2}$ ab. Liegt der Wärmefluss aus dem Kern oberhalb dieses Bereiches, so ist eine thermisch getriebene Konvektionsbewegung im Kern möglich.

Eine andere Antriebsmöglichkeit der Konvektion besteht im chemischen Auftrieb. Kühlt der Kern ab, unterschreitet die Temperaturverteilung im Kern zu einem Zeitpunkt die Liquiduskurve der Metalllegierung, so dass ein fester innerer Kern ausfriert. Beim Ausfrieren, des inneren Kerns wird zusätzliche latente Wärme frei, die die thermische Konvektion unterstützen kann. Bedeutender ist jedoch, dass das Eisen im Marskern einige Prozent Schwefel enthält, der feste Eisenkern jedoch aus nahezu reinem Eisen besteht. Beim Ausfrieren des inneren Kerns reichert sich das leichte Element im verbleibenden flüssigen Bereich an. Dies hat einerseits Auswirkungen auf die Schmelztemperatur, die durch den höheren Schwefelanteil gesenkt wird, so dass das Anwachsen des Kerns etwas verlangsamt wird. Andererseits hat der Schwefel eine geringere Dichte als sein umgebendes Material, so dass er aufsteigen und eine chemische Konvektionsbewegung antreiben kann. Diese chemische Konvektionsbewegung ist insbesondere dann möglich, wenn der Wärmefluss an der KMG zu gering ist, um thermische Konvektion zu ermöglichen.

Mit diesen Mechanismen ergeben sich verschiedene Szenarien für die zeitliche Entwicklung des Dynamos im Mars und das aktuelle Fehlen eines globalen Magnetfeldes (Stevenson 2001). Im ersten Fall ist Mars zu Beginn sehr heiß und hat einen gänzlich geschmolzenen Kern. Das System kühlt zu Beginn stark ab, die Mantelkonvektion ist heftig und führt in dieser Zeit genug Wärme vom Kern ab, so dass der Temperaturgradient im Kern so groß ist, dass das Kernmaterial thermisch konvektiert und ein Magnetfeld erzeugt wird. Mit der Zeit nimmt die Kühlrate des Planeten ab und der Wärmefluss an der KMG 
sinkt unter den Wärmefluss entlang der Adiabate, so dass die Konvektion im Kern und mit ihr der Dynamoprozess stoppt. Ein innerer Kern existiert nach diesem Modell heute noch nicht. Allerdings ist es möglich, dass die Dynamoaktivität zu einem zukünftigen Zeitpunkt wieder einsetzt, wenn das System so weit abgekühlt ist, dass sich ein fester Kern bildet und chemische Konvektion einsetzt.

Im zweiten Modell wird angenommen, dass der Schwefelgehalt sehr gering ist, so dass sehr früh in der Entwicklung des Planeten ein innerer Kern gebildet wird, der sehr schnell wächst. Unter diesen Annahmen ist es möglich, zunächst einen Kern mit einem noch relativ kleinen festen Kern und einem ausreichend großen flüssigen Anteil zu haben, in dem chemische Konvektion (eventuell anfänglich von thermischer Konvektion unterstützt) einen Dynamoprozess erlaubt. Kühlt der Planet weiter ab, wächst der innere Kern stark an, so dass die verbleibende flüssige Schicht zu dünn wird, um in ihr ein Magnetfeld zu erzeugen. Nach diesem Modell ist der Marskern heute ganz oder weitgehend fest, so dass kein Dynamoprozess möglich ist.

Nimmo und Stevenson (2000) schlagen ein drittes Szenario vor, in dem eine frühe Phase der Plattentektonik in Betracht gezogen wird. In diesem Modell ist der Schwefelgehalt des Kern nur von geringer Bedeutung. Zunächst ist der Kern zumindest in einer ausreichend dicken Schicht flüssig, ein innerer Kern kann, muss aber nicht, vorhanden sein. In der anfänglichen Phase nimmt die Marsoberfläche an der Konvektionsbewegung des Mantels teil, so dass z.B. Lithosphärenteile in den Mantel abtauchen und zu einer effizienten Kühlung des Systems beitragen. Während dieser Phase kann der Kern ausreichend gekühlt werden, um thermische Konvektion im Inneren und damit einen Dynamo zu ermöglichen. Mit der Zeit -eventuell in einem sehr kurzen Zeitraum- ändert sich der Konvektionsstil, in eine Konvektionsbewegung, wie sie heute auf dem Mars vorliegt, an der die Marsoberfläche nicht mehr teil nimmt, sondern die Lithosphäre eine feste, stagnierende Schicht um den Planeten bildet. Ohne Bewegung der Oberfläche ist der Wärmetransport weit weniger effektiv, so dass es zu einer starken Verlangsamung des Abkühlens, eventuell durch die radioaktiven Elemente sogar zu einem Aufheizen des Systems kommt. Durch den weniger effizienten Wärmetransport, kann der nötige Temperaturgradient im Kern nicht mehr aufrecht gehalten werden, so dass die Konvektion und damit der Dynamo ausstirbt. Sollte sich das System aufheizen bzw. zumindest der Kern nicht weiter abkühlen, wächst auch der innere Kern nicht weiter, so dass auch chemische Konvektion nicht möglich ist. Einen Mechanismus, der zum schnellen Aussterben der Plattentektonik führen kann, geben Nimmo und Stevenson (2000) nicht an. Hinweise auf eine mögliche, frühe Phase der Plattentektonik finden sich in einem Streifenmuster in der Magnetisierung in den alten südlichen Hochländern (Connerney et al. 1999).

Da die innere Struktur des Mars und insbesondere des Kerns nicht bekannt ist, fehlen bislang Daten, die einige der Modelle klar ausschließen. Thermische Evolutionsmodelle für Mars können den Wärmefluss an der KMG simulieren und die Lebensdauer eines thermisch getriebenen Dynamos durch den Vergleich mit dem Wärmefluss entlang der Adiabaten bestimmen. Außerdem lässt sich die Temperaturentwicklung an der KMG modellieren. Wird diese Temperatur mit geeigneten Annahmen ins Innere des Kerns fortgesetzt, lässt sich aus dem Vergleich einer geeigneten Schmelzkurve der Eisenlegierung und des interpolierten Temperaturprofils die Existenz und Größe eines inneren, festen Kerns bestimmen. 


\subsection{Thermische Evolutionsmodelle und Zielsetzung}

Thermische Evolutionsmodelle versuchen, die thermische Entwicklung eines Planeten zu beschreiben und heutige Beobachtungsdaten zu erklären. Für die thermische Entwicklung eines Planeten ist die Mantelkonvektion von großer Bedeutung. Das eigentlich feste Silikatgestein eines Planetenmantels bewegt sich unter Druck und mechanischen Spannungen auf geologischen Zeitskalen und lässt sich als äußerst zähe Flüssigkeit verstehen. Interne Wärmequellen und der Temperaturkontrast zwischen Oberfläche und Kern können dieses Material in eine Konvektionsbewegung versetzen. Diese Konvektionsbewegung transportiert Wärme aus dem Inneren des Planeten nach außen. Die Heftigkeit der Konvektion wird weitgehend durch die Viskosität des Materials gesteuert. Während des Abkühlens des Planeten steigt die Viskosität an und die Konvektionsstärke nimmt ab. Die Viskosität spielt zudem in den oberflächennahen kalten Schichten eine bedeutende Rolle. Hier ist die Viskosität so hoch, dass keine Bewegung mehr möglich ist und sich eine stagnierende Schicht, eine kohärente Platte, ausbildet. In dieser Schicht, die hier als Lithosphäre verstanden wird, kann Wärme lediglich durch Diffusion bzw. Wärmeleitung transportiert werden. Dieser Mechanismus ist weniger effektiv als der Transport durch Konvektion. Das Ausbilden der Lithosphäre hat somit einen starken Einfluss auf die thermische Entwicklung des Planeten.

Leider erlaubt die Nichtlinearität der zugrunde liegenden Gleichungen nicht, von dem 'Jetzt-Zustand' in die Vergangenheit zu rechnen. Stattdessen muss eine geeignete Startsituation angenommen werden, von der die Modelle mit dem Ziel vorwärts gerechnet werden, möglichst viele Eigenschaften des heutigen Planeten wiedergeben. Die Startsituation beschreibt den Planeten kurz nach seiner Entstehung und für die vorliegenden Marsmodelle nach der Differentiation in den Silikatmantel und den Eisenkern. Das Starttemperaturprofil berücksichtigt Energie, die bei der Akkretion des Planeten durch den Zusammenstoß vieler kleinerer Planetesimale aufgenommen wird, sowie Energie, die bei der Differentiation eines zunächst homogenen Körpers in verschiedene Schichten mit unterschiedlicher Dichte entsteht. Für Mars deutet die Analyse von charakteristischen Isotopenverhältnissen darauf hin, dass der Akkretions- und Differentiationsprozess sehr schnell erfolgt ist (10 - $30 \mathrm{Ma}$ (Lee und Halliday 1997), 13 $\pm 2 \mathrm{Ma}$ (Kleine et al. 2002)). In der weiteren Entwicklung nimmt der Mantel Wärme vom Kern auf, der dadurch abkühlt, und transportiert sie zur Oberfläche. Der Zerfall der radioaktiven Elemente produziert zusätzliche Wärme im Mantel, die abgeführt werden muss. Diese Wärmeproduktionsrate nimmt im Lauf der Zeit ab.

Mit Hilfe von Evolutionsmodellen, die die Konvektionsströmung und ihre Entwicklung simulieren, kann z.B. das Konvektionsmuster im Marsmantel nach einer Evolution von 4.5 Ga untersucht werden. Im Strömungsmuster lässt sich erkennen, ob nach diesem Zeitraum noch warme Aufströme im Marsmantel existieren, die aktiven Vulkanismus an der Oberfläche hervorrufen. Es ist möglich, die Anzahl der Aufströme und die Entwicklung ihrer Positionen zu beobachten und mögliche Mechanismen für die starke Konzentration des Vulkanismus auf dem Mars zu untersuchen. In den Modellen kann z.B. der Einfluss der verschiedenen Phasengrenzen, insbesondere des kernnahen endothermen Übergangs getestet werden. Die Simulationen geben Aufschluss über die Temperaturentwicklung im Mantel, die z.B. auch für die Produktion von Schmelzen wichtig ist. Es ist möglich, die Dicke der Lithosphäre abzuschätzen und das Wachstum im Lauf der Zeit zu beschreiben. 
Der Wärmefluss aus dem Kern kann zur Bestimmung der Existenz und Lebensdauer eines thermisch getriebenen Dynamos herangezogen werden und die Temperaturentwicklung des Kern ermöglicht Aussagen über die Existenz und Größe eines festen inneren Kerns. Die numerischen Modelle bieten auch die Möglichkeit, den Einfluss verschiedener Parameter auf diese Daten und Entwicklungen zu testen.

Zielsetzung dieser Arbeit sind möglichst realitätsnahe Evolutionsmodelle der Konvektion im Marsmantel und der thermischen Entwicklung des Planeten, die die oben aufgezeigten Fragestellungen beantworten.

Die thermische Entwicklung des Planeten steht bei vielen bisherigen Konvektionsmodellen für Mars nicht im Vordergrund. Ein Ziel vieler Modelle ist die Erklärung bzw. die Suche nach einem Mechanismus für die starke Konzentration des Vulkanismus auf dem Mars. In diesem Zusammenhang wird der Einfluss einer kernnahen endothermen Phasengrenze auf das Konvektionsmuster untersucht. Weinstein (1995) benutzt dazu das Modell einer inkompressiblen, isoviskosen Flüssigkeit in Zylindergeometrie. Die Temperaturrandbedingungen sind konstant und auch die interne Heizrate zeitunabhängig. In diesen Simulationen stirbt das kleinskalige Konvektionsmuster aus, wenn die endotherme Phasengrenze nahe genug an der KMG liegt. Der Bereich unterhalb der Phasengrenze stagniert, so dass die Dicke der thermischen Grenzschicht anwächst. Es entsteht so ein Konvektionsmuster mit nur einem Plume. Erste dreidimensionale, sphärische Konvektionmodelle für Mars, die das besondere Konvektionsmuster mit wenigen bzw. hier nur einem Aufstrom erklären können, wurden von Harder und Christensen (1996) vorgestellt. Diese Modelle erreichen die starke Reduzierung der Plumeanzahl ebenso durch die endotherme Phasengrenze zwischen $\gamma$-Spinell und Perowskit und Magnesiowüstit in der Nähe der KMG und der thermischen Grenzschicht. Ähnlich wie die Modelle von Weinstein verwenden Harder und Christensen (1996) die Boussinesq-Approximation (vgl. Kapitel (3.2)) und vernachlässigen im Zusammenhang mit der Phasengrenze Temperatureffekte durch latente Wärme. Außerdem werden konstante Materialparameter, insbesondere eine konstante Viskosität über den gesamten Mantel angenommen. Die Temperaturrandbedingungen sind auch hier isotherm und zeitlich konstant. Dieses Modell wird in Harder (1998) genauer analysiert und auf seine Robustheit getestet, indem u.a. die Position der Phasengrenze und ihr Beitrag zu den Auftriebskräften variiert wird.

Breuer et al. (1998) veröffentlichen hingegen Modelle für die Mantelkonvektion im Mars, die die Nicht-Boussinesq-Effekte (Kapitel (3.2)) der latenten Wärme berücksichtigen und unterstreichen die Bedeutung der exothermen Phasengrenzen zwischen $\alpha$ - und $\beta$-Spinell sowie zwischen $\beta$ - und $\gamma$-Spinell. Die freiwerdende latente Wärme reduziert die Temperaturdifferenz zwischen Plume und umgebendem Gestein in der Tiefe der Phasengrenze und vermindert so den Auftrieb. In ihren Modellen gelingt allein durch die exothermen Phasengrenzen eine Reduktion der Anzahl der Aufströme, die aber weniger effektiv ist als im Fall mit der endothermen Phasengrenze. Im Gegensatz zu Harder wird das Mantelgestein als kompressible Flüssigkeit aufgefasst und eine tiefenabhängige (temperaturunabhängige) Viskosität verwendet. Anders als Harder und Weinstein, die eine feste mechanische Randbedingung annehmen, soll hier ein starker Viskositätsgradient in der oberflächennahen Schicht, die stagnierende Oberfläche des Mars beschreiben. Harder (2000) zeigt, dass allein die Änderung in der Randbedingung die Anzahl der Aufströme reduziert, so dass die Rolle der exothermen Phasengrenzen hierbei fraglich bleibt.

Andere Konvektionsmodelle für Mars untersuchen die inneren Vorgänge, die zur Dicho- 
tomie beitragen können. Zhong und Zuber (2001) finden z.B., dass bei einer geschichteten Viskosität mit einer rheologisch weichen Asthenosphäre ein Konvektionsmuster mit der Kugelflächenfunktionen von Grad $l=1$ bevorzugt wird. Diese spezielle Konvektionsmuster kann zu den Unterschieden zwischen der nördlichen und südlichen Hemisphäre beitragen. Die mineralischen Phasengrenzen werden in diesem Modell außer Betracht gelassen. Die thermische Evolution wird hier nur durch ein Abnehmen der internen Heizrate berücksichtigt, die Kerntemperatur ist zeitlich konstant. Wie weit die freien mechanischen Randbedindungen das Ergebnis beeinflussen, wird nicht analysiert.

In Zusammenhang mit der Dichotomie wird diskutiert, dass die verschiedenen Krustenregionen unterschiedlich stark mit radioaktiven Elementen angereichert sind. Dabei ist die Heizrate in der südlichen Hemisphäre erhöht und auf der Nordhalbkugel verringert. Wüllner und Harder (1998) untersuchen, welchen Einfluss diese oberflächennahe, lateral variierende Heizrate auf das Konvektionsmuster hat und finden eine Fokussierung der Aufströme unter den stärker angereichterten Regionen und der Abströme unter den an Wärmequellen verarmten Regionen. Für Mars würden sich daher Aufströme unter der alten, südlichen Hemisphäre konzentrieren und die Lithosphäre wäre dort dünner. Dies steht im Widerspruch zu den Beobachtungen und dem Vulkanismusvorkommen auf der Nordhalbkugel, so dass bessere Daten für die Krustenstruktur und Zusammensetzung erforderlich sind, um die Richtigkeit der Annahme zu überprüfen. Auch diese Modelle in einer 2D/3D-Kugelschale in der Boussinesq-Näherung vernachlässigen die Phasengrenzen und das säkulare Abkühlen des Planeten, berücksichtigen allerdings eine Lithosphäre mit konstanter Dicke durch einen starken Viskositätsanstieg nahe der Oberfläche.

Die thermische Evolution des Planeten wird in den bis hierhin vorgestellten Modellen nicht betrachtet. Die thermische Geschichte des Mars ist bisher weitgehend in parametrisierten Modellen untersucht worden. Diese parametrisierten Modell lösen nicht die strömungsmechanischen Gleichungen und können somit keine Auskunft über das Konvektionsmuster geben, sondern zeigen globale Trends der Evolution auf. Der Wärmetransport wird parametrisiert, indem geeignete Skalierungsgesetze die Abhängigkeit des Wärmeflusses von der Konvektionsstärke beschreiben. Stevenson et al. (1983) nutzen parametrisierte Modelle, um das Vorhandensein bzw. das Fehlen von Magnetfeldern für die einzelnen Planeten zu verstehen. In ihren Modellen für Mars unterschreitet der Wärmefluss aus dem Kern zwischen 1.0 und 1.2 Ga den Wert, der für die Konvektion im Kern und einen thermischen Dynamo notwendig ist. Bei einem Schwefelgehalt von 10 $\%$ friert ein innerer Kern aus, der nach 4.5 Ga einen Radius von 952 km hat und chemische Konvektion zulässt. Bei höheren Schwefelgehalten (25\%) kann kein innerer Kern gebildet werden. Schubert und Spohn (1990) analysieren mit einem gegenüber Stevenson et al. (1983) erweiterten Modell, das das Wachstum der Lithosphäre berücksichtigt, den Einfluss von Schwefel auf die thermische Entwicklung des Mars. Sie zeigen, dass die Manteltemperatur, der Wärmefluss an der Oberfläche und die Lithosphärendicke kaum vom Schwefelgehalt beeinflusst werden. Das Ausfrieren eines festen Kerns wird hingegen stark vom Schwefelgehalt und der Mantelviskosität und damit der Konvektionsstärke beeinflusst. Höhere Viskositäten erfordern einen geringeren Schwefelgehalt, um einen festen Kern bilden zu können. Schubert und Spohn (1990) erklären das heutige Fehlen eines Magnetfeldes auf dem Mars damit, dass der Marskern bei einem Schwefelgehalt von weniger als $16 \%$ komplett ausgefroren ist und keine Flüssigkeitsschicht für eine ausreichend starke Konvektion mehr hat. Liegt der Schwefelgehalt über $16 \%$, folgern Schubert und 
Spohn (1990), dass noch kein innerer Kern gebildet worden ist und der Wärmefluss aus dem Kern zu gering ist. Der Einfluss der Krustenbildung und der Konzentration der radioaktiven Elementen auf die thermische Entwicklung wird in Spohn (1991) untersucht. In diesen Modellen fallen der Wärmefluss und die Temperatur typischerweise zunächst monoton exponentiell ab und die Viskosität nimmt zu. Nach dieser Phase des starken Abkühlens kühlt der Planet deutlich langsamer ab. Die Modelle liefern Manteltemperaturen zwischen 1600 und $1800 \mathrm{~K}$, einen Wärmefluss an der Oberfläche zwischen 20 und 35 $\mathrm{mW} / \mathrm{m}^{2}$ und Abschätzungen für die rheologische Lithosphäre zwischen 100 und $400 \mathrm{~km}$ (Spohn et al. 1998).

Ein Problem vieler parametrisierten Modelle ist, dass sie den Effekt einer stark temperaturabhängigen Viskosität nicht ausreichend berücksichtigen. Bei solchen Rheologien bildet sich eine stagnierende Schicht unterhalb der kalten Oberfläche aus, die der konvektierenden Schicht überlagert ist. Durch die stagnierende Schicht sind u.a. der die Konvektion treibende Temperaturkontrast in der konvektierenden Schicht und die Viskositätsbedingungen verändert. Die Art der Beschreibung des Übergangs zwischen Lithosphäre und konvektierendem Mantel in den parametrisierten Modellen hat großen Einfluss auf die Ergebnisse (Grasset und Parmentier 1998, Spohn et al. 2001a). Spohn et al. (2001a) stellen die Ergebnisse verschiedener Modelle gegenüber.

Nimmo und Stevenson (2000) beziehen in ihre parametrisierten Modellen eine kurze Phase der Plattentektonik ein und untersuchen den Effekt auf die thermische Entwicklung und die Lebensdauer des Dynamos. Sie zeigen, dass die Plattentektonik neben dem Wärmefluss an der Oberfläche auch den Wärmefluss aus dem Kern erhöht und in dieser Zeit einen thermisch getriebenen Dynamo möglich macht. Die Modelle von Williams und Nimmo (2004) überprüfen den Einfluss der Kerntemperatur und der Existenz von radioaktiven Elementen im Kern auf die Lebenszeit des Dynamos. Sie kommen zu dem Ergebnis, dass ein um $150 \mathrm{~K}$ heißerer Kern (gegenüber dem Mantel) einen frühen Dynamo auch ohne Plattentektonik ermöglicht. Der Einfluss der radioaktiven Wärme im Kern erweist sich als gering.

Parametrisierte Modelle können neben den Schwierigkeiten bei stark temperaturabhängigen Rheologien die Effekte der Phasengrenzen, die in den Konvektionsmodellen deutlich geworden sind, insbesondere den Einfluss auf den Wärmefluss an der KMG und seine Zeitabhängigkeit (dem Abklingen überlagerte Variationen durch die Strömung) nicht beschreiben.

Dynamische Evolutionsmodelle, die das volle strömungsmechanische Problem betrachten und dabei die säkulare Abkühlung des Systems berücksichtigen, sind z.B. von Billich (1995) für den Mond berechnet worden. Conzelmann (1999) zeigt Ergebnisse achsensymetrischer, dynamischer Evolutionsmodelle für Merkur (vgl. Kapitel (6)). Für die Erde sind ebenfalls einige Simulationen zur thermischen Entwicklung durchgeführt worden, die das Abkühlen des Kerns und die Abnahme der internen Heizrate berücksichtigen. Zwei- und dreidimensionale kartesische Modelle mit einer temperaturabhängigen Viskosität, die auch die Hauptphasengrenzen in der Erde in Betracht ziehen, werden z.B. von Yuen et al. (1995) vorgestellt. Butler et al. (2005) führen achsensymmetrische Simulationen durch, die neben den Phasengrenzen und einer temperaturabhängigen Viskosität die Temperatureffekte beim Ausfrieren eines inneren Kerns einbeziehen. Konvektionsmodelle für Mars, die das Abkühlen des Planeten mit in Betracht ziehen, werden in Buske (2002) vorgestellt. Diese dreidimensionalen sphärischen Modelle in der Boussinesq- 
Approximation sind gegenüber den Modellen von Harder und Christensen (1996) realitätsgetreuer, indem sie eine tiefenabhängige, druck- und temperaturbestimmte Viskosität nach dem Arrheniusgesetz (vgl. Kapitel (3.5)) annehmen. Das Viskositätsgesetz erlaubt die Simulation einer Lithosphäre und berücksichtigt den Viskositätsanstieg im abkühlenden System, der die Konvektion schwächt und die Abkühlrate des Planeten beeinflusst. Der Kern in diesen Modellen kühlt entsprechend des Wärmeflusses aus dem Kern in den Mantel ab und auch die interne Heizrate verringert sich mit der Zeit. Diese Modelle starten von einem Temperaturprofil mit möglichst hohen Temperaturen im Inneren und dünnen Grenzschichten, das die Situation nach der Differentiation des Planeten beschreiben soll. Der Einfluss der kernnahen, endothermen Phasengrenze auf das Konvektionsmuster und die thermische Evolution wird untersucht. In diesen realitätsgetreueren Modellen wird die Anzahl der Aufströme durch die endotherme Phasengrenze stark (auf zwei) reduziert. Nach $4.5 \mathrm{Ga}$ ist der Temperaturkonstrast an der KMG groß genug um plumeförmige Aufströme zuzulassen, die an der Oberfläche Vulkanismus hervorrufen können. Die Phasengrenze verringert den Wärmefluss aus dem Kern und verlangsamt das Abkühlen desselben. Der Einfluss der Phasengrenze auf die Lithosphärendicke von $290 \mathrm{~km}$ nach 4.5 Ga ist gering.

Ziel der vorliegenden Arbeit ist es, die Modelle darüber hinaus zu erweitern und den Einfluss verschiedener thermischer Größen und deren Temperatur- und Tiefenabhängigkeit auf das Konvektionsmuster, die thermische Evolution und die Existenz und Lebensdauer eines Dynamos zu untersuchen.

Zunächst wird der Einfluss des Viskositätsprofils durch Variation des Aktivierungsvolumens, das die Druckabhängigkeit der Viskosität beschreibt, untersucht. Das Viskositätsprofil beeinflusst direkt die Konvektionsstärke bzw. die radiale Verteilung der Bewegungsenergie und kann zusammen mit dem Effekt auf die Lithosphärendicke eine starke Bedeutung für die thermische Entwicklung haben. Das Aktivierungsvolumen ist experimentell bisher nur mit großen Unsicherheiten bestimmt (Kapitel (3.5)), so dass der Einfluss über einen bestimmten Bereich getestet wird. Außerdem beobachten Christensen und Yuen (1985), dass die Bedeutung der endothermen Phasengrenze von der lokalen Rayleighzahl abhängt und deutlich geschwächt wird, wenn z.B. die Viskosität in der entsprechenden Tiefe zu klein ist. Dies könnte Auswirkungen auf das Konvektionsmuster im Marsmantel haben.

Im nächsten Schritt werden die Modelle durch die Tiefenabhängigkeit des thermischen Ausdehnungskoeffizienten $\alpha$ erweitert. Im tiefenabhängigen (normierten) Profil sind die Werte für $\alpha$ an der KMG kleiner als im Fall mit konstanten Parametern. Dies kann die thermische Grenzschicht stabilisieren (Hansen et al. 1991, 1993) und so das Konvektionsmuster verändern. Zudem kann die Entwicklung der Temperatur und des Wärmeflusses durch eine mögliche Verlagerung der Bereiche mit starker Konvektion beeinflusst werden.

Desweiteren wird die thermische Leitfähigkeit in Anlehnung an die Modelle von Hofmeister (1999) abhängig von der gemittelten Temperatur und dem hydrostatischen Druck beschrieben. Dadurch ergeben sich Profile mit einem relativen Minimum der Leitfähigkeit in der Übergangszone zur Lithosphäre, so dass das Abkühlen des Planeten verlangsamt werden könnte. Für die Erde beobachten van den Berg et al. (2002) (van den Berg und Yuen 2002, van den Berg et al. 2004) diesen Einfluss auf die Abkühlrate. Der starke Anstieg der thermischen Leitfähigkeit mit der Tiefe führt bei Modellen für die Erde zu einem 
Stabilisieren der thermischen Grenzschicht und der Konvektionsbewegung (Dubuffet und Yuen 2000, Dubuffet et al. 2002). Der gegenüber der Erde abgeschwächte Effekt könnte das Konvektionsmuster im Marsmantel verändern.

Der Einfluss der Kombination eines tiefenabhängigen Ausdehungskoeffizienten und einer druck- und temperaturbestimmten thermischen Leitfähigkeit wird im Anschluss untersucht. In diese Modellen werden auch die exothermen Phasengrenzen einbezogen und es wird modelliert, wie sich die Positionen der Phasengrenzen während des Abkühlens des Planeten verändern und welchen Einfluss diese zeitlich variable Position hat. Zusätzlich wird in diesen Modellen die Kerntemperatur im Startprofil variiert, um den Einfluss auf den Wärmefluss und die Stabilität der thermischen Grenzschicht zu testen.

Die nächste Erweiterung betrifft die Nicht-Boussinesq-Effekte wie latente Wärme, Temperatureffekte durch adiabatische Kompression und Dekompression sowie Reibungswärme. Insbesondere ist interessant, wie weit sich die latente Wärme (auch der exothermen Phasengrenzen) auf das Konvektionsmuster auswirken kann. In den erweiterten, vollständigen Boussinesq-Modellen ist die Temperaturentwicklung an der KMG von großer Bedeutung, da ihre Fortsetzung in das Kerninnere Aufschluss über die Existenz und Größe eines inneren festen Kerns geben kann.

Das folgende Kapitel beschreibt das mathematische Modell und stellt die Druck- und Temperaturabhängigkeit der Viskosität und der thermischen Leitfähigkeit sowie das Profil des thermischen Ausdehnungskoeffizienten vor. 



\section{Theoretische Grundlagen}

Das folgende Kapitel beschreibt das mathematische Modell der Konvektionsströmung und der thermischen Evolution des Mantels. Es berücksichtigt die säkulare Abkühlung, mögliche Phasengrenzen sowie die Tiefenabhängigkeit der Viskosität, der thermischen Leitfähigkeit und des thermischen Ausdehnungskoeffizienten.

\subsection{Grundgleichungen der Strömungsmechanik}

Das zunächst einmal feste Silikatgestein unterliegt im Planetenmantel hohen mechanischen Spannungen, die dazu führen, dass innerhalb der Silikatkristalle, Fehlstellen, Verunreinigungen und Versetzungen durch den Kristall wandern und das Material verformen. Es kommt zu einer Kriechbewegung im Festkörper, die erlaubt, das Material unter diesen Bedingungen wie eine äußerst zähe Flüssigkeit aufzufassen und durch die Gleichungen der Strömungsmechanik zu beschreiben. Die folgenden Grundgleichungen ergeben sich aus Erhaltungsbedingungen für Masse, Impuls und Energie und gelten pro Fluidvolumeneinheit $V$ (Schubert et al. 2001, chap. 6):

Die Erhaltung der Masse wird durch die Kontinuitätsgleichung beschrieben:

$$
\frac{\partial \rho}{\partial t}+\vec{\nabla} \cdot(\rho \vec{v})=0
$$

Dabei symbolisiert $\rho$ die Dichte, $\vec{v}$ die Geschwindigkeit der Flüssigkeit und $t$ die Zeit.

Die Impulserhaltung führt zur Navier-Stokes-Gleichung:

$$
\rho \frac{D \vec{v}}{D t}=-\vec{\nabla} p+\vec{\nabla} \cdot \underline{\underline{\tau}}+\vec{f}
$$

$\frac{D}{D t}=\frac{\partial}{\partial t}+\vec{v} \cdot \vec{\nabla}$ bezeichnet die substantielle Ableitung. Damit wird die Änderung der Impulsdichte im Euler'schen System d.h. in einem von einem externen, ruhenden Beobachter betrachteten System beschrieben. $p$ steht für den Druck und $\underline{\underline{\tau}}$ für den deviatorischen Spannungstensor, der über $\tau_{i j}=\sigma_{i j}+p \delta_{i j}$ mit dem Druck $p$ und dem Spannungstensor $\underline{\underline{\sigma}}$ verbunden ist ( $\delta_{i j}$ : Kroneckersymbol). Der Spannungstensor beschreibt die Kräfte, die das umgebende Material auf die Oberfläche des betrachteten Volumens ausüben. $\vec{f}$ ist eine allgemeine Volumenkraftdichte, die im Folgenden nur durch die Schwerkraft aufgebracht wird. Es gilt: $\vec{f}=\rho \vec{g}$ mit $\vec{g}$ dem Schwerebeschleunigungsvektor.

In einem viskosen Medium führen mechanische Spannungen zu einer kontinuierlich fortschreitenden Deformation des Materials. Diese Verformung wird mit dem Deformationsratentensor $\underline{\underline{\dot{\epsilon}}}=\frac{1}{2}\left(\vec{\nabla} \vec{v}+\{\vec{\nabla} \vec{v}\}^{T}\right)\left(\{\}^{T}\right.$ : transponierter Tensor) beschrieben. Der Druck, der 
isotrope Anteil des Spannungstensors, führt dabei nur zu einer endlichen aber nicht fortschreitenden Volumenänderung, so dass die Deformationsraten durch die deviatorischen Spannungen bestimmt sind. In einem Newton'schen Medium gilt eine lineare Beziehung zwischen dem Deformationsratentensor und dem deviatorischen Spannungstensor. Für ein zusätzlich isotropes Medium mit Scherviskosität $\eta$ ist folgende Relation gültig:

$$
\underline{\underline{\tau}}=2 \eta\left(\underline{\underline{\dot{\epsilon}}}-\frac{1}{3}(\vec{\nabla} \cdot \vec{v}) \underline{\underline{1}}\right)
$$

mit dem Einheitstensor $\underline{\underline{1}}$. Die Volumenviskosität, die den viskosen Widerstand des Mediums gegen Volumenänderungen beschreibt, wird bei den zu betrachtenden Prozessen vernachlässigt.

Die Energieerhaltung wird im ersten Hauptsatz der Thermodynamik beschrieben. Danach ist in einem abgeschlossenen Volumen V die Änderung der inneren Energie $u$ (pro Masse) gleich der Summe aus am System geleisteten Arbeit und der pro Volumen zu- oder abgeführten bzw. erzeugten Wärme.

$$
\rho \frac{D u}{D t}=\underline{\underline{\sigma}} \cdot \underline{\underline{\dot{\epsilon}}}-\vec{\nabla} \cdot \vec{q}+Q
$$

$\underline{\underline{\sigma}} \cdot \underline{\underline{\epsilon}}$ beschreibt die am System pro Volumen verrichtete mechanische Leistung, $\vec{q}$ die Wärmeflussdichte und $Q$ die Wärmeproduktionsrate pro Volumen, die z.B. die Wärmeproduktion durch den Zerfall von radioaktiven Elementen bzw. im Fall von Phasengrenzen auch die latente Wärme beinhaltet.

Spaltet man im Term für die mechanischen Leistung den Druckterm ab, indem man zum deviatorischen Spannungstensor $\tau$ übergeht, wird die Gleichung den thermodynamischen Relationen für Gase ähnlicher. Die Wärmeflussdichte wird durch das Fourier-Gesetz ersetzt, nach dem die Wärmeflussdichte proportional zum Temperaturgradienten $\vec{\nabla} T$ ist. Die thermischen Leitfähigkeit $\lambda$ ist die Proportionalitätskonstante:

$$
\vec{q}=-\lambda \vec{\nabla} T
$$

Damit gilt:

$$
\rho \frac{D u}{D t}=-p(\vec{\nabla} \cdot \vec{v})+\underline{\underline{\tau}} \cdot \underline{\underline{\dot{\epsilon}}}+\vec{\nabla} \cdot(\lambda \vec{\nabla} T)+Q
$$

Der erste Term entspricht dabei der mechanischen Leistung durch Volumenänderung $((p d V)$ in der Formulierung der thermodynamischen Gesetze für Gase). Der zweite, stets positive Summand beschreibt die Wärmeerzeugung durch mechanische Reibung. Mit dem zweiten Hauptsatz der Thermodynamik $T d S=d u+p d V$ und mit den bekannten thermodynamischen Relationen für die Entropie $S$ lässt sich die Gleichung in eine Gleichung für die Temperatur $T$ umwandeln:

$$
\rho c_{p}\left(\frac{D}{D t} T-\frac{\alpha T}{\rho c_{p}} \frac{D}{D t} p\right)=\underline{\underline{\tau}} \cdot \underline{\underline{\dot{\epsilon}}}+\vec{\nabla} \cdot(\lambda \vec{\nabla} T)+Q
$$

mit der spezifischen Wärmekapazität bei konstantem Druck $c_{p}$ und dem thermischen Ausdehnungskoeffizienten $\alpha$. Die zeitliche Änderung der Temperatur ist, neben den oben erwähnten Effekten auf der rechten Seite der Gleichung, bestimmt durch Temperatureffekte durch Advektion (enthalten in der substantiellen Ableitung der Temperatur) sowie Einflüsse durch adiabatische Kompression bzw. Dekompression (zweiter Term auf der linken Seite). 


\subsection{Boussinesq- und erweiterte Boussinesq-Näherung}

Die Boussinesq-Näherung bzw. die erweiterte Boussinesq-Näherung ermöglicht eine Vereinfachung der strömungsmechanischen Gleichungen. Dazu werden folgenden Annahmen gemacht: Die Dichte kann in allen Termen mit Ausnahme des Auftriebsterms wie eine Konstante behandelt werden, d.h. das Mantelgestein kann als inkompressible (Ausnahme im Auftriebsterm) Flüssigkeit aufgefasst werden. Insbesondere hängt die Dichte nicht vom Druck ab und die Temperaturabhängigkeit im Auftriebsterm ist linear. Die erweiterte Boussinesq-Näherung berücksichtigt aber im Gegensatz zur einfachen BoussinesqNäherung die adiabatische Temperaturzunahme mit der Tiefe sowie Temperatureffekte durch viskose Reibung und latente Wärme im Fall von Phasengrenzen.

Im weiteren Verlauf wird nun die Wärmeproduktionsrate pro Volumen $Q$ als Summe der Beiträge durch radioaktive Elemente $H$ und durch latente Wärme $Q_{L}$ betrachtet.

Mit dieser Näherung nehmen die Grundgleichungen folgende Gestalt an:

$$
\begin{gathered}
\vec{\nabla} \cdot \vec{v}=0 \\
\rho_{0} \frac{D}{D t} \vec{v}=-\vec{\nabla} p+\vec{\nabla} \cdot \underline{\underline{\tau}}+\rho \vec{g} \\
\frac{D}{D t} T-\frac{\alpha T}{\rho_{0} c_{p}} \frac{D}{D t} p=\frac{1}{\rho_{0} c_{p}}\left(\vec{\nabla} \cdot(\lambda \vec{\nabla} T)+\underline{\underline{\tau}} \cdot \underline{\underline{\dot{\epsilon}}}+H+Q_{L}\right)
\end{gathered}
$$

Das Fließgesetz (3.3) vereinfacht sich zu:

$$
\underline{\underline{\tau}}=2 \eta \underline{\underline{\dot{\epsilon}}}
$$

\subsection{Zustandsgleichung}

Die Zunahme der Dichte im Mantel wird im Wesentlichen durch hydrostatische Kompression verursacht. Dadurch nimmt die Dichte im Mantel um etwa $65 \%$ zwischen der Oberfläche und der Kern-Mantel-Grenze (KMG) zu. Dichteänderung durch Druck und Temperaturänderung, die durch die Konvektion verursacht werden, sind im Verhältnis zu der gemittelten Dichte des Mantel klein. Das rechtfertigt eine lineare Zustandsgleichung (Schubert et al. 2001, Kap. 6.10). Die Druckabhängigkeit der Dichte wird sowohl in der Boussinesq-, wie auch in der erweiterten Boussinesq-Näherung vernachlässigt. Ohne Phasengrenze gilt somit folgende Zustandsgleichung:

$$
\rho=\rho_{0}-\alpha \rho_{0}\left(T-T_{0}\right)
$$

$\rho_{0}=\rho\left(T_{0}\right)$ ist die Dichte im Referenzfall mit $T=T_{0}$.

Um die Effekte der Phasengrenze berücksichtigen zu können, wird die Phasenfunktion $\Gamma$ eingeführt (Christensen und Yuen 1985). Die Phasenfunktion $\Gamma$ bescheibt den relativen Anteil der dichteren Phase und ist im Idealfall (eines univarianten Phasenübergangs) eine Stufenfunktion, die als Funktion des Drucks $p$ und der Temperatur $T$ die Werte 0 oder 1 
annimmt. Für die Stufenfunktion gilt:

$$
\Gamma= \begin{cases}1 & \text { unterhalb der Phasengrenze } \\ 0 & \text { oberhalb der Phasengrenze }\end{cases}
$$

Die Druck- und Temperaturbedingungen des Phasenübergangs sind durch die Phasengrenzkurve (Clausius-Clapeyron-Kurve) bestimmt (Abschnitt (2.3.3)). Für den Umwandlungsdruck $p_{\text {Tr }}$ gilt in Abhängigkeit von der Temperatur:

$$
p_{\operatorname{Tr}}=p_{\operatorname{Tr}, 0}+\gamma\left(T-T_{0}\right)
$$

darin kennzeichnet $p_{\operatorname{Tr}, 0}$ den Phasenübergangsdruck bei der Referenztemperatur $T_{0}$ und $\gamma$ die Steigung der Clausius-Clapeyron-Kurve.

Damit lässt sich der reduzierte Druck bzw. "Überdruck" $\pi$ definieren:

$$
\pi=p-p_{\operatorname{Tr}}=p-p_{\operatorname{Tr}, 0}-\gamma\left(T-T_{0}\right)
$$

Beim Phasenübergangsdruck $p=p_{\text {Tr }}$ gilt: $\pi=0$. Zur Vereinfachung wird bei der Berechnung des reduzierten Drucks nur der hydrostatische Anteil des Drucks betrachtet und nicht hydrostatische Anteile werden vernachlässigt. Im Idealfall der Stufenfunktion nimmt die Phasenfunktion folgende Form an:

$$
\Gamma(\pi)= \begin{cases}1 & \pi \geq 0 \\ 0 & \pi<0\end{cases}
$$

Damit ergibt sich folgende Zustandsfunktion für die Dichte:

$$
\rho=\rho_{0}\left[1-\alpha\left(T-T_{0}\right)+\Gamma \frac{\triangle \rho}{\rho_{0}}\right]
$$

mit dem Dichtekonstrast der beiden Phasen $\triangle \rho$.

Mit der Definition des thermischen Ausdehnungskoeffizienten $\alpha$ :

$$
\alpha=\frac{1}{V} \frac{\partial V}{\partial T} \quad \text { bzw. } \quad \alpha=-\frac{1}{\rho_{0}} \frac{\partial \rho}{\partial T}
$$

lässt sich aus der Zustandsgleichung ein effektiver thermischer Ausdehungskoeffizient $\alpha_{\text {eff }}$ für den Fall mit Phasengrenze berechnen:

$$
\alpha_{\mathrm{eff}}=\alpha-\frac{\triangle \rho}{\rho_{0}} \frac{\partial \Gamma}{\partial T}=\alpha-\frac{\triangle \rho}{\rho_{0}} \frac{d \Gamma}{d \pi} \frac{\partial \pi}{\partial T}=\alpha+\gamma \frac{\triangle \rho}{\rho_{0}} \frac{d \Gamma}{d \pi}
$$

Im Fall der Stufenfunktion stellt $d \Gamma / d \pi$ einen Delta-Peak dar.

In der vorliegenden Arbeit wird das Problem vereinfacht, indem keine laterale Änderung von $d \Gamma / d \pi$ bzw. $\pi$ zugelassen wird, sondern der Effekt der Phasengrenze auf der ganzen Kugelschale konzentriert in einer Tiefe wirkt (der 'Überdruck' $\pi$ hängt quasi von einer über die Kugelschale gemittelten Temperatur ab). Diese Tiefe muss ggf. in jedem Zeitschritt neu bestimmt werden, wenn die Modelle berücksichtigen, dass die Position der Phasengrenze beim Abkühlen des Planeten dem veränderten (über Kugelschalen gemittelten) Temperaturprofil angepasst werden muss (vgl. Kapitel (4.5)). Die Auftriebseffekte 
durch das Aufwölben z.B. einer endothermen Phasengrenze in einem Plume werden simuliert, indem im Idealfall auf einem einzigen radialen Level (auf der ganzen Kugelschale) der Wert des thermischen Ausdehnungskoeffizienten von seinem eigentlichen Wert abweicht. $\alpha_{\text {eff }}$ ist in dieser Näherung über den Mantel konstant bzw. folgt einem vorgegebenen Profil (Abschnitt (3.6)) und ändert in der Tiefe der Phasengrenze auf der ganzen Kugelschale sprunghaft seinen Wert. Im Fall der endothermen Phasengrenze ist $\alpha_{\text {eff }}$ dort kleiner. In dieser Darstellung durch $\alpha_{\text {eff }}$ wird deutlich, dass der relative Beitrag der Phasengrenze zu den Auftriebskräften temperaturunabhängig ist. Die Temperatur der Anomalie beeinflusst jedoch den absoluten Beitrag der Phasengrenze.

Numerisch ist es nicht möglich, den Idealfall der Stufenfunktion bzw. des Delta-Peaks zu realisieren. Hier muss der Phasenübergang so über eine endliche Breite "verschmiert" werden, dass auch erste Ableitung $d \Gamma / d \pi$ endliche Werte annimmt und numerisch handhabbar wird. Dabei muss die Normierung

$$
\int_{r_{i}}^{r_{a}} \frac{d \Gamma}{d \pi} d r=1
$$

berücksichtigt werden. Die numerische Realisation wird in Buske (2002, Kap. 4.2) vorgestellt. Bei mehreren Phasengrenzen wird dieses Verfahren in den jeweiligen Tiefen der Phasengrenzen angewandt.

\subsection{Entdimensionalisieren der Gleichungen}

In diesem Abschnitt werden die Gleichungen (3.8) - (3.10) und (3.17) entdimensionaliert, indem die verwendeten Variablen jeweils durch das Produkt eines dimensionslosen Wertes mit einem für das System typischen Wert ersetzt werden. So werden z.B. alle Längen $r$ mit der Manteldicke $d$ als charakteristische Systemgröße skaliert. Die dabei neu eingeführten, mit einem Strich (') gekennzeichneten Größen sind dimensionslos und von der Größenordnung 1. Die Anzahl der Kontrollparameter wird so reduziert, die Gleichungen werden übersichtlicher, numerischer besser handhabbar und universeller, da beim Zurückskalieren mehrere Möglichkeiten gegeben sind.

Es gelten folgende Definitionen (Christensen 1984) :

\begin{tabular}{|c|c|}
\hline Länge & $r=d \cdot r^{\prime}$ \\
\hline Zeit & $t=\frac{d^{2}}{\kappa} \cdot t^{\prime}$ \\
\hline Geschwindigkeit & $\vec{v}=\frac{\kappa}{d} \cdot \overrightarrow{v^{\prime}}$ \\
\hline Spannung & $\underline{\underline{\sigma}}=\frac{\kappa \eta_{\mathrm{ref}}}{d^{2}} \cdot \underline{\underline{\sigma^{\prime}}}$ \\
\hline Druck & $p=\frac{\kappa \eta_{\mathrm{ref}}}{d^{2}} \cdot p^{\prime}$ \\
\hline reduzierter hydr. Druck & $\pi=\rho_{0} g d \cdot \pi^{\prime}$ \\
\hline Temperatur & $T=\Delta T \cdot T^{\prime}+T_{\text {surf }}$ \\
\hline interne Heizrate & $H=\frac{\lambda \Delta T}{d^{2}} \cdot H^{\prime}$ \\
\hline
\end{tabular}


Die thermische Diffusivität $\kappa$ ist dabei definiert als $\kappa=\lambda_{\text {ref }} / c \rho_{0}$. Im Fall einer variierenden thermischen Leitfähigkeit steht $\lambda_{\text {ref }}$ für den Referenzwert der Leitfähigkeit. Ähnlich bezeichnet $\eta_{\text {ref }}$ die Referenzviskosität bei einer tiefenabhängigen Viskosität (vgl. später). $\Delta T$ ist die Temperaturdifferenz zwischen äußerem und innerem Rand und $T_{\text {surf }}$ die Oberflächentemperatur.

Die Energie pro Gewichtseinheit durch latente Wärme $q_{L}$ lässt sich aus der Steigung der Clausius-Clapeyron-Kurve $\gamma$ wie folgt bestimmen:

$$
q_{L}=\gamma \frac{T_{a} \Delta \rho}{\rho_{0}^{2}}
$$

wobei $T_{a}$ die absolute Temperatur kennzeichnet. Für die frei werdende Energie pro Volumen $Q_{L}$ während des Phasenübergangs ergibt sich:

$$
Q_{L}=\rho_{0} q_{L} \frac{D \Gamma}{D t}
$$

Setzt man die Zustandsgleichung (3.17) mit dem effektiven Ausdehnungskoeffizienten $\alpha_{\text {eff }}$ (3.19) im Auftriebsterm von (3.9) ein und berücksichtigt, dass der dabei auftretende Ausdruck $\rho_{0} \vec{g}$ mit dem hydrostatischen Anteil des Druckgradienten $\vec{\nabla} p_{\text {hydr }}=\rho_{0} \vec{g}$ zusammengefasst werden kann ( $p^{\prime}$ bezeichnet dann den nicht hydrostatischen Druck), so nehmen die Gleichungen (3.8) - (3.10) mit der oben genannten Skalierung folgende Form an:

$$
\begin{aligned}
& \vec{\nabla}^{\prime} \cdot \vec{v}^{\prime}=0 \\
& \frac{1}{P r}\left(\frac{D}{D t^{\prime}} \vec{v}^{\prime}\right)=-\vec{\nabla}^{\prime} p^{\prime}+\vec{\nabla}^{\prime} \cdot \underline{\underline{\tau}}^{\prime}+\alpha^{\prime} R a\left(1+P \frac{d \Gamma}{d \pi^{\prime}}\right) T^{\prime} \vec{e}_{r} \\
& \frac{D}{D t^{\prime}} T^{\prime}+\alpha^{\prime} D i v_{r}\left(T^{\prime}+T_{\text {surf }}^{\prime}\right)=\vec{\nabla}^{\prime} \cdot\left(\lambda^{\prime} \vec{\nabla}^{\prime} T^{\prime}\right)+ \\
& \frac{D i}{R a} \underline{\tau^{\prime}} \cdot \underline{\underline{\epsilon}}^{\prime}+H^{\prime}+P D i\left(T^{\prime}+T_{\text {surf }}^{\prime}\right) \frac{D \Gamma^{\prime}}{D t^{\prime}}
\end{aligned}
$$

$\overrightarrow{e_{r}}$ bezeichnet den Einheitsvektor in radialer Richtung (positiv nach außen). Im Falle eines tiefenabhängigen thermischen Ausdehnungskoeffizienten $\alpha$ bzw. einer tiefenabhängigen thermischen Leitfähigkeit $\lambda$ gilt: $\alpha^{\prime}=\alpha / \alpha_{\text {ref }}$ bzw. $\lambda^{\prime}=\lambda / \lambda_{\text {ref }}$, wobei $\alpha_{\text {ref }}$ bzw. $\lambda_{\text {ref }}$ die jeweiligen Referenzwerte sind. Bei konstantem $\alpha$ bzw. $\lambda$ gilt im Folgenden $\alpha^{\prime}=1$ bzw. $\lambda^{\prime}=1$.

Nach Christensen und Yuen (1985) lässt sich die Wärmeleitungsgleichung folgendermaßen umformen. Die Striche (') enfallen dabei wieder (mit Ausnahme von $\alpha^{\prime}$ und $\lambda^{\prime}$, die nur bei nicht konstanten Parametern von 1 verschieden sind) und die Größen gelten als dimensionslos.

$$
\frac{D \Gamma}{D t}=\frac{d \Gamma}{d \pi} \frac{D \pi}{D t}=\frac{d \Gamma}{d \pi}\left(\frac{\partial \pi}{\partial T} \frac{D T}{D t}+\frac{\partial \pi}{\partial r} v_{r}\right)=-\frac{d \Gamma}{d \pi}\left(\gamma \frac{D T}{D t}+v_{r}\right)
$$

Damit ergibt sich:

$$
\begin{array}{r}
{\left[1+\frac{d \Gamma}{d \pi} \gamma P D i\left(T+T_{\text {surf }}\right)\right] \frac{D T}{D t}+\alpha^{\prime}\left(1+P \frac{d \Gamma}{d \pi}\right)\left(T+T_{\text {surf }}\right) D i v_{r}=} \\
\vec{\nabla} \cdot\left(\lambda^{\prime} \vec{\nabla} T\right)+\frac{D i}{R a} \underline{\underline{\tau}} \cdot \underline{\underline{\dot{\epsilon}}}+H
\end{array}
$$


Neben dem bereits eingeführten $\alpha_{\text {eff }}=\alpha\left(1+P \frac{d \Gamma}{d \pi}\right)$ lässt sich der Ausdruck $c_{\mathrm{p} \text {,eff }}=$ $c_{p}\left[1+\frac{d \Gamma}{d \pi} \gamma P D i\left(T+T_{\text {surf }}\right)\right]$ als effektive Wärmekapazität interpretieren, die in der Tiefe der Phasenumwandlung ihren Wert vergrößert. Ein Verringern des Wertes von $c_{\mathrm{p}, \mathrm{eff}}$ wie bei $\alpha_{\text {eff }}$ im Falle einer endothermen Phasengrenze ist hier nicht möglich, da die Steigung der Clausius-Clapeyron-Kurve quadratisch in die Gleichung eingeht.

In den Gleichungen treten folgende dimensionslose Kenngrößen auf:

$\begin{array}{rlrl} & \text { Prandtlzahl } & \operatorname{Pr} & =\frac{\eta_{\mathrm{ref}}}{\kappa \rho} \\ & \text { Rayleighzahl } & R a & =\frac{\alpha_{\mathrm{ref}} \rho_{0} g \Delta T d^{3}}{\kappa \eta_{\mathrm{ref}}} \\ & \text { Phasenparameter } & P & =\frac{\Delta \rho \gamma}{\alpha_{\mathrm{ref}} \rho_{0}^{2} g d} \\ \text { Dissipationszahl } & D i & =\frac{\alpha_{\mathrm{ref}} g d}{c_{p}}\end{array}$

Die Prandtlzahl $\operatorname{Pr}$ beschreibt den relativen Einfluss der Trägheitskräfte. Aufgrund der hohen Mantelviskosität von $\eta_{\text {ref }}=O\left(10^{21}\right)$ ist die Prandtlzahl selbst von der Größenordung $\operatorname{Pr}=O\left(10^{23}\right)$. Da die Trägheitskräfte mit dem Kehrwert der Prandtlzahl in die Gleichung eingehen, ist ihr Einfluss gering, so dass sie vernachlässigt werden können. Physikalisch bedeutet dies, dass die Strömung sich instantan den neuen Temperatur- und Druckbedingungen anpasst.

Die Rayleighzahl $R a$ beschreibt das Verhältnis von konvektionsfördernden zu konvektionshemmenden Parametern und ist somit ein Maß für die Konvektionsstärke. Für den Marsmantel lässt sich ein Wert von $R a_{\text {Mars }}=5.3 \cdot 10^{6}$ abschätzen. Im Falle tiefenabhängiger bzw. temperaturabhängiger Parameter wie Viskosität, thermischen Ausdehnungskoeffizienten bzw. thermischer Leitfähigkeit (vgl. folgende Kapitel), ist es wichtig, Referenzwerte zu definieren, die z.B. in die Rayleighzahl eingehen. Die Referenzviskosität soll in der halben Manteltiefe bei einer typischen Manteltemperatur von $1573 \mathrm{~K}$ erreicht werden. Ebenso werden die Profile der tiefenabhängigen thermischen Leitfähigkeit bzw. des thermischen Ausdehnungkoeffizienten so normiert, dass der Referenzwert in der Mantelmitte bei $1573 \mathrm{~K}$ gilt.

Der Phasenparameter P charakterisiert die Phasengrenze und beschreibt die Bedeutung der Auftriebseffekte, die durch das Aufwölben der Phasengrenze entstehen, relativ zu den thermischen Auftriebseffekten. Der Fall $|P|=1$ bedeutet dementsprechend, dass beide Auftriebsmechanismen gleich großen Einfluss ausüben. Ein positiver Phasenparamter kennzeichnet eine exotherme Phasengrenze (zusätzlicher Auftrieb durch die Phasengrenze), ein negativer eine endotherme (verringerter Auftrieb). Auch ein vom Betrag kleiner Phasenparameter kann einen signifikanten Einfluss auf die Strömung haben, da diese Auftriebseffekte im Gegensatz zu den thermischen Effekten stark lokalisiert sind. Unter Verwendung von $P=0.0$ ergeben sich die Gleichungen in einem Fluid mit nur einer Phase. Mit den in Abschnitt (2.3.3) vorgestellten Werten für den Dichtekontrast und die Clausius-Clayperon-Steigung ergeben sich Phasenparameter für die einzelnen Übergänge von: $P_{\alpha \beta}=0.27230, P_{\beta \gamma}=0.32420$ und $P_{\gamma p v}=-0.27230$. Durch die geringere Manteldicke des Mars und die geringere Schwerebeschleunigung sind die Phasenparameter betragsmäßig größer als für die Erde, was einen größeren Einfluß der Phasengrenzen auf 
die Konvektion im Mars vermuten lässt. Der Einfluss eines negativeren Wertes für $P_{\gamma p v}$, wie er sich z.B. durch einen größeren Dichtekontrast ergeben würde, ist in Ansätzen in Buske (2002) untersucht worden.

Für die Lokalisation der Phasengrenze sorgt der Ausdruck $d \Gamma / d \pi$, der im Fall einer Stufenfunktion durch einen Delta-Peak dargestellt wird und nur in der Tiefe der Phasengrenze einen von Null verschiedenen Wert annimmt.

Der letze dimensionslose Parameter ist die Dissipationszahl $D i$, die die Bedeutung der Temperaturänderung durch Nicht-Boussinesq-Effekte (adiabatische Temperaturzunahme mit der Tiefe, latente Wärme und viskose Reibung) angibt. Mit den später für Mars angenommenen Parametern ergibt sich eine Dissipationszahl von $D i=0.1445$, was einen schwachen Einfluss der Nicht-Boussinesq-Effekte erwarten lässt. Die Gleichungen der Boussinesq-Näherung ergeben sich aus den obigen Gleichungen mit $D i=0.0$. Damit wird auch deutlich, dass latente Wärme im Gegensatz zu den Effekten durch das Aufwölben der Phasengrenze ein Nicht-Boussinesq-Effekt ist. Möchte man im Falle einer Phasengrenze die latente Wärme mitberücksichtigen, müssen auch die Temperatureffekte durch viskose Reibung und adiabatische Kompression in Betracht bezogen werden, da alle Beiträge mit der Dissipationszahl skalieren. Andernfalls entsteht ein Ungleichgewicht an Quellen und Senken der thermischen Energie. (Christensen und Yuen 1985, Christensen 1995). Als Vereinfachung kann der Einfluss der Phasengrenze auf die spezifische Wärmekapazität vernachlässigt werden, da $\gamma P D i<<1$ und somit der Beitrag gering ist (Christensen 1995).

Damit bekommen die Gleichungen die folgende Gestalt:

$$
\begin{gathered}
\vec{\nabla} \cdot \vec{v}=0 \\
\vec{\nabla} p=\vec{\nabla} \cdot \underline{\underline{\tau}}+\alpha^{\prime} R a\left(1+P \frac{d \Gamma}{d \pi}\right) T \overrightarrow{e_{r}} \\
\frac{D T}{D t}+\alpha^{\prime}\left(1+P \frac{d \Gamma}{d \pi}\right)\left(T+T_{\text {surf }}\right) D i v_{r}=\vec{\nabla} \cdot\left(\lambda^{\prime} \vec{\nabla} T\right)+\frac{D i}{R a} \underline{\underline{\tau}} \cdot \underline{\underline{\epsilon}}+H
\end{gathered}
$$

\subsection{Viskosität}

Auch unterhalb der Schmelztemperatur ist es möglich, das dann noch feste kristalline Gestein im Mantel unter hohen mechanischen Spannungen zu verformen und in eine Kriechbewegung zu versetzen. Dabei bewegen sich Gitterfehler wie z.B. Leerstellen und Versetzungen durch das Material. Man unterscheidet im Wesentlichen zwei Kriechmechanismen: das Diffusionskriechen und das Dislokationskriechen. Die Effektivität der beiden Mechanismen hängt von den Druck- und Temperaturbedingungen sowie von Eigenschaften der vorliegenden Gesteine ab.

Beim Diffusionskriechen verformt sich der Kristall, indem Leerstellen fortschreitend auf benachbarte Gitterplätze wandern und in der Gegenbewegung die Atome den vorher freien, benachbarten Platz einnehmen. Leerstellen (Punktdefekte) entstehen, indem ein Atom in der Nähe der Oberfläche von seinem Gitterplatz im Inneren in eine neue Atomlage an der Oberfläche gesetzt wird. Die Wahrscheinlichkeit der Erzeugung oder Vernichtung 
von Leerstellen hängt von den Spannungen ab, die auf den Seiten eines Kristalls angreifen. Sind diese Spannugen nicht auf allen Seiten gleich, kommt es zu Unterschieden in der Konzentration der Leerstellen, die durch Diffusion ausgeglichen werden. Die Diffusionsbewegung von Leerstellen und Atomen ist thermisch aktiviert, der Diffusionskoeffizient $D$ wird durch einen Arrheniusterm beschrieben:

$$
D=D_{0} \exp \left(-\frac{\left(E^{*}+p V^{*}\right)}{R T}\right)
$$

Dabei ist $E^{*}$ die Aktivierungsenergie pro Mol, die sowohl die Energie zur Erzeugung einer Leerstelle als auch die nötige Potentialbarriere zwischen Leerstelle und besetztem Gitterplatz enthält. Das Aktivierungsvolumen pro Mol $V^{*}$ berücksichtigt, mit dem Druck multipliziert, dass unter Druck das Erzeugen von Leerstellen mehr Energie erfordert und die Energiebarriere zum Nachbaratom höher ist. Der Exponentialausdruck ergibt sich direkt aus der Boltzmannverteilung, die den Anteil der Atome mit einer bestimmten Energie bei der Temperatur $T$ angibt. $D_{0}$ enthält die Sprungfrequenz, die Häufigkeit der Übergänge auf den Nachbarplatz, $R$ ist die allgemeine Gaskonstante.

Neben dem Diffusionskriechen entsteht ein in vielen Fällen effektiverer Kriechmechanismus, wenn an Stelle von Punktdefekten (z.B. Leerstellen), Versetzungen (Liniendefekte), z.B. zusätzliche Gitterebenen, durch den Kristall wandern. Versetzungen können in verschiedenen Gitterebenen unter einer Scherspannung durch den Kristall gleiten, was nur eine geringfügige Umordnung der Atome erforderlich macht. Gleitet eine Versetzung auf ihrer Gleitebene durch den ganzen Kristall, führt dies zu einer Verformung des Kristalls um eine Gittereinheit. Das Gleiten durch den Kristall kann durch Kristalldefekte wie Fremdatome und andere Versetzungen gestoppt werden. Diese Blockierung kann aber wieder gelöst werden, indem die Versetzung auf eine höhere Gleitebene klettert. Dazu ist es erforderlich, entlang der Versetzung eine Linie von Atomen zu entfernen bzw. hinzuzufügen. Dies ist z.B. durch Leerstellen im Gitter möglich, die zur Versetzung hindiffundieren. Dieser Diffusionsprozess ist maßgebend für die Rate des Versetzungskriechens und wird durch die Temperatur- und Druckbedingungen nach dem obigen Arrheniusgesetz beschrieben (Christensen 2000). Die Verformbarkeit des Materials bzw. seine Viskosität hängt damit von dem Diffussionskoeffizienten ab: $\eta(D)$.

Experimente und Theorie liefern eine allgemeine Beziehung zwischen Spannungen und Deformationsrate, die für beide Kriechmechanismen gültig ist (Schubert et al. 2001):

$$
\underline{\underline{\epsilon}}=\frac{1}{2 \eta} \underline{\underline{\tau}}=A\left(\frac{\tau_{I I}}{\mu}\right)^{n-1}\left(\frac{b}{d}\right)^{m} \exp \left(-\frac{\left(E^{*}+p V^{*}\right)}{R T}\right) \underline{\underline{\tau}}
$$

Dabei ist $A$ ein Normalisierungsfaktor, $\tau_{I I}$ die Wurzel aus der zweiten Invarianten des Spannungstensors. $\mu$ bezeichnet den Schermodul, $d$ die Korngröße und $b$ den Betrag des Burgesvektors. Der Burgesvekor beschreibt die relative Atombewegung, wenn eine Versetzung sich durch den Kristall bewegt. Mit seiner Hilfe lässt sich die Form der Versetzung charakterisieren (z.B. Christensen (2000), Schubert et al. (2001)).

In Abhängigkeit vom dominierenden Kriechmechanismus variieren die Werte für $m$ und $n$. Für das Diffusionskriechen sind typische Werte $n=1$ und $m=2.5$ bzw. $n=3.5$ und $m=0$ für den Dislokationsmechanismus. Damit ist die Relation zwischen Spannung und Deformationsrate im Fall des Diffusionskriechens linear und die Viskosität unabhängig 
von der Spannung. Allerdings besteht durch den Ausdruck $d^{m}$ eine starke Abhängigkeit der Viskosität von der Korngröße, so dass die Gesteine um so besser verformbar sind, je feinkristalliner sie sind. Im Gegensatz dazu ist die Beziehung zwischen Spannung und Dehnungsrate im Fall des Dislokationskriechens stark nicht linear. Eine Abhängigkeit von der Korngröße entfällt jedoch.

In einer realistischen Beschreibung des Mantelmaterials sind beide Mechanismen zu berücksichtigen, wobei der jeweilige Beitrag von den vorliegenden Spannungs- und Temperaturbedingungen abhängt und von der Korngröße beeinflusst wird. Darüber hinaus haben der Wassergehalt und das Vorhandensein von Schmelzen einen Einfluss auf das Fließverhalten.

Labormessungen zeigen, dass im Gegensatz zur Theorie die Werte für das Aktivierungsvolumen $V^{*}$ und die Aktivierungsenergie $E^{*}$ im Arrheniusterm von der verwendeten Rheologie abhängen. Auch hier spielt der Wassergehalt eine signifikante Rolle.

\begin{tabular}{|l|c|c|c|c|c|}
\hline & $\mathrm{A}[1 / \mathrm{s}]$ & $\mathrm{n}$ & $\mathrm{m}$ & $E^{*}[\mathrm{~kJ} / \mathrm{mol}]$ & $V^{*}\left[\mathrm{~cm}^{3} / \mathrm{mol}\right]$ \\
\hline Dislokationskriechen & & & & & \\
\hline trockener Olivin & $3.5 \cdot 10^{22}$ & 3.5 & 0.0 & 540 & $15-25$ \\
\hline wassergesättigter Olivin & $2.0 \cdot 10^{18}$ & 3.0 & 0.0 & 430 & $10-20$ \\
\hline Diffusionskriechen & & & & & \\
\hline trockener Olivin & $8.7 \cdot 10^{15}$ & 1.0 & 2.0 & 300 & 6 \\
\hline wassergesättigter Olivin & $5.3 \cdot 10^{15}$ & 1.0 & 3.0 & 240 & 5 \\
\hline
\end{tabular}

Tabelle 3.1: Viskositätsparameter für Olivin. Korngröße: $d=3 \mathrm{~mm}$, Betrag des Burgesvektors: $b=0.55 \mathrm{~nm}$, Schermodul: $\mu=80 \mathrm{GPa}$ (Karato und Wu 1993).

Die Parameter für Olivin, die Karato und Wu (1993) für ihre Betrachtung der Rheologie des oberen Mantels zusammenstellen, sind in Tabelle (3.1) angeben. Karato und Wu (1993) weisen darauf hin, dass es einerseits nur wenige Messungen des Aktivierungsvolumen beim Dislokationsmechanismus gibt und andererseits diese Messungen mit großen Messunsicherheiten behaftet sind, so dass sie dieses vergleichsweise große Intervall angeben. Für das Aktivierungsvolumen in wasserhaltigen Zustand fehlen die Labordaten, so dass Karato und Wu (1993) das Aktivierungsvolumen für wasserhaltigen Olivin aus dem Wert für trockenen Olivin ableiten.

Für das Konvektionsmodell wird vereinfacht, eine Newton'sche Rheologie, ein linearer Zusammenhang $(n=1)$ zwischen Spannung und Dehnung angenommen und der Einfluß der Korngröße vernachlässigt, so dass mit $\eta=\tau_{i j} /\left(2 \dot{\epsilon}_{i j}\right)$ gilt:

$$
\eta=A^{\prime} \exp \left(\frac{E^{*}+p V^{*}}{R T}\right)
$$

Dabei ist $A^{\prime}$ eine von der Korngröße unabhängige Konstante. Desweiteren wird die Druckabhängigkeit auf den hydrostatischen Druckanteil reduziert und die Temperaturabhängigkeit vereinfacht, indem statt der Temperatur $T(r, \theta, \phi)$ die über Kugelschalen mit gleichem Radius $r$ gemittelte Temperatur $\langle T(r)>$ verwendet wird. Die Viskosität variiert so lediglich über die Tiefe und ist auf jeder Kugeloberfläche konstant. SunderPlaßmann (1996) zeigt in seiner Diplomarbeit, dass zumindest im kartesischen 2D-Fall 
eine gute Übereinstimmung des Konvektionsmuster zwischen dem Fall mit obiger Vereinfachung und dem Fall, in dem eine vollständige Temperaturabhängigkeit benutzt wird, besteht. Damit wird folgendes Viskositätsgesetz mit dem Normalisierungsfaktor $\eta_{0}$ und dem Planeten- bzw. Außenradius $r_{a}$ verwendet:

$$
\eta(r,<T(r)>)=\eta_{0} \exp \left(\frac{E^{*}+\rho_{0} g\left(r_{a}-r\right) V^{*}}{R<T(r)>}\right)
$$

bzw. in entdimensionalisierter Form:

$$
\eta^{\prime}\left(r^{\prime},<T^{\prime}\left(r^{\prime}\right)>\right)=\eta_{0}^{\prime} \exp \left(\frac{E^{\prime *}+\left(r_{a}^{\prime}-r^{\prime}\right) V^{\prime *}}{<T^{\prime}\left(r^{\prime}\right)>+T_{\text {surf }}^{\prime}}\right)
$$

mit

$$
V^{*}=\frac{V^{*} \rho g d}{R \Delta T} \quad E^{* *}=\frac{E^{*}}{R \Delta T} \quad T_{\text {surf }}^{\prime}=\frac{T_{\text {surf }}}{\Delta T} \quad \eta_{0}^{\prime}=\frac{\eta_{0}}{\eta_{\text {ref }}}
$$

(Die Striche $\left({ }^{\prime}\right)$ entfallen im Folgenden wieder.)

Christensen (1984) vergleicht in Modellrechnungen die Effekte linearer und nicht linearer Fließgesetze und zeigt, dass die Newtonsche Rheologie eine geeignete Parametrisierung liefert, wenn die Parameter $E^{*}$ und $V^{*}$ für das Dislokationskriechen um jeweils einen Faktor $0.3-0.5$ reduziert werden. Damit ergeben sich für trockenen Olivin Werte von: $E^{*}=162-270 \mathrm{~kJ} / \mathrm{mol}$ und $V^{*}=4.5-12.5 \mathrm{~cm}^{3} / \mathrm{mol}$. Sehr hohe Werte für $E^{*} \mathrm{bzw}$. $V^{*}$ führen zu starken Gradienten der Viskosität, insbesondere in den thermischen Grenzschichten nahe der KMG und der Oberfläche. Diese starken Gradienten können zu numerischen Problemen führen, so dass die Parameterwahl unter Umständen durch die Numerik begrenzt ist. Für Mars werden folgende Parameter verwendet: $E^{*}=187.5 \mathrm{~kJ} / \mathrm{mol}$ und $V^{*}=3 \mathrm{~cm}^{3} / \mathrm{mol}$. Der Wert für das Aktivierungsvolumen ist aus den erwähnten numerischen Gründen relativ klein gewählt. In Kapitel (5.1) wird die Größe dieses Parameters variiert (auch vergrößert) und der Einfluss auf das Konvektionsmuster und die thermische Evolution untersucht.

Als Referenzviskosität $\eta_{\text {ref }}$ wird der Wert in halber Manteltiefe bei einer typischen Manteltemperatur von $1573 \mathrm{~K}$ benutzt: $\eta_{\text {ref }}=\eta\left(r=r_{i}+\frac{d}{2}, T=1573 \mathrm{~K}\right)=3.3 \cdot 10^{21} \mathrm{~Pa} \mathrm{~s}$. Die Viskosität im Marsmantel kann eventuell über den Werten für die Erde liegen, da der Marsmantel vermutlich weniger Wasser enthält. Variationen von $E^{*}$ und $V^{*}$ mit der Tiefe durch z.B. die Phasengrenzen und den damit verbundenen Veränderungen in den Eigenschaften und möglicherweise im Deformationsverhalten (Sammis et al. 1977, Meade und Jeanloz 1990, Karato und Li 1992) werden im Rahmen dieser Näherung vernachlässigt. Bei der numerischen Behandlung der Viskosität ist zu berücksichtigen, dass in der kalten Lithosphäre sehr hohe Viskositäten und Gradienten der Viskosität auftreten, die zu numerischen Instabilitäten führen. Da die Geschwindigkeiten ab einer bestimmten Viskosität verschwindend klein sind, ist es nicht erforderlich, die Viskosität weit über diesen Wert steigen zu lassen. Für die numerische Realisation ist es sinnvoll, die Viskosität durch einen 'cutoff' zu beschränken. Ist dieser 'cutoff' hoch genug gewählt, werden die Ergebnisse durch diesen Eingriff kaum beeinflusst. Im verwendeten Programm wird ein 'cutoff' in der Temperatur verwendet, so dass die Viskosität nicht mit $\langle T(r)\rangle$ berechnet wird, sondern mit einer nach unten durch $T_{\text {cutoff }}$ beschränkten Temperatur $T_{\text {cut }}(r)$, die sich aus 
folgender Gleichung ergibt:

$$
\left(T_{\text {cut }}(r)\right)^{6}=(<T(r)>)^{6}+\left(T_{\text {cutoff }}\right)^{6}
$$

Dieses 'cutoff'-Verfahren wirkt sich stark auf die niedrigen Temperaturen aus und ermöglicht einen glatten Übergang in den 'cutoff'-Wert und lässt dabei die mittleren und hohen Temperaturen weitgehend unberührt.

Eine temperatur- und druckabhängige Viskosität ist in den thermischen Evolutionsmodellen von großer Bedeutung. In der kalten oberen thermischen Grenzschicht erreicht die Viskosität sehr hohe Werte, die Bewegungen in diesem Bereich unmöglich machen, so dass Wärmetransport nur durch Wärmeleitung möglich ist und der Wärmeverlust des Planeten verlangsamt wird. Der Effekt wird umso größer je dicker die Lithosphäre wird. Auch im restlichen Mantel nimmt die Viskosität während des Abkühlvorgangs des Planeten zu, was die Konvektionsbewegung schwächt und ebenfalls die Abkühlrate des Mantels senkt. Die Druckabhängigkeit der Viskosität wird besonders deutlich im isothermen Bereich des Temperaturprofils. Sie erzeugt eine niedrigviskose Zone unterhalb der oberflächennahen thermischen Grenzschicht und sorgt dafür, dass die Viskosität mit der Tiefe zunimmt und erst in der kernnahen thermischen Grenzschicht wieder abfällt. Die durch die Druckabhängigkeit höheren Viskositäten im unteren Mantel sind besonders wichtig für das Abkühlen des Kerns und haben einen wichtigen Einfluss auf die Wirkung der kernnahen endothermen Phasengrenze (vgl. Kapitel (5.1)).

\subsection{Thermischer Ausdehnungskoeffizient}

Der thermische Ausdehnungskoeffizient $\alpha$ bestimmt im Konvektionsproblem die Größe der konvektionstreibenden Auftriebskräfte. Deshalb hat seine Variation im Mantel Einfluss auf die thermische Evolution und das Konvektionsmuster. Die Variation des thermischen Ausdehnungkoeffizienten mit dem Druck wird mit Hilfe des Anderson-GrüneisenParameters $\delta_{T}$ beschrieben (Anderson 1967, Birch 1968).

$$
\delta_{T}=-\left(\frac{\ln \alpha}{\ln \rho}\right)_{T}
$$

Chopelas und Boehler (1989) haben mit Hilfe von Hochdruckexperimenten an Olivin und Magnesiumoxiden diesen Parameter bestimmt:

$$
\left(\frac{\ln \alpha}{\ln V}\right)_{T}=5.5 \pm 0.5
$$

Ähliche Werte werden auch von Reynard und Price (1990) in theoretischen, atomistischen Modellen gefunden.

Vernachlässigt man, dass $\delta_{T}$ von $\rho / \rho_{0}$ abhängig $^{1}$ ist, führt die Integration von Gleichung (3.49) zu:

$$
\left(\frac{\alpha}{\alpha_{0}}\right)=\left(\frac{\rho}{\rho_{0}}\right)^{-\delta_{T}}
$$

\footnotetext{
${ }^{1}$ Chopelas und Boehler (1992) zeigen die Druck- und schwache Temperaturabhängigkeit von $\delta_{T}$. Anderson et al. (1992) fi nden ebenfalls eine Variation von $\delta_{T}$
} 
Dabei bezeichnen die mit 0 indizierten Werte, die entsprechenden Parameter bei Normbedingungen. Chopelas und Boehler (1989) zeigen, dass bei hohen Drücken die Temperaturabhängigkeit des thermischen Ausdehnungskoeffizienten vernachlässigbar ist, so dass sie hier nicht berücksichtigt wird.

Die Adams-Williamson-Gleichung beschreibt, wie die Dichte mit dem hydrostatischen Druck zunimmt:

$$
\frac{1}{\rho} \frac{d \rho}{d p}=-\frac{1}{K_{0}^{*}} \quad \text { bzw. } \quad \frac{1}{\rho} \frac{d \rho}{d r}=-\frac{\rho g}{K_{0}^{*}}
$$

bzw. in entdimensionalisierter Form (ohne ')

$$
\frac{1}{\rho} \frac{d \rho}{d r}=-\frac{\rho g d}{K_{0}^{*}}
$$

$K_{0}^{*}$ ist dabei der Kompressionsmodul, der in dieser Gleichung nicht konstant ist (gekenzeichnet mit $\left(^{*}\right)$ ). Der Kompressionsmodul kann aus den seimischen Geschwindigkeiten bestimmt werden. Zur Integration von Gleichung (3.53) verwenden Leitch et al. (1991) die Näherung, dass $K_{0}^{*}$ ungefähr proportional zu $\left(\rho / \rho_{0}\right)^{3}$ ist:

$$
K_{0}^{*} \approx K_{0}\left(\frac{\rho}{\rho_{0}}\right)^{3}
$$

Setzt man diese Annahmen in Gleichung (3.53) ein, erhält man folgendes Dichteprofil:

$$
\rho=\rho_{0}\left(1+2 \frac{\rho_{0} g d}{K_{0}}\left(r_{a}-r\right)\right)^{0.5}
$$

bzw. mit $\delta_{T}=6$ folgendes Profil für den thermischen Ausdehnungskoeffizienten:

$$
\alpha(r)=\alpha_{0}\left(1+2 \frac{\rho_{0} g d}{K_{0}}\left(r_{a}-r\right)\right)^{-3}
$$

$\alpha_{0}$ wird zur Normalisierung so gewählt, dass der Referenzwert $\alpha_{\text {ref }}$ in der halben Manteltiefe bei $r=\left(r_{a}-r_{i}\right) / 2$ erreicht wird. Für die Marsmodelle ergibt sich $\rho_{0} g d / K_{0}=0.16$, so dass der thermische Ausdehnungskoeffizient zwischen KMG und Oberfläche um den Faktor $\alpha\left(r_{a}\right) / \alpha\left(r_{i}\right)=2.3$ zunimmt. Sohl und Spohn (1997) betrachten Strukturmodelle für das Innere des Mars und berechnen u.a. thermodynamische Parameter. Werden in ihrem Profil des thermischen Expansionskoeffiezienten die Effekte der Phasengrenze vernachlässigt, ergibt sich ungefähr der gleiche Wert für $\alpha\left(r_{a}\right) / \alpha\left(r_{i}\right)$. Abbildung (5.15) zeigt das verwendete Profil. Der Einfluss der Phasengrenze wird im Profil nach Gleichung (3.56) nicht weiter berücksichtigt. Die Auftriebseffekte bei Phasenübergängen werden durch den beschriebenen effektiven thermischen Ausdehnungskoeffizienten $\alpha_{\text {eff }}$ in Betracht gezogen.

\subsection{Thermische Leitfähigkeit}

Die thermische Leitfähigkeit $\lambda$ bestimmt als Proportionalitätsfaktor im Fourier-Gesetz (Gleichung (3.5)) die Wärmeflussdichte in Abhängigkeit vom Temperaturgradienten. Sie beschreibt den Beitrag des diffusiven Wärmetransports. In der thermischen Entwicklung 
spielt ihre Größe insbesondere in der Lithosphäre und an der KMG eine wichtige Rolle. In der Lithosphäre ist sie bedeutsam für die Effektivität des Wärmetransport durch die stagnierende Schicht. Im Evolutionsmodell beeinflusst der Wert von $\lambda$ an der KMG die Abkühlrate des Kerns, die proportional zum Wärmefluss aus dem Kern in den Mantel ist (vgl. Abschnitt (3.9)).

Die thermische Leitfähigkeit setzt sich aus einem Beitrag des Kristallgitters durch Gitterschwingungen (Phononen), der vor allem bei niedrigen Temperaturen von Bedeutung ist, und einem Beitrag bei hohem Druck und hohen Temperaturen durch Wärmestrahlung zusammen. Der Beitrag der Phononen zur thermischen Leitfähigkeit wird durch steigenden Druck oder niedrigere Temperaturen erhöht, da einerseits die Frequenz der Gitterschwingungen steigt und die Dichtezunahme zu einer größeren Kollisionswahrscheinlichkeit mit benachbarten Atomen oder Gitterfehlern führt. Der Beitrag der Wärmestrahlung ist vom Druck unabhängig. Hofmeister (1999) leitet folgendes Modell für die thermische Leitfähigkeit des Mantels aus Messungen der Lebensdauer von Phononen anhand des Reflexionsvermögens im Infrarotbereich ab.

$$
\lambda(p, T)=\lambda_{298}\left(\frac{298}{T}\right)^{a} \exp \left[-\left(4 \gamma+\frac{1}{3}\right) \int_{298}^{T} \alpha(\theta) d \theta\right]\left(1+\frac{K_{0}^{\prime} p}{K_{0}}\right)+f(T)
$$

mit dem Anteil durch Wärmestrahlung $f(T)$ in Gegenwart von $\mathrm{Fe}^{2+}$ :

$$
f(T)=0.01753-1.0365 \cdot 10^{-4} T+2.2451 \cdot 10^{-7} T^{2}-3.403 \cdot 10^{-11} T^{3}
$$

Dabei haben die Symbole folgende Bedeutung: $\lambda_{298}$ ist der Wert der thermischen Leitfähigkeit an der Oberfläche bei $T=298 \mathrm{~K}$ und dient der Normierung, $a$ ist ein FitParameter, der vom Typ der chemischen Bindung abhängt und für Silikate den Wert $a=0.3$ hat ( $a=0.9$ für Oxide). $\gamma$ ist der Grüneisenparameter, $K_{0}$ der Kompressionsmodul und $K_{0}^{\prime}=d K_{0} / d p$.

Für die numerische Realisation werden einige Vereinfachungen gemacht. Ähnlich wie bei der Viskosität wird hier ebenfalls eine laterale Variation vermieden, indem statt der Temperatur $T$ erneut die über Kugelschalen mit gleichem Radius gemittelte Temperatur $\langle T(r)\rangle$ verwendet wird. Die Druckabhängigkeit wird durch den hydrostatischen Druckanteil vertreten. Zudem wird vereinfacht ein konstanter, thermischer Ausdehnungkoeffizient $\alpha_{\text {ref }}$ angenommen. Ebenso sind die $K_{0}$ und $K_{0}^{\prime}=d K_{0} / d p$ druck- und temperaturunabhängig. Während in der ursprünglichen Arbeit von Hofmeister (1999) das Profil der thermischen Leitfähigkeit für verschiedene Minerale berechnet worden ist und das Gesamtprofil sich aus einer je nach Druck-und Temperaturbedingungen gewichteten Summe der einzelnen Mineralbeiträge ergibt, wird hier vereinfacht ein Profil berechnet. Für den Fit-Parameter $a$ gilt dabei der Wert für Silikate. Die übrigen Materialparameter sind so gewählt bzw. gemittelt, dass sie im Rahmen ihrer Messgenauigkeit (vgl. Harder (1998)) Olivin bzw. Spinell darstellen. Der größte Teil des Marsmantels besteht aus Olivin/Spinell, der Phasenübergang zum Perowskit nahe der KMG wird bei der Berechnung von $\lambda$ in dieser Vereinfachung nicht berücksichtigt. Für Perowskit ist insbesondere der Kompressionsmodul mit $K_{0}=261 \mathrm{GPa}$ größer als für Olivin/Spinell $K_{0}=160 \mathrm{GPa}$, was bei gleichen Temperatur- und Druckbedingungen zu einer etwas geringeren thermischen Leitfähigkeit unterhalb der Phasengrenze führen würde. Beim Anteil der Wärmestrahlung wird berücksichtigt, dass der Marsmantel reich an $\mathrm{FeO}$ ist (Spohn et al. 1998) und somit $\mathrm{Fe}^{2+}$-Ionen 
erhält. Damit ergibt sich folgende vereinfachte Beziehung:

$$
\begin{aligned}
\lambda(r,<T(r)>)= & \lambda_{298}\left(\frac{298}{<T(r)>}\right)^{a} \exp \left[-\left(4 \gamma+\frac{1}{3}\right)\right. \\
& \left(\alpha_{\mathrm{ref}}(<T(r)>-298)\right]\left(1+\frac{K_{0}^{\prime}\left(\rho_{0} g\left(r_{a}-r\right)\right)}{K_{0}}\right) \\
& +f(<T(r)>)
\end{aligned}
$$

mit dem Anteil durch Wärmestrahlung $f(T)$ :

$$
\begin{aligned}
f(<T(r)>)= & 0.01753-1.0365 \cdot 10^{-4}<T(r)>+ \\
& 2.2451 \cdot 10^{-7}<T(r)>^{2}-3.403 \cdot 10^{-11}<T(r)>^{3}
\end{aligned}
$$

Es werden, soweit nicht explizit geändert, folgende Materialparameter zur Berechnung der thermischen Leitfähigkeit im Marsmantel verwendet: $\lambda_{298}=4.7 \mathrm{~W} / \mathrm{mK}, a=0.3$, $\alpha_{\text {ref }}=2.0 \cdot 10^{-5} 1 / \mathrm{K}, \gamma=1.2, K_{0}=160 \mathrm{GPa}$ und $d K_{0} / d p=5$ (siehe z.B. Harder (1998), Hofmeister (1999) oder Sohl und Spohn (1997)).

In der dimensionslosen Darstellung wird das Profil so normiert, dass $\lambda^{\prime}$ in halber Tiefe und bei $T=1573 \mathrm{~K}$ den Wert 1 hat $\left(r_{i}\right.$ : Radius der KMG):

$$
\lambda^{\prime}(r,<T(r)>)=\frac{\lambda(r,<T(r)>)}{\lambda\left(0.5\left(r_{a}-r_{i}\right), 1573 \mathrm{~K}\right)}
$$

Yanagawa et al. (2004) vergleichen in einem kartesischen 2D-Fall in der BoussinesqNäherung einige Modelle mit vollständiger Temperaturabhängigkeit der thermischen Leitfähigkeit, in denen sowohl eine tiefenabhängige als auch eine horizontale Variation möglich ist, mit Modellen, die nur eine von der horizontal gemittelten Temperatur abhängige Leitfähigkeit berücksichtigen. Das Profil der thermischen Leitfähigkeit ist in vereinfachter Form an den Phononenanteil von Hofmeister (1999) $(a=0.9)$ angelehnt. Sie kommen in diesem Beispiel zu dem Ergebnis, dass keine signifikanten Unterschiede in der Struktur der Konvektionszellen, der Abkühlrate, gemittelten Temperatur und den Geschwindigkeiten durch das Vernachlässigen der lateralen Variation entstehen. In ihrem Modellen sind diese Größen zumindest bei ausreichend hohen Rayleighzahlen durch die Tiefenabhängigkeit der thermischen Leitfähigkeit bestimmt.

Mit dem Starttemperaturprofil ergibt sich der folgende Verlauf (Abbildung (5.26)): Vom Oberflächenwert fällt die thermische Leitfähigkeit in der thermischen Grenzschicht stark ab und durchläuft ein Minimum, wenn die Temperatur in den isothermen Bereich übergeht. Durch die Druckabhängigkeit steigt die Leitfähigkeit dann an und erreicht ein relatives Maximum bevor sie in der unteren thermischen Grenzschicht wieder abfällt.

\subsection{Interne Wärmequellen}

Das Mantelgestein der terrestrischen Planeten enthält langlebige radioaktive Elemente wie ${ }^{235} \mathrm{U},{ }^{238} \mathrm{U},{ }^{232} \mathrm{Th}$ und ${ }^{40} \mathrm{~K}$ als Spurenelemente, die durch ihren Zerfall Wärme produzieren. Die anfängliche Konzentration $C_{0}^{i}$ vor $t=4.5 \mathrm{Ga}$ eines jeden Isotops $i$ mit der Zerfallskonstante $\lambda_{i}^{*}$ kann mit dem Zerfallsgesetz aus der heutigen Konzentration $C_{p}^{i}$ bestimmt werden:

$$
C_{0}^{i}=C_{p}^{i} \exp \left(\lambda_{i}^{*} t\right)
$$


Mit der Wärmeproduktionsrate $h_{i}$ pro Volumen der einzelnen Isotope kann die durch radioaktiven Zerfall erzeugte Wärme bestimmt werden:

$$
H(t)=\sum_{i=1}^{4} a_{i} C_{0}^{i} h_{i} \exp \left(-\lambda_{i}^{*} t\right)
$$

$a_{i}$ gibt dabei den Anteil des radioaktiven Isotops am Gesamtgehalt des Elementes im Mantel an.

Im Modell unterbleibt zur Vereinfachung die Aufschlüsselung nach den einzelnen Elementen. Es wird eine gemittelte Wärmeproduktionsrate und eine mittlere Zerfallszeit benutzt.

$$
H(t)=H_{0} \cdot \exp \left(-\lambda^{*} t\right)
$$

Bei einer gemittelten Zerfallszeit von $\lambda^{*}=0.434(\mathrm{Ga})^{-1}$ ist die Heizrate nach $4.5 \mathrm{Ga}$ auf ein Siebtel des Startwertes gefallen. Bei einer chondritischen Zusammensetzung des Marsmantels ergibt sich eine heutige Wärmeproduktionsrate von $H_{\text {chond. }}$ (heute) $=1.95$. $10^{-8} \mathrm{Wm}^{-3}$ (Harder und Christensen 1996) bzw. nach dem Zerfallsgesetz eine Heizrate von $H_{\text {chond. }}(t=0)=1.365 \cdot 10^{-7} \mathrm{Wm}^{-3}$ vor $4.5 \mathrm{Ga}$. Hohe interne Heizraten zu Beginn der Evolution führen bei hohen Rayleighzahlen zu numerischen Instabilitäten, so dass ein Kompromiss zwischen der numerischen Machbarkeit und der Realitätstreue der Parameter gefunden werden muss. In den Evolutionsmodellen für Mars wird $H_{0}=1.836 \cdot 10^{-8} \mathrm{Wm}^{-3}$ benutzt. Dieser Wert entspricht etwa $13.5 \%$ des chondritischen Wertes und simuliert so einen zu stark an radioaktiven Elementen verarmten Mantel. Dadurch wird der Mantel zu schwach von innen beheizt und kühlt schneller ab. Die Heizrate ist dennoch hoch genug, um gegenüber der Wärme aus dem Kern eine bedeutende Wärmequelle darzustellen. Eine gewisse Reduktion gegenüber dem chondritischen Wert ist sinnvoll, da die radioaktiven Elemente lithophil sind und sich in der Kruste anreichern und somit nicht zum Heizen des gesamten Mantels beitragen. Im Modell ist die Heizrate homogen über den Mantel verteilt. Die vernachlässigte Aufschlüsselung nach den einzelnen Isotopen führt u.a. dazu, dass die anfängliche Heizrate im Modell unterschätzt wird, da der starke Beitrag durch den Zerfall des sehr kurzlebigen ${ }^{40} \mathrm{~K}$ gedämpft wird.

\subsection{Rand- und Startbedingungen}

Um die Gleichungen (3.40) - (3.42) zu lösen, müssen Randbedingungen an das Temperatur- und Geschwindigkeitsfeld gestellt werden. Für die Radialkomponente der Geschwindigkeit $v_{r}$ muss gefordert werden, dass $v_{r}\left(r_{i}, t\right)=v_{r}\left(r_{a}, t\right)=0$ gilt, da kein Material durch die Grenzflächen in den Mantel zu- bzw. aus dem Mantel abströmen kann. Bei den weiteren Forderungen an das Geschwindigkeitsfeld wird berücksichtigt, dass Mars heute keine Plattentektonik hat, sondern ein sogenannter Ein-Platten-Planet ist. Um diese Beobachtung realistisch zu simulieren, muss die Geschwindigkeit an der Oberfläche verschwinden, was durch die 'no-slip'-Randbedingung gewährleistet wird.

$$
\vec{v}\left(r_{a}, t\right)=0
$$

Harder (2000) zeigt, dass eine 'free-slip'-Randbedingung an der Oberfläche bei nicht ausreichend hohem Viskositätskontrast in der Lithosphäre bzw. eine weiche, halb-bewegliche 
Lithosphäre zu einer Reduzierung der Anzahl der Aufströme führen kann. Dieser Effekt überlagert sich dem Einfluss der Phasengrenze. Die 'no-slip'-Randbedingung vermeidet diesen Einfluss auf das Konvektionsmuster.

An der KMG wird die Bedingung des freien Gleitens bzw. eine 'free-slip'-Bedingung verwendet. Dabei müssen neben der Radialkomponente der Geschwindigkeit die Scherspannungen -die horizontalen Komponenten des Spannungstensors- an der Oberfläche verschwinden:

$$
v_{r}\left(r_{i}, t\right)=0 \quad \sigma_{r \theta}\left(r_{i}, t\right)=\sigma_{r \phi}\left(r_{i}, t\right)=0
$$

Die Beziehung zwischen Spannungen und Dehnungsraten (Gleichung (3.11)) liefert dann folgende Bedingung für die Geschwindigkeit in Kugelkoordinaten:

$$
v_{r}\left(r_{i}, t\right)=\left.0 \quad \frac{\partial}{\partial r}\left(\frac{v_{\theta}}{r}\right)\right|_{\left(r=r_{i}, t\right)}=\left.\frac{\partial}{\partial r}\left(\frac{v_{\phi}}{r}\right)\right|_{\left(r=r_{i}, t\right)}=0
$$

Bei der Temperatur an der Oberfläche wird eine Änderung mit der Zeit vernachlässigt und sie wird als konstant angenommen:

$$
T\left(r_{a}, t\right)=T_{\text {surf }} \quad \text { bzw. entdimensionalisiert } \quad T^{\prime}\left(r_{a}, t\right)=0
$$

Für die Temperatur an der KMG $T\left(r_{i}, t\right)=T_{c}(t)$ hingegen wird berücksichtigt, dass der Kern langsam abkühlt, indem er Wärme an den Mantel abgibt. Die Änderung der Energie des Kerns lässt sich durch folgende Bilanz beschreiben:

$$
C_{\mathrm{Kern}} \rho_{\mathrm{Kern}} V_{\mathrm{Kern}} \frac{d T_{c}(t)}{d t}=A_{\mathrm{Kern}} \lambda\left(r_{i}\right) \frac{d T_{c}(t)}{d r}+H_{\mathrm{Kern}} V_{\mathrm{Kern}}
$$

Dabei ist $C_{\text {Kern }}$ die spezifische Wärmekapazität (bei konstantem Druck) des Kerns, $\rho_{\text {Kern }}$ die Dichte des Kernmaterials, $V_{\text {Kern }}=4 / 3 \pi r_{i}^{3}$ das Volumen des Kerns, $A_{\text {Kern }}=4 \pi r_{i}^{3}$ entsprechend die Oberfläche des Kerns und $H_{\text {Kern }}$ die Wärmeproduktionsrate pro Volumen durch interne Wärmequellen im Kern. $C_{\text {Kern }}$ wird etwas größer gewählt als der Materialwert und korrigiert so, dass $T_{c}$ kleiner als die volumengemittelte Temperatur im Kern ist. Es wird angenommen, dass das Material im Kern heftiger konvektiert als der Mantel, so dass die Oberfläche des Kerns isotherm ist und keine lateralen Temperaturvariationen auf der Randfläche auftreten. Das Abkühlen des Kerns wird dann bestimmt durch den Temperaturgradienten an der KMG, der seinerseits von der Konvektion im Mantel abhängt. Interne Wärmequellen im Kern wie z.B. radioaktive Elemente werden vernachlässigt $\left(H_{\text {Kern }}=0\right)$. Desweiteren bleiben thermische Effekte durch das mögliche Ausfrieren eines inneren Kerns hier unberücksichtigt. Diese Annahme wird durch die Betrachtung des Kerns in Kapitel (5.6) gerechtfertigt. Entdimensionaliert man die Leistungsbilanz (Striche $\left({ }^{\prime}\right)$ wieder weggelassen) nach dem beschriebenen Schema, ergibt sich folgende Differentialgleichung, aus der in jedem Zeitschritt die aktuelle Temperatur an der KMG berechnet werden muss.

$$
\frac{d T_{c}(t)}{d t}=3 \frac{\lambda\left(r_{i}\right)}{\kappa C_{\text {Kern }} \rho_{\text {Kern }}} \frac{1}{r_{i}} \frac{d T_{c}(t)}{d r}=3 \frac{\lambda\left(r_{i}\right)}{\lambda_{\text {ref }}} \frac{\rho_{\text {Mantel }} C_{\text {Mantel }}}{\rho_{\text {Kern }} C_{\text {Kern }}} \frac{1}{r_{i}} \frac{d T_{c}(t)}{d r}
$$

Diese Differentialgleichung wird in jeden Zeitschritt mit einem einfachen Euler-EinschrittVerfahren integriert. Die Implementierung in den numerischen Code und ein geeignetes 
Testverfahren wird in Buske (2002) beschrieben.

Zur Berechnung der thermischen Evolution muss ein Starttemperaturfeld vorgegeben werden. Als Startprofil dient ein Grenzschichtprofil, das durch dünne Grenzschichten an der KMG (Dicke: $s_{\text {bot }}$ ) und an der Oberfläche (Dicke: $s_{\text {top }}$ ) und hohe Temperaturen im Inneren gekennzeichnet ist. Dieses Profil soll stark vereinfacht die Situation kurz nach der Akkretion des Planeten und seiner Differentiation in Mantel und Eisenkern beschreiben. Mathematisch hat es folgende Gestalt (dimensionslose Größen):

$$
T(r, 0)=\left\{\begin{array}{lc}
T_{\text {int }} \sin \left((\pi / 2)\left(r_{a}-r\right) / s_{\text {top }}\right) & \left(r_{a}-r\right) / s_{\text {top }}<1 \\
T_{c}(0)-\left(T_{c}(0)-T_{\text {int }}\right) \sin \left((\pi / 2)\left(r-r_{i}\right) / s_{\text {bot }}\right) & \left(r-r_{i}\right) / s_{\text {bot }}<1 \\
T_{\text {int }} & \text { sonst }
\end{array}\right.
$$

$T_{\text {int }}$ ist dabei die anfängliche Temperatur im Mantelinneren, die auch durch die numerische Machbarkeit beschränkt ist. Abbildung (5.1) zeigt einen Verlauf dieses Profils. Für die Simulationen werden folgende Werte verwendet: $s_{\text {top }}=306 \mathrm{~km}, s_{\text {bot }}=204 \mathrm{~km}$ und $T_{\mathrm{int}}=1751 \mathrm{~K}$. Bei zu hohen Starttemperaturen im Inneren oder zu dünnen Grenzschichten führt die Heftigkeit der Konvektion bzw. ein zu großer Gradient in der Viskosität zu numerischen Instabilitäten.

Diesem Profil werden Störungen aufgegeben, die in radialer Richtung durch ein gerades Polynom sechster Ordnung beschrieben werden. In lateraler Richtung wird eine Störung aufgeprägt, die durch zufällige Auswahl von Moden und Amplituden der Kugelflächenfunktion bestimmt wird. Diese Startkonfiguration wird bei den verschiedenen Simulationen nicht variiert. Die zufällige Störung gewährleistet das Vorhandensein auch kleinskaliger Störungen und repräsentiert die Tatsache, dass die wirkliche Starttemperaturverteilung im Planeten nicht bekannt ist. Die aufgebrachte Störung führt zum Einsatz der Konvektion. 


\section{Numerische Methoden}

In diesem Kapitel werden die Erweiterungen des numerischen Programms und deren Tests kurz beschrieben. Der Code, der die Gleichungen (3.40) - (3.42) zusammen mit den Randbedingungen löst, ist aus dem Dynamocode von Glatzmaier und Christensen entstanden. In Glatzmaier (1984) wird das zugrunde liegende numerische Verfahren angewandt auf das Problem von konvektionsbetriebenen Dynamos in Sternen (kompressibles Medium, anelastische Näherung) beschrieben. Christensen hat den Code später für den Dynamoprozess im flüssigen Eisenkern der Erde (inkompressibles Medium in der Boussinesq-Approximation) umformuliert. Zur Lösung des Mantelkonvektionsproblems kann der Code weiter vereinfacht werden, indem nur der Konvektionsteil betrachtet wird und die Erzeugung bzw. Vernichtung von Magnetfeld sowie der Einfluss von magnetischen Kräften vernachlässigt werden. Desweiteren entfällt der Beitrag der Trägheitskräfte aufgrund der hohen Prandtlzahl. Damit liegt ein semispektraler Code vor, der die strömungsmechanischen Gleichungen ohne Phasengrenzen, bei konstanten Materialparametern in der Boussinesq-Näherung in einer Kugelschale löst. Die Implementation einer Phasengrenze, der tiefenabhängigen, druck-und temperaturbestimmten Viskosität und der zeitabhängigen Temperaturrandbedingung an der KMG wird in Buske (2002) vorgestellt.

\subsection{Grundlagen}

In diesem Abschnitt soll das numerische Verfahren grob skizziert werden. Bei der numerischen Umsetzung der Gleichungen wird ausgenutzt, dass das Geschwindigkeitsfeld nach Gleichung (3.40) divergenzfrei ist und sich somit durch einen poloidalen und einen toroidalen Anteil darstellen lässt. Zu beiden Vektorfeldern gibt es ein skalares Potential $W$ für den poloidalen und $Z$ für den toroidalen Beitrag, so dass sich die Geschwindigkeit schreiben lässt als:

$$
\vec{v}=\vec{\nabla} \times \vec{\nabla} \times\left(W \vec{e}_{r}\right)+\vec{\nabla} \times\left(Z \vec{e}_{r}\right)
$$

Diese Darstellung erfüllt implizit die Massenerhaltung. Das toroidale Geschwindigkeitsfeld hat keine radiale Komponente. Im Fall einer tiefenabhängigen, lateral aber konstanten Viskosität ist das toroidale Feld unabhängig vom Auftriebsterm und der Temperatur, da das toroidale Feld keinen Beitrag zur Radialkomponente der Stokes-Gleichung liefert (Billich 1995). Dies wird bei Betrachtung der viskosen Kräfte in der Stokes-Gleichung (3.41) deutlich:

$$
\vec{\nabla} \cdot \underline{\underline{\tau}}=\vec{\nabla} \cdot(2 \eta \underline{\underline{\dot{\epsilon}}})=\eta \Delta \vec{v}+2 \underline{\underline{\dot{\epsilon}}} \cdot \vec{\nabla} \eta=-\eta(\vec{\nabla} \times \vec{\nabla} \times \vec{v})+2 \underline{\underline{\dot{\epsilon}}} \cdot \vec{\nabla} \eta
$$


Für den toroidalen Beitrag zur Radialkomponente gilt:

$$
\left[-\eta\left(\vec{\nabla} \times \vec{\nabla} \times \vec{v}^{Z}\right)+2 \underline{\underline{\dot{\epsilon}}}^{Z} \cdot \vec{\nabla} \eta\right] \cdot \vec{e}_{r}=\left[-\eta \vec{\nabla} \times \vec{\nabla} \times\left(\vec{\nabla} \times\left(Z \vec{e}_{r}\right)\right)+2 \underline{\underline{\dot{\epsilon}}}^{Z} \cdot\left(\frac{\partial \eta}{\partial r}\right) \vec{e}_{r}\right] \cdot \vec{e}_{r}
$$

Der erste Term auf der rechten Seite lässt sich umformen zu:

$$
\left[-\eta \vec{\nabla} \times \vec{\nabla} \times\left(\vec{\nabla} \times\left(Z \vec{e}_{r}\right)\right)\right] \cdot \vec{e}_{r}=\left[\eta \vec{\nabla} \times(\Delta Z) \vec{e}_{r}\right] \cdot \vec{e}_{r}=0
$$

Die Radialkomponente des toroidalen Geschwindikeitsfeldes verschwindet, so dass für den zweiten Ausdruck folgt:

$$
\left[2 \underline{\underline{\epsilon}}^{Z} \cdot\left(\frac{\partial \eta}{\partial r}\right) \vec{e}_{r}\right] \cdot \vec{e}_{r}=2 \dot{\epsilon}_{r r}^{Z} \frac{\partial \eta}{\partial r}=0
$$

Das toroidale Feld trägt somit nicht zur Radialkomponente bei und ist vom Auftriebsterm abgekoppelt. Da die Auftriebskräfte aber die Reibungseffeke balancieren, muss der toroidale Anteil der Reibungskräfte verschwinden bzw. $\underline{\underline{\epsilon}}^{Z}=0$ gelten. Das toroidale Feld beschreibt daher eine starre Festkörperrotation, die hier vernachlässigt werden kann und es gilt: $Z=0$.

So bleiben die Gleichungen (3.41) - (3.42), um die skalaren Felder $T, p$ und $W$ zu bestimmen. In der Kugelschale werden die Felder in lateraler Richtung durch vollständig normierte Kugelflächenfunktion $Y_{l}^{m}(\theta, \phi)$ mit Grad $l$ und Ordnung $m$ dargestellt. Für die Kugelflächenfunktionen gilt mit den zugeordneten Legendre-Polynomen $P_{l}^{m}$ :

$$
Y_{l}^{m}(\theta, \phi)=\frac{1}{\sqrt{2 \pi}} \exp (i m \phi) P_{l}^{m}(\cos \theta)
$$

Die räumliche Auflösung in lateraler Richtung wird durch den maximalen Grad $l_{\max }$ und die maximale Ordnung $m_{\max }$ der verwendeten Kugelflächenfunktion beschränkt. Die radiale Abhängigkeit wird durch Tschebyscheff-Polynome $T_{n}(r)$ beschrieben, die folgendermaßen auf dem Intervall [-1,1] definiert sind:

$$
T_{n}(x)=\cos (n \arccos x)
$$

Die Gitterpunkte in radialer Richtung werden aus den $\mathrm{x}$-Werten $x_{k}$ der Extrema des Tschebyscheff-Polynoms mit dem höchsten $n=N$ bestimmt. Diese Extrema liegen bei:

$$
x_{k}=\cos \left(\frac{k \pi}{N}\right) \quad 0 \leq k \leq N
$$

Die radialen Level werden durch eine Transformation auf das Intervall [-1,1] abgbildet. Für eine lineare Transformation gilt:

$$
x=\frac{2 r-r_{a}-r_{i}}{r_{a}-r_{i}}
$$

Hier sind die Gitterpunkte stark an den Rändern konzentriert. Dies führt zu einer hohen Punktdichte auch in der Lithosphäre, in der die Geschwindigkeiten nahezu verschwinden, so dass keine hohe numerische Auflösung benötigt wird. Hingegen werden viele radiale Gitterpunkte im Bereich der starken Viskositätsgradienten benötigt. Tilgner (1999) 
schlägt eine nicht lineare Abbildungsfunktion zwischen den festen Tschebyscheff-Gitterpunkten $x_{k}$ und den radialen Leveln vor, die erlaubt, die Gitterpunkte in numerisch sinnvollen Regionen zu konzentrieren. Diese Abbildungsfunktion muss umkehrbar und mehrfach differenzierbar sein. Auch die Umkehrfunktion sollte differenzierbar sein, da die Ableitung bei der Integration zur Bestimmung von Werten, wie z.B. der kinetischen Energie, gebraucht wird. Eine geringfügige Verbesserung der numerischen Stabilität konnte in den Evolutionsmodellen mit einem Polynom achter Ordnung erzielt werden:

$$
x_{k}=\sum_{i=0}^{8} a_{i} r_{k}^{i} \quad 0<k<N \quad\left(a_{i} \text { : siehe Anhang }\right)
$$

Die radialen Level müssen hier so bestimmt werden, dass $x_{k}$ den Tschebyscheff-Gitterpunkten entspricht ( $r_{i}$ und $r_{a}$ liegen zusätzlich fest durch: $r_{a}-r_{i}=1$ und $r_{i} / r_{a}=0.4$ (für Mars)). Bei radialen Ableitungen ist die Kettenregel zu berücksichtigen, was dazu führt, dass bei einer nicht linearen Abbildungsfunktion nicht konstante Ableitungen $d x / d r$ bzw. entsprechend höhere Ableitungen auftreten.

Die nicht lineare Abbildungsfunktion verbessert die numerische Stabilität im Evolutionsfall nur geringfügig, da einerseits durch die Anforderungen an die Abbildungsfunktion nicht jede Punktverteilung realisiert werden kann und zu starke Gradienten in der Abbildungsfunktion selbst ihrerseits zu numerischen Problemen führen. Erschwerend kommt im Evolutionsmodell hinzu, dass der Bereich hoher Viskositätsgradienten, der einer hohen numerischen Auflösung bedarf, sich mit der Zeit durch die Abkühlung des Systems verschiebt und so ggf. ein zeitlich variables Gitter benötigt wird.

In der Darstellung durch Kugelflächenfunktionen und Tschebyscheff-Polynome ergibt sich z.B. für $W$ folgende Form:

$$
W(t, r, \theta, \phi)=\sum_{l, m} W_{l}^{m}(r, t) Y_{l}^{m}(\theta, \phi)
$$

mit

$$
W_{l}^{m}(r, t)=\left(\frac{2}{N}\right)^{\frac{1}{2}} \sum_{n=0}^{N}{ }^{\prime \prime} W_{l n}^{m}(t) T_{n}(r)
$$

Hier kennzeichnet $\left({ }^{\prime \prime}\right)$, dass die Terme mit $n=0$ und $n=N$ zur richtigen Normierung mit $\frac{1}{2}$ multipliziert werden.

Für das numerische Lösungsverfahren werden die Wärmeleitungsgleichung (3.42), die radiale Komponente der Stokes-Gleichung (3.41) sowie deren horizontale Divergenz herangezogen. Nachdem die Geschwindigkeit durch das skalare Potential $W$ ausgedrückt ist, werden alle Größen durch die Entwicklung nach Kugelflächenfunktionen ersetzt. Als Zeitschrittverfahren werden das explizite Adams-Bashforth-Verfahren zweiter Ordnung und das implizite Crank-Nicolson-Verfahren zweiter Ordnung für die Diffusionsterme verwendet. Die gesuchten Tschebyscheff-Koeffizienten z.B. $W_{l}^{m}$ werden mit der Tschebyscheff-Kollokations-Methode bestimmt, indem die zeitlich diskretisierten, in $l$ und $m$ entkoppelten Gleichungen in den Tschebyscheff-Raum $W_{l n}^{m}$ transformiert werden und dann an den inneren Kollokationspunkten $x_{k}$ (an den Randpunkte gelten die Randbedingungen) gelöst werden. Im Tschebyscheff-Raum werden die benötigten Ableitungen berechnet, bevor die Rücktransformation in den radialen Raum erfolgt. 
Die Berechnung von Produkten in den nicht linearen Termen, wie z.B. im Advektionsterm, erfolgt nicht im Funktionenraum. Im Funktionenraum sind die Produkte zweier Größen als das Produkt zweier (theoretisch unendlichen) Summen zu berechnen bzw. das Integral einer Faltung ist zu bestimmen. Diese erfordert einen hohen mathematischen und rechnerischen Aufwand, der verringert werden kann, indem diese Produkte nicht im Funktionenraum berechnet werden, sondern nach einer Transformation in den Ortsraum für jeden Gitterpunkt bestimmt werden. Das so erhaltene Produkt wird dann in den Funktionenraum zurück transformiert. Für die hierzu notwendigen Tranformationen sowie für die Transformation in den Tschebyscheff-Raum stehen leistungsfähige Algorithmen (z.B. Fast-Fourier-Transformation) zur Verfügung.

Spektrale Methoden sind finite Differenzen Methoden insofern überlegen, dass zum Erreichen der gleichen Genauigkeit eine geringere Auflösung erforderlich ist und die Darstellung durch orthogonale Funktionen Informationen auf der ganzen Kugelschale liefert und nicht nur an vorbestimmten Gitterpunkten. Hinzu kommt, dass Ableitungen im Funktionenraum exakt berechnet werden können. Details und Beispiele finden sich bei Glatzmaier (1984) und Tilgner (1999).

\subsection{Gitterparameter: räumliche Auflösung}

Für die Simulationen werden folgende Parameter für das Gitter und die räumliche Auflösung benutzt: Die Anzahl der Tschebyscheff-Gitterpunkte beträgt 145. Es werden 109 Tschebyscheff-Polynome verwendet. In $\phi$-Richtung enthält das Gitter 288 Punkte, in $\theta$ Richtung 144 Punkte. Für den maximalen Grad bzw. die maximale Ordnung der verwendeten Kugelflächenfunktion gilt: $l_{\max }=m_{\max }=120$. Die Anzahl der Gitterpunkte übersteigt sowohl in radialer Richtung als auch in den Winkelrichtungen die Ordnung der verwendeten Funktionen. Dies vermeidet bzw. verringert sogenannte 'aliasing'-Effekte. Beim 'aliasing' werden bei den Transformationen zwischen Ortraum und Funktionenraum höhere Moden, die bei der Multiplikation entstehen, nicht als solche erkannt, wenn sie nicht durch genügend Gitterpunkte abgedeckt werden.

\subsection{Tiefenabhängiges $\lambda$}

Bei Verwendung einer tiefenabhängigen thermischen Leitfähigkeit muss bei der Behandlung des Diffusionsterms ein zusätzlicher Ausdruck berücksichtigt werden. Bei konstanter thermischer Leitfähigkeit hat der Diffusionsterm folgende Gestalt:

$$
\vec{\nabla} \cdot(\lambda \vec{\nabla} T)=\lambda \Delta T
$$

Im Modell (siehe Abschnitt (3.7)) variiert die thermische Leitfähigkeit in Abhängigkeit von der horizontal gemittelten Temperatur und dem hydrostatischen Druck mit der Tiefe (Gleichung (3.59)). Sie ist lateral konstant. Für diesen Fall gilt:

$$
\vec{\nabla} \cdot(\lambda(r) \vec{\nabla} T)=(\vec{\nabla} \lambda(r)) \cdot \vec{\nabla} T+\lambda(r) \Delta T=\left(\frac{\partial \lambda(r)}{\partial r}\right)\left(\frac{\partial T}{\partial r}\right)+\lambda(r) \Delta T
$$

Dabei wird zur Übersichtlichkeit $\lambda(r,<T(r)>)$ mit $\lambda(r)$ abgekürzt. Das Profil $\lambda(r,<$ $T(r)>$ ) wird in jedem Zeitschritt für die entsprechende Temperaturverteilung neu nach 
Gleichung (3.61) berechnet. Die Bestimmung der radialen Ableitung erfolgt aufgrund der größeren Genauigkeit im Tschebyscheffraum. Die thermische Leitfähigkeit ändert sich in jedem Zeitschritt, so dass die auftretenden Matrizen in jedem Zeitschritt neu berechnet und invertiert werden müssen.

Die Implementierung einer radialabhängigen Leitfähigkeit kann getestet werden, indem ein einfaches tiefenabhängiges Profil gewählt wird, für das die zeitunabhängige Wärmediffusionsgleichung analytisch gelöst werden kann. Mit dem Profil $\lambda(r)=1 / r^{2}$ ergibt sich z.B. für den zu betrachtenden radialen Anteil der zeitunabhängigen Wärmeleitungsgleichung:

$$
0=\vec{\nabla} \cdot(\lambda(r) \vec{\nabla} T)=\frac{1}{r^{2}} \frac{\partial}{\partial r}\left(\lambda(r) r^{2} \frac{\partial T}{\partial r}\right)=\frac{1}{r^{2}} \frac{\partial^{2} T}{\partial r^{2}}
$$

Mit den Randbedingungen: $T\left(r_{a}\right)=0$ und $T\left(r_{i}\right)=1$ findet man als Lösung:

$$
T(r)=-r+r_{i}+1 \quad \text { mit } \quad\left(r_{a}-r_{i}=1\right)
$$

Als Test initialisiert man das Temperaturfeld mit einem beliebigen (von dem Profil nach Gleichung (4.15) abweichenden) Startprofil und integriert ohne Berechnung der konvektiven Beiträge $(R a=0)$ solange, bis ein stationärer Zustand erreicht wird. Das so berechnete Profil stimmt in den durchgeführten Tests mit der Gerade nach Gleichung (4.15) überein.

\subsection{Erweiterte Boussinesq-Näherung}

In der erweiterten Boussinesq-Näherung treten in der Wärmeleitungsgleichung (3.42) zusätzliche Terme auf, die mit der Dissipationszahl (Gleichung (3.39)) skalieren und in der Boussinesq-Näherung vernachlässigt werden. Der Ausdruck, der die adiabatischen Effekte beschreibt, enthält das Produkt von Radialgeschwindigkeit $v_{r}$ und Temperatur $T$. Der Reibungsterm besteht ebenfalls aus einer Summe von Produkten, hier werden Geschwindigkeitskomponenten bzw. Ableitungen (nach den Raumkoordinaten) derselben miteinander multipliziert und dann addiert. Die Berechnung von Produkten im Funktionenraum ist, wie oben erwähnt, aufwendig und wird durch die Transformation in den Ortsraum vermieden. In der Boussinesq-Näherung wird diese Vorgehensweise bereits auf den Advektionsterm angewandt, so dass die notwendigen Transformationsroutinen vorhanden sind. Der Ausdruck für die adiabatische Kompression/Dekompression und die latente Wärme, lässt sich mit dem effektiven thermischen Ausdehnungskoeffizienten $\alpha_{\text {eff }}$ vereinfachen zu:

$$
\alpha^{\prime}\left(1+P \frac{d \Gamma}{d \pi}\right)\left(T+T_{\text {surf }}\right) D i v_{r}=D i \alpha_{\text {eff }}\left(T+T_{\text {surf }}\right) v_{r}
$$

Für den Reibungsterm gilt in Kugelkoordinaten:

$$
\begin{aligned}
\underline{\underline{\tau}} \cdot \underline{\underline{\dot{\epsilon}}}= & \eta(r)\left[2\left\{\left(\frac{\partial v_{r}}{\partial r}\right)^{2}+\left(\frac{1}{r} \frac{\partial v_{\theta}}{\partial \theta}+\frac{v_{r}}{r}\right)^{2}+\left(\frac{1}{r \sin \theta} \frac{\partial v_{\phi}}{\partial \phi}+\frac{v_{r}}{r}+\frac{v_{\theta} \cot \theta}{r}\right)^{2}\right\}+\right. \\
& \left\{\frac{1}{r \sin \theta} \frac{\partial v_{\theta}}{\partial \phi}+\frac{\sin \theta}{r} \frac{\partial}{\partial \theta}\left(\frac{v_{\phi}}{\sin \theta}\right)\right\}^{2}+\left\{\frac{1}{r \sin \theta} \frac{\partial v_{r}}{\partial \phi}+r \frac{\partial}{\partial r}\left(\frac{v_{\phi}}{r}\right)\right\}^{2}+ \\
& \left.\left\{r \frac{\partial}{\partial r}\left(\frac{v_{\theta}}{r}\right)+\frac{1}{r} \frac{\partial v_{r}}{\partial \theta}\right\}^{2}\right]
\end{aligned}
$$


Diese beiden Ausdrücke werden in jedem Zeitschritt für alle Gitterpunkte im Ortsraum berechnet und wie der nicht lineare Advektionsterm nach dem expliziten Adams-Bashforth-Verfahren in der Zeit integriert.

Die durchgeführten Programmänderungen werden u.a. überprüft, indem das Programm auf interne Konsistenz getestet wird (Christensen und Yuen 1985). In der Leistungsbilanz muss die im gesamten Volumen erzeugte mechanische Energie (pro Zeiteinheit) mit der Reibungswärme in der ganzen Kugelschale übereinstimmen. Die Stokesgleichung (3.41) beschreibt die Änderung des Impulses mit der Zeit. Die Multiplikation mit $\vec{v}$ führt zur Leistungsbilanz. Integriert man diese mit Hilfe der partiellen Integration über das ganze Volumen, ergibt sich bei festen Rändern die zu überprüfende Bedingung:

$$
\int R a \alpha_{\mathrm{eff}}^{\prime} T v_{r} d V=\int \underline{\underline{\tau}} \cdot \underline{\underline{\dot{\epsilon}}} d V
$$

Die Werte dieser Integrale weichen bei den Tests um weniger als $1.0 \%$ voneinander ab.

\subsection{Bestimmung der Tiefe der Phasengrenze}

Die Lage einer Phasengrenze ist durch die Druck- und Temperaturbedingungen bestimmt. In der numerischen Simulation werden laterale Temperaturunterschiede nicht berücksichtigt und die Tiefe der Phasenumwandlung wird aus dem horizontal gemittelten Temperaturprofil bestimmt. Kühlt das System im Laufe der Evolution ab, ändert sich die Position der Phasengrenze. Bei der Simulation einer solchen temperaturabhängigen Tiefe der Phasengrenze wird in jedem Zeitschritt die Phasenumwandlungstiefe aus dem Schnittpunkt des über Kugelschalen mit gleichem Radius gemittelten Temperaturprofils mit der entsprechenden Phasengrenzkurve (Gleichungen (2.1) - (2.3)) bestimmt. Dies geschieht mit der Methode des nächsten Nachbarn, indem für jede Temperatur des gemittelten Profils entsprechend der Phasengrenzkurve (Clausius-Clayperon-Kurven) der nötige Umwandlungsdruck bestimmt wird. Dieser Umwandlungsdruck wird mit dem hydrostatischen Druck in der entsprechenden Tiefe verglichen. Die Lage der Phasengrenze bestimmt sich durch den radialen Gitterpunkt, an dem der Unterschied zwischen Umwandlungsdruck und hydrostatischem Druck minimal ist. 


\section{Ergebnisse der Evolutionsmodelle für Mars}

In diesem Kapitel werden die Ergebnisse der Evolutionsrechnungen für Mars vorgestellt. Dabei wird in den Abschnitten (5.1) - (5.4) die Boussinesq-Approximation verwendet und in Kapitel (5.5) der Einfluss der Nicht-Boussinesq-Effekte auf die thermische Evolution und das Konvektionsmuster analysiert. Alle Simulationen starten im Boussinesq-Fall mit dem gleichen Temperaturprofil und stellen die gleichen Randbedingungen an das Geschwindigkeitsfeld und die Temperatur. Das Starttemperaturfeld und die Randbedingung sind in Kapitel (3.9) beschrieben. Die weiteren verwendeten Modellparameter, die in den Kapiteln (2) bzw. (3) diskutiert worden sind, fasst Tabelle (A.2) im Anhang zusammen. In Abschnitt (5.1) wird der Einfluss des Aktivierungsvolumens in der Viskosität auf das Konvektionsmuster und die thermische Entwicklung untersucht sowie das Zusammenspiel mit der kernnahen endothermen Phasengrenze analysiert. Alle übrigen Materialparameter sind zunächst homogen. In Unterkapitel (5.2) werden unter Berücksichtigung einer temperatur- und druckabhängigen Viskosität Ergebnisse verschiedener Profile des thermischen Ausdehnungskoeffizienten, insbesondere auch des Profils im Mars gezeigt. Auch hier wird die Rolle des endothermen kernnahen Phasenübergangs betrachtet. Im folgenden Abschnitt (5.3) werden bei gleichem Viskositätsprofil und konstantem $\alpha$ die Unterschiede im Konvektionsmuster bzw. der thermischen Entwicklung durch eine tiefenabhängige, druck- und temperaturbestimmte thermische Leitfähigkeit mit und ohne endotherme Phasengrenze vorgestellt. Die Modelle werden bei unverändertem Viskositätsparametern durch die Kombination von $\alpha(r)$ und $\lambda(r)$, wie sie sich für Mars ergeben, sowie die zusätzlichen exothermen Phasengrenzen in Abschnitt (5.4.1) realitätsgetreuer gestaltet. Der Einfluss der Startkerntemperatur auf diese Ergebnisse wird in Abschnitt (5.4.2) getestet.

Bei der Auswertung der verschiedenen Simulationen ist das Konvektionsmuster, insbesondere die Anzahl der Aufströme, interessant, um die starke Konzentration des Vulkanismus auf dem Mars zu erklären. Darüber hinaus hat die thermische Entwicklung des Planeten eine große Bedeutung, z.B. für die Aktivität des Vulkanismus oder den Dynamoprozess. Der Wärmefluss aus dem Kern lierfert wichtige Hinweise auf die Existenz und Lebensdauer eines thermisch getriebenen Dynamos im Mars. Die Lithosphäre ist für die thermische Entwicklung des Planeten bedeutsam, so dass auch ihre Entwicklung beobachtet wird. Da die kernnahe endotherme Phasengrenze nur im Fall eines vergleichsweise kleinen Kerns existiert, wird ihrer Rolle besondere Aufmerksamkeit zuteil. 


\subsection{Einfluss des Aktivierungsvolumens}

Das Viskositätsgesetz nach Arrhenius (siehe Kapitel (3.5)) enthält als Materialparameter die Aktivierungsenergie $E^{*}$ und das Aktivierungsvolumen $V^{*}$ :

$$
\eta(r)=\eta_{0} \exp \left(\frac{E^{*}+\rho_{0} g\left(r_{a}-r\right) V^{*}}{R<T(r)>}\right)
$$

Das Aktivierungsvolumen beschreibt dabei die Druckabhängigkeit der Viskosität. Besonders das Aktivierungsvolumen ist durch Labormessungen nur unzureichend genau bestimmt. Die Angaben schwanken je nach Kriechmechanismus und Wassergehalt zwischen $5 \mathrm{~kJ} \mathrm{~mol}^{-1}<V^{*}<25 \mathrm{~kJ} \mathrm{~mol}^{-1}$. Typisch für trockenen Olivin beim Diffusionskriechen ist ein Wert von $V^{*}=6 \mathrm{~kJ} \mathrm{~mol}^{-1}$ (vgl. Abschnitt (3.5)). Da ein großes Aktivierungsvolumen zu starken Gradienten der Viskosität beiträgt, die numerisch schwer handhabbar sind, wird in den meisten Rechnungen ein vergleichsweise kleiner Wert von $V^{*}=3 \mathrm{~kJ} \mathrm{~mol}^{-1}$ verwendet. In diesem Kapitel soll der Einfluss des Aktivierungsvolumens auf das Konvektionsmuster und die thermische Entwicklung untersucht werden. Insbesondere das Zusammenspiel von Phasengrenze und Viskosität soll analysiert werden. Christensen und Yuen (1985) untersuchen die grundsätzliche Wirkung einer endothermen Phasengrenze auf die Konvektion in kartesischen 2D-Modellen. Sie finden, dass der Einfluss der Phasengrenze mit der lokalen Rayleighzahl zunimmt. In ihren Modellen befindet sich die Phasengrenze in halber Tiefe des Simulationsgebiets, und es stellt sich die Frage, ob ein solcher Effekt auch bei einer sehr dünnen Perowskitschicht zu beobachten ist.

Abbildung (5.1) zeigt das Anfangsviskositätsprofil für verschiedene Aktivierungsvolumina zusammen mit dem verwendeten Anfangstemperaturprofil. Die Viskosität steigt innerhalb der kernnahen thermischen Grenzschicht zunächst von einem Minimum an der KMG an und erreicht ein relatives Maximum. Je größer das Aktivierungsvolumen ist, desto höher sind die Viskositäten innerhalb der thermischen Grenzschicht, also auch in der Tiefe der Phasengrenze und um so deutlicher fällt das relative Maximum aus. Im isothermen Mantelbereich wird die Druckabhängigkeit der Viskosität besonders deutlich. Sie bestimmt den Abfall der Viskosität mit abnehmenden Druck in Richtung Oberfläche. Mit steigendem Aktivierungsvolumen nimmt der Viskositätsgradient (im Betrag) zu und es entsteht ein relatives Minimum unter der oberflächennahen thermischen Grenzschicht. Je größer das Aktivierungsvolumen ist, desto ausgeprägter ist diese niedrigviskose Zone. In der oberflächennahen kalten thermischen Grenzschicht steigt die Viskosität stark an, wobei der Maximalwert durch einen 'cutoff' (siehe Abschnitt (3.5)) beschränkt wird. Die Viskositätsprofile sind so normiert, dass in halber Tiefe $\left(r_{1 / 2}=2380 \mathrm{~km}\right)$ bei $T=1573 \mathrm{~K}$ der Referenzwert von $3.3 \cdot 10^{21} \mathrm{~Pa}$ s erreicht wird. Für $V^{*}=1.5 \mathrm{~kJ} \mathrm{~mol}^{-1}$ wurde die 'cutoff'-Temperatur (vgl. Kapitel (3.5)) nicht dem veränderten Wert des Aktivierungsvolumens angepasst, so dass die 'cutoff'-Viskosität hier etwas höher liegt. Vergleiche in den beiden anderen Fällen zwischen angepasster und beibehaltener 'cutoff'-Temperatur zeigen aber keine signifikanten Veränderungen in der thermischen Entwicklung oder im Konvektionsmuster.

Die verschiedenen Viskositätsprofile haben einen deutlichen Einfluss auf das Konvektionsmuster, das für den Zeitpunkt nach 4.5 Ga für die einzelnen Simulationen in Abbildung (5.2) dargestellt ist. Die linke Graphik zeigt jeweils die Radialgeschwindigkeit in halber Tiefe und die rechte Teilabbildung einen meridionalen Schnitt durch die Temperaturan- 


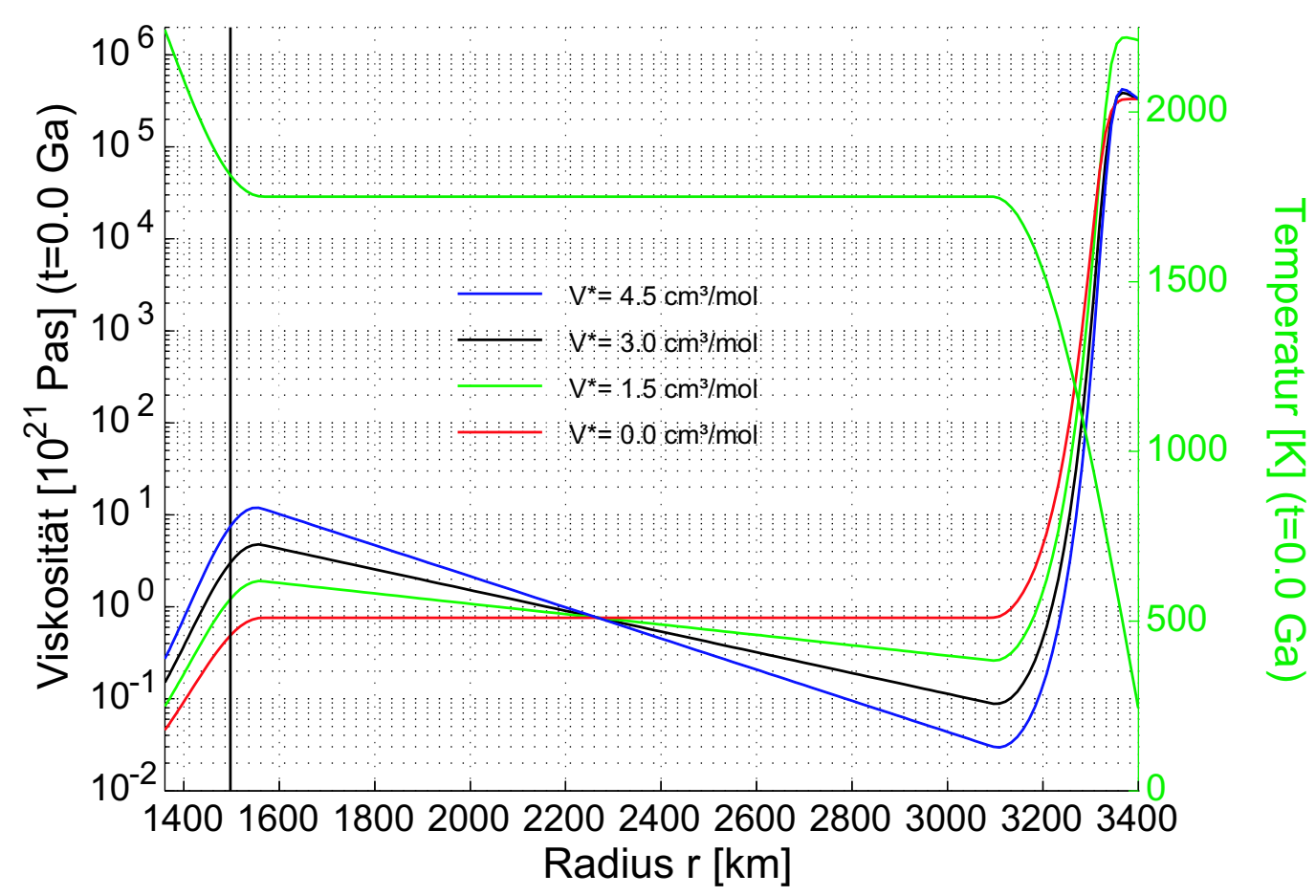

Abbildung 5.1: Einfluss des Aktivierungsvolumens auf das Viskositätsprofil in der Starttemperaturverteilung. Die Lage der endothermen Phasengrenze ist durch die schwarze Linie markiert.

omalie $(T-<T(r)>)$ in einem Aufstrom. Die genaue Lage des Schnitts ist durch den Längengrad $\phi$ angegeben. Abbildung (5.2a) stellt den Fall ohne endotherme Phasengrenze $(P=0.0)$ bei dem Referenzwert für das Aktivierungsvolumen von $V^{*}=3.0 \mathrm{~kJ} \mathrm{~mol}^{-1}$ dar. In der Radialgeschwindigkeit in halber Tiefe sind nach $4.5 \mathrm{Ga}$ sechs konzentrierte Aufströme erkennbar. Der Schnitt durch die Temperaturanomalie $(T-<T(r)>$ ) verdeutlicht, dass die Aufströme nach 4.5 Ga eine deutlich positive Temperaturanomalie aufweisen. Die zeitliche Entwicklung des Konvektionsmusters (Abbildung (5.3)) zeigt, dass die nach $4.5 \mathrm{Ga}$ sichtbaren Aufströme bereits früh in der Evolution vorhanden sind und dadurch gestärkt werden, dass andere Plumes mit ihnen verschmelzen. Nach etwa 2.0 bis $2.5 \mathrm{Ga}$ haben die Auf- und Abströme die Anordnung eingenommen, die nach $4.5 \mathrm{Ga}$ zu beobachten ist. Ab diesem Zeitpunkt schwächt sich lediglich die Geschwindigkeitsamplitude ab.

In Abbildung (5.2c) ist bei gleichem Aktivierungsvolumen die kernnahe endotherme Phasengrenze $(P=-0.2723)$ berücksichtigt worden. Die Anzahl der Aufströme nach $4.5 \mathrm{Ga}$ ist deutlich reduziert gegenüber dem Fall ohne Phasengrenze. Lediglich zwei Aufströme dominieren das Konvektionsmuster. Die Geschwindigkeit der Konvektionsbewegung nach $4.5 \mathrm{Ga}$ ist höher als im Fall ohne Phasengrenze. Auch die Temperaturanomalie im Aufstrom fällt stärker aus. Die Entwicklung zu diesem Konvektionsmuster ist in (Abbildung (5.4)) aufgezeigt. Aus dem zunächst kleinskaligen Muster entsteht nach etwa 2.5 bis 3.0 Ga die Situation mit nur zwei konzentrierten Aufströmen, die sich danach nicht 
a) $V^{*}=3.0 \mathrm{~cm}^{3} / \mathrm{mol}(P=0.0)$
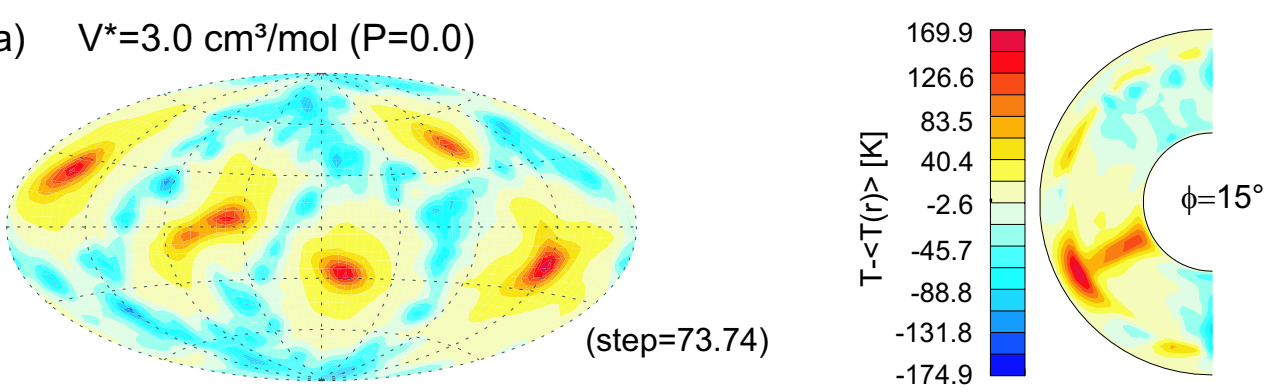

b) $V^{*}=4.5 \mathrm{~cm}^{3} / \mathrm{mol}(\mathrm{P}=-0.2723)$
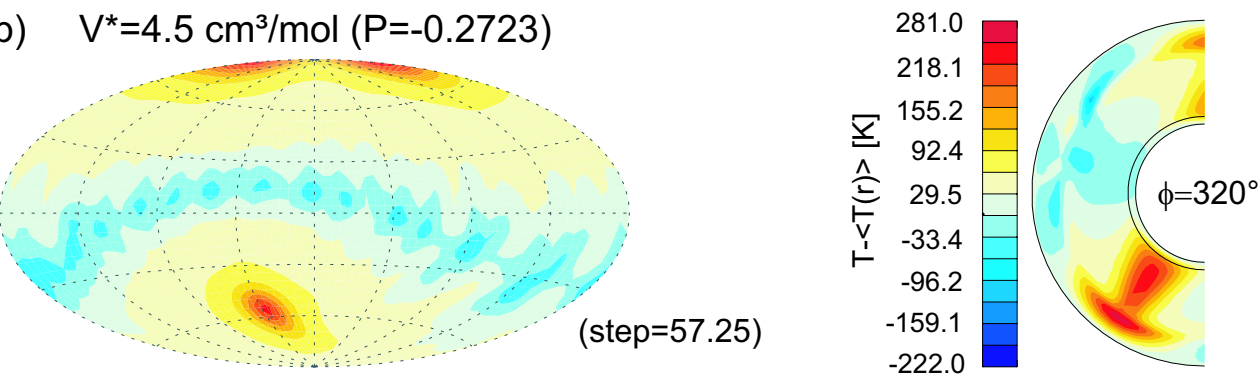

c) $V^{*}=3.0 \mathrm{~cm}^{3} / \mathrm{mol}(P=-0.2723)$
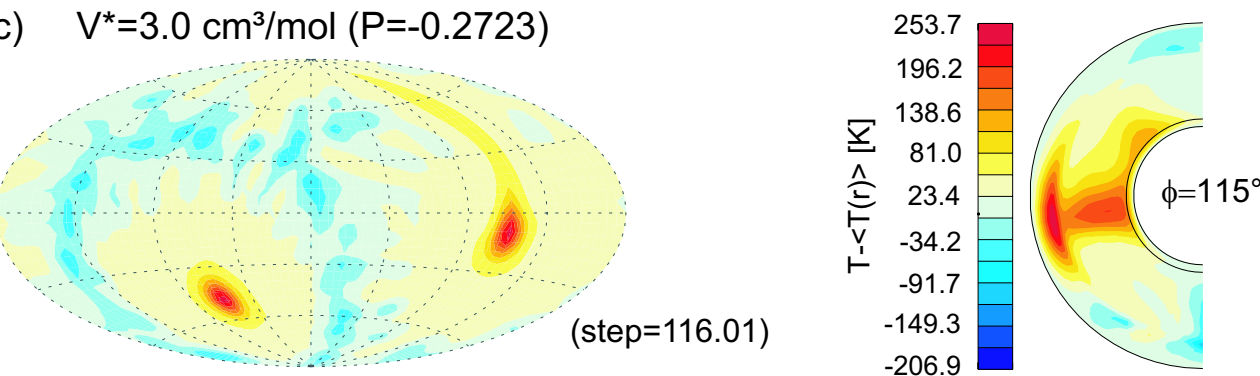

d) $V^{*}=1.5 \mathrm{~cm}^{3} / \mathrm{mol}(P=-0.2723)$
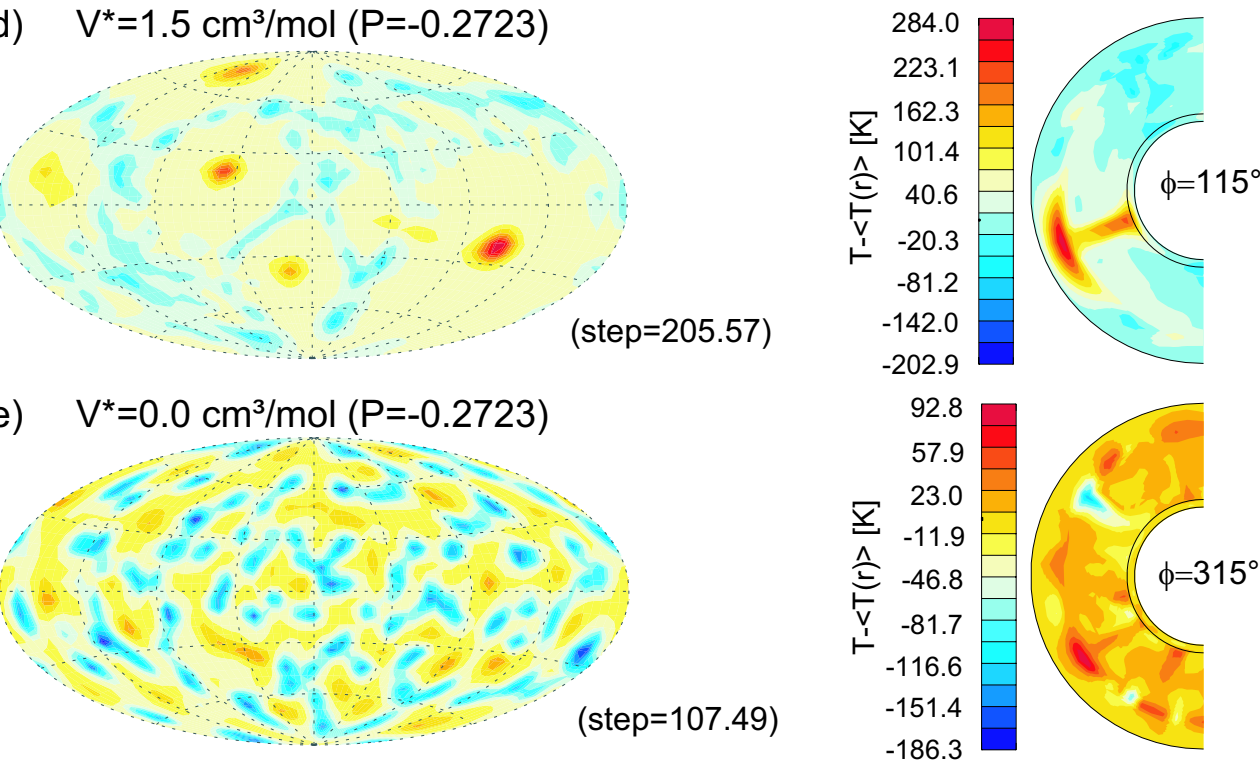

e) $V^{*}=0.0 \mathrm{~cm}^{3} / \mathrm{mol}(P=-0.2723)$

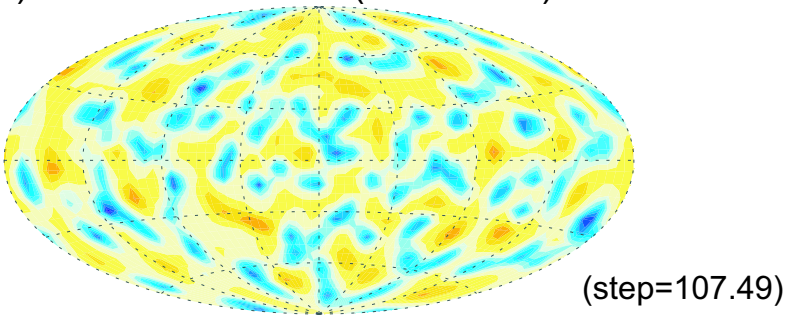

$-186.3$

Abbildung 5.2: Einfluss des Aktivierungsvolumens auf das Konvektionsmuster nach 4.5 Ga: Links: Radialgeschwindigkeit in halber Tiefe (rot: Aufströme, blau: Abströme). Der Contourstep der Geschwindigkeit ist angegeben: step $=100$ entspricht $0.14 \mathrm{~cm} / \mathrm{a}$. Rechts: Temperaturanomalie $(T-<T(r)>)$ in $[\mathrm{K}]$ in einem Aufstrom. 
a) $t=0.5 \mathrm{Ga}$

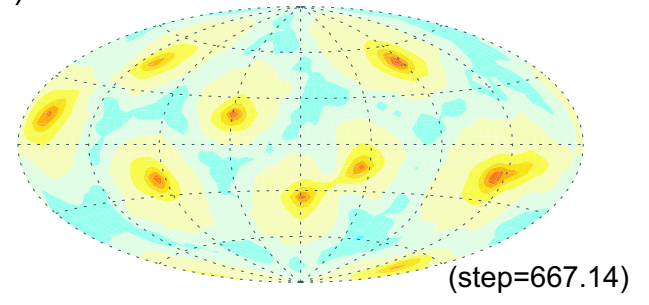

c) $\mathrm{t}=1.5 \mathrm{Ga}$

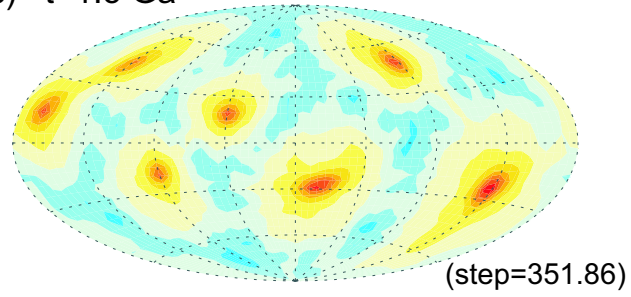

e) $\mathrm{t}=2.5 \mathrm{Ga}$

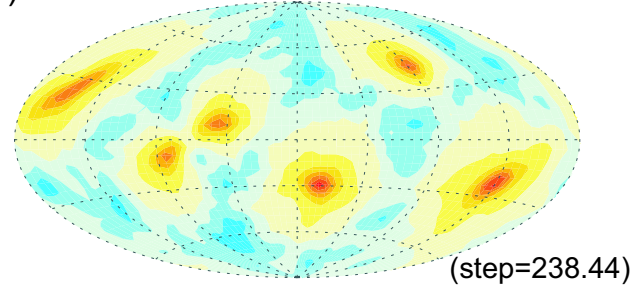

g) $\mathrm{t}=3.5 \mathrm{Ga}$

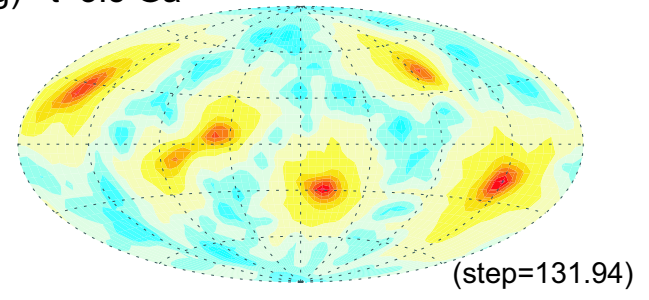

b) $\mathrm{t}=1.0 \mathrm{Ga}$

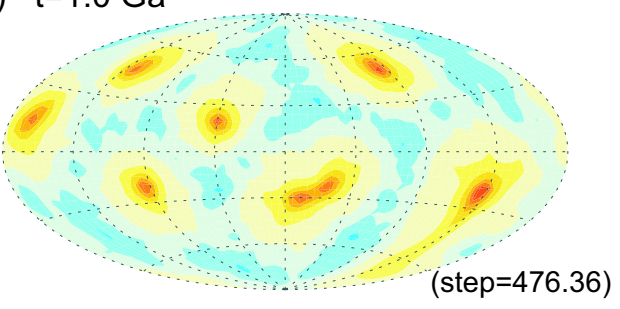

d) $t=2.0 \mathrm{Ga}$

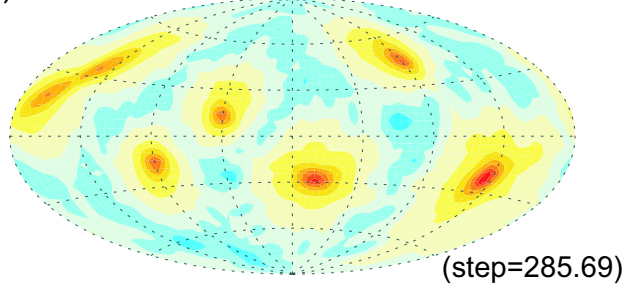

f) $t=3.0 \mathrm{Ga}$

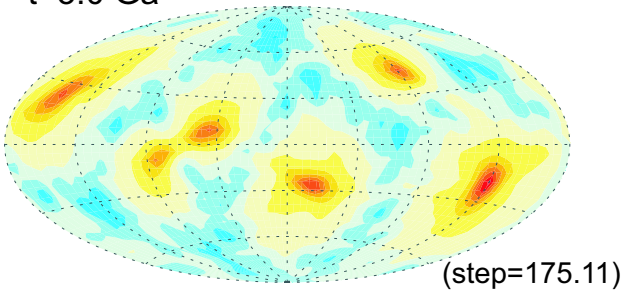

h) $\mathrm{t}=4.0 \mathrm{Ga}$

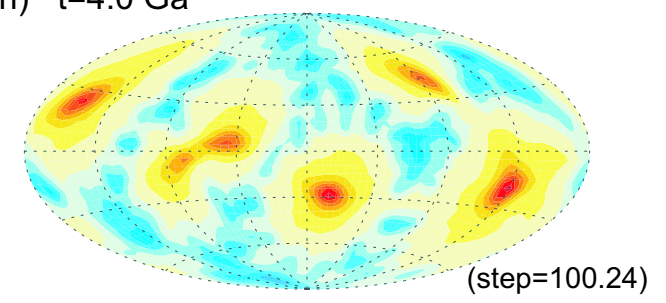

i) $\mathrm{t}=4.5 \mathrm{Ga}$

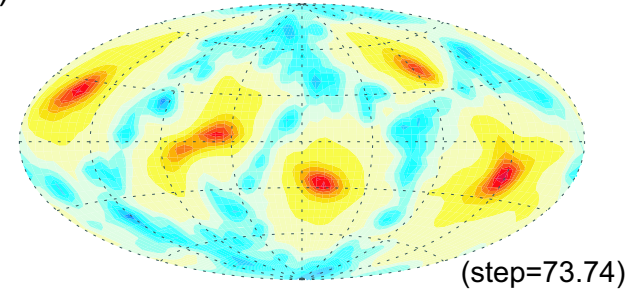

Abbildung 5.3: Zeitliche Entwicklung des Konvektionsmusters im Referenzfall mit $V^{*}=$ $3.0 \mathrm{~kJ} \mathrm{~mol}^{-1}$ ohne endotherme Phasengrenze $(P=0.0)$ : Radialgeschwindigkeit in halber Tiefe (rot: Aufströme, blau: Abströme). Der Contourstep der Geschwindigkeit ist angegeben: step=100 entspricht $0.14 \mathrm{~cm} / \mathrm{a}$. 
a) $\mathrm{t}=0.5 \mathrm{Ga}$

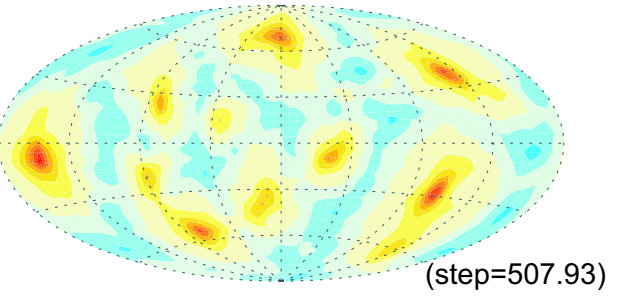

c) $\mathrm{t}=1.5 \mathrm{Ga}$

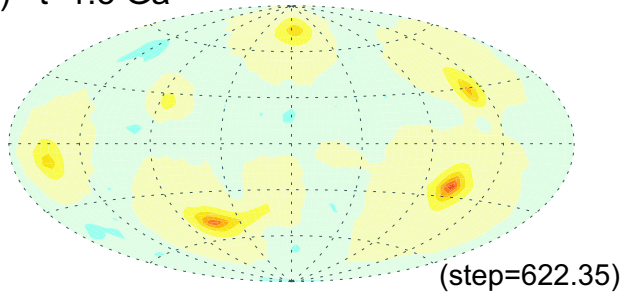

e) $\mathrm{t}=2.5 \mathrm{Ga}$

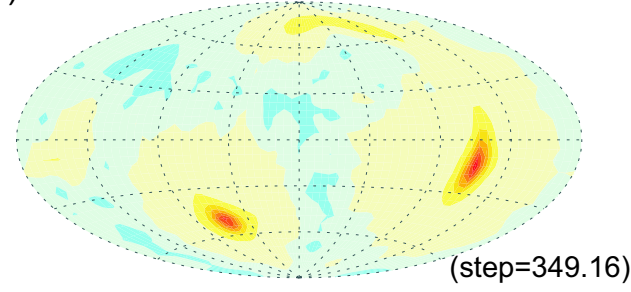

g) $\mathrm{t}=3.5 \mathrm{Ga}$

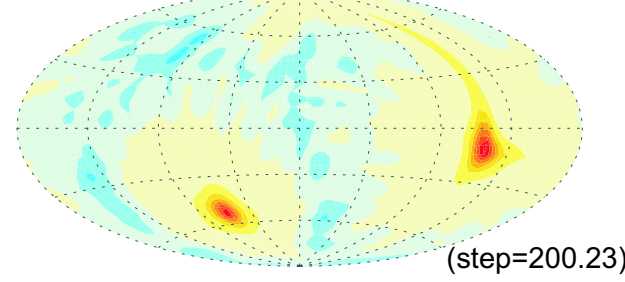

b) $\mathrm{t}=1.0 \mathrm{Ga}$

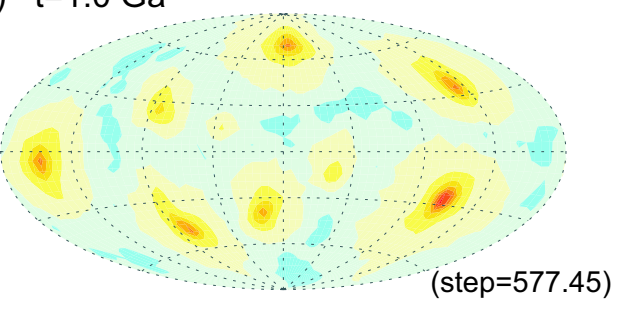

d) $t=2.0 \mathrm{Ga}$

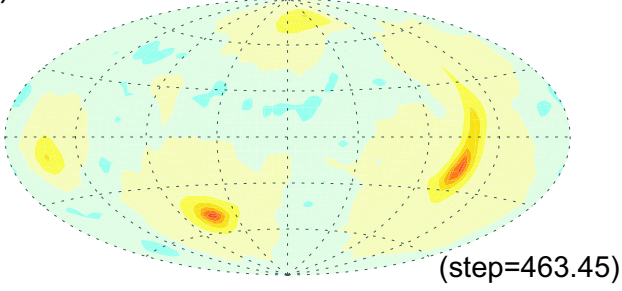

f) $t=3.0 \mathrm{Ga}$

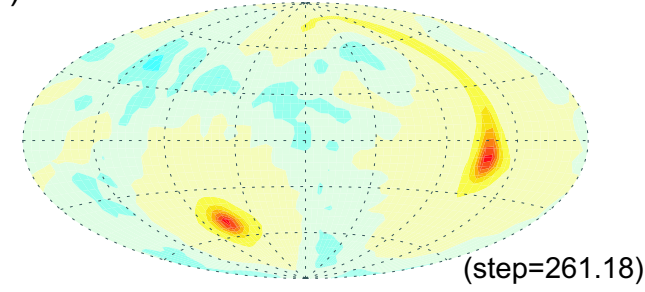

h) $\mathrm{t}=4.0 \mathrm{Ga}$

i) $\mathrm{t}=4.5 \mathrm{Ga}$

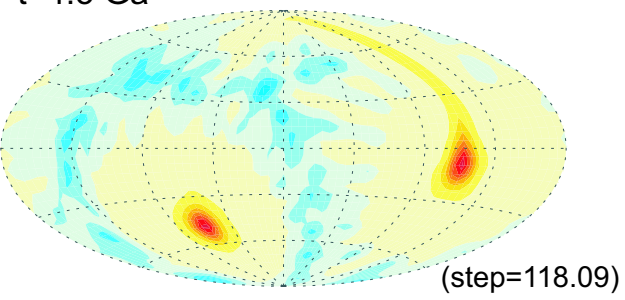

Abbildung 5.4: Zeitliche Entwicklung des Konvektionsmusters im Referenzfall mit $V^{*}=$ $3.0 \mathrm{~kJ} \mathrm{~mol}^{-1}$ mit endothermer Phasengrenze $(P=-0.2723)$ : Radialgeschwindigkeit in halber Tiefe (rot: Aufströme, blau: Abströme). Der Contourstep der Geschwindigkeit ist angegeben: step $=100$ entspricht $0.14 \mathrm{~cm} / \mathrm{a}$. 
mehr in der Form ändert. Ähnlich wie im Fall ohne Phasengrenze sind die verbleibenden Plumes schon zu Beginn vorhanden und nahezu ortsfest. Die Anzahl der Aufströme nimmt einerseits durch das Verschmelzen von Aufströmen und andererseits durch das Aussterben von schwächeren Plumes ab. Bei diesem Aktivierungsvolumen reduziert also die kernnahe endotherme Phasengrenze die Anzahl der Aufströme deutlich.

Bei einem höheren Aktivierungsvolumen $\left(V^{*}=4.5 \mathrm{~kJ} \mathrm{~mol}^{-1}\right)$ stellt sich bei Berücksichtigung der Phasengrenze (Abbildung (5.2b)) ebenfalls ein Konvektionsmuster mit nur zwei Aufströmen ein. Die Positionen der Aufströme sind gegenüber dem vorher betrachteten Referenzfall $\left(V^{*}=3.0 \mathrm{~kJ} \mathrm{~mol}^{-1}\right)$ verändert, ein starker Aufstrom befindet sich am nördlichen Pol. Dieses Konvektionsmuster wird schon zwischen 1.6 und $2.1 \mathrm{Ga}$ und damit früher als im Referenzfall erreicht, die Radialgeschwindigkeiten sind jedoch hier geringer. Die positive Temperaturanomalie im Schnitt durch den Aufstrom ist etwas stärker als im Referenzfall.

Ein Verringern des Aktivierungsvolumens führt zu einer Zunahme der Anzahl der Aufströme. Bei $V^{*}=1.5 \mathrm{~kJ} \mathrm{~mol}^{-1}$ und Berücksichtigung der Phasengrenze sind in Abbildung (5.2d) sechs Aufströme erkennbar, von denen drei schwächer ausfallen. Der Schnitt durch das Temperaturfeld in einem starken Aufstrom zeigt eine ausgeprägte warme Anomalie. Bei den bisher untersuchten Simulationen stellt die Abbildung der Radialgeschwindigkeit bei $4.5 \mathrm{Ga}$ ein Muster dar, dessen Form sich über einen längeren Zeitraum nicht mehr verändert hat und lediglich in der Amplitude schwächer wird. Im Fall $V^{*}=1.5 \mathrm{~kJ} \mathrm{~mol}^{-1}$ zeigt ein Weiterintegrieren der Simulation, dass die schwächeren Aufströme aussterben und zwischen 8 und 8.75 Ga ein Muster mit nur drei Aufströmen entsteht.

Abbildung (5.2e) stellt die Situation dar, in der die Druckabhängigkeit komplett vernachlässigt worden ist $\left(V^{*}=0.0 \mathrm{~kJ} \mathrm{~mol}^{-1}\right)$. Hier entsteht trotz endothermer Phasengrenze ein sehr kleinskaliges Konvektionsmuster mit einer Vielzahl von Aufströmen, die deutlich schwächer und weniger konzentriert sind als im Referenzfall. Die Abströme, die in den vorher diskutierten Fällen weitgehend in langgestreckten Bändern angeordnet waren, sind jetzt kleinskaliger. Die Geschwindigkeitsamplitude der Abströme übersteigt die der Aufströme und die Temperaturanomalie im Aufstrom ist deutlich schächer als in den übrigen Fällen. Der Maximalwert der negativen Temperaturanomalien (unterer Grenzwert des Farbbalkens) übersteigt betragsmäßig den Wert für die positiven Anomalien und verdeutlicht die Dominanz der Abströme.

Der unterschiedliche Einfluss der Phasengrenze in Abhängigkeit vom Aktivierungsvolumen ist auch in den radialen Profilen der Radialgeschwindigkeit erkennbar. Abbildung (5.5) stellt diese Profile zu Beginn der Entwicklung nach 0.5 Ga dar. In der Nähe der Oberfläche zeigt sich zu diesem frühen Zeitpunkt der Einfluss der niedrigviskosen Zone. Je ausgeprägter dieser Bereich ist, desto höher ist dort die Radialgeschwindigkeit und desto dünner wird die stagnierende Lithosphäre. Der Bereich der hohen Geschwindigkeiten verschiebt sich mit zunehmenden Aktivierungsvolumen in Richtung Oberfläche. Für den Einfluss der Phasengrenze auf das Konvektionsmuster ist der Bereich in der Tiefe des Phasenübergangs bedeutsamer. Bei $V^{*}=1.5 \mathrm{~kJ} \mathrm{~mol}^{-1}$ und deutlicher für $V^{*}=0.0 \mathrm{~kJ} \mathrm{~mol}^{-1}$ bildet sich ein klares Minimum in der Tiefe der Phasengrenze aus. Dieses Minimum in der Radialgeschwindigkeit ist gleichbedeutend mit einem Minimum im Massenfluss durch die Phasengrenze, so dass die Konvektion hier in zwei voneinander nahezu unabhängigen Schichten stattfindet, die durch die Phasengrenze getrennt werden. Die konvektions- 


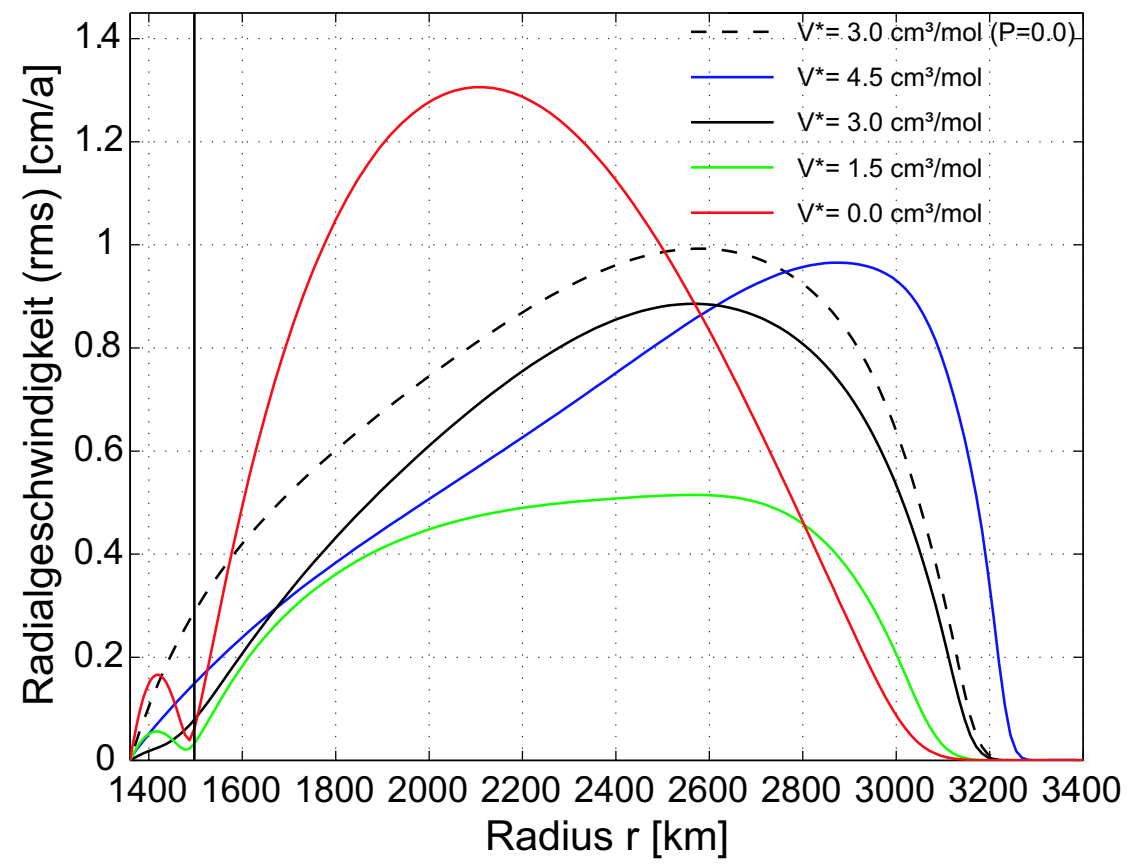

Abbildung 5.5: Einfluss des Aktivierungsvolumens auf das Profil der Radialgeschwindigkeit bei $t=0.5 \mathrm{Ga}$. Die schwarze Linie markiert die Lage der endothermen Phasengrenze.

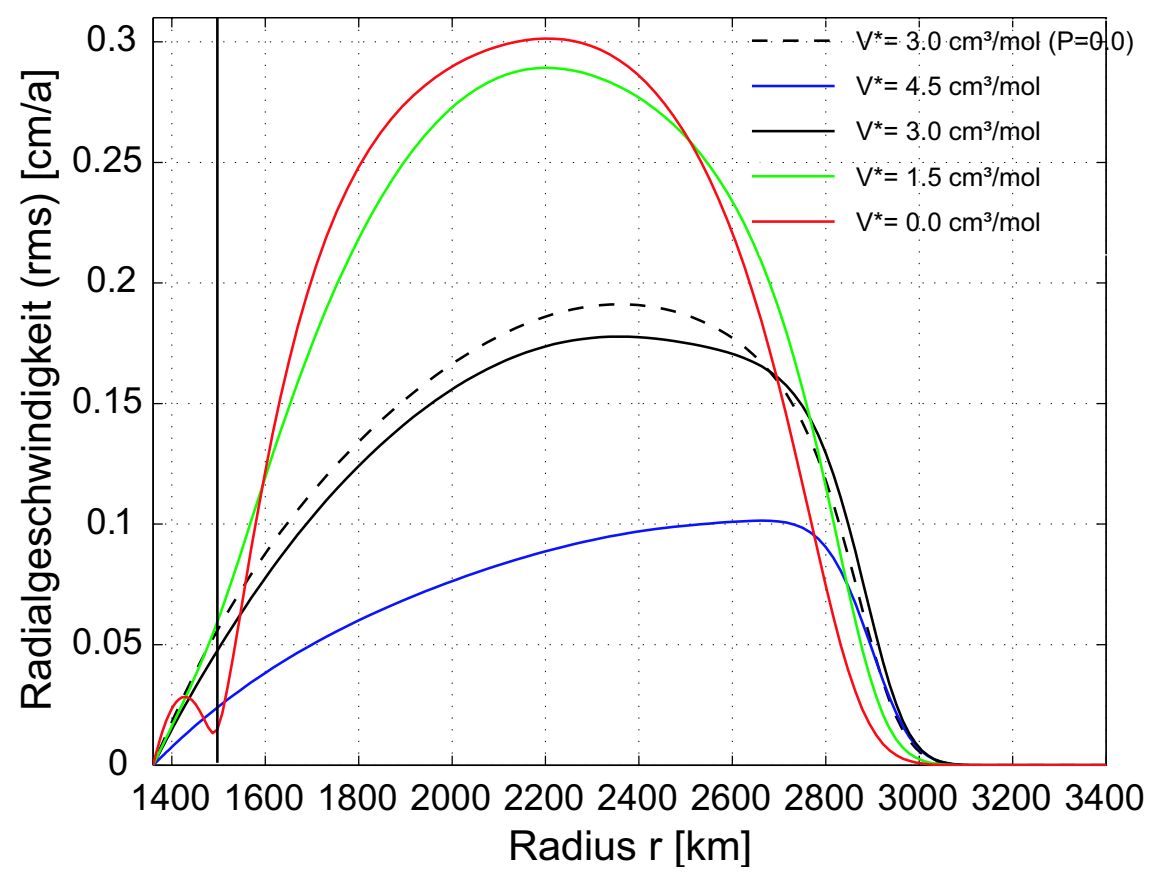

Abbildung 5.6: Einfluss des Aktivierungsvolumens auf das Profil der Radialgeschwindigkeit bei $t=4.5 \mathrm{Ga}$. Die schwarze Linie markiert die Lage der endothermen Phasengrenze. 
hemmende Wirkung der endothermen Phasengrenze ist bei den niedrigen Viskositäten so stark, dass nur sehr wenig Material zwischen den beiden Schichten ausgetauscht werden kann. Christensen und Yuen (1985) begründen vergleichbare Ergebnisse damit, dass aufgrund der niedrigen Viskositäten die viskose Kopplung sehr schwach ist. Für die obere Schicht entstehen die Aufströme somit erst oberhalb der Phasengrenze, so dass sie durch diese nicht behindert werden können und ein Konvektionsmuster mit vielen, auch schwächeren Aufströmen möglich ist.

Abbildung (5.6) zeigt, dass der Einfluss des Aktivierungsvolumens auf die Dicke der stagnierenden Schicht nach 4.5 Ga wesentlich geringer ist. Die Geschwindigkeiten sind bei $V^{*}=4.5 \mathrm{~kJ} \mathrm{~mol}^{-1}$ deutlich kleiner, wohingegen beim Absenken des Aktivierungsvolumens höhere Geschwindigkeiten zu beobachten sind. In der Nähe der Phasengrenze bleibt das Minimum für $V^{*}=0.0 \mathrm{~kJ} \mathrm{~mol}^{-1}$ bestehen, ist aber bei $V^{*}=1.5 \mathrm{~kJ} \mathrm{~mol}^{-1}$ verschwunden. Bei vernachlässigter Druckabhängigkeit der Viskosität findet die Konvektion während der ganzen Evolution in zwei durch die Phasengrenze getrennte Schichten statt, so dass die Anzahl der Aufströme in der oberen Schicht nicht durch die Phasengrenze reduziert werden kann. Durch die Teilung des Mantels in zwei Bereiche ist die Dicke der oberen Konvektionsschicht geringer. Dies unterstützt das Ausbilden eines kleinskaligen Konvektionsmusters. Im Fall von $V^{*}=1.5 \mathrm{~kJ} \mathrm{~mol}^{-1}$ erreicht die Viskosität während des Abkühlens ausreichend hohe Werte, um die viskose Kopplung durch die Phasengrenze hindurch herzustellen und die geschichtete Konvektion aufzuheben. Damit kann die Phasengrenze auf die Aufströme wirken und deren Anzahl reduzieren. Da die Phasengrenze nur zeitlich verzögert wirksam werden kann und die Konvektion durch die weniger ausgeprägte niedrigviskose Zone bei $V^{*}=1.5 \mathrm{~kJ} \mathrm{~mol}^{-1}$ im Vergleich zum Referenzfall über etwa 3.5 Ga schwächer ist (Abbildung (5.10)), ist es möglich, dass sich das Konvektionsmuster nach $4.5 \mathrm{Ga}$ weiterhin verändert und die Anzahl der Plumes noch reduziert wird. Zur späteren Reduzierung der Plumes trägt auch das weitere Abkühlen und die damit verbundene Abschwächung der Konvektion bei, wodurch ebenfalls ein großkaliges Konvektionsmuster bevorzugt wird.

Die Konvektion in der unteren kernnahen Schicht bei vernachlässigter Druckabhängigkeit wird in der Form des Temperaturprofils nach 0.5 Ga (Abbildung (5.7)) deutlich. Hier bildet sich ein für die Konvektion typisches Temperaturprofil mit einem isothermen Bereich und thermischen Grenzschichten aus. In den Grenzschichten nimmt die Temperatur stark ab. Die Phasengrenze wirkt als starke Barriere für den konvektiven Wärmetransport. Die Wärme, die vom Kern abgeben wird, kann nicht effizient abtransportiert werden, so dass sich der Bereich unter der Phasengrenze im Vergleich zum Referenzfall aufheizt. Die Abbildung zeigt, dass oberhalb der Phasengrenze nur ein geringer Anteil der ursprünglichen thermischen Grenzschicht zur Verfügung steht. Der verbleibende Temperaturkontrast ist sehr gering, so dass die Entstehung von Plumes in der oberen Schicht erschwert wird. Dies trägt zur Dominanz der Abströme und zur geringen positiven Temperaturanomalie der Aufströme bei. Das Erhöhen des Aktivierungsvolumens $\left(V^{*}=4.5 \mathrm{~kJ} \mathrm{~mol}^{-1}\right)$ führt schon zu diesem frühen Zeitpunkt zu deutlich niedrigen Temperaturen im Mantel. Der isotherme Bereich erstreckt sich durch die dünnere stagnierende Schicht weiter in Richtung Oberfläche.

Nach 4.5 Ga zeigen die Profile der gemittelten Temperatur (Abbildung (5.8)), dass der Planet um so schneller abkühlt, je größer das Aktivierungsvolumen ist. Im geschichteten Fall sind die Geschwindigkeiten unterhalb der Phasengrenze (Abbildung (5.6)) jetzt so ge- 


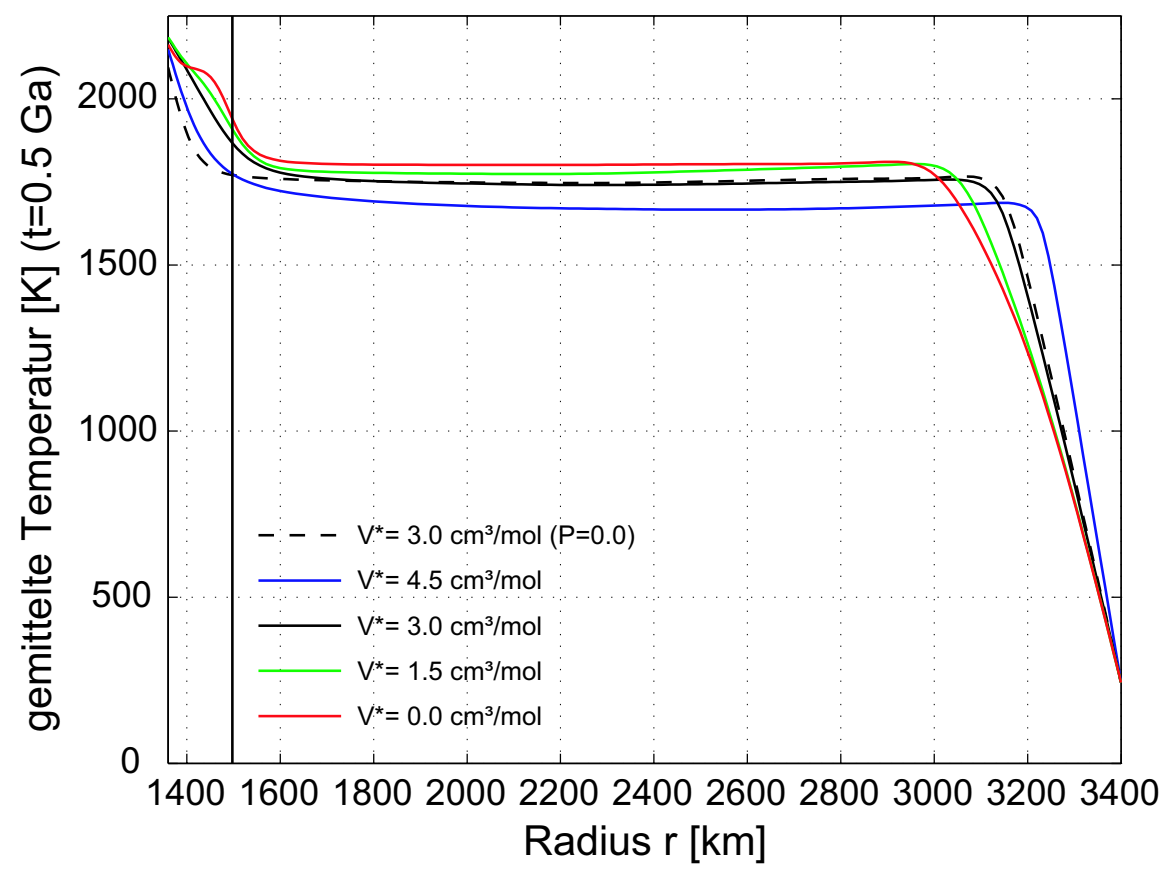

Abbildung 5.7: Einfluss des Aktivierungsvolumens auf das Temperaturprofil bei $t=0.5 \mathrm{Ga}$. Die schwarze Linie markiert die Lage der endothermen Phasengrenze.

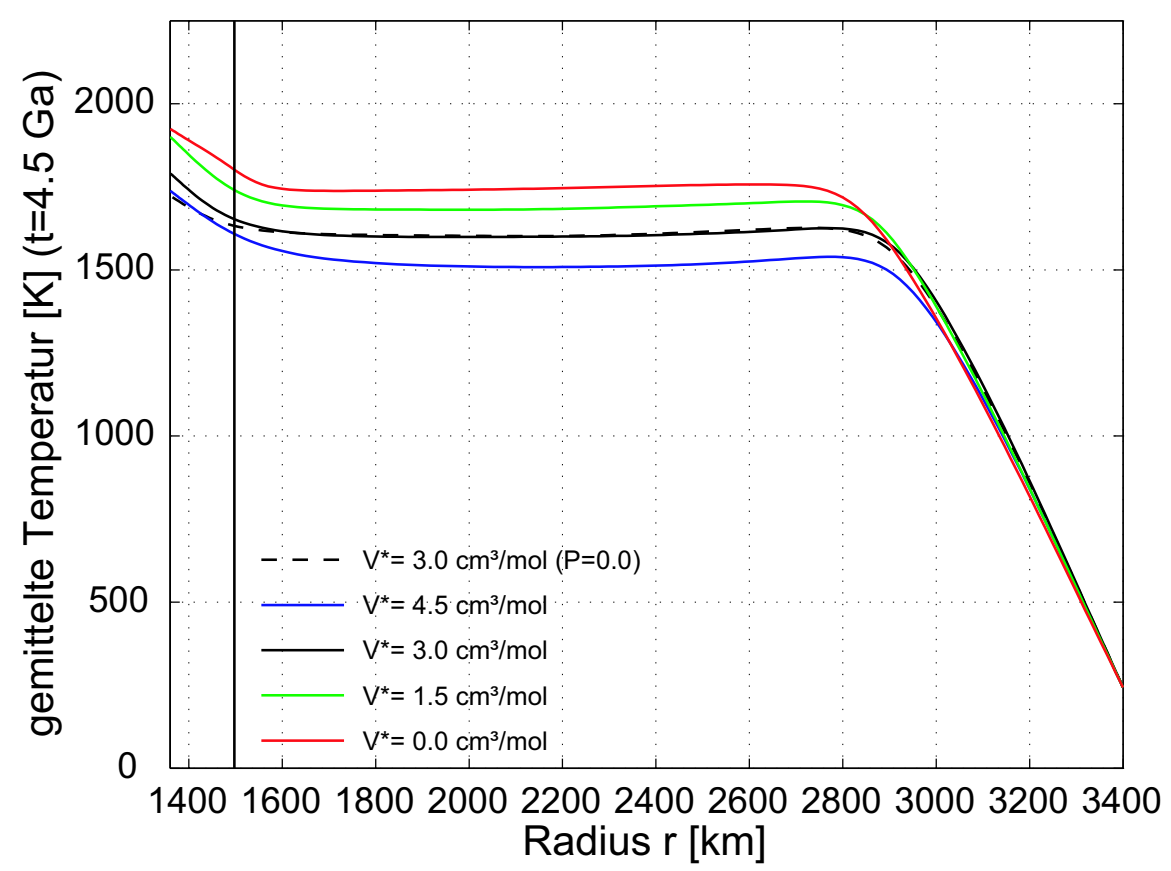

Abbildung 5.8: Einfluss des Aktivierungsvolumens auf das Temperaturprofil bei $t=4.5 \mathrm{Ga}$. Die schwarze Linie markiert die Lage der endothermen Phasengrenze. 
ring, dass das anfängliche Konvektionsprofil nicht mehr sichtbar ist. Der Temperaturkontrast direkt über der Phasengrenze ist aber weiterhin gering. Im Fall $V^{*}=4.5 \mathrm{~kJ} \mathrm{~mol}^{-1}$ ist die kernnahe thermische Grenzschicht gegenüber dem Referenzfall etwas dicker. Der größere Temperaturunterschied zwischen Kern und Mantel kann zu der beobachteten höheren Temperaturanomalie im Aufstrom beitragen. In der Nähe der Oberfläche ist der Einfluss des Aktivierungsvolumens analog zum Profil der Radialgeschwindigkeit (Abbildung (5.6)) zu diesen Zeitpunkt deutlich geringer.

In Abbildung (5.9) sind die Viskositätsprofile nach 4.5 Ga dargestellt. Das unterschied-

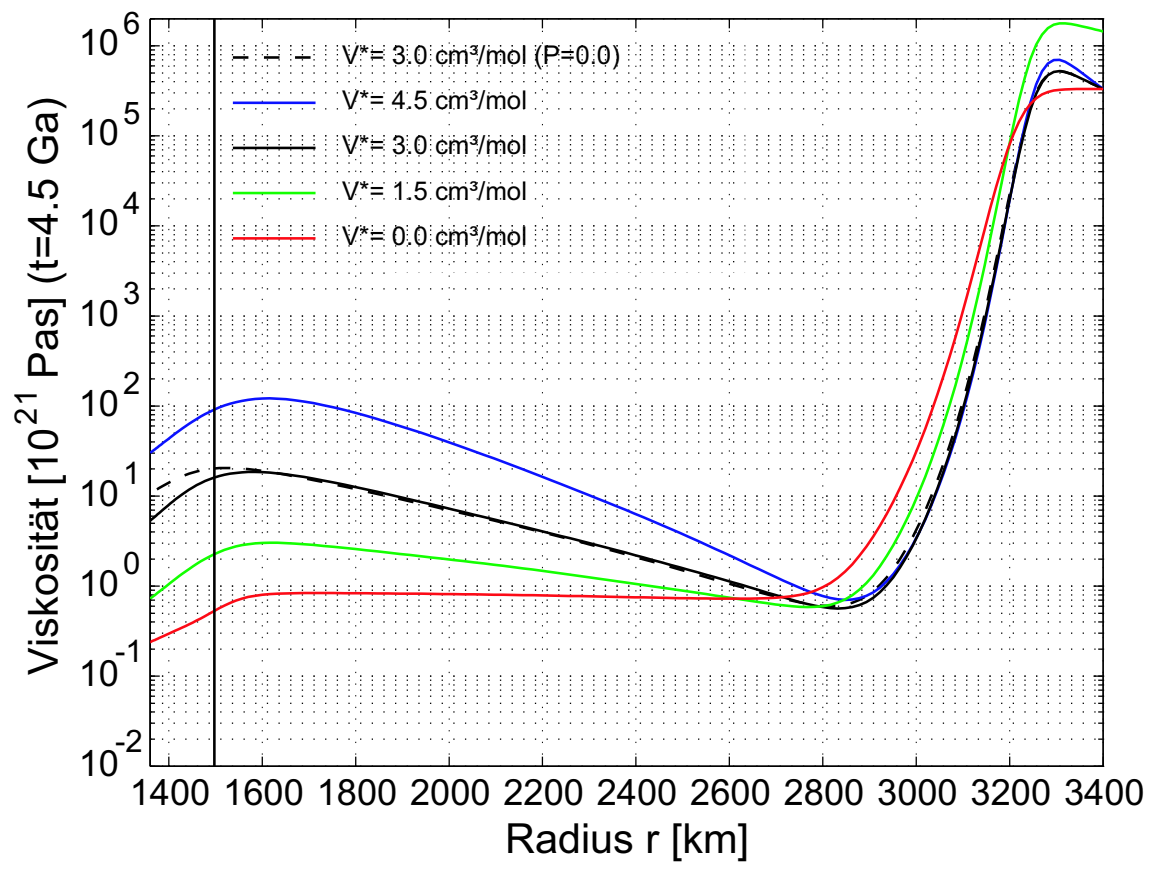

Abbildung 5.9: Viskositätsprofile nach 4.5 Ga für die unterschiedlichen Aktivierungsvolumina. Die Tiefe der endothermen Phasengrenze ist durch die schwarze Linie markiert.

liche Abkühlen des Planeten führt dazu, dass sich die Profile trotz unterschiedlichem Aktivierungsvolumen im oberflächennahen Bereich ähnlicher sind. Insbesondere der Minimalwert der Viskosität unterhalb der thermischen Grenzschicht stimmt nahezu überein. Dies erklärt die geringeren Unterschiede in der Dicke der stagnierenden Schicht im Radialgeschwindigkeitsprofil nach 4.5 Ga. Die höheren Viskositäten bei höherem Aktivierungsvolumen lassen die beobachteten geringeren Geschwindigkeiten im Konvektionsmuster plausibel erscheinen. In der Nähe der KMG führen die hohen Viskositäten bei $V^{*}=4.5 \mathrm{~kJ} \mathrm{~mol}^{-1}$ ebenfalls zu geringeren Geschwindigkeiten und tragen zum Verdicken der thermischen Grenzschicht bei.

Das Abkühlverhalten in Abhängigkeit vom Aktivierungsvolumen lässt sich aus dem Zusammenspiel der Unterschiede in der Konvektionsstärke mit den Unterschieden in der Dicke der stagnierenden Schicht erklären. In der Lithosphäre ist die Viskosität so hoch, dass Wärme nur durch Wärmeleitung transportiert werden kann. Die Konvektionsstärke ist in Abbildung (5.10) durch das über das Volumen gemittelte Quadrat der Geschwin- 


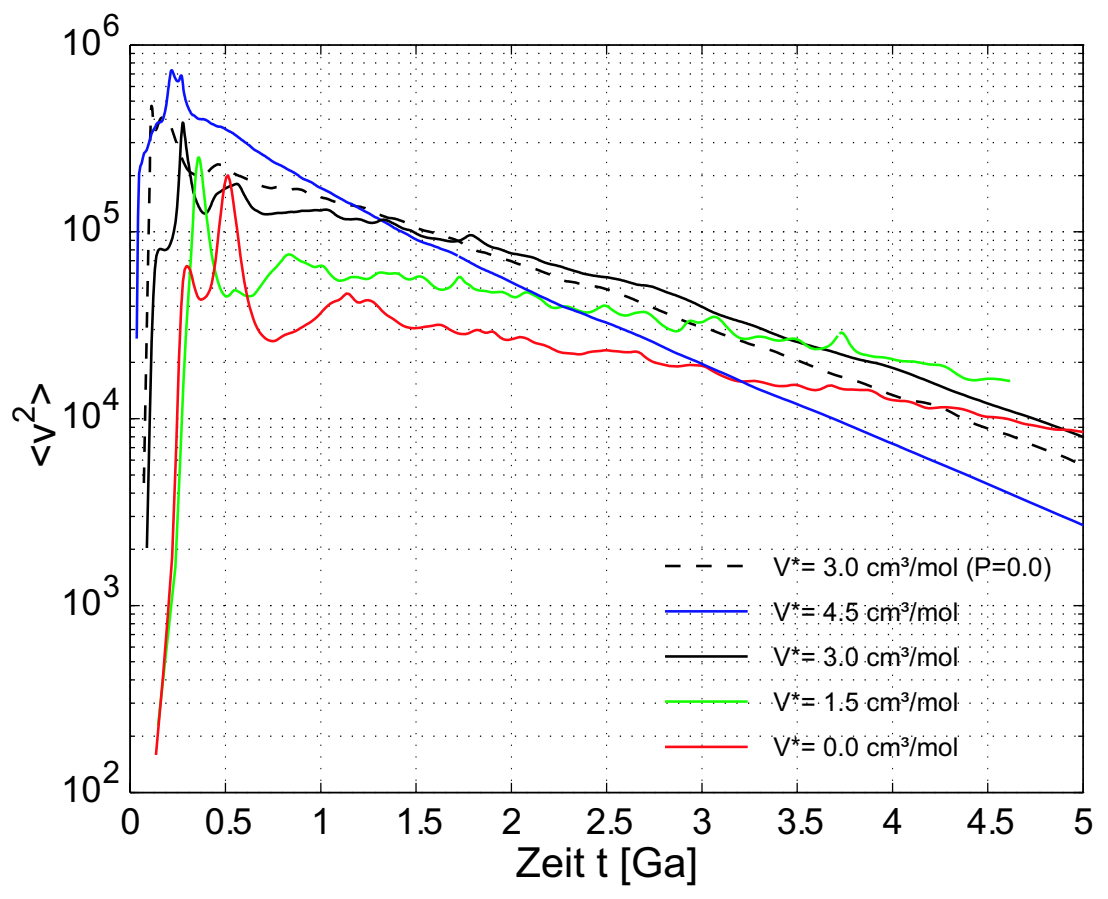

Abbildung 5.10: Einfluss des Aktivierungsvolumens auf die zeitliche Entwicklung der Konvektionsstärke.

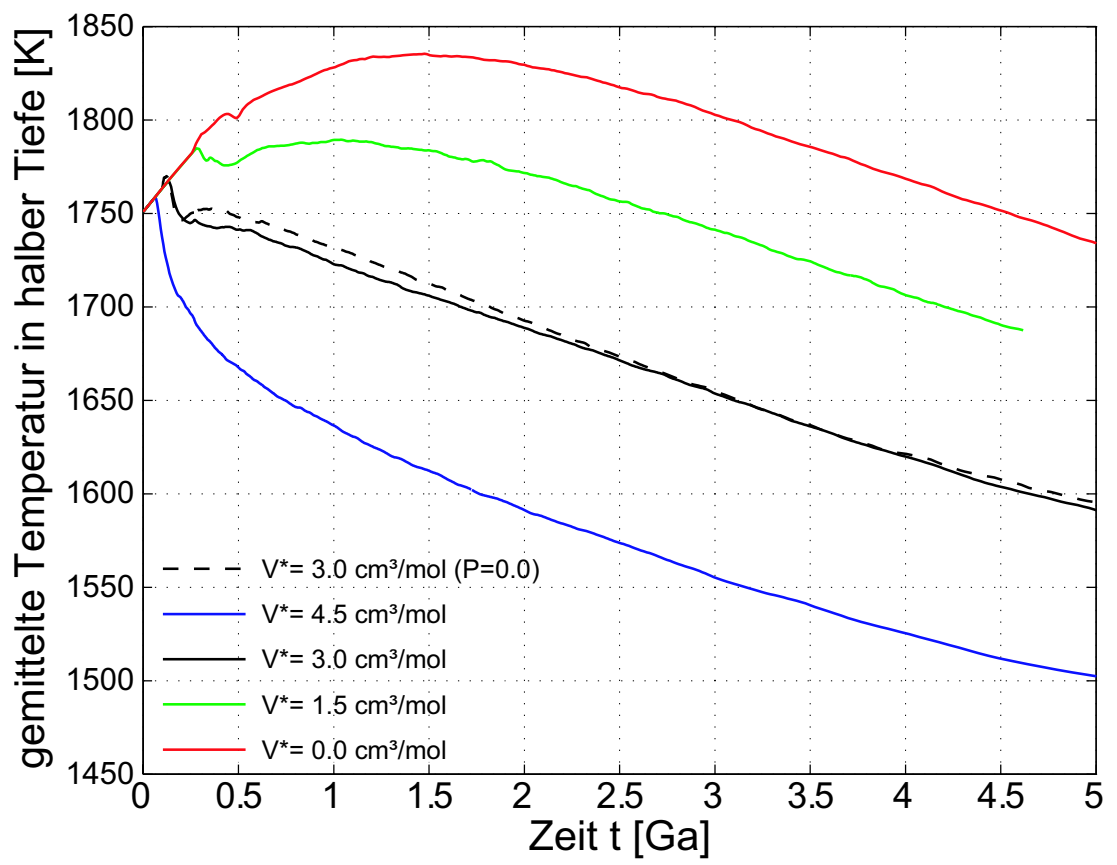

Abbildung 5.11: Einfluss des Aktivierungsvolumens auf die zeitliche Entwicklung der Temperatur in halber Tiefe. 


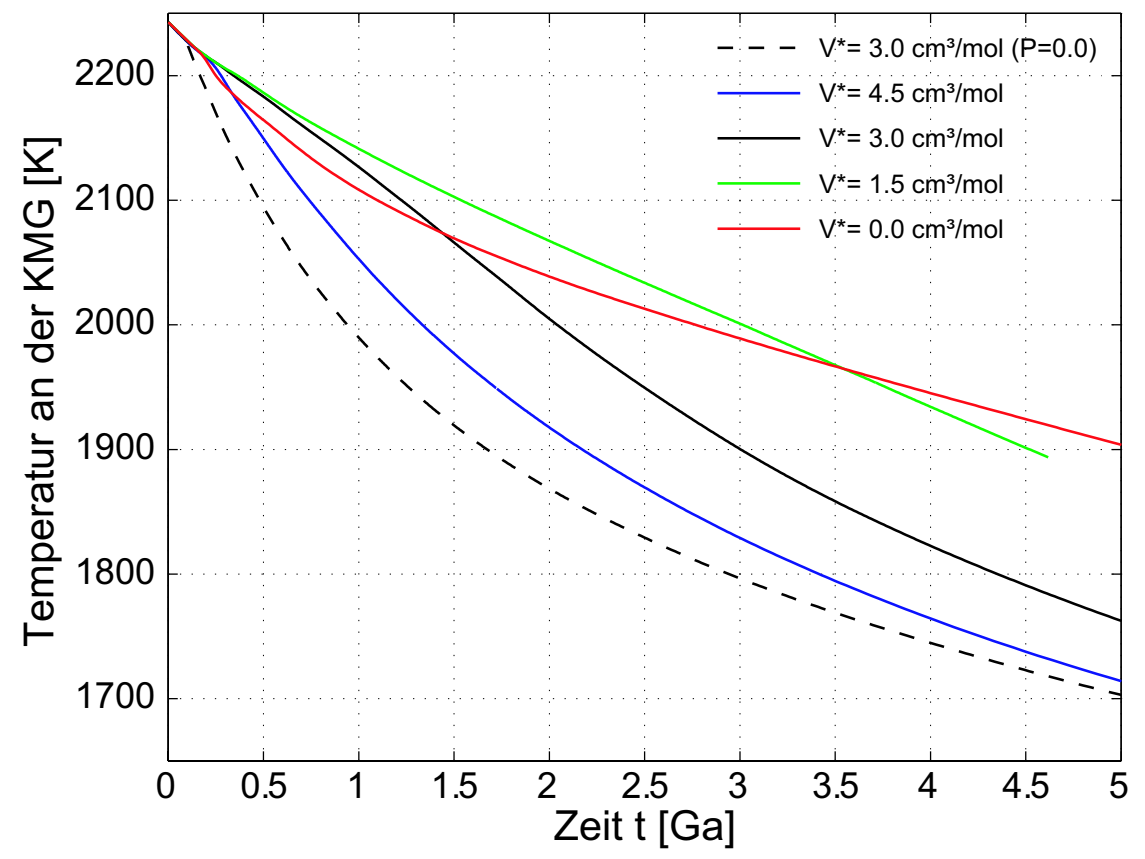

Abbildung 5.12: Einfluss des Aktivierungsvolumens auf die zeitliche Entwicklung der Temperatur an der KMG.

digkeit dargestellt. Das Erhöhen des Aktivierungsvolumens führt durch das Verstärken des niedrigviskosen Bereichs anfänglich zu einer sehr starken Konvektionsbewegung. Da die stagnierende Schicht in diesem Fall sehr dünn ist, kann der Planet effektiv Wärme nach außen abführen und kühlt stark ab. Dies wird in der zeitlichen Entwicklung der Temperatur in halber Tiefe (Abbildung (5.11)) bzw. an der KMG (Abbildung (5.12)) und im Temperaturprofil nach 0.5 Ga (Abbildung (5.8)) deutlich. Das Abkühlen des Planeten erhöht die Viskosität, so dass die Konvektionsstärke abnimmt und die stagnierende Schicht wächst. Zusammen schwächen diese beiden Effekte das Abkühlen des Planeten. Das erhöhte Aktivierungsvolumen steigert die Temperaturabhängigkeit der Viskosität, so dass die Konvektionsstärke stärker abnimmt. Die angesprochenen hohen Viskositäten in der Nähe der KMG schwächen die Abkühlrate des Kerns.

Ein Absenken des Aktivierungsvolumens führt analog durch die höheren Viskositäten im oberflächennahen Bereich zu einem Abschwächen der Konvektionsstärke und zu einer dickeren stagnierenden Schicht, so dass die Wärme weniger effektiv abgegeben werden kann und die Temperaturen in der Mantelmitte zunächst ansteigen. Das Ansteigen der Temperaturen und die damit sinkende Viskosität erklären das verspätete Auftreten des anfänglichen Maximums in der Konvektionsstärke. Bei dem verringerten Aktivierungsvolumen von $V^{*}=1.5 \mathrm{~kJ} \mathrm{~mol}^{-1}$ kühlt der Kern durch die abgeschwächte Konvektion langsamer als im Referenzfall ab. Im Fall der geschichteten Konvektion $\left(V^{*}=0.0 \mathrm{~kJ} \mathrm{~mol}^{-1}\right)$ setzt sich dieser Trend jedoch nicht direkt fort. Durch die Konvektion unterhalb der Phasengrenze kühlt der Kern in diesem Fall zunächst schneller als im Referenzfall ab. Dann verlangsamt sich das Abkühlen durch das Abschwächen der Konvektion und durch den 
geringer werdenden Temperaturunterschied zwischen Kern und Mantelinneren. Der Vergleich der Simulationen mit und ohne Phasengrenze bei $V^{*}=3.0 \mathrm{~kJ} \mathrm{~mol}^{-1}$ zeigt, dass der Einfluss der Phasengrenze auf die Temperaturentwicklung in halber Tiefe gering ist, wohingegen an der KMG das Abkühlen des Kerns durch die Phasengrenze verlangsamt wird.

Die Lithosphärendicke kann aus den numerischen Simulationen anhand der radialen Geschwindigkeitsprofile bestimmt werden und wird als der Bereich definiert, in dem im radialen Profil sowohl der rms-Wert der Tangential- als auch der Radialgeschwindigkeit (siehe Anhang (A.3)) verschwinden bzw. einen vorgegebenen Wert unterschreiten. In dieser Arbeit wird dieser Schwellenwert über die Pecletzahl $P e=v d / \kappa=1.0$ festgelegt. Die Pecletzahl setzt die Bedeutung der Advektion ins Verhältnis zum Beitrag der Diffusion und entspricht der dimensionslosen Geschwindigkeit $(P e=1.0$ entspricht einer Geschwindigkeit von $0.0139 \mathrm{~mm} / \mathrm{a})$. Bei $P e=v d / \kappa \leq 1.0$ gewinnt der Wärmetransport durch Diffusion an Bedeutung und kennzeichnet die Lithosphäre. Der absolute Wert der Lithosphärendicke hängt vom verwendeten Schwellenwert ab (siehe Anhang).

Das Verringern des Aktivierungsvolumens führt zu höheren Viskositäten in der Nähe der

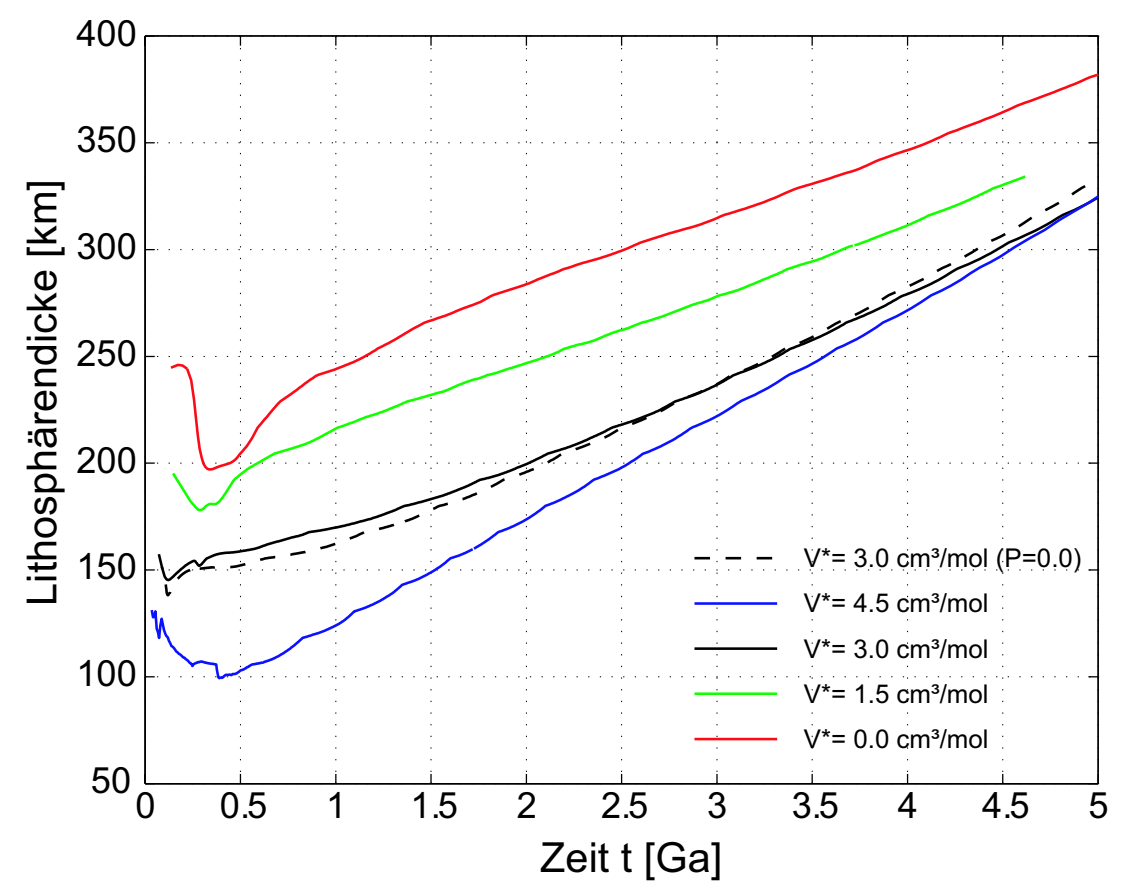

Abbildung 5.13: Einfluss des Aktivierungsvolumens auf die zeitliche Entwicklung der Lithosphärendicke.

Oberfläche und zu einer dickeren Lithosphäre. Analog erzeugt ein höheres Aktivierungsvolumen zunächst eine deutlich dünnere Lithosphäre. Durch das stärkere Abkühlen im Lauf der Zeit und die erhöhte Temperaturabhängigkeit der Viskosität wird aber nach 4.5 Ga etwa dieselbe Lithosphärendicke wie im Referenzfall erreicht. Die Lithosphärendicken liegen zwischen $300 \mathrm{~km}\left(V^{*}=4.5 \mathrm{~kJ} \mathrm{~mol}^{-1}\right)$ und $367 \mathrm{~km}\left(V^{*}=0.0 \mathrm{~kJ} \mathrm{~mol}^{-1}\right)$. Ein Vergleich der bestimmten Lithosphärendicke mit Messdaten erfolgt in der Diskussion 
(Kapitel (7)).

Die Temperaturentwicklung des Planeten spiegelt sich im Wärmefluss wieder. Abbildung

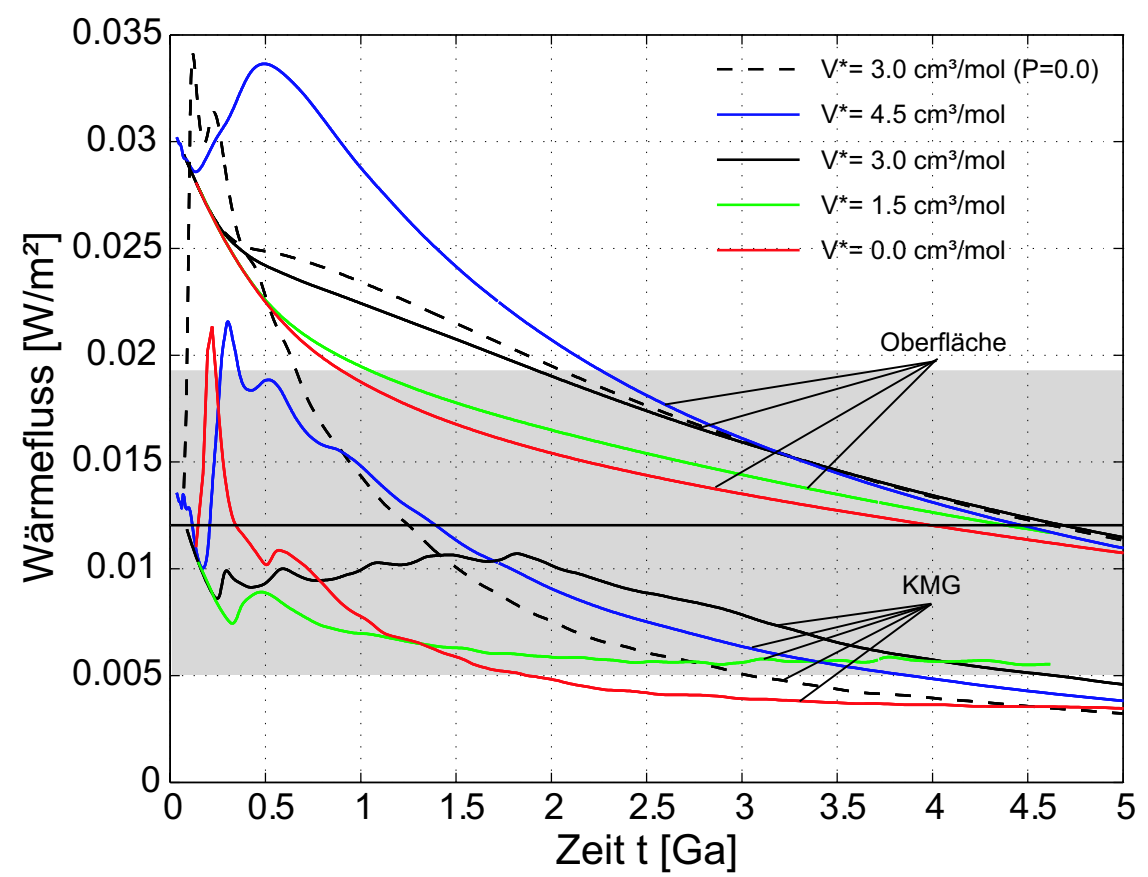

Abbildung 5.14: Einfluss des Aktivierungsvolumens auf die zeitliche Entwicklung der Wärmeflusses. Der grau schattierte Bereich markiert den Wertebereich für den Wärmefluss entlang der Kernadiabate (Nimmo und Stevenson 2000). Die schwarze Linie zeigt zur Orientierung den Mittelwert dieses Bereichs.

(5.14) zeigt die Entwicklung des Wärmeflusses an der Oberfläche und an der KMG. An der Oberfläche führt die dünne Lithosphäre im Zusammenspiel mit der anfänglich starken Konvektion in Fall eines erhöhten Aktivierungsvolumens nach kurzer Zeit zu einem im Vergleich zum Referenzfall deutlich erhöhten Wärmefluss, der während der Evolution abnimmt und nach 4.5 Ga nur geringfügig unterhalb des Wärmeflusses im Referenzfall liegt. Der Wärmefluss an der Oberfläche bei erniedrigtem Aktivierungsvolumen liegt nach kurzer Zeit unter den Werten im Referenzfall. Die Zeitspanne, die bis zum Abweichen vom Referenzfall vergeht, hängt von der Dicke der anfänglichen Lithosphäre $a b$, da die durch die Konvektion herantransportierte Wärme durch die Lithosphäre zur Oberfläche diffundieren muss. Nach 4.5 Ga liegt der Wärmefluss an der Oberfläche zwischen $11.4 \mathrm{~mW} / \mathrm{m}^{2}$ $\left(V^{*}=0.0 \mathrm{~kJ} \mathrm{~mol}^{-1}\right)$ und $12.4 \mathrm{~mW} / \mathrm{m}^{2}$ im Referenzfall.

An der KMG bestimmt weitgehend die Konvektionsstärke den Wärmefluss. Etwas Besonderes ist wiederum der Fall der geschichteten Konvektion $\left(V^{*}=0.0 \mathrm{~kJ} \mathrm{~mol}^{-1}\right)$. Obwohl die volumen-gemittelte Konvektionsstärke zu Beginn relativ schwach ist, führt die Konvektion in der dünnen, kernnahen, unteren Schicht zu einem erhöhten Wärmefluss. Der Wärmefluss aus dem Kern ist bedeutsam für die Möglichkeit eines thermisch getriebenen Dynamos im Mars. Damit thermische Konvektion im Kern einen Dynamo treiben kann, muss der Wärmefluss aus dem Kern größer sein als der Wärmefluss entlang der 
Kernadiabate (Kapitel (2.5)). Der Wärmefluss entlang der Kernadiabate ist nur unzureichend bekannt, da viele Materialparameter des Eisens, insbesondere bei einem erhöhten Schwefelgehalt bei den Druck- und Temperaturbedingungen im Marskern mit großen Unsicherheiten behaftet sind. Nimmo und Stevenson (2000) schlagen den in Abbildung (5.14) grau schattierten Wertebereich für den Wärmefluss entlang der Adiabate vor. Zur besseren Orientierung wird ein mittlerer Wert von $F_{\mathrm{m}}=12 \mathrm{~mW} / \mathrm{m}^{2}$ angenommen. In den Simulationen mit kernnaher endothermer Phasengrenze liegt der Wärmefluss bei $V^{*}=4.5 \mathrm{~kJ} \mathrm{~mol}^{-1}$ durch die stärkere Konvektion bis etwa $1.4 \mathrm{Ga}$ über diesem Wert. Im Fall der geschichteten Konvektion übersteigt der Wärmefluss diesen Mittelwert bis etwa $0.35 \mathrm{Ga}$. In diesen Fällen wäre in den genannten Zeiträumen ein thermisch getriebener Dynamo möglich. Die geringe Breite des Maximums erlaubt im Fall der geschichteten Konvektion relativ kurze Aktivitätszeiten für den Dynamo. Anhand der Simulationen ist es allerdings schwierig den genauen Einsatz der Dynamoaktivität festzulegen, da der anfängliche Abfall des Wärmeflusses und der daraufhin einsetzende Wiederanstieg von der Startkonfiguration (z.B. Störungsamplitude und -geometrie) abhängen kann. Im Referenzfall (mit Phasengrenze) bzw. beim Verringern des Aktivierungsvolumens ist ein thermisch getriebener Dynamo unwahrscheinlich. Eine Lebensdauer von $1.4 \mathrm{Ga}$ im Fall von $V^{*}=4.5 \mathrm{~kJ} \mathrm{~mol}^{-1}$ überschreitet deutlich die Abschätzungen aus den fehlenden Magnetisierungen in den großen Einschlagkratern wie z.B. dem Hellas Basin, die eine Lebensdauer des Dynamos im Mars von ungefähr 0.5 Ga nahelegen (Kapitel (2.5)). Dies ist wahrscheinlicher mit einem adiabatischen Wärmefluss von $17-18 \mathrm{~mW} / \mathrm{m}^{2} \mathrm{zu}$ erreichen. Um die Lebenszeit des Dynamos mit Hilfe der Simulationen besser einzuschränken, ist eine genauere Kenntnis der Materialparameter nötig. Der heutige Wärmefluss aus dem Kern ist in allen Modellen zu gering, um einen thermisch getriebenen Dynamo zu ermöglichen.

Zusammenfassend führt ein niedrigeres Aktivierungsvolumen zu niedrigeren Viskositäten an der KMG und im Bereich der Phasengrenze sowie zu höheren Viskositäten bzw. einer schwächer ausgeprägten niedrigviskosen Zone unterhalb der oberflächennahen thermischen Grenzschicht. Die niedrigen Viskositäten in der Tiefe der Phasengrenze verstärken den konvektionshemmenden Effekt der Phasengrenze und machen die Phasengrenze zu einer nahezu undurchlässigen Barriere für die Konvektionsströmung, so dass diese in zwei unabhängigen Schichten stattfindet. In der oberen Schicht steht die Phasengrenze dann nicht mehr zur Reduzierung der Anzahl der Aufströme zur Verfügung, so dass ein Konvektionsmuster mit vielen, schwächeren Auströmen möglich wird. Dieser Effekt kann je nach Aktivierungsvolumen über den ganzen Evolutionszeitraum auftreten oder nach einem gewissen Zeitraum durch das Erhöhen der Viskosität beim Abkühlen des Systems abklingen. Damit die Phasengrenze die Anzahl der Aufströme reduzieren kann, darf also eine Mindestviskosität nicht unterschritten werden. Ein niedrigeres Aktivierungsvolumen führt zu einer geringeren Kühlrate des Planeten. Durch ein höheres Aktivierungsvolumen wird die niedrigviskose Zone in der Nähe der Oberfläche verstärkt. Dies führt insbesondere zu Beginn der Evolution zu einer dünneren Lithosphäre und zu einer stärkeren Konvektionsbewegung, so dass der Kern und der Planetenmantel schneller abkühlen. Das schnellere Abkühlen des Kerns bzw. der durch die heftigere Konvektion erhöhte Wärmefluss an der KMG machen einen frühen thermisch getriebenen Dynamo im Mars auch im Fall mit Phasengrenze wahrscheinlicher. Der heutige Wärmefluss aus dem Kern ist zu gering, um einen thermisch getriebenen Dynamo zu ermöglichen. 


\subsection{Einfluss verschiedener Profile von $\alpha(r)$}

Der thermische Ausdehnungskoeffizient $\alpha$ ist in den bisherigen Modellen vereinfacht als konstant angenommen worden. Wie in Kapitel (3.6) näher erläutert wird, variiert er mit der Tiefe als Funktion des Drucks. Da der Expansionskoeffizient die konvektionstreibenden Auftriebskräfte beeinflusst, kann seine Tiefenabhängigkeit möglicherweise das Konvektionsmuster, die Rolle der Phasengrenze und die thermische Entwicklung des Planeten verändern. Dies soll in diesem Kapitel anhand verschiedener Profile untersucht werden. Abbildung (5.15) zeigt die verschiedenen betrachteten Profile des thermischen Ausdeh-

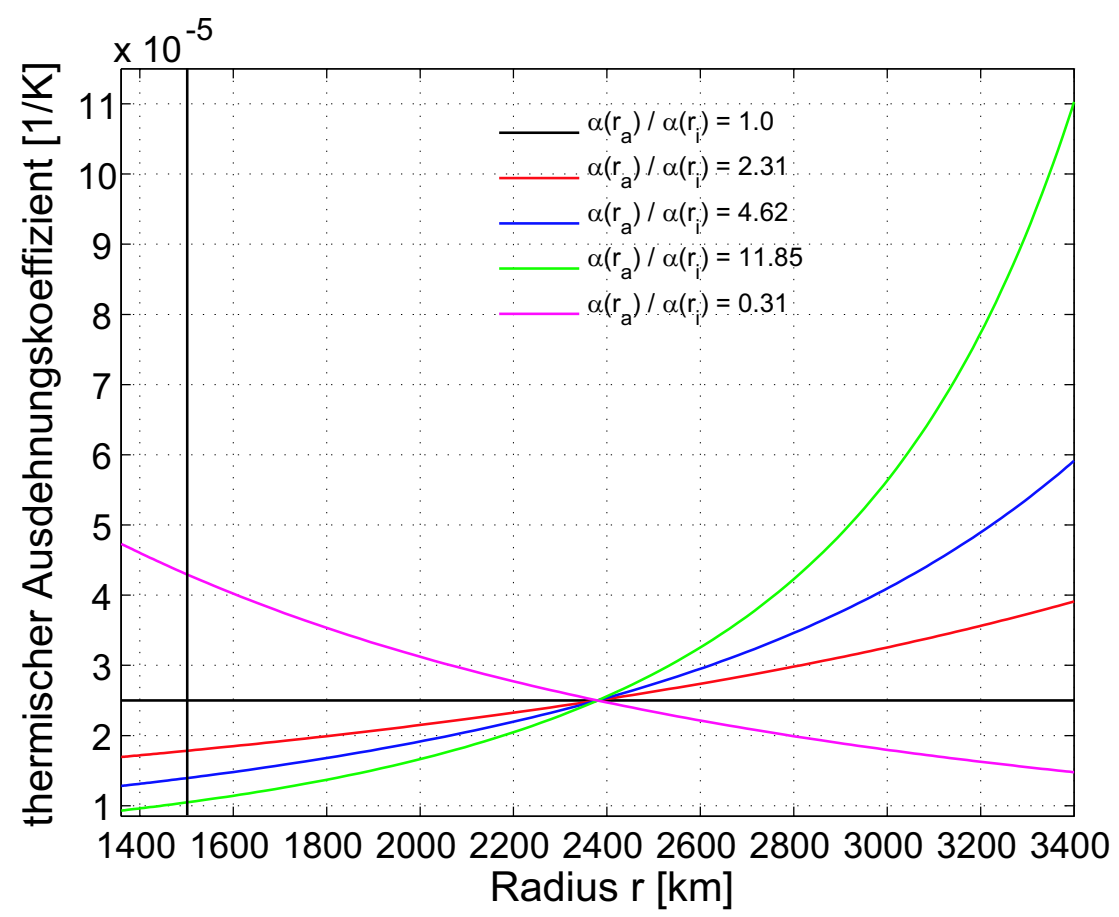

Abbildung 5.15: Radiale Profile des thermischen Ausdehnungskoeffizienten. Die Lage der endothermen Phasengrenze ist durch die schwarze Linie markiert.

nungskoeffizienten, die nach Gleichung (3.56) berechnet werden. Als Referenzfall gilt der Fall mit konstantem $\alpha$. Die Profile sind so normiert, dass in halber Tiefe der Referenzwert erreicht wird. Dem Polynom (3.56) entsprechend, nimmt der Wert des thermischen Ausdehnungskoeffizienten von der Oberfläche zur KMG ab, wobei die Abweichungen vom Referenzfall an der KMG kleiner als an der Oberfläche sind. Die Wahl der auftretenden Materialparameterkombination bestimmt das Verhältnis $\alpha\left(r_{a}\right) / \alpha\left(r_{i}\right)$, das hier zur Charakterisierung der Profile verwendet wird. Mit Ausnahme der magentafarbenen Kurve, die den unphysikalischen Fall eines mit der Tiefe zunehmenden Ausdehnungskoeffizienten repräsentiert und zu einem besseren Verständnis des tiefenabhängigen Parameters beitragen soll, ist $\alpha\left(r_{a}\right) / \alpha\left(r_{i}\right)$ größer als 1. Für Mars ergibt sich mit den verwendeten Daten nach Gleichung (3.56) eine Zunahme des Ausdehnungskoeffizienten von der KMG zur Oberfläche von $\alpha\left(r_{a}\right) / \alpha\left(r_{i}\right)=2.3$. Für die Erde gilt ungefähr $\alpha\left(r_{a}\right) / \alpha\left(r_{i}\right)=11.85$ (Leitch et al. 1991). 
Die Konvektionsmuster, die sich unter Verwendung der unterschiedlichen Profile nach 4.5 Ga einstellen, sind in Abbildung (5.16) in den verschiedenen Zeilen durch die Radialgeschwindigkeit in halber Tiefe dargestellt. Dabei vergleichen die beiden Spalten die Situation bei Vernachlässigung einer kernnahen endothermen Phasengrenze (linke Spalte, $P=0.0$ ) mit der Situation, die diesen Phasenübergang mit in Betracht zieht (rechte Spalte, $P=-0.2723$ ). Die erste Zeile zeigt den Refenzfall bei konstantem thermischen Ausdehnungskoeffizient. Dieser Fall ist bereits im Zusammenhang mit dem Einfluss des Aktivierungsvolumens gezeigt worden und ist hier zur besseren Vergleichbarkeit nochmals aufgeführt. Ein tiefenabhängiges Profil des thermischen Ausdehungskoeffizienten mit $\alpha\left(r_{a}\right) / \alpha\left(r_{i}\right)=2.3$ führt bei Vernachlässigung der Phasengrenze zu einem Konvektionsmuster mit vier Aufströmen, von denen einer deutlich schwächer als die drei übrigen ist. Dieses Muster, in dem die Anzahl der Plumes gegenüber dem Referenzfall ohne Phasengrenze zurückgegangen ist, hat sich nach ungefähr $2.5 \mathrm{Ga}$ etabliert. Das Einbeziehen der endothermen Phasengrenze bei diesem $\alpha$-Profil ergibt eine Anordnung mit drei Aufströmen, die zwischen 1.3 und 1.9 Ga erreicht wird und sich anschließend lediglich in der Amplitude abschwächt. Wird die Tiefenabhängigkeit unter Berücksichtigung der Phasengrenze weiter verstärkt $\left(\alpha\left(r_{a}\right) / \alpha\left(r_{i}\right)=4.6\right)$, so verändert sich das Konvektionsmuster gegenüber dem vorher betrachteten Fall lediglich durch ein Abschwächen der Amplitude der Geschwindigkeit. Erhöht man die radiale Abhängigkeit weiter, so ergeben sich fast identische Konvektionsmuster mit vier Aufströmen für den Fall mit Phasengrenze und den Fall, der den Phasenübergang vernachlässigt. Die Aufströme sind nahezu symmetrisch zum Äquator und zum Meridian bei $\phi=45^{\circ}$. Auch die Geschwindigkeitsamplituden werden durch die Phasengrenze kaum beeinflusst. Lediglich die Zeitspanne, die vergeht, bis sich dieses Konvektionsmuster etabliert, ist unterschiedlich: Ohne Phasengrenze dauert dies etwa 1.5 - 2.0 Ga, wohingegen bei Berücksichtigung des Mineralübergangs nur 0.5 - 0.6 Ga vergehen. Die Verwendung des unphysikalischen Profils mit einem erhöhten Ausdehungskoffizienten in der Nähe der KMG erzeugt, wenn die Phasengrenze nicht in Betracht gezogen wird, ein Konvektionsmuster mit sechs deutlichen Aufströmen, das sich zwischen 2.5 und 3.0 Ga einstellt. Die Phasengrenze reduziert die Anzahl der Aufströme auf drei, von denen der polnahe Plume etwas schwächer als die übrigen ist. Die Aufströme benötigen zwischen 3.5 und 4.0 Ga, um diese Anordnung einzunehmen. Insbesondere bei der Aufstromgeschwindigkeit entsteht durch die Phasengrenze ein starker Unterschied. Ein tiefenabhängiger thermischer Ausdehungskoeffizient mit kleineren Werten in der Nähe der KMG kann ohne kernnahe endotherme Phasengrenze zu einer Reduzierung der Anzahl der Aufströme führen. Allerdings ist dieser Mechanismus weniger effektiv als die Phasengrenze im Referenzfall und setzt sich bei einem weiteren Absenken von $\alpha$ im kernnahen Bereich nicht fort. Der Einfluss der Phasengrenze wird durch die Tiefenabhängigkeit von $\alpha$ (bei $\alpha\left(r_{a}\right) / \alpha\left(r_{i}\right)>1$ ) abgeschwächt.

In den physikalisch sinnvollen $\alpha$-Profilen nimmt $\alpha$ an der KMG kleinere Werte an. Ein kleinerer thermischer Ausdehnungskoeffizient hemmt auch ohne Phasengrenze Instabilitäten in der thermischen Grenzschicht und schwächere Plumes in ihrer Aufwärtsbewegung durch ein Verringern des Auftriebs. Die angesammelte Wärme steigt dann konzentriert in wenigen starken Plumes auf. Diese wenigen Aufströme werden bei ihrem Aufstieg durch den nach oben zunehmenden Ausdehnungskoeffizienten weiter gestärkt. Sie sind meist stark genug, um die Phasengrenze zu durchdringen, so dass ihr Einfluss verringert wird. Die Phasengrenze hilft jedoch, die Endkonfiguration früher zu erreichen. Hinzu 
a) $\alpha\left(r_{a}\right) / \alpha\left(r_{i}\right)=1$

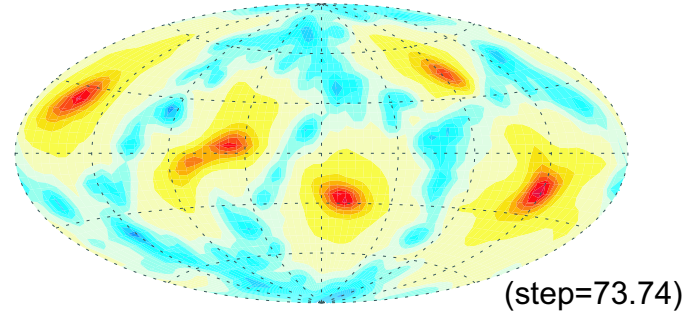

b) $\alpha\left(r_{a}\right) / \alpha\left(r_{i}\right)=2.31$

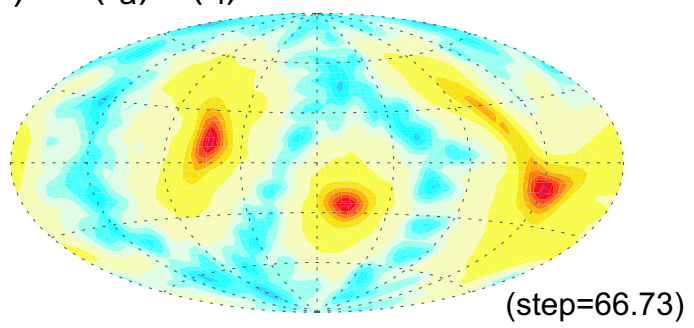

c) $\alpha\left(r_{a}\right) / \alpha\left(r_{i}\right)=4.62$

d) $\alpha\left(r_{a}\right) / \alpha\left(r_{i}\right)=11.85$

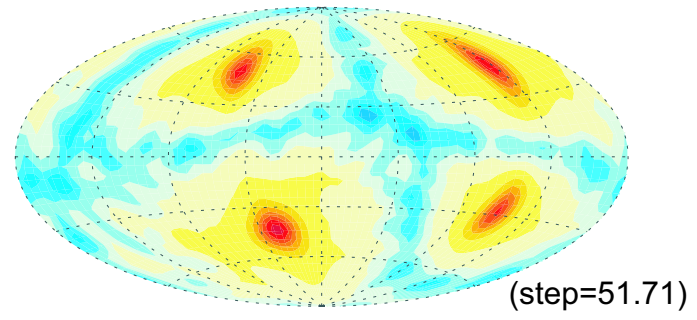

e) $\alpha\left(r_{a}\right) / \alpha\left(r_{i}\right)=0.3$

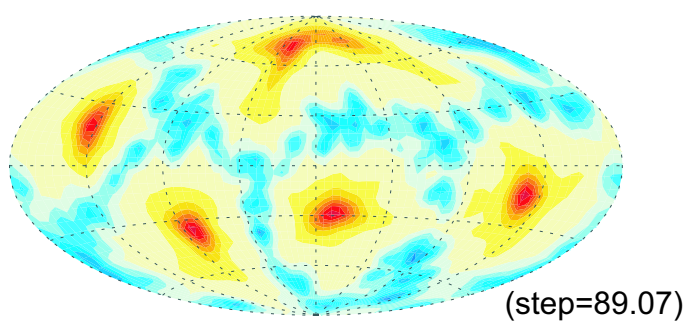

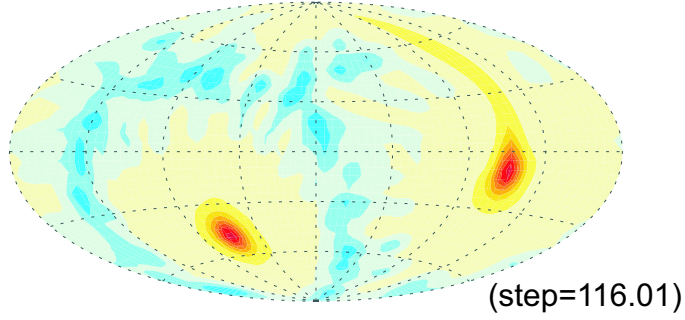

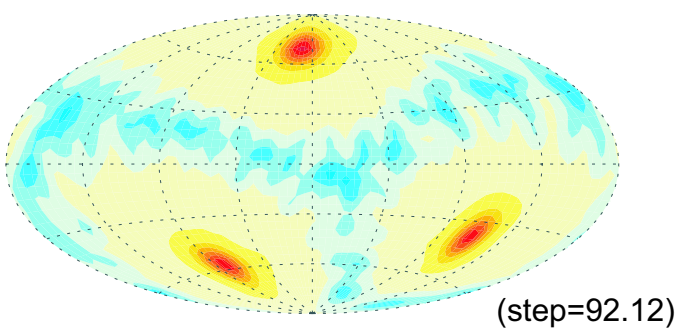

$($ step $=92.12)$
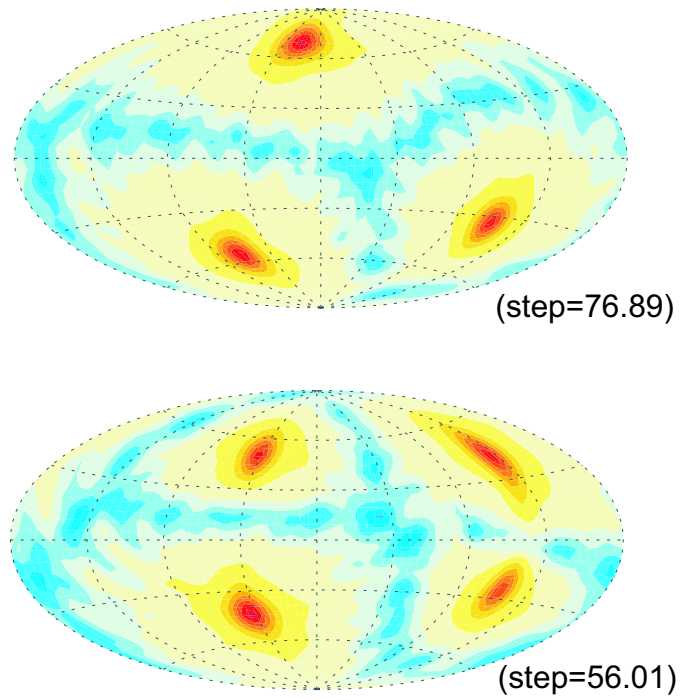

(step $=56.01)$

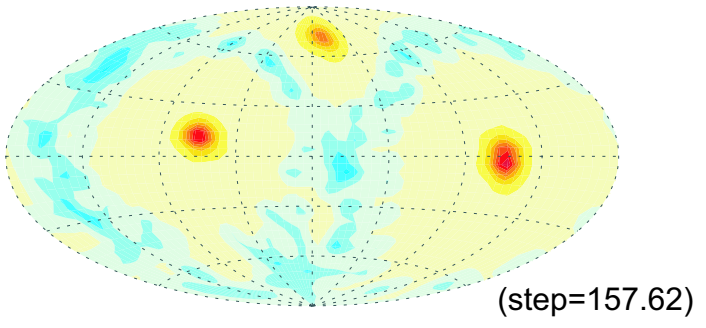

Abbildung 5.16: Einfluss der verschiedenen Profile des thermischen Ausdehungskoeffizienten auf das Konvektionsmuster $(t=4.5 \mathrm{Ga})$ : Radialgeschwindigkeit in halber Tiefe (rot: Aufströme, blau: Abströme). Die linke Spalte zeigt die Situation ohne Phasengrenze $(P=0.0)$, in der rechten Spalte wird die kernnahe endotherme Phasengrenze berücksichtigt $(P=-0.2723)$. Der Contourstep der Geschwindigkeit ist angegeben: $s t e p=100$ entspricht $0.14 \mathrm{~cm} / \mathrm{a}$. 
kommt der Einfluss der Abströme. Durch den deutlich erhöhten Expansionskoeffizienten in der oberen Mantelhälfte wird der Einfluss der Abströme auf das Konvektionsmuster verstärkt, so dass sie das Konvektionsmuster vorgeben und die Position und Anzahl der Plumes zu einer mehr passiven Reaktion auf die Abströme wird. Der Trend, die Anzahl der Aufströme allein durch ein Absenken von $\alpha$ an der KMG (ohne Phasengrenze) zu reduzieren, setzt sich so nicht zwangsläufig fort. Instabilitäten in der kernnahen, thermischen Grenzschicht, auf die die Phasengrenze Einfluss nehmen kann, spielen bei diesem Mechanismus nur eine untergeordnete Rolle, so dass der Einfluss des Phasenübergangs klein ist und sich nur zu Beginn der Evolution bei den anfänglich vielen Aufströmen auswirkt.

Hansen et al. (1991) untersuchen in einem kartesischen zweidimensionalen Modell den Einfluss eines tiefenabhängigen thermischen Ausdehnungskoeffizienten auf die Konvektion in einem inkompressiblen Mantel (erweiterte Boussinesq-Näherung, ohne thermische Evolution). Auch sie finden sowohl im isoviskosen Medium (Hansen et al. 1991) als auch im Fall einer tiefenabhängigen Viskosität (Hansen et al. 1993) ein Stabilisieren der kernnahen thermischen Grenzschicht durch $\alpha(r)$ und ein großskaliges Konvektionsmuster mit wenigen, starken, stationären Aufströmen. Diese starken, konzentrierten Aufströme verhindern, dass innerhalb der thermischen Grenzschicht Wärme angesammelt wird und weitere Instabilitäten entstehen.

Abbildung (5.17) zeigt die radialen Profile der Radialgeschwindigkeit zum einen frühen

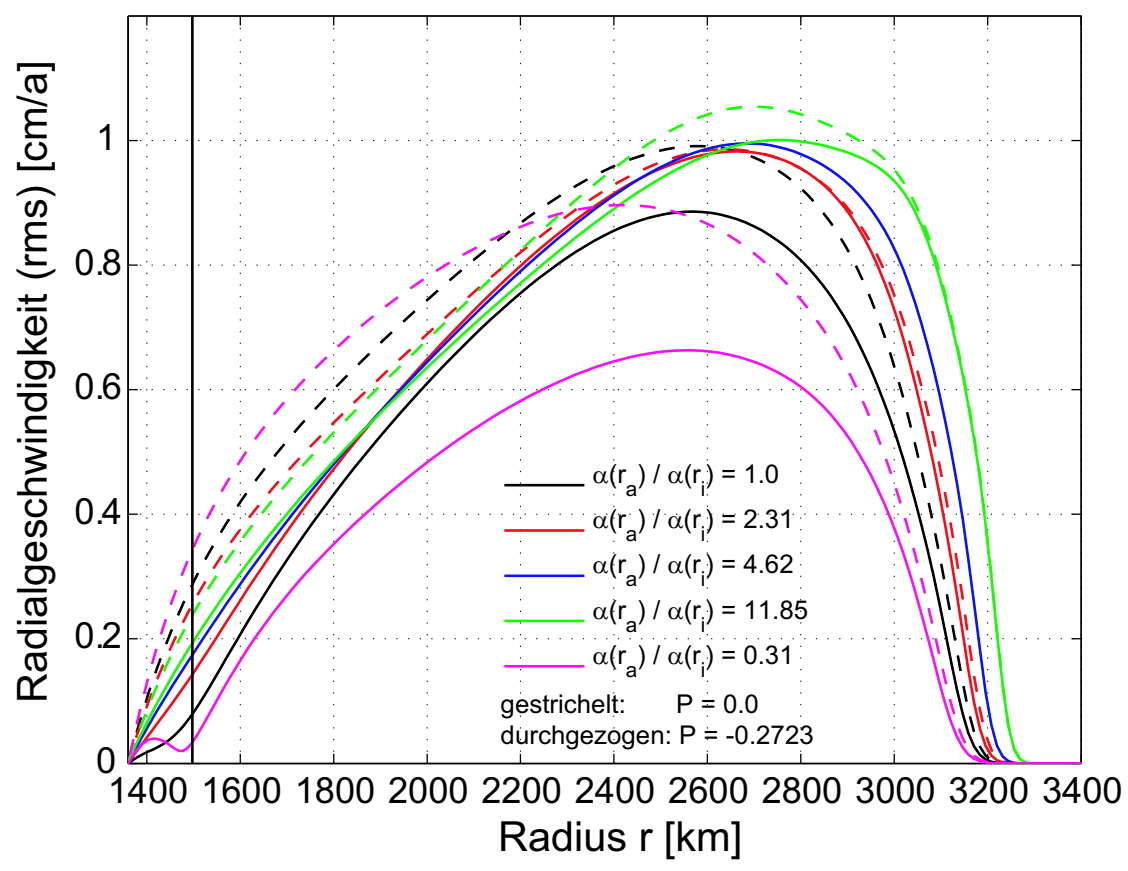

Abbildung 5.17: Einfluss der verschiedenen $\alpha(r)$ auf das Profil der Radialgeschwindigkeit bei $t=0.5 \mathrm{Ga}$. Die schwarze Linie markiert die Lage der endothermen Phasengrenze.

Zeitpunkt in der Entwicklung bei $t=0.5 \mathrm{Ga}$. Zu diesem Zeitpunkt wird deutlich, dass die Geschwindigkeitsprofile (bei $\alpha\left(r_{a}\right) / \alpha\left(r_{i}\right)>1$ ) in Richtung der hohen thermischen 


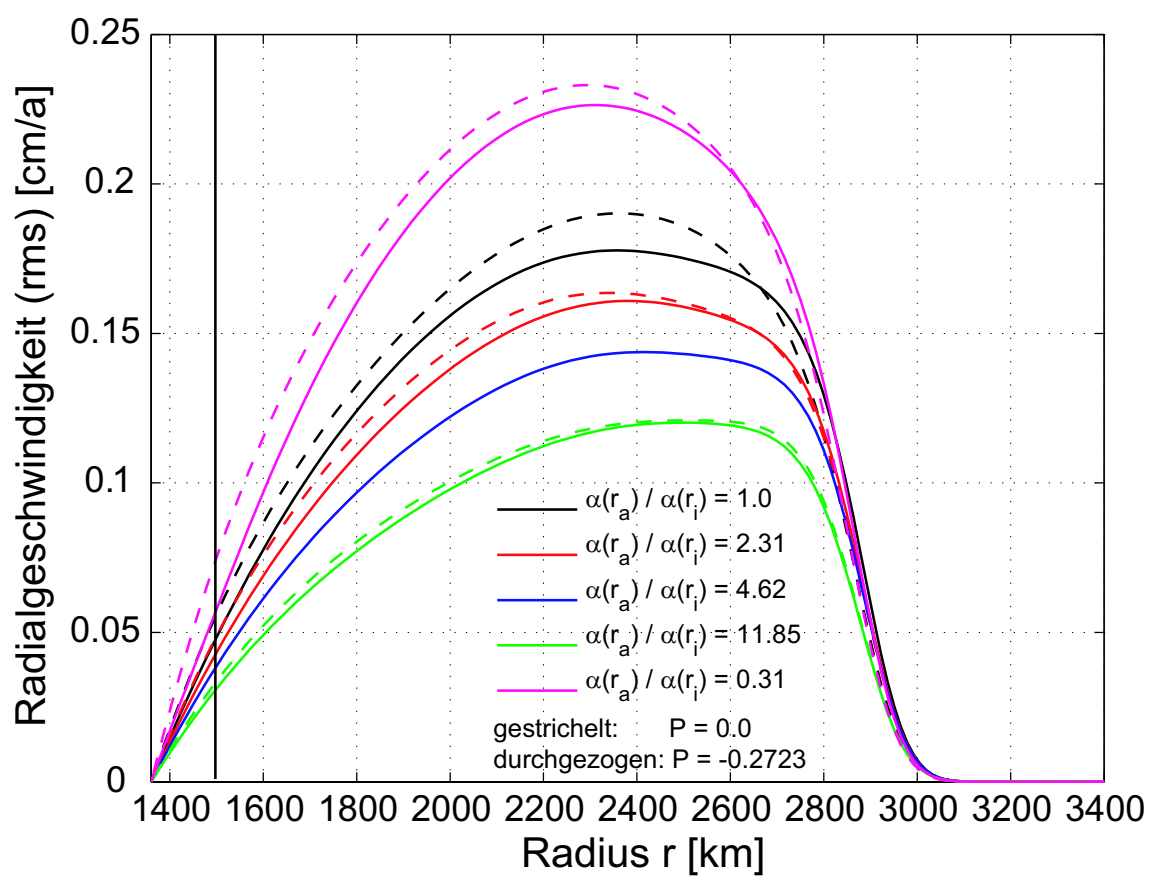

Abbildung 5.18: Einfluss der verschiedenen $\alpha(r)$ auf das Profil der Radialgeschwindigkeit bei $t=4.5 \mathrm{Ga}$. Die schwarze Linie markiert die Lage der endothermen Phasengrenze.

Ausdehnungskoeffizienten in der Nähe der Oberfläche verschoben sind. Die Radialgeschwindigkeit ist umso höher, je größer das Verhältnis $\alpha\left(r_{a}\right) / \alpha\left(r_{i}\right)$ ist. Gleichzeitig nimmt die Dicke der Lithosphäre mit diesem Verhältnis ab. Das Berücksichtigen der Phasengrenze (durchgezogene Linien) führt zu etwas geringeren Geschwindigkeiten, wobei aber im oberflächennahen Bereich der Einfluss der Phasengrenze mit steigendem $\alpha\left(r_{a}\right) / \alpha\left(r_{i}\right)$ geringer wird. Wird die endotherme Phasengrenze vernachlässigt (gestrichelte Linien), so sind auch im kernnahen Bereich die Radialgeschwindigkeiten umso höher, je größer der thermische Ausdehnungskoeffizient ist. Die größten Geschwindigkeiten ergeben sich in diesem Bereich für das Profil mit $\alpha\left(r_{a}\right) / \alpha\left(r_{i}\right)=0.3$. Da die Unterschiede zwischen den verschiedenen $\alpha(r)$-Profilen an der KMG geringer sind als in der oberen Mantelhälfte, sind die Unterschiede in der Radialgeschwindigkeit kleiner. Das Einbeziehen der Phasengrenze kehrt das Bild jedoch so um, dass nun im kernnahen Bereich und insbesondere in der Tiefe der Phasengrenze die Geschwindigkeit mit abnehmendem $\alpha$ steigt. Im unphysikalischen Profil mit den größten $\alpha$-Werten in dieser Region tritt sogar ein deutliches Minimum in der Tiefe der Phasengrenze auf. Ähnlich wie im Fall der erniedrigten Viskosität durch das Verringern des Aktivierungsvolumens (Kapitel (5.1)) führt ein Erhöhen des Ausdehnungskoeffizienten zu einem Anstieg der lokalen Rayleighzahl (Rayleighzahl, in deren Berechnung die lokalen Parameter an Stelle der Referenzwerte eingehen), einem Absenken der viskosen Kopplung in der Strömung und zu einem verstärkten, konvektionshemmenden Wirken der endothermen Phasengrenze. Analog reduziert ein verringerter Ausdehnungskoeffizient den Einfluss der Phasengrenze und lässt höhere Geschwindigkeiten zu. Die zumindest zeitweilige Schichtung der Konvektion im 
Profil mit $\alpha\left(r_{a}\right) / \alpha\left(r_{i}\right)=0.3$ kann dazu beitragen, dass sich das Konvektionsmuster über einen vergleichsweise langen Zeitraum verändert hat und eventuell noch weiter verändert. Nach 4.5 Ga haben die unterschiedlichen Abkühlraten und der damit verbundene Anstieg der Viskosität dazu geführt, dass im oberflächennahen Bereich die Radialgeschwindigkeitsprofile sehr ähnlich sind (Abbildung (5.18)). Die Abnahme der Konvektionsstärke durch den temperaturbedingten Viskositätsanstieg führt zu niedrigeren Radialgeschwindigkeiten in der Mantelmitte mit steigendem Kontrast $\alpha\left(r_{a}\right) / \alpha\left(r_{i}\right)$. Dies gilt nun auch für den Bereich an der KMG und in der Tiefe der Phasengrenze ungeachtet der Phasengrenze. Insbesondere das Minimum für das Profil $\alpha\left(r_{a}\right) / \alpha\left(r_{i}\right)=0.3$ ist nicht mehr vorhanden. Die Viskosität ist soweit angestiegen, dass eine ausreichende viskose Kopplung besteht und den Einfluss der Phasengrenze abschwächt. Ohne die endotherme Phasengrenze liegen die Geschwindigkeiten in weiten Teilen des Mantels oberhalb der Profile, die bei Berücksichtigung der Phasengrenze entstehen. Der Unterschied nimmt aber mit steigen$\operatorname{dem} \alpha\left(r_{a}\right) / \alpha\left(r_{i}\right)$ deutlich ab.

Abbildung (5.19) zeigt das gemittelte Temperaturprofil nach $4.5 \mathrm{Ga}$. Innerhalb der Figur

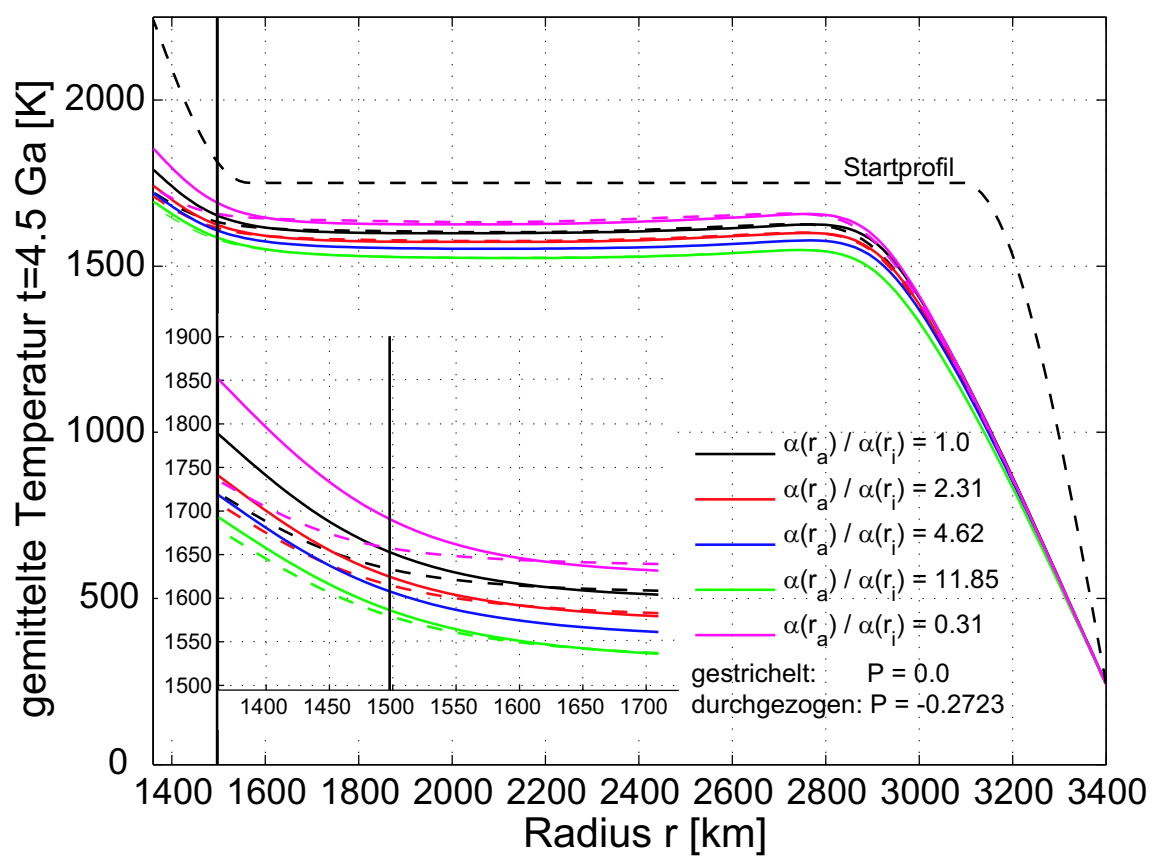

Abbildung 5.19: Einfluss der verschiedenen $\alpha(r)$ auf das Temperaturprofil nach 4.5 Ga. Die schwarze Linie markiert die Lage der endothermen Phasengrenze.

ist der kernnahe Bereich zusätzlich vergrößert dargestellt. In der Nähe der Oberfläche ist der Einfluss der verschiedenen Profile des thermischen Ausdehungskoeffizienten gering. Im übrigen Mantel führt ein Verstärken des $\alpha\left(r_{a}\right) / \alpha\left(r_{i}\right)$-Kontrasts zu niedrigeren Temperaturen. In der Mantelmitte ist die Veränderung der Temperaturen durch die Phasengrenze sehr klein. An der KMG und in der Nähe der Phasengrenze sind die Temperaturen höher, wenn die endotherme Phasengrenze mitsimuliert wird. Dies ist umso stärker der Fall je größer die der Ausdehnungskoeffizient in dieser Region ist. 
Die Entwicklung zu diesem Profil kann mit Hilfe der Konvektionsstärke erklärt werden

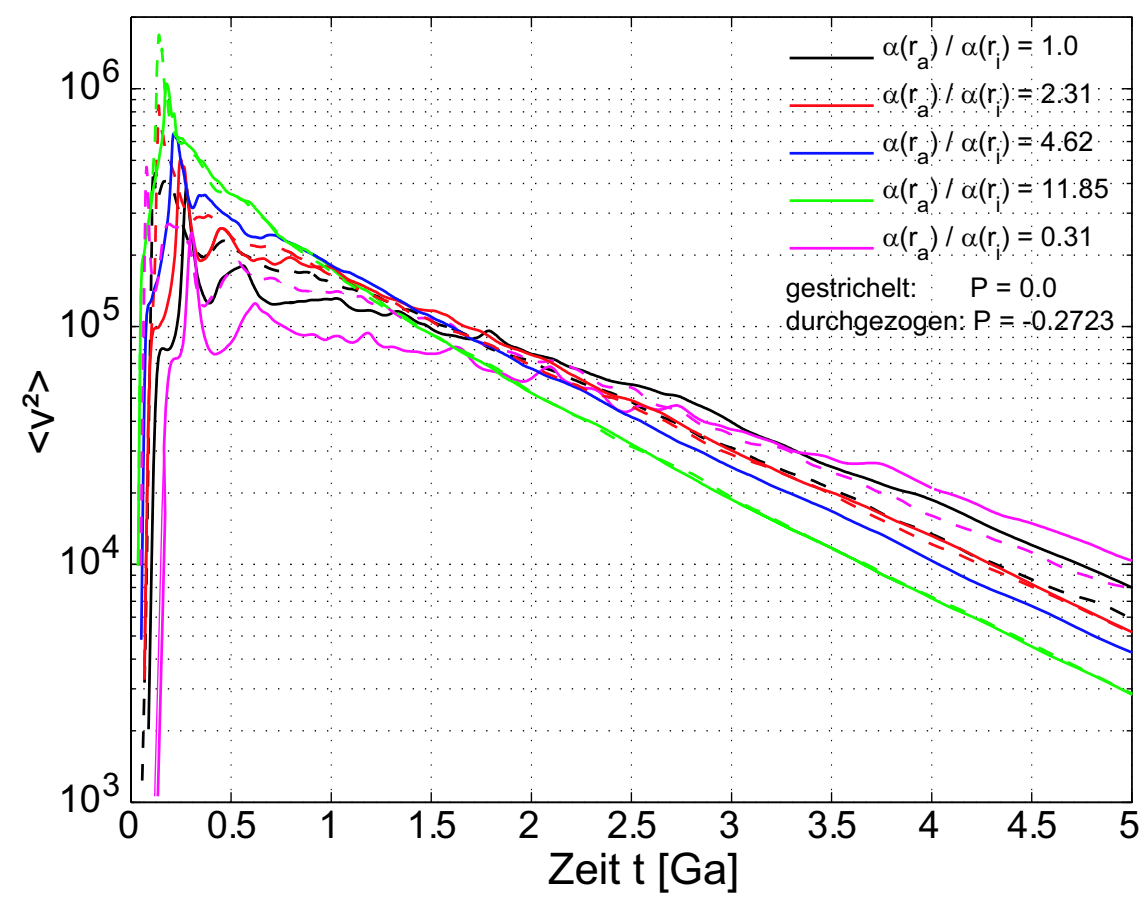

Abbildung 5.20: Einfluss der verschiedenen $\alpha(r)$ auf die Konvektionsstärke.

(Abbildung (5.20)). Die hohen Werte des thermischen Ausdehungskoeffizienten in der oberen Mantelhälfte führen zu einer sehr starken Konvektionsbewegung zu Beginn der Evolution, insbesondere im oberflächennahen Bereich (siehe Abbildung (5.17)) -ähnlich wie zuvor die verstärkte niedrigviskose Zone bei erhöhtem Aktivierungsvolumen. Zusammen mit der dünnen Lithosphäre kann der Mantel so effektiv abkühlen, was sich auch in der Temperaturentwicklung in halber Tiefe (Abbildung (5.21)) widerspiegelt. Durch die schwächere Konvektion und dickere Lithosphäre heizt sich der mittlere Mantel im Fall $\alpha\left(r_{a}\right) / \alpha\left(r_{i}\right)=0.3$ stärker als im Referenzfall auf. Die Konvektionsstärke wird in der Evolution einerseits durch die steigende Viskosität abgeschwächt und anderseits nimmt die stagnierende Lithosphäre zunehmend den Bereichs mit hohem, konvektionstreibenden Ausdehnungskoeffizienten ein. Sowohl bei der Konvektionsstärke als auch bei der Temperaturentwicklung in halber Tiefe macht sich die Phasengrenze lediglich bei den Profilen mit höheren $\alpha$-Werten an der KMG und in der Tiefe der Phasengrenze bemerkbar, wobei der Temperatureffekt in halber Tiefe sehr klein ist. Die heftigere Konvektion zu Beginn der Evolution bei $\alpha\left(r_{a}\right) / \alpha\left(r_{i}\right)>1$ zusammen mit dem Unterdrücken der Instabilitäten in der thermischen Grenzschicht erklärt, warum sich das Konvektionsmuster in kürzerer Zeit stabilisiert. Umgekehrt vergeht im Fall $\alpha\left(r_{a}\right) / \alpha\left(r_{i}\right)=0.3$ zumindest im Fall mit Phasengrenze mehr Zeit.

An der KMG wirkt sich die endotherme Phasengrenze deutlicher auf die Temperaturentwicklung (Abbildung (5.22)) aus. Ohne Phasengrenze kühlt der Kern mit dem unphysikalischen Profil (erhöhte $\alpha$-Werten in der Nähe der KMG) zu Beginn geringfügig stärker ab. In diesem Fall ist die Konvektion zu Beginn in der Nähe des Kerns vergleichsweise stark. Da die Konvektion im übrigen Mantel aber schwächer ist und die Wärme durch 


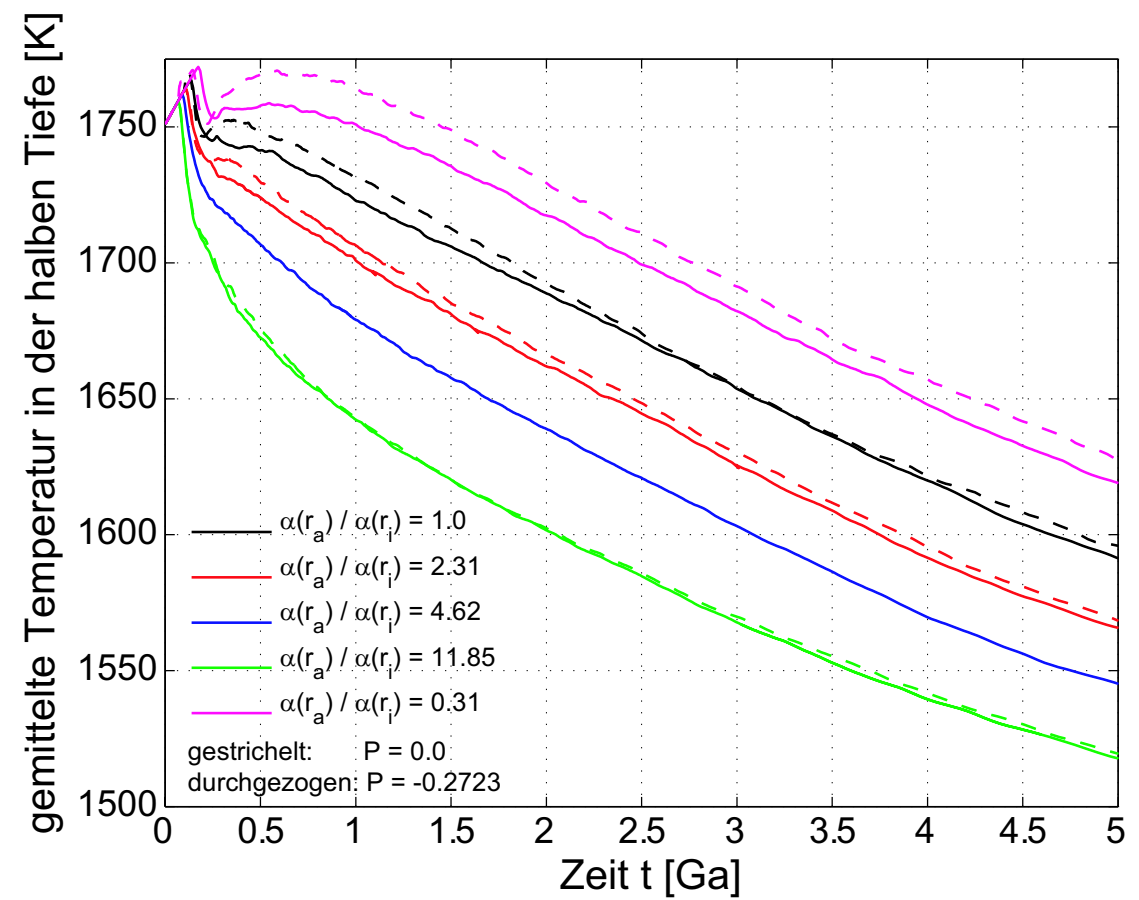

Abbildung 5.21: Einfluss der verschiedenen $\alpha(r)$ auf die Evolution der Temperatur in halber Tiefe.

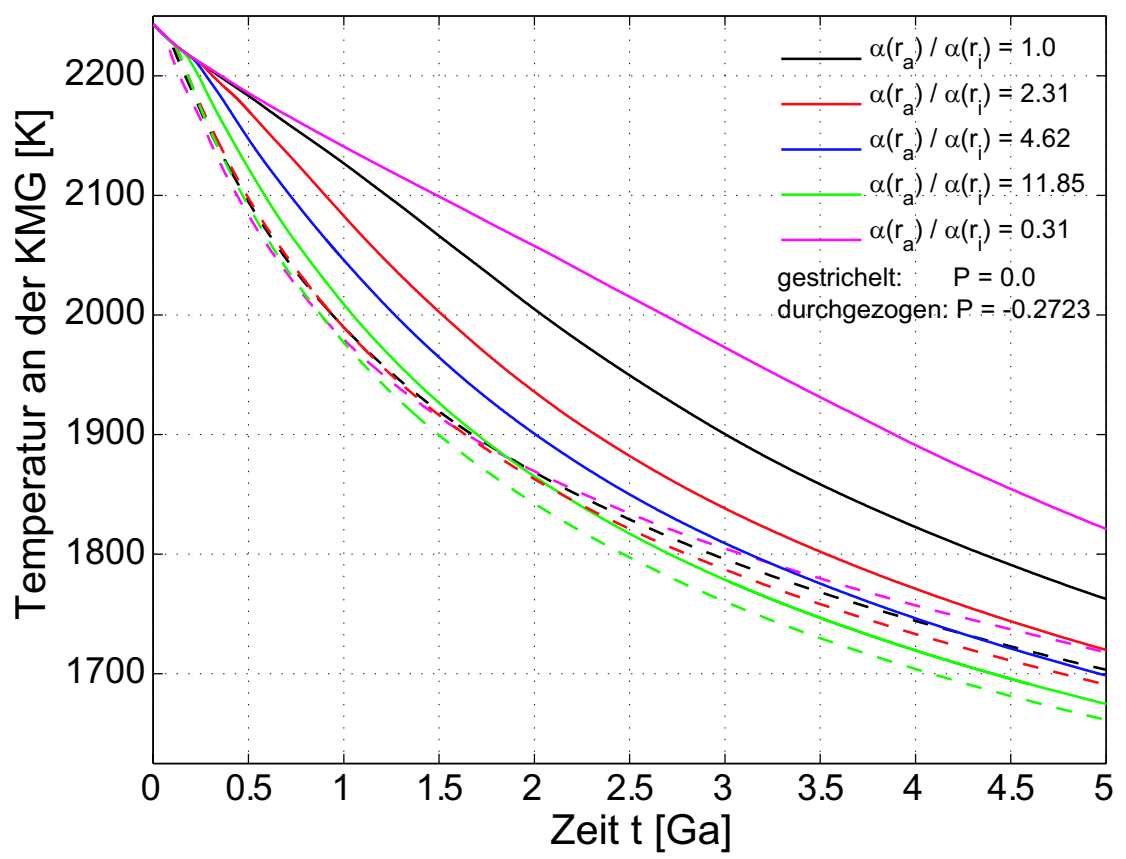

Abbildung 5.22: Einfluss der verschiedenen $\alpha(r)$ auf die Evolution der Temperatur an der KMG. 
die dickere Lithosphäre nicht abgegeben werden kann, sinkt der Wärmefluss (Abbildung (5.23)) aus dem Kern mit der Zeit und die Kühlrate verringert sich. Bei den übrigen Profilen kühlt der Kern schneller als im Referenzfall ab, da die Konvektion zu Beginn stärker ist und die Wärme durch die vergleichsweise starke Konvektion in der Nähe der Oberfläche nach außen abgeführt werden kann (Abbildung (5.24)). Dies erlaubt einen im Vergleich zum Referenzfall höheren Wärmefluss aus dem Kern. Die Phasengrenze hemmt das Abkühlen des Kerns umso deutlicher je größer der thermische Ausdehnungskoeffizient in der Nähe der KMG und der Phasengrenze ist bzw. sinkt der Einfluss, wenn $\alpha(r)$ hier kleinere Werte annimmt.

Die zu Beginn verstärke Konvektion bei den Profilen mit $\alpha\left(r_{a}\right) / \alpha\left(r_{i}\right)>1$ und der da-

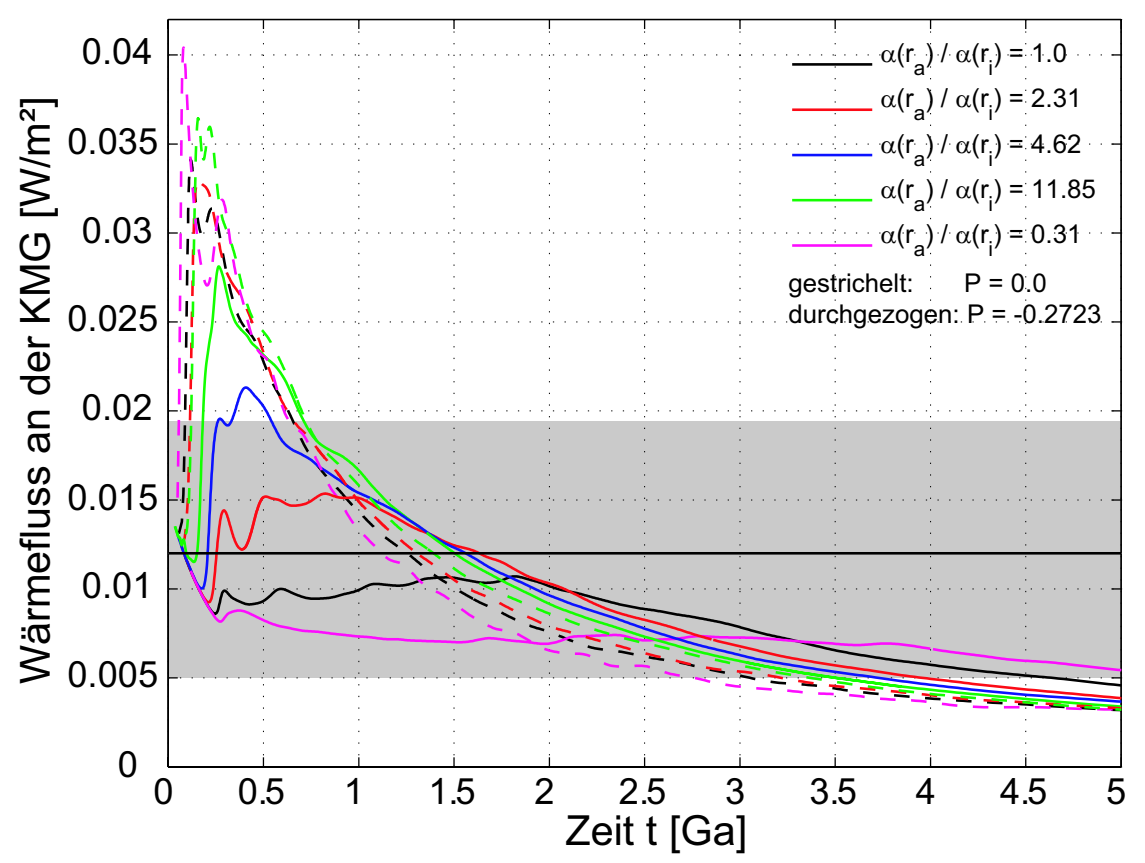

Abbildung 5.23: Einfluss der verschiedenen $\alpha(r)$ auf die Entwicklung des Wärmeflusses an der KMG. Der grau unterlegte Bereich kennzeichnet den Wertebereich des Wärmeflusses entlang der Adiabate im Marskern (Nimmo und Stevenson 2000). Die schwarze Linie zeigt zur Orientierung den Mittelwert dieses Bereichs.

mit verbundene erhöhte Wärmefluss aus dem Kern auch im Fall mit einer endothermen Phasengrenze steigert die Wahrscheinlichkeit eines thermisch getriebenen Dynamos im Mars. Der Wärmefluss an der KMG liegt für das Profil mit $\alpha\left(r_{a}\right) / \alpha\left(r_{i}\right)=2.31$, das für Mars angenommen wird, in der Zeit zwischen 0.25 und $1.63 \mathrm{Ga}$ über $F_{\mathrm{m}}=12 \mathrm{~mW} / \mathrm{m}^{2}$, so dass ein thermisch getriebener Dynamo möglich sein könnte. Ähnlich wie beim erhöhten Wärmefluss durch das erhöhte Aktivierungsvolumen stimmt das Zeitfenster und die Intervalllänge nicht mit den Beobachtungen überein. Ein weiteres Erhöhen des Kontrasts $\alpha\left(r_{a}\right) / \alpha\left(r_{i}\right)$ zeigt allerdings, dass die Breite des Peaks mit besonders hohem Wärmefluss abnimmt, so dass kürzere Zeitintervalle für eine mögliche Aktivität eines thermisch getriebenen Dynamos erreichbar sind. In den Modellen liegt der Wärmefluss aus dem Kern 
nach 4.5 Ga unter dem Wärmefluss entlang der Kernadiabate, so dass heute kein thermisch getriebener Dynamo aktiv sein kann.

Abbildung (5.24) stellt den Wärmefluss an der Oberfläche dar. Die unterschiedlichen

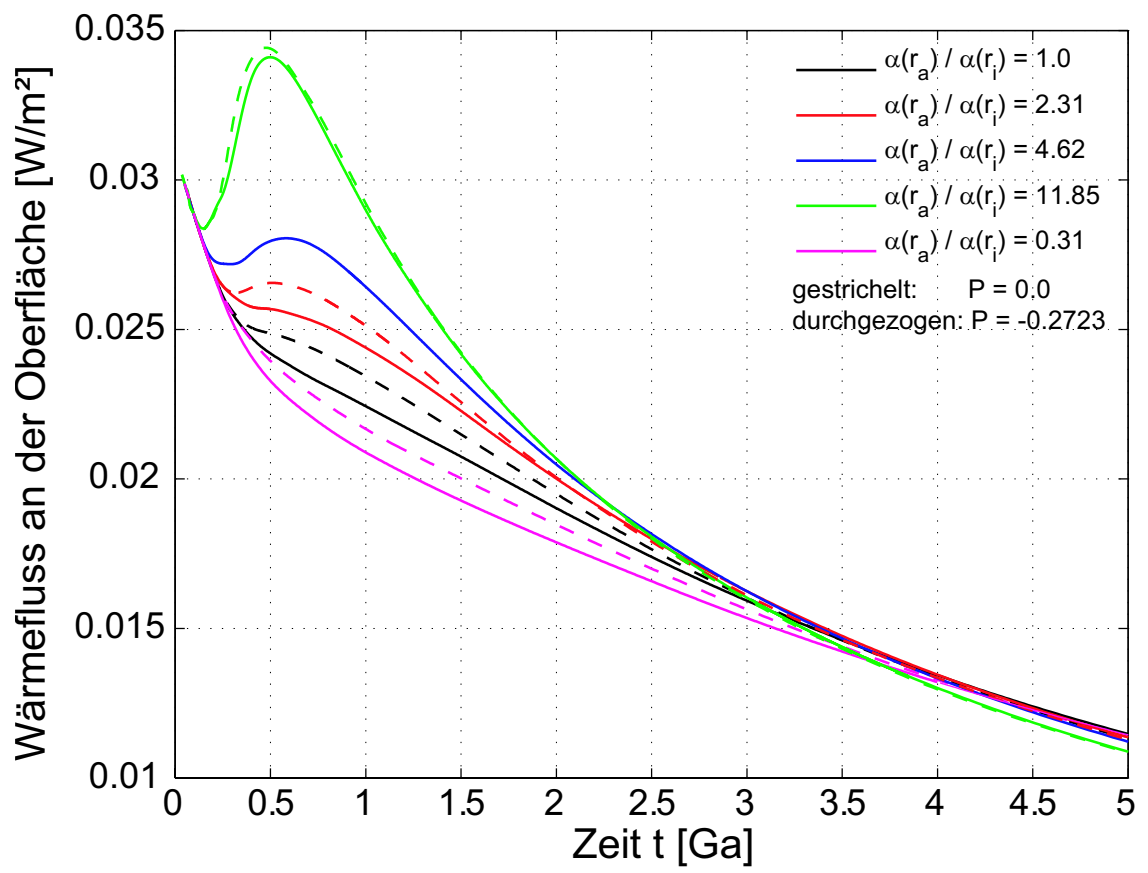

Abbildung 5.24: Einfluss der verschiedenen $\alpha(r)$ auf die Entwicklung des Wärmeflusses an der Oberfläche.

Profile haben auf den "heutigen" Wärmefluss nach einer Evolution von 4.5 Ga einen geringen Einfluss. Ähnliches gilt für die Lithosphärendicke, die in Abbildung (5.25) gezeigt ist. Sie variiert nach 4.5 Ga für die physikalisch sinnvollen Profile zwischen 300 und 308 $\mathrm{km}$.

Zusammenfassend führt ein tiefenabhängiger thermischer Expansionskoeffizient mit kleineren Werten (im Vergleich zum Referenzfall) an der KMG zu einer Stabilisierung der thermischen Grenzschicht und auch ohne Phasengrenze zu einer Reduktion der Anzahl der Aufströme. Die kalten Abströme werden durch den erhöhten Ausdehnungskoeffizienten in der Nähe der Oberfläche für das Konvektionsmuster bedeutender und bestimmen die Position und Anzahl der Aufströme mit. Der Einfluss der Phasengrenze sinkt, wenn der Ausdehnungskoeffizient im kernnahen Bereich kleiner wird, da das Konvektionsmuster weniger durch die Instabilitäten in der thermischen Grenzschicht, auf die die Phasengrenze wirken könnte, bestimmt wird. Auch für die thermische Entwicklung wird die Phasengrenze weniger bedeutsam, wenn mit dem thermischen Ausdehungskoeffizienten auch die lokale Rayleighzahl im Bereich um den Übergang abnimmt. Die höheren Werte des thermischen Ausdehnungskoeffizienten in der oberen Mantelhälfte führen zu einer anfänglich dünneren Lithosphäre und einer stärkeren Konvektionsbewegung, so dass der Planet effektiver abkühlen kann. Der Einfluss auf die heutige Lithosphärendicke ist klein. Mit einer Tiefenabhängigkeit des Ausdehnungskoeffizienten, wie sie für den Mars ange- 


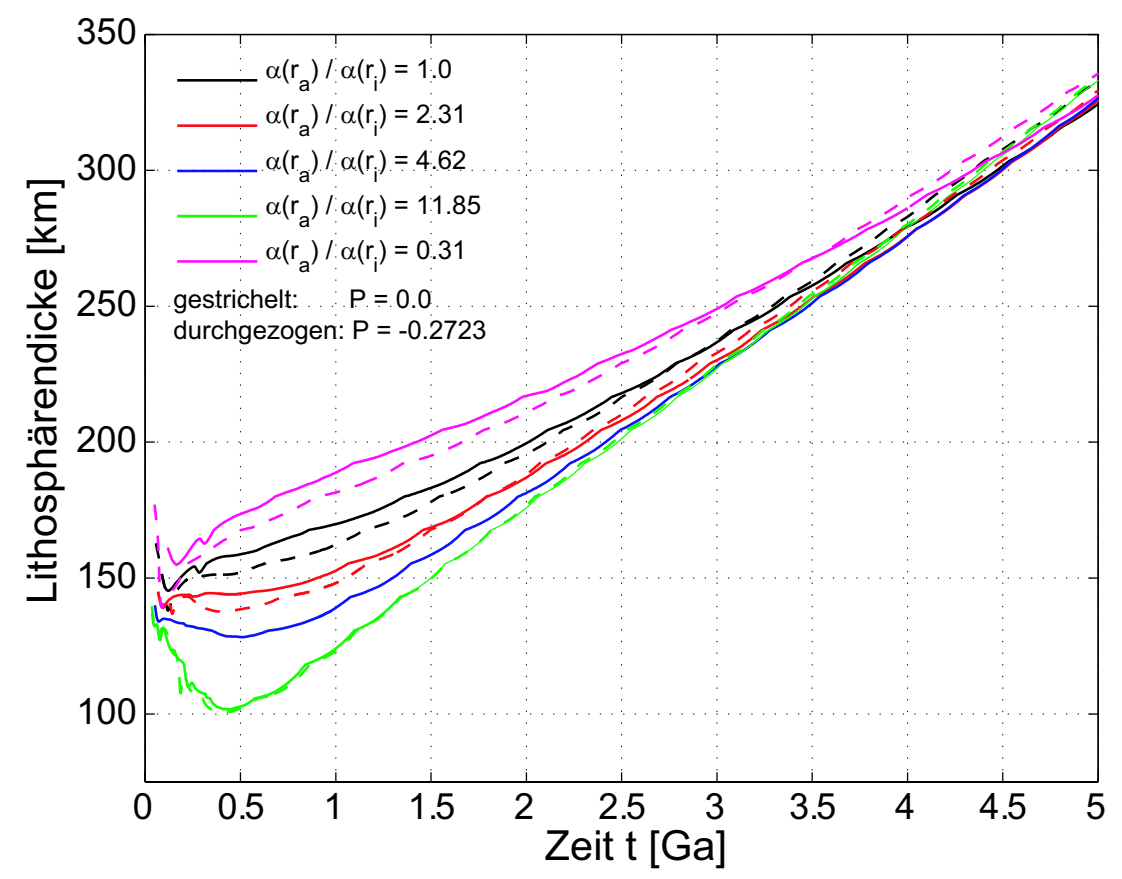

Abbildung 5.25: Einfluss der verschiedenen $\alpha(r)$ auf die Entwicklung der Lithosphärendicke.

nommen wird, ergibt sich bei Berücksichtigung der Phasengrenze ein Konvektionsmuster mit drei Aufströmen, das die Konzentration des Vulkanismus in zwei Zentren nicht vollends erklären kann. Die Temperatur an der KMG sinkt durch das tiefenabhängige Profil um etwa $50 \mathrm{~K}$ in der Mantelmitte um ca. $25 \mathrm{~K}$ im Vergleich zum Referenzfall. Der Wärmefluss an der KMG ist zu Beginn der Evolution gegenüber dem Fall mit konstantem $\alpha$ erhöht, so dass die Wahrscheinlichkeit eines frühen, thermisch getriebenen Dynamos gesteigert wird.

\subsection{Einfluss verschiedener Profile von $\lambda(r)$}

Hofmeister (1999) hat aus Messungen der Lebensdauer von Phononen ein Modell der thermischen Leitfähigkeit des Mantels abgeleitet. Dieses Modell und seine Vereinfachungen für das Konvektionsmodell sind in Kapitel (3.7) genauer beschrieben. In diesem Abschnitt wird der Einfluss einer tiefenabhängigen, druck- und temperaturbestimmten thermischen Leitfähigkeit auf das Konvektionsmuster und die thermische Entwicklung untersucht. Neben dem Profil, wie es sich nach Gleichung (3.61) für Mars ergibt, wird ein weiteres Profil (Profil 2) betrachtet, bei dem Parameter der Erde verwendet werden $\left(d=3000 \mathrm{~km}, K_{0}=261 \mathrm{GPa}\right.$ und $\left.g=9.8 \mathrm{~m} / \mathrm{s}^{2}\right)$ und das eine stärkere Druckabhängigkeit aufweist. Abbildung (5.26) zeigt diese Profile zum Zeitpunkt $t=0.0 \mathrm{Ga}$ mit dem Starttemperaturprofil, das gegenüber den vorherigen Rechnungen nicht verändert worden ist. Die Profile sind so normiert, dass in halber Tiefe bei $T=1573 \mathrm{~K}$ der Referenzwert erreicht wird. Diese Normierung liefert für Profil 2 sehr kleine Werte der thermischen Leit- 


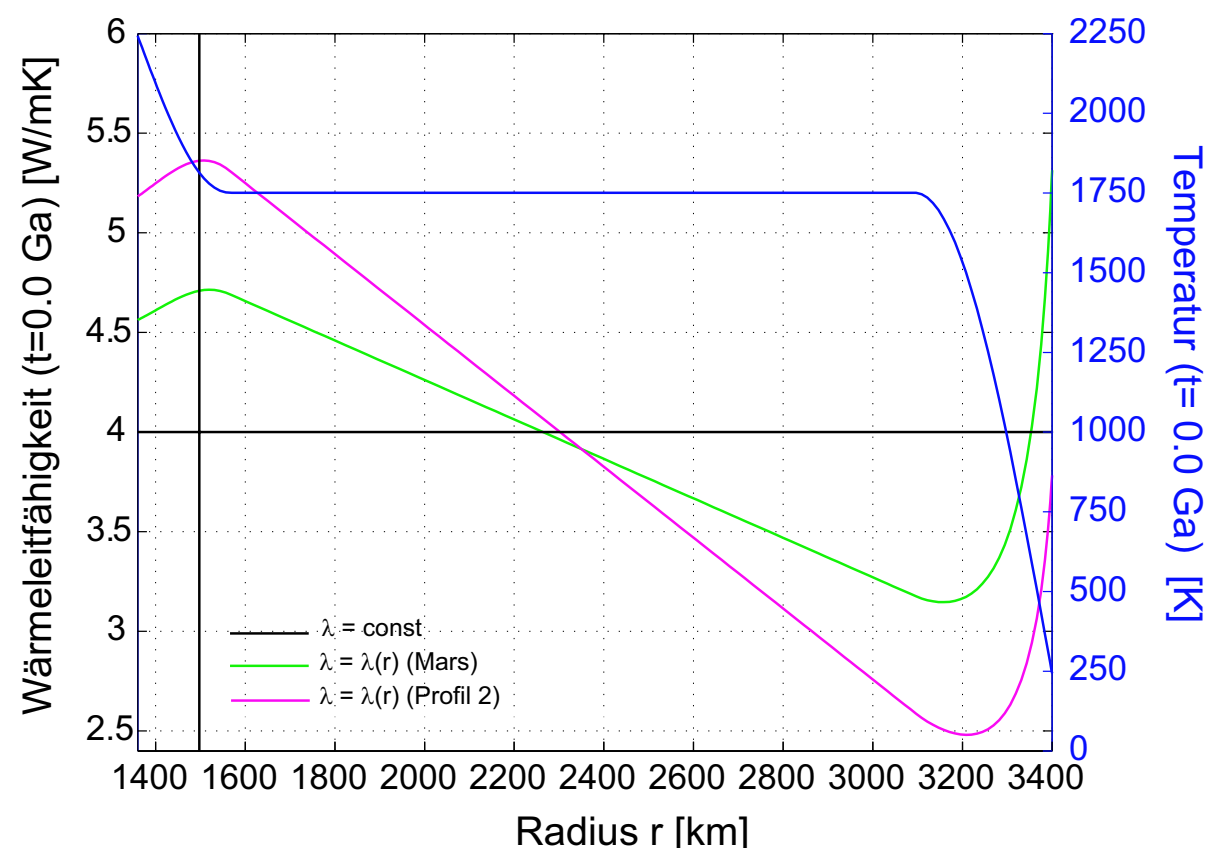

Abbildung 5.26: Profile der thermischen Leitfähigkeit $\lambda(r)$ zum Zeitpunkt $t=0.0 \mathrm{Ga}$ zusammen mit dem verwendeten Starttemperaturprofil.

fähigkeit an der Oberfläche. Das druck- und temperaturabhängige $\lambda$-Profil führt durch die Druckabhängigkeit in der unteren Mantelhälfte zu höheren Werten der thermischen Leitfähigkeit. Innerhalb der kernnahen thermischen Grenzschicht fallen die Werte zur KMG leicht ab. In der oberen Mantelhälfte ist die thermische Leitfähigkeit zunächst kleiner als im Referenzfall mit konstantem $\lambda$ und es bildet sich unterhalb der thermischen Grenzschicht ein deutliches Minimum aus, bevor $\lambda$ innerhalb der Grenzschicht in Richtung Oberfläche wieder ansteigt. Für Mars ergeben sich an der Oberfläche deutlich höhere Werte als im Referenzfall, für Profil 2 liegt der Wert an der Oberfläche etwas unter dem Referenzwert.

In Abbildung (5.27) ist das Konvektionsmuster nach 4.5 Ga für die Simulationen mit den verschiedenen Profilen durch die Radialgeschwindigkeit in halber Tiefe dargestellt. Die beiden Spalten unterscheiden erneut zwischen dem Fall mit kernnaher endothermer Phasengrenze $(P=-0.2723)$ in der rechten Spalte und dem Fall ohne Phasengrenze $(P=0.0)$ in der linken Spalte. Die erste Zeile zeigt zur besseren Vergleichbarkeit wieder den Referenzfall mit konstantem $\lambda$. Die Tiefenabhängigkeit von $\lambda$, wie sie für den Mars angenommen wird, beeinflusst das Konvektionsmuster nach 4.5 Ga im Fall ohne Phasengrenze nur schwach. Lediglich der Aufstrom bei $\phi=90^{\circ}$ auf der Nordhemisphäre ist in seiner Amplitude geringfügig schwächer und etwas weiter östlich in Richtung des Äquators gewandert. Auch in der Entwicklung zu diesem Konvektionsmuster gibt es keine signifikanten Unterschiede. Mit kernnaher endothermer Phasengrenze entsteht bei diesem $\lambda$-Profil zwischen 2.0 und $2.5 \mathrm{Ga}$ ein Konvektionsmuster mit drei Aufströmen. Dieses Konvektionsmuster wird etwas früher als im Referenzfall erreicht und die Geschwindigkeitsamplitude ist geringfügig höher. Die Evolution zu diesem Konvektionsmuster erfolgt 
a) $\lambda=$ const

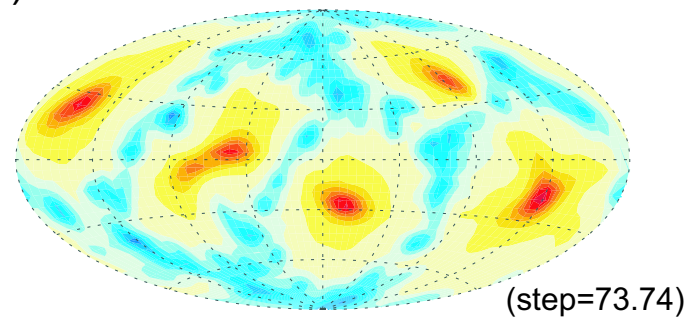

b) $\lambda=\lambda(r)$ (Mars)

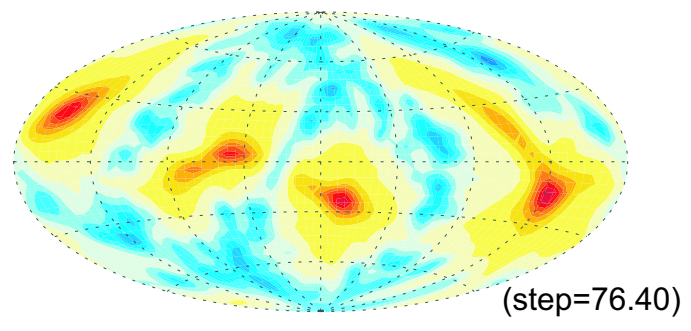

c) $\lambda=\lambda(r)$ (Profil 2)

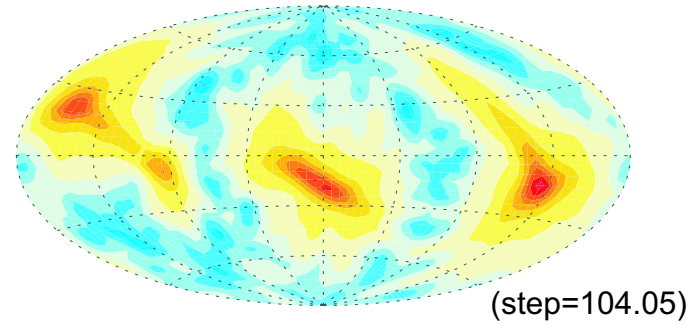

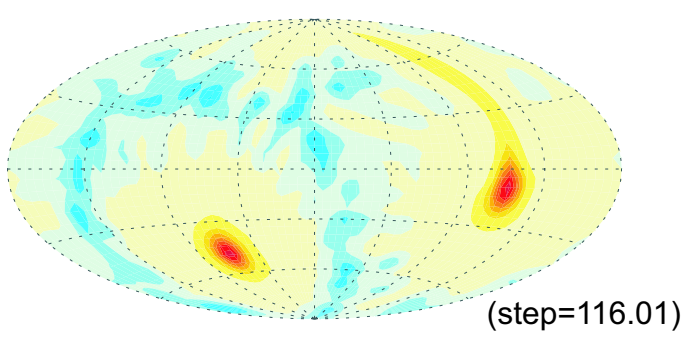

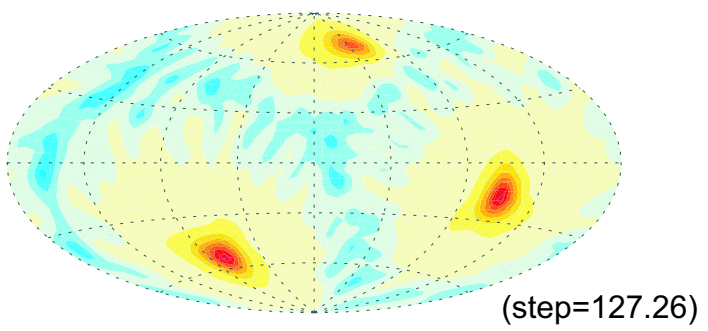

$($ step $=127.26)$

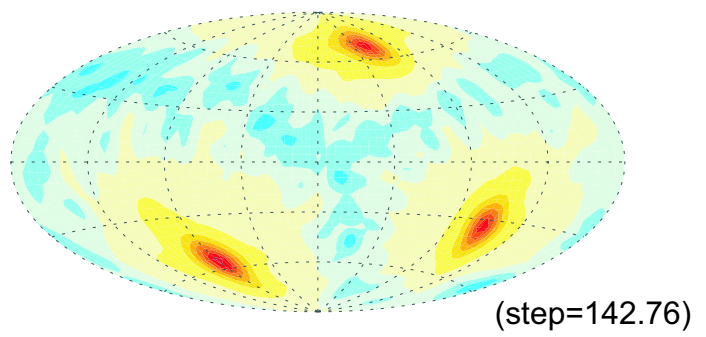

Abbildung 5.27: Einfluss der beiden Profile der thermischen Leitfähigkeit auf das Konvektionsmuster $(t=4.5 \mathrm{Ga})$ : Radialgeschwindigkeit in halber Tiefe (rot: Aufströme, blau: Abströme). Die linke Spalte zeigt die Situation ohne Phasengrenze $(P=0.0)$, in der rechten Spalte wird die kernnahe endotherme Phasengrenze berücksichtigt $(P=-0.2723)$. Der Contourstep der Geschwindigkeit ist angegeben: step $=100$ entspricht $0.14 \mathrm{~cm} / \mathrm{a}$.

sehr ähnlich wie im Referenzfall, lediglich der Aufstrom am Pol, der sich im Referenzfall abschwächt und ausstirbt, bleibt hier bestehen. Mit einer verstärkten Druckabhängigkeit (Profil 2) entsteht ohne Phasengrenze nach 4.5 Ga ein Konvektionsmuster, in dem nur noch vier voneinander getrennte Aufströme erkennbar sind, von denen einer deutlich schwächer ist als die übrigen. Die Geschwindigkeitsamplitude der Aufströme ist höher als im Referenzfall. Im Referenzfall wird zwischen 2.0 und $2.5 \mathrm{Ga}$ ein Konvektionsmuster mit sechs Aufströmen beobachtet (Abbildung (5.3)), von denen alle bis auf zwei voneinander durch Abströme getrennt werden. Dieses Muster bleibt bis zum Ende der Evolution so erhalten. Für Profil 2 wird ein sehr ähnliches Muster zwischen 1.5 und 2.0 Ga erreicht. Allerdings brechen hier in der weiteren Evolution die trennenden Abstrombänder auf und die Aufströme bewegen sich aufeinander zu und vereinigen sich, so dass die Anzahl nach 4.5 Ga reduziert ist. Mit Phasengrenze wird mit Profil 2 wiederum ein Konvektionsmuster mit drei Aufströmen erzeugt, das sich schon nach 1.5 - 2.0 Ga etabliert hat. Dabei 
sind die drei verbleibenden Aufströme bereits früh in der Evolution deutlich stärker als die übrigen, die sehr schnell schwächer werden und aussterben. Die starken Aufströme verändern ihre Postion bis zum Ende der Evolution nicht. Im Referenzfall ist die Dominanz der später verbleibenden Aufströme in dem frühen Stadium nicht so deutlich und die schwächeren Aufströme überdauern einen längeren Zeitraum (Abbildung (5.4)).

Der Einfluss des Profils $\lambda(r)$, wie es sich mit den Marsparametern ergibt, auf das Konvektionsmuster ist insbesondere ohne Phasengrenze gering. Im Fall mit Phasengrenze tragen die erhöhten Diffusivitäten an der KMG eventuell dazu bei, kleinere Temperaturanomalien oder Instabilitäten durch Wärmediffusion zu schwächen, verbleibende Aufströme sind stark genug, um die Phasengrenze zu durchdringen, so dass der Effekt der Phasengrenze geringer ist. Die Geschwindigkeiten in der Nähe der KMG sind durch die Phasengrenze besonders zu Beginn der Evolution geringer, so dass die Diffusion eine größere Rolle spielen kann. Dies scheint im Fall mit Phasengrenze bei Profil 2 in verstärkter Form der Fall zu sein, so dass schwächere Abströme durch Diffusion schneller aussterben. Bei Vernachlässigung der Phasengrenze führt der mit der Tiefe stärker zunehmende Diffusionskoeffizient (Profil 2) zu einem Abschwächen der Abströme (durch Wärmeleitung), was vielleicht das Aufbrechen der Abstrombänder und das Vereinen von Plumes vereinfachen kann. Die durch die höheren Temperaturen (siehe unten) stärkere Konvektion unterstützt dies zusätzlich.

Dubuffet und Yuen (2000) finden in isoviskosen, dreidimensionalen (kartesischen) Modellen (Boussinesq-Näherung) ein Stabilisieren der Konvektionsströmung und der Grenzschichten durch eine realistische (vollständig) temperatur- und druckabhängige thermische Leitfähigkeit. Die Modelle benutzen Parameter für den unteren Erdmantel $(\mathrm{Ra}=5$. $10^{5}$ ). Später untersuchen Dubuffet et al. (2002) in zwei- und dreidimensionalen, kartesischen isoviskosen Konvektionsmodellen in der Boussinesq-Näherung (im Parameterbereich der Erde) systematisch den Einfluss der einzelnen Beiträge zur thermischen Leitfähigkeit, insbesondere berücksichtigen sie die vollständige Temperaturabhängigkeit der thermischen Leitfähigkeit, die die Nichtlinearität der Gleichung erhöht. Vernachlässigen sie diese zusätzliche Nichtlinearität und nehmen ein rein tiefenabhängiges (linear mit der Tiefe ansteigendes) Profil der Leitfähigkeit an, so beobachten sie einen stabilisierenden Effekt auf die Konvektion, der der einer tiefenabhängigen Viskosität oder eines tiefenabhängigen Ausdehnungskoeffizienten ähnlich ist. In den vorliegenden Modellen wird ebenfalls ein (zeitabhängiges) tiefenabhängiges Profil verwendet, das im konvektierenden Bereich von dem linearen Anstieg der Leitfähigkeit mit dem Druck dominiert ist, so dass die Ergebnisse von Dubuffet et al. (2002) obige Beobachtungen unterstützen können. Abbildung (5.28) zeigt das Profil der gemittelten Temperatur nach 4.5 Ga für die Simulationen mit den unterschiedlichen Profilen der thermischen Leitfähigkeit. Der kernnahe Bereich ist zusätzlich vergrößert dargestellt. Besonders auffällig ist der Unterschied in der Nähe der Oberfläche bei Profil 2. Die niedrigen Werte der thermischen Leitfähigkeit führen bei Profil 2 zu einem deutlich stärkeren Temperaturgradienten. Der isotherme Mantelbereich dehnt sich etwa $100 \mathrm{~km}$ weiter in Richtung Oberfläche aus. Zudem sind die Temperaturen hier höher als im Referenzfall. Die schwächere Druckabhängigkeit, wie sie für Mars gilt, führt in unmittelbarer Nähe der Oberfläche zu etwas geringeren Temperaturen als im Referenzfall. Der Anstieg ist in diesem Fall etwas steiler und die Temperaturen in halber Tiefe sind geringfügig höher als im Referenzfall. In der Mantelmitte und im oberflächennahen Bereich ist der Einfluss der Phasengrenze gering. Dies wird ebenfalls 


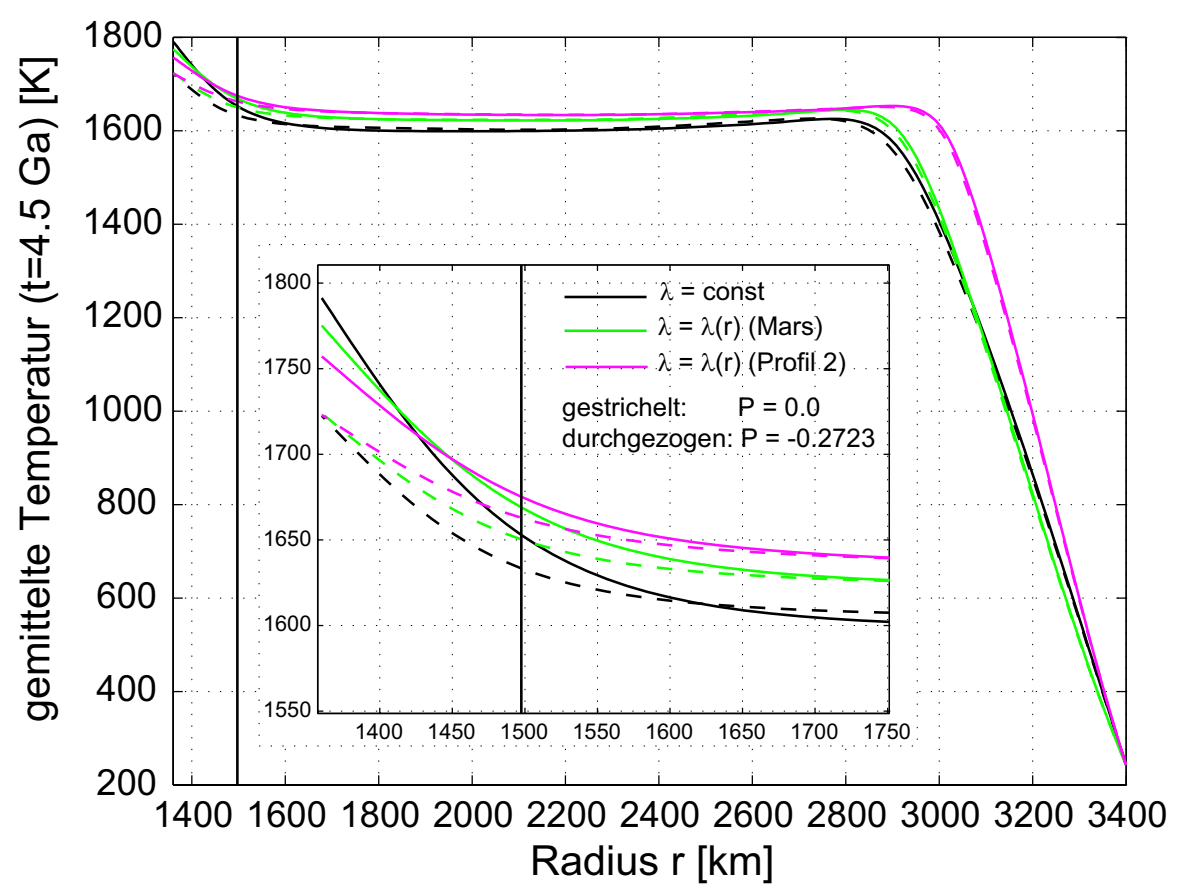

Abbildung 5.28: Einfluss der beiden Profile der thermischen Leitfähigkeit auf das gemittelte Temperaturprofil nach 4.5 Ga. Die schwarze Linie markiert die Position der Phasengrenze.

bei Betrachtung der Temperaturentwicklung in halber Tiefe in Abbildung (5.29) deutlich. Durch die Wahl der Tiefenabhängigkeit von $\lambda$ wird der Einfluss der Phasengrenze auf die Temperaturentwicklung in halber Tiefe nicht beeinflusst. Allerdings werden mit dem Profil für Mars in der Mantelmitte etwas höhere Temperaturen (ca. $20 \mathrm{~K}$ über dem Referenzfall) erreicht. Mit Profil 2 ist der Einfluss stärker, so dass die Temperaturen $30 \mathrm{~K}$ über den Temperaturen im Referenzfall liegen. Durch die niedrigen Wärmeleitfähigkeiten in der Nähe der Oberfläche und insbesondere beim Übergang zur Lithosphäre, in der Wärme nur durch Diffusion transportiert werden kann, kann der Mantel die Wärme weniger effizient abgeben. Zusammen mit dem zumindest zu Beginn höheren Wärmefluss aus dem Kern in den Mantel führt dies zu einem Erhöhen der Temperaturen.

Ein verlangsamtes Abkühlen des Planeten durch eine druck- und (vollständig) temperaturabhängige thermische Leitfähigkeit und die damit verbundene niedrigleitfähige Zone beobachten auch van den Berg et al. (2002), van den Berg und Yuen (2002) für den isoviskosen Fall bzw. van den Berg et al. (2004) für den Fall einer druck- und temperaturabhängigen Viskosität. Diese Evolutionsmodelle in zweidimensionaler kartesischer Geometrie (erweiterte Boussinesq-Approximation) für den Erdmantel vernachlässigen den Wärmefluss aus dem Kern. Wird die Temperaturabhängigkeit der Leitfähigkeit vernachlässigt und nur die lineare Druckabhängigkeit in Betracht gezogen, ist der Unterschied in der Abkühlrate (verglichen mit dem Fall einer konstanten Leitfähigkeit) geringer. Dies verdeutlicht die Bedeutung der Temperaturabhängigkeit der thermischen Leitfähigkeit für die thermische Entwicklung (van den Berg et al. 2004). Aufgrund diverser Unterschie- 


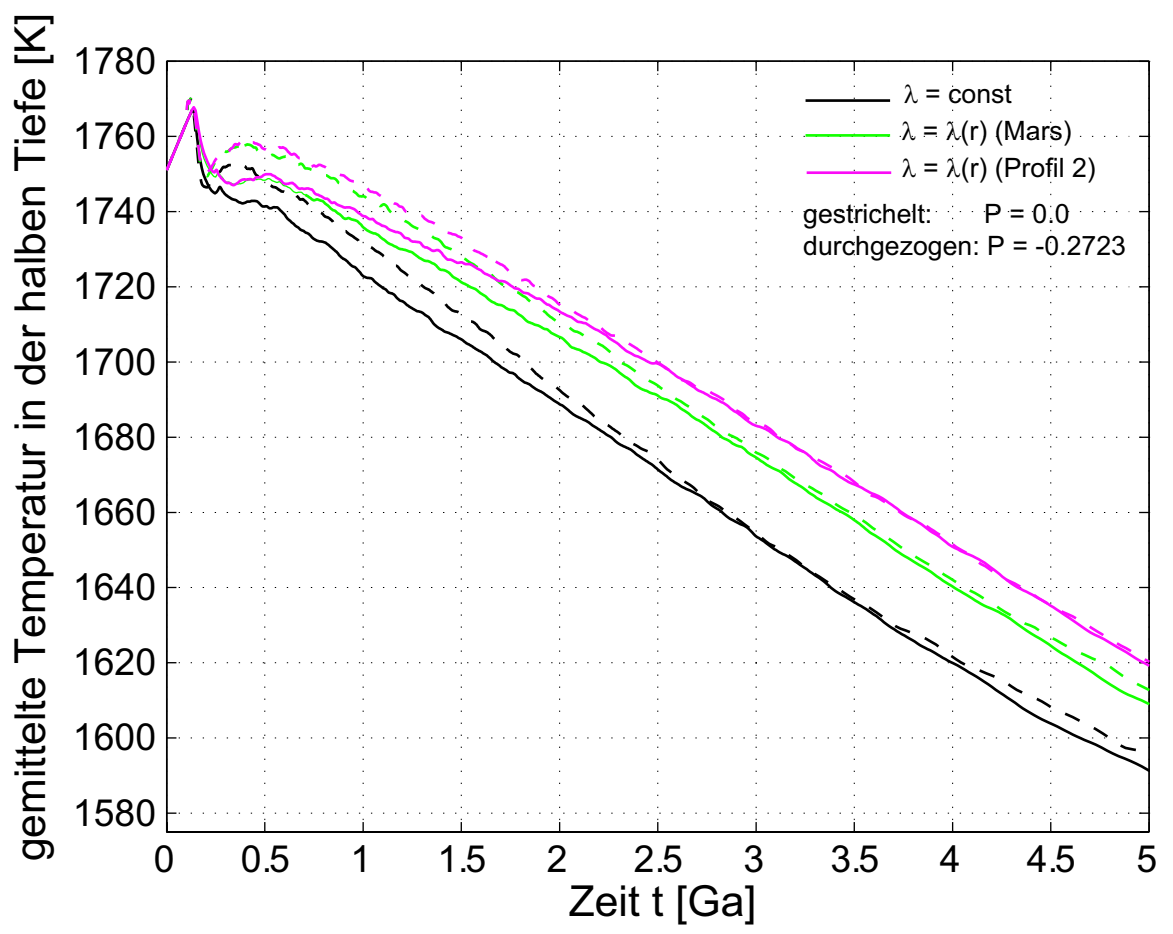

Abbildung 5.29: Einfluss der beiden Profile der thermischen Leitfähigkeit auf die Temperaturentwicklung in halber Tiefe.

de zu den vorliegenden Modellen (Geometrie (van den Berg und Yuen 2002), Parameter (Erde-Mars), Näherungsweise (erweiterte - einfache Boussinesq-Näherung), Temperaturrandbedingung an der KMG) können die Temperaturunterschiede durch die Einführung einer realistischen thermischen Leitfähigkeit nicht verglichen werden. In den vorliegenden Modellen ist der Einfluss der Tiefenabhängigkeit der thermischen Leitfähigkeit deutlich geringer.

Die Profile der thermischen Leitfähigkeit am Ende der Evolution sind in Abbildung (5.30) gezeigt. Gegenüber den Startprofilen sind die Minima in der Nähe der Oberfläche entsprechend der Temperaturprofile etwas in Richtung Mantelmitte verschoben und schwächer ausgeprägt. Auch an der KMG sieht man den Einfluss des im Vergleich zum Startprofil abgeschwächten Temperaturgradienten.

An der KMG führen die höheren thermischen Leitfähigkeiten im Fall ohne Phasengrenze zunächst zu einem geringfügig schnelleren Abkühlen des Kerns (Abbildung (5.31)) . Bei $t=2.0 \mathrm{Ga}$ besteht zwischen den Temperaturen an der KMG im Referenzfall und bei Profil 2 ein Unterschied von lediglich 20 K. Später wird der Unterschied sogar kleiner, so dass sich nach $4.5 \mathrm{Ga}$ die gleiche Temperatur von $1723 \mathrm{~K}$ für alle Profile einstellt. Da der Mantel bei einem tiefenabhängigen $\lambda$ etwas wärmer ist, sinkt der Temperaturgradient in der kernnahen thermischen Grenzschicht und der Wärmefluss (Abbildung (5.32)) verringert sich trotz höherer Leitfähigkeit, so dass sich die Temperaturen angleichen. Mit der endothermen Phasengrenze kühlt der Kern während der ganzen Evolution bei den tiefenabhängigen Profilen, insbesondere bei Profil 2 stärker ab als im Referenzfall. Am Ende der Evolution liegen die Temperaturen an der KMG bei Profil 2 etwa 34 K unter den 


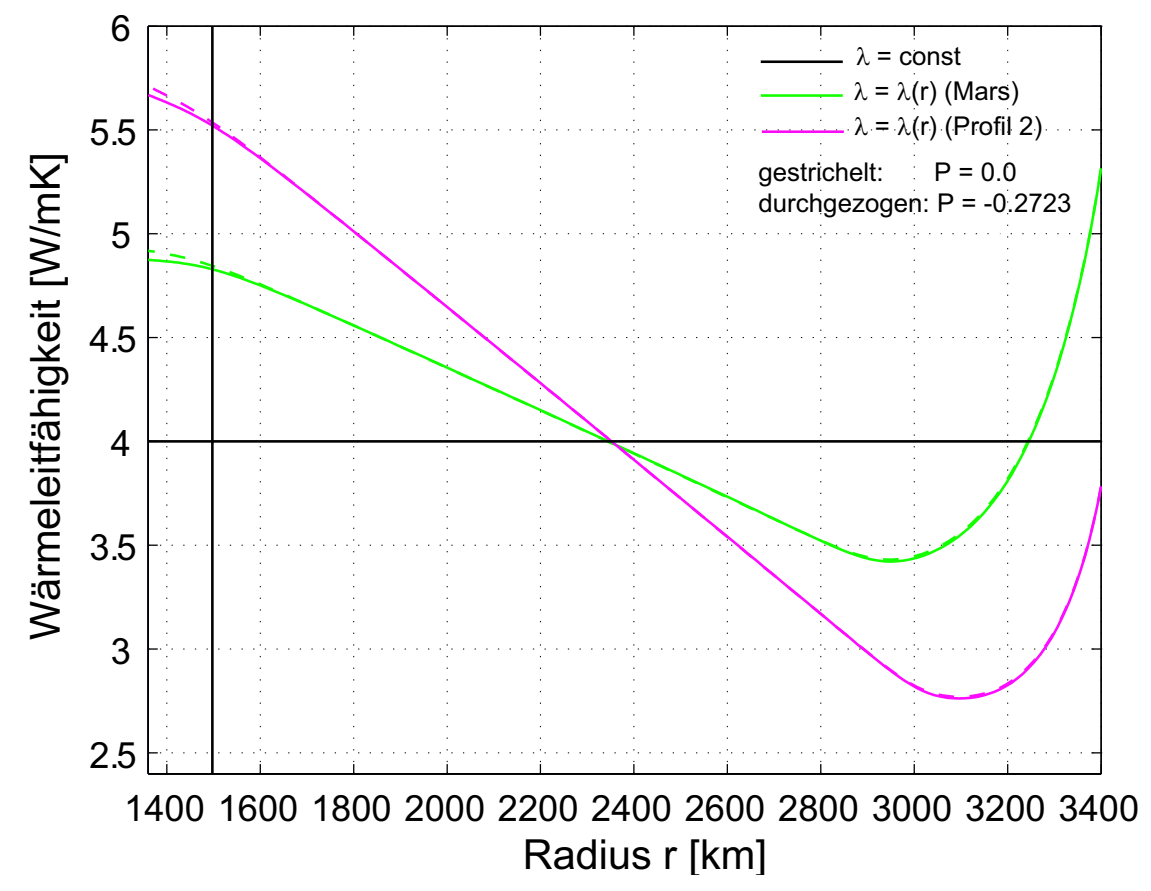

Abbildung 5.30: Profile der thermischen Leitfähigkeit nach 4.5 Ga.

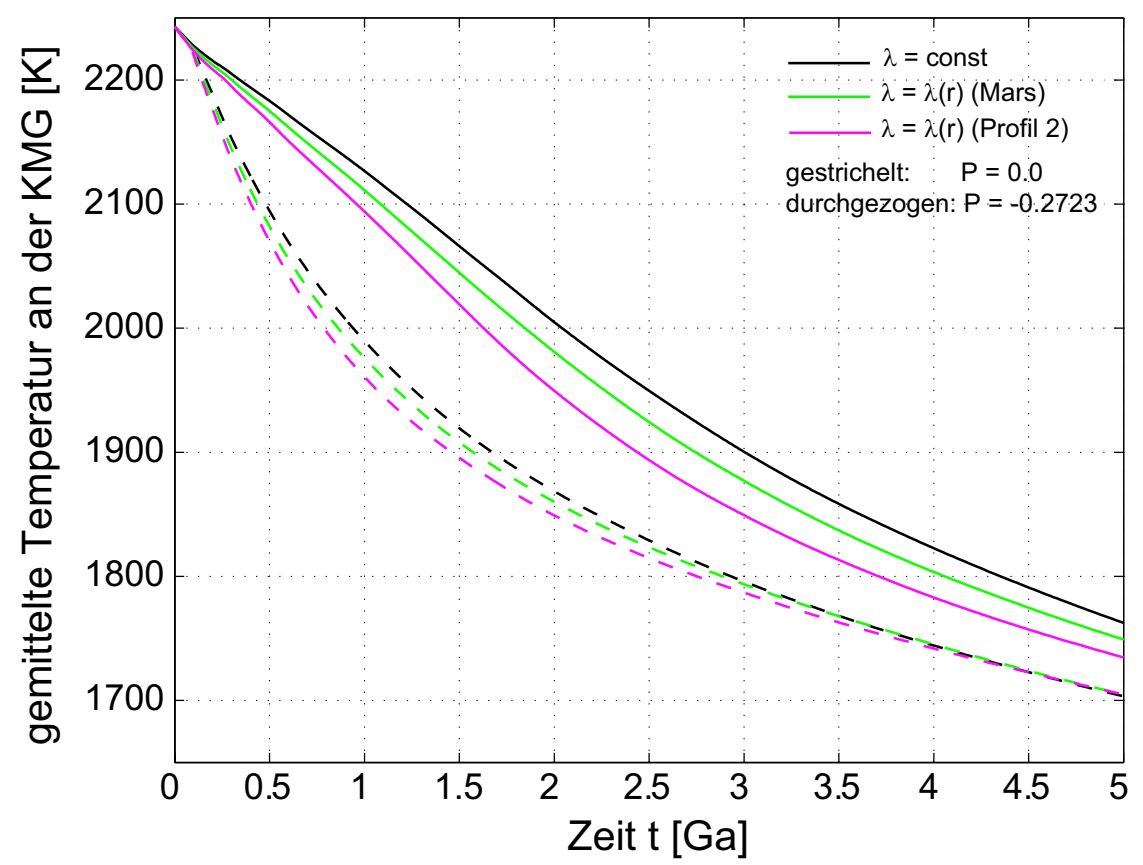

Abbildung 5.31: Einfluss der beiden Profile der thermischen Leitfähigkeit auf die Temperaturentwicklung an der KMG. 


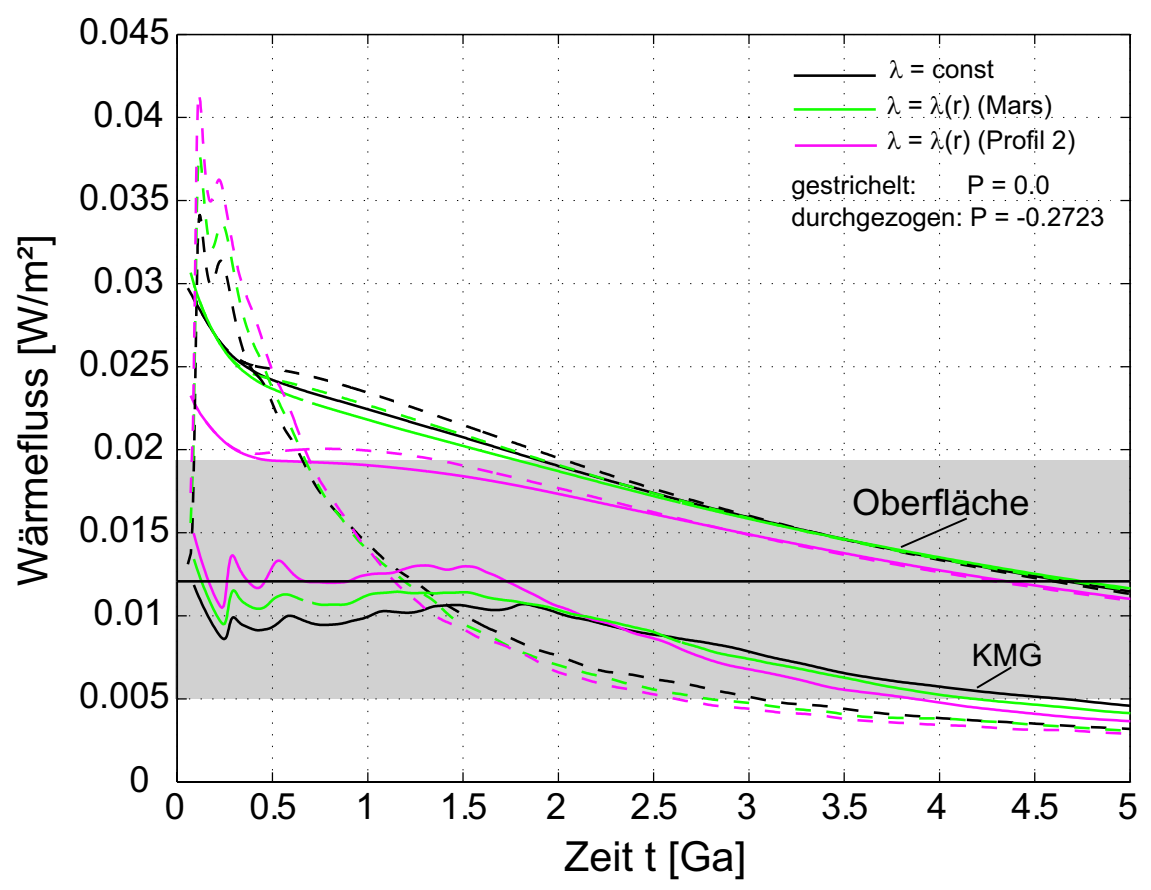

Abbildung 5.32: Einfluss der verschiedenen $\lambda(r)$ auf die Entwicklung des Wärmeflusses an der KMG und der Oberfläche. Der grau unterlegte Bereich kennzeichnet den Wertebereich des Wärmeflusses entlang der Adiabate im Marskern (Nimmo und Stevenson 2000). Die schwarze Linie zeigt zur Orientierung den Mittelwert dieses Bereichs.

Werten im Referenzfall (1790 K). Zu dem stärkeren Abkühlen bei Profil 2 trägt auch der schwächere Einfluss der Phasengrenze bei, die das Abkühlen verlangsamt. Der Einfluss ist für das $\lambda$-Profil des Mars etwas kleiner, so dass sich hier nach 4.5 Ga ein Temperaturunterschied von $15 \mathrm{~K}$ gegenüber der Referenzfall ergibt. Der Temperaturunterschied durch die Phasengrenze nimmt durch die Tiefenabhängigkeit von $\lambda$ ab.

Der Wärmefluss an der Oberfläche ist zu Beginn der Evolution durch die niedrigen Werte von $\lambda$ (Profil 2) kleiner als im Referenzfall. Am Ende der Evolution ist der Unterschied jedoch gering, da der Temperaturgradient bei Profil 2 deutlich stärker ist.

An der KMG wird der Wärmefluss sowohl mit also auch ohne Phasengrenze durch die erhöhten thermischen Leitfähigkeiten zu Beginn der Evolution gegenüber dem Referenzfall etwas erhöht. Vergleicht man den Wärmefluss an der KMG mit dem Wärmefluss entlang der Kernadiabate (grau hinterlegte Bereich) und orientiert sich am Mittelwert $\left(F_{\mathrm{m}}=12 \mathrm{~mW} / \mathrm{m}^{2}\right)$, so reicht dieses Erhöhen bei dem $\lambda$-Profil, das für Mars angenommen wird, nicht aus, diesen Wert zu überschreiten und einen thermisch getriebenen Dynamo zu ermöglichen. Bei Profil 2 liegt der Wärmefluss zwischen 0.1 und $1.7 \mathrm{Ga}$ geringfügig über diesem Mittelwert, allerdings beschreibt dieses Profil nicht die Situation im Mars und zudem stimmt erneut die Lebensdauer eines möglichen Dynamos nicht mit den Beobachtungen überein. Der "heutige" Wärmefluss ist zu gering, um einen thermisch getriebenen Dynamo zu ermöglichen.

Der Effekt der unterschiedlichen $\lambda$-Profile auf die Lithosphärendicke ist in Abbildung 


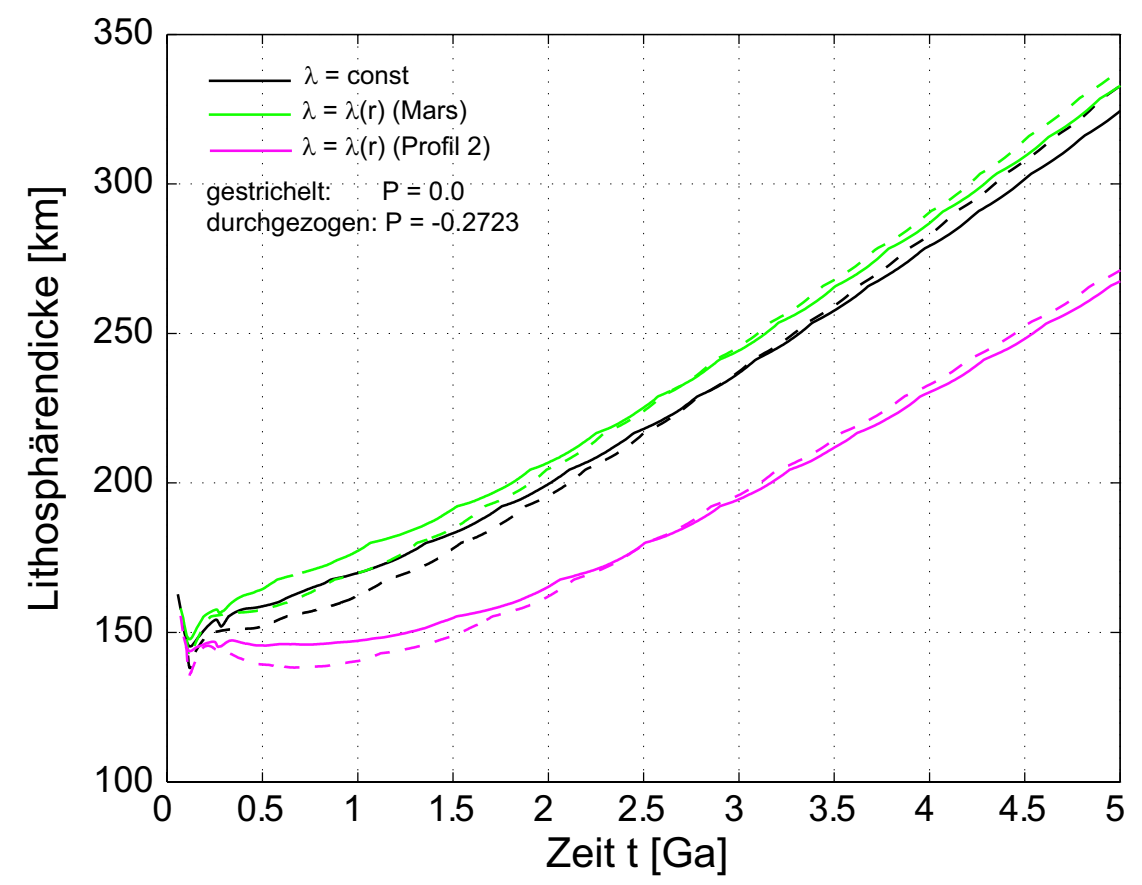

Abbildung 5.33: Einfluss der verschiedenen $\lambda(r)$ auf die Entwicklung der Lithosphärendicke

(5.33) gezeigt. Das $\lambda$-Profil für Mars führt zu einer geringfügig dickeren Lithosphäre (ohne Phasengrenze: $315 \mathrm{~km}$, mit Phasengrenze $309 \mathrm{~km}$ ), die sich durch die etwas niedrigeren Temperaturen in diesem Bereich (siehe Abbildung (5.28)) erklärt. Hingegen ergibt sich bei Profil 2 durch das ausgeprägtere Minimum, die kleineren Werte der thermischen Leitfähigkeit an der Oberfläche und das damit verbundenen Aufheizen im oberen Mantel eine deutlich (etwa $50 \mathrm{~km}$ ) dünnere Lithosphäre. Aufgrund der geringen thermischen Leitfähigkeit in der Lithosphäre kühlt der Planet trotz vergleichsweise dünner Lithosphäre und stärkerer Konvektion (durch höhere Temperaturen) langsamer ab.

Insgesamt ist der Effekt einer tiefenabhängigen thermischen Leitfähigkeit mit den Parametern für den Mars auf die thermische Entwicklung und das Konvektionsmuster sehr schwach. Eine verstärkt tiefenabhängige, druck- und temperaturbestimmte thermische Leitfähigkeit kann auch ohne Phasengrenze zu einer Reduktion der Aufströme und einem Stabilisieren der kernnahen thermischen Grenzschicht führen. Der Einfluss der Phasengrenze auf die thermische Entwicklung wird ähnlich wie bei einem tiefenabhängigen thermischen Ausdehnungskoeffizienten reduziert. Der Mantel kühlt durch das Ausbilden einer Zone mit niedriger Leitfähigkeit und einen erhöhten Wärmefluss aus dem Kern etwas langsamer ab. Das anfängliche Erhöhen des Wärmeflusses an der KMG ist aber schwach und erhöht die Wahrscheinlichkeit für einen thermisch getriebenen Dynamo nur unwesentlich. Insbesondere bleibt der Peak im Wärmefluss sehr breit, so das es schwer ist, eine kurze Lebensdauer des Marsdynamos zu erklären. Der Wärmefluss nach $4.5 \mathrm{Ga}$ ist zu gering, um einen aktuellen, thermisch getriebenen Dynamo zu erlauben. 


\subsection{Einfluss der Kombination von $\alpha(r)$ und $\lambda(r)$}

\subsubsection{Modelle für Mars mit $\alpha(r), \lambda(r)$ und zusätzlichen exothermen Phasengrenzen}

Nachdem in den vorherigen Abschnitten nacheinander die Effekte eines tiefenabhängigen thermischen Ausdehnungskoeffizienten und einer tiefenabhängigen, druck- und temperaturbestimmten thermischen Leitfähigkeit untersucht worden sind, wird in diesem Kapitel der Einfluss der Kombination beider Tiefenabhängigkeiten betrachtet. Dabei werden die Profile jeweils so gewählt, dass sie auf den Mars zutreffen. Um die Modelle darüber hinaus realitätsgetreuer zu gestalten, werden in weiteren Simulationen die exothermen Phasengrenzen zwischen $\alpha$ - und $\beta$-Spinell sowie zwischen $\beta$ - und $\gamma$-Spinell berücksichtigt. Im letzten Schritt sind die Positionen der einzelnen Phasengrenzen im Lauf der Zeit nicht mehr fest, sondern verändern sich mit der Zeit entsprechend der Temperaturentwicklung. In dieser Simulation wird in jedem Zeitschritt der Schnittpunkt der jeweiligen Clausius-Clayperon-Kurve mit dem gemittelten Temperaturprofil berechnet und die Tiefe der einzelnen Phasengrenzen neu bestimmt.

Die Konvektionsmuster, die sich nach 4.5 Ga durch Kombination von $\alpha(r)$ und $\lambda(r)$ und die zusätzlichen exothermen Phasengrenzen ergeben, sind in Abbildung (5.34) durch die Radialgeschwindigkeit in der Mantelmitte dargestellt. In der linken Spalte ist die kernnahe endotherme Phasengrenze vernachlässigt, während sie in der rechten Spalte berücksichtigt wird. Die erste Zeile zeigt zum Vergleich den Referenzfall mit konstanten Materialparametern. Ohne die endotherme Phasengrenze ergibt sich bei gleichzeitiger Berücksichtigung von $\alpha(r)$ und $\lambda(r)$ ein Konvektionsmuster mit drei Aufströmen. Dieses Konvektionsmuster weicht auch in der Geschwindigkeitsamplitude nur geringfügig vom Fall $\alpha(r), \lambda=$ const (Abbildung (5.16 a)) ab. Lediglich der vierte Plume, der im Fall $\alpha(r), \lambda=$ const sehr schwach ist, verschmilzt hier mit dem benachbarten Aufstrom. Das Gleiche gilt für die Simulation, die die endotherme Phasengrenze berücksichtigt. Das Konvektionsmuster ist nahezu identisch zur Simulation mit $\alpha(r), \lambda=$ const. Im Fall $\alpha=$ const, $\lambda=\lambda(r)$ ergibt sich zwar ebenfalls eine Anordnung mit drei Aufströmen, allerdings ist hier die Geschwindigkeitsamplitude höher. Die Anzahl der Aufströme wird bei $\alpha(r)$ und $\lambda(r)$ durch die endotherme Phasengrenze nicht mehr beeinflusst, die Positionen der Aufströme sind leicht verändert und die Amplitude ist mit endothermer Phasengrenze höher. Die Entwicklung zu diesem Muster vollzieht sich mit Phasengrenze schneller. Hier werden etwa 1.5 Ga benötigt (Abbildung (5.36)), während ohne Phasengrenze ca. 2.5 Ga vergehen (Abbildung (5.35)). Simuliert man auch den Effekt der beiden exothermen Phasenübergänge mit, so entsteht ohne kernnahe endotherme Phasengrenze ähnlich zum vorherigen Fall ein Muster mit drei deutlich voneinander getrennten Plumes. Dieses Muster benötigt ebenfalls 2.0 - 2.5 Ga, um sich zu entwickeln, allerdings sind die Geschwindigkeiten deutlich höher. Mit Hilfe der endothermen Phasengrenze reduziert sich die Anzahl der Aufströme auf zwei, von denen der eine gegenüber dem Referenzfall etwas in Richtung Norden verschoben ist. Die Amplitude ist dagegen deutlich höher als im Referenzfall und das Muster wird zwischen 1.5 und 2.0 Ga (Abbildung (5.37)) und somit früher als im Referenzfall erreicht. Entfernt man in diesem Fall nach 2.0 Ga die endotherme Phasengrenze, so verändert sich das Konvektionsmuster nicht signifikant gegenüber dem Fall, der die endotherme Phasengrenze während der ganzen Entwicklung 
a) $\alpha=$ const, $\lambda=$ const
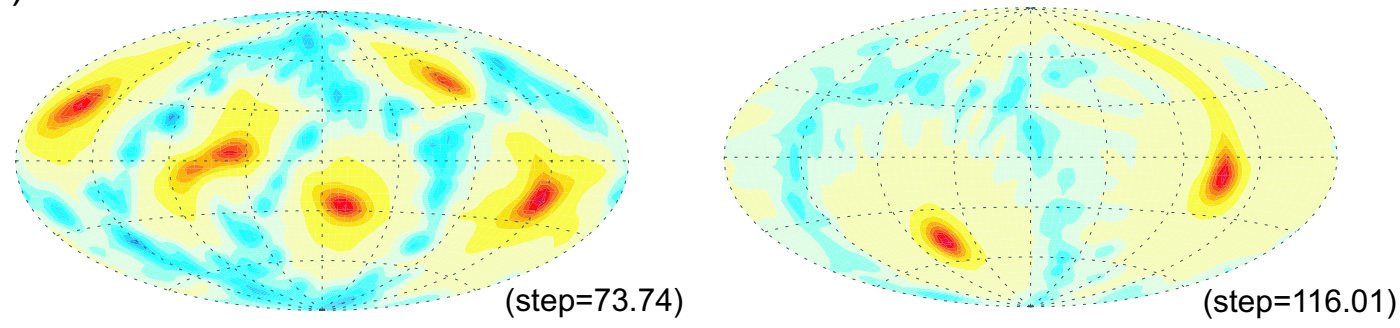

b) $\alpha=\alpha(r), \lambda=\lambda(r)$
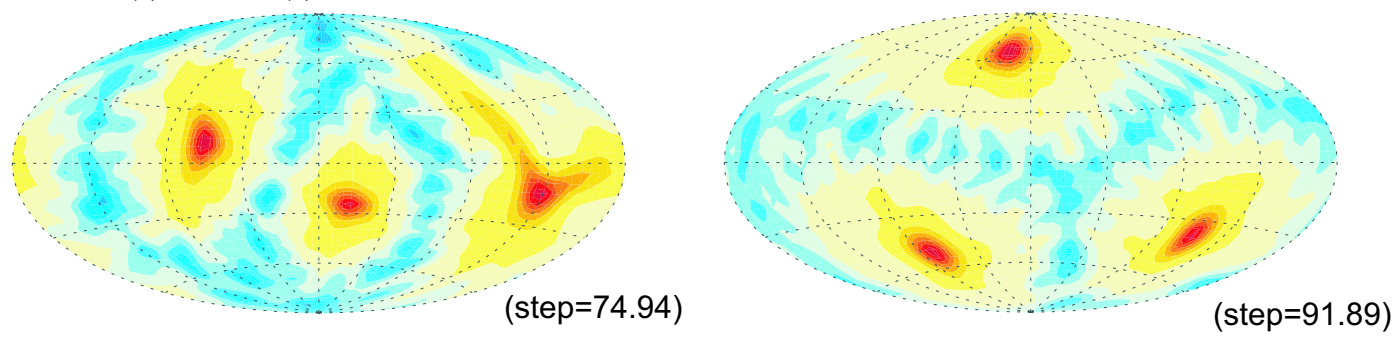

c) $\alpha=\alpha(r), \lambda=\lambda(r)$, zwei exotherme Phasengrenzen
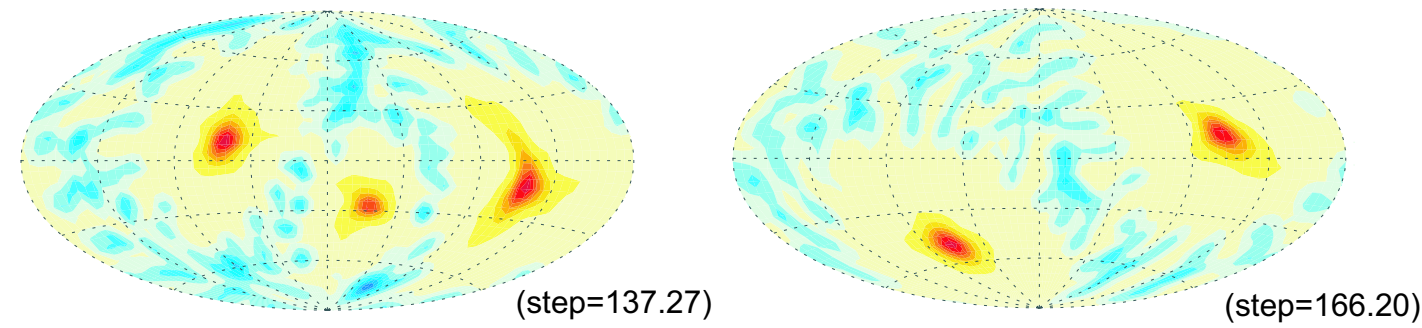

d) $\alpha=\alpha(r), \lambda=\lambda(r)$, zeitlich veränderliche Position der Phasengrenzen

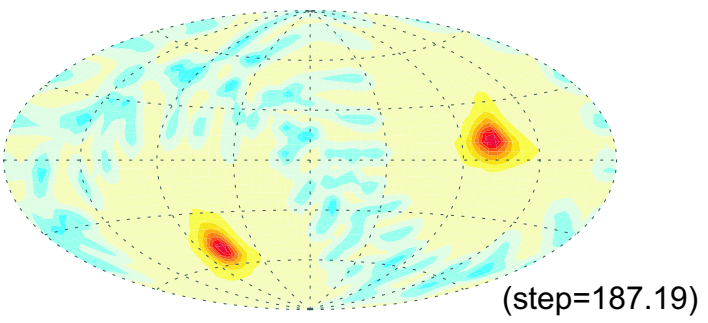

Abbildung 5.34: Konvektionsmuster nach 4.5 Ga bei Kombination von $\alpha(r)$ und $\lambda(r)$ und zusätzlichen exothermen Phasengrenzen: Radialgeschwindigkeit in halber Tiefe (rot: Aufströme, blau: Abströme). Die linke Spalte zeigt die Situation ohne die kernnahe endotherme Phasengrenze, in der rechten Spalte wird die kernnahe endotherme Phasengrenze berïcksichtigt. Der Contourstep der Geschwindigkeit ist angegeben: step=100 entspricht $0.14 \mathrm{~cm} / \mathrm{a}$. 
a) $t=0.5 \mathrm{Ga}$

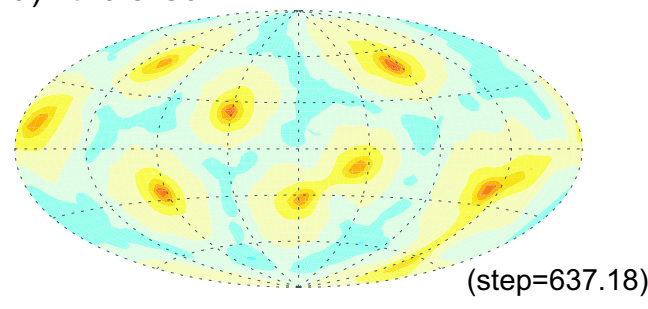

c) $\mathrm{t}=1.5 \mathrm{Ga}$

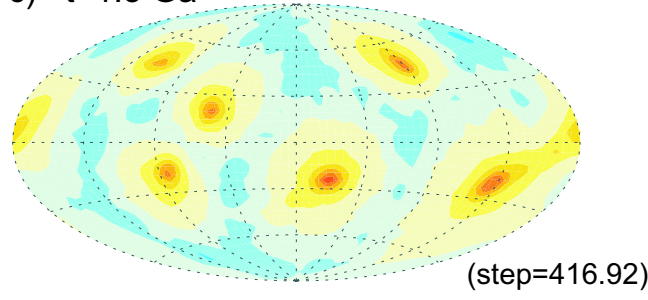

e) $\mathrm{t}=2.5 \mathrm{Ga}$

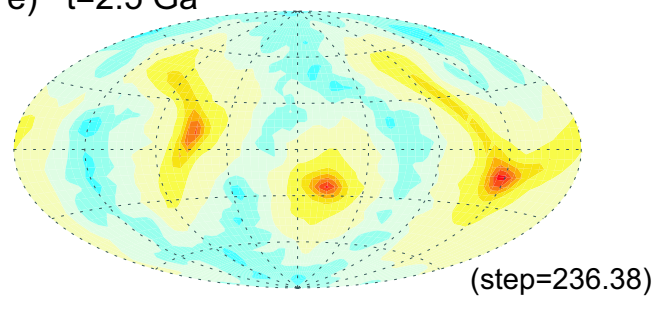

g) $\mathrm{t}=3.5 \mathrm{Ga}$

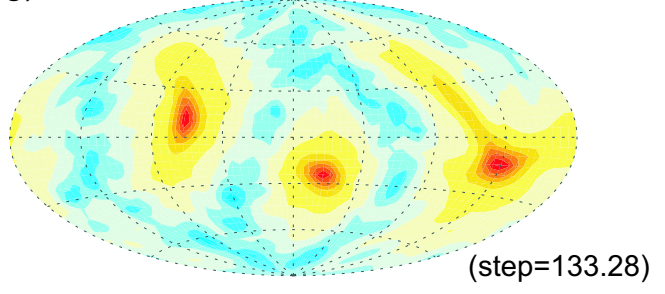

b) $\mathrm{t}=1.0 \mathrm{Ga}$

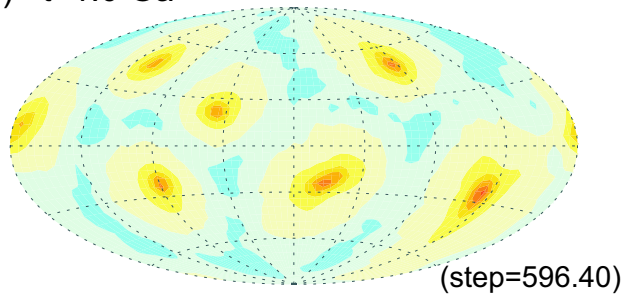

d) $t=2.0 \mathrm{Ga}$

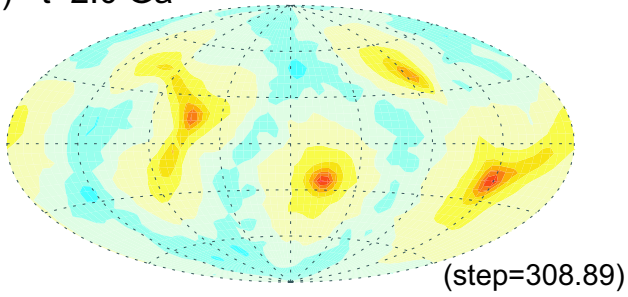

f) $t=3.0 \mathrm{Ga}$

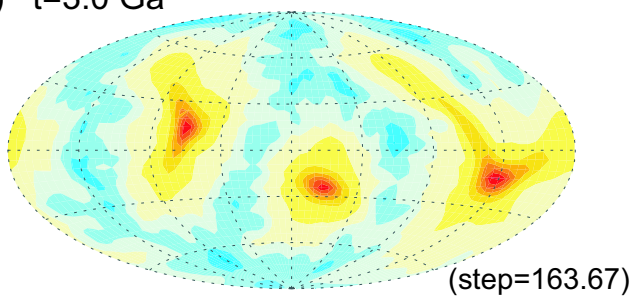

h) $\mathrm{t}=4.0 \mathrm{Ga}$

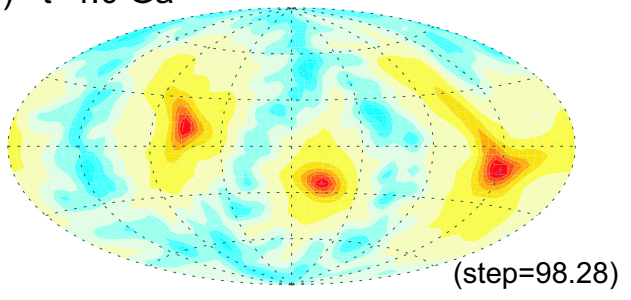

i) $\mathrm{t}=4.5 \mathrm{Ga}$

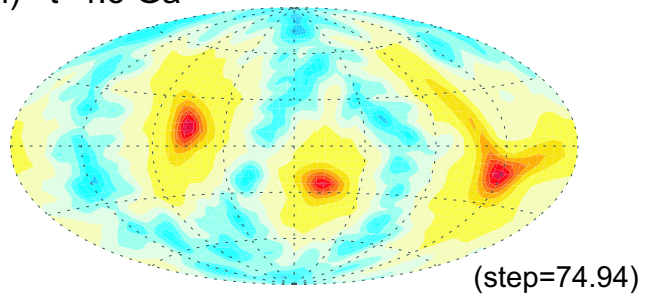

Abbildung 5.35: Zeitliche Entwicklung des Konvektionsmusters bei Kombination von $\alpha(r)$ und $\lambda(r)$ ohne Phasengrenzen: Radialgeschwindigkeit in halber Tiefe (rot: Aufströme, blau: Abströme). Der Contourstep der Geschwindigkeit ist angegeben: step=100 entspricht $0.14 \mathrm{~cm} / \mathrm{a}$. 
a) $t=0.54 \mathrm{Ga}$

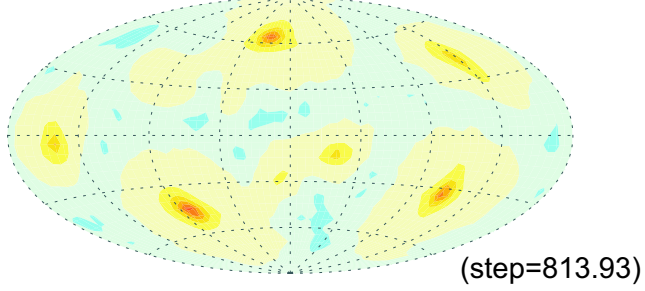

c) $t=1.06 \mathrm{Ga}$

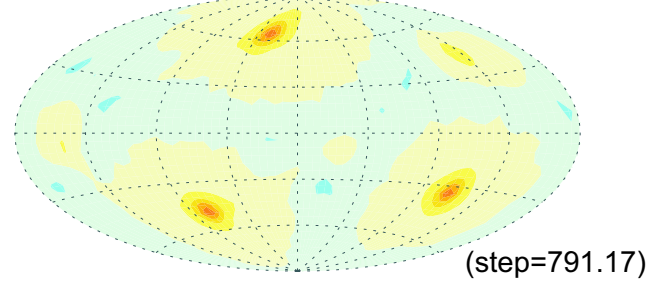

e) $\mathrm{t}=1.49 \mathrm{Ga}$

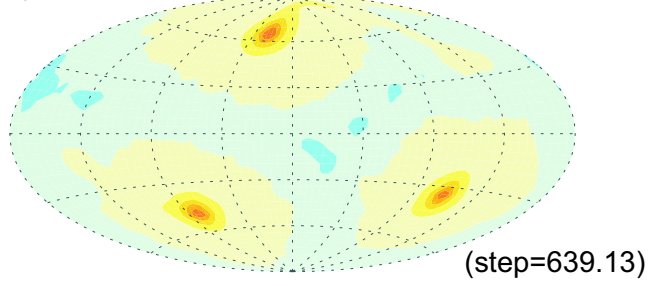

g) $t=2.12 \mathrm{Ga}$

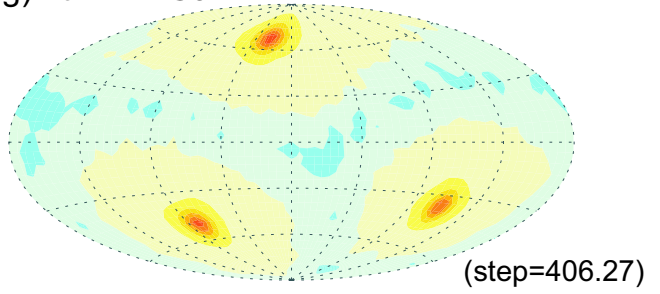

b) $t=0.71 \mathrm{Ga}$

$($ step $=941.17)$

d) $t=1.23 \mathrm{Ga}$

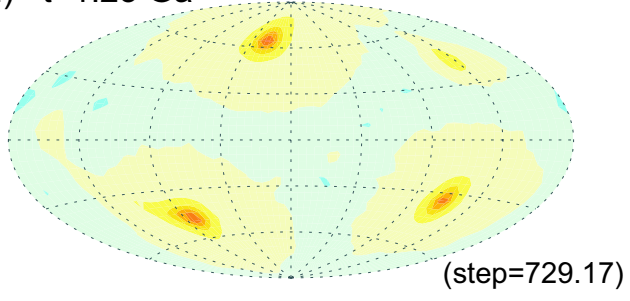

f) $t=1.74 \mathrm{Ga}$

(step=532.47)

h) $t=3.23 \mathrm{Ga}$

i) $\mathrm{t}=4.5 \mathrm{Ga}$

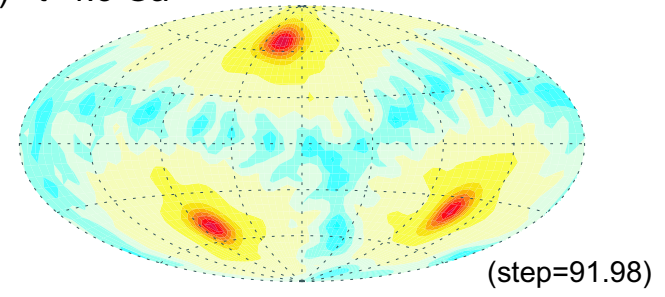

Abbildung 5.36: Zeitliche Entwicklung des Konvektionsmusters bei Kombination von $\alpha(r)$ und $\lambda(r)$ mit endothermer Phasengrenze $(P=-0.2723)$ : Radialgeschwindigkeit in halber Tiefe (rot: Aufströme, blau: Abströme). Der Contourstep der Geschwindigkeit ist angegeben: step $=100$ entspricht $0.14 \mathrm{~cm} / \mathrm{a}$. 
a) $t=0.5 \mathrm{Ga}$

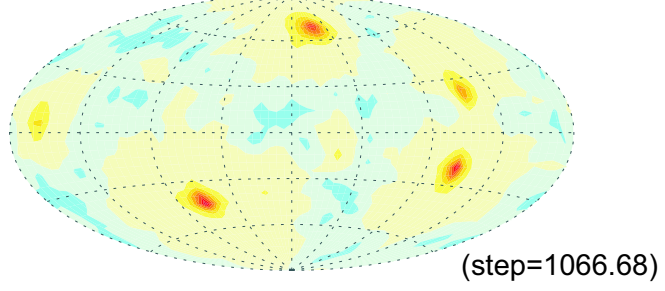

c) $t=1.5 \mathrm{Ga}$

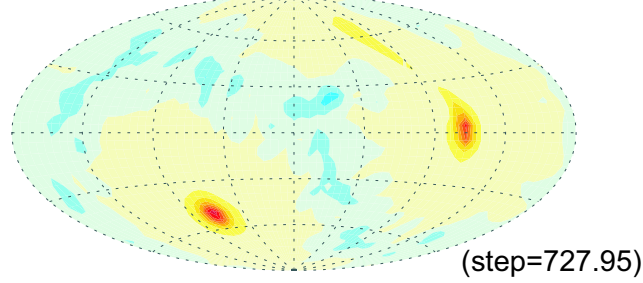

e) $\mathrm{t}=2.5 \mathrm{Ga}$

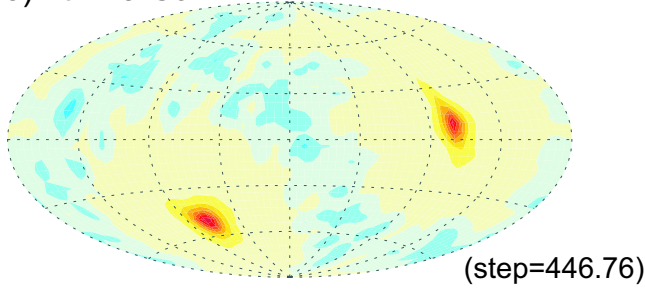

g) $t=3.5 \mathrm{Ga}$

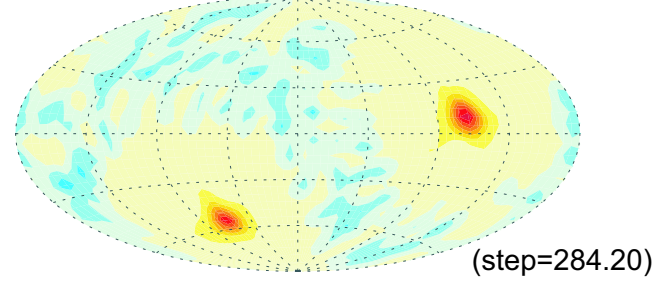

b) $t=1.0 \mathrm{Ga}$

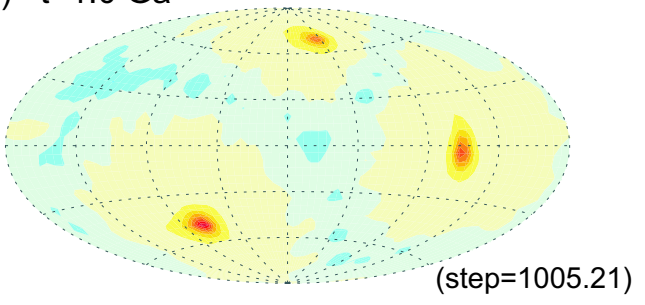

d) $t=2.0 \mathrm{Ga}$

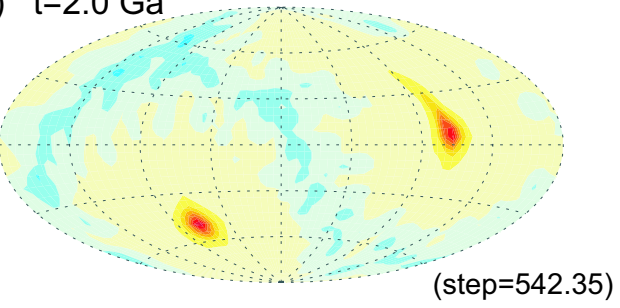

f) $t=3.0 \mathrm{Ga}$

(step=329.49)

h) $\mathrm{t}=4.0 \mathrm{Ga}$

i) $t=4.5 \mathrm{Ga}$

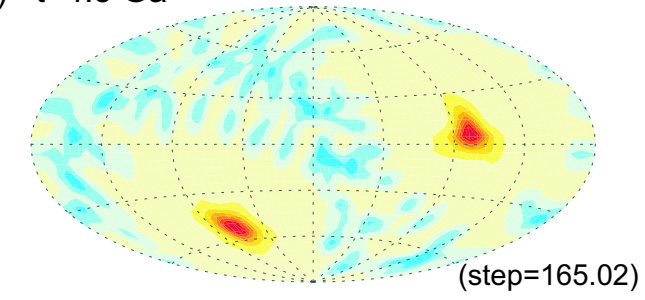

Abbildung 5.37: Zeitliche Entwicklung des Konvektionsmusters bei Kombination von $\alpha(r)$ und $\lambda(r)$ mit endothermer und exothermen Phasengrenzen: Radialgeschwindigkeit in halber Tiefe (rot: Aufströme, blau: Abströme). Der Contourstep der Geschwindigkeit ist angegeben: step $=100$ entspricht $0.14 \mathrm{~cm} / \mathrm{a}$. 
in Betracht zieht. Lediglich die Amplitude schwächt sich leicht ab. Das zeitliche Verändern der Position der Phasengrenze hat keinen signifikanten Einfluss auf die Anordnung von Auf- und Abströmen. In der Augenblickaufnahme ist die Radialgeschwindigkeit in diesem Fall $13 \%$ höher als im Fall mit zeitlich festen Positionen der Phasenübergänge. In Abschnitt (5.3) ist gezeigt worden, dass der Einfluss von $\lambda(r)$ mit den Parametern für Mars auf das Konvektionsmuster sehr gering ist. Dies bestätigt sich ebenfalls bei der Kombination von $\alpha(r)$ und $\lambda(r)$, so dass das Konvektionsmuster durch $\alpha(r)$ bestimmt erscheint. Der Einfluss der kernnahen Phasengrenze ist bei der Kombination von $\alpha(r)$ und $\lambda(r)$ geringer. Die exothermen Phasengrenzen verstärken die Konvektionsbewegung, was die in diesem Fall höheren Geschwindigkeitsamplituden in halber Tiefe, in direkter Nähe zu den exothermen Phasengrenzen erklärt (Abbildung (5.38)). Der Unterschied zwischen zwei bzw. drei Aufströmen im Fall mit endothermer Phasengrenze ist gering und wird im Wesentlichen durch das Verhalten des polnahen Plumes bestimmt. In den Simulationen mit nur einem tiefenabhängigen Parameter scheint dieser Plume stabilisiert zu sein. Im Referenzfall wird er im Lauf der Zeit schwächer, das Abstromband zu dem östlichen Plume bricht auf und der polnahe Plume verschmilzt mit diesem Aufstrom (Abbildung (5.4)). Bei den exothermen Phasengrenzen bewegt sich der polnahe Aufstrom durch die stärkere Konvektion im Vergleich zum Referenzfall vom Pol weg und verschmilzt ebenso mit den übrigen, so dass die Anzahl reduziert wird (Abbildung (5.37)). Der Unterschied hängt jedoch wahrscheinlich sehr stark von den exakten Bedingungen direkt nach dem Start ab, die durch die exothermen Phasengrenzen verändert werden, und kann nicht eindeutig mit einem verstärkten Wirken der endothermen Phasengrenzen erklärt werden. Das Konvektionsmuster ändert sich nicht signifikant, wenn man nach $2.0 \mathrm{Ga}$ die endotherme Phasengrenze entfernt, was die Stabilität diese Musters zeigt und verdeutlicht, dass die Phasengrenze in diesen Stadium der Entwicklung keine entscheidende Rolle mehr spielt.

In der Temperaturentwicklung in halber Tiefe (Abbildung (5.39)) kompensieren sich bei Kombination von $\alpha(r)$ und $\lambda(r)$ die einzelnen Effekte nahezu. Ohne die exothermen Phasengrenzen kühlt der Mantel in halber Tiefe unwesentlich schneller ab. Der Temperaturunterschied zum Referenzfall beträgt bei $t=4.5 \mathrm{Ga}$ nur $5 \mathrm{~K}$. Das Berücksichtigen der exothermen Phasengrenzen bzw. die zeitliche Variation der Position der Phasengrenzen liefert etwas höhere Temperaturen als im Referenzfall. Allerdings ist auch hier der Unterschied von $5 \mathrm{~K}$ bei $t=4.5 \mathrm{Ga}$ nicht signifikant. Bei der magentafarbenen Kurve ist die Position des Phasenübergangs zwischen $\beta$ - und $\gamma$-Spinell von $r_{\beta \gamma}=2053 \mathrm{~km} \mathrm{zu}$ $r_{\beta \gamma}=2117 \mathrm{~km}$ in Richtung Oberfläche verschoben, um einen besseren Vergleich mit dem Fall der beweglichen Phasengrenze zu gewährleisten. Der Einfluss dieser Positionsänderung ist allerdings vernachlässigbar (rote und magentafarbene Kurve kommen fast zur Deckung), so dass im Weiteren keine Unterscheidung zwischen beiden Fällen gemacht wird. Die zeitabhängige Position der Phasengrenzen ist in Abbildung (5.40) dargestellt.

An der KMG führen sowohl $\alpha(r)$ als auch $\lambda(r)$ zu einem schnelleren Abkühlen des Kerns, so dass sich die Einflüsse beider Tiefenabhängigkeiten hier zu Beginn der Evolution verstärken können (Abbildung (5.41)). Das Vernachlässigen der endothermen Phasengrenze führt bei gleichzeitiger Berücksichtigung von $\alpha(r)$ und $\lambda(r)$ nach $4.5 \mathrm{Ga}$ zu etwa $15 \mathrm{~K}$ niedrigeren Temperaturen gegenüber dem Fall mit konstanten Materialparametern. Die exothermen Phasengrenzen verstärken das Abkühlen durch die heftigere Konvektion darüber hinaus, so dass sich Temperaturunterschiede nach $4.5 \mathrm{Ga}$ von $23 \mathrm{~K}$ einstellen. Diese Temperaturunterschiede durch die tiefenabhängigen Parameter bzw. die exo- 


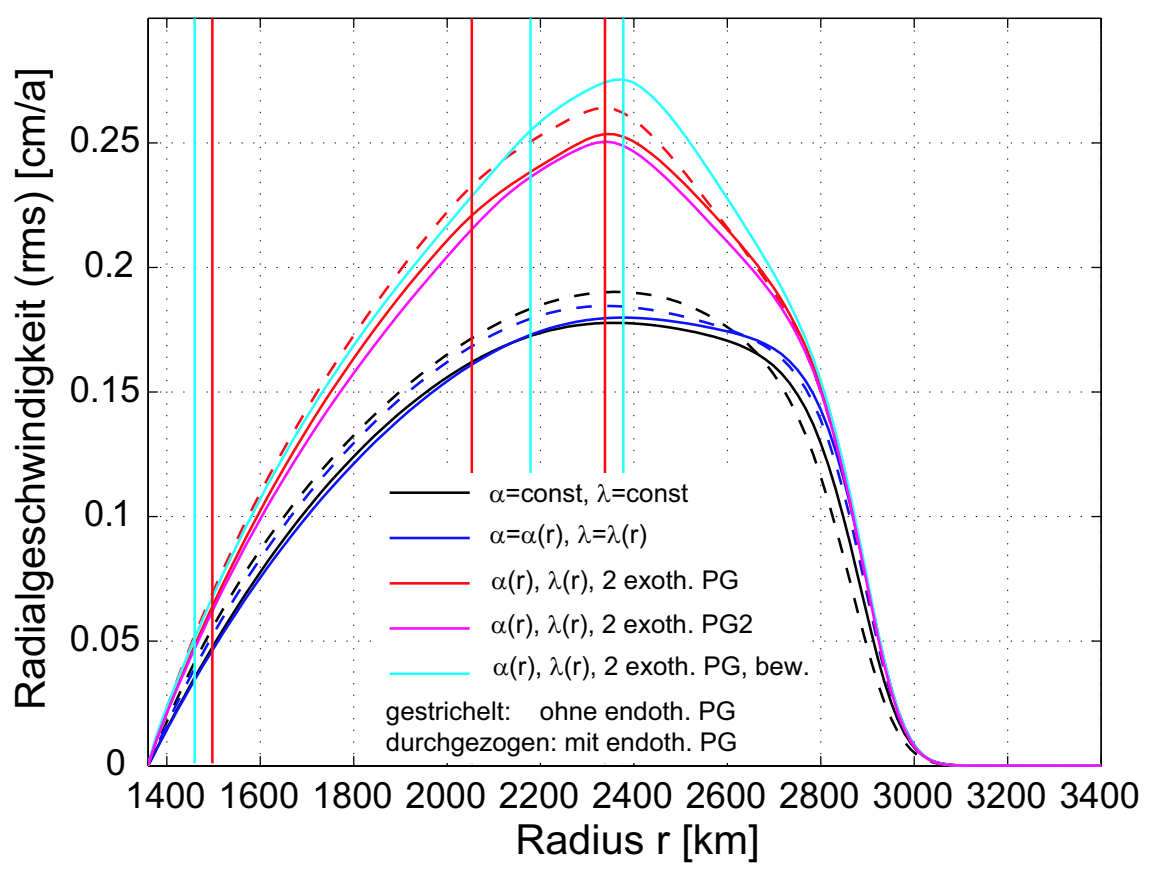

Abbildung 5.38: Einfluss der Kombination von $\alpha(r)$ und $\lambda(r)$ und der exothermen Phasengrenzen auf das Profil der Radialgeschwindigkeit nach 4.5 Ga. Die Lage der einzelnen Phasengrenzen (nach $4.5 \mathrm{Ga}$ ) ist durch die senkrechten Linien markiert.

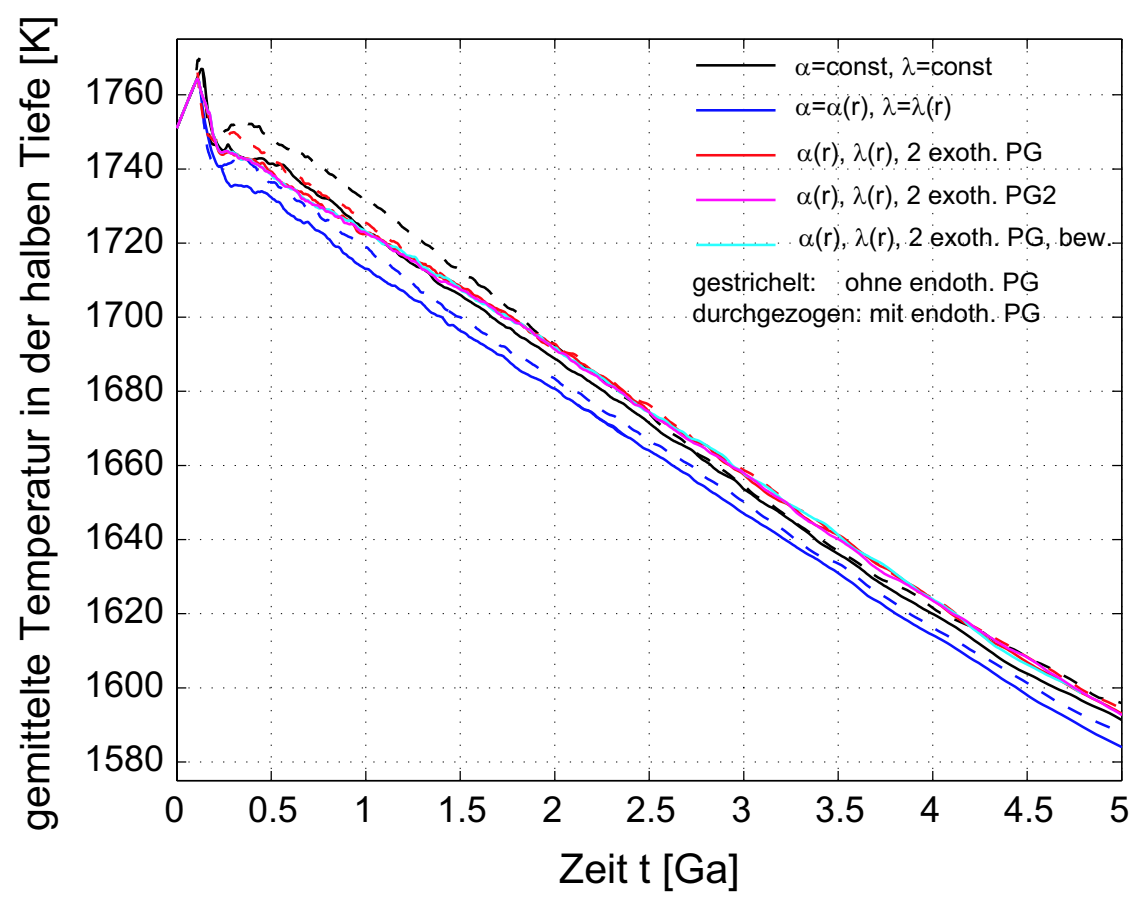

Abbildung 5.39: Einfluss der Kombination von $\alpha(r)$ und $\lambda(r)$ und der exothermen Phasengrenzen auf die Entwicklung der Temperatur in halber Tiefe. 


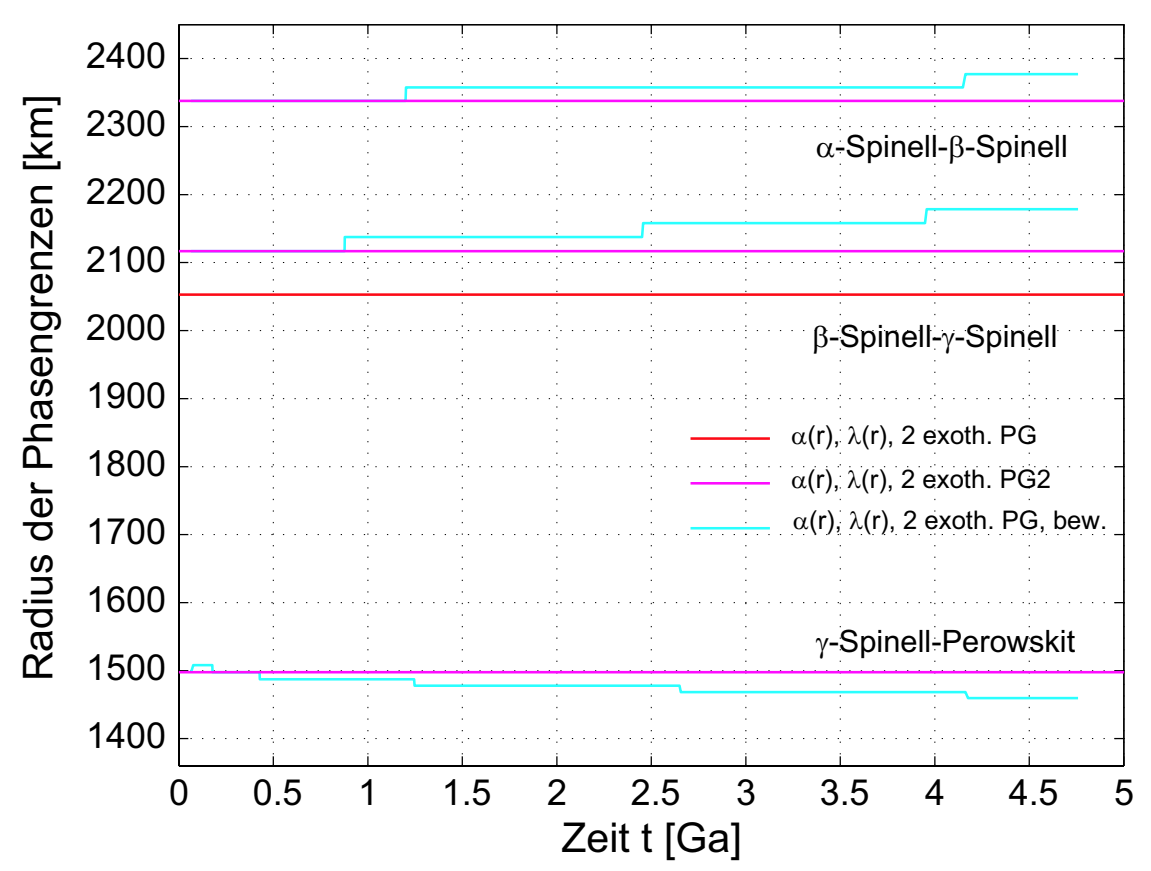

Abbildung 5.40: Zeitliche Entwicklung der Position der Phasengrenzen. Im magentafarbenen Fall ist die exotherme Phasengrenze zwischen $\beta$ - und $\gamma$-Spinell in Richtung Oberfläche verschoben, so dass die Position der Starttiefe im Fall der beweglichen Phasengrenze entspricht. Ansonsten stimmen die rot und magenta markierten Positionen überein.

thermen Phasengrenzen sind sehr klein. Durch die Tiefenabhängigkeit von $\alpha(r)$ sind sie jedoch stärker als im Fall $\lambda(r)$, wo am Ende nahezu gleiche Temperaturen erreicht werden. Deutlicher wird der Einfluss der Erweiterungen unter Einbeziehung der endothermen Phasengrenze. Hier führt die Kombination von $\alpha(r)$ und $\lambda(r)$ zu einem Temperaturunterschied von $55 \mathrm{~K}$ gegenüber dem Referenzfall, die exothermen Phasengrenzen vergröBern diesen Unterschied auf 75 K. Die zeitliche Veränderung der Lage der Phasengrenzen hat nur einen geringen Einfluss. Der Effekt von $\alpha(r)$ und $\lambda(r)$ im Fall mit endothermer Phasengrenze ist deutlicher, da sie nicht nur direkt den Wärmetransport beeinflussen, sondern auch die Wirkung der Phasengrenze selbst reduzieren. Dies wird daran deutlich, dass der Temperaturunterschied durch die endotherme Phasengrenze bei der Kombination von $\alpha(r)$ und $\lambda(r)$ kleiner als im Fall mit konstanten Materialparametern ist. Die "heutige" Temperatur an der KMG (nach 4.5 Ga) im Fall ohne Phasengrenzen bei konstanten Materialparametern unterscheidet sich kaum vom "realitätsgetreuen" Fall mit drei, zeitlich in ihrer Position veränderlichen Phasenübergängen und tiefenabhängigen Parametern. Die genaue zeitliche Entwicklung hängt jedoch von der Existenz der Phasenübergänge und der Tiefenabhängigkeit der Parameter ab.

Das Abkühlverhalten des Kerns spiegelt sich auch im Wärmefluss an der KMG wieder. Entsprechend der Temperaturentwicklung an der KMG führen sowohl $\alpha(r)$ als auch $\lambda(r)$ einzeln zu Beginn der Evolution zu einem Erhöhen des Wärmeflusses aus dem Kern. Die gleichzeitige Berücksichtigung beider Tiefenabhängigkeiten verstärkt diesen Effekt, 


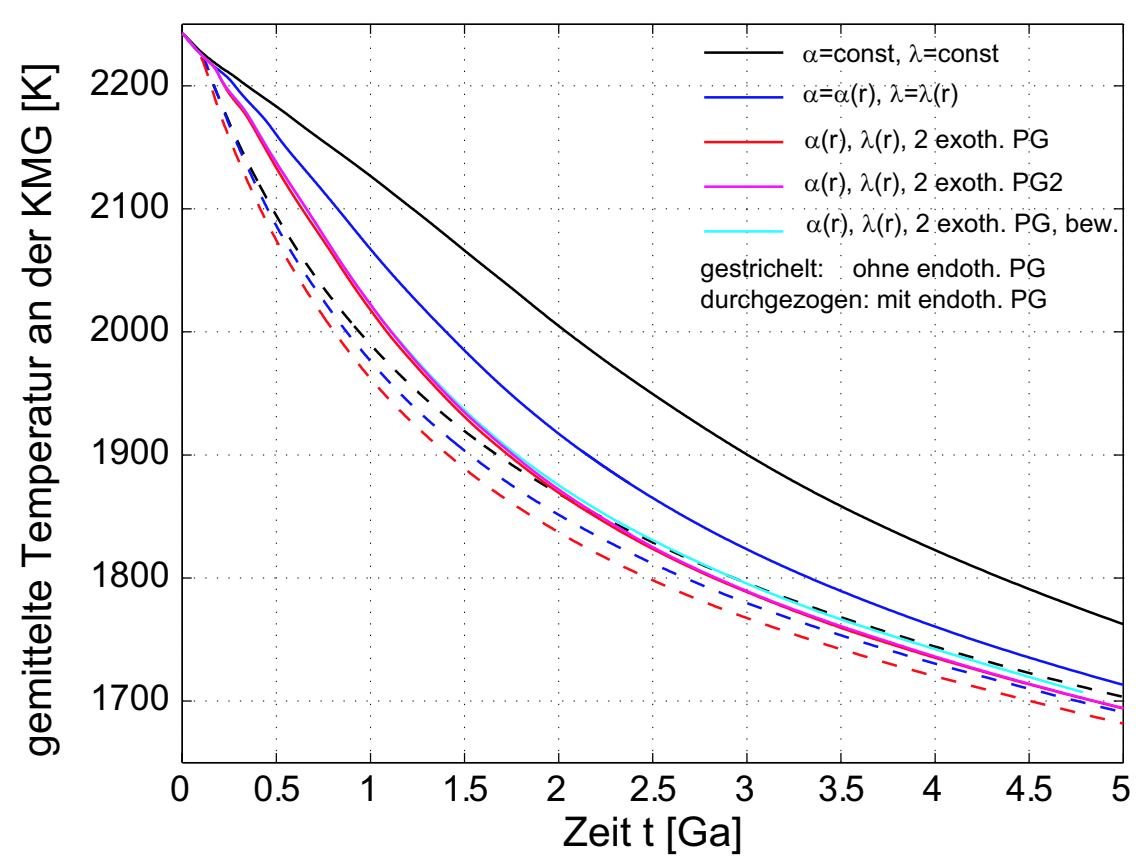

Abbildung 5.41: Einfluss der Kombination von $\alpha(r)$ und $\lambda(r)$ und der exothermen Phasengrenzen auf die Entwicklung der Temperatur an der KMG

wie Abbildung (5.42) zeigt. Die exothermen Phasengrenzen erhöhen den Wärmefluss nochmals. Gegen Ende der Evolution ist allerdings der Unterschied im Wärmefluss zwischen den Fällen ohne endotherme Phasengrenze und den vollständigen Modellen (drei Phasengrenzen, $\alpha(r)$ und $\lambda(r)$ ) sehr klein. Lediglich im Referenzfall mit endothermem Phasenübergang und konstanten thermischen Parametern ist der Wärmefluss geringfügig höher. Das Erhöhen des Wärmeflusses aus dem Kern unterstützt die Möglichkeit eines thermisch getriebenen Dynamos in der frühen Evolution. Selbst im Fall mit endothermer Phasengrenze überschreiten alle Simulationen den Mittelwert des Parameterbereichs für den Wärmefluss entlang der Adiabate von $12 \mathrm{~mW} / \mathrm{m}^{2}$. Ohne die exothermen Phasenübergänge gilt dies über einen Zeitraum von $1.7 \mathrm{Ga}$, mit diesen für 1.6 Ga. Bei Verstärkung der Konvektion mit Hilfe der exothermen Phasenübergänge wird zudem der obere Grenzwert des adiabatischen Wärmeflusses überschritten. Sollte sich folglich der obere Bereich des Intervalls für den adiabatischen Wärmefluss als richtig erweisen, so würde das Erreichen der hohen Werte und die kürzere Lebenszeit des Dynamos für diese Modelle sprechen. Bei Kombination von $\alpha(r)$ und $\lambda(r)$ ist der Einfluss der endothermen Phasengrenze auf das Konvektionsmuster nicht mehr so dominant, bzw. auch ohne diesen Übergang ist die Anzahl der Aufströme gegenüber dem Referenzfall reduziert. In beiden Fällen wird ein Konvektionsmuster mit drei Aufströmen erreicht. Der Unterschied zwischen drei Aufströmen und zwei Aufstömen, die durch die Vulkanismusverteilung nahegelegt werden, ist klein. Ohne die endotherme Phasengrenze ist der Wärmefluss bei Kombination von $\alpha(r)$ und $\lambda(r)$ in einem deutlich kürzeren Zeitintervall stark erhöht. Dies führt bei einem hohen Wert für den adiabatischen Wärmefluss zu einer Lebenszeit des Dynamos von etwa $0.7 \mathrm{Ga}$, was mit den Beobachtungen vereinbar ist. Auch bei Kombination von $\alpha(r)$ und 


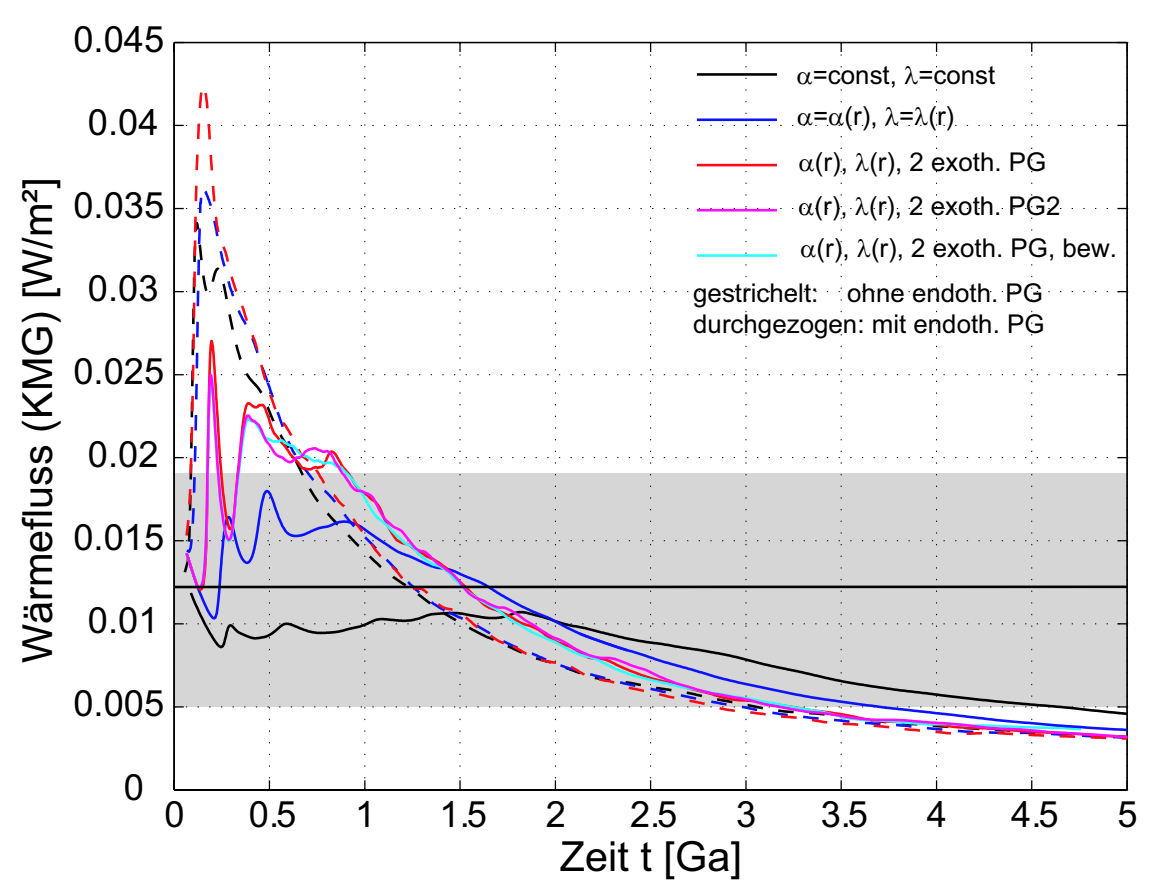

Abbildung 5.42: Einfluss der Kombination von $\alpha(r)$ und $\lambda(r)$ und der exothermen Phasengrenzen auf die Entwicklung des Wärmeflusses an der KMG. Der grau unterlegte Bereich kennzeichnet den Wertebereich des Wärmeflusses entlang der Adiabate im Marskern (Nimmo und Stevenson 2000). Die schwarze Linie zeigt zur Orientierung den Mittelwert dieses Bereichs.

$\lambda(r)$ und Berücksichtigung der Effekte der exothermen Phasengrenzen ist der Wärmefluss nach 4.5 Ga zu klein, um durch thermische Konvektion einen Dynamo anzutreiben.

Der Wärmefluss an der Oberfläche wird nach $4.5 \mathrm{Ga}$ durch die Tiefenabhängigkeit der thermischen Parameter bzw. die verschiedenen Kombinationen der Phasengrenzen nicht signifikant beeinflusst und liegt zwischen 12.3 und $12.5 \mathrm{~mW} / \mathrm{m}^{2}$.

In der zeitlichen Entwicklung der Lithosphärendicke (Abbildung (5.43)) überwiegt bei der Kombination von $\alpha(r)$ und $\lambda(r)$ zu Beginn der Evolution der Einfluss von $\alpha(r)$, der im Vergleich zum Referenzfall zu einer etwas dünneren Lithosphäre führt. Nach $4.5 \mathrm{Ga}$ werden durch die kombinierten Tiefenabhängigkeiten bzw. die zusätzlichen exothermen Phasengrenzen nahezu identische Lithosphärendicken erreicht, die mit etwa $310 \mathrm{~km} \mathrm{ca}$. $10 \mathrm{~km}$ stärker sind als im Referenzfall mit endothermer Phasengrenze. (Im oberflächennahen Bereich ist der Temperaturgradient wie in Abbildung (5.28) durch $\lambda(r)$ verändert und führt zu dieser Zunahme der Lithosphärendicke.)

Zusammenfassend lässt sich festhalten, dass bei der gleichzeitigen Berücksichtigung von $\alpha(r)$ und $\lambda(r)$ der Einfluss der endothermen Phasengrenze auf das Konvektionsmuster sinkt. In beiden Fällen entsteht ein Konvektionsmuster mit drei Aufströmen, so dass die Kombination von $\alpha(r)$ und $\lambda(r)$ ohne endotherme Phasengrenze die Anzahl der Aufströme deutlich reduziert. Die zusätzlichen exothermen Phasengrenzen wirken sich insbesondere auf die Geschwindigkeitsamplitude aus. Auf das Konvektionsmuster im Fall ohne 


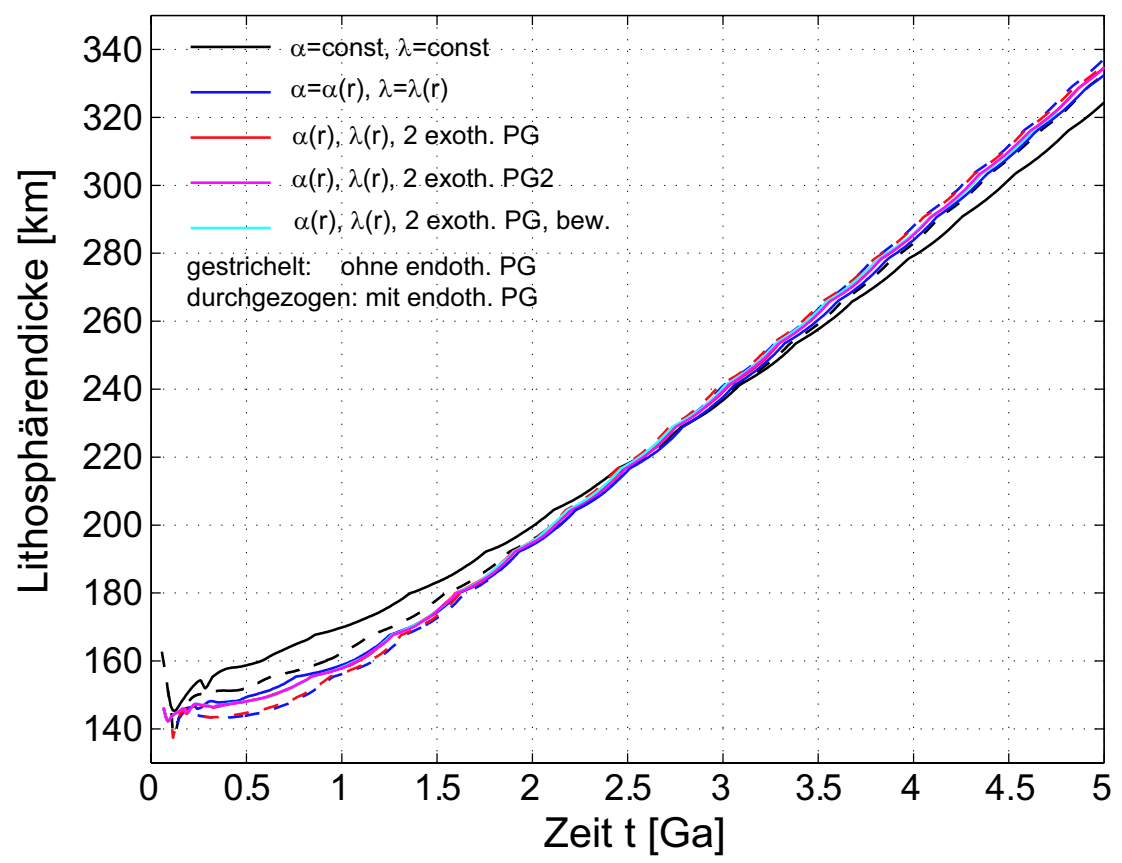

Abbildung 5.43: Einfluss der Kombination von $\alpha(r)$ und $\lambda(r)$ und der exothermen Phasengrenzen auf der Lithosphärendicke.

endotherme Phasengrenze haben sie keinen Einfluss, wohingegen sich mit dem Übergang zum Perowskit ein Muster mit zwei Aufströmen einstellt, das gut mit dem in zwei Regionen konzentrierten Vulkanismus auf dem Mars korreliert. An dieser Konfiguration ändert die zeitliche Variation der Position der Phasengrenzen bzw. ein Entfernen der endothermen Phasengrenzen nach Einstellen dieses Musters nichts. Bei der Temperaturentwicklung in halber Tiefe kompensieren sich die entgegengesetzt wirkenden Einflüsse von $\alpha(r)$ und $\lambda(r)$, so dass in diesem Bereich durch die Kombination keine signikanten Unterschiede zu den Simulationen mit konstanten Materialparametern entstehen. An der KMG wirken sowohl $\alpha(r)$ als auch $\lambda(r)$ so, dass das Abkühlen beschleunigt wird. Ohne endotherme Phasengrenze sind die Unterschiede zum Referenzfall nach $4.5 \mathrm{Ga}$ jedoch klein, wobei sie deutlicher ausfallen, wenn dieser Phasenübergang berücksichtigt wird. Der direkte Einfluss der endothermen Phasengrenze ist bei der Kombination von $\alpha(r)$ und $\lambda(r)$ folglich geringer. Das schnellere Abkühlen wird durch einen höheren Wärmefluss aus dem Kern hervorgerufen, der durch die exothermen Phasengrenzen deutlich gesteigert wird. Auch bei Berücksichtigung der endothermen Phasengrenze ist es nun möglich, zu Beginn der Evolution einen thermisch getriebenen Dynamo im Mars zu realisieren, auch wenn der Wärmefluss entlang der Adiabate an der oberen Grenze des realistischen Bereichs liegt. Wird die Anzahl der Plumes durch alleinige Kombination von $\alpha(r)$ und $\lambda(r)$ reduziert und die endothermen Phasengrenzen vernachlässigt, so erlaubt das kürzere Zeitintervall des erhöhten Wärmeflusses eine kürzere Lebensdauer des Dynamos, die den Beobachtungen nahe kommt. Die Lithosphärendicke wird durch die kombinierte Tiefenabhängigkeit von $\alpha(r)$ und $\lambda(r)$ nur schwach beeinflusst und beträgt etwa $310 \mathrm{~km}$. 


\subsubsection{Einfluss der Kerntemperatur im Startprofi I bei Modellen mit $\alpha(r)$ und $\lambda(r)$}

Im vorangegangen Kapitel ist gezeigt geworden, dass der Einfluss der Phasengrenze bei Kombination von $\alpha(r)$ und $\lambda(r)$ sowohl auf das Konvektionsmuster als auch auf die thermische Entwicklung kleiner ist, als im Fall mit konstanten Parameter. Beide Tiefenabhängigkeiten schwächen Instabilitäten an der KMG und verlagern die Konvektion in Richtung Oberfläche bzw. Verstärken den Einfluss der kalten Abströme (insbesondere $\alpha(r)$ ). Durch Variation der Startkerntemperatur wird in diesem Abschnitt verdeutlicht, dass der Einfluss der endothermen Phasengrenze auch bei Kombination von $\alpha(r)$ und $\lambda(r)$, wie sie für Mars angenommen werden, nicht vernachlässigbar ist, wenn thermische Instabilitäten an der KMG für die Konvektion bedeutsam sind. Desweiteren führt eine höhere Starttemperatur an der KMG, die letztlich eine unbekannte Größe ist, zu einem stärkeren Temperaturgradienten und einem erhöhten Wärmefluss aus dem Kern, der für die Frage nach der Existenz und Lebensdauer eines thermisch getriebenen Dynamos wichtig ist. Außerdem wird untersucht wie die thermische Entwicklung durch die Starttemperatur beeinflusst wird.

Die Starttemperaturprofile mit den unterschiedlichen Kerntemperaturen sind in Abbil-

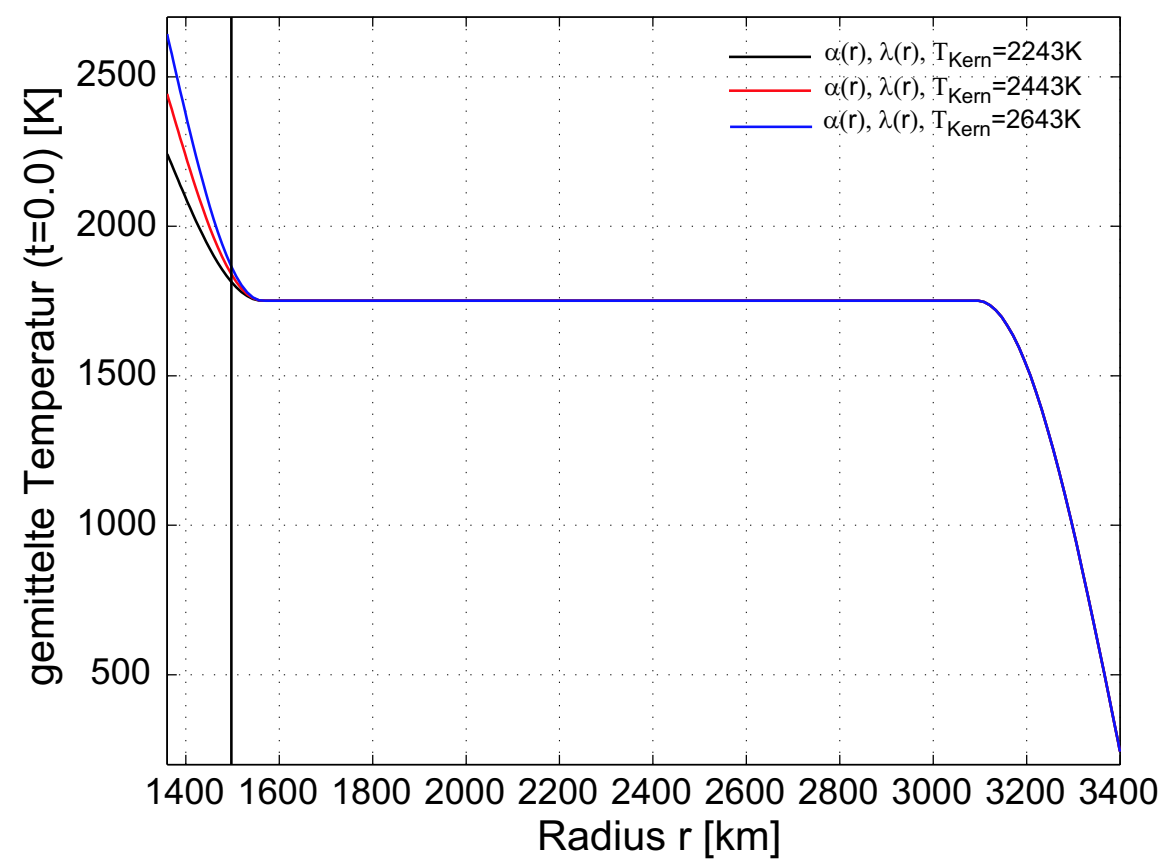

Abbildung 5.44: Starttemperaturprofile mit unterschiedlichen Kerntemperaturen. Die schwarze Linie markiert die Lage der endothermen Phasengrenze.

dung (5.44) dargestellt. Die thermische Leitfähigkeit an der KMG variiert durch diese Temperaturänderung zwischen 4.38 und $4.57 \mathrm{~W} / \mathrm{mK}$ (im Referenzfall mit $2243 \mathrm{~K}$ ). Der Effekt auf die Viskosität ist deutlicher. Sie liegt zwischen $0.0174 \cdot 10^{21}$ und $0.1492 \cdot 10^{21}$ Pa s (im Referenzfall mit 2243 K). 
a) $\alpha=\alpha(r), \lambda=\lambda(r)$, Kerntemperatur (t=0.0 Ga): $2243 \mathrm{~K}$
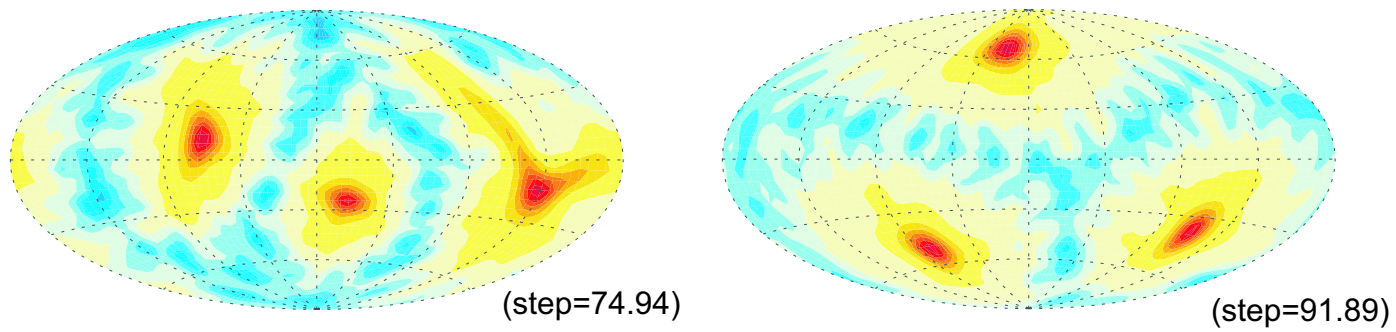

b) $\alpha=\alpha(r), \lambda=\lambda(r)$, Kerntemperatur $(t=0.0 \mathrm{Ga})$ : $2443 \mathrm{~K}$
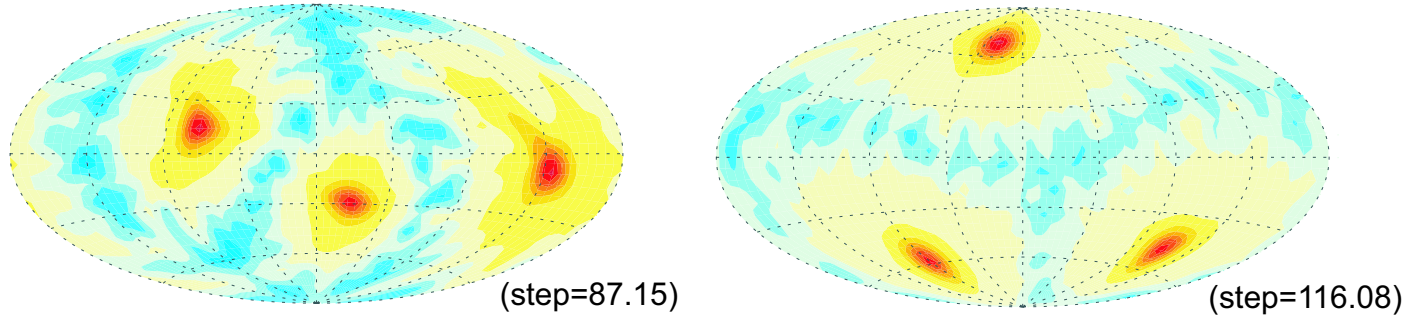

c) $\alpha=\alpha(r), \lambda=\lambda(r)$, Kerntemperatur (t=0.0 Ga): $2643 \mathrm{~K}$
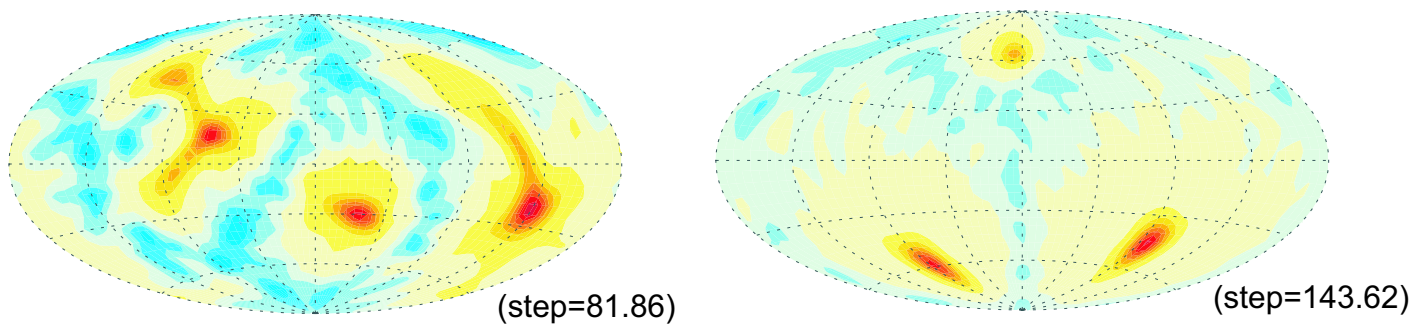

Abbildung 5.45: Konvektionsmuster nach 4.5 Ga bei Kombination von $\alpha(r)$ und $\lambda(r)$ und unterschiedlichen Kerntemperaturen im Startprofil: Radialgeschwindigkeit in halber Tiefe (rot: Aufströme, blau: Abströme). Die linke Spalte zeigt die Situation ohne die kernnahe endotherme Phasengrenze, in der rechten Spalte wird die kernnahe endotherme Phasengrenze berücksichtigt. Der Contourstep der Geschwindigkeit ist angegeben: step=100 entspricht $0.14 \mathrm{~cm} / \mathrm{a}$. 
Die Konvektionsmuster, die sich für die unterschiedlichen Temperaturprofile nach 4.5 Ga einstellen sind in Abbildung (5.45) zusammengestellt. Die erste Zeile zeigt den Referenzfall mit Berücksichtigung von $\alpha(r)$ und $\lambda(r)$ bei der Startkerntemperatur von 2243 $\mathrm{K}$, der im vorherigen Kapitel vorgestellt worden ist. Die beiden Spalten unterscheiden erneut zwischen dem Fall mit endothermer Phasengrenze (rechte Spalte) und der Situation bei Vernachlässigung dieses Übergangs (linke Spalte). Das Erhöhen der Kerntemperatur im Startprofil um $200 \mathrm{~K}$ beeinflusst das Konvektionsmuster nach $4.5 \mathrm{Ga}$ nur geringfügig. Sowohl mit als auch ohne endotherme Phasengrenze entsteht das bekannte Konvektionsmuster mit drei Aufströmen. Die Geschwindigkeiten sind etwas höher als im Referenzfall und der Unterschied in der Geschwindigkeitsamplitude durch die Phasengrenze ist etwas größer. Ein weiteres Anheben der Kerntemperatur führt jedoch auch bei Kombination von $\alpha(r)$ und $\lambda(r)$ zu einem unterschiedlichen Konvektionsmuster in Abhängigkeit von der Berücksichtigung der endothermen Phasengrenzen. Ohne diese Phasengrenze entsteht ein Konvektionsmuster nach $4.5 \mathrm{Ga}$ mit vier bis fünf Plumes, von denen zwei schwächer sind als die übrigen. Mit der kernnahen endothermen Phasengrenze stellt sich wiederum ein Konvektionsmuster mit drei Aufströmen ein, die durch Abstrombänder voneinander getrennt sind. Der polnahe Aufstrom hat jedoch eine geringere radiale Aufstiegsgeschwindigkeit. Der Geschwindigkeitsunterschied zwischen dem Fall mit bzw. ohne Phasengrenze ist durch das Erhöhen der Starttemperatur ebenfalls angestiegen.

Die höhere Startkerntemperatur bzw. der stärkere Temperaturgradient an der KMG fördert zusammen mit der verringerten Viskosität Instabilitäten in der Grenzschicht an der KMG. Auf diese kann die kernnahe endotherme Phasengrenze wirken, so dass ihr Einfluss wieder gestärkt wird. Zumindest im Fall mit einer Kerntemperatur von $2643 \mathrm{~K}$ zu Beginn entstehen deutliche Unterschiede im Konvektionsmuster. Hier zeigt sich die Phasengrenze als der effektivere (gegenüber der Kombination von $\alpha(r)$ und $\lambda(r)$ ) Mechanismus, um die Anzahl der Aufströme zu reduzieren.

Der durch die Änderung des Starttemperaturprofils verstärkte Einfluss der Phasengrenze zeigt sich besonders deutlich in der Temperaturentwicklung an der KMG in Abbildung (5.46). Ohne Phasengrenze führt der stark unterschiedliche Wärmefluss aus dem Kern zu Beginn dazu, dass die Temperaturen an der KMG sich im Lauf der Evolution trotz unterschiedlicher Starttemperaturen angleichen und nach $4.5 \mathrm{Ga}$ in einem engen Intervall zwischen 1710 K (Starttemperatur: $2243 \mathrm{~K}$ ) und $1723 \mathrm{~K}$ (Starttemperatur: $2643 \mathrm{~K}$ ) liegen. Mit kernnaher endothermer Phasengrenze liegen die Temperaturen durch den unterschiedlichen Beitrag der Phasengrenze in einem weiteren Temperaturbereich zwischen 1735 K (Starttemperatur: 2243 K) und 1809 K (Starttemperatur: 2643 K). Der Einfluss des Starttemperaturprofils auf die Entwicklung der Temperatur in halber Tiefe ist sehr gering, so dass nach $4.5 \mathrm{Ga}$ die Temperaturen für die verschiedenen Simulationen in diesem Bereich im Intervall $1601 \pm 3.5 \mathrm{~K}$ liegen.

Im Temperaturprofil nach 4.5 Ga (Abbildung (5.47)) ist im Wesentlichen der Bereich in der Nähe der KMG durch die beschriebenen Unterschiede in der Kerntemperatur von den verschiedenen Startprofilen und der Phasengrenze beeinflusst. In der Nähe der Oberfläche führt eine höhere Startkerntemperatur zu geringfügig höheren Temperaturen, so dass die Lithosphäre nach $4.5 \mathrm{Ga}$ in diesen Fällen etwas dünner ausfällt (Abbildung (5.48)). Die Lithosphärendicken liegen zwischen 297 km (Starttemperatur: $2643 \mathrm{~K}$, mit Phasengrenze) und $312 \mathrm{~km}$ (Referenzfall ohne Phasengrenze). Der anfänglich sehr hohe Wärmefluss (Abbildung (5.49)) aus dem Kern bzw. der starke Wärmeeintrag in den Mantel beim 


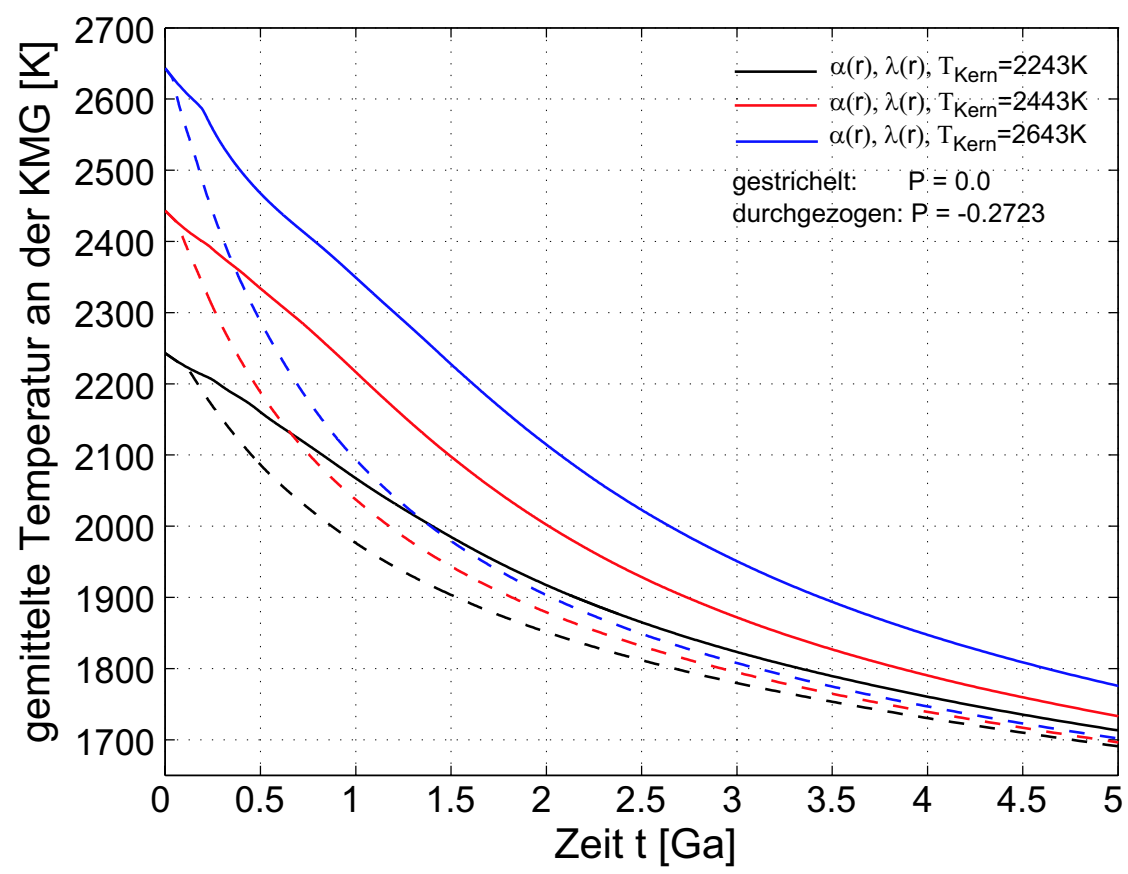

Abbildung 5.46: Entwicklung der Temperatur an der KMG bei unterschiedlicher Starttemperatur des Kerns.

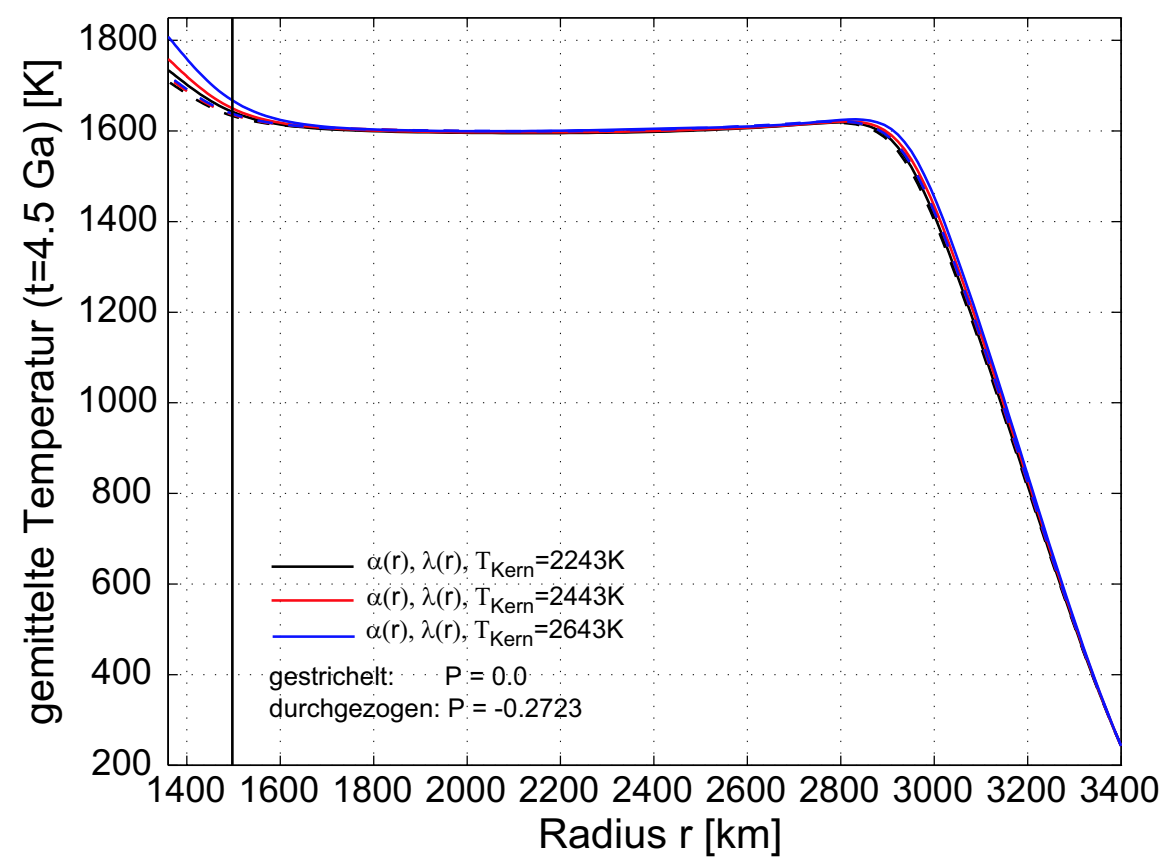

Abbildung 5.47: Einfluss der verschiedenen Startkerntemperaturen auf das Temperaturprofil nach 4.5 Ga. Die schwarze Linie markiert die Lage der endothermen Phasengrenze. 


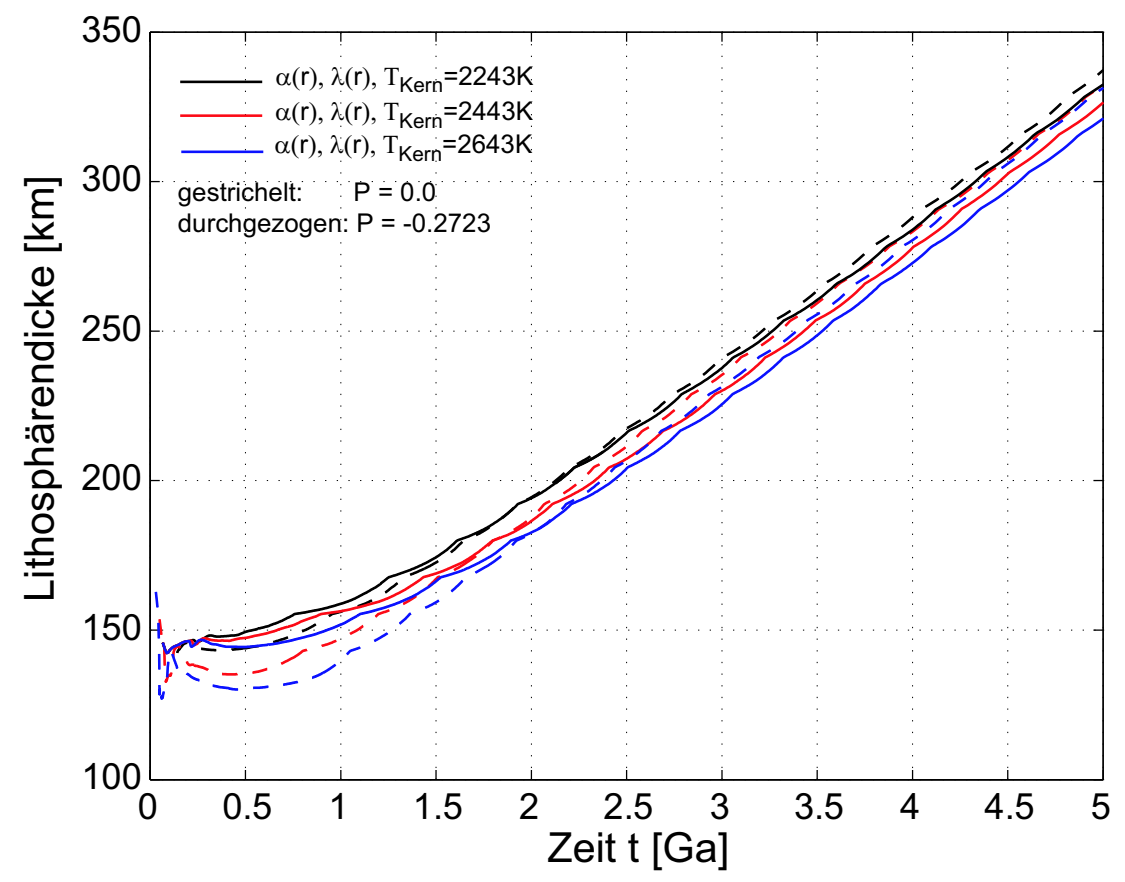

Abbildung 5.48: Entwicklung der Lithosphärendicke bei unterschiedlicher Starttemperatur des Kerns.

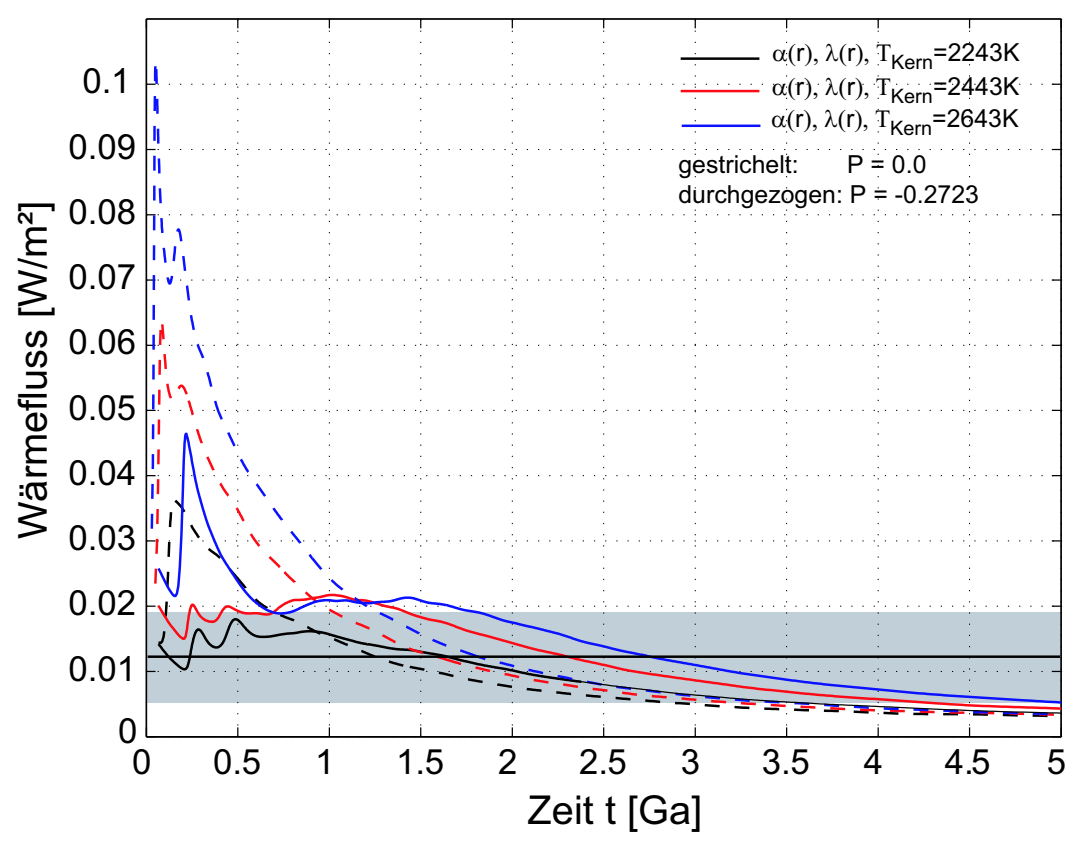

Abbildung 5.49: Entwicklung des Wärmeflusses an der KMG bei unterschiedlicher Starttemperatur des Kerns. Der grau unterlegte Bereich kennzeichnet den Wertebereich des Wärmeflusses entlang der Adiabate im Marskern (Nimmo und Stevenson 2000). Die schwarze Linie zeigt zur Orientierung den Mittelwert dieses Bereichs. 


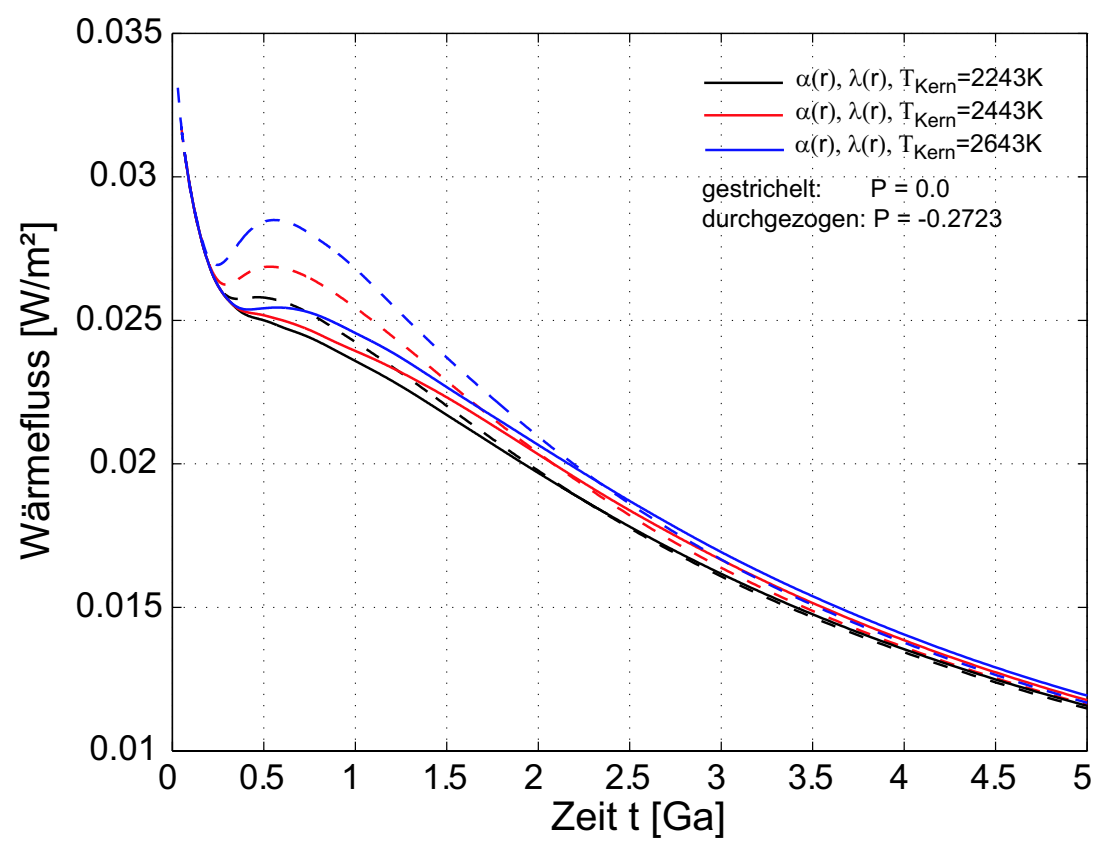

Abbildung 5.50: Entwicklung des Wärmeflusses an der Oberfläche bei unterschiedlicher Starttemperatur des Kerns.

Erhöhen der Starttemperatur an der KMG macht sich insbesondere ohne Phasengrenze zu Beginn der Evolution durch eine dünnere Lithosphäre bemerkbar. Etwa zeitgleich zur dünneren Lithosphäre ist der Wärmefluss an der Oberfläche (Abbilung (5.50)) erhöht. Es besteht eine zeitliche Verzögerung zwischen dem Peak im Wärmefluss an der KMG und dem Ausdünnen der Lithosphäre bzw. dem hohen Wärmefluss an der Oberfläche, da die Wärme durch den Mantel und die schon vorhandene Lithosphäre (hier nur durch Diffusion) transportiert werden muss. Dies führt darüber hinaus dazu, dass das Wärmesignal an der Oberfläche glatter ist. Der Oberflächenwärmefluss liegt nach 4.5 Ga zwischen $12.4 \mathrm{~mW} / \mathrm{m}^{2}$ (Refenzfall ohne Phasengrenze) und $12.9 \mathrm{~mW} / \mathrm{m}^{2}$ (Starttemperatur: $2643 \mathrm{~K}$, mit Phasengrenze).

An der KMG liegt der Wärmefluss bei allen Rechnungen mit erhöhter Starttemperatur zeitweilig über dem oberen Schwellenwert für den adiabatischen Wärmefluss. Ohne Phasengrenze ist dies für 1.0 bzw. 1.3 Ga bei (Starttemperatur: $2643 \mathrm{~K}$ ) der Fall. Mit Phasengrenze und einer Starttemperatur von $2443 \mathrm{~K}$ wird dieser Wert im Zeitintervall zwischen 0.7 und $1.4 \mathrm{Ga}$ überschritten. Ähnlich wie im Referenzfall bildet sich kein scharfes Maximum aus, dass eine kurze Lebenszeit des Dynamos erlaubt. Dies ist jedoch der Fall für den anfänglich sehr heißen Kern (2643 K). Hier liegt der Wärmefluss für 0.7 Ga oberhalb des oberen Schwellenwertes. Allerdings ist bei der Betrachtung des adiabatischen Wärmeflusses hier noch nicht berücksichtigt, dass der adiabatische Temperaturgradient im Kern linear von der Kerntemperatur abhängt und mit dieser ansteigt. Bei höheren Kerntemperaturen muss ein entsprechend höherer adiabatischer Wärmefluss angenommen werden. Das Erhöhen der Startkerntemperatur um 18 \% von 2243 auf 2643 $\mathrm{K}$ verschiebt den Bereich des adiabatischen Wärmeflusses zu $5.9-22.4 \mathrm{~mW} / \mathrm{m}^{2}$. Für die 
sehr hohe Kerntemperatur übersteigt der Wärmefluss zu Beginn auch diese höheren Werte. Ein aktueller thermisch getriebener Dynamo ist unwahrscheinlich.

Mit Hilfe von Akkretions- und Differentiationsmodellen ist zu prüfen, ob der angenommene große Temperaturunterschied zwischen Kern und Mantel möglich ist. Schubert und Spohn (1990) nehmen in ihren parametrisierten Evolutionsmodellen für Mars höhere Starttemperaturen von $3000 \mathrm{~K}$ für den Kern an, allerdings ist der Unterschied zur Manteltemperatur bei $2573 \mathrm{~K}$ im Bereich des hier betrachteten Referenzfalls.

Das Anheben der Kerntemperatur bzw. des Temperaturgradienten an der KMG fördert Instabilitäten in der thermischen Grenzschicht, so dass ohne die endotherme Phasengrenze zumindest bei einer sehr hohen Temperatur von $2643 \mathrm{~K}$ bei der Kombination von $\alpha(r)$ und $\lambda(r)$ wieder mehr Aufströme möglich sind. Die endotherme Phasengrenze führt in diesem Fall zu einer effizienten Reduzierung der Anzahl der Aufströme und spielt zumindest für die thermische Entwicklung an der KMG eine bedeutende Rolle. Der Einfluss der Startkerntemperatur und der Phasengrenze auf die Temperaturentwicklung im übrigen Mantel ist gering. Ein deutlicher Effekt entsteht beim Wärmefluss an der KMG, der durch den stärkeren Temperaturgradienten angehoben wird und so die Lebenszeit eines thermisch getriebenen Dynamos in der Frühphase der Entwicklung beeinflusst.

\subsection{Einfluss der Nicht-Boussinesq-Effekte}

\subsubsection{Konstante thermische Parameter}

In den bisherigen Simulationen wurde die Boussinesq-Näherung verwendet, in deren Rahmen man Temperatureffekte durch viskose Reibung, adiabatische Kompression und Dekompression sowie durch latente Wärme im Zusammenhang mit den Phasenübergängen vernachlässigt. Diese Temperatureinflüsse werden in diesem Kapitel in der erweiterten ("extended") Boussinesq-Näherung berücksichtigt. Dazu wird in den Gleichungen (3.40) - (3.42) die Dissipationszahl für Mars von $D i=0.1445$ (anstelle von $D i=0.0 \mathrm{im}$ Boussinesq-Fall) verwendet. Der thermische Ausdehnungskoeffizient $\alpha$ sowie die thermische Leitfähigkeit $\lambda$ sind zunächst über den Mantel konstant. Es werden Modelle ohne Phasengrenzen, mit der endothermen Phasengrenze, mit den zwei exothermen Phasengrenzen und Modelle mit allen drei Phasengrenzen untersucht. Insbesondere stellt sich die Frage, wieweit die Effekte der latenten Wärme das Konvektionsmuster beeinflussen. Reduziert die endotherme Phasengrenze die Anzahl der Plumes weiterhin effektiv, wenn die freiwerdende latente Wärme das Aufsteigen des warmen Materials fördert? Kann die latente Wärme, die beim Durchgang der Plumes durch exotherme Phasengrenzen aufgenommen werden muss, die Anzahl der Aufströme reduzieren?

Um eine Vergleichbarkeit zwischen den Simulationen in der Boussinesq- und in der erweiterten Boussinesq-Näherung zu erzielen, muss das bisherige Temperaturstartprofil durch Berücksichtigung der adiabatischen Temperaturzunahme sowie ggf. der latenten Wärme der Phasenübergänge angepasst werden. Dies geschieht, indem unterhalb der oberflächennahen thermischen Grenzschicht $\left(r_{1}\right)$, die Temperaturänderung entlang der Adiabate auf das vorher verwendete Temperaturprofil aufaddiert wird. Die Temperaturbeitrag der Adiabate $\Delta T_{\text {adiabat }}(r)$ wird durch das Integral über den adiabatischen Tempe- 
raturgradienten bestimmt (dimensionsbehaftete Größen):

$$
\Delta T_{\text {adiabat }}(r)=\int_{r_{1}}^{r} \frac{\alpha_{\text {eff }} g T\left(r^{\prime}\right)}{C_{p}} d r^{\prime}
$$

Die Verwendung von $\alpha_{\text {eff }}$ berücksichtigt auch den Beitrag der latenten Wärme der Phasengrenzen. Für eine mittlere Manteltemperatur von $1751 \mathrm{~K}$ im Startprofil hat der adiabatische Temperaturgradient im Mars einen Wert von $0.124 \mathrm{~K} / \mathrm{km}$.

Wird das Temperaturprofil nicht angepasst, so ist die Startsituation im extended Boussi-

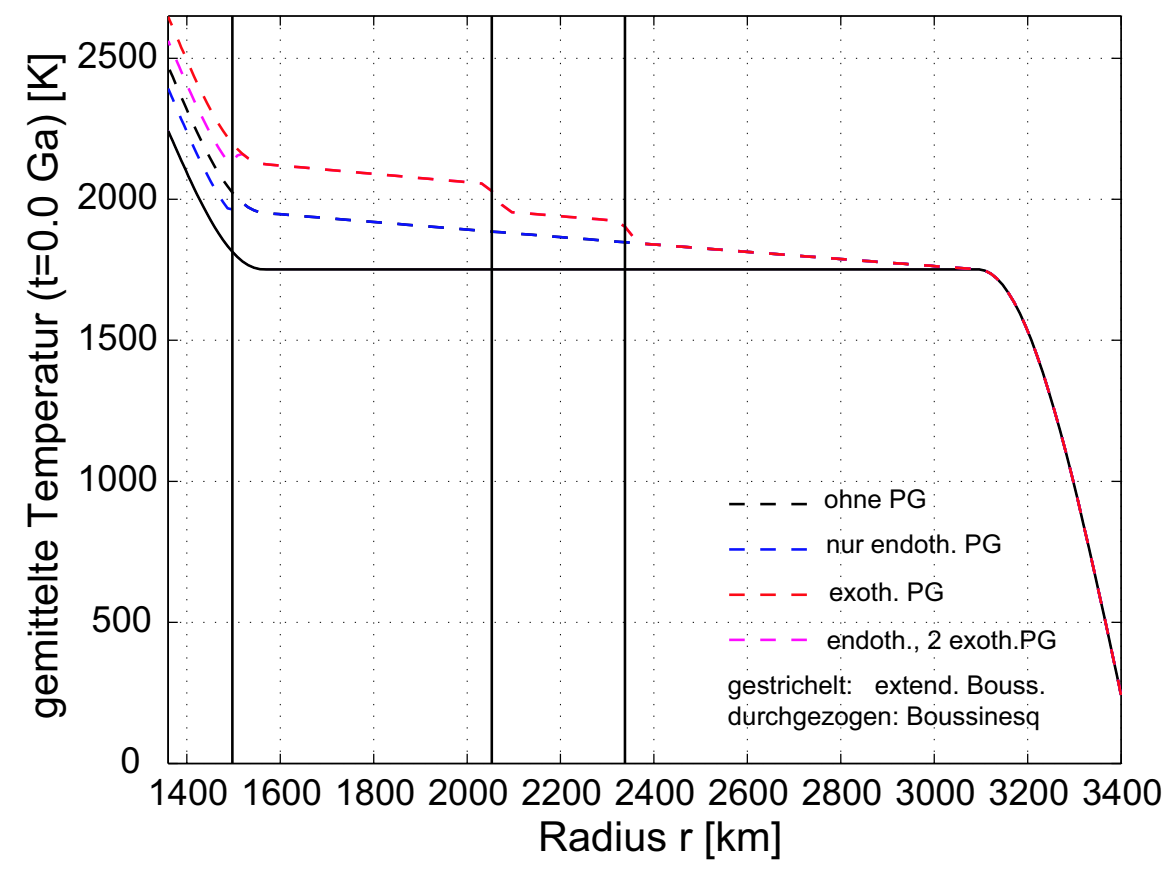

Abbildung 5.51: Anpassung der Starttemperaturprofile in der erweiterten BoussinesqNäherung. Die senkrechten, schwarzen Linien markieren die Positionen der einzelnen Phasengrenzen.

nesq-Fall unteradiabatisch, so dass die Konvektion deutlich später einsetzt, nachdem sich das System durch die vorhandenen internen Wärmequellen und den Wärmefluss aus dem Kern aufgeheizt hat. Die Anpassung des Temperaturprofils führt dazu, dass der anfängliche Temperaturkontrast $\Delta T$ zwischen KMG und Oberfläche verändert wird (vgl. Tabelle (5.1)). Dies ist bei der Skalierung bzw. Entdimensionalisierung zu beachten. Die angepassten Starttemperaturprofile sind in Abbildung (5.51) dargestellt. Die Profile für die Modelle mit endothermer Phasengrenze zeigen einen Temperatursprung durch die latente Wärme im Übergang zur bzw. in der thermischen Grenzschicht. Wie sich später zeigt, ist der Einfluss der latenten Wärme an dieser Stelle gering, so dass sie bei der Anpassung vernachlässigt werden könnte.

Die veränderten Temperaturprofile führen mit den bisher verwendeten Viskositätsparametern zu niedrigeren Viskositäten, die die thermische Entwicklung des Planeten und das Konvektionsmuster beeinflussen. Durch diesen Einfluss auf die Viskosität ist der direkte 


\begin{tabular}{|l|c|c|c|c|}
\hline & Rayleigzahl & $\Delta T[\mathrm{~K}]$ & $E^{*}[\mathrm{~kJ} / \mathrm{mol}]$ & $V^{*}\left[\mathrm{~cm}^{3} / \mathrm{mol}\right]$ \\
\hline Boussinesq & $8.93 \cdot 10^{6}$ & 2000 & 187.5 & 3.0 \\
\hline extended Boussinesq & & & & \\
\hline ohne Phasengrenzen (PG) & $9.95 \cdot 10^{6}$ & 2229 & 187.5 & 4.53 \\
\hline endotherme PG & $9.60 \cdot 10^{6}$ & 2150 & 187.5 & 4.53 \\
\hline zwei exotherme PG & $10.74 \cdot 10^{6}$ & 2405 & 187.5 & 6.31 \\
\hline endo- und exotherme PG & $10.35 \cdot 10^{6}$ & 2319 & 187.5 & 6.31 \\
\hline
\end{tabular}

Tabelle 5.1: Anpassung der Modellparameter in der erweiterten Boussinesq-Näherung. Die Rayleighzahl gilt mit der lokalen Viskosität von $6.65 \cdot 10^{20} \mathrm{~Pa}$ s bei $t=0.0 \mathrm{Ga}$ und $r=2318 \mathrm{~km}$. Der Temperaturkontrast ist für $t=0.0 \mathrm{Ga}$ angegeben.

Beitrag der Nicht-Boussinesq-Effekte schwer zu erkennen. Eine Anpassung der Viskositätsparameter in der erweiterten Boussinesq-Näherung versucht, diese Viskositätseffekte zu minimieren, damit die Nicht-Boussinesq-Effekte klarer hervortreten. Die Viskositäts-

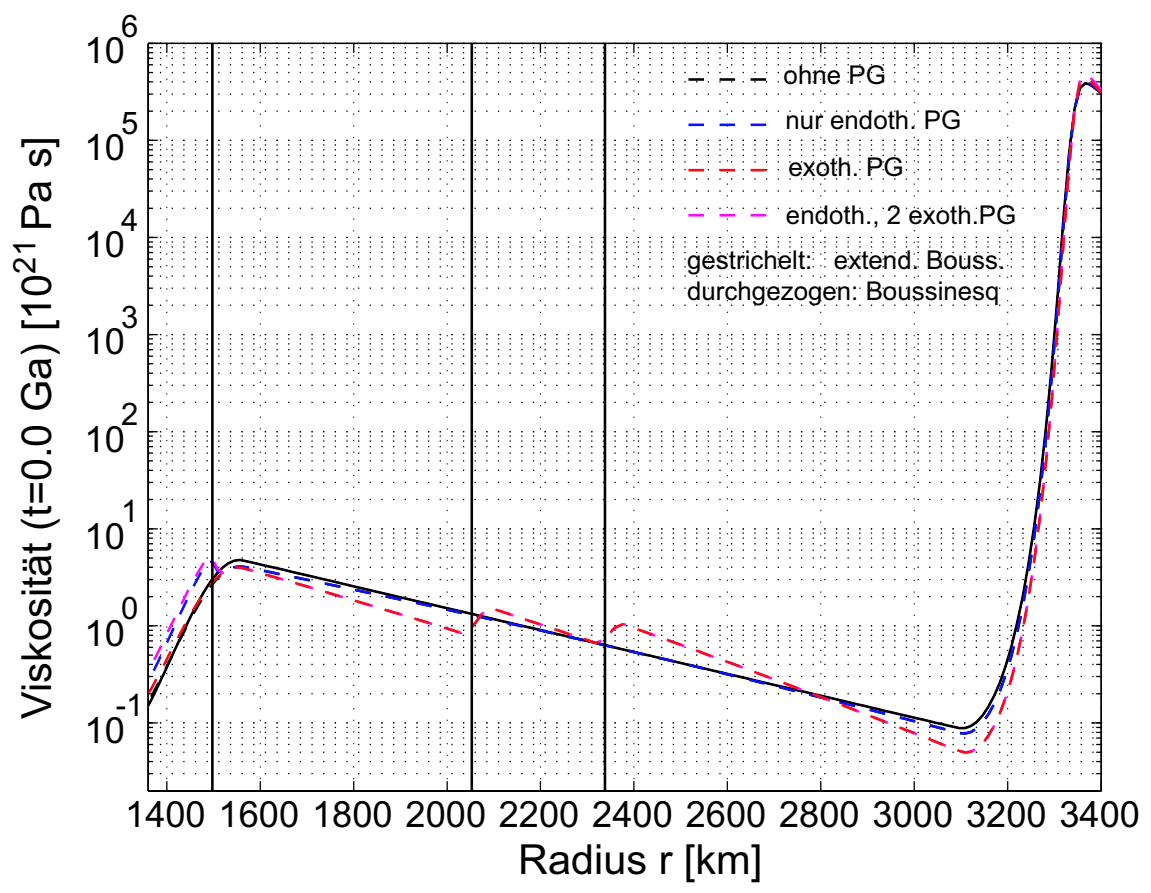

Abbildung 5.52: Anpassung der Viskositätsprofile an die veränderten Starttemperaturprofile in der erweiterten Boussinesq-Näherung. Die senkrechten, schwarzen Linien markieren die Positionen der einzelnen Phasengrenzen.

parameter in der erweiterten Boussinesq-Näherung werden so gewählt, dass die Anfangsviskositätsprofile mit den Profilen in der Boussinesq-Näherung übereinstimmen. Dazu wird das Aktivierungsvolumen verändert und die Profile werden so normiert, dass sie in der Startkonfiguration bei $r=2318 \mathrm{~km}$ übereinstimmen (der exotherme Phasenübergang zwischen $\alpha$ - und $\beta$ - Spinell macht eine Normierung in halber Tiefe schwierig). Die ver- 
wendeten Werte für das Aktivierungsvolumen sind in Tabelle (5.1) angeben. Diese Werte liegen in einem akzeptablen Bereich für Olivin (siehe Kapitel (3.5)), wenn man die Unsicherheiten in der Materialgröße und der Parametrisierung der Rheologie berücksichtigt. Abbildung (5.52) zeigt die angepassten Startviskositätsprofile.

Die Temperaturprofile nehmen durch die latente Wärme der Phasengrenzen einen komplexen Verlauf an, so dass die Anpassung des Viskositätsprofils nicht perfekt erfolgen kann. Die Viskositätsänderung durch den Temperatursprung in der Nähe der KMG durch die latente Wärme kann nicht kompensiert werden, so dass in der erweiterten BoussinesqNäherung in diesem Bereich höhere Viskositäten auftreten als im Startprofil der Boussinesq-Näherung. Für die Simulationen, die die exothermen Phasengrenzen berücksichtigen, hat sich bei Versuchen mit verschiedenen Aktivierungsvolumina der angegebene Wert als am besten geeignet herausgestellt. Die Viskositätseffekte können durch die Anpassung der Parameter lediglich minimiert und nicht komplett eliminiert werden. Insbesondere in den Simulationen mit den exothermen Phasengrenzen sind Einflüsse durch die Viskositätseffekte zu erwarten, da hier trotz Anpassung große Viskositätsunterschiede verbleiben. Auch die höheren Viskositäten in der Nähe der KMG in Zusammenhang mit der endothermen Phasengrenze können zu Unterschieden in der Entwicklung des Boussinesq- und des erweiterten Boussinesq-Modells führen, ohne direkt einen NichtBoussinesq-Effekt darzustellen.

In Abbildung (5.53) sind die Konvektionsmuster für die Modelle mit Berücksichtigung verschiedener Phasengrenzen im Boussinesq- und im erweiterten Boussinesq-Fall gegenüber gestellt. Bei den Simulationen in der linken Spalte ist die endotherme Phasengrenze in der Nähe der KMG vernachlässigt, während die Modelle in der rechten Spalte diesen Phasenübergang berücksichtigen. Im Fall ohne Phasengrenzen gibt es keine signifikanten Unterschiede im Konvektionsmuster zwischen der Boussinesq- und der erweiterten Boussinesq-Näherung. Beide Ergebnisse zeigen sechs Aufströme, die weitestgehend durch Abströme voneinander getrennt sind. Im erweiterten Boussinesq-Fall sind lediglich an zwei weiteren Stellen die trennenden Abstrombänder aufgebrochen. Die Positionen und die Geschwindigkeitsamplituden stimmen in beiden Näherungen gut überein und auch in der Entwicklung zu diesem Konvektionsmuster gibt es keine deutlichen Unterschiede. Ähnliches gilt für die Modelle, die nur die kernnahe endotherme Phasengrenze berücksichtigen. Sowohl in der Boussinesq- als auch in der erweiterten Boussinesq-Näherung dominieren zwei konzentrierte Aufströme das Konvektionsmuster. Bei Berücksichtigung der Nicht-Boussinesq-Effekte ist die Amplitude der Geschwindigkeit etwas höher und die Positionen der Aufströme sind so verändert, dass sie jetzt antisymmetrisch zum Äquator und symmetrisch zum Nullmeridian liegen.

Werden nur die exothermen Phasenübergänge in die Simulation einbezogen, so sind die Unterschiede zwischen den beiden betrachteten Näherungen etwas größer. Im Boussinesq-Fall entsteht, sehr ähnlich zum Modell ohne Phasengrenzen, ein Muster mit sechs Aufströmen, deren Positionen auf der Westhalbkugel etwas gegenüber der Situation ohne Phasengrenzen verschoben sind. Die konvektionsfördernden exothermen Phasengrenzen rufen deutlich höhere Geschwindigkeiten hervor. Im erweiterten Boussinesq-Fall ist die Anzahl der Aufströme geringer und es stabilisiert sich ein Konvektionsmuster mit lediglich fünf Plumes, von denen einer deutlich schwächer ist als die übrigen. Die Geschwindigkeiten sind gegenüber dem Boussinesq-Fall bedeutend geringer. Allerdings werden in beiden Näherungen etwa $1.5 \mathrm{Ga}$ benötigt, um diese Muster zu etablieren, die danach 
a) Boussinesq-Näherung
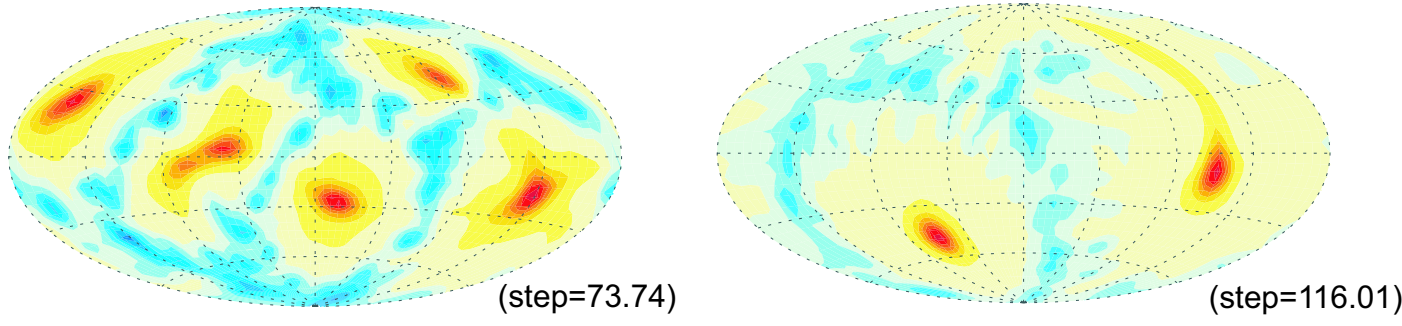

b) Erweiterte Boussinesq-Näherung
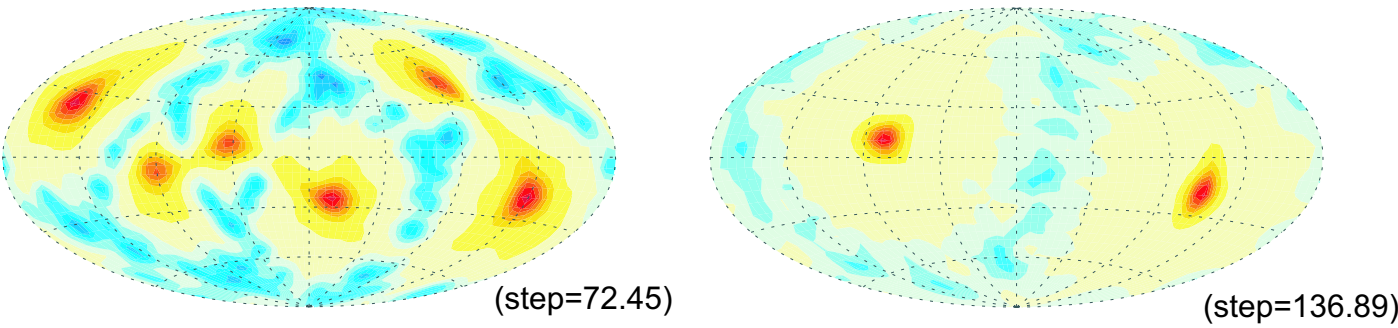

c) Boussinesq-Näherung, zwei exotherme Phasengrenzen
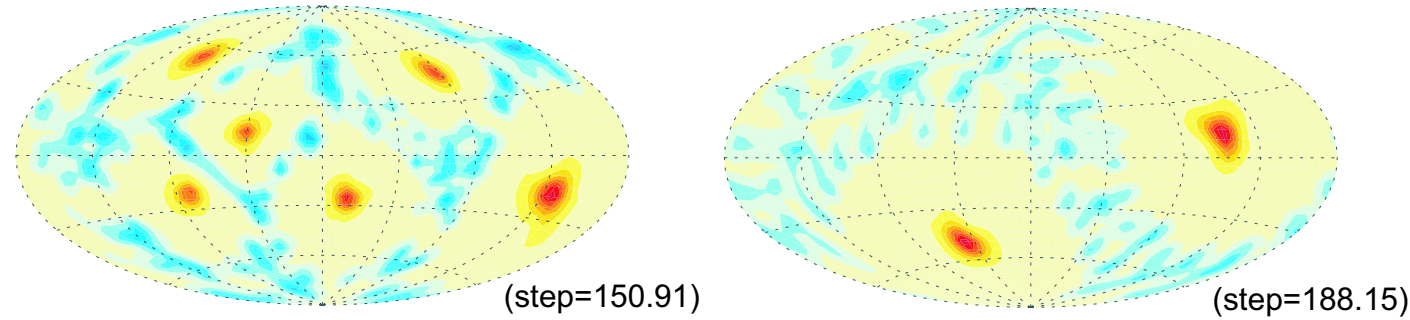

d) Erweiterte Boussinesq-Näherung, zwei exotherme Phasengrenzen
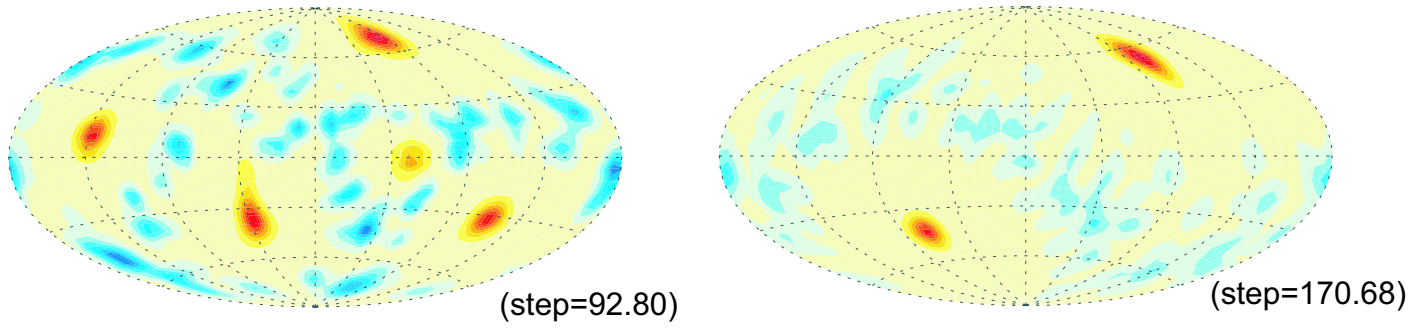

Abbildung 5.53: Vergleich der Konvektionsmuster nach $4.5 \mathrm{Ga}$ im Boussinesq-Fall und im erweiterten Boussinesq-Fall: Radialgeschwindigkeit in halber Tiefe (rot: Aufströme, blau: Abströme). Die linke Spalte zeigt die Situation ohne die kernnahe endotherme Phasengrenze, in der rechten Spalte wird die kernnahe endotherme Phasengrenze berücksichtigt. Der Contourstep der Geschwindigkeit ist angegeben: step=100 entspricht $0.14 \mathrm{~cm} / \mathrm{a}$. 
nur in der Amplitude abnehmen. Insbesondere der schwächere Aufstrom im erweiterten Boussinesq-Fall hat auch zu früheren Zeitpunkten der Evolution $(\mathrm{ab} \sim 1.0 \mathrm{Ga}$ ) eine geringere Aufstromgeschwindigkeit. Der Vergleich der beiden Näherungen bei Berücksichtigung aller drei Phasengrenzen zeigt, dass der Einfluss auf das Konvektionsmuster hier gering ist. In beiden Fällen entstehen zwei Aufströme, wobei in der erweiterten Boussinesq-Näherung erneut die Symmetrie zum Äquator bei gleichzeitiger Antisymmetrie zum Nullmeridian deutlich wird. Im Boussinesq-Fall dauert es 1 - $1.5 \mathrm{Ga}$, dieses Muster zu stabilisieren, während in der erweiterten Näherung 2.5 - 3.0 Ga vergehen. Die Geschwindigkeitsamplitude im erweiterten Boussinesq-Fall ist geringfügig reduziert gegenüber der einfachen Boussinesq-Näherung, aber in beiden Fällen deutlich höher als im Fall, der die exothermen Phasengrenzen vernachlässigt.

Die Simulationen ohne die exothermen Phasengrenzen zeigen, dass der Einfluss der NichtBoussinesq-Effekte auf das Konvektionsmuster nach 4.5 Ga gering ist. Ohne Phasengrenzen kompensiert die Viskositätsanpassung den Effekt der adiabatischen Temperaturzunahme weitgehend. Latente Wärme tritt in diesem Fall nicht auf und die Temperaturerhöhung durch viskose Reibung ist vernachlässigbar klein (siehe weiter unten), so dass sie sich nicht auf das Konvektionsmuster auswirkt. Auch bei Berücksichtigung der endothermen Phasengrenze kann die latente Wärme das Konvektionsmuster nicht verändern. Beim Durchgang eines Plumes durch eine endotherme Phasengrenze wird im Prinzip latente Wärme frei, die den Plume erwärmt und sein Aufsteigen fördert. Gleichzeitig wird das Aufwölben der Phasengrenze, das die Aufwärtsbewegung behindert, in dem durch die latente Wärme heißeren Plume verstärkt (vgl. Kapitel (2.4)). Somit steht nur ein Teil der latenten Wärme, der durch den Faktor $(1+P d \Gamma / d \pi)$ (endothermer Phasenübergang: $P<0$ ) im Auftriebsterm (Gleichung (3.41)) bestimmt wird, als zusätzlicher Auftrieb zur Verfügung. Die Simulationen zeigen, dass dieser auftriebsfördernde Beitrag der latenten Wärme des kernnahen endothermen Phasenübergangs zu schwach ist, um die Anzahl der Aufströme nach $4.5 \mathrm{Ga}$ zu erhöhen bzw. das Konvektionsmuster signifikant zu verändern. In den Temperaturprofilen nach 4.5 Ga (Abbildung (5.55)) ist kein Temperaturbeitrag der latenten Wärme dieses Übergangs in der Nähe der thermischen Grenzschicht erkennbar, wodurch der schwache Effekt der latenten Wärme in dieser Tiefe unterstrichen wird. Die latente Wärme kann nur dann eine Rolle spielen, wenn die Temperaturverteilung direkt durch die Konvektionsbewegung bestimmt wird und sich signifikante Materialmengen durch die Phasengrenze bewegen. In der Nähe der thermischen Grenzschicht sind die Radialgeschwindigkeiten und der damit verbundene Massenfluss jedoch sehr gering.

Durchstößt ein Plume exotherme Phasengrenzen, so wird beim Phasenübergang latente Wärme aufgenommen, so dass der Plume abkühlt, einen Teil seines Auftriebs verliert und die die Aufwärtsbewegung unterstützende Wölbung der Phasengrenze reduziert wird. Die Simulationen zeigen, dass die latente Wärme (bei Vernachlässigung der endothermen Phasegrenze) die Anzahl der Aufströme durch diesen Mechanismus reduzieren kann. Die Reduktion der Aufströme von sechs auf fünf durch die latente Wärme ist allerdings weniger effektiv als die Reduktion durch das Aufwölben der endothermen Phasengrenze, die ein Muster mit zwei Aufströmen ermöglicht.

Der Einfluss der latenten Wärme der exothermen Phasengrenzen ist in diesen Simulationen größer als der der endothermen Phasengrenze, da einerseits in mittleren Manteltiefen die Massenflussdichte höher ist als in der Tiefe der kernnahen endothermen Phasengrenze und zusätzlich, zumindest für den Übergang zwischen $\beta$ - und $\gamma$ - Spinell der Phasenpa- 
rameter größer ist. Hinzu kommt, dass die latente Wärme der exothermen Phasenübergänge auch die Wölbung der Phasengrenze so schwächt, dass zwei Wirkungsweisen den Aufstieg eines Plumes hemmen. Durch den positiven Phasenparameter im Auftriebsterm verstärkt die Phasengrenze den Einfluss der latenten Wärme auf den Auftrieb.

Die in Abbildung (5.53) beobachtete, geringere Geschwindigkeitsamplitude im Vergleich zum Boussinesq-Fall kann durch den konvektionshemmenden Effekt der latenten Wärme der exothermen Phasengrenzen beeinflusst werden. Stärker ist jedoch hier der Effekt der Viskosität. Die beschriebene Anpassung der Viskositätsparameter ist in Simulationen mit den exothermen Phasengrenzen nur unzureichend möglich, so dass sich nach $4.5 \mathrm{Ga}$ starke Unterschiede herausbilden (Abbildung (5.58)). Ein Einfluss auf das Konvektionsmuster kann nicht ausgeschlossen werden.

Der Unterschied durch die verschiedenen Näherungen bei der Berücksichtigung der drei Phasengrenzen ist gering. Die Anzahl der vorhandenen starken Plumes kann auch durch die latente Wärme der exothermen Phasengrenzen nicht beeinflusst werden. Auch hier kann sich das beschriebene Problem bei der Anpassung der Viskositätsparameter auf die Geschwindigkeitsamplitude und die Zeitspanne bis zum Erreichen des stabilen Konvektionsmusters auswirken.

Die Temperaturstruktur der Aufströme nach 4.5 Ga ist in Abbildung (5.54) durch die

a) ohne Phasengrenzen
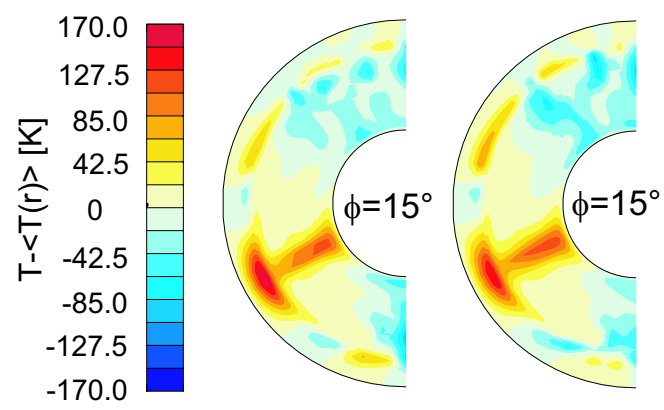

c) zwei exotherme Phasengrenzen
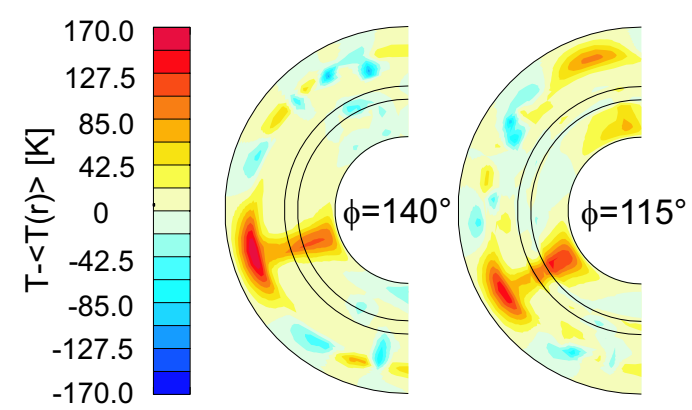

d) endo- und exotherme Phasengrenzen
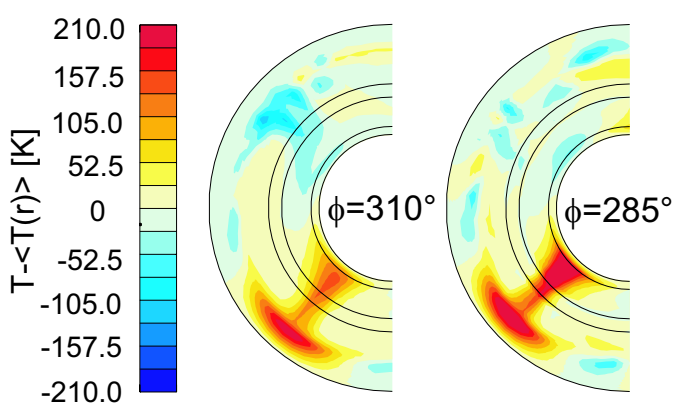

Abbildung 5.54: Vergleich der Temperaturanomalie $(T-<T(r)>)[\mathrm{K}]$ der Aufströme nach 4.5 Ga im Boussinesq-Fall und im erweiterten Boussinesq-Fall. Für die verschiedenen Kombinationen der Phasengrenzen zeigt der linke Schnitt jeweils den Boussinesq-Fall und der rechte Schnitt den erweiterten Boussinesq-Fall.

Temperaturanomalie $(T-<T(r)>)$ dargestellt. In jeder Teilabbildung zeigt der linke 
Schnitt die Situation im Boussinesq-Fall und der rechte Teil das Ergebnis der erweiterten Boussinesq-Näherung. Die Lage der Schnitte ist durch den angegebenen Längengrad $\phi$ charakterisiert. Bei Betrachtung der Temperaturanomalie $(T-<T(r)>)$ sind starke Effekte der Manteladiabate und der latenten Wärme im erweiterten Boussinesq-Fall subtrahiert worden, so dass die Temperaturanomalie in beiden Näherungen vergleichbarer sind. Wie schon beim Konvektionsmuster zeigen sich auch in den Temperaturanomalien der Plumes kaum Unterschiede zwischen den beiden Näherungen, wenn alle Phasengrenzen vernachlässigt werden. Bei der Simulation mit nur einer endothermen Phasengrenze sind die Temperaturanomalien größer als im Fall ohne Phasengrenzen. Die Erweiterung der Boussinesq-Näherung ändert die Plumeform nicht signifikant. Allerdings ist die Temperaturanomalie im erweiterten Boussinesq-Fall im Plumeinneren etwas größer. Im warmen Aufstrom ist der Beitrag der adiabatischen Kompression bzw. Dekompression (Gleichung (3.42)) größer als im mittleren Temperaturprofil, das subtrahiert worden ist. Dieser Effekt führt zu den höheren Temperaturen. Da die Temperaturen bei den Simulationen mit endothermer Phasengrenze höher sind als im Fall ohne Phasengrenzen, ist der Beitrag hier größer. Zusätzliche kleinere Einflüsse durch latente Wärme bzw. die nicht optimale Kompensation der Viskositätseffekte sind nicht auszuschließen.

Bei den Modellen mit zwei exothermen Phasengrenzen ändert sich die Amplitude und Form der Temperaturanomalie nicht signifikant gegenüber dem Fall ohne Phasengrenzen. In der erweiterten Näherung wird der Plume beim Durchgang durch die exothermen Phasengrenzen eingeschnürt bzw. wird insbesondere beim Durchgang durch die Grenze zwischen $\beta$ - und $\gamma$ - Spinell dünner und die positive Temperaturanomalie schwächt sich durch die aufzubringende latente Wärme ab. In der Tiefe des Phasenübergangs bildet sich auch in einiger Entfernung vom Aufstrom eine schwache, negative Anomalie aus, die dadurch entsteht, dass um den Plume herum Material aufsteigt (Abbildung (5.53)) und ebenfalls latente Wärme aufnehmen muss. Der Effekt der latenten Wärme trägt zur Temperaturanomalie des Plumes bei, obwohl das gemittelte Temperaturprofil subtrahiert worden ist, da die Beiträge der latenten Wärme, wie die adiabatischen Temperatureffekte im heißen Plume größer sind als im Mittel (Gleichung (3.42)).

Bei der Kombination von endothermen und exothermen Phasenübergängen in der erweiterten Boussinesq-Näherung sind erneut das Einschnüren in der Tiefe der exothermen Phasengrenzen und die etwas höheren Temperaturen im Plumeinneren sichtbar, die ebenfalls in der Simulation mit der endothermen Phasengrenze aufgetreten sind. Auch hier wird die Geometrie des Aufstroms durch die erweiterte Boussinesq-Näherung nicht verändert.

In Abbildung (5.55) sind die Profile der gemittelten Temperatur nach 4.5 Ga dargestellt. Der Temperaturunterschied zwischen den Simulationen in der Boussinesq- und erweiterten Boussinesq-Näherung wird durch die adiabatische Temperaturzunahme und die latente Wärme der exothermen Phasengrenzen bestimmt. Dies wird deutlich, wenn in den Temperaturprofilen der erweiterten Boussinesq-Näherung die Manteladiabate subtrahiert wird. In den Abbildungen (5.56) und (5.57) ist dies beispielhaft für die Simulationen ohne Phasengrenzen und die Modelle mit drei Phasengrenzen gezeigt. Abbildung (5.56) zeigt schwarz gestrichelt das Temperaturprofil nach $4.5 \mathrm{Ga}$ in der erweiterten Näherung im Fall ohne Phasengrenzen und mit der durchgezogenen schwarzen Linie das Profil zur gleichen Zeit im Boussinesq-Fall. Wird die adiabatische Temperaturänderung nach Gleichung (5.1) (rote Linie: Manteladiabate) vom Profil in der erweiterten Näherung abgezo- 


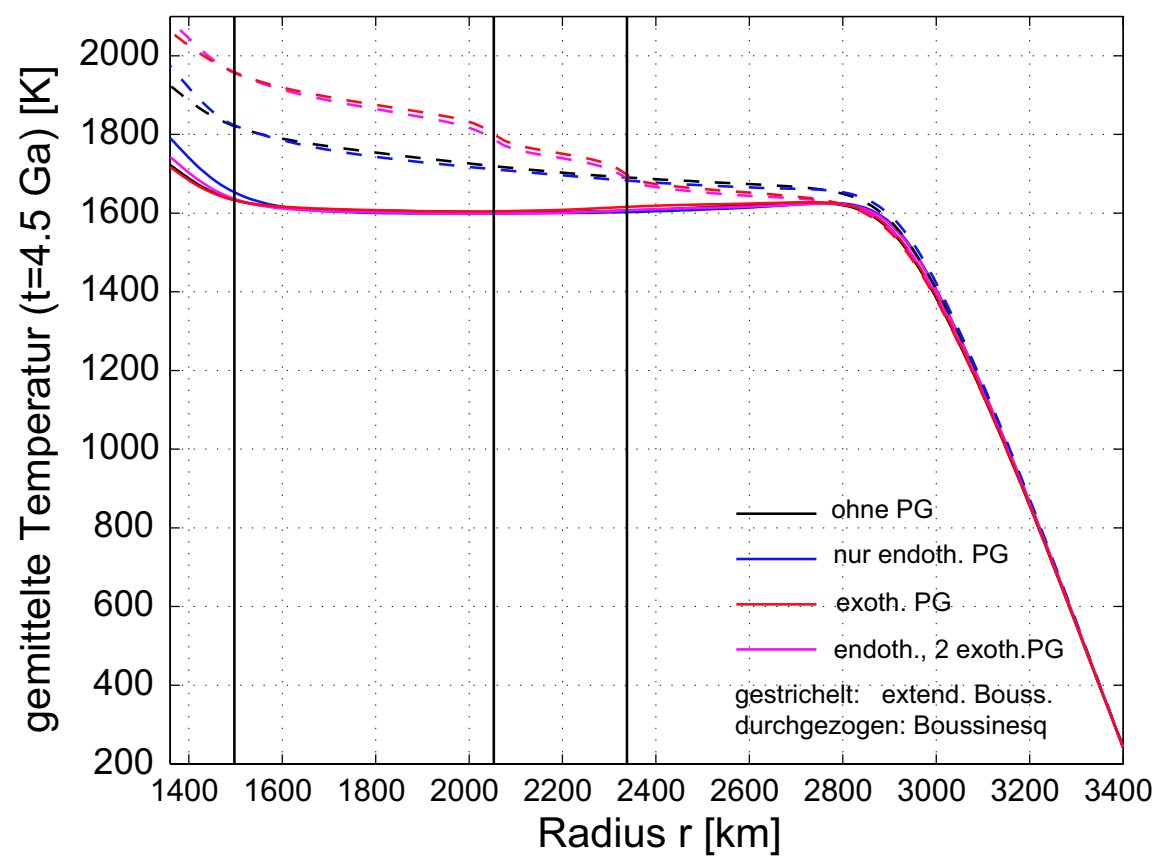

Abbildung 5.55: Profile der gemittelten Temperatur nach $4.5 \mathrm{Ga}$ in der Boussinesq- und der erweiterten Boussinesq-Näherung. Die senkrechten schwarzen Linien markieren die Tiefen der Phasengrenzen.

gen, so ergibt sich die cyanfarbene Kurve, die nahezu mit der Kurve des Boussinesq-Falls zur Deckung kommt. Die Temperaturunterschiede zwischen beiden Näherungen entstehen somit durch den adiabatischen Beitrag, die Effekte der viskosen Reibung sind vernachlässigbar. In Abbildung (5.57) ist dieselbe Vorgehensweise für die Modelle mit drei Phasengrenzen dargestellt. Auch hier stimmt das korrigierte Profil weitgehend mit dem Profil im Boussinesq-Fall überein. Allerdings ist der Temperaturanstieg durch die latente Wärme in den Tiefen der exothermen Phasengrenzen im gemittelten Profil der erweiterten Boussinesq-Näherung weniger stark und diffuser als in der theoretisch berechneten Manteladiabate, so dass sich im korrigierten Profil Minima ausbilden. Aus 'Ausschmieren' des Temperatursprungs der latenten Wärme ist durch Wärmediffusion zu erklären, die bei der Berechnung der theoretischen Manteladiabate nicht berücksichtigt wird.

In Abbildung (5.58) ist das Viskositätsprofil nach 4.5 Ga dargestellt. Obwohl die obigen Betrachtungen zeigen, dass die Temperaturunterschiede durch die Adiabate dominiert werden und die Anpassung des Aktivierungsvolumes diese Effekte kompensieren soll, zeigen sich deutlich Unterschiede in den Viskositäten, insbesondere bei den Fällen mit den exothermen Phasengrenzen. Diese Unterschiede verdeutlichen die Probleme bei der Kompensation der Viskositätseffeke und tragen u.a. zu den teilweise starken Geschwindigkeitsunterschieden, die in Abbildung (5.53) zu beobachten sind, bei.

Die Temperaturentwicklung in halber Tiefe für die verschiedenen Simulationen in beiden Näherungen ist in Abbildung (5.59) zu sehen. Die zeitliche Entwicklung wird durch die beiden Näherungen nicht signifikant beeinflusst, so dass ein nahezu konstanter Off- 


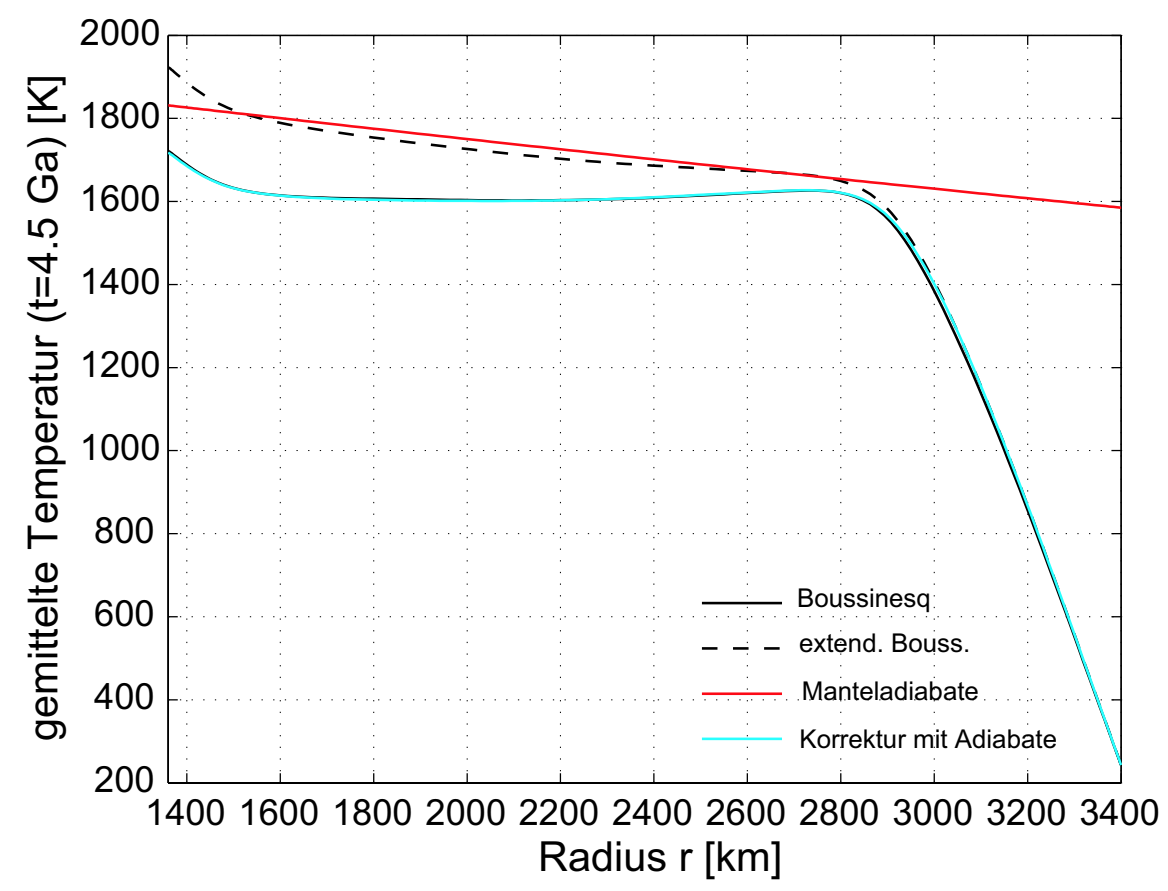

Abbildung 5.56: Kompensation der adiabatischen Temperaturzunahme im Temperaturprofil nach $4.5 \mathrm{Ga}$ in der erweiterten Boussinesq-Näherung für die Simulationen ohne Phasengrenzen.

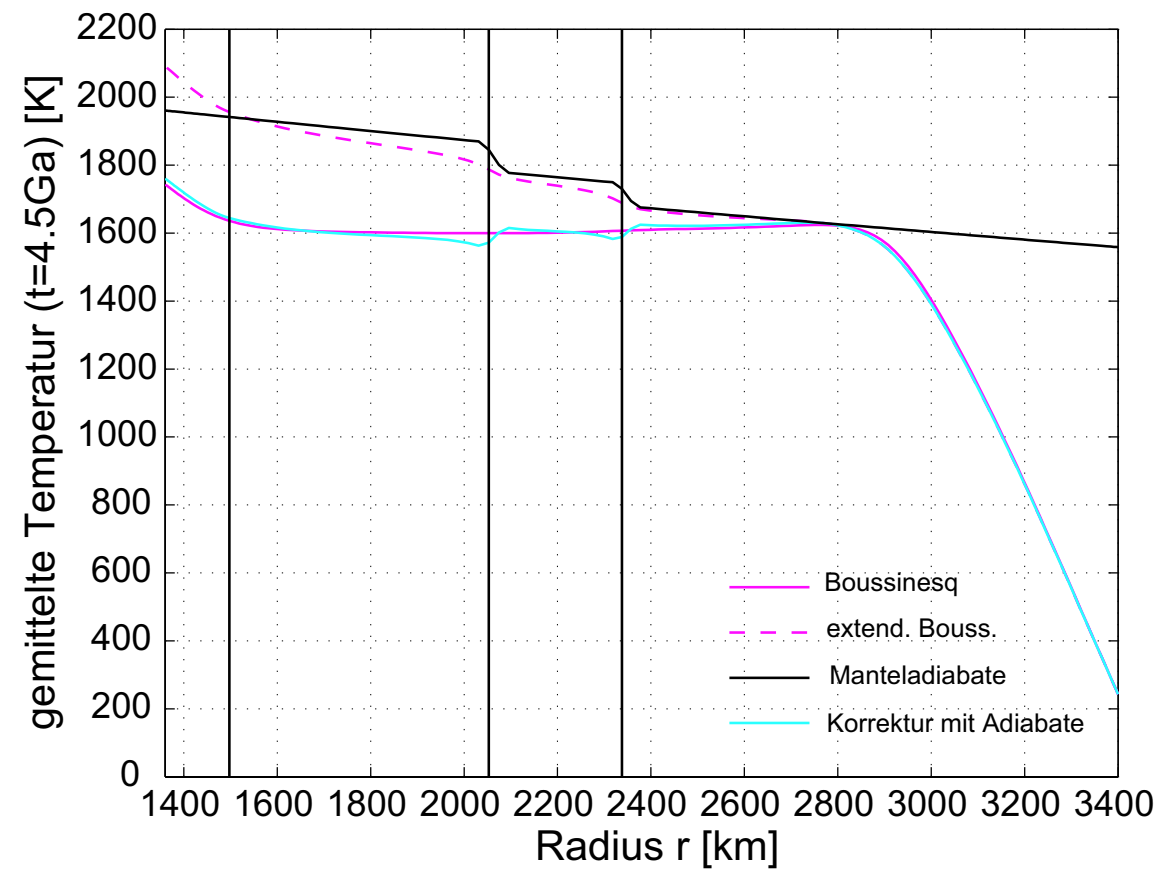

Abbildung 5.57: Kompensation der adiabatischen Temperaturzunahme im Temperaturprofil nach $4.5 \mathrm{Ga}$ in der erweiterten Boussinesq-Näherung mit drei Phasengrenzen. 


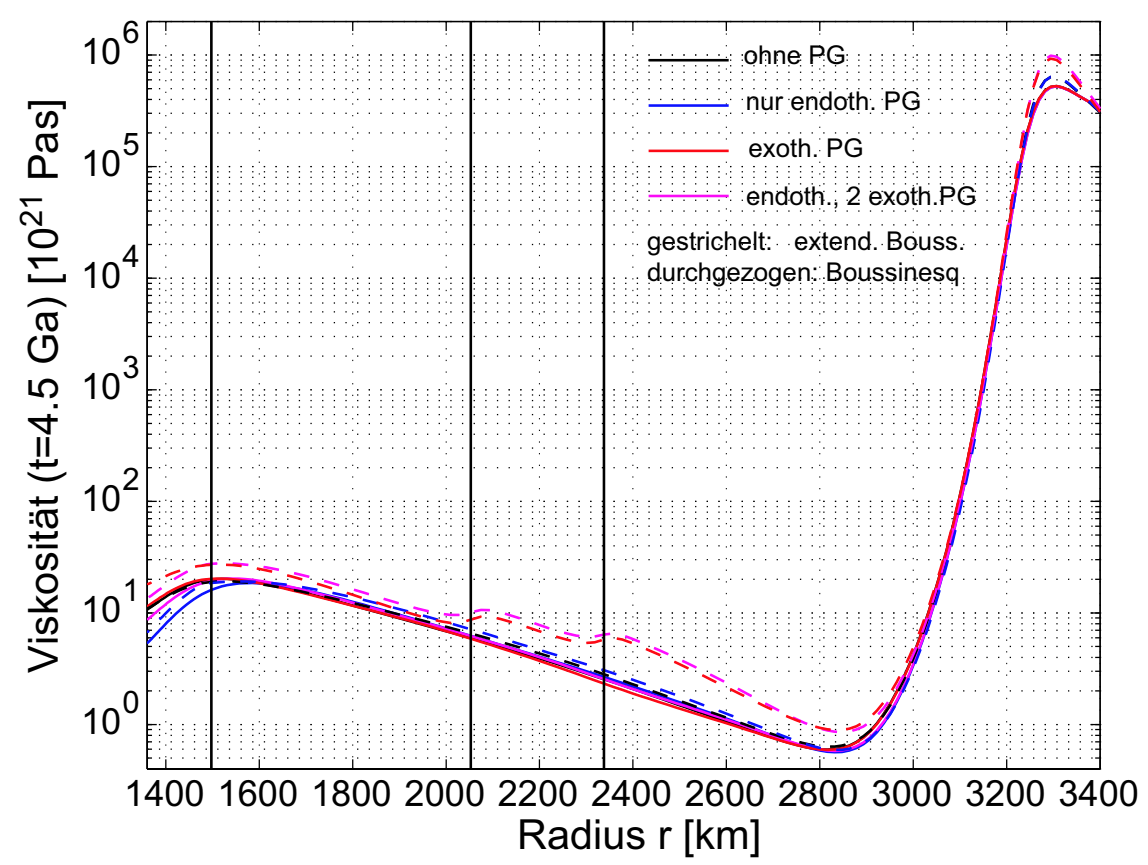

Abbildung 5.58: Viskositätsprofile nach $4.5 \mathrm{Ga}$ in der Boussinesq- und der erweiterten Boussinesq-Näherung. Die senkrechten schwarzen Linien markieren die Tiefen der Phasengrenzen.

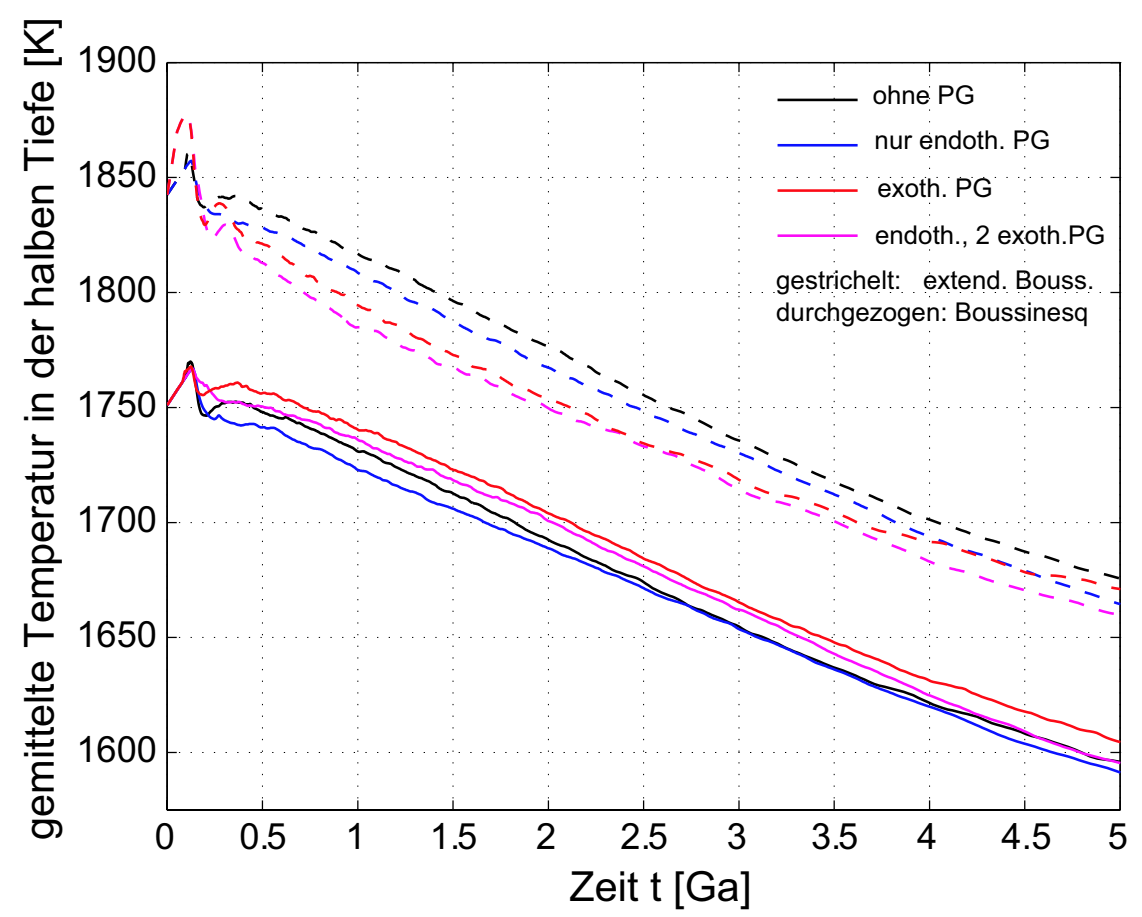

Abbildung 5.59: Einfluss der verschiedenen Näherungen auf die Entwicklung der Temperatur in halber Tiefe. 
set zwischen den entsprechenden Kurven in beiden Näherungen besteht, der durch die adiabatische Temperaturzunahme bestimmt ist. Der Temperaturoffset liegt nach $4.5 \mathrm{Ga}$ zwischen $60 \mathrm{~K}$ in den Simulationen mit drei Phasengrenzen und $80 \mathrm{~K}$ ohne Phasengrenzen.

Analog wird auch die zeitliche Entwicklung der Temperatur an der KMG (Abbildung

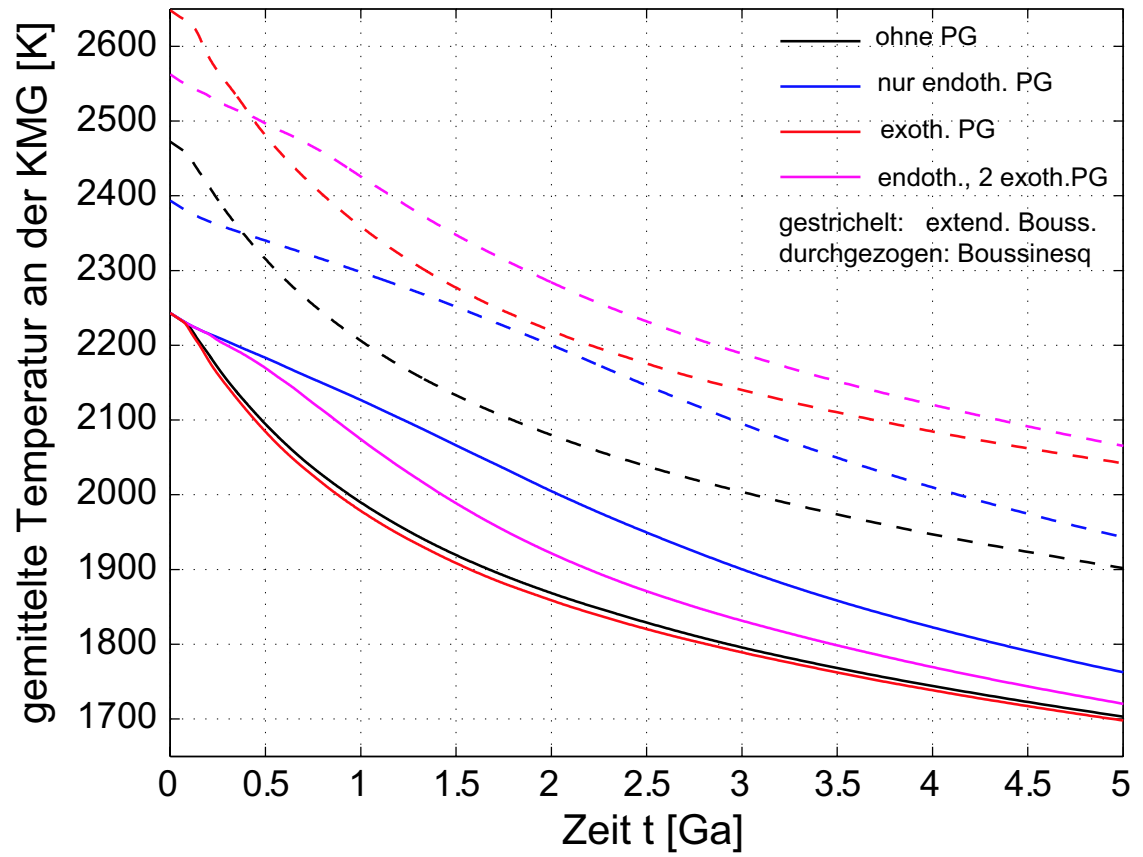

Abbildung 5.60: Einfluss der verschiedenen Näherungen auf die Entwicklung der Temperatur an der KMG.

(5.60)) durch die Erweiterung der Näherung nicht signifikant verändert. Ähnlich wie in der halben Manteltiefe verlaufen die Kurven der Simulationen mit der gleichen Kombination der Phasengrenzen in beiden Näherungen nahezu parallel. Der Offset ist weitgehend durch die unterschiedliche Kerntemperatur beim Start bzw. die Manteladiabate gegeben. In den Abbildungen (5.56) und (5.57) ergeben sich nach Subtraktion der Manteladiabate von den Temperaturprofilen der erweiterten Näherung nach $4.5 \mathrm{Ga}$ Temperaturunterschiede an der KMG von $5 \mathrm{~K}$ (ohne Phasengrenzen) bzw. $15 \mathrm{~K}$ (drei Phasengrenzen) relativ zum Boussinesq-Fall. Diese kleinen Unterschiede zeigen, dass der Offset zwischen den Temperaturen in beiden Näherungen durch die Adiabate dominiert ist. In der erweiterten Boussinesq-Näherung liegen die Kerntemperaturen nach 4.5 Ga zwischen $1923 \mathrm{~K}$ (ohne Phasengrenzen) und $2091 \mathrm{~K}$ (mit drei Phasengrenzen). Diese Temperaturen werden in Kapitel (5.6) bei der Betrachtung der Temperaturen im Kern verwendet.

In Abbildung (5.61) ist der Wärmefluss an der KMG für die verschiedenen Kombinationen der Phasengrenzen in beiden Näherungen dargestellt. Ohne Phasengrenzen stimmen die Kurven in beiden Näherungen weitgehend überein. Zu Beginn ist der Wärmefluss in der erweiterten Näherung etwas höher. Die exothermen Phasengrenzen erhöhen den Wärmefluss, wobei dies in der erweiterten Boussinesq-Näherung verstärkt der Fall ist. Bei Be- 


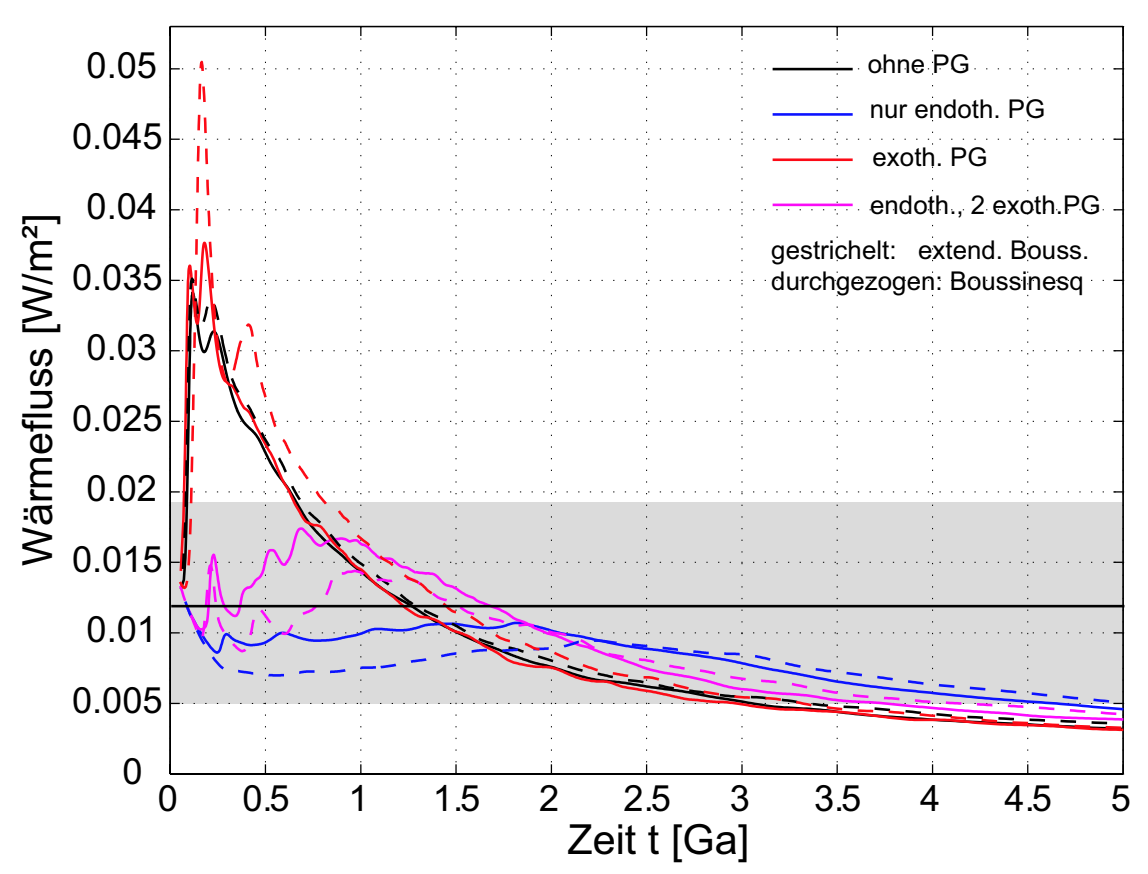

Abbildung 5.61: Einfluss der verschiedenen Näherungen auf die Entwicklung des Wärmeflusses an der KMG. Der grau unterlegte Bereich kennzeichnet den Wertebereich des Wärmeflusses entlang der Adiabate im Marskern (Nimmo und Stevenson 2000). Die schwarze Linie zeigt zur Orientierung den Mittelwert dieses Bereichs.

rücksichtigung der endothermen Phasengrenze führt die extended Boussinesq-Näherung sowohl bei nur einer Phasengrenze als auch im Fall mit drei Phasenübergängen zu einem reduzierten Wärmefluss, so dass die Wahrscheinlichkeit eines Dynamos zu Beginn der Entwicklung nur für niedrige adiabatische Wärmeflüsse gegeben ist. Der heutige Wärmefluss ist zu gering, um einen thermischen Dynamo anzutreiben. Die Unterschiede in der zeitlichen Entwicklung im Wärmefluss sind auf die unvollständige Viskositätsanpassung zurückzuführen bzw. stark von ihr beeinflusst. Die niedrigen Viskositäten im unteren Mantel, die in den Simulationen mit den exothermen Phasengrenzen auftreten, fördern den Wärmefluss aus dem Kern. Im Umkehrschluss hemmen die höheren Anfangsviskositäten im kernnahen Bereich in den Anfangsprofilen mit endothermer Phasengrenze den Wärmefluss. Die hier beobachteten Unterschiede gehen im Wesentlichen nicht auf die Nicht-Boussinesq-Effekte zurück.

Das Gleiche gilt für die Entwicklung der Lithosphärendicke, deren zeitliche Entwicklung in Abbildung (5.62) dargestellt ist. Nach 4.5 Ga liegen die Lithosphärendicken in allen Simulationen in einem engen Bereich zwischen 298 und 308 km, der Unterschied durch die verschiedenen Näherungen ist vernachlässigbar.

Insgesamt hat der Vergleich der Simulationen in der Boussinesq-Näherung und der erweiterten Boussinesq-Näherung gezeigt, dass viskose Reibung im Mars bei der kleinen Dissipationszahl keinen signifikanten Einfluss hat und Temperatureffekte im Wesentlichen durch die adiabatische Temperaturzunahme mit der Tiefe und die latente Wärme 


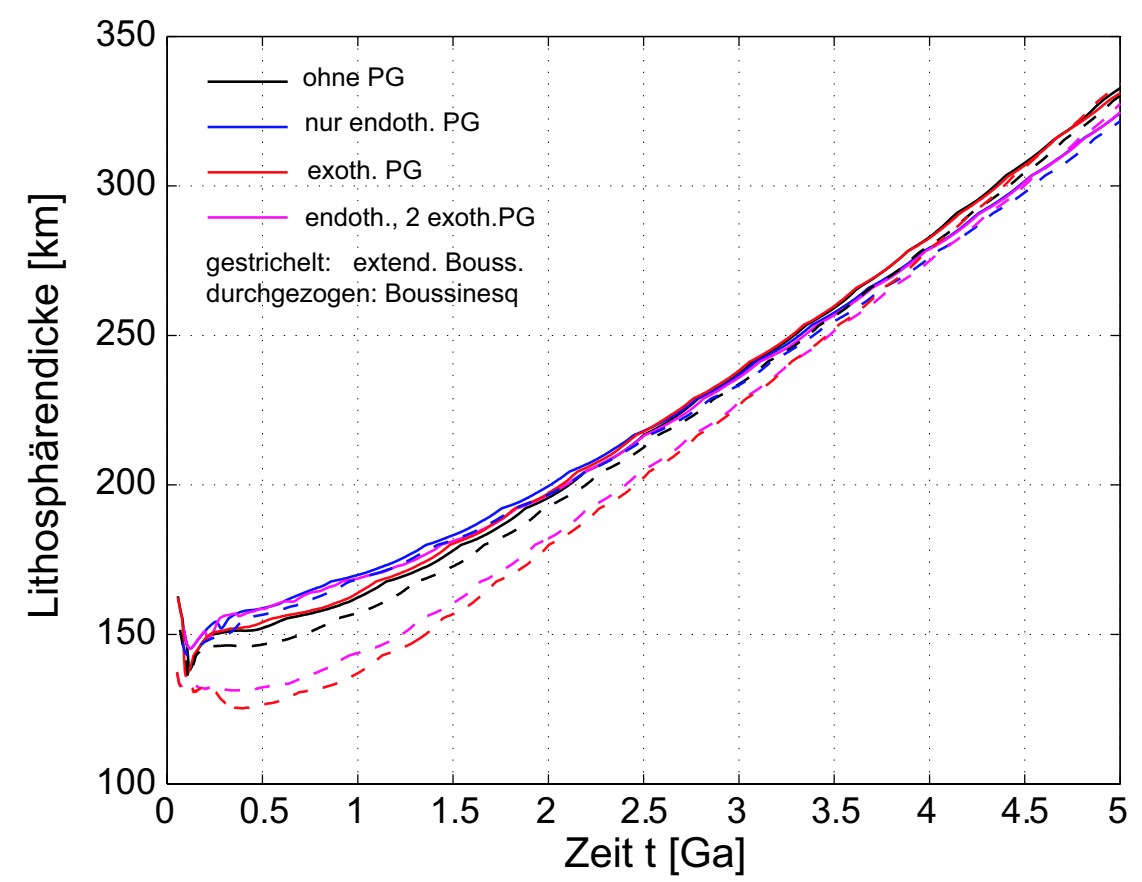

Abbildung 5.62: Einfluss der verschiedenen Näherungen auf die Entwicklung der Lithosphärendicke.

gegeben sind. Werden diese Temperatureffekte auf die Viskosität durch geeignete Wahl der Viskositätsparameter kompensiert, so sind die Unterschiede in der thermischen Entwicklung und im Konvektionsmuster bei Simulationen ohne Phasengrenzen oder mit nur einer kernnahen endothermen Phasengrenze sehr gering. Die latente Wärme des kernnahen Übergangs spielt keine signifikante Rolle. Die exothermen Phasengrenzen im mittleren Mantel haben einen deutlicheren Effekt und können bei Vernachlässigen der endothermen Phasengrenze die Anzahl der Plumes, denen die latente Wärme einen Teil ihres Auftriebs nimmt, reduzieren. Allerdings ist dieser Mechanismus weit weniger effizient als das Aufwölben der kernnahen endothermen Phasengrenzen. Bei Kombination aller drei Phasengrenzen führt die Erweiterung der Boussinesq-Näherung zu keiner deutlichen Veränderung im Konvektionsmuster. Eine vollständige Trennung zwischen den NichtBoussinesq-Effekten und den Viskositätseffekten durch die veränderte Temperaturverteilung gelingt nicht. Dies führt zu kleineren Unterschieden zwischen den Modellen in der Boussinesq- und der erweiterten Boussinesq-Näherung, die nicht auf direkte NichtBoussinesq-Effekte zurückgehen.

\subsubsection{Druck- und temperaturabhängige thermische Parameter}

In diesem Kapitel werden Modelle vorgestellt, die im Rahmen der erweiterten BousinesqNäherung auch die Tiefenabhängigkeit des thermischen Ausdehnungskoeffizienten $\alpha$ sowie die Druck- und Temperaturabhängigkeit der thermischen Leitfähigkeit $\lambda$ berücksichtigen. Durch die Variation von $\alpha$ und $\lambda$ sind keine prinzipiellen Unterschiede zum vorher- 
gehenden Abschnitt zu erwarten, dennoch stellen diese Simulationen die umfassendesten Modelle in dieser Arbeit dar, so dass das Konvektionsmuster und die Temperaturstruktur kurz beschrieben werden soll. Es werden nur zwei Modelle gezeigt, die sich durch die Existenz bzw. Vernachlässigung der kernnahen endothermen Phasengrenze unterscheiden. Bei den Simulationen, die auch die exothermen Phasengrenzen einbeziehen, führt die Berechnung der viskosen Reibung bei den hier auftretenden hohen Geschwindigkeiten bzw. Geschwindigkeitsgradienten beim Übergang zur Lithosphäre zu Beginn der Evolution zu numerischen Problemen, so dass diese Modellrechnungen nicht erfolgreich durchgeführt worden konnten.

Bei der Anpassung des Starttemperaturprofils muss die Tiefenabhängigkeit von $\alpha$ bei der Integration des adiabatischen Temperaturgradienten (Gleichungen (5.1)) berücksichtigt werden. Zusätzlich zur Viskosität muss auch die thermische Leitfähigkeit durch Anpassung des Kompressionsmoduls $K_{0}$ bzw. der Druckabhängigkeit dem neuen Starttemperaturprofil angeglichen werden. Sowohl Viskosität als auch die thermischen Leitfähigkeit werden so normiert, dass sie beim entsprechenden Starttemperaturprofil in halber Tiefe $(r=2380 \mathrm{~km})$ übereinstimmen.

Abbildung (5.63) zeigt die Starttemperaturprofile zusammen mit den angepassten Pro-

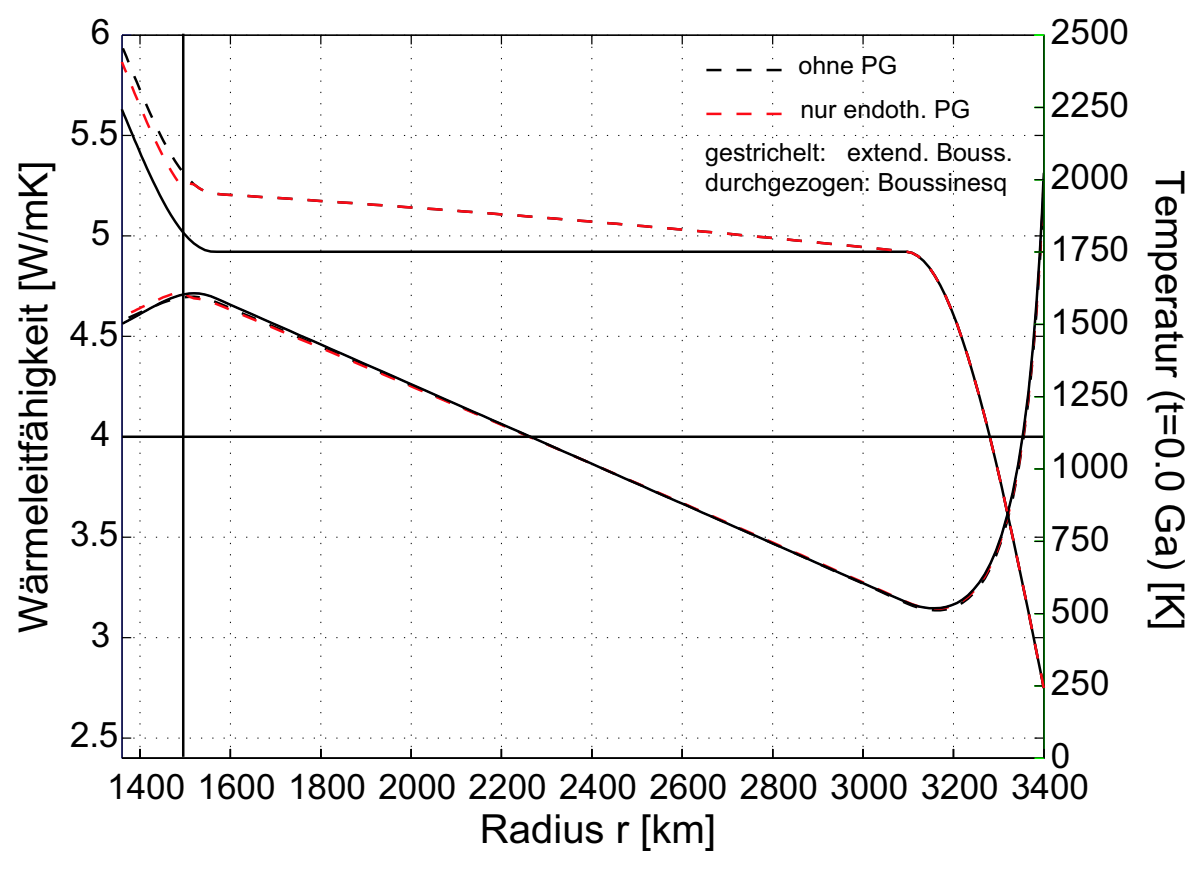

Abbildung 5.63: Anpassung der thermischen Leitfähigkeit an die Starttemperaturprofile in der erweiterten Boussinesq-Näherung. Die senkrechte, schwarze Linie markiert die Position der endothermen Phasengrenze.

filen der thermischen Leitfähigkeit $\lambda$. Tabelle (5.2) gibt einen Überblick über die Modellparameter, die verändert worden sind, um den adiabatischen Temperaturgradienten zu kompensieren. Die Aktivierungsenergie im Viskositätsgesetz bleibt wie bei den Simulationen mit konstanten Parametern unverändert.

Im vorherigen Abschnitt ist deutlich geworden, dass der Einfluss der Nicht-Boussinesq- 


\begin{tabular}{|l|c|c|c|c|}
\hline & Rayleigzahl & $\Delta T[\mathrm{~K}]$ & $K_{0}[\mathrm{GPa}]$ & $V^{*}\left[\mathrm{~cm}^{3} / \mathrm{mol}\right]$ \\
\hline Boussinesq & $10.70 \cdot 10^{6}$ & 2000 & 160 & 3.0 \\
\hline extended Boussinesq & & & & \\
\hline ohne Phasengrenzen (PG) & $11.88 \cdot 10^{6}$ & 2220 & 145 & 4.53 \\
\hline mit endothermer PG & $11.58 \cdot 10^{6}$ & 2164 & 147 & 4.53 \\
\hline
\end{tabular}

Tabelle 5.2: Anpassung der Modellparameter in der erweiterten Boussinesq-Näherung. Die Rayleighzahl gilt mit der lokalen Viskosität von $5.71 \cdot 10^{20} \mathrm{~Pa}$ s und der thermischen Leitfähigkeit von $3.89 \mathrm{~W} / \mathrm{mK}$ bei $t=0.0 \mathrm{Ga}$ und $r=2380 \mathrm{~km}$. Der Temperaturkonstrast ist für $t=0.0 \mathrm{Ga}$ angegeben.

Effekte auf das Konvektionsmuster und die thermische Entwicklung, insbesondere im Fall ohne Phasengrenzen bei Kompensation der Viskositätseffekte der Adiabate sehr klein ist. Dies bestätigt sich, wenn die Tiefenabhängigkeit bzw. Druck- und Temperaturabhängigkeit von $\alpha$ und $\lambda$ in die Modelle einbezogen wird, wie die folgenden Abbildungen verdeutlichen.

Abbildung (5.64) zeigt das Konvektionsmuster nach 4.5 Ga dargestellt durch die Radialgeschwindigkeit in halber Tiefe. In den Simulationen auf der linken Seite sind keine Phasengrenzen berücksichtigt worden, während in der rechten Spalte die kernnahe endotherme Phasengrenze mitmodelliert worden ist. Für beide Spalten ist der Unterschied zwi-

a) Boussinesq-Näherung
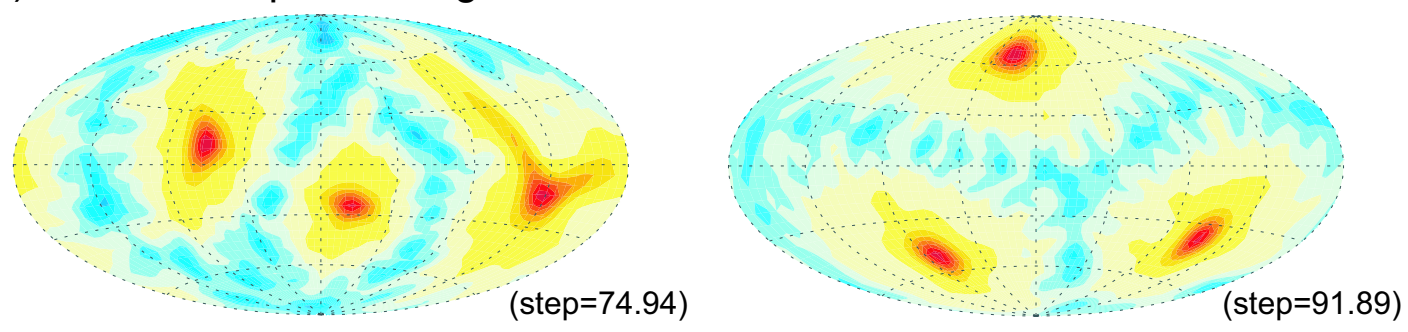

b) Erweiterte Boussinesq-Näherung
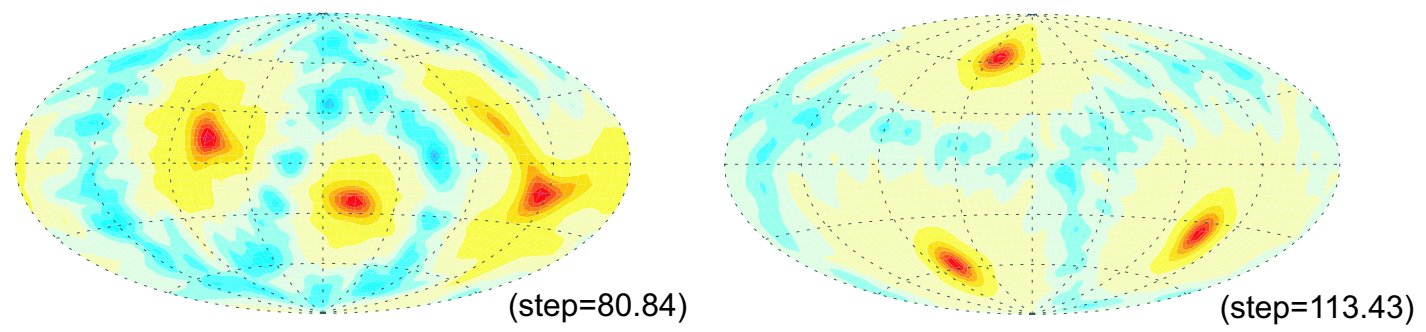

Abbildung 5.64: Vergleich der Konvektionsmuster nach 4.5 Ga im Boussinesq-Fall und im erweiterten Boussinesq-Fall bei tiefenabhängigen thermischen Parametern $\alpha(r)$ und $\lambda(r)$ : Radialgeschwindigkeit in halber Tiefe (rot: Aufströme, blau: Abströme). Die linke Spalte zeigt die Situation ohne die kernnahe endotherme Phasengrenze, in der rechten Spalte wird die kernnahe endotherme Phasengrenze berücksichtigt. Der Contourstep der Geschwindigkeit ist angegeben: $\mathrm{step}=100$ entspricht $0.14 \mathrm{~cm} / \mathrm{a}$. 
schen der Boussinesq-Näherung und der erweiterten Boussinesq-Näherung vernachlässigbar. In allen Simulationen bilden sich drei starke Aufströme aus, deren Position durch das Einbeziehen der Nicht-Boussinesq-Effekte nicht verändert wird. Auch in der Entwicklung zu diesem Konvektionsmuster entstehen, wie zuvor bei den konstanten Materialparametern, keine deutlichen Unterschiede durch die erweiterte Näherung. Ohne endotherme Phasengrenze bildet sich das Muster nach etwa $2.5 \mathrm{Ga}$ heraus mit dieser Phasengrenze zwischen 1.5 und $2.0 \mathrm{Ga}$.

Die geometrische Form und Temperaturstruktur der Aufströme ist in Abbildung (5.65)

a) ohne Phasengrenzen
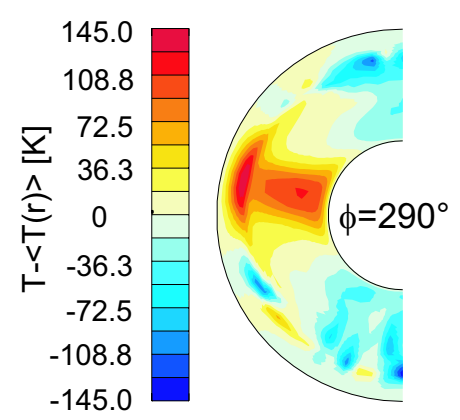

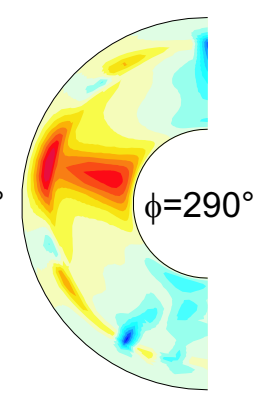

b) endotherme Phasengrenze

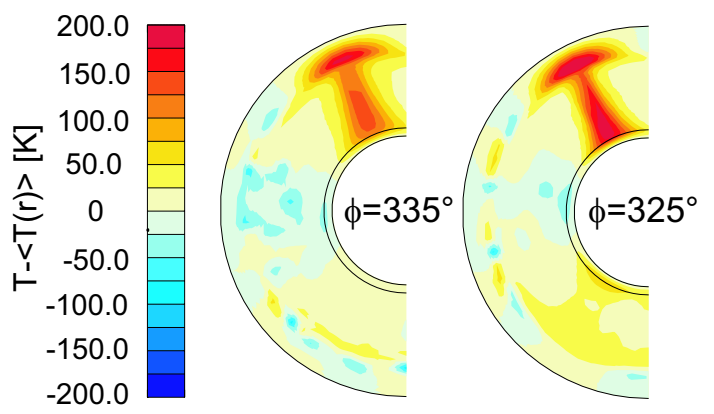

Abbildung 5.65: Vergleich der Temperaturanomalie $(T-<T(r)>)[\mathrm{K}]$ der Aufströme nach 4.5 Ga im Boussinesq-Fall und im erweiterten Boussinesq-Fall bei tiefenabhängigen thermischen Parametern. Der linke Schnitt zeigt jeweils den Boussinesq- der Rechte den erweiterten Boussinesq-Fall.

durch die Temperaturanomalie $(T-<T(r)>)$ verdeutlicht. In Übereinstimmung mit den Beobachtungen bei konstanten thermischen Parametern beeinflussen die Nicht-Boussinesq-Effekte die Geometrie und Temperaturanomalie im Fall ohne endotherme Phasengrenze nicht signifikant. Im Fall mit endothermer Phasengrenze ist die Temperaturanomalie im Aufstrom etwas höher (vgl. Kapitel (5.5.1)). Im Vergleich zu den konstanten thermischen Parametern sind die Amplituden der Temperaturanomalie bei den tiefenabhängigen Parametern geringer.

Abbildung (5.66) zeigt die Temperaturprofile nach 4.5 Ga. Der Temperaturunterschied zwischen den beiden Näherungen geht, wie im Fall der konstanten Parameter, im Wesentlichen auf den Beitrag der Adiabate zurück. Nach Subtraktion der Manteladiabate stimmen die Temperaturprofile in der erweiterten Näherung gut mit den Profilen in der einfachen Boussinesq-Näherung überein. Abbildung (5.67) zeigt diese Korrektur für den Fall mit endothermer Phasengrenze. Die Temperatur an der KMG beträgt in der erweiterten Näherung nach $4.5 \mathrm{Ga} 1920 \mathrm{~K}$ ohne endotherme Phasengrenze und $1930 \mathrm{~K}$ mit endothermer Phasengrenze.

Für die Temperaturentwicklung in halber Tiefe ergibt sich analog zu den Simulationen mit konstanten Parametern ein nahezu konstanter Offset zwischen der extended BoussinesqNäherung und der einfachen Betrachtungsweise.

Der Wärmefluss an der KMG ist in Abbildung (5.68) dargestellt. In Übereinstimmung mit den Modellen bei konstanten thermischen Parametern beeinflusst die Erweiterung der Näherung (bei Anpassung der Viskositätsparameter) den Wärmefluss im Fall ohne endother- 


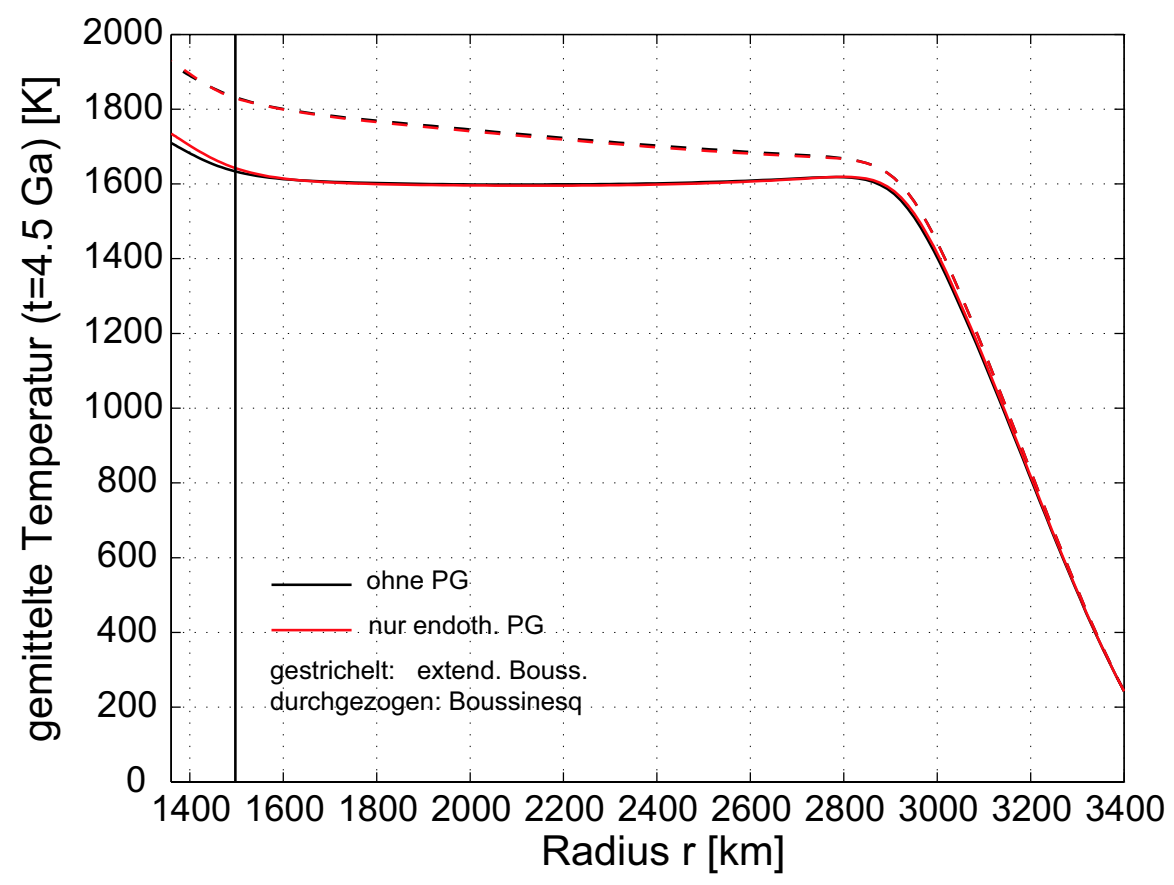

Abbildung 5.66: Profile der gemittelten Temperatur nach $4.5 \mathrm{Ga}$ in der Boussinesq- und der erweiterten Boussinesq-Näherung bei tiefenabhängigen thermischen Parametern. Die senkrechte schwarzen Linie markiert die Tiefe der endothermen Phasengrenze.

me Phasengrenze nicht und führt mit der endothermen Phasengrenze zu einem reduzierten Wärmefluss. Allerdings liegt auch dieser zeitweillig über dem Mittelwert $F_{\mathrm{m}}$, so dass ein thermisch getriebener Dynamo über diesem Zeitraum möglich wäre. Für die Lebensdauer des Dynamos bleibt das Problem des breiten Maximums, das im Zusammenhang mit den tiefenabhängigen Parametern ausführlich diskutiert worden ist. Die Unterschiede in der Entwicklung in der erweiterten Näherung sind u.a. von der nicht kompletten Kompensation der Viskositätseffekte verursacht.

Die Lithosphärendicke wird auch bei tiefenabhängigen thermischen Parametern nicht durch die Nicht-Boussinesq-Effekte verändert.

Der Einfluss der Nicht-Boussinesq-Effekte auf das Konvektionsmuster und die thermische Evolution ist auch bei Berücksichtigung die Tiefenabhängigkeit der thermische Parameter $\alpha$ und $\lambda$ gering, wenn die Viskositätseffekte der Adiabate geeignet kompensiert werden. Der wesentliche Temperaturunterschied entsteht durch den Beitrag der Adiabate. Das Konvektionsmuster bleibt unverändert. 


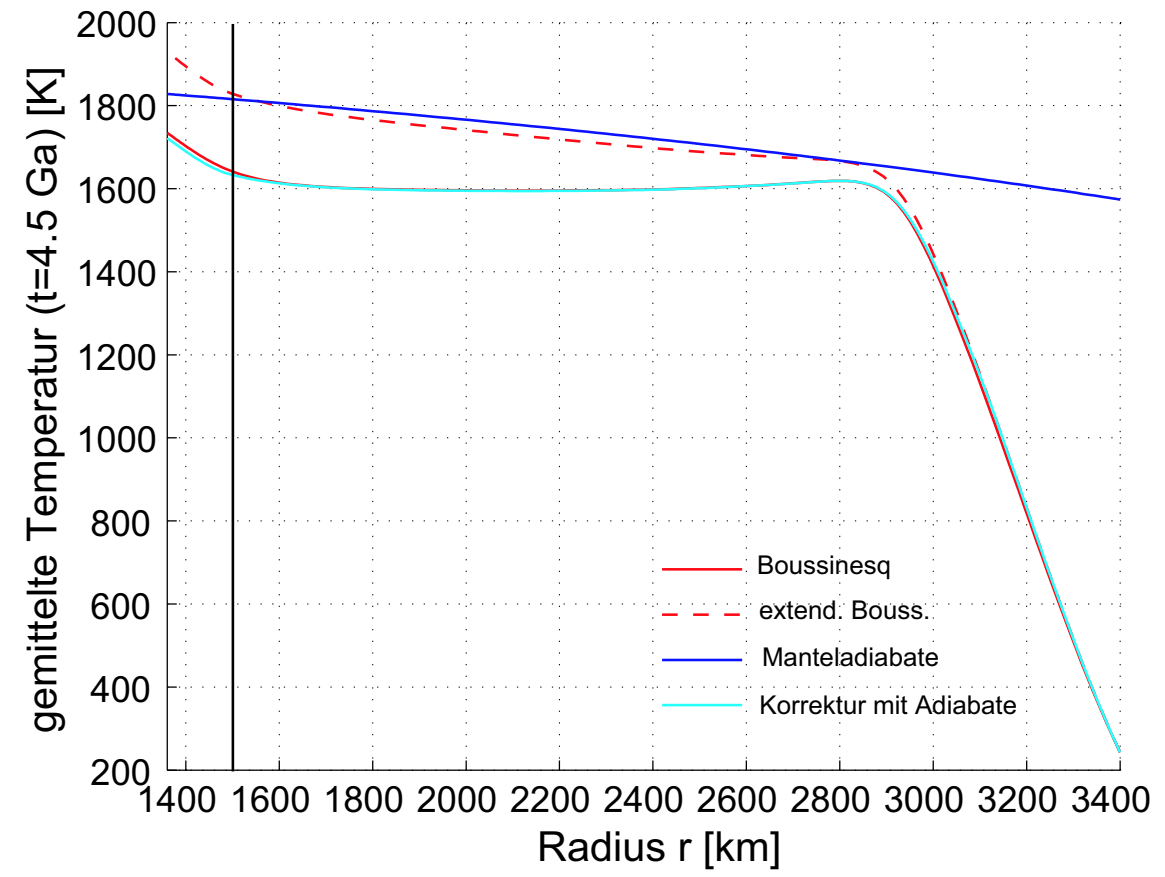

Abbildung 5.67: Kompensation der adiabatischen Temperaturzunahme im Temperaturprofil nach 4.5 Ga in der erweiterten Boussinesq-Näherung für die Simulationen mit kernnaher endothermer Phasengrenze bei tiefenabhängigen thermischen Parametern. 


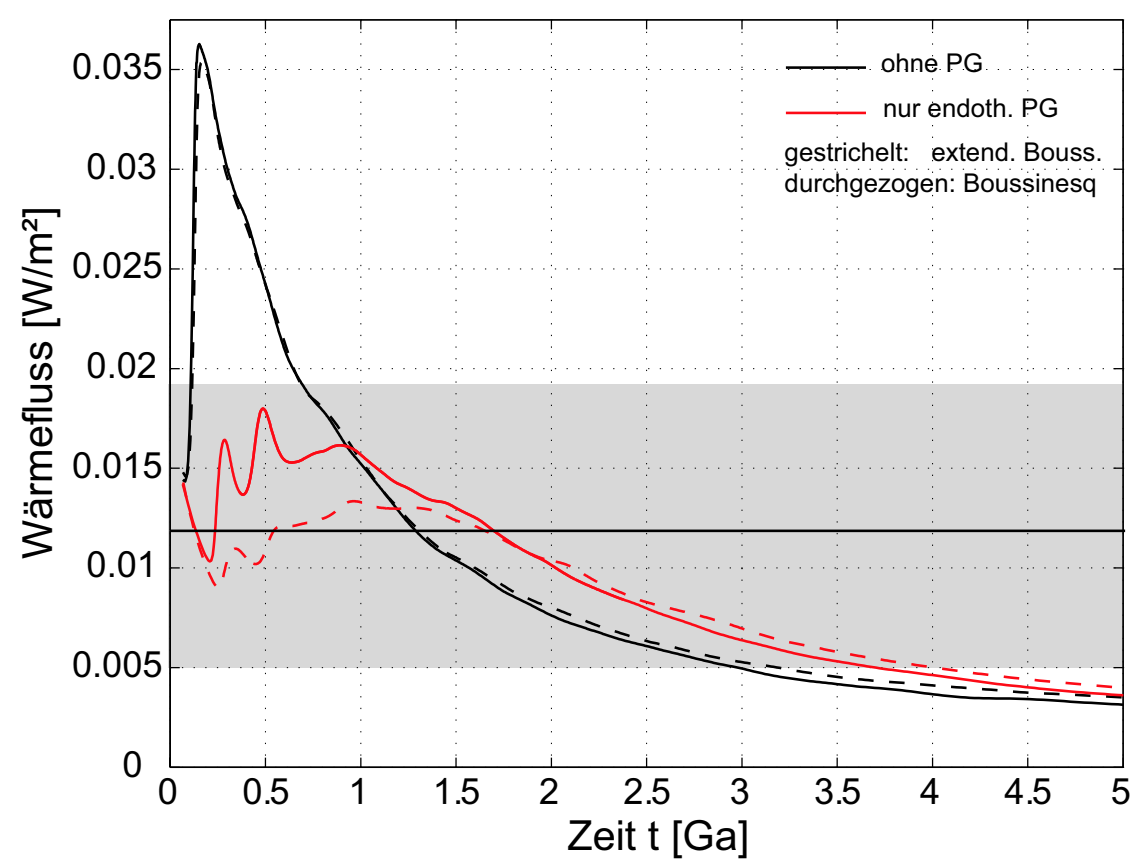

Abbildung 5.68: Einfluss der verschiedenen Näherungen auf die Entwicklung des Wärmeflusses an der KMG bei tiefenabhängigen thermischen Parametern. Der grau unterlegte Bereich kennzeichnet den Wertebereich des Wärmeflusses entlang der Adiabate im Marskern (Nimmo und Stevenson 2000). Die schwarze Linie zeigt zur Orientierung den Mittelwert dieses Bereichs. 


\subsection{Der Aufbau des Marskerns}

In den vorangegangenen Abschnitten ist die Existenz und Lebensdauer eines Dynamos im Mars im Zusammenhang mit dem Wärmefluss aus dem Marskern diskutiert worden, der, falls er den Wärmefluss entlang der Kernadiabate übersteigt, einen thermisch getriebenen Dynamo ermöglichen könnte. Dabei ist angenommen worden, dass ein ausreichender Anteil des Marskerns flüssig ist. Ein zweiter Mechanismus, der einen Dynamo antreiben könnte, besteht in der chemischen Konvektion, die beim Ausfrieren eines inneren festen Kerns einsetzt. Der Eisenkern des Mars enthält Schwefel, der beim Ausfrieren eines inneren, reinen Eisenkerns frei wird und als leichte Komponente aufsteigen kann. Dadurch setzt er ähnlich wie die durch höhere Temperaturen weniger dichte Komponente in der thermischen Wärmeströmung eine Konvektionsbewegung in Gang.

Da seismologische Daten für Mars bisher fehlen und die vorliegenden Daten nicht ausreichen, die Größe und innere Struktur des Marskerns zu bestimmen, ist die Frage, ob der Kern komplett flüssig, fest oder in Teilen flüssig ist, nicht eindeutig beantwortet (vgl. Abschnitte (2.3.1), (2.3.4) und (2.5)). Mit Hilfe der Temperaturentwicklung an der KMG aus den vorliegenden Modellen und der geeigneten Schmelzkurven für Eisen mit dem entsprechenden Schwefelzusatz kann ein Strukturmodell des Marskerns abgeleitet werden und die Frage nach einer möglichen Existenz eines inneren Kerns beantwortet werden.

Die Schmelzkurve für reines Eisen ist von Boehler 1986 für den Bereich bis $40 \mathrm{GPa}$

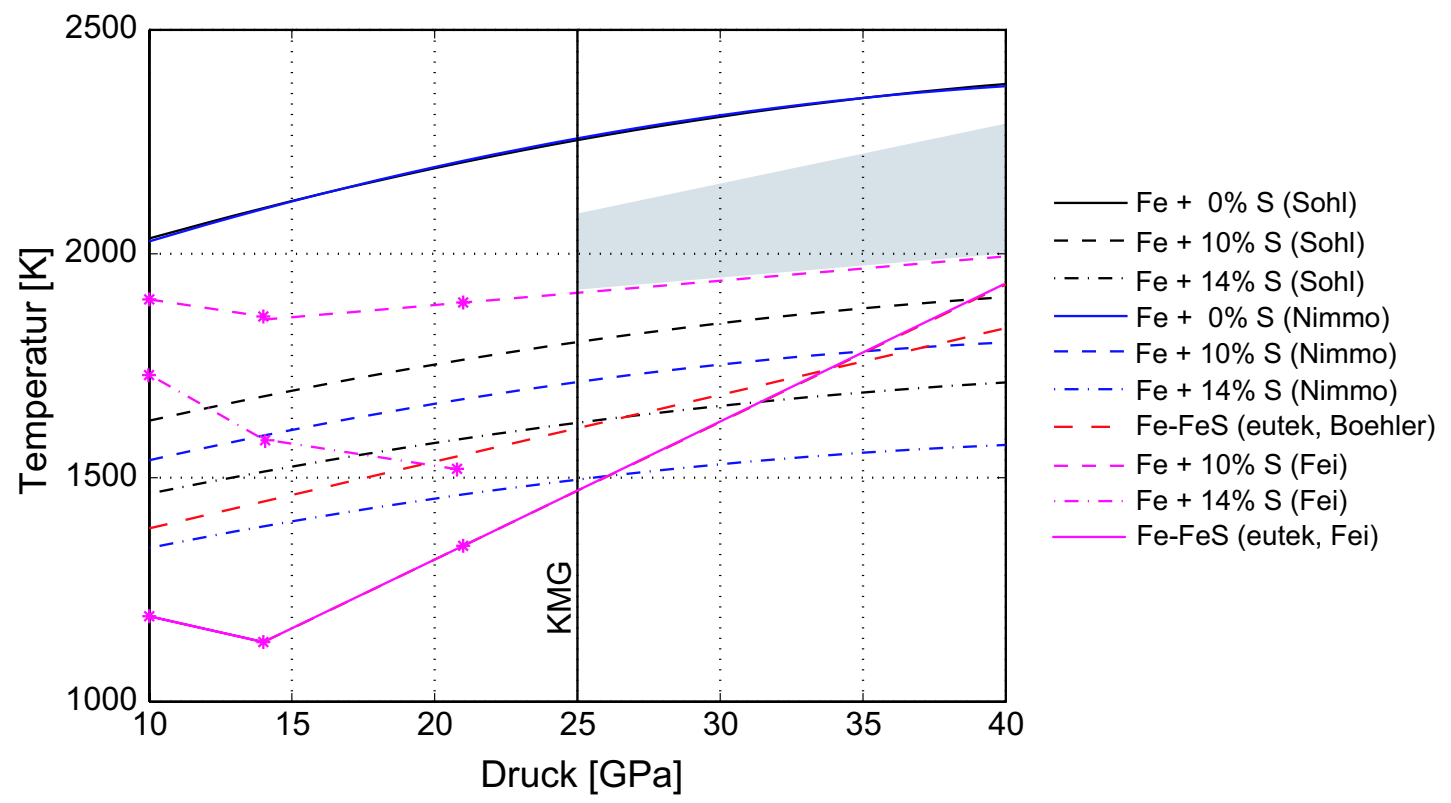

Abbildung 5.69: Schmelzkurven für Eisen mit verschiedenen Anteilen von Schwefel aus verschiedenen Modellen bzw. Messungen. Der unterlegte Bereich zeigt, den Temperaturbereich der Kernadiabate.

in einer Diamantpresse mit Hilfe eines widerstandgeheizten Eisendrahtes gemessen worden (Boehler 1986). Diese Daten sind später mit veränderten Techniken (lasergeheizte Diamantpresse) von Boehler selbst (Boehler et al. 1990) aber auch von anderen Gruppen bestätigt worden und auf einen weiteren Druckbereich ausgedehnt worden (z.B. Boeh- 
ler (1993)), um eine geeignete Interpolation für den Druck im Erdkern zu erzielen. Einen Überblick über die Ergebnisse der verschiedenen Gruppen und einen Einblick in die messtechnischen Verfahren und deren Probleme geben die Review-Artikel von Boehler (1996a, 2000).

Leichte Elemente wie S, O und Ni senken die Schmelztemperatur ab. Diese Absenkung kann prinzipiell experimentell gemessen werden oder aus thermodynamischen Größen berechnet werden. Der Einfluss von Sauerstoff auf die Schmelztemperatur bzw. die Schmelztemperatur von $\mathrm{FeO}$ wird in Boehler $(1992,1993)$ untersucht. Für Mars wird Schwefel als leichte Komponente im Kern favorisiert. Die Schmelzkurve von FeS und der eutektischen Legierung von Fe-FeS ist in Boehler (1992) bzw. Boehler (1996b) dargestellt. Die Schmelzkurve für das Eutektikum ist dabei bis zu einem Druck von $62 \mathrm{GPa}$ vermessen worden. In Abbildung (5.69) ist die Schmelzkurve des Eutektikums nach den Daten von Boehler vereinfacht eingetragen.

Die Bestimmung der Schmelztemperatur in einer nicht eutektischen Zusammensetzung mit herkömmlichen optischen Methoden ist schwierig, da in einem Mehrkomponentensystem zunächst nur ein kleiner Anteil schmilzt, der schwer zu beobachten ist, so dass diese Methode die Schmelztemperaturen überschätzt. Eine empfindlichere Methode ist die Beobachtung von Interferenzmustern, die mit einem Laser erzeugt werden und sich beim Eintritt der Schmelze stark ändern. Bislang gibt es nur sehr wenige Daten, die die Schmelzkurve von Eisen mit einem geringen Schwefelanteil untersucht haben.

Fei und Bertka (1996) sowie Fei et al. (1997) haben die Schmelzbedingungen im Fe-FeSSystem bei Drücken von 1 bar, $10 \mathrm{GPa}$ und $14 \mathrm{GPa}$ und später auch bei $21 \mathrm{GPa}$ (Fei et al. 2000a,b) analysiert. Die Schmelztemperaturen, die sie für einen Schwefelgehalt von 10 $\%$ und $14 \%$ bzw. für das Eutektikum bestimmen, sind in Abbildung (5.69) dargestellt. Es besteht eine Diskrepanz zu den Daten von Boehler für das Eutektikum, die von den Autoren nicht diskutiert wird. Die Daten für einen Schwefelgehalt von $10 \%$ und für das Eutektikum sind in den Druckbereich des Marskerns linear interpoliert worden. Da bei höheren Drücken die Steigung der Schmelzkurve abnimmt, überschätzt die Kurve vermutlich die Temperaturen in diesem Druckbereich.

Die Druckabhängigkeit der Schmelztemperatur $T_{\mathrm{m}}$ von reinem Eisen sowie von Eisen mit einigen Prozent Schwefel wird in der Literatur nach dem Lindemann-Gesetz parametrisiert: Sohl und Spohn (1997) verwenden folgende Gleichung, in der $x_{\mathrm{s}}$ den prozentualen Schwefelgehalt angibt und $p$ für den Druck steht.

$$
T_{\mathrm{m}}=1809\left(1-2 x_{\mathrm{s}}\right)\left(1.0145+1.215 \cdot 10^{-11} p-1.159 \cdot 10^{-22} p^{2}\right)
$$

Williams und Nimmo (2004) verändern die Parameter leicht und benutzen:

$$
T_{\mathrm{m}}=1811\left(1-2.41 x_{\mathrm{s}}\right)\left(1.0+1.335 \cdot 10^{-11} p-1.394 \cdot 10^{-22} p^{2}\right)
$$

Die Schmelzkurven die sich damit für reines Eisen ergeben, sind in Abbildung (5.69) aufgenommen. Beide Parametrisierungen stimmen gut überein. Allerdings ist der Temperaturanstieg zwischen 25 und $40 \mathrm{GPa}$ etwas zu flach verglichen mit den Messdaten von Boehler, der bei $40 \mathrm{GPa}$ eine Schmelztemperatur von $2500 \mathrm{~K}$ findet. Neben den Kurven für reines Eisen sind auch die Kurven für Eisen mit $10 \%$ bzw. $14 \% \mathrm{~S}$ in beiden Parametrisierungen in Abbildung (5.69) gezeigt.

Um Rückschlüsse auf die innere Struktur des Kerns und seinen Aggregatzustand zu erhalten, müssen diese Schmelzkurven mit den Temperaturen im Marskern verglichen werden. 
In der erweiterten Boussinesq-Näherung liegen die Kerntemperaturen nach 4.5 Ga bei konstanten thermischen Parametern zwischen 1920 K (ohne Phasengrenzen) und 2092 $\mathrm{K}$, wenn alle drei Phasengrenzen berücksichtigt werden. Die Kerntemperaturen für die Simulationen mit tiefenabhängigen Parametern liegen innerhalb dieses Intervalls. Folgen die Temperaturen im Kern einer Adiabate, so ergibt sich mit den diskutierten Unsicherheiten bei den Parametern des adiabatischen Gradienten im Kern (vgl. Abschnitt (2.5)) der schattierte Bereich in Abbildung (5.69). Für die obere Grenzkurve sind die Werte für $\alpha, g$ und $C_{p}$ im Rahmen der Unsicherheiten so angenommen, dass der adiabatische Gradient maximiert wird, für die untere Kurve sind sie entsprechend so gewählt, dass die Temperaturzunahme im Kern minimiert ist. Es wird berücksichtigt, dass die Schwerebeschleunigung linear zum Zentrum des Planeten abnimmt.

In einem reinen Eisenkern liegt die Schmelztemperatur oberhalb des Temperaturbereichs der Adiabate. In diesem Fall wäre der Kern komplett ausgefroren bzw. fest. Die Kerntemperaturen liegen jedoch über den parametrisierten Schmelzkurven (sowohl nach Sohl und Spohn (1997) als auch nach Williams und Nimmo (2004)) für einen Schwefelgehalt von $10 \%$ bzw. $14 \%$ und über der Kurve des Eutektikums nach Boehler, so dass der Kern in diesen Fällen gänzlich flüssig ist und bisher keinen festen inneren Kern ausgebildet hat.

Die Interpolation der Messungen von Fei et al. (1997, 2000b) über einen weiten Druckbereich ist mit großen Unsicherheiten behaftet. Die Schmelzkurve für einen Schwefelgehalt von $10 \%$ liegt geringfügig unter der Grenzkurve für die adiabatischen Temperaturen. Die Kurven verlaufen fast parallel, so dass geringe Temperaturunterschiede unter Umständen zu großen Unterschieden in der Kerngröße führen können und sinnvolle Aussagen nur möglich sind, wenn der Messbereich zu höheren Drücken ausgeweitet wird, die Unsicherheiten verringert und die Unstimmigkeiten zu den Daten von Boehler geklärt werden. Williams und Nimmo (2004) schätzen die heutige Kerntemperatur im Mars ab, indem sie annehmen, dass sich die Temperaturen im oberen Mantel von Mars und Erde nicht stark unterscheiden. Die Manteladiabate, ausgehend von dieser Temperatur, liefert eine Abschätzung der Temperatur an der KMG von $2000 \mathrm{~K}$, die mit den vorgestellten Simulationen übereinstimmt. Ihr Vergleich von Kernadiabate und Schmelzkurve bei einem Schwefelgehalt von $14.2 \%$ liefert ebenfalls einen gänzlich flüssigen Kern.

Die Adiabate stellt (bei Vernachlässigung der Grenzschichten) den größtmöglichen Temperaturanstieg im flüssigen Kern dar. Das andere Extrem wäre eine konstante Temperaturverteilung im Kern. Allerdings verändert dies die Lage des Temperaturbereichs relativ zu den verlässlichen Schmelzkurven und die Aussagen über die innere Struktur nicht.

Für die Realisation eines Dynamos im Mars bedeutet ein komplett flüssiger Kern bzw. das Fehlen eines inneren Kerns, dass ein chemisch getriebener Dynamo bei einem Schwefelgehalt von mindestens $10 \%$ nicht möglich ist. Allerdings kann das Material in diesem flüssigen Kern thermisch konvektieren, wenn der Mantel genug Wärme vom Kern abtransportiert und einen ausreichend hohen Temperaturgradienten im Kern aufrecht erhält. Die zeitliche Entwicklung des Marsdynamos stellt sich damit wie folgt dar: Zu Beginn der Evolution hat Mars einen thermisch getriebenen Dynamo, der das Magnetfeld für die starke Krustenmagnetisierung erzeugt hat. In dieser Zeit übersteigt der Wärmefluss aus dem Kern den Wärmefluss entlang der Adiabate im Kern. Das genaue Zeitfenster für die Aktivität des Dynamos hängt entscheidend vom Wert des Wärmeflusses entlang der Kernadiabate ab. In den Simulationsreihen sind verschiedene Konstellationen vorgestellt worden, in denen ein erhöhter Wärmefluss aus dem Kern möglich ist. Viele Modelle ha- 
ben allerdings, insbesondere bei Berücksichtigung der endothermen Phasengrenze, das Problem eines relativ breiten Maximums im Wärmefluss, dass kurze Zeiten der Dynamoaktivität, wie sie durch das Fehlen der Magnetisierung in den großen Einschlagkratern nahegelegt wird, erschwert. Nach dieser Phase des erhöhten Wärmeflusses aus dem Kern und der damit verbundenen Dynamoaktivität sinkt der Wärmefluss an der KMG durch den Abkühlprozess des Planeten und das Abschwächen der Mantelkonvektion. Der Wärmefluss fällt unter den Wärmefluss entlang der Kernadiabate und der Marsdynamo stirbt aus. Der Kern ist bis heute noch nicht soweit abgekühlt, dass sich ein innerer Kern bildet und chemische Konvektion zulässt. Im Moment fehlt somit im Mars ein Antriebsmechanismus für den Dynamoprozess, der ein globales Magnetfeld erzeugen könnte. In der weiteren Entwicklung des Planeten könnte der Dynamoprozess neu beginnen, wenn durch das Ausfrieren des inneren Kerns und das Aufsteigen der leichten Elemente genug mechanische Energie erzeugt wird. 


\section{Thermische Evolutionsmodelle für Merkur}

\subsection{Innerer Aufbau und Magnetfeld des Merkur}

Merkur ist der sonnennächste und mit einem Radius von $r_{a}=2439 \pm 1 \mathrm{~km}$ der kleinste terrestrische Planet. Seine mittlere Dichte von $5430 \pm 10 \mathrm{~kg} / \mathrm{m}^{3}$ (Anderson et al. 1987) ist deutlich höher als die Dichte der übrigen erdähnlichen Planeten und legt eine sehr eisenreiche Zusammensetzung bzw. einen großen Eisenkern nahe. Mit Hilfe der beiden Mariner 10 Vorbeiflüge (März 1974 und März 1975) sind die Koeffizienten $C_{2}^{2}=$ $(1.0 \pm 0.5) \cdot 10^{-5}$ und $J_{2}=(6.0 \pm 2.0) \cdot 10^{-5}$ (Anderson et al. 1987) des Schwerefeldes bestimmt worden. Der große Wert von $J_{2}$ deutet bei der langsamen Rotation des Planeten (58.6462 Tage) auf einen nicht hydrostatischen Beitrag zur polaren Abflachung des Planeten hin. Mit der bisherigen Datenlage des Schwerefeldes sind geeignete Korrekturen für diesen nicht hydrostatischen Anteil nicht möglich, so dass weder das Trägheitsmoment noch das erste Dichtemoment für Merkur bestimmt werden können. Grobe Abschätzungen aus $J_{2}$ liefern einen Wert für das Trägheitsmoment um die Rotationsachse von $C=0.34 R M^{2}$, wobei $R$ der volumen-gemittelte Oberflächenradius ist und $M$ die Planetenmasse (Spohn et al. 2001b). Damit fehlen für Merkur wichtige Daten, die die innere Struktur des Planeten bestimmen können bzw. Modellannahmen einschränken. Diese Modelle werden für Merkur nur durch die Planetenmasse, den Radius und allgemeine Annahmen über die chemische Zusammensetzung des Planeten bzw. über den strukturellen Aufbau (Kruste, Mantel, eisenreicher Kern) von erdähnlichen Planeten beschränkt.

Die geplante ESA-Mission BepiColombo zum Merkur soll wesentlich zur Lösung dieses Problems beitragen und Aufschluss über den inneren Aufbau des Planeten geben. Im Rahmen dieser Mission soll das Schwerefeld mit hoher Genauigkeit vermessen werden und mit Hilfe eines Laseraltimeters die Topographie sehr genau bestimmt werden. Zudem sollen Daten mit hoher Präzision zur Rate und Amplitude der Libration der Rotationsachse und ihrer Neigung gewonnen werden. Aus diesen Messungen lässt sich dann das Trägheitsmoment um die Rotationsachse sowie das Trägheitsmoment einer festen äußeren Schicht (um die Rotationsachse) sehr genau bestimmen. Das Verhältnis dieser beiden Werte kann Auskunft geben, ob Merkur einen flüssigen oder zumindest teilweise flüssigen Kern hat. Zudem liefert das Trägheitsmoment eine wichtige Beschränkung bei der Modellierung der inneren Struktur. Das Laseraltimeter soll ferner in der Lage sein, die Verformung des Planeten durch die Tiden, die die Sonne hervorruft, zu vermessen. Mit diesen Daten lässt sich die Love-Zahl $k_{2}$ bestimmen, die ebenfalls Aussagen über die innere Struktur (u.a. Kerngröße, Radius eines inneren Kerns) zulässt. Die Daten aus dem 
Schwerefeld ermöglichen eventuell Aussagen über die Topographie oder Welligkeit der KMG bzw. über Variationen der Krustendicke (Spohn et al. 2001b). Spohn et al. (2001b) geben einen Überblick, welche neuen Erkenntnisse durch die Mission BepiColombo möglich sind und welche Genauigkeiten bei den Messungen angestrebt werden bzw. erforderlich sind.

Zum heutigen Zeitpunkt fehlen diese Daten jedoch und die Modelle sind weniger gut beschränkt. Die hohe mittlere Dichte des Planeten (bei Normbedingungen ohne Kompression: $5300 \pm 10 \mathrm{~kg} / \mathrm{m}^{3}$ ) lässt auf einen extrem großen Eisenkerns mit einem Radius zwischen 1800 und $1900 \mathrm{~km}$ schließen (Spohn et al. 2001b). Kosmochemische Argumentationen, die die Entstehung des Merkur an seiner heutigen Position im Sonnensystem vorraussetzen, schlagen einen geringen Schwefelanteil (0.5 - 5 Gew.-\%) im Kern vor (Goettel 1988). Der Silikatmantel ist auf eine sehr dünne Schicht von nur 500 - 700 km beschränkt. Aufgrund des niedrigen Drucks treten wahrscheinlich keine Phasenübergänge im Mantelmaterial auf.

Harder und Schubert (2001) haben mit Hilfe von Monte-Carlo-Simulationen eine große Anzahl von Strukturmodellen für Merkur berechnet und insbesondere den Schwefelgehalt des Kerns variiert. Diese Modelle zeigen deutlich, dass die Bestimmung des Trägheitsmoments wichtig ist, um die Variationsbreite der Modellangaben zu beschränken. Die Existenz und Größe eines inneren Kerns lässt sich mit diesen Modellen nicht eindeutig bestimmen.

Die Frage nach der Struktur des Kerns ist auch für Merkur im Zusammenhang mit dem Magnetfeld und einem Dynamoprozess interessant. Während der Mariner 10 Mission ist das Magnetfeld des Merkurs in zwei Vorbeiflügen gemessen worden. Die Daten belegen ein (im Vergleich zur Erde) schwaches, intern erzeugtes, globales Magnetfeld mit einer Dipolfeldstärke von $\sim 300 \mathrm{nT}$ (die Dipolachse ist $\sim 10^{\circ}$ gegen die Rotationsachse geneigt) (Connerney und Ness 1988). Der Wert des Dipolmoments ist mit großen Unsicherheiten behaftet (Connerney und Ness 1988). Damit dieses Feld durch einen hydrodynamischen Dynamo erzeugt werden kann, muss ein ausreichender Anteil des Kerns flüssig sein und ein Mechanismus zum Antrieb der Konvektionsbewegung bestehen. Thermische Evolutionsmodelle, die die Entwicklung eines inneren Kerns mitberücksichtigen, können bei der Beantwortung dieser Frage helfen. Anhand der Modelle lässt sich die Existenz und Größe eines inneren Kerns bestimmen. Bildet sich ein innerer Kern aus, so kann chemische Konvektion einen Dynamo antreiben. Ein Vergleich des Wärmeflusses an der KMG mit dem Wärmefluss entlang der Kernadiabate im Modell kann auch Aufschluss über die Möglichkeit eines thermisch getriebenen Dynamos geben.

Eine weitere Beobachtung, die durch thermische Evolutionsmodelle bestätigt werden kann, ist die Abnahme des Planetenradius mit der Zeit. Radarbeobachtungen und Daten der Mariner 10 Missionen zeigen Verwerfungszonen, aus deren Anzahl, Höhen und Längen die Abnahme des Planetenradius auf etwa 1 - 2 km abgeschätzt werden kann. Diese Abnahme wird auf das Abkühlen des Planeten und das Ausfrieren eines inneren Kerns zurückgeführt (Melosh und McKinnon 1988). Der genaue Wert dieser Abnahme ist allerdings mit Unsicherheiten verbunden, da bislang nicht die gesamte Merkuroberfläche vermessen werden konnte (Strom 1997). Auch der zeitliche Einsatz der Entstehung dieser Verwerfungszonen ist nicht genau bekannt. Geologische Beobachtungen legen einen Beginn der Bildung nach dem 'heavy bombardment' vor etwa 3.8 Ga nahe (Strom 1997). Stevenson et al. (1983) stellen neben den parametrisierten Modellen für Mars auch Evolu- 
tionsmodelle für Merkur vor. Sie betrachten Modelle mit verschiedenem Anfangsschwefelgehalt des Kerns zwischen 1 und 5 Gew.-\%. In allen Modellen setzt das Ausfrieren eines inneren Kerns früh (zwischen 0.23 und $0.6 \mathrm{Ga}$ ) in der Entwicklung ein und es entstehen innere Kerne mit Radien zwischen 1408 und 1762 km. In allen Fällen ist der Wärmefluss aus dem Kern nach kurzer Zeit zu gering, um thermische Konvektion zu gewährleisten. Allerdings ist in allen Simulationen chemische Konvektion möglich, so dass zumindest in den Fällen mit einer ausreichend dicken Flüssigkeitsschicht ein hydrodynamischer Dynamo zur Erzeugung des Magnetfeldes nicht auszuschließen ist.

Die Daten für den Kern von Stevenson et al. (1983) werden von den Modellen von Spohn (1991) bestätigt, in denen eine realistischere Beschreibung der Lithosphäre verwendet wird. Schubert et al. (1988) verwenden eine steifere Rheologie als Stevenson et al. (1983) und kommen in Abhängigkeit vom Schwefelgehalt zu Größen für den inneren Kern zwischen 780 und 1730 km. Das Kernwachsum setzt für einen Schwefelgehalt von 5 Gew.-\% erst nach $2.5 \mathrm{Ga}$ ein $(0.7 \mathrm{Ga}$ bei $1 \mathrm{Gew} .-\%)$. Der Wärmefluss reicht nach $4.5 \mathrm{Ga}$ nicht mehr aus, einen thermisch getriebenen Dynamo zu realisieren. Schubert et al. (1988) berechnen in ihren Modellen auch die Abnahme des Planetenradius während des Abkühlens und ermitteln Werte zwischen 6 km (5 Gew.-\% Schwefel) und $10 \mathrm{~km}$ (0.2 Gew.-\% Schwefel).

Neuere parametrisierte Evolutionsmodelle für Merkur werden von Hauck et al. (2004) vorgestellt. Die Schmelzbedingungen für die Fe-FeS-Legierung im Kern werden in diesen Modellen den jüngeren experimentellen Ergebnissen von Fei et al. (1997) bzw. Fei et al. (2000b) angepasst. Außerdem unterscheiden sich die Modelle von denen von Stevenson et al. (1983) bzw. Schubert et al. (1988) durch die unterschiedliche Behandlung der Lithosphäre und die Berücksichtigung der Energiebeiträge durch partielles Schmelzen im Mantel. Die Modelle untersuchen den Einfluss der partiellen Schmelzen im Mantel, der Mantelrheologie, der anfänglichen Temperaturverteilung, der internen Wärmeproduktion und des Schwefelgehalts im Kern auf die Entwicklung des Planeten. In Simulationen mit einem Schwefelgehalt von > 6.5 Gew.-\% im Kern, einer Thorium-dominierten internen Wärmeproduktionsrate und einer Mantelrheologie für trockenen Olivin gelingt es, die Abnahme des Planetenradius auf 1 bis $2 \mathrm{~km}$ zu beschränken. Gleichzeitig ist in diesen Modellen chemische Konvektion im Kern als Antriebsmechanismus für einen Dynamo möglich, so dass das beobachtete, intern erzeugte Magnetfeld erklärt werden kann. Modelle, in denen der Wärmefluss aus dem Kern ausreichend überadiabatisch ist, um einen thermisch getriebenen Dynamo zu realisieren, liefern Änderungen im Planetenradius die sehr weit von den Beobachtungen abweichen.

Erste achsensymmetrische Konvektionsmodelle für Merkur, die eine druck- und temperaturabhängige Viskosität beinhalten und die thermische Evolution simulieren, werden von Conzelmann (1999) berechnet. In Zusammenhang mit der thermischen Entwicklung wird der Einfluss verschiedener Viskositätsparameter, des Schwefelgehalts und der Anfangskerntemperatur auf die thermische Entwicklung und das Ausbilden eines inneren Kerns untersucht. Zudem werden Rechnungen, die von einem (festen) Grenzschichttemperaturprofil gestartetet werden, mit Simulationen, die einen vorher berechneten Gleichgewichtszustand als Ausgangssituation annehmen, verglichen.

In Anlehnung und Erweiterung der Arbeit von Conzelmann (1999) werden im vorliegenden Kapitel Evolutionsrechnungen für Merkur vorgestellt. In zunächst achsensymmetrischen Modellen wird der Einfluss der Temperaturverteilung im Startprofil auf die thermische Entwicklung und das Ausbilden eines festen inneren Kerns untersucht. Eine zweite 
Modellreihe analysiert den Einfluss des anfänglichen Schwefelgehalts und der Parametrisierung der Schmelzkurve. Die genaue Schmelzkurve für Eisen mit einigen Prozent Schwefel ist bei Kernbedingungen noch nicht vermessen worden und somit nur unzureichend bekannt. Deshalb werden verschiedene mögliche Parametrisierungen verwendet und ihr Einfluss auf die Entwicklung des inneren Kerns simuliert. Im letzten Abschnitt erfolgt dann der Vergleich zwischen achsensymmetrischen und vollständig dreidimensionalen Evolutionsmodellen bei verschiedenen Rayleighzahlen.

\subsection{Modellierung des Kerns}

Die thermischen Evolutionsmodelle für Merkur sollen das mögliche Ausfrieren eines inneren Kerns und sein Wachsen im Lauf der Entwicklung mitsimulieren. Beim Ausfrieren des Kerns wird latente Wärme frei und das Aufsteigen der leichten Komponente, des Schwefels, setzt gravitative Energie frei. Diese beiden Energiebeiträge müssen in der Energiebilanz des Kerns berücksichtigt werden. Für Merkur werden, wie für Mars, interne Heizquellen im Kern (z.B. radioaktive Elemente) vernachlässigt. Merkur wird durch die Tiden der Sonne verformt, so dass Dissipationsenergie erzeugt wird. Schubert et al. (1988) berücksichtigen diesen Beitrag in der Energiebilanz für den Kern. Allerdings zeigt sich, dass dieser Beitrag nur bei sehr großen, inneren Kernen und für ein kleines Intervall von Kernviskositäten (abhängig von der Größe der Eisenkörner) signifikant ist, so dass er hier vernachlässigt wird.

Die Größe des inneren Kerns kann durch einen Vergleich zwischen der Schmelztemperatur des Kernmaterials aus Eisen und einigen Prozent Schwefel mit der Temperaturverteilung im Kern bestimmt werden. Die folgende Betrachtungsweise entspricht dem Vorgehen von Schubert et al. (1988) und Conzelmann (1999). Die Schmelztemperatur des Kernmaterials $T_{m}(r)$ wird mit Hilfe des Lindemann-Schmelzgesetzes in Abhängigkeit vom Druck im Kern $P(r)$ und dem Schwefelgehalt $x$ parametrisiert (vgl. Kapitel (5.6), Stevenson et al. (1983), Sohl und Spohn (1997)):

$$
T_{m}(r)=T_{m 0}\left(1-\alpha_{S} x\right)\left(1.0145+T_{m 1} P(r)+T_{m 2} P^{2}(r)\right)
$$

$T_{m 0}, \alpha_{S}, T_{m 1}$ und $T_{m 2}$ sind darin Konstanten. Die Werte dieser Fitparameter sind in Tabelle (6.1) angegeben. Die genaue Schmelzkurve für Eisen mit einigen Prozent Schwefel

\begin{tabular}{|l|c|c|c|c|}
\hline & $T_{m 0}$ & $\alpha_{S}$ & $T_{m 1}$ & $T_{m 2}$ \\
\hline Schmelzkurve I & $1809 \mathrm{~K}$ & 2 & $1.215 \cdot 10^{-11} \mathrm{~Pa}^{-1}$ & $-8 \cdot 10^{-23} \mathrm{~Pa}^{-2}$ \\
\hline Schmelzkurve II & $1809 \mathrm{~K}$ & 2.4 & $1.54 \cdot 10^{-11} \mathrm{~Pa}^{-1}$ & $-11.7 \cdot 10^{-23} \mathrm{~Pa}^{-2}$ \\
\hline
\end{tabular}

Tabelle 6.1: Fitparameter für die Schmelzkurve des Eisens mit Schwefelbeimischungen.

ist bislang noch nicht für die Druck- und Temperaturbedingungen im Merkurkern bestimmt worden (Boehler, persönliche Mitteilung), so dass die genaue Schmelzpunkterniedrigung durch geringe Schwefelbeimischungen in diesem Druckbereich nicht bekannt ist. Messungen am Eutektikum zeigen, dass sich bei sehr hohen Drücken (z.B. im Erdkern) die Schmelzkurven von reinem Eisen und der eutektischen Verbindung annähern 
und die Schmelzpunkterniedrigung vernachlässigbar wird (Boehler 1996b). Im Rahmen dieser Arbeit werden zwei verschiedene Parametrisierungen der Schmelzkurve betrachtet (vgl. Tabelle (6.1)). Die Parameter für 'Schmelzkurve I' stimmen mit den von Conzelmann (1999) benutzten Werten überein und sind an die Labordaten von Boehler (1996a,c) angepasst. Die mit diesem Parametersatz ('Schmelzkurve I') parametrisierten Schmelzkurven sind für einen Schwefelgehalt von 2 Gew.-\% sowie für reines Eisen zusammen mit der Kernadiabate (siehe unten) für zwei verschiedene Temperaturen an der KMG in Abbildung (6.1) dargestellt. Der gezeigte Druckbereich entspricht dem Druckintervall im Merkurkern. Die Schmelztemperatur im Zentrum des Planeten (40 GPa) wird durch den Schwefelgehalt von 2 Gew.-\% in dieser Parametrisierung um etwa $100 \mathrm{~K}$ erniedrigt. Für die höhere Adiabate ist der Kern bei 2 Gew.-\% Schwefel komplett flüssig.

In der zweiten Parametrisierung ('Schmelzkurve II') sind die Parameter an Hauck et al. (2004) angelehnt, die die experimentellen Ergebnisse für die Schmelzbedingungen der FeFeS-Legierung unter Berücksichtigung der Hochdruckphasen von Fei et al. (1997) bzw. Fei et al. (2000b) anfitten. Diese Parametrisierung wird in Abschnitt (6.3.2) im Zusammenhang mit dem Einfluss des Schwefelgehaltes verwendet. Abbildung (6.15) vergleicht die Schmelzkurven mit beiden Parametrisierungen für verschiedene Schwefelanteile. Bei gleichem Schwefelgehalt liegen die Schmelztemperaturen mit der zweiten Parametrisierung ('Schmelzkurve II') deutlich höher.

Für das Temperaturprofil im Kerninneren $T_{\text {Kern }}(r)$ wird angenommen, dass es ausgehend

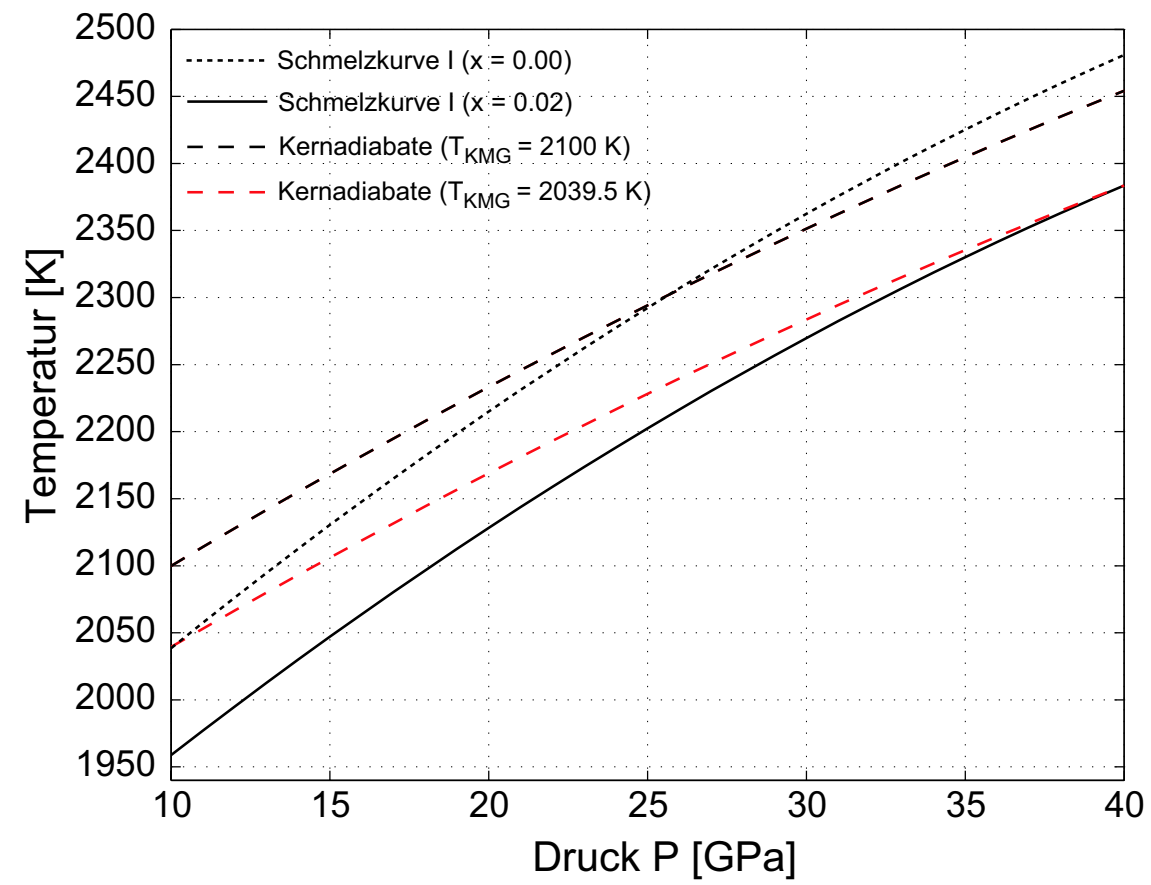

Abbildung 6.1: Parametrisierte Schmelzkurven ('Schmelzkurve I') für reines Eisen $(x=0.0)$ und Eisen mit 2 Gew.-\% Schwefel $(x=0.02)$ sowie Kernadiabaten mit $T_{\mathrm{KMG}}=2100 \mathrm{~K}$ und $T_{\mathrm{KMG}}=2040 \mathrm{~K}$.

von der Temperatur $T_{\mathrm{KMG}}\left(T_{c}(t)=T_{\mathrm{KMG}}(t)\right)$ an der Kernoberfläche der Kernadiabate 
folgt. Nichtadiabatische Temperatureffekte bzw. Grenzschichten werden dabei vernachlässigt. Die Kernadiabate ist durch folgende Beziehung (Stevenson et al. 1983) gegeben:

$$
T_{\mathrm{Kern}}(r)=T_{\mathrm{KMG}}(t)\left(\frac{1+T_{a 1} P(r)+T_{a 2} P^{2}(r)}{1+T_{a 1} P_{\mathrm{KMG}}+T_{a 2} P_{\mathrm{KMG}}^{2}}\right)
$$

$P_{\mathrm{KMG}}$ ist dabei der Druck an der KMG und $T_{a 1}, T_{a 2}$ sind Konstanten, deren Werte (Stevenson et al. 1983) in Tabelle (A.3) im Anhang gegeben sind.

Die Starttemperatur an der KMG $T_{\mathrm{KMG}}(0)$ ist so hoch, dass der Kern zu Beginn ganz geschmolzen ist. Kühlt der Kern im Lauf der Evolution ab und fällt die Temperatur im Planetenzentrum unter die Schmelztemperatur, so bildet sich ein fester innerer Kern aus (vgl. Abbildung (6.1)). Der Radius lässt sich aus dem Druck $P_{i K}$ bestimmen, bei dem die Kernadiabate die Schmelzkurve schneidet. Dieser hydrostatische Druck lässt sich auf dem Druck $P_{z}$ im Planetenzentrum zurückführen:

$$
P_{i K}=P_{z}-\frac{1}{2} \rho_{\text {Kern }} g_{\mathrm{KMG}} \frac{r_{i K}^{2}}{r_{i}}
$$

$\rho_{\text {Kern }}$ ist die als homogen angenommene Dichte des Kernmaterials, $r_{i}$ der Kernradius und $g_{\mathrm{KMG}}$ der Wert der Schwerebeschleunigung an der KMG. Dichteunterschiede zwischen dem inneren festen Kern und dem flüssigen Kern werden dabei vernachlässigt (Stevenson et al. 1983). Damit kann der Radius des inneren Kerns $r_{i K}$ bestimmt werden:

$$
r_{i K}=\sqrt{\frac{2\left(P_{z}-P_{i k}\right) r_{i}}{\rho_{\mathrm{Kern}} g_{\mathrm{KMG}}}}
$$

Für die Schwerebeschleunigung an der KMG $\left(g_{\mathrm{KMG}}\right)$ gilt dabei:

$$
g_{\mathrm{KMG}}=g \rho_{\mathrm{Kern}}\left(\frac{r_{i} / r_{a}}{\rho_{\mathrm{Kern}}\left(r_{i} / r_{a}\right)^{3}+\rho_{0}\left(1-\left(r_{i} / r_{a}\right)^{3}\right)}\right)
$$

Hier ist $g$ die Oberflächenwert der Schwerebeschleunigung und $\rho_{0}$ die Manteldichte. Die Änderung des Radius des inneren Kerns mit der Temperatur an der KMG $d r_{i K} / d T_{\mathrm{KMG}}$ kann berechnet werden, indem die Gleichungen (6.1) und (6.2) beim Übergangsdruck zum inneren Kern gleichgesetzt werden und der Ausdruck nach $d / d T_{\mathrm{KMG}}$ differenziert wird. Damit kann die zeitliche Änderung der Größe des inneren Kerns nach folgender Beziehung berechnet werden:

$$
\frac{d r_{i K}}{d t}=\frac{d r_{i K}}{d T_{\mathrm{KMG}}} \frac{d T_{\mathrm{KMG}}}{d t}
$$

Die zeitliche Änderung der Masse des inneren Kerns $m$ kann damit bestimmt werden:

$$
\frac{d m}{d t}=4 \pi r_{i K}^{2} \rho_{\mathrm{Kern}} \frac{d r_{i K}}{d t}=4 \pi r_{i K}^{2} \rho_{\mathrm{Kern}} \frac{d r_{i K}}{d T_{\mathrm{KMG}}} \frac{d T_{\mathrm{KMG}}}{d t}
$$

Durch das Wachsen des inneren Kerns, der aus reinem Eisen besteht, reichert sich der Schwefel im verbleibenden flüssigen Teil des Kerns an, so dass die Schmelztemperatur 
sinkt. Dies kann in der Parametrisierung der Schmelzkurve (Gleichung (6.1)) berücksichtigt werden. Der Schwefelanteil $x$ beträgt (Stevenson et al. 1983):

$$
x=x_{0} \frac{M_{\text {Kern }}}{M_{\text {Kern }}-m}=\frac{x_{0} r_{i}^{3}}{r_{i}^{3}-r_{i K}^{3}}
$$

$x_{0}$ ist dabei der Schwefelanteil zu Beginn (ohne inneren Kern) und $M_{\text {Kern }}$ ist die Gesamtmasse des Kerns.

Beim Ausfrieren eines inneren Kerns wird pro Masseneinheit die latente Wärme $L$ frei. Der Wert der latenten Wärme hängt von der Schmelztemperatur und der Schmelzentropie ab. Vereinfacht wird in den Modellen jedoch ein konstanter Wert von $L=2.5 \cdot 10^{5} \mathrm{~J} / \mathrm{kg}$ für die latente Wärme pro Masseneinheit verwendet.

Bei der Bildung des festen inneren Kerns reichert sich der Schwefel zunächst in einer Grenzschicht um den inneren Kern an. Da der Schwefel relativ zu dem ihn umgebenden Material leichter ist, steigt er auf und vermischt sich mit dem übrigen Kernmaterial. Durch diese chemische Konvektionsbewegung wird Gravitationsenergie freigesetzt. Schubert et al. (1988) haben diese Gravitationsenergie pro Masseneinheit unter Berücksichtigung der Dichteunterschiede zwischen äußerem und inneren Kern berechnet:

$$
E_{G}=\frac{2 \pi G r_{i}^{2} x_{0} \Delta \rho}{\left(1-\xi^{3}\right)^{2}}\left(\frac{\rho_{\text {Kern }}}{\rho_{S}}\right)\left[\frac{1}{5}\left(1-\xi^{5}\right)-\frac{\xi^{2}}{3}\left(1-\xi^{3}\right)\right]
$$

dabei ist $G$ die Gravitationskonstante, $\Delta \rho$ der Dichteunterschied zwischen Eisen und Schwefel und $\xi=r_{i K} / r_{i}$. Durch das Wachsen eines inneren Kerns wird damit folgende Leistung frei:

$$
P_{G}=E_{G} \frac{d m}{d t}+L \frac{d m}{d t}
$$

Diese zusätzliche Leistung muss nun in der Leistungsbilanz für den Kern (vgl. Gleichung (3.68)) berücksichtigt werden. Die Temperaturänderung an der KMG wird durch den Wärmefluss aus dem Kern und die bei der Bildung des inneren Kerns produzierten Wärme bestimmt:

$$
V_{\mathrm{Kern}} \rho_{\mathrm{Kern}} C_{\mathrm{Kern}} \frac{d T_{\mathrm{KMG}}}{d t}=A_{\mathrm{Kern}} \lambda \frac{d T_{\mathrm{KMG}}}{d r}-\left(L+E_{G}\right) \frac{d m}{d t}
$$

( $C_{\text {Kern }}$ ist so gewählt, dass $T_{\mathrm{KMG}}$ statt der volumen-gemittelten Temperatur im Kern verwendet werden kann.) Mit dem Ausdruck für die zeitliche Änderung der Masse des inneren Kerns (Gleichung (6.7)) gilt dann für die zeitliche Entwicklung der Temperatur an der KMG:

$$
\frac{d T_{\mathrm{KMG}}}{d t}=\frac{A_{\mathrm{Kern}} \lambda\left(d T_{\mathrm{KMG}} / d r\right)}{V_{\mathrm{Kern}} \rho_{\mathrm{Kern}} C_{\mathrm{Kern}}-\left(L+E_{G}\right) 4 \pi r_{i}^{2} \rho_{\mathrm{Kern}} d r_{i} / d T_{\mathrm{KMG}}}
$$

Die Gleichungen (6.6) und (6.12) bilden ein System von zwei gekoppelten Differentialgleichungen, die mit dem Runge-Kutta-Verfahren vierter Ordnung gelöst werden. Die Größe des Zeitschritts wird durch die Wachstumsrate des inneren Kerns beschränkt. Zum Test der Implementation wird die Energiebilanz des Kerns überprüft.

Ähnlich wie die Evolutionsmodelle für Mars starten die Simulationen für Merkur von einem Grenzschichtprofil mit dünnen Grenzschichten und hohen Temperaturen im Inneren, das vereinfacht die Situation nach der Akkretion und Differentiation beschreiben 
soll (Abbildung (6.2)). Insbesondere ist die Temperatur an der KMG zu Beginn mit 2100 K so hoch gewählt, dass der gesamte Kern flüssig ist. Die Geometrie der überlagerten Störungen bleibt gegenüber den Modellen für Mars unverändert (Kapitel (3.9)). Die interne Heizrate beträgt $H_{0}=5.2373 \cdot 10^{-8} \mathrm{~W} / \mathrm{m}^{3}$ zu Beginn. Akkretionsmodelle für Merkur weisen auf hohe Verlustraten für Kalium und Uran bei der Entstehung des Planeten hin, so dass wahrscheinlich Thorium das dominierende radioaktive Element ist (Cameron et al. (1988), Conzelmann (1999)). Wie in den Marsmodellen (vgl. Kapitel (3.8)) wird auch in den Simulationen für Merkur nicht zwischen den einzelnen radioaktiven Elementen unterschieden. Für die Zerfallszeit wird hier der Wert für Thorium verwendet $\left(\lambda^{*}=0.0495(\mathrm{Ga})^{-1}\right)$.

Die Viskositätsparameter entsprechen der Rheologie I bei Conzelmann (1999) mit $E^{*}=$ $466 \mathrm{~kJ} / \mathrm{mol} \mathrm{K}, V^{*}=8.02 \mathrm{~cm}^{3} / \mathrm{mol}$. Die Referenzviskosität von $\eta_{\text {ref }}=8.1 \cdot 10^{22} \mathrm{~Pa} \mathrm{~s}$ wird in halber Manteltiefe bei einer Temperatur von 1573 K erreicht. Schubert et al. (1988) merken an, dass der Merkurmantel wahrscheinlich steifer ist und bei gleichen Druck- und Temperaturbedingungen, wie in der Erde höhere Viskositäten zu erwarten sind.

Das 'cutoff'-Verfahren für die Viskosität ist ebenfalls dem Verfahren bei Conzelmann (1999) angeglichen:

$$
\eta(r)=\frac{\eta_{0} \eta_{\infty}}{\eta_{\infty} \exp \left(-\frac{E^{*}+\rho_{0} g\left(r_{a}-r\right) V^{*}}{R<T(r)>}\right)+\eta_{0}}+\eta_{1}
$$

Dabei ist $\eta_{\infty}$ der obere und $\eta_{1}$ der untere Grenzwert der Viskosität (siehe Tabelle (A.3) im Anhang). Die Rayleighzahl bei Normbedingungen beträgt damit $R a=698$. Die verwendeten Materialparameter sind in Tabelle (A.3) im Anhang zusammengestellt und der Arbeit von Conzelmann (1999) bzw. den dortigen Quellen entnommen.

Merkur ist wie Mars ein Ein-Platten-Planet, so dass dieselben mechanischen Randbedingungen (Oberfläche: 'no-slip', KMG: 'free-slip', (Kapitel (3.9)) verwendet werden. (Conzelmann (1999) nimmt beide Grenzflächen als frei Ränder an.) Die Temperatur an der KMG wird nach Gleichung (6.12), wie beschrieben, in jedem Zeitschritt neu bestimmt, wohingegen die Temperatur $T_{0}$ an der Oberfläche konstant $440 \mathrm{~K}$ beträgt.

Die Gitterparameter für die räumliche Auflösung sind unverändert gegenüber den Marssimulationen (Kapitel (4.2)). Allerdings wird eine lineare Abbildungsfunktion zwischen den radialen Leveln und den Tschebyscheff-Gitterpunkten verwendet (Gleichung (4.8)).

\subsection{Ergebnisse der Evolutionsmodelle für Merkur}

\subsubsection{Einfluss des Temperaturstartprofi ls}

In diesem Abschnitt wird der Einfluss des Starttemperaturprofils auf die thermische Entwicklung und das Ausfrieren eines inneren Kerns untersucht. Die Startprofile der ersten Gruppe (durchgezogene Linien in den folgenden Abbildungen) unterscheiden sich durch die Temperatur im isothermen Bereich, die zwischen 1600 und $1900 \mathrm{~K}$ in Schritten von $100 \mathrm{~K}$ variiert wird. Die Kerntemperatur bei $t=0.0 \mathrm{Ga}$ liegt in dieser Gruppe bei $2100 \mathrm{~K}$. Die Modelle der zweiten Gruppe (gestrichelte Linien in Abbildung (6.2)) unterscheidet die Anfangstemperatur an der KMG. Sie liegt zwischen 2100 und 2400 K, wobei die Temperatur des isothermen Bereichs $1600 \mathrm{~K}$ ist. Abbildung (6.2) zeigt die unterschiedlichen 
betrachteten Starttemperaturprofile und Abbildung (6.3) die entsprechenden Profile der Viskosität. Als Referenzfall gilt das Profil mit $1600 \mathrm{~K}$ in der halben Manteltiefe und einer Startkerntemperatur von $2100 \mathrm{~K}$.

In den Simulationen in diesem Abschnitt wird die Schmelzkurve mit den Fitparametern

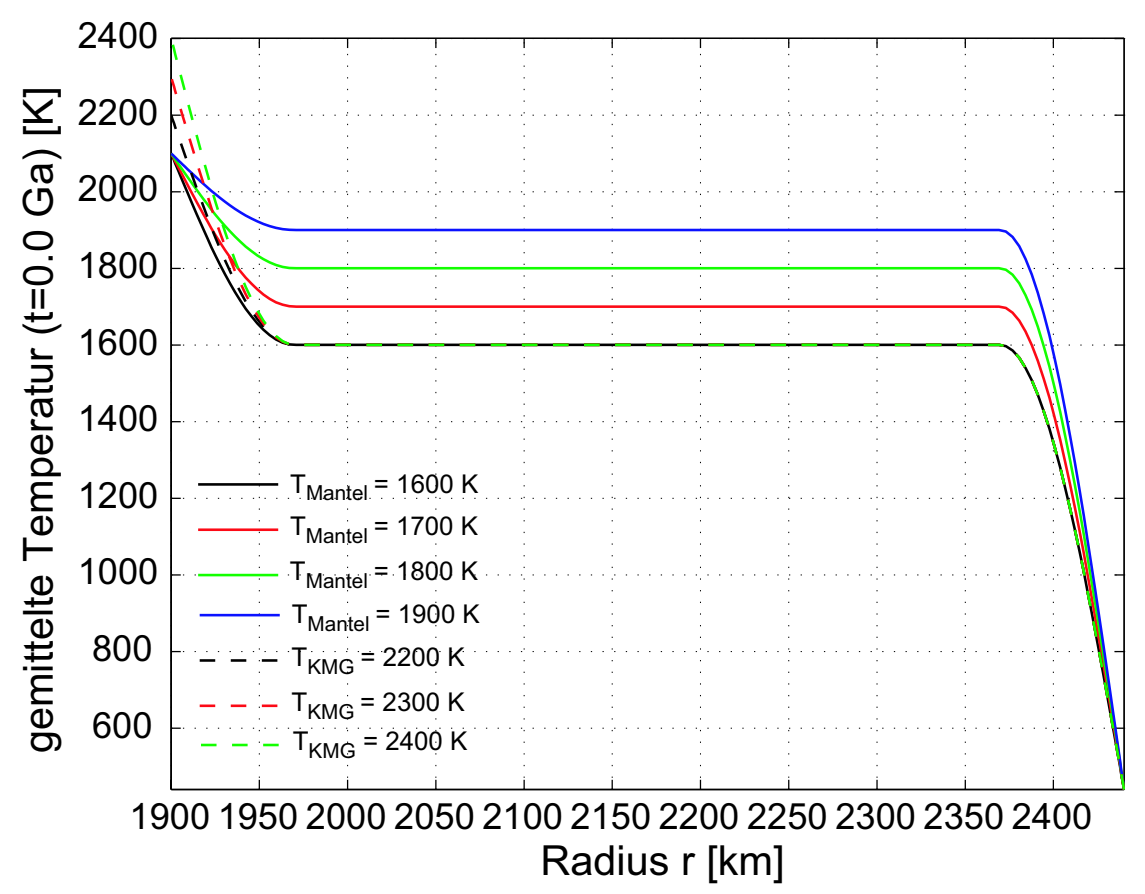

Abbildung 6.2: Radiale Profile der gemittelten Temperatur zum Zeitpunkt $t=0.0$ Ga.

für 'Schmelzkurve I' (Tabelle (6.1)) parametrisiert. Der anfängliche Schwefelgehalt beträgt $x_{0}=0.02$ (2 Gew.-\%). Die Referenz-Rayleighzahl ist 698 .

In Abbildung (6.4) ist der Einfluss des Starttemperaturprofils auf die zeitliche Entwicklung eines festen inneren Kerns dargestellt. Das Erhöhen der Manteltemperatur führt tendenziell zu einem verspäteten Einsetzen des Kernwachstums, so dass bei einer $300 \mathrm{~K}$ höheren Manteltemperatur der innere Kern erst nach $1.45 \mathrm{Ga}$ und damit etwa $0.71 \mathrm{Ga}$ später als im Referenzfall auszufrieren beginnt. Dieser verspätete Einsatz ist auf die unterschiedlichen Abkühlraten des Kerns (Abbildung (6.5)) durch die verschiedenen anfänglichen Manteltemperaturen zurückzuführen. Höhere Manteltemperaturen verringern den Temperaturgradienten an der KMG und reduzieren den anfänglichen Wärmefluss aus dem Kern (Abbildung (6.6)), so dass zunächst der Kern im Referenzfall am schnellsten abkühlt. Die höheren Manteltemperaturen führen durch die geringeren Viskositäten zu einem früheren Einsatz der Konvektion, der durch ein relatives Maximum im Wärmefluss an der KMG (Abbildung (6.6)) und damit verbunden einer starken Änderung in der Abkühlrate des Kerns (Abbildung (6.5)) deutlich wird. Das frühere Einsetzen der Konvektion kompensiert zeitweilig das anfänglich langsamere Abkühlen. Bei $T_{\text {Mantel }}(0)=1700 \mathrm{~K}$ ist dieser Effekt so stark, dass die zur Ausbildung des festen inneren Kerns nötigen Temperatur hier noch vor dem Referenzfall erreicht wird. In den übrigen Fällen mit erhöhter Mantelstarttemperatur kann die Konvektion aufgrund des geringen Temperaturkontrasts zwischen 


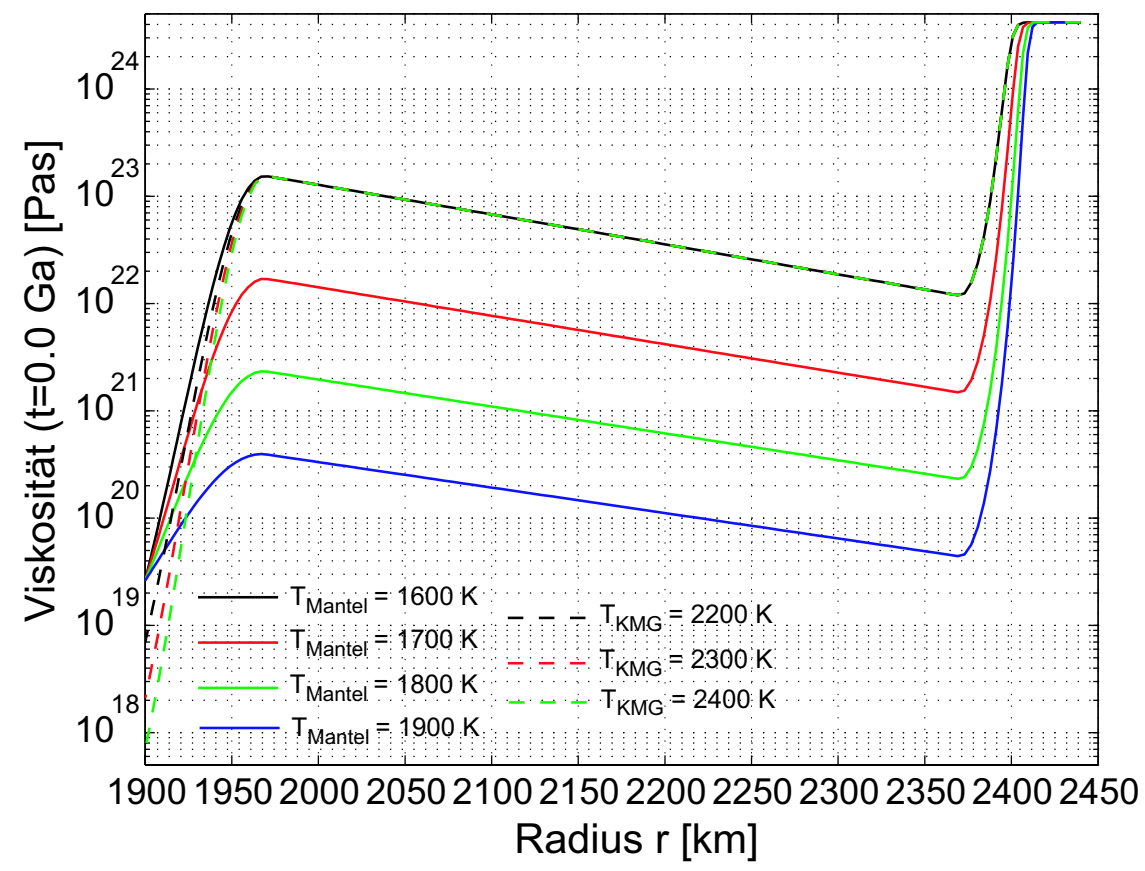

Abbildung 6.3: Radiale Profile der Viskosität zum Zeitpunkt $t=0.0 \mathrm{Ga}$.

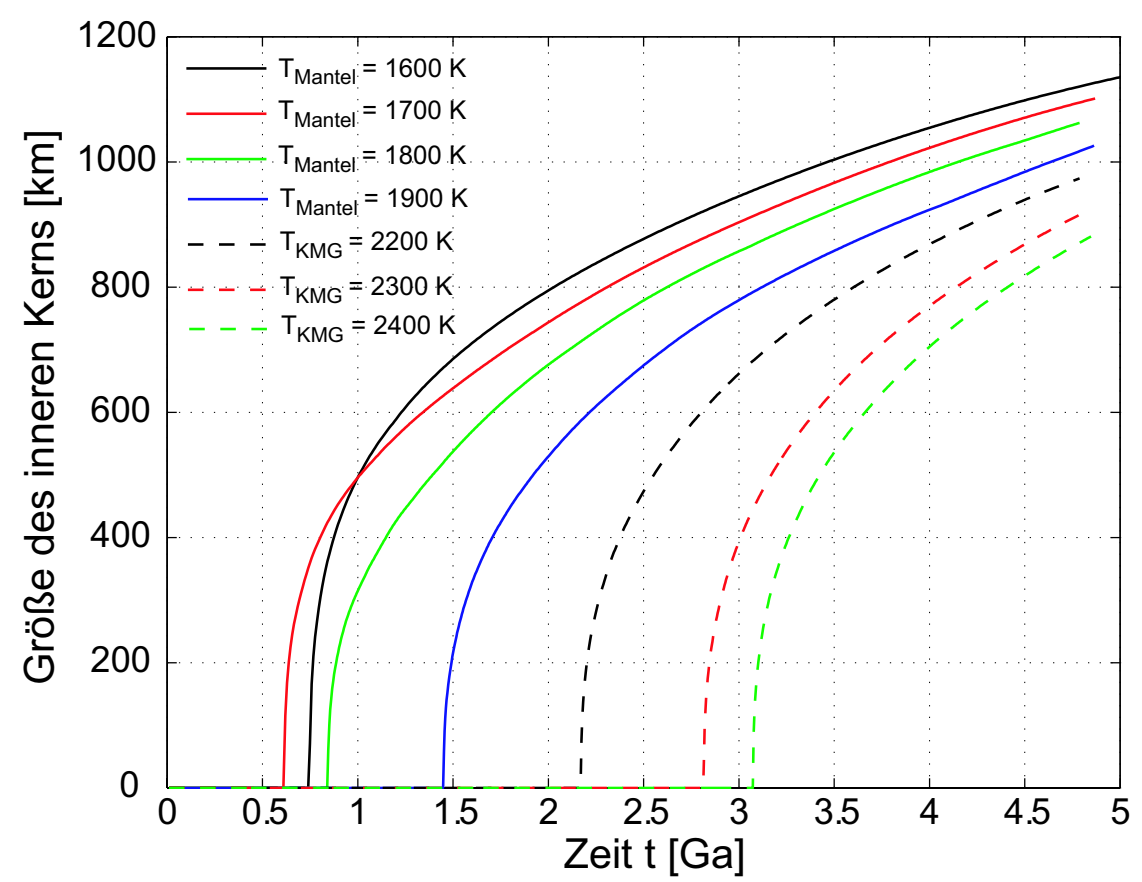

Abbildung 6.4: Zeitliche Entwicklung der Größe des festen inneren Kerns. 
Kern und Mantel die Wärme nicht effektiv vom Kern abtransportieren, so dass das Erhöhen der Manteltemperatur zu geringeren Abkühlraten des Kerns und damit zu einem verspäteten Ausbilden des festen inneren Kerns führt. Mit einer höheren Startmanteltemperatur liegt die volumen-gemittelte Temperatur (Abbildung (6.7)) während der gesamten Entwicklung etwas höher als im Referenzfall. Die damit verbundenen Unterschiede in der Viskosität und der Konvektionsstärke führen zu etwas unterschiedlichen Abkühlraten des Kerns und beeinflussen die Wachstumsrate derart, dass sich die Größen des inneren Kerns im Lauf der Evolution annähern. Nach 4.5 Ga liegen sie im Bereich zwischen 984 und $1099 \mathrm{~km}$.

Deutlicher ist der Einfluss der anfänglichen Kerntemperatur. Bei einer Erhöhung der Kerntemperatur um $300 \mathrm{~K}$ verzögert sich der Einsatz der Kernbildung um $2.33 \mathrm{Ga}$, so dass der innere Kern erst nach $3.07 \mathrm{Ga}$ zu wachsen beginnt. Die verkürzte Wachstumszeit führt zu kleineren inneren Kernen mit Radien zwischen $818 \mathrm{~km}\left(T_{\mathrm{KMG}}(0)=2400 \mathrm{~K}\right)$ und $940 \mathrm{~km}\left(T_{\mathrm{KMG}}(0)=2200 \mathrm{~K}\right)$ nach $4.5 \mathrm{Ga}$.

Die Evolution der Temperatur an der KMG (Abbildung (6.5)) verdeutlicht nochmals die

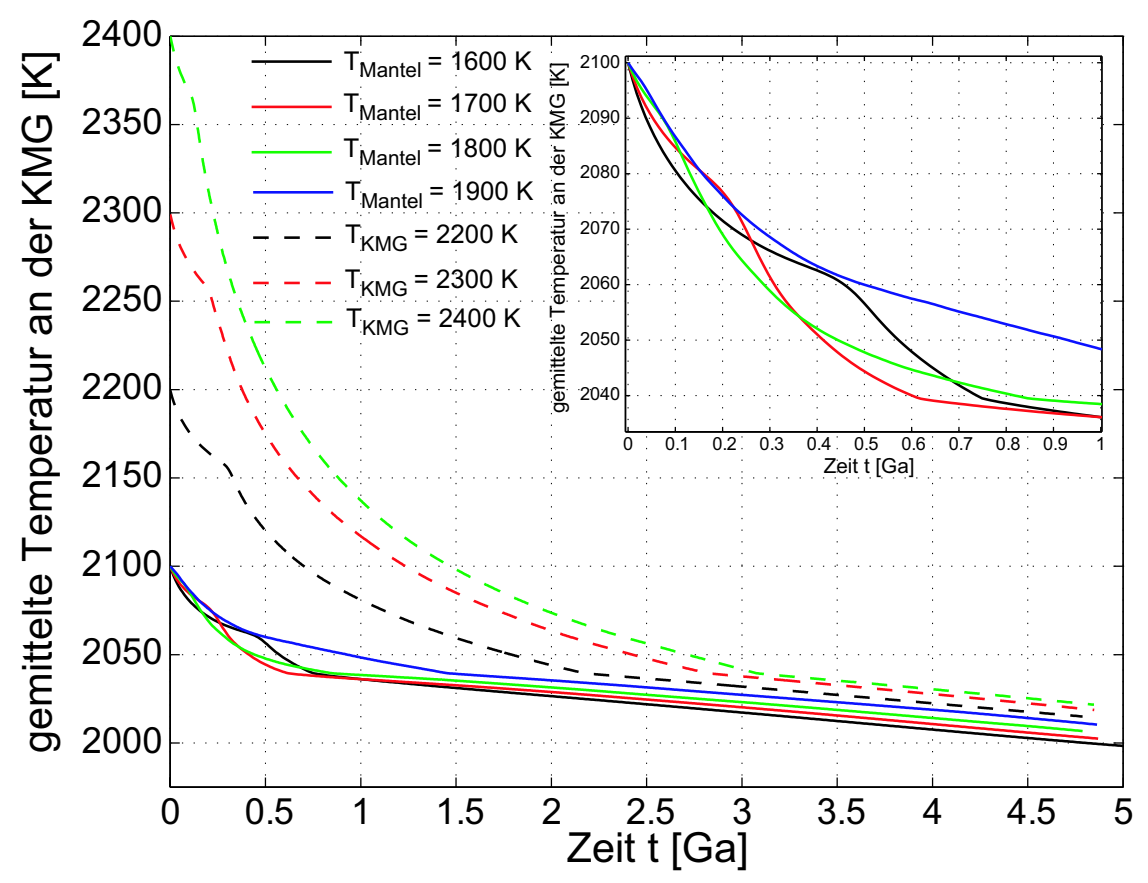

Abbildung 6.5: Zeitliche Entwicklung der Temperatur an der KMG. Das Zeitintervall von 0 bis $1 \mathrm{Ga}$ ist oben rechts zusätzlich vergrößert dargestellt.

Unterschiede in der Entwicklung der Kerngröße durch die unterschiedlichen Startprofile. Die in den meisten Kurven in den ersten $0.5 \mathrm{Ga}$ zu beobachtende sprunghafte Änderung der Steigung zeigt den Einsatz der Konvektion an. Mit dem Einsetzen des Wachstums des festen inneren Kerns nimmt die Abkühlrate des Kerns durch die freiwerdende latente und gravitative Energie ab. Nach 4.5 Ga unterscheiden sich die Temperaturen an der KMG in den verschiedenen Startmodellen nicht mehr stark. Für die verschiedenen Mantelstarttemperaturen liegt die Kerntemperatur im Intervall von 2003 K bis 2014 K. Das Erhöhen 
der Anfangskerntemperatur um $300 \mathrm{~K}$ führt nach $4.5 \mathrm{Ga}$ zu einem Unterschied von $22 \mathrm{~K}$. Der Radius des inneren Kerns nimmt in allen Fällen nach $4.5 \mathrm{Ga}$ weniger als $60 \%$ des

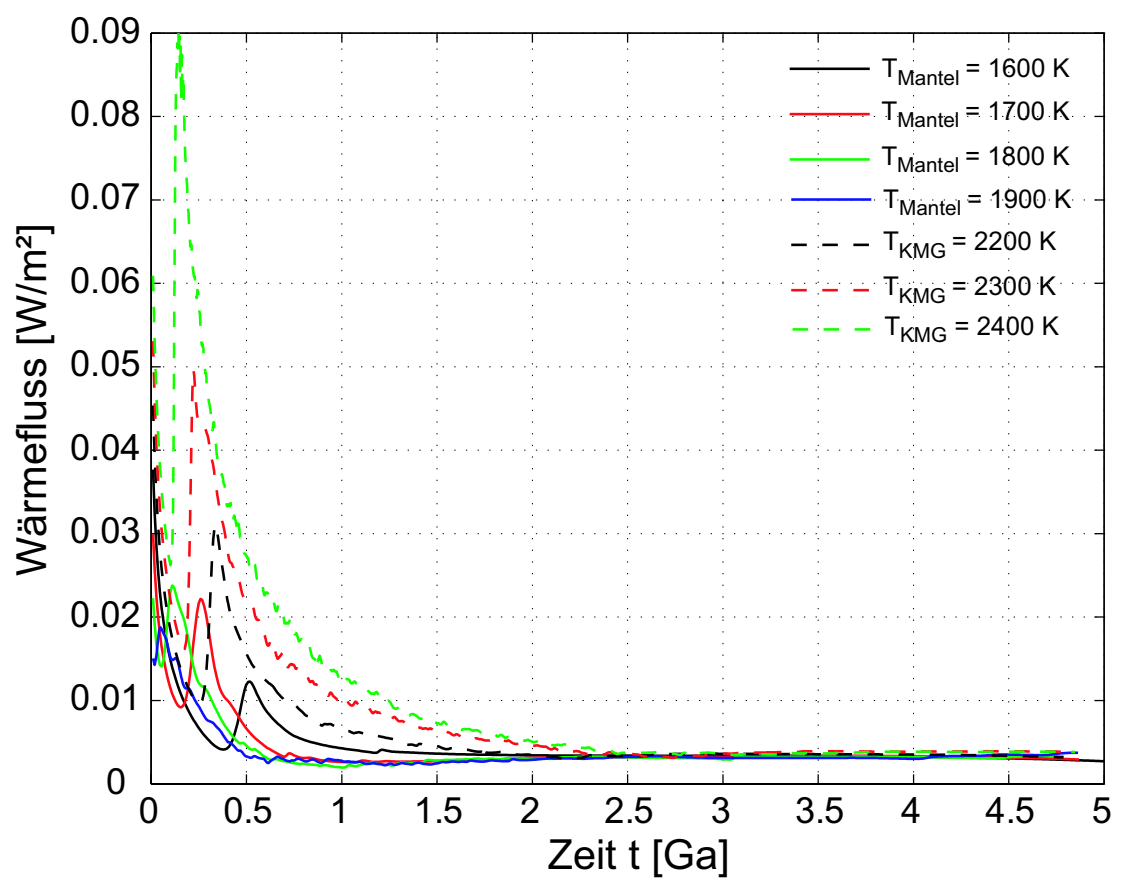

Abbildung 6.6: Zeitliche Entwicklung des Wärmeflusses an der KMG.

Gesamtkernradius ein. Der Merkurkern besitzt somit einen flüssigen äußeren Anteil, in dem eventuell chemische Konvektion einen Dynamo antreiben kann. Ein thermisch getriebener Dynamo zum heutigen Zeitpunkt ist nach den vorliegenden Modellen unwahrscheinlich, da der Wärmefluss aus dem Kern (Abbildung (6.6)) mit $3.1-3.9 \mathrm{~mW} / \mathrm{m}^{2}$ verglichen mit dem Wärmefluss durch Wärmeleitung entlang der Kernadiabate zu klein ist. Schubert et al. (1988) nehmen einen Wärmefluss von $11 \mathrm{~mW} / \mathrm{m}^{2}$ entlang der Kernadiabate im Merkur an. Die im Zusammenhang mit dem Wärmefluss entlang der Kernadiabate im Mars diskutierten Unsicherheiten der Parameter (Kapitel (2.5)) sind für Merkur wahrscheinlich ähnlich. Der geringere Druck an der KMG führt zu etwas höheren Werten des thermischen Ausdehnungskoeffizienten. Der geringere Schwefelanteil lässt eventuell auch höhere thermische Leitfähigkeiten zu. Das von Nimmo und Stevenson (2000) angegebene Intervall von $F_{\text {Kern,ad }}=5-19 \mathrm{~mW} \mathrm{~m}^{-2}$ ist so groß, dass der Wärmefluss der Kernadiabate im Merkur wahrscheinlich in diesem Intervall bzw. im höheren Bereich dieses Intervalls liegt. Mit allen betrachteten Startprofilen fällt der Wärmefluss an der KMG zunächst ab und durchläuft danach mit dem Einsatz der Konvektion ein Maximum. Mit steigender Kerntemperatur ist dieses Maximum stärker ausgeprägt. Mit einer höheren Startmanteltemperatur setzt die Konvektion durch die niedrigeren Viskositäten früher ein. Das Maximum übersteigt in allen Fällen $11 \mathrm{~mW} / \mathrm{m}^{2}$, so dass eine Phase mit einem thermisch getriebenen Dynamo möglich wäre. Nach diesem Maximum nimmt der Wärmefluss monoton ab und erreicht die oben erwähnten geringen Werte.

An der Oberfläche fällt der Wärmefluss in den ersten $0.5 \mathrm{Ga}$ sehr stark von $112 \mathrm{~mW} / \mathrm{m}^{2}$ 


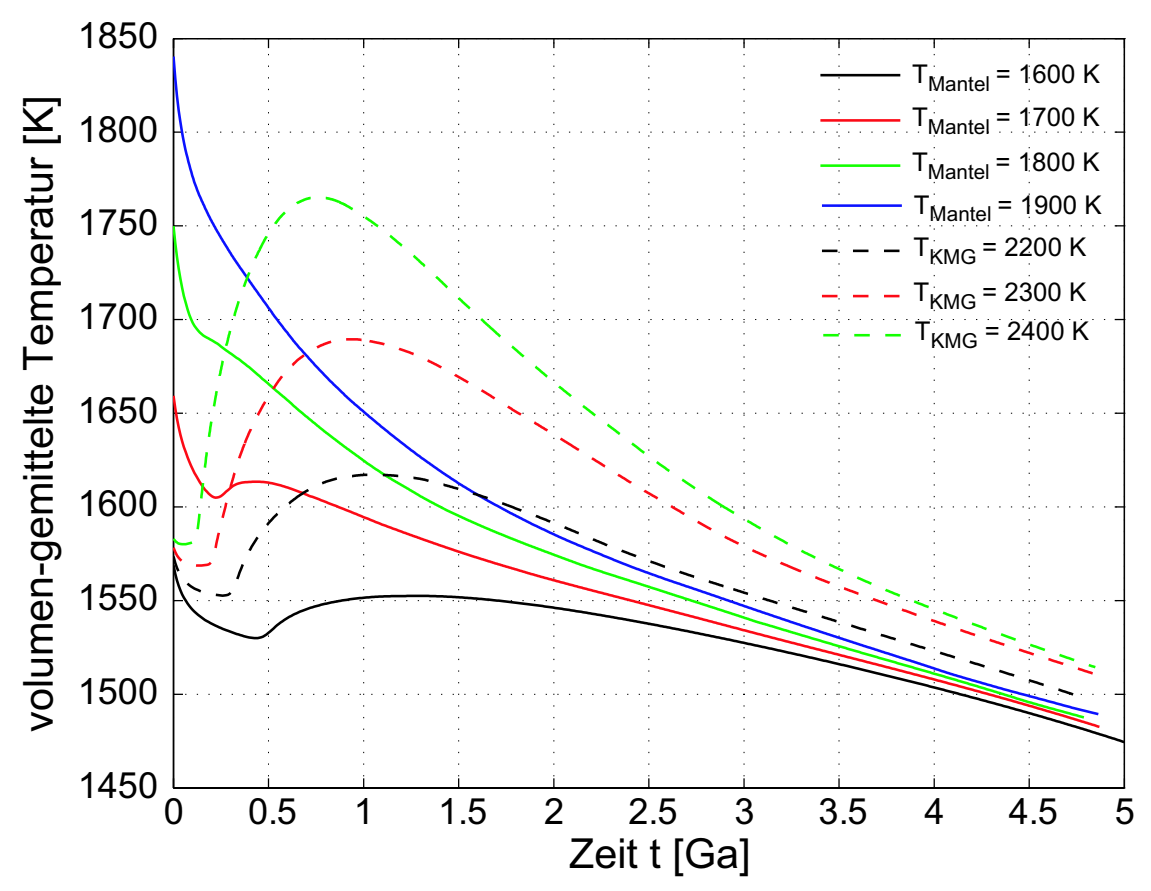

Abbildung 6.7: Zeitliche Entwicklung der volumen-gemittelten Temperatur.

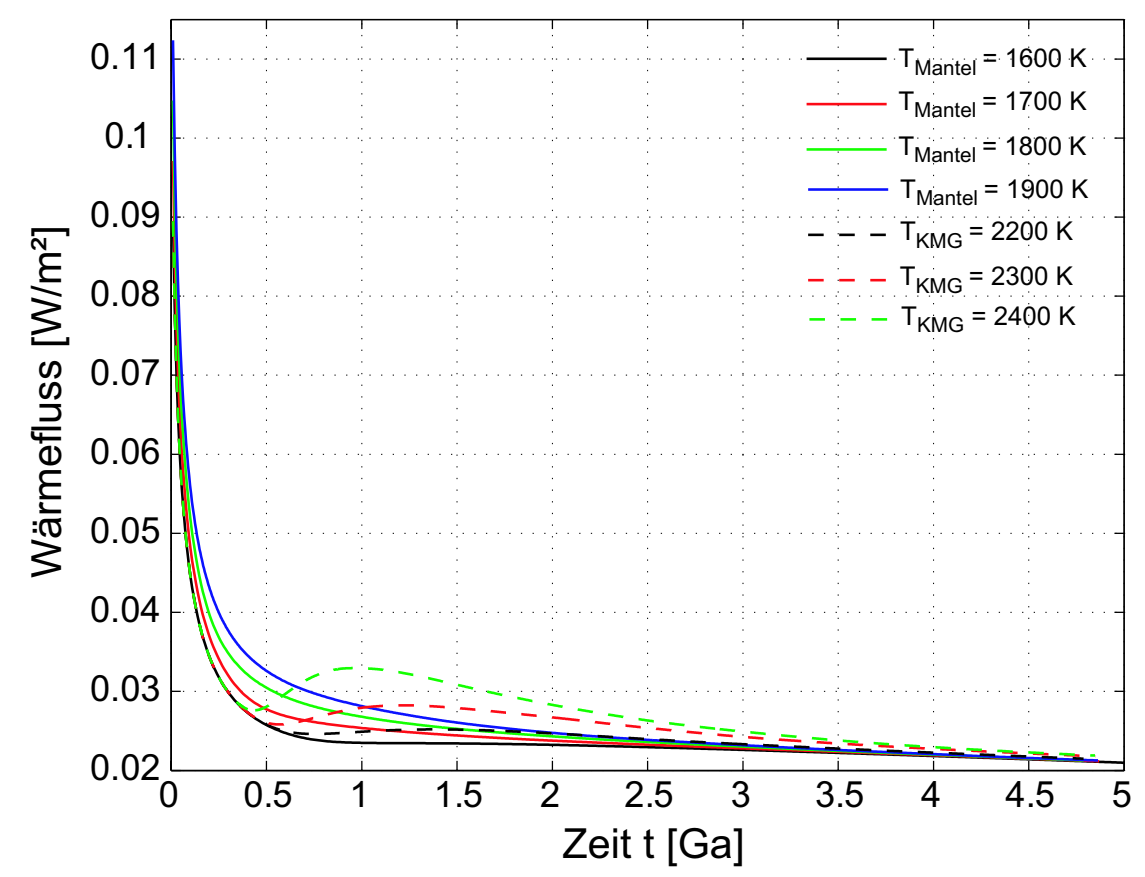

Abbildung 6.8: Zeitliche Entwicklung des Wärmeflusses an der Oberfläche. 
$\left(T_{\text {Mantel }}(0)=1900 \mathrm{~K}\right)$ bzw. $90 \mathrm{~mW} / \mathrm{m}^{2}$ (Referenzfall, Modelle mit verschiedenen Kernstarttemperaturen) auf Werte zwischen $32.6\left(T_{\text {Mantel }}(0)=1900 \mathrm{~K}\right)$ und $25.8 \mathrm{~mW} / \mathrm{m}^{2}$ (Referenzfall) ab. Danach verringert sich der Wärmefluss nur noch geringfügig, so dass die Werte für alle Startprofile nach $4.5 \mathrm{Ga}$ in dem engen Intervall $21.9 \pm 0.5 \mathrm{~mW} / \mathrm{m}^{2}$ liegen. Beim Erhöhen der Kerntemperatur steigt der Wärmefluss an der Oberfläche zwischen 0.5 und $1.0 \mathrm{Ga}$ nochmals an, bevor er monoton abfällt. Der anfänglich hohe Wärmefluss aus dem Kern führt zu höheren Temperaturen im Mantel (Abbildung (6.7)), so dass die geringeren Viskositäten die Konvektion verstärken und zeitverzögert zum erhöhten Wärmefluss an der Oberfläche führen.

Die Konvektion im Merkurmantel ist nach 4.5 Ga auf eine sehr dünne Schicht nahe

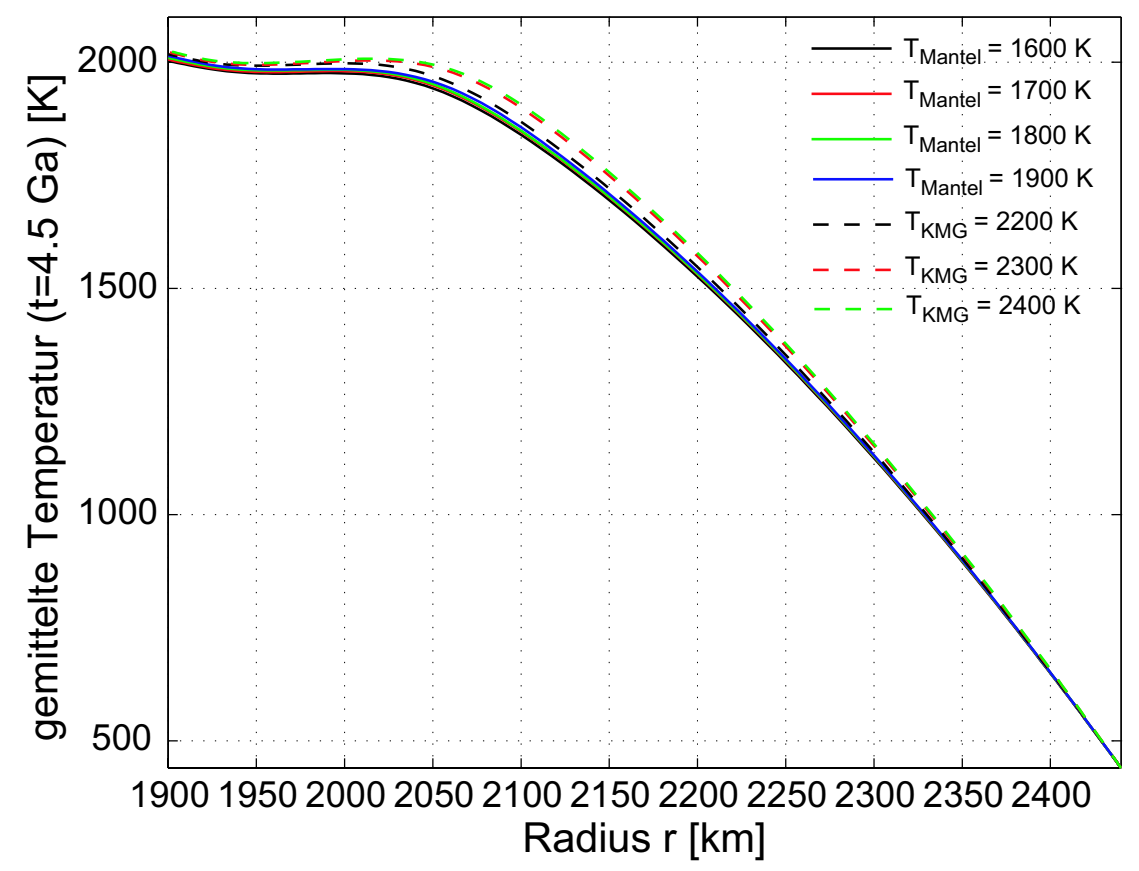

Abbildung 6.9: Radiale Profile der gemittelten Temperatur nach 4.5 Ga für die unterschiedlichen Startprofile.

der KMG beschränkt. Dies zeigen sowohl die radialen Temperaturprofile in Abbildung (6.9) als auch die meridionalen Schnitte des Temperaturfelds (Abbildungen (6.12) und (6.13)). Der Temperaturgradient an der KMG ist nur noch gering. Die isotherme Schicht, die das Innere des konvektierenden Bereichs kennzeichnet, ist vergleichsweise dünn und erstreckt sich am Ende der Evolution über ca. $150 \mathrm{~km}$. Höhere Anfangskerntemperaturen lassen eine etwas dickere Schicht zu. Der Temperaturunterschied durch die unterschiedlichen Startprofile in dieser Schicht ist gering und liegt bei etwa $20 \mathrm{~K}(r=1950 \mathrm{~km})$. Diese geringen Unterschiede am Ende der Evolution werden durch die volumen-gemittelte Temperatur (Abbildung (6.7)) ebenfalls belegt. Die Temperaturprofile werden durch eine sehr dicke thermische Grenzschicht unter der Oberfläche dominiert, die mehr als die Hälfte der Manteldicke einnimmt.

Die zeitliche Entwicklung der Temperaturprofile wird in Abbildung (6.10) für das Start- 


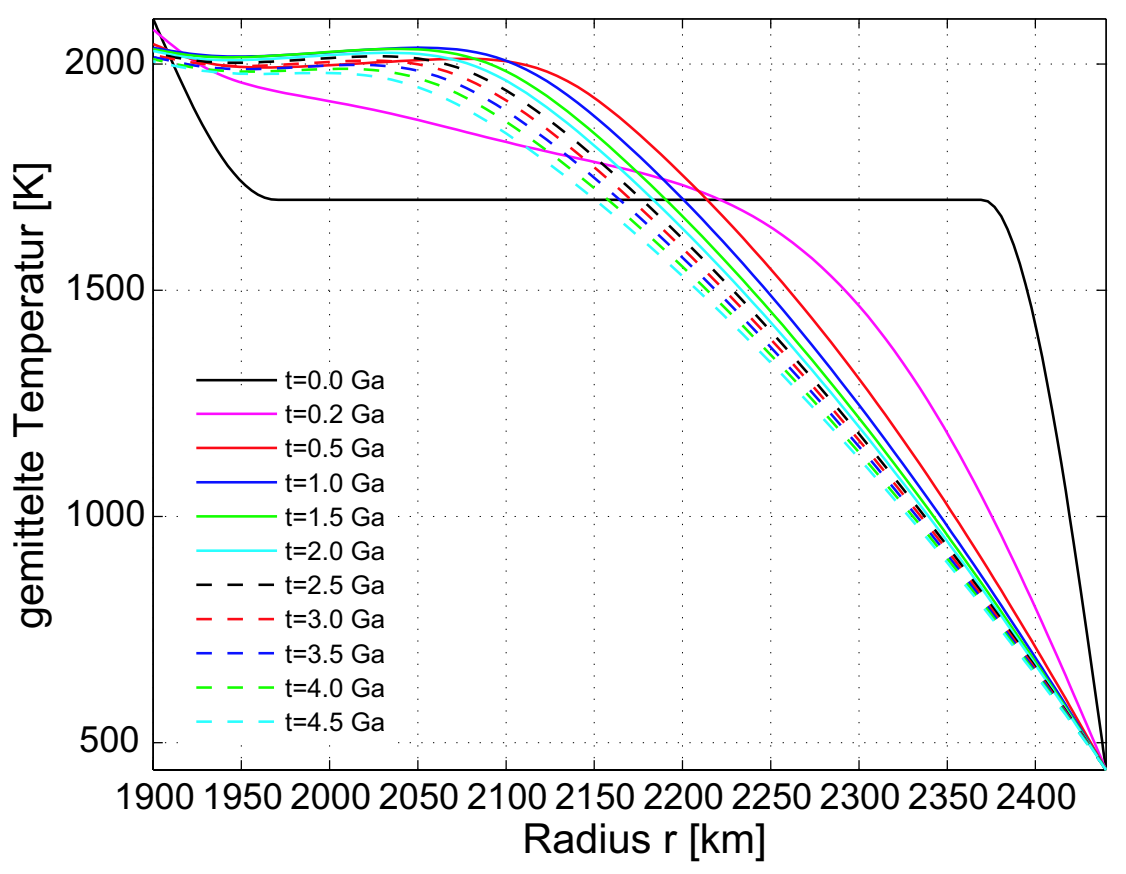

Abbildung 6.10: Zeitliche Entwicklung des radialen Temperaturprofils für das Startprofil mit $T_{\mathrm{KMG}}(0)=2100 \mathrm{~K}$ und $T_{\text {Mantel }}(0)=1700 \mathrm{~K}$. Die Legende gibt die Zuordnung zu den unterschiedlichen Zeitpunkten an.

profil mit $T_{\mathrm{KMG}}(0)=2100 \mathrm{~K}$ und $T_{\text {Mantel }}(0)=1700 \mathrm{~K}$ dargestellt. Vom Startprofil ausgehend heizt sich der untere Mantel auf, wobei gleichzeitig im oberflächennahen Bereich Wärme abgeben wird, so dass sich schon nach 0.5 bis $1.0 \mathrm{Ga}$ das charakterische Profil mit der dominanten dicken oberen Grenzschicht eingestellt hat. Das System kühlt ab, indem sich diese Grenzschicht weiter verdickt. Das schnelle Ausbilden der dicken Grenzschicht geht mit dem starken Abfall im Wärmefluss an der Oberfläche einher. Conzelmann (1999) beobachtet das schnelle Ausbilden der dicken thermischen Grenzschicht zu Beginn auch in Gleichgewichtsmodellen und schließt daraus, dass dieser Vorgang in der Frühphase der Evolution nicht direkt aus dem Abkühlen des Planeten resultiert, sondern vom Startprofil und der Rheologie abhängt.

Die Lithosphärendicke wird in den Modellen für Merkur analog zu den Marsmodellen mit Hilfe der Peclet-Zahl definiert (Kapitel (A.2)). Der Grenzwert der Geschwindigkeit $(P e=1)$ beträgt $0.0584 \mathrm{~mm} / \mathrm{a}$. Abbildung (6.11) zeigt die zeitliche Entwicklung der Lithosphärendicke. Mit Hilfe der Geschwindigkeiten kann die Lithosphäre erst nach Einsatz der Konvektion bestimmt werden, so dass die Entwicklung hier nach 0.5 Ga gezeigt wird. Der starke Wärmeeintrag in den Mantel bei einer hohen Kerntemperatur zu Beginn ermöglicht anfänglich eine dünnere Lithosphäre, die auch im Lauf der Evolution dünner als im Referenzfall bleibt. Bei einer Startkerntemperatur von $2400 \mathrm{~K}$ beträgt die Lithosphärendicke nach 4.5 Ga 278 km. Der Einfluss der unterschiedlichen Startmanteltemperaturen ist nicht so ausgeprägt. Hier werden Lithosphärendicken von $293 \pm 1 \mathrm{~km}$ erreicht. 


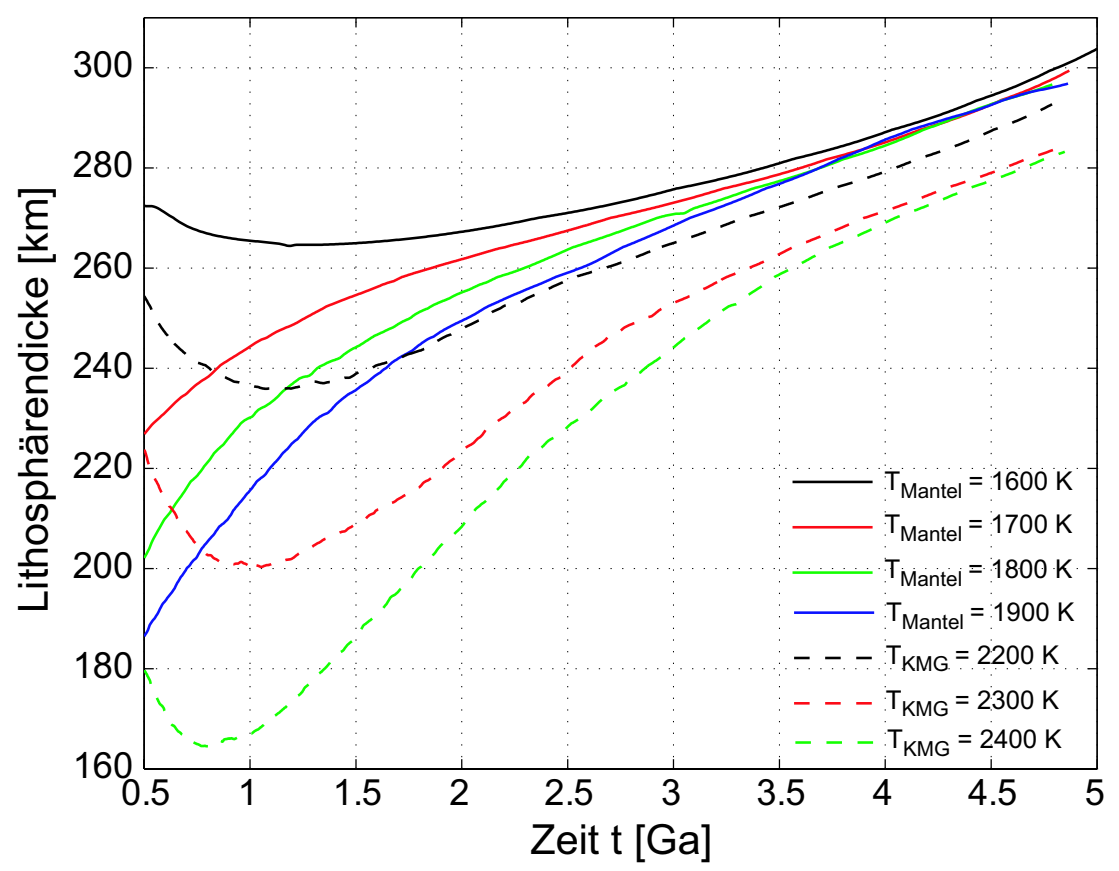

Abbildung 6.11: Zeitliche Entwicklung der Lithosphärendicke bei den unterschiedlichen Startprofilen.

Das Konvektionsmuster mit dieser dicken Lithosphäre ist durch Schnitte durch das Temperaturfeld und die Temperaturanomalie $(T-<T(r)>$ ) nach 4.5 Ga in Abbildung (6.12) für die Simulationen mit unterschiedlicher Manteltemperatur und in Abbildung (6.13) für die unterschiedlichen Kerntemperaturen im Startprofil dargestellt. Die unterschiedlichen Manteltemperaturen im Startprofil haben keinen starken Einfluss auf das Konvektionsmuster nach $4.5 \mathrm{Ga}$. Für alle Profile bildet sich ein Muster mit 10 bis 11 Auf- bzw. 10 bis 12 Abströmen ähnlicher Struktur aus. Lediglich im Fall mit der anfänglich höchsten Manteltemperatur unterscheiden sich die einzelnen Auf- und Abströme etwas stärker und sind weniger regelmäßig angeordnet. Die Variation der anfänglichen Kerntemperatur wirkt sich ebenfalls nicht stark auf das Konvektionsmuster aus. Auch hier sind 8 bis 10 Auf- bzw. 9 bis 10 Abströme erkennbar. Die absoluten Temperaturen sind in diesen Simulationen allerdings etwas höher. Mit ansteigender Kernstarttemperatur sind die Aufund Abströme etwas ungleichmäßiger. In allen Modellen ist die Temperaturanomalie der Abströme deutlich stärker als die der Aufströme, so dass die Abströme das Konvektionsmuster vorgeben. Der geringere Temperaturkonstrast an der KMG, insbesondere in den Simulationen mit niedrigen Startkerntemperaturen schwächt die Aufströme.

Das Abkühlen führt zusammen mit dem Ausfrieren eines festen inneren Kerns zu einer Volumenänderung im Planeten, die sich durch eine Veränderung im Radius bemerkbar macht. Diese Veränderung des Radius kann auf der Oberfläche durch Verwerfungen beobachtetet werden. Im Anhang (A.5) ist die Berechnung dieser Radiusänderung beschrieben. Abbildung (6.14) zeigt die Abnahme des Planetenradius mit der Zeit. Das starke Abkühlen des Kerns bei hohen Startkerntemperaturen führt zu großen Radiusänderungen 

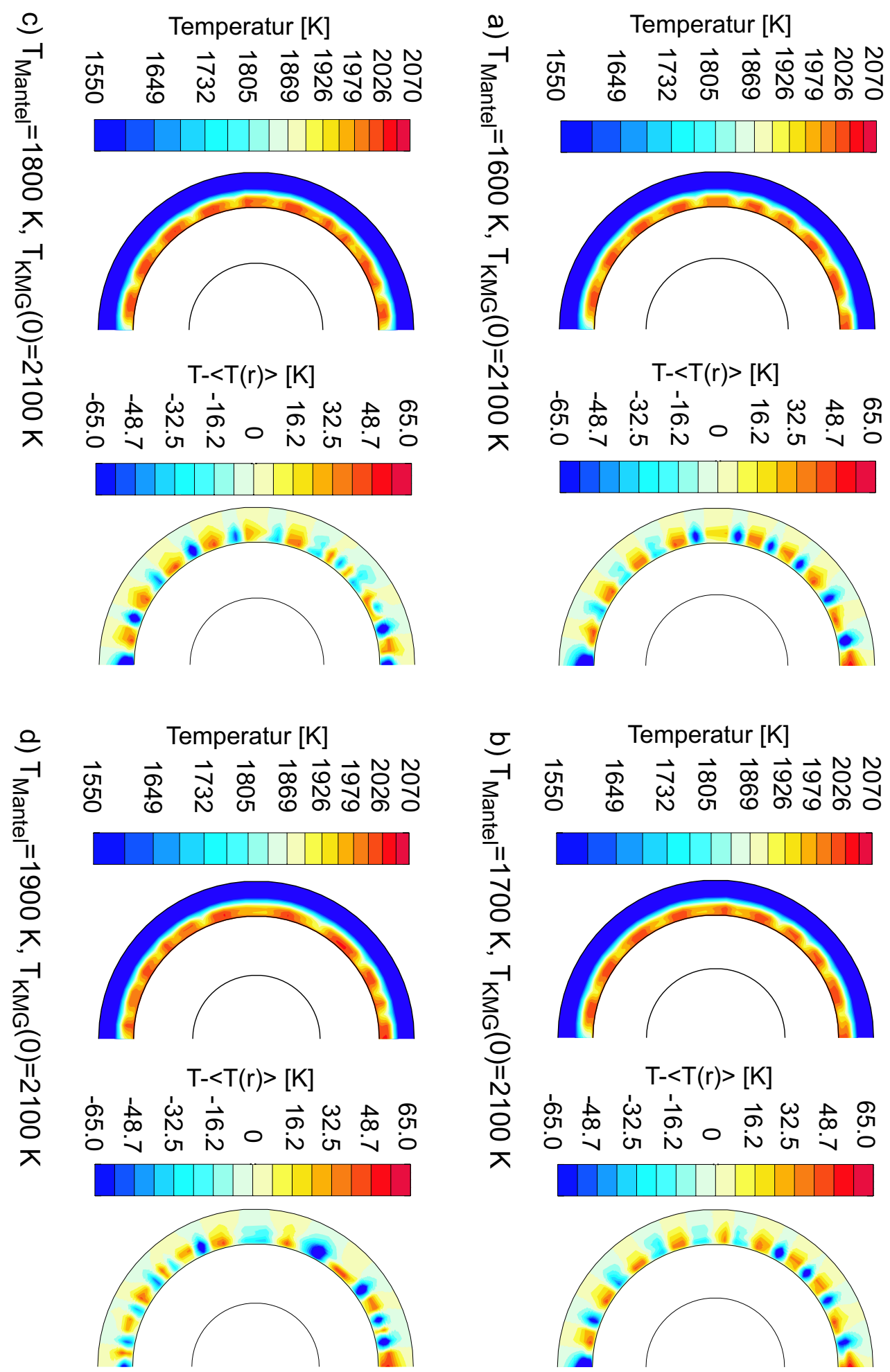

Abbildung 6.12: Schnitte durch das Temperaturfeld und die Temperaturanomalie $(T-<T(r)>)$ nach 4.5 Ga für die Simulationen mit den unterschiedlichen Manteltemperaturen im Startprofil. 

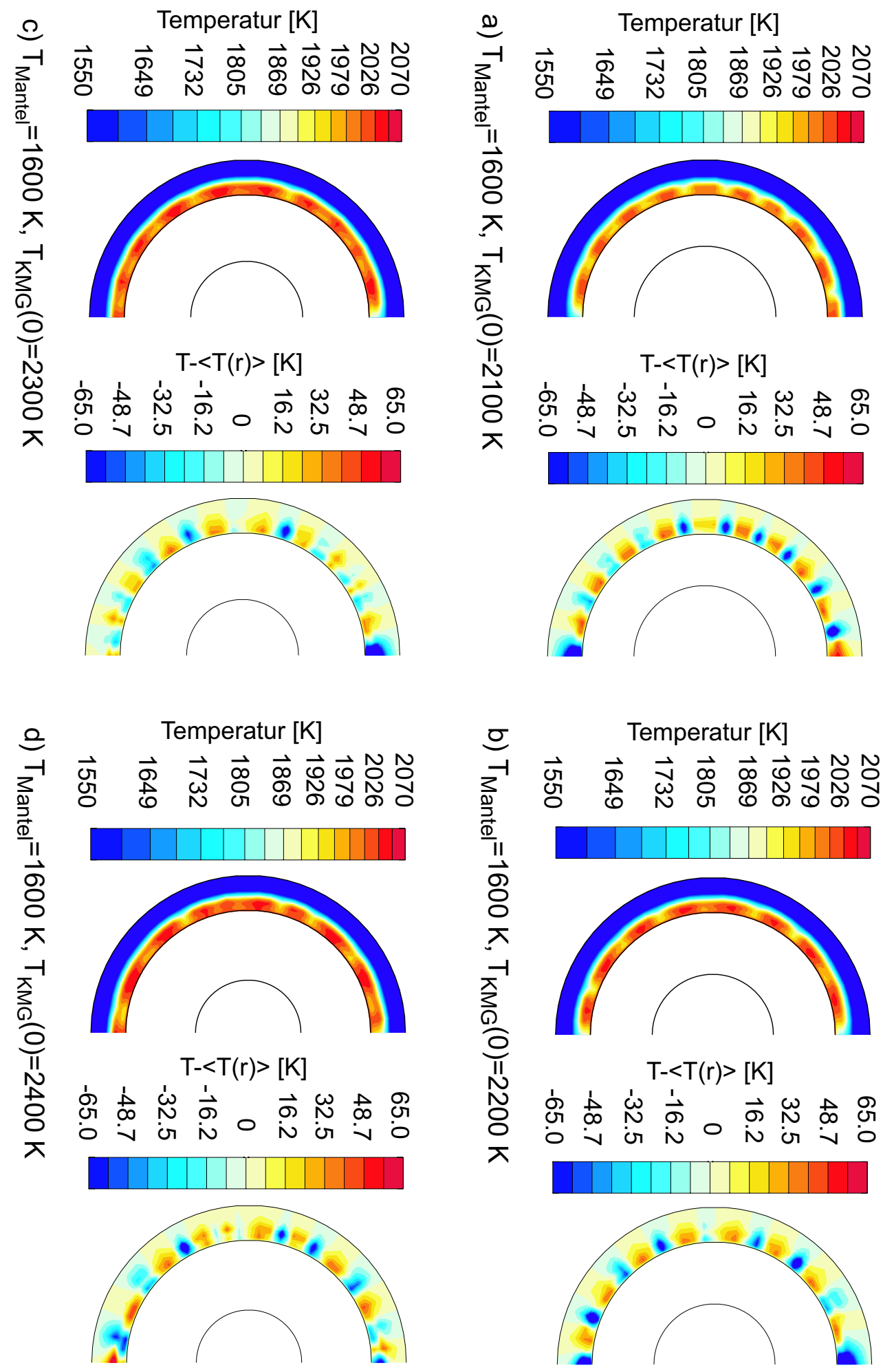

Abbildung 6.13: Schnitte durch das Temperaturfeld und die Temperaturanomalie $(T-<T(r)>)$ nach 4.5 Ga für die Simulationen mit den unterschiedlichen Kerntemperaturen im Startprofil 


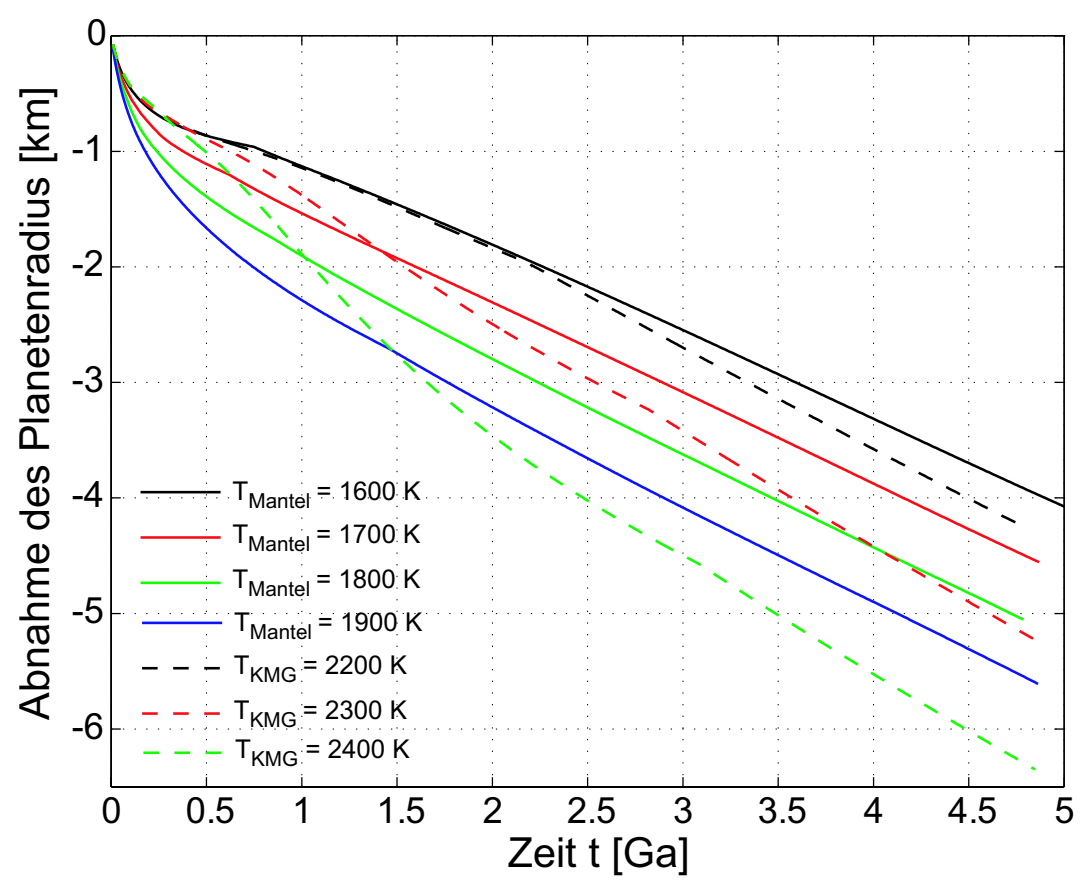

Abbildung 6.14: Abnahme des Planetenradius mit der Zeit.

von bis zu $6.0 \mathrm{~km}$. Bei den unterschiedlichen Startmanteltemperaturen werden nach 4.5 Ga sehr ähnliche Kerntemperaturen und Radien für den inneren Kern erreicht, so dass im Wesentlichen das verschieden starke Abkühlen des Mantels für die Unterschiede in der Änderung des Planetenradius veranwortlich ist. Die unterschiedlichen Manteltemperaturmodelle können Änderungen zwischen 3.7 und $5.3 \mathrm{~km}$ bedingen. Wird berücksichtigt, dass die Verwerfungen lediglich die Änderung des Planetenradius nach dem Ende des 'heavy bombardments' (vor etwa $3.8 \mathrm{Ga}$ ) wiedergeben (Schubert et al. 1988), verringert sich die Abnahme des Planetenradius für die unterschiedlichen Startmanteltemperaturen auf Werte zwischen 2.8 und $3.4 \mathrm{~km}$ bzw. bei der höchsten Startkerntemperatur auf $4.7 \mathrm{~km}$. Die aus den Simulationen bestimmten Radiusänderungen liegen alle über den Wert von 1 bis $2 \mathrm{~km}$, der aus der Vermessung von Verwerfungen gewonnen worden ist. Insbesondere bei anfänglich hohen Kerntemperaturen und sehr hohen Manteltemperaturen werden die Beobachtungsdaten nicht wiedergegeben.

\subsubsection{Einfluss des Schwefelgehalts im Kern}

In diesem Abschnitt wird der Einfluss des Schwefelgehalts auf die Entwicklung eines inneren Kerns für die beiden in Abschnitt (6.2) beschriebenen Parametrisierungen der Schmelzkurve (vgl. Tabelle (6.1)) untersucht. Es werden neben dem oben verwendeten Schwefelgehalt von $x_{0}=0.02$ eine schwächere Schwefelkonzentration von $x_{0}=0.01$ und zwei höhere Konzentrationen von $x_{0}=0.03$ und $x_{0}=0.04$ in Betracht gezogen. Alle Modelle in diesem Abschnitt werden von dem Startprofil mit $T_{\text {Mantel }}(0)=1700 \mathrm{~K}$ 
$\left(T_{\mathrm{KMG}}(0)=2100 \mathrm{~K}\right)$ (Abbildung (6.2)) gestartet und die Referenz-Rayleighzahl beträgt unverändert 698.

Abbildung (6.15) zeigt die Schmelzkurven beider Parametrisierungen für die verschie-

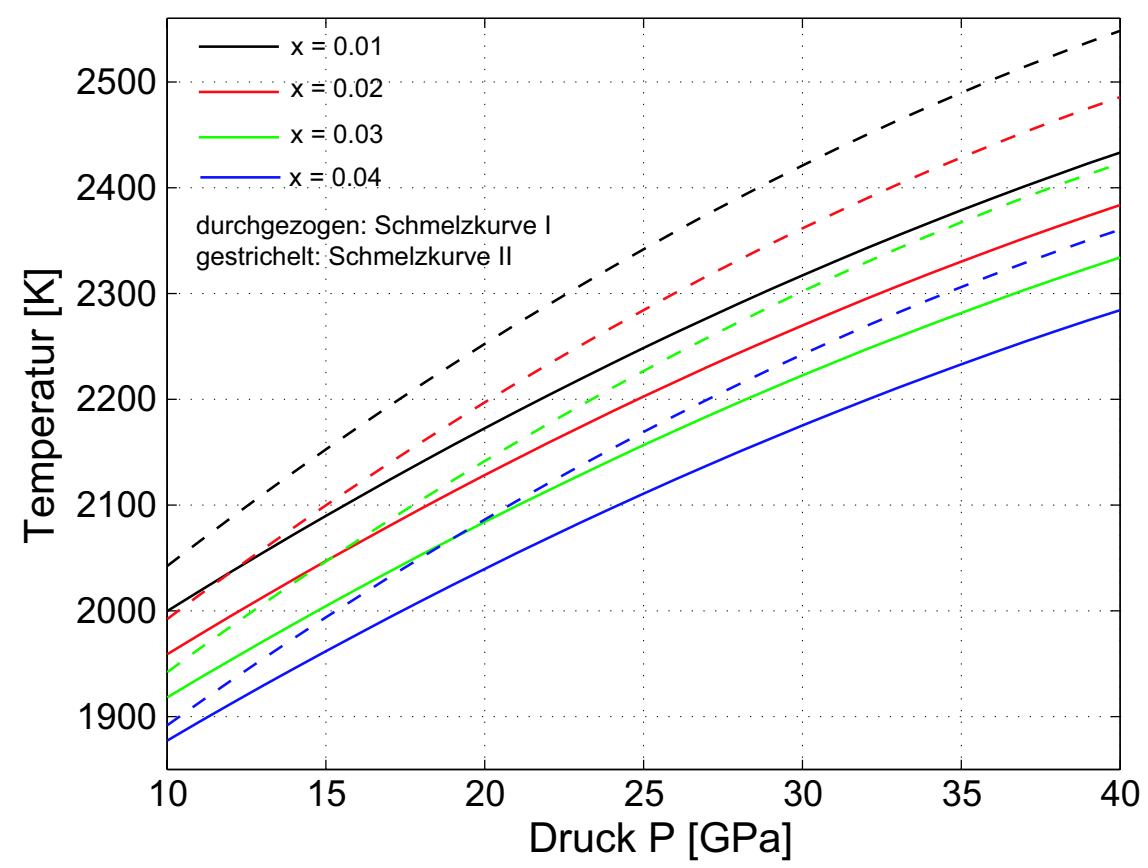

Abbildung 6.15: Schmelzkurven für Eisen mit unterschiedlichem Schwefelgehalt für beide Parametrisierungen (vgl. Tabelle (6.1)).

denen Ausgangsschwefelgehalte. Das Erhöhen des Schwefelanteils senkt die Schmelztemperatur deutlich ab. In der zweiten Parametrisierung ('Schmelzkurve II') liegen die Schmelztemperaturen bei gleichem Schwefelgehalt höher und der Einfluss des Schwefelanteils ist größer. Bei einem Schwefelbeitrag von 1 bzw. 2 Gew.-\% übersteigen die Schmelztemperaturen im Zentrum die Temperaturen der Anfangsadiabate, so dass zu Beginn nicht der gesamte Kern flüssig ist. Um eine bessere Vergleichbarkeit mit den übrigen Rechnungen zu erzielen, werden bei dieser Parametrisierung der Schmelzkurve ('Schmelzkurve II') nur die Modelle mit höherem Schwefelgehalt vorgestellt.

Die unterschiedlichen Entwicklungen der Kerngröße sind in Abbildung (6.16) dargestellt. Die Reduzierung des Schwefelanteils führt durch die höheren Schmelztemperaturen zu einem früheren Einsatz des Kernwachstums. Bei einer Halbierung des Schwefelgehalts auf 1 Gew.- \% beginnt die Bildung des inneren Kerns (bei 'Schmelzkurve I') schon nach 0.13 Ga und damit etwa $0.48 \mathrm{Ga}$ früher als im Referenzfall mit 2 Gew.-\%. Nach $4.5 \mathrm{Ga}$ ist der Radius des inneren Kerns mit $1358 \mathrm{~km} 287 \mathrm{~km}$ größer als im Fall mit $x_{0}=0.02$. Analog verzögert sich das Wachstum des inneren Kerns bei einem höheren Schwefelgehalt und den damit verbundenen niedrigeren Schmelztemperaturen. Bei $x_{0}=0.03$ friert der feste innere Kern erst ab 3.14 Ga und damit etwas 2.53 Ga später als im Referenzfall aus. Nach $4.5 \mathrm{Ga}$ ist seine Größe mit $610 \mathrm{~km}$ deutlich geringer. Wird der Schwefelgehalt bei 'Schmelzkurve I' weiter auf $x_{0}=0.04$ erhöht, kühlt der Kern während der Evolution 


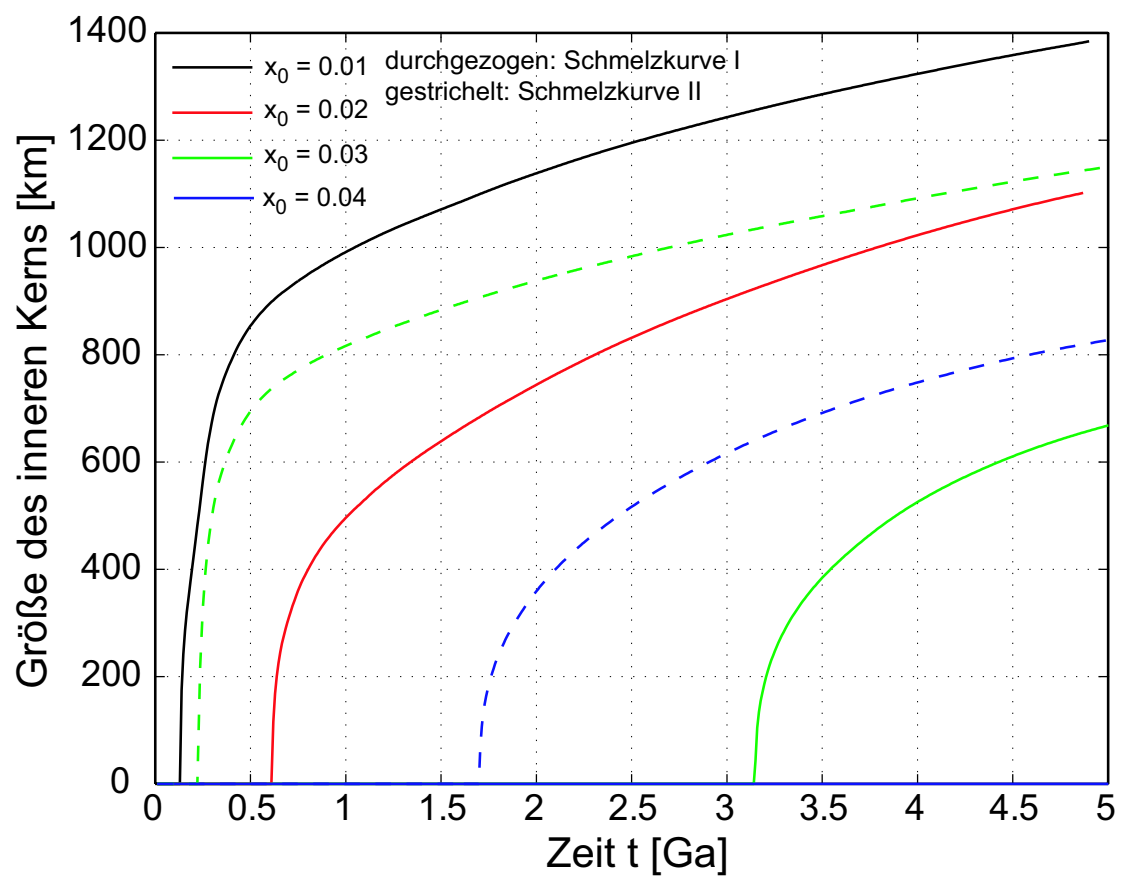

Abbildung 6.16: Einfluss des anfänglichen Schwefelgehalts und der verwendeten Parametrisierung der Schmelzkurve auf die Entwicklung des inneren Kerns.

nicht ausreichend stark ab, um die niedrigen Schmelztemperaturen zu unterschreiten. In diesem Fall bildet sich kein fester innerer Kern, der gesamte Kern bleibt flüssig. Die Situation verändert sich jedoch, wenn die Parametrisierung der 'Schmelzkurve II' mit den höheren Schmelztemperaturen verwendet wird. Mit dieser Parametrisierung bildet sich bei einem Schwefelgehalt von $x_{0}=0.03$ schon nach 0.22 Ga ein fester innerer Kern, dessen Größe nach $4.5 \mathrm{Ga} 1123$ km erreicht. Auch bei einem Schwefelanteil von 4 Gew.-\% ist hier das Wachsen eines inneren Kerns möglich. Das Ausbilden des inneren Kerns beginnt nach $1.7 \mathrm{Ga}$ und führt zu einer heutigen Größe von $793 \mathrm{~km}$. Die zeitliche Entwicklung des inneren Kerns und seine Größe nach 4.5 Ga hängt somit stark vom Schwefelgehalt und der genauen Parametrisierung der Schmelzkurve ab.

Das zeitlich versetzte Beginnen des Kernwachstums und die unterschiedlichen Größen des inneren Kerns bzw. dessen Zuwachsraten beeinflussen die Entwicklung der Temperatur an der KMG. Das Abkühlen des Kerns (Abbildung (6.17)) wird durch die beim Ausbilden des festen inneren Kerns freiwerdende latente Wärme und gravitative Energie gebremst. Für 'Schmelzkurve I' entsteht so durch die unterschiedlichen Schwefelgehalte nach 4.5 Ga ein Temperaturunterschied an der KMG von $43 \mathrm{~K}$ zwischen dem Modellen mit $x_{0}=0.01$ und $x_{0}=0.04$.

Die unterschiedlichen Temperaturen wirken sich auch auf den Wärmefluss an der KMG (Abbildung (6.18)) aus. Die höheren Temperaturen (Abbildungen (6.17) und (6.19)) und niedrigeren Viskositäten bei größeren inneren Kernen ermöglichen einen stärkeren Temperaturgradienten an der KMG und somit einen höheren Wärmefluss aus dem Kern. Allerdings ist der Wärmefluss in diesen Modellen mit Werten zwischen $1.0-3.7 \mathrm{~mW} / \mathrm{m}^{2}$ 


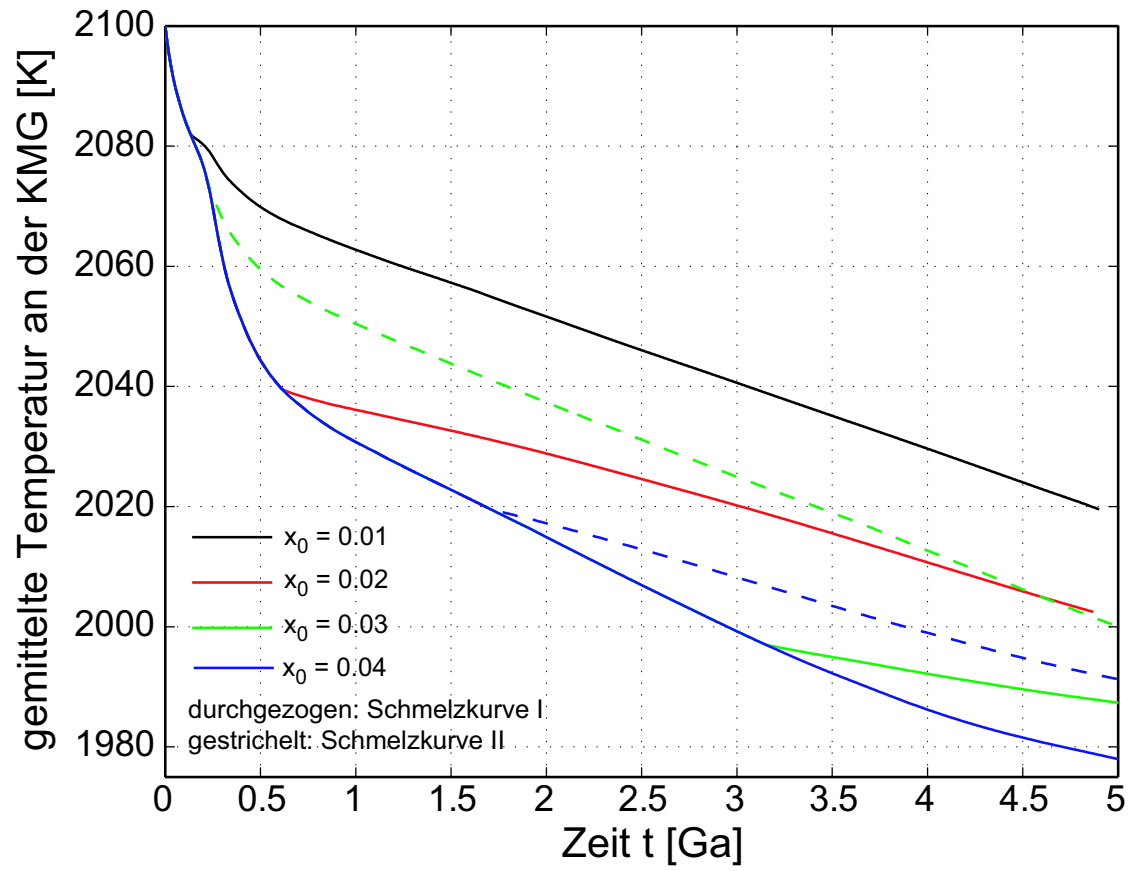

Abbildung 6.17: Einfluss des anfänglichen Schwefelgehalts und der verwendeten Parametrisierung der Schmelzkurve auf die Entwicklung der Temperatur an der KMG.

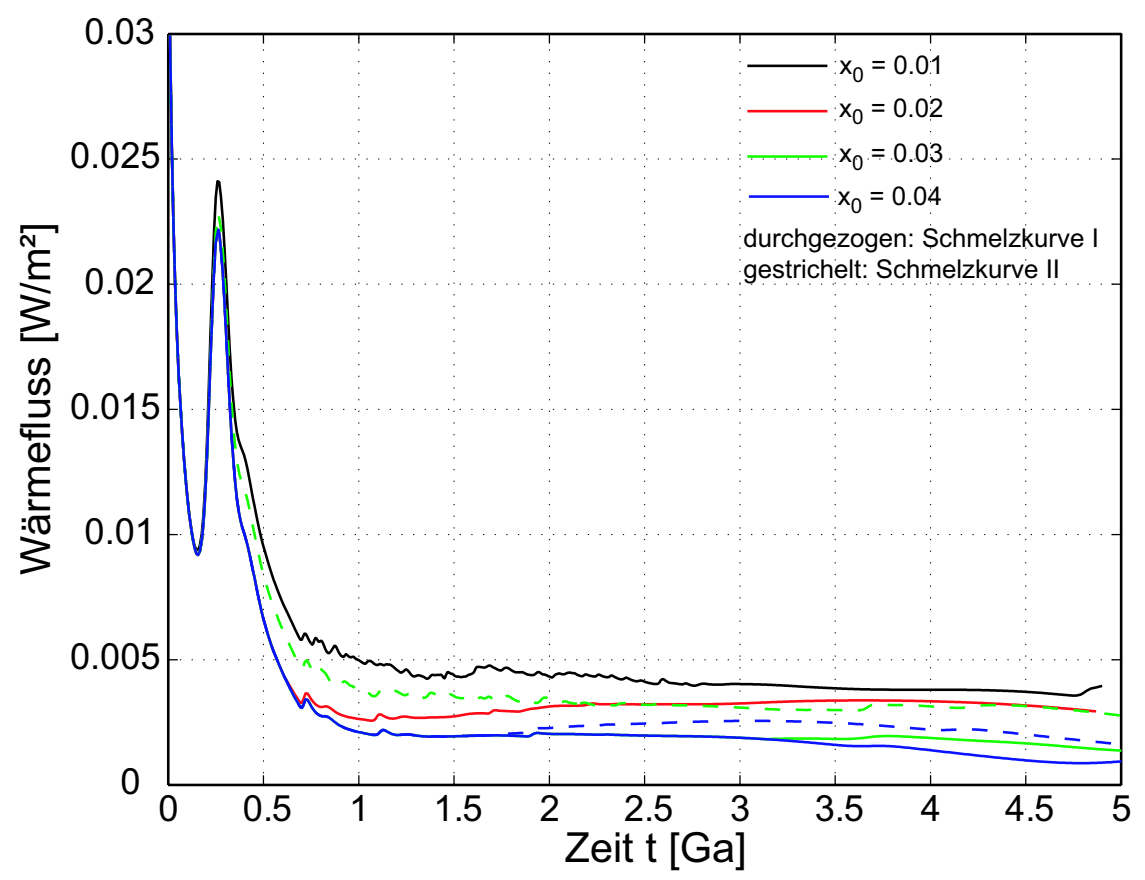

Abbildung 6.18: Einfluss des anfänglichen Schwefelgehalts und der verwendeten Parametrisierung der Schmelzkurve auf die Entwicklung des Wärmeflusses an der KMG. 
im Vergleich zum Wärmefluss entlang der Kernadiabate $\left(11 \mathrm{~mW} / \mathrm{m}^{2}\right.$ (Schubert et al. 1988)) ebenfalls zu gering, um einen thermisch getriebenen Dynamo zu realisieren. Bei $x_{0}=0.04$ ('Schmelzkurve I') scheitert auch ein chemisch getriebener Dynamo, da kein innerer Kern ausfriert. In diesem Modell kann das beobachtete Magnetfeld nicht durch einen hydrodynamischen Dynamoprozess im Inneren erklärt werden.

Der Wärmefluss an der Oberfläche wird durch die angenommenen, unterschiedlichen Schmelzkurven nicht signifikant beeinflusst. Nach $4.5 \mathrm{Ga}$ liegt der Wärmefluss für die unterschiedlichen Modelle in einem engen Intervall zwischen 20.8 und $21.8 \mathrm{~mW} / \mathrm{m}^{2}$.

Der höhere Wärmeeintrag aus dem Kern in den Mantel führt auch im Volumenmittel zu höheren Temperaturen. Bei 'Schmelzkurve I' liegt die volumen-gemittelte Temperatur nach $4.5 \mathrm{Ga}$ bei $x_{0}=0.01 \mathrm{mit} 1516 \mathrm{~K}$ deutlich über der Temperatur von $1456 \mathrm{~K}$ bei $x_{0}=0.04$. Die höheren Temperaturen fördern durch die niedrigeren Viskositäten die Konvektion, so dass die Wärme effektiver vom Kern abtransportiert werden kann.

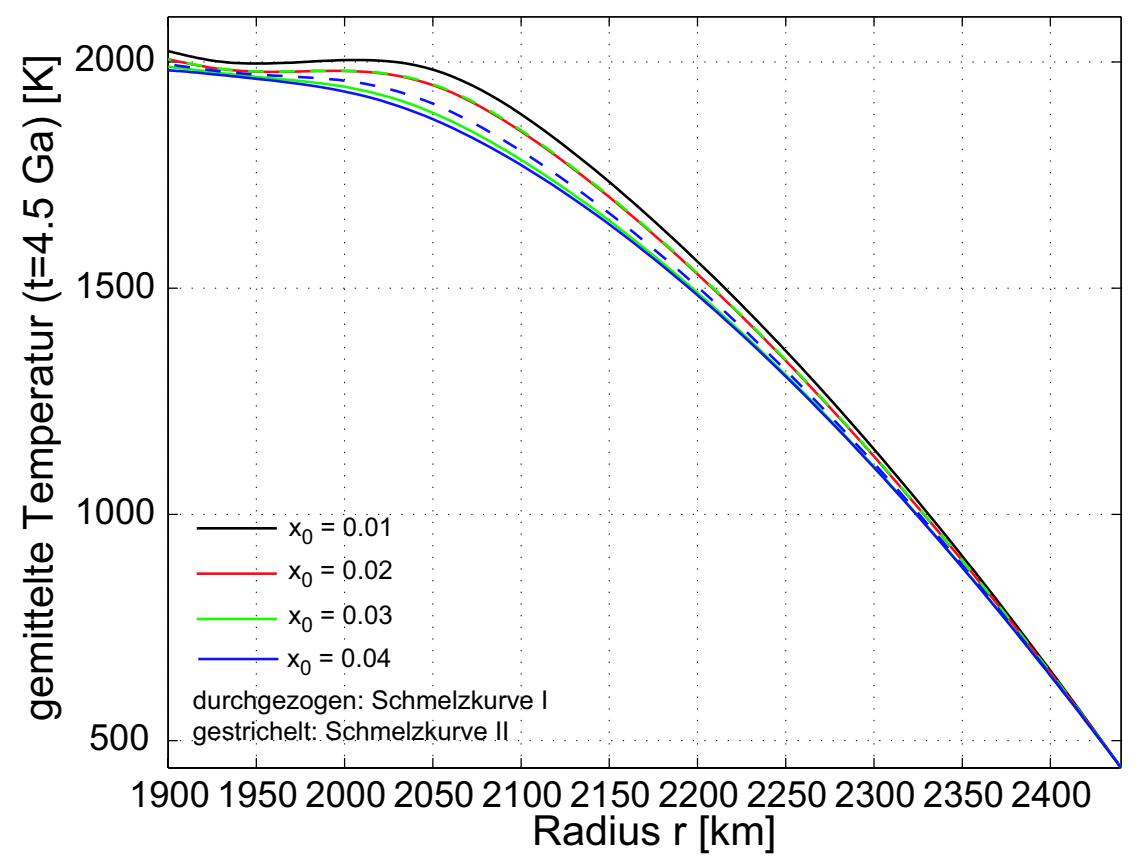

Abbildung 6.19: Einfluss des anfänglichen Schwefelgehalts und der verwendeten Parametrisierung der Schmelzkurve auf die Temperaturprofil nach 4.5 Ga.

Das Temperaturprofil nach 4.5 Ga in Abbildung (6.19) fasst die Entwicklung zusammen. Die Form der Profile bei $x_{0}=0.04$ ('Schmelzkurve I') und $x_{0}=0.03$ ('Schmelzkurve I') deutet an, dass die Konvektion nach $4.5 \mathrm{Ga}$ in diesem Fällen sehr schwach ist. Die Bedeutung der Konvektion in der Entwicklung wird durch den Vergleich mit einem rein konduktiven Modell in Abschnitt (6.3.3) (z.B. Abbildung (6.26)) verdeutlicht. Die etwas unterschiedliche Dicke der oberflächennahen thermischen Grenzschicht spiegelt sich in der Lithosphärendicke wieder. Nach 4.5 Ga liegen die Lithosphärendicken zwischen 283 $\mathrm{km}\left(x_{0}=0.01\right)$ und $322 \mathrm{~km}\left(x_{0}=0.04\right)$ bei 'Schmelzkurve I' bzw. $292 \mathrm{~km}\left(x_{0}=0.03\right)$ und $308 \mathrm{~km}\left(x_{0}=0.04\right)$ bei 'Schmelzkurve II'. 


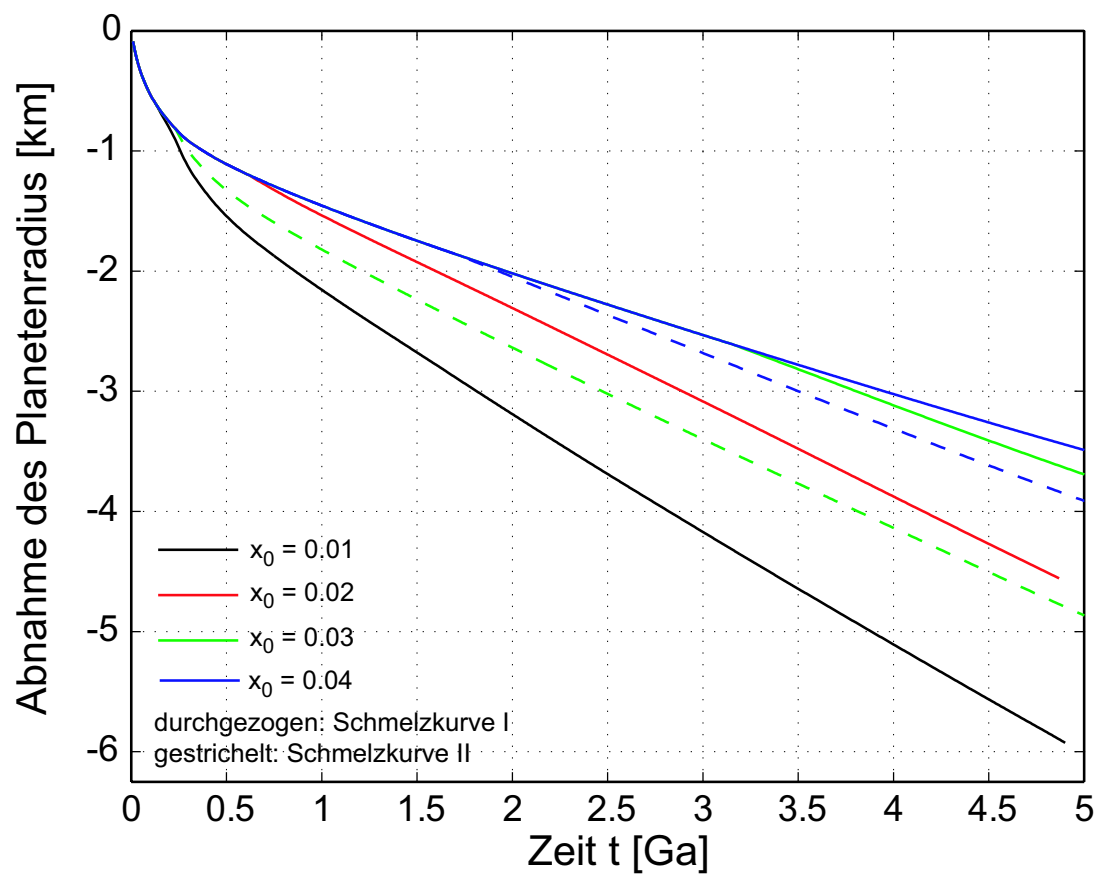

Abbildung 6.20: Einfluss des anfänglichen Schwefelgehalts und der verwendeten Parametrisierung der Schmelzkurve auf die Abnahme des Planetenradius.

Abbildung (6.20) zeigt den Einfluss der Schmelzbedingungen bzw. der damit verbundenen unterschiedlichen Größen des inneren Kerns auf die Abnahme des Planetenradius. Die deutlich kleineren inneren Kerne bei 'Schmelzkurve I' und einem Schwefelgehalt von 3 bzw. 4 Gew.-\% lassen den Planeten mit nur 3.4 bzw. 3.3 km deutlich weniger schrumpfen als im Referenzfall mit 2 Gew.-\% (4.3 km). Betrachtet man hier nur die Veränderung in den letzen $3.8 \mathrm{Ga}$, liegen die Werte mit $2.1 \mathrm{bzw}$. $2.0 \mathrm{~km}$ in der Nähe der Daten aus Vermessung der Verwerfungen, die eine Abnahme zwischen 1 und $2 \mathrm{~km}$ nahe legen. Bei Verringern des Schwefelgehalts $\left(x_{0}=0.01\right.$, 'Schmelzkurve I') weicht die Veränderung des Planetenradius mit 5.6 bzw. 3.8 km (in den letzten $3.8 \mathrm{Ga}$ ) stärker von den Messdaten ab. Bei gleichem Schwefelgehalt entstehen bei Verwendung der zweiten Parametrisierung ('Schmelzkurve II') größere innere Kerne, die die Abnahme des Planetenradius verstärken (4.5 bzw. $2.9 \mathrm{~km}\left(x_{0}=0.03\right), 3.6$ bzw. $\left.2.3 \mathrm{~km}\left(x_{0}=0.04\right)\right)$. Trotz der stärkeren Abnahme des Planetenradius bei 'Schmelzkurve II' liegen die Werte für die letzten 3.8 Ga in der Nähe der Messdaten. Ein höhere Schwefelgehalt kann dazu beitragen, die beobachteten geringen Schrumpfungsraten des Planeten zu erklären.

Der Schwefelgehalt und die genaue Parametrisierung der Schmelzkurve haben einen deutlichen Einfluss auf die Entwicklung und Größe des inneren Kerns. Höhere Schwefelgehalte führen zu kleineren inneren Kernen und zu einer geringeren Abnahme des Planetenradius. Allerdings hängt es schon bei einem Schwefelgehalt von 4 Gew.- $\%$ von der genauen Schmelzkurve ab, ob überhaupt ein innerer Kern entsteht. Ohne das Ausbilden eines inneren Kerns fehlt jedoch ein Antriebsmechanismus für einen hydrodynamischen Dynamo im Inneren, der das beobachtete Magnetfeld erklären kann. Aufgrund des geringen 
Wärmeflusses aus dem Kern ist ein thermisch getriebener Dynamo nicht möglich. Um die Schmelzbedingungen von Eisen mit einigen Gewichtsprozent Schwefel bei Druckund Temperaturbedingungen im Merkurkern besser festlegen zu können, sind erweiterte Labormessungen erforderlich.

\subsubsection{Dreidimensionale Evolutionsmodelle für das Merkurinnere}

In diesem Kapitel werden die oben untersuchten achsensymmetrischen Evolutionsmodelle für Merkur mit vollständig dreidimensionalen Simulationen verglichen. Für die Schmelzkurve wird wie in Abschnitt (6.3.1) die Parametrisierung 'Schmelzkurve I' verwendet. Der anfängliche Schwefelgehalt beträgt 2 Gew.-\%. Es werden Simulationen mit drei verschiedenen Rayleighzahlen $(R a=698, R a=3490$ und $R a=6980)$ vorgestellt. Die Referenzviskosität in halber Tiefe bei einer Temperatur von $1573 \mathrm{~K}$ entspricht bei der 5-fach höheren Rayleighzahl $\eta_{\text {ref }}=1.6 \cdot 10^{22}$ Pa s bzw. $\eta_{\text {ref }}=8.1 \cdot 10^{21}$ Pa s bei der 10-fach höheren Rayleighzahl. Um die Bedeutung der Konvektion, insbesondere bei der kleineren Rayleighzahl abzuschätzen, werden zum Vergleich rein konduktive Modellrechnungen $(R a=0)$ durchgeführt. Für das Starttemperaturprofil gilt in allen Simulationen: $T_{\text {Mantel }}(0)=1700 \mathrm{~K}$ und $T_{\mathrm{KMG}}(0)=2100 \mathrm{~K}$ (Abbildung (6.2)).

Die Entwicklung des inneren festen Kerns für die verschiedenen Modelle ist in Abbil-

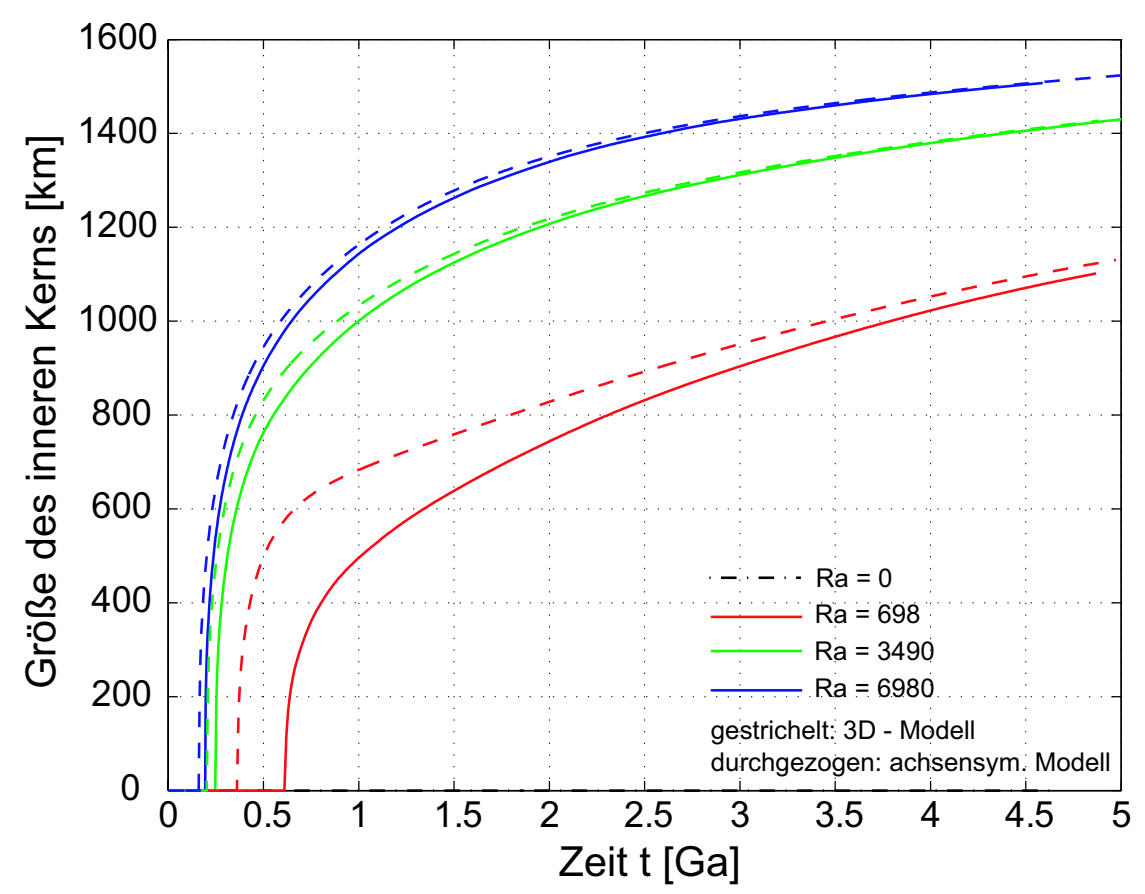

Abbildung 6.21: Entwicklung des inneren Kerns bei unterschiedlichen Rayleighzahlen in achsensymetrischen und vollständig dreidimensionalen Evolutionsmodellen.

dung (6.21) gezeigt. Die rote, durchgezogene Linie stellt den Referenzfall mit einer Rayleighzahl von $698 \mathrm{im}$ achsensymmetrischen Modell dar. Im vollständig dreidimensionalen Modell (gestrichelte Kurve) setzt das Kernwachstum schon bei 0.36 Ga und damit 0.25 
Ga früher ein. Allerdings führen unterschiedliche Wachstumsraten zu einer Annäherung der Kurven für die unterschiedlichen Dimensionen des Modells, so dass die Größe des inneren Kerns nach $4.5 \mathrm{Ga}$ um weniger als $25 \mathrm{~km}$ abweicht.

Diese unterschiedliche Entwicklung lässt bei der Betrachtung des Wärmeflusses an der

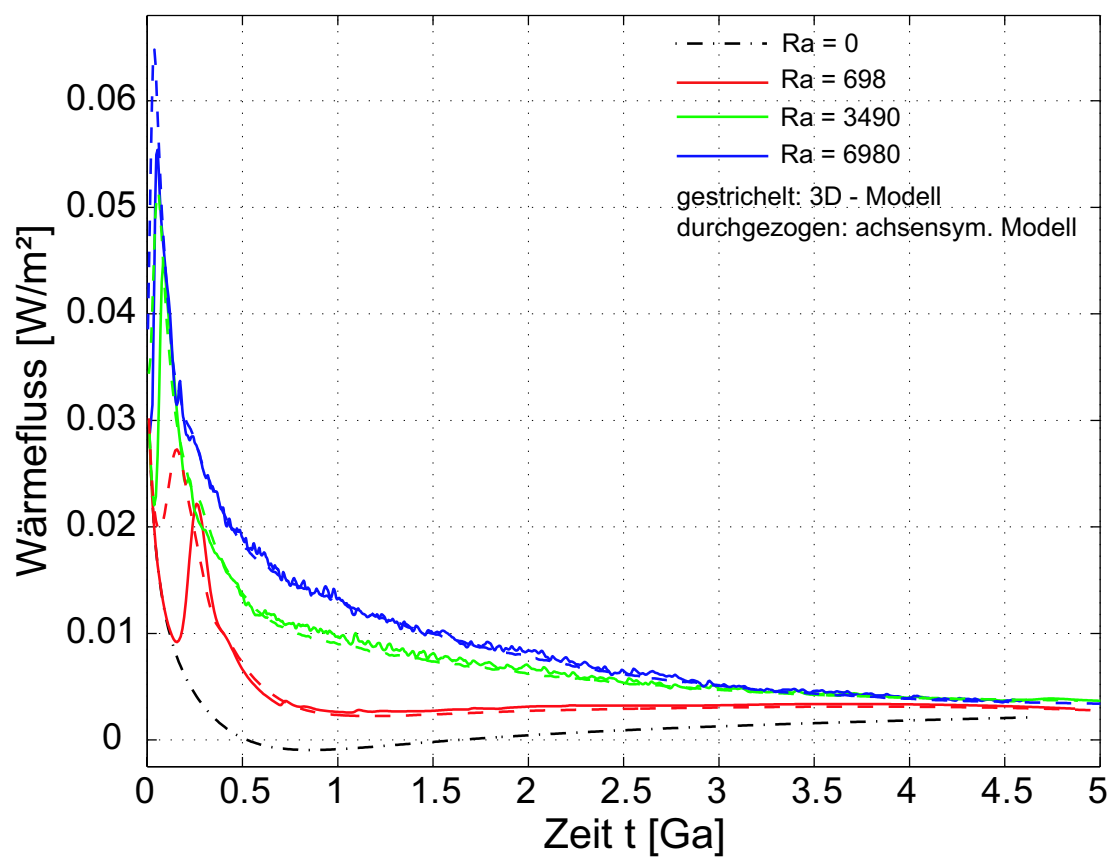

Abbildung 6.22: Entwicklung des Wärmeflusses an der KMG bei unterschiedlichen Rayleighzahlen in achsensymetrischen und vollständig dreidimensionalen Evolutionsmodellen.

KMG (Abbildung (6.22)) verstehen. Im dreidimensionalen Fall wird bei einer Rayleighzahl von 698 (rot gestrichelte Kurve) das relative Maximum des Wärmeflusses, das mit dem Einsatz der Konvektion verbunden ist, früher erreicht. Dadurch kühlt der Kern schneller ab und ein vorgezogener Beginn des Ausfrierens des inneren Kerns ist möglich. Die Wachstumsrate ist an den Wärmefluss gekoppelt, so dass der Kern im dreidimensionalen Fall zu Beginn schneller wächst (bei Einsatz des Kernwachstums ist der Wärmefluss höher als im achsensymmetrischen Fall). Das frühere Einsetzen der Konvektion kann mit einer Änderung der Störungsgeometrie und Amplitude verbunden sein. Im beiden Modellgeometrien besteht die anfängliche Störung aus zufällig ausgewählten Moden der Kugelflächenfunktionen. Die unterschiedlichen Einsatzzeiten der Konvektion im 2D- und 3DModell sprechen dafür, dass die entsprechenden Moden unterschiedlich stark anwachsen oder nicht gleichberechtigt initialisiert wurden. Bei der niedrigen Rayleighzahl wächst die Störung über einen im Vergleich zum Fall mit $R a=3490$ bzw. $R a=6980$ langen Zeitraum an, so dass der Einsatz der Konvektion stärker von den Details der initialisierten Störung abhängt als bei den größeren Rayleighzahlen.

Wird die Rayleighzahl erhöht (grüne und blaue Kurve), verringert sich der Unterschied in der Entwicklung des inneren Kerns zwischen dem achsensymmetrischen Modell und 
dem vollständig dreidimensionalen Modell. Bei einer Rayleighzahl von 3490 besteht nur noch ein zeitlicher Versatz von $0.04 \mathrm{Ga}$ und bei der höchsten Rayleighzahl von nur noch $0.03 \mathrm{Ga}$. Bei den höheren Rayleighzahlen kühlt der Kern durch die heftigere Konvektion schneller aus, so dass das Kernwachstum früher beginnt und mit einem Radius von 1406 km $(R a=3490)$ bzw. 1504 km $(R a=6980)$ deutlich größere innere Kerne entstehen. Der Unterschied in der Größe des inneren Kerns nach 4.5 Ga durch die Geometrie des Modells ist bei den höheren Rayleighzahlen nicht signifikant. Für die achsensymmetrischen Modelle liefert eine zusätzliche Simulation mit $R a=17450$ einen noch größeren inneren Kern mit einem Radius von 1591 km. Die rein konduktiven Modellsimulationen sind achsensymmetrisch und durch die strich-punktierten Linien dargestellt. Der Kern kühlt in diesen Modellen so langsam ab, dass sich im betrachteten Zeitraum kein innerer Kern ausbilden kann.

Die Entwicklung des Wärmeflusses an der KMG (Abbildung (6.22)) verläuft bis auf den erwähnten Unterschied im Einsatz der Konvektion bei den dreidimensionalen und den achsensymmetrischen Simulationen sehr ähnlich. Der Vergleich mit der konduktiven Lösung zeigt, dass die Konvektion im Fall mit $R a=698 \mathrm{zu}$ Beginn der Entwicklung eine untergeordnete Rolle spielt und der Wärmetransport zunächst durch Wärmeleitung dominiert ist. Im achsensymmetrischen Fall weicht der Wärmefluss nach etwa $0.15 \mathrm{Ga}$ (im dreidimensionalen Fall nach $0.05 \mathrm{Ga}$ ) allerdings deutlich vom konduktiven Verlauf ab. Durch Wärmeleitung allein kann der Mantel, der auch durch die radioaktiven Quellen geheizt wird, die Wärme nicht effektiv vom Kern abführen, so dass dieser sich zwischenzeitlich durch einen negativen Wärmefluss wieder aufheizt. Auch nach Durchlaufen des Maximums liegt der Wärmefluss bei $R a=698$ deutlich über dem konduktiven Modell und zeigt die Bedeutung der Konvektion an. Das Erhöhen der Rayleighzahl führt zu einem früheren Einsatz der Konvektion und steigert den Wärmefluss während der ganzen Entwicklung. Allerdings liegt der Wärmefluss heute in allen Modellen in einem engen Intervall von 3 bis $3.8 \mathrm{~mW} / \mathrm{m}^{2}$ ( $2 \mathrm{~mW} / \mathrm{m}^{2}$, konduktive Lösung) unterhalb des Wärmeflusses entlang der Adiabate $\left(11 \mathrm{~mW} / \mathrm{m}^{2}\right.$ (Schubert et al. 1988)), so dass ein thermisch getriebener Dynamo unwahrscheinlich ist. Dies gilt ebenfalls für das achsensymmetrische Modell mit $R a=17450$, bei dem der Wärmefluss nach 4.5 Ga bei $3.3 \mathrm{~mW} / \mathrm{m}^{2}$ liegt.

Der Einfluss der unterschiedlichen Rayleigzahlen und der resultierenden starken Unterschiede in der Temperaturverteilung (Abbildungen (6.24) und (6.25)) sind in der zeitlichen Entwicklung des Wärmeflusses an der Oberfläche sichtbar. Die höheren Rayleighzahlen führen zu einem schnelleren Abkühlen des Kerns und zu einem höheren Wärmeeintrag in den Mantel. Durch die niedrigeren Viskositäten kann die verstärkte Konvektion die Wärme effektiv bis zur Lithosphäre transportieren. Der Wärmefluss durchläuft so bei der höchsten Rayleighzahl nach dem anfänglich starken Abfall ein schwaches relatives Maximum (bei etwa $0.7 \mathrm{Ga}$ ). Nach $4.5 \mathrm{Ga}$ liegt der Wärmefluss für alle Modelle in einem engen Intervall von $21.9 \pm 0.5 \mathrm{~mW} / \mathrm{m}^{2}\left(20.3 \mathrm{~mW} / \mathrm{m}^{2}(R a=0)\right)$. Auch der Wärmefluss bei der stark erhöhten Rayleighzahl $(R a=17450)$ liegt in diesem Intervall. Die Geometrie des Modellraums ist nur für $R a=698$ bei sehr unterschiedlicher Größe des inneren Kerns sichtbar und selbst da sehr gering. In den ersten $0.5 \mathrm{Ga}$ enspricht der Wärmefluss $(R a=698)$ an der Oberfläche etwa dem konduktiven Wärmefluss, danach wird auch an der Oberfläche der Wärmefluss durch die Konvektion erhöht. Aufgrund der dicken Lithosphäre, in der die Wärme nur durch Wärmeleitung transportiert werden kann, stellt der konduktive Wärmefluss jedoch den dominanten Beitrag dar. 


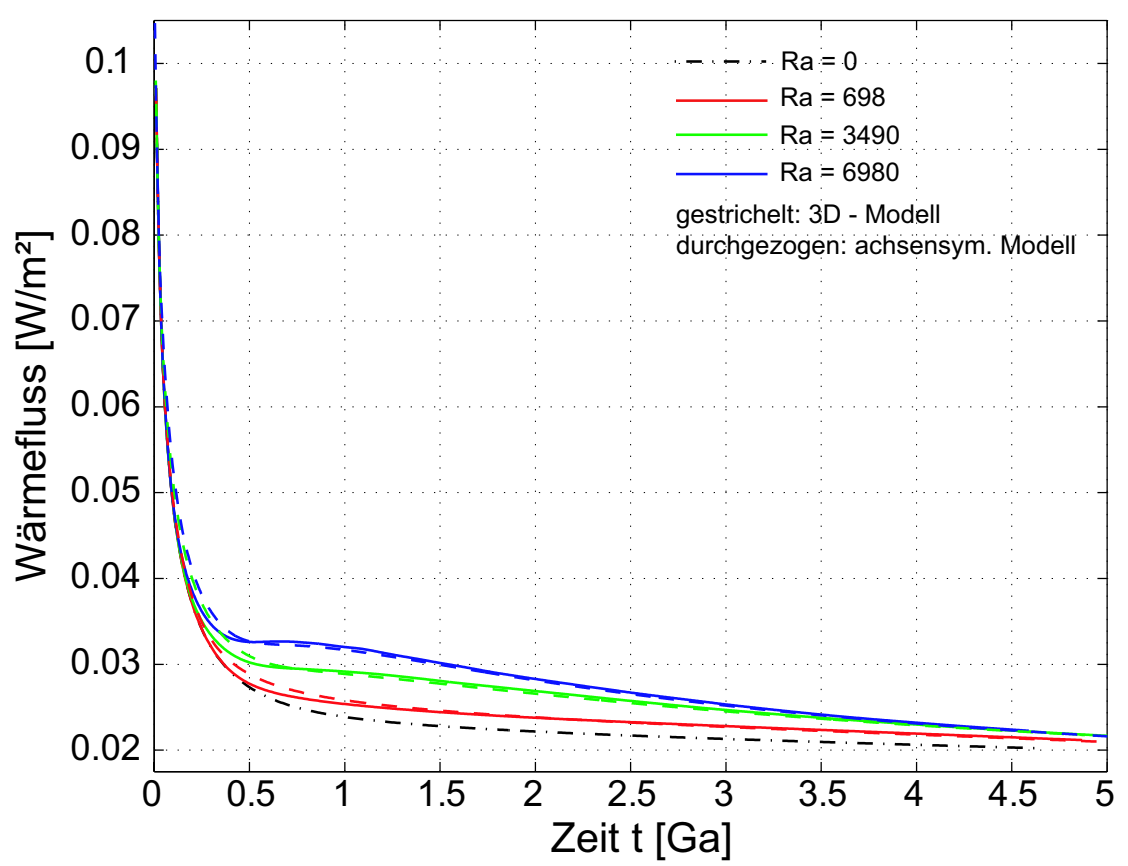

Abbildung 6.23: Entwicklung des Wärmeflusses an der Oberfläche bei unterschiedlichen Rayleighzahlen in achsensymetrischen und vollständig dreidimensionalen Evolutionsmodellen.

Abbildung (6.24) zeigt die Temperaturentwicklung an der KMG. Beim Erhöhen der Rayleighzahl kühlt der Kern schneller ab und der Unterschied zwischen dem vollständig dreidimensionalen und dem achsensymmetrischen Modell ist vernachlässigbar. Im Modell mit $R a=698$ ist der Einsatz der Konvektion in der Änderung der Steigung zu Beginn sichtbar. Der unterschiedliche Einsatz im achsensymmetrischen und dreidimensionalen Modell führt zu kleinen Unterschieden in der Temperaturentwicklung an der KMG, die aber im Lauf der Zeit abnehmen. Nach 4.5 Ga liegt die Temperatur an der KMG für $R a=698$ bei $2006 \mathrm{~K}$ (achsensymmetrisches Modell) und der Unterschied zwischen achsensymmetrischen und vollständig dreidimensionalem Modell beträgt lediglich $3 \mathrm{~K}$. Für die höheren Rayleighzahlen wird dieser Unterschied noch kleiner. Für $R a=3490$ liegt die Temperatur nach $4.5 \mathrm{Ga}$ bei $1945 \mathrm{~K}$ und für $R a=6980$ bei $1910 \mathrm{~K}$ ( $R a=17450$ : 1864 K). Im Modell, das nur den Wärmetransport durch Wärmeleitung simuliert, kühlt der Kern deutlich langsamer ab und heizt sich sogar zwischenzeitlich wieder auf, da der Mantel die Wärme nicht abführen kann. Nach 4.5 Ga beträgt die Temperatur $2050 \mathrm{~K}$.

In der volumen-gemittelten Temperatur, die in Abbildung (6.25) gezeigt ist, sowie im Temperaturprofil nach $4.5 \mathrm{Ga}$ finden sich ebenfalls keine signifikanten Unterschiede durch die Erweiterung des achsensymmetrischen Modells zum vollständig dreidimensionalen Modell. Der größere Wärmeeintrag aus dem Kern durch die heftigere Konvektion (Erhöhen der Rayleighzahl) und die beim Ausbilden der größeren inneren Kerne freiwerdende Energie ermöglicht zeitweillig eine im Volumenmittel höhere Temperatur. Nach 4.5 Ga ist der Unterschied in der volumen-gemittelten Temperatur durch die Rayleighzahl (ohne 


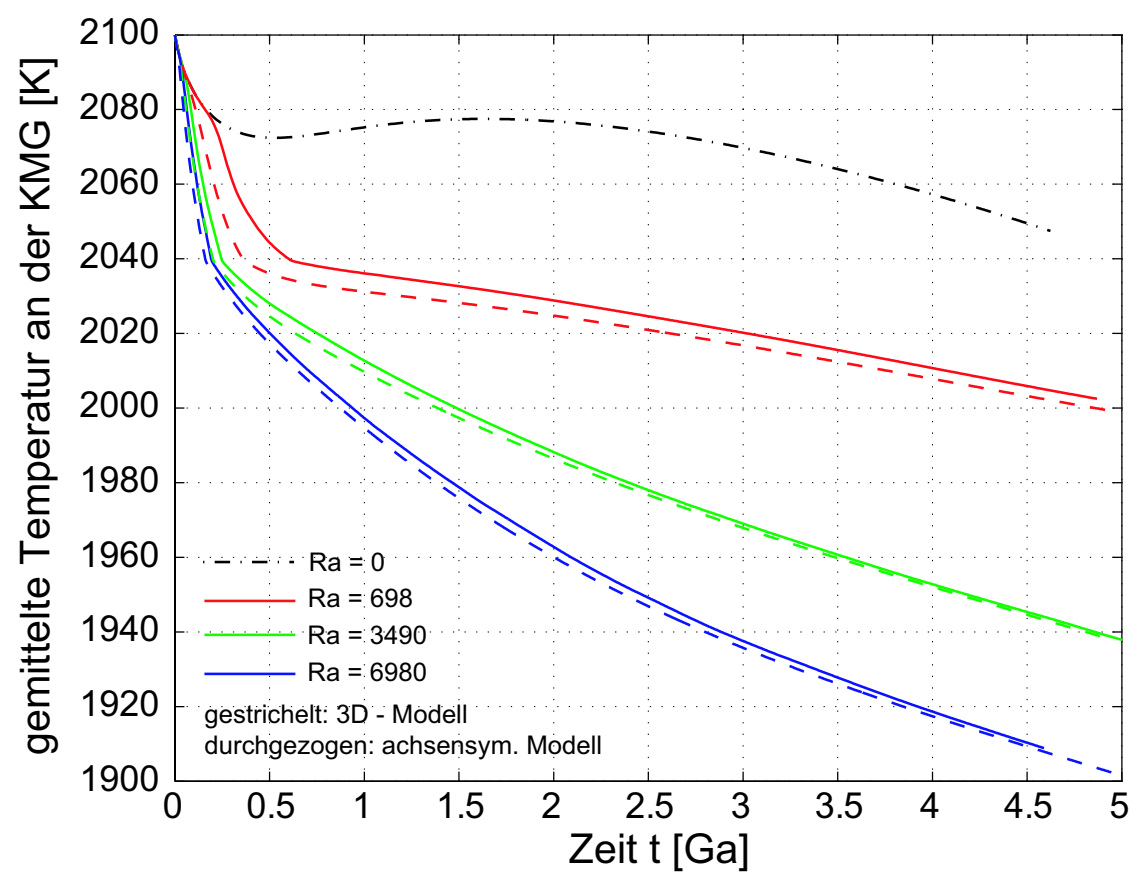

Abbildung 6.24: Entwicklung der Temperatur an der KMG bei unterschiedlichen Rayleighzahlen in achsensymetrischen und vollständig dreidimensionalen Evolutionsmodellen.

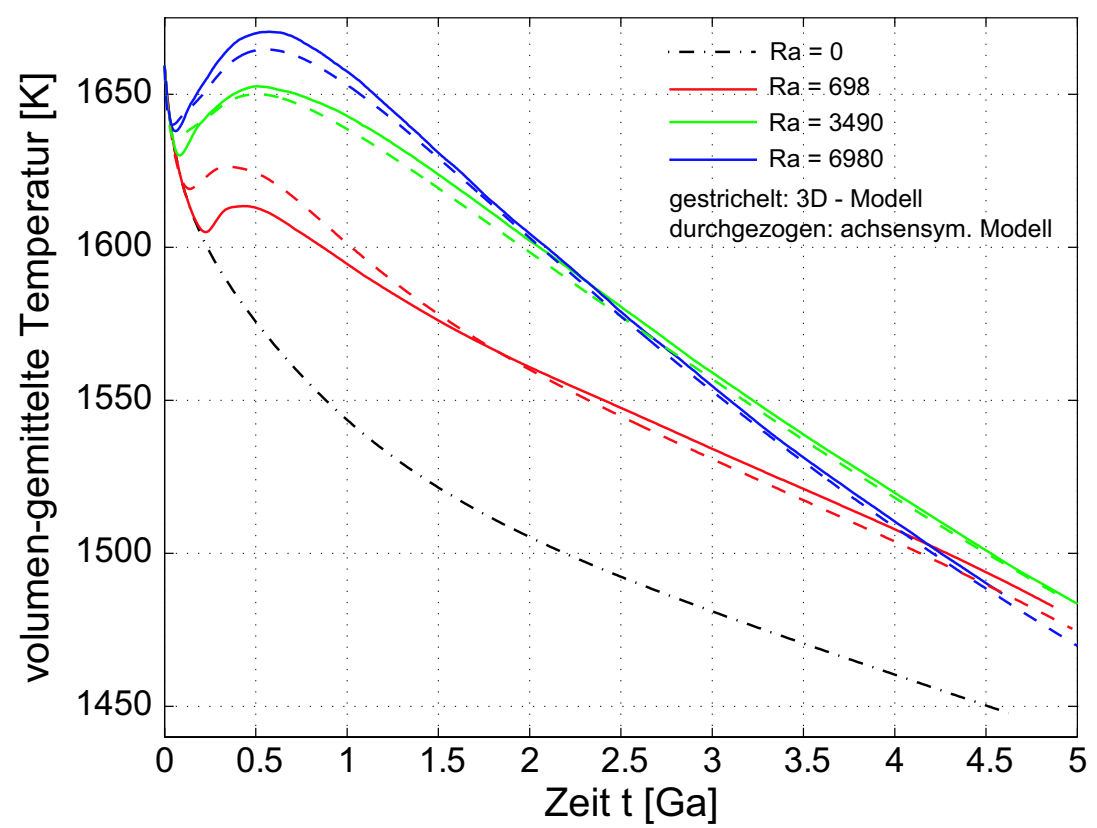

Abbildung 6.25: Entwicklung der volumen-gemittelten Temperatur bei unterschiedlichen Rayleighzahlen in achsensymetrischen und vollständig dreidimensionalen Evolutionsmodellen. 


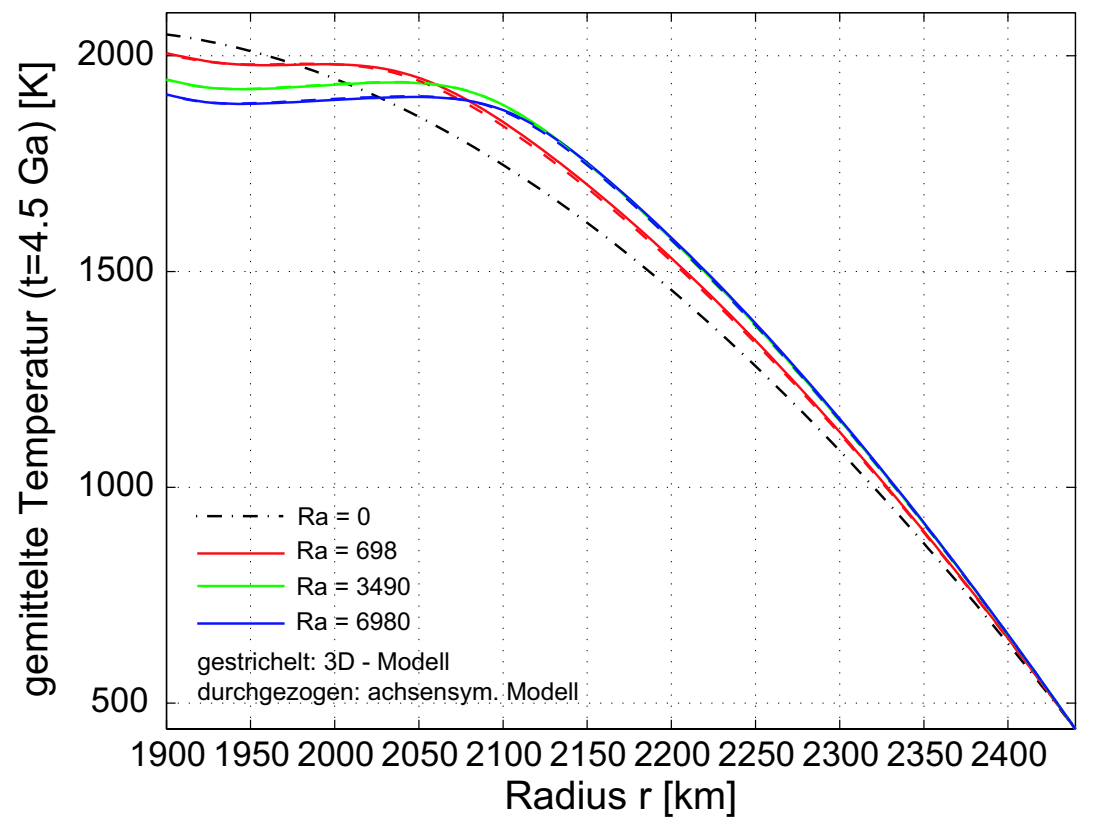

Abbildung 6.26: Temperaturprofile nach 4.5 Ga bei unterschiedlichen Rayleighzahlen in achsensymetrischen und vollständig dreidimensionalen Evolutionsmodellen.

den konduktiven Fall) allerdings nur noch gering (1495 \pm 6 K). Während der Evolution liegt die volumen-gemittelte Temperatur im konduktiven Fall deutlich unter den Temperaturen, die im Fall mit $R a=698$ erreicht werden (nach $4.5 \mathrm{Ga}: 1450 \mathrm{~K}$ ).

Das Temperaturprofil nach 4.5 Ga (Abbildung (6.26)) zeigt, dass die thermische Grenzschicht an der KMG in allen Simulationen sehr dünn und der Temperaturkontrast äußerst gering ist. Das Erhöhen der Rayleighzahl dehnt den isothermen, konvektierenden Bereich in Richtung Oberfläche aus, so dass die oberflächennahe thermische Grenzschicht etwas dünner ist. Die dünnere Grenzschicht erklärt zusammen mit den niedrigeren Temperaturen im Inneren die sehr ähnlichen volumen-gemittelten Temperaturen nach $4.5 \mathrm{Ga}$. Nach 4.5 Ga wird der Unterschied im Temperaturprofil zwischen dem Wärmeleitungsmodell und dem Modell mit der kleinen Rayleighzahl besonders im kernnahen Bereich deutlich. Der Einfluss der Rayleighzahl auf die Dicke der oberen thermischen Grenzschicht spiegelt sich in der Entwicklung der Lithosphärendicke (Abbildung (6.27)) wider. Bei einer Rayleighzahl von 698 beträgt die Lithosphärendicke 296 km. Für $R a=3490$ ergibt sich eine Dicke von $260 \mathrm{~km}$ und bei der höchsten angenommenen Rayleighzahl ( $R a=6980)$ eine Dicke von $252 \mathrm{~km}$. Der Einfluss der Modellgeometrie ist sehr gering ( $R a=698$ : $3.5 \mathrm{~km}$ ). Die Bestimmung der Lithosphärendicke mit Hilfe der Geschwindigkeitsprofile ist für das rein konduktive Modell nicht möglich.

Die Abnahme des Planetenradius durch das Abkühlen des Planeten ist in Abbildung (6.28) gezeigt. Bei $R a=698$ reduziert sich der Planetenradius in $4.5 \mathrm{Ga}$ um 4.3 bzw. 3.0 $\mathrm{km}$, wenn nur der Zeitraum nach dem 'heavy bombardment' $(3.8 \mathrm{Ga})$ betrachtet wird. Der Unterschied zwischen den vollständig dreidimensionalen und den achsensymmetrischen Modellen liegt bei etwa 190 m. Bei den größeren Rayleighzahlen kühlt das ganze System 


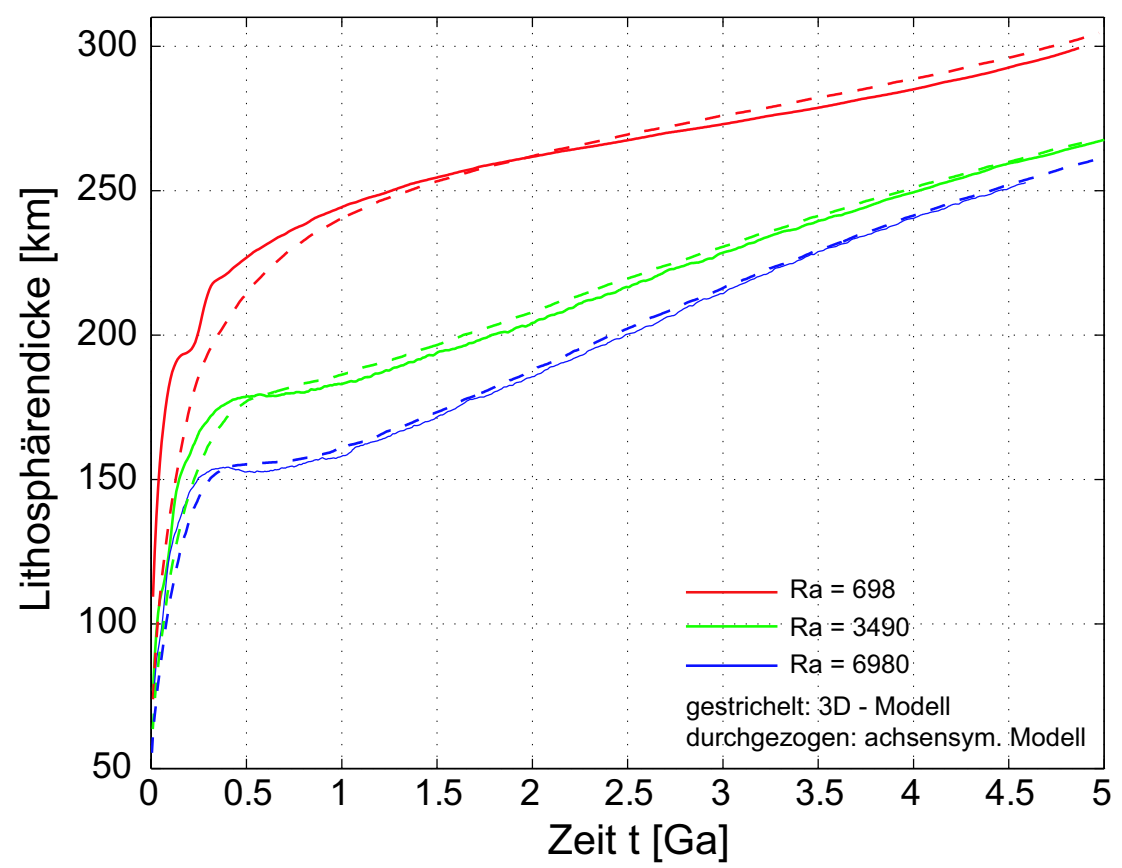

Abbildung 6.27: Entwicklung der Lithosphärendicke bei unterschiedlichen Rayleighzahlen in achsensymetrischen und vollständig dreidimensionalen Evolutionsmodellen.

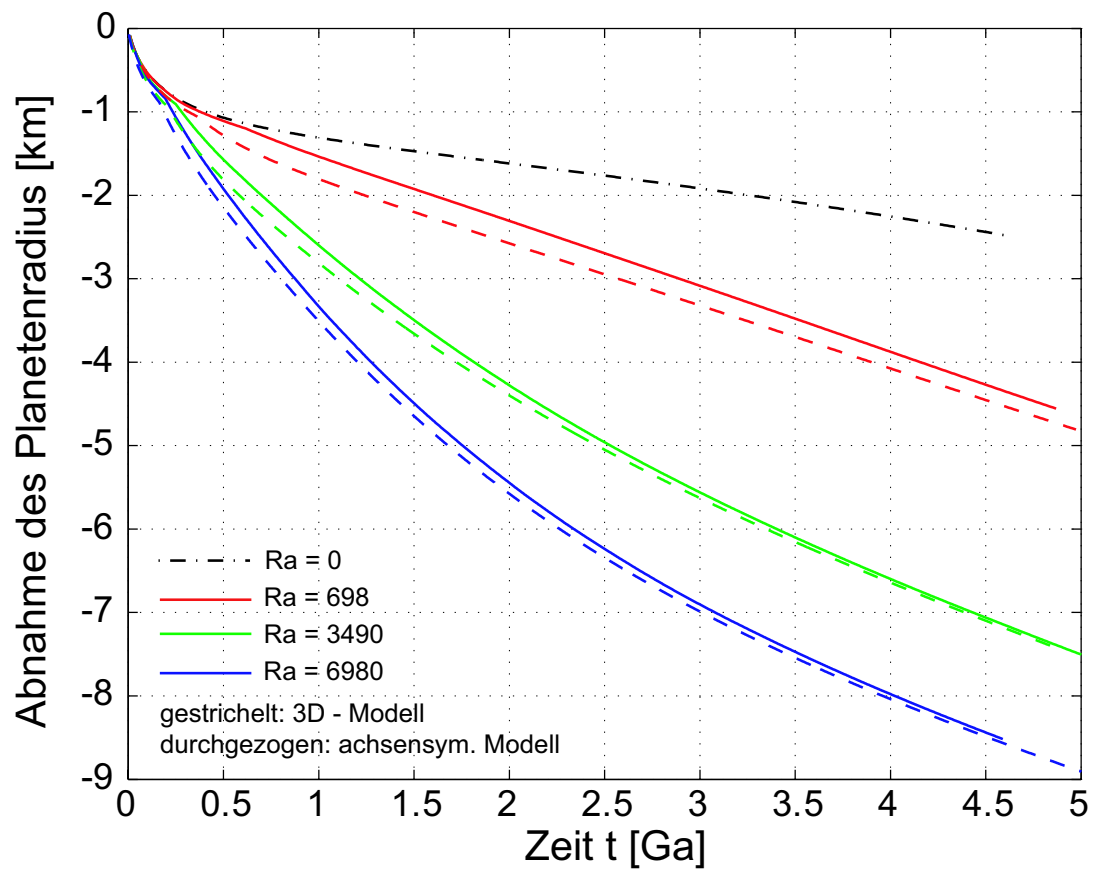

Abbildung 6.28: Abnahme des Planetenradius mit der Zeit. 
scheller aus und es bilden sich größere innere Kerne, so dass die Schrumpfungsraten deutlich größer sind. Bei $R a=3490$ nimmt der Planetenradius um $7 \mathrm{~km}(5 \mathrm{~km}$ in den letzten $3.8 \mathrm{Ga}$ ) und bei der höchsten Rayleighzahl um $8.4 \mathrm{~km}$ bzw. $5.9 \mathrm{~km}$ in den letzten $3.8 \mathrm{Ga}$ ab. Der Einfluss der Erweiterung vom achsensymmetrischen zum vollständig dreidimensionalen Modell ist vernachlässigbar. Verglichen mit den Beobachtungsdaten von 1 bis 2 $\mathrm{km}$ sind diese Schrumpfungsraten insbesondere bei den höheren Rayleighzahlen deutlich zu groß, selbst wenn die Radiusveränderung in den ersten $0.7 \mathrm{Ga}$ der Entwicklung, die unter Umständen nicht durch die Messungen wiedergegeben wird, vernachlässigt wird. Die geringste Schrumpfung des Planeten wird im Fall der reinen Wärmeleitung erziehlt und beträgt nur 2.4 bzw. $1.2 \mathrm{~km}$.

Das Konvektionsmuster nach 4.5 Ga für die dreidimensionalen Simulationen ist in $\mathrm{Ab}$ -

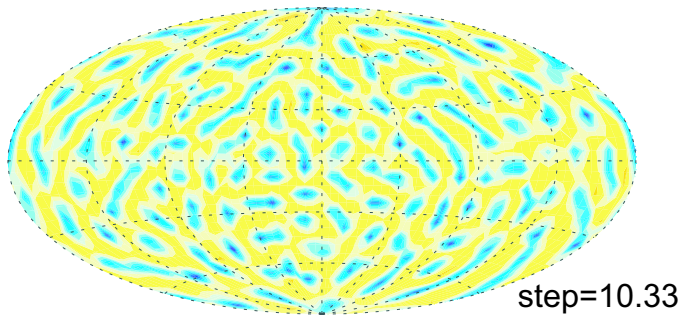

a) $\mathrm{Ra}=698$

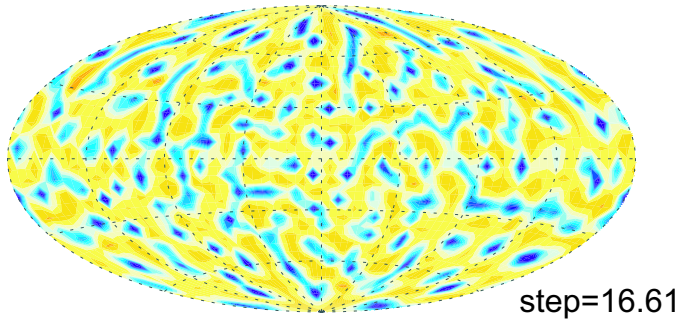

b) $\mathrm{Ra}=3490$

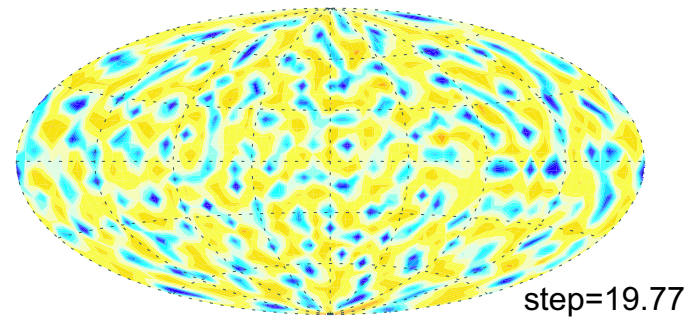

c) $\mathrm{Ra}=6980$

Abbildung 6.29: Konvektionsmuster der dreidimensionalen Simulationen nach 4.5 Ga: Radialgeschwindigkeit bei $r=1996$ km (blau: Abströme, rot: Aufströme). Der Contourstep der Geschwindigkeit ist angegeben: step=100 entspricht $0.58 \mathrm{~cm} / \mathrm{a}$.

bildung (6.29) durch die Radialgeschwindigkeit bei $r=1996 \mathrm{~km}$ dargestellt. Diese Tiefe liegt im isothermen Bereich des Temperaturprofils unterhalb der thermischen Grenzschicht und somit im konvektierenden Teil des Mantels. Das Konvektionsmuster in allen drei betrachteten Fällen ist sehr kleinskalig und durch die schnelleren Abströme dominiert. Das Anheben der Rayleighzahl führt lediglich zu einem Anstieg der Geschwindigkeitsamplitude und wirkt sich kaum auf die Struktur des Konvektionsmusters aus.

Abbildung (6.30) zeigt die Schnitte durch das Temperaturfeld nach $4.5 \mathrm{Ga}$ bzw. durch die Temperaturanomalie $(T-<T(r)>$ ) für die achsensymmetrischen Modelle und Abbildung (6.31) analog für die vollständig dreidimensionalen Simulationen. Für die dreidimensionalen Modelle liegen diese Schnitte bei $\phi=350^{\circ}(R a=698, R a=3490)$ bzw. $\phi=220^{\circ}$ für $R a=6980$. Wie die Analyse der Temperaturprofile bzw. der gemittelten Größen nahe legt, sind keine signikanten Unterschiede in der Amplitude der Temperatur bzw. der Temperaturanomalie zwischen dem zwei- und dreidimensionalen Fall zu be- 


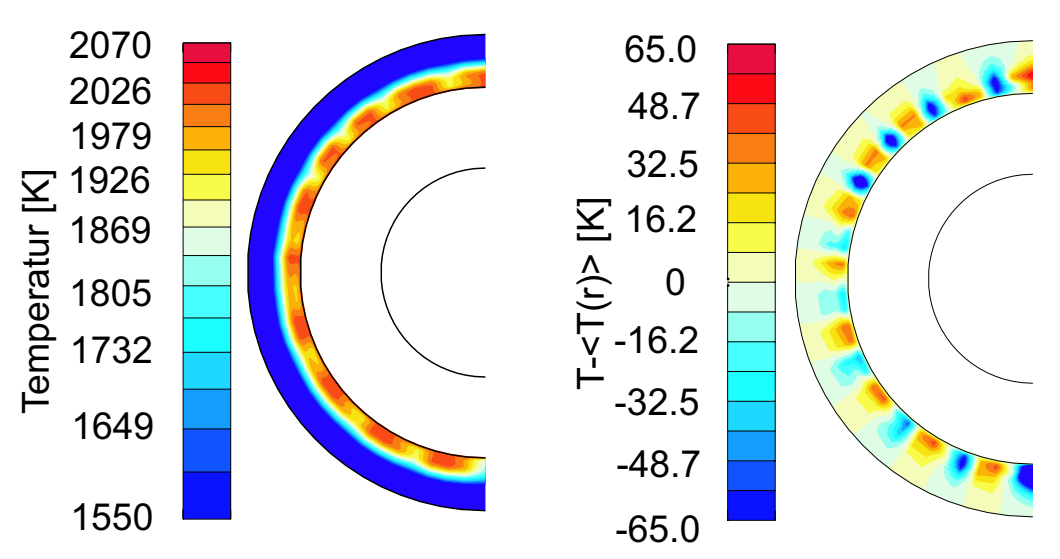

a) $\mathrm{Ra}=698$, achsensymmetrisch
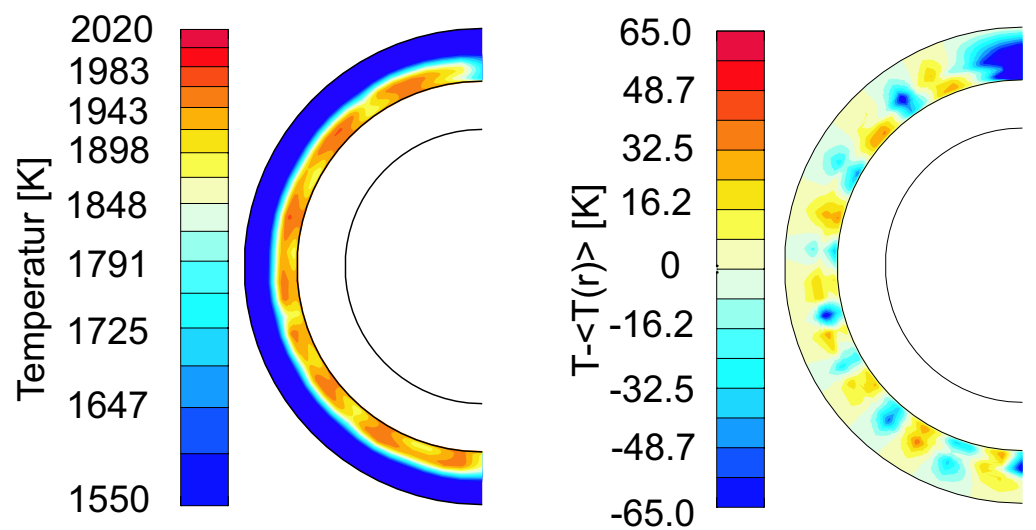

b) $\mathrm{Ra}=3490$, achsensymmetrisch
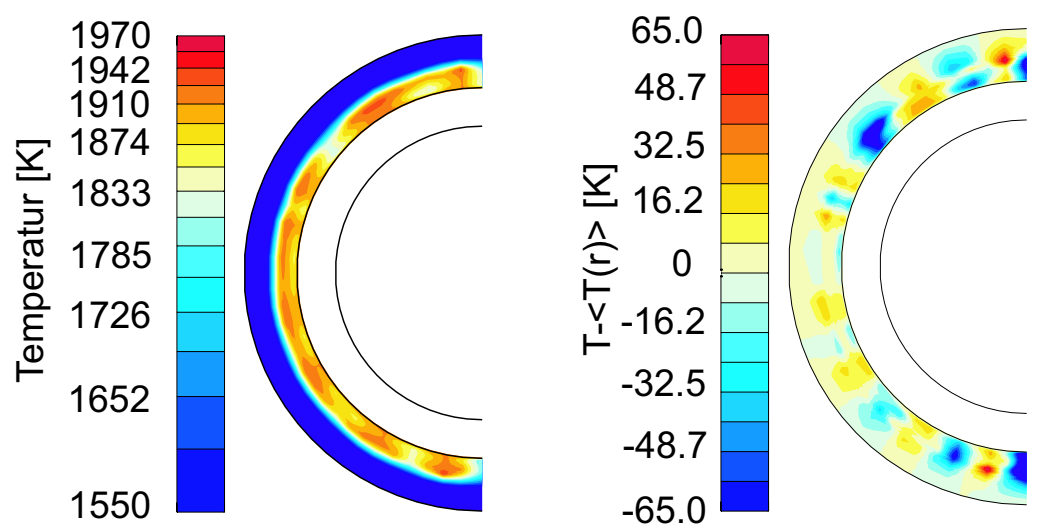

c) $R a=6980$, achsensymmetrisch

Abbildung 6.30: Schnitte durch das Temperaturfeld und die Temperaturanomalie $(T-<T(r)>)$ nach $4.5 \mathrm{Ga}$ für die achsensymmetrischen Modelle bei verschiedenen Rayleighzahlen. 

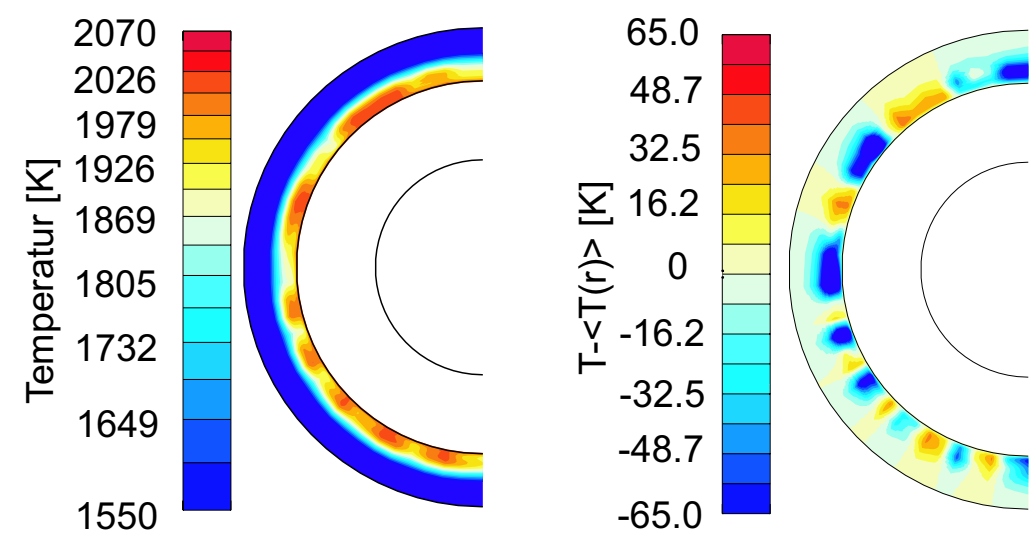

a) $\mathrm{Ra}=698,3 \mathrm{D}$
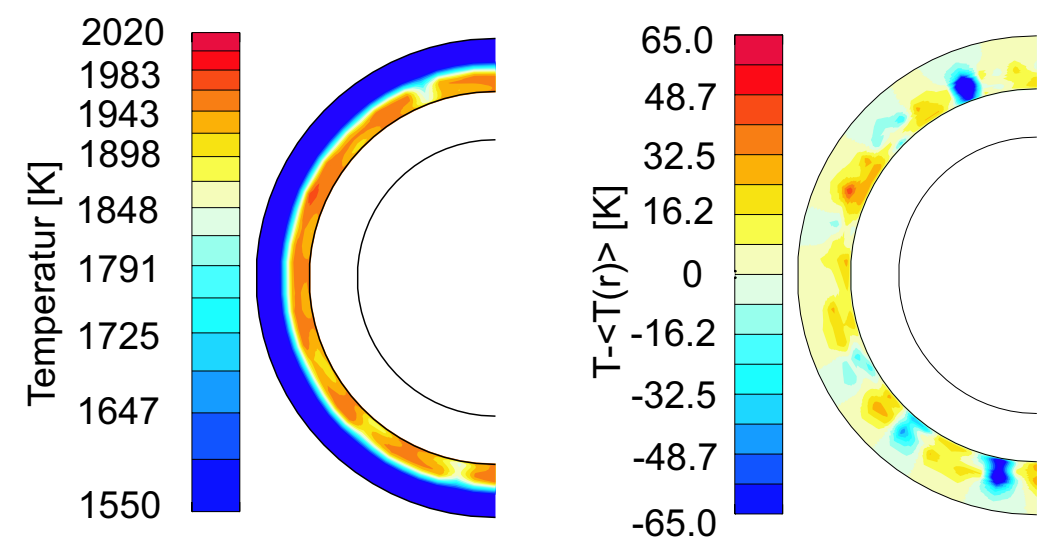

b) $R a=3490,3 D$
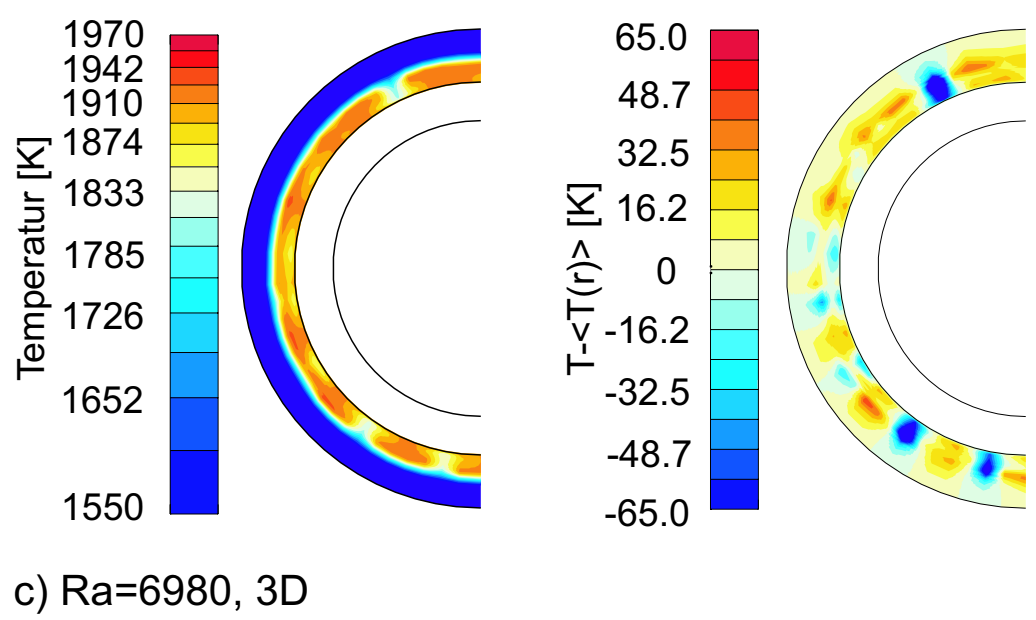

Abbildung 6.31: Schnitte durch das Temperaturfeld und die Temperaturanomalie $(T-<T(r)>)$ nach $4.5 \mathrm{Ga}$ für die vollständig dreidimensionalen Modelle bei verschiedenen Rayleighzahlen. Die Schnitte liegen bei $\phi=350^{\circ}(R a=698, R a=3490)$ bzw. $\phi=220^{\circ}(R a=6980)$. 
obachten. In allen Fällen ist die Dominanz der kalten Abströme in der Darstellung der Temperaturanomalie erkennbar. In den achsensymmetrischen Modellen wird beim Anstieg der Rayleighzahl die Anzahl der Aufströme von $10(R a=698)$ auf 8 ( $R a=3490$, $R a=6980$ ) reduziert. Zudem nimmt die Temperaturanomalie der Aufströme deutlich ab und die Dominanz der Abströme wird verstärkt. Der geringe Temperaturgradient an der KMG nach 4.5 Ga (Abbildung (6.26)) schwächt die Aufströme.

In der thermischen Entwicklung ist der Unterschied zwischen achsensymmetrischen und vollständig dreidimensionalen Modellen gering. Die Konvektionsstärke hat einen signifikanten Effekt auf die Evolution der Temperaturverteilung und die Entwicklung und Größe des inneren Kerns. Hier ist es wichtig, bessere Abschätzungen für die Viskosität zu gewinnen.

\subsubsection{Zusammenstellung weiterer Simulationsergebnisse}

\subsubsection{Variation des Aktivierungsvolumens}

Bei einer Rayleighzahl von $R a=6980$ ist der Einfluss des Aktivierungsvolumens auf das Kernwachstum und die thermische Entwicklung untersucht worden. Die Viskositätsprofile sind so normiert, dass sie im Startprofil $\left(T_{\text {Mantel }}(0)=1700 \mathrm{~K}, T_{\mathrm{KMG}}(0)=2100 \mathrm{~K}\right)$ etwa in halber Tiefe $(r=2153 \mathrm{~km})$ übereinstimmen. Bei allen Simulationen wird ein anfänglicher Schwefelgehalt von 2 Gew.-\% angenommen und die Schmelzkurve durch den ersten Parametersatz (siehe Tabelle (6.1), 'Schmelzkurve I') beschrieben.

In Tabelle (6.2) sind wichtige Größen nach 4.5 Ga zusammengestellt. Die Simulationser-

\begin{tabular}{|l|c|c|c|c|c|}
\hline$V^{*}\left[\mathrm{~cm}^{3} / \mathrm{mol}\right]$ & 0.0 & 8.02 & 12.02 & 16.04 & 20.03 \\
\hline Einsatz des Kernwachstums [Ga] & 0.15 & 0.19 & 0.22 & 0.24 & 0.26 \\
\hline Größe des inneren Kerns [km] & 1486 & 1504 & 1506 & 1510 & 1509 \\
\hline Temperatur an der KMG [K] & 1918 & 1910 & 1909 & 1908 & 1908 \\
\hline volumen-gem.Temperatur [K] & 1500 & 1490 & 1488 & 1483 & 1480 \\
\hline Wärmefluss (KMG) $\left[\mathrm{mW} / \mathrm{m}^{2}\right]$ & 4.0 & 3.6 & 3.5 & 3.3 & 3.2 \\
\hline Wärmefluss (Oberfläche) $\left[\mathrm{mW} / \mathrm{m}^{2}\right]$ & 22.6 & 22.4 & 22.4 & 22.3 & 22.2 \\
\hline Lithosphärendicke $[\mathrm{km}]$ & 252 & 251 & 249 & 247 & 245 \\
\hline Abnahme des Planetenradius $[\mathrm{km}]$ & -8.1 & -8.4 & -8.5 & -8.6 & -8.6 \\
\hline
\end{tabular}

Tabelle 6.2: Ergebnisse achsensymmetrischer Simulationen mit verschiedenen Aktivierungsvolumina. Bis auf den Einsatz des Kernwachstums gelten die Daten nach 4.5 Ga.

gebnisse zeigen, dass die Variation des Aktivierungsvolumens keinen signifikanten Effekt auf das Kernwachstum und die thermische Entwicklung hat. Dies gilt insbesondere für die Daten nach 4.5 Ga. In den Marsmodellen führt ein Erhöhen des Aktivierungsvolumens durch das stärker ausgeprägte Viskositätsminimum in der Nähe der Oberfläche zu einem schnelleren Auskühlen des Planeten (Kapitel (5.1)). In den Merkurmodellen ist der Einfluss des Aktivierungsvolumens wesentlich schwächer, da die niedrigviskose Zone nach kurzer Zeit Teil der dicken oberen Grenzschicht wird, die nicht an der Konvektionsbewegung teilnimmt. Der verbleibende Viskositätsunterschied durch das Aktivierungsvolumen 
kann die thermische Entwicklung weniger stark beeinflussen. So variiert der Wärmefluss an der KMG nach 4.5 Ga nicht stark mit dem Aktivierungsvolumen und ist in allen Simulationen zu gering, um einen thermisch getriebenen Dynamo zu realisieren. 


\section{Zusammenfassung und Diskussion}

\subsection{Evolutionsmodelle für Mars}

In der vorliegenden Arbeit sind erweiterte Konvektionsmodelle zur thermischen Entwicklung des Marsinneren vorgestellt worden. Diese Modelle versuchen, das besondere Konvektionsmuster im Marsmantel mit nur wenigen starken Aufströmen zu erklären. Dieses Konvektionsmuster könnte zu dem konzentrierten Vulkanismus an der Oberfläche führen. Ein weiteres Ziel ist es, die Entstehung der starken Krustenmagnetisierung innerhalb der ersten $0.5 \mathrm{Ga}$ der Entwicklung zu verstehen und eine Erklärung für das heutige Fehlen eines globalen Magnetfeldes zu geben. Im Einzelnen ist der Einfluss der Druckabhängigkeit der Viskosität, der Tiefenabhängigkeit des thermischen Ausdehnungskoeffizienten, der Druck- und Temperaturabhängigkeit der thermischen Leitfähigkeit, der exothermen Phasengrenzen sowie der Nicht-Boussinesq-Effekte (Temperatureffekte durch latente Wärme, adiabatische Kompression und viskose Reibung) auf das Konvektionsmuster und die thermische Evolution des Mars untersucht worden. Der Rolle der kernnahen endothermen Phasengrenze, die nur im Fall eines kleinen Marskerns möglich ist, gilt besondere Aufmerksamkeit.

Der kernnahe endotherme Phasenübergang von $\gamma$-Spinell zu Perowskit und Magnesiowüstit führt bei konstanten thermischen Parametern $(\alpha, \lambda)$ und einer temperatur- und druckabhängigen Viskosität im Lauf der Entwicklung zu einer Reduzierung der Anzahl der Aufströme. Nach 2.5 bis $3.0 \mathrm{Ga}$ ist das Konvektionsmuster von zwei starken Plumes geprägt. Die Anordnung von Auf- und Abströmen verändert sich danach nicht mehr und die nach 4.5 Ga bestehenden konzentrierten Aufströme könnten den heute auf dem Mars auf zwei Regionen beschränkten Vulkanismus erklären. Ohne den endothermenn Phasenübergang überleben sechs Aufströme die Evolution und lassen einen weiter verteilten Vulkanismus erwarten. Diese Wirkung der endothermen Phasengrenze ist jedoch abhängig von der Viskosität in der Tiefe des Übergangs. In Modellen mit einer reduzierten bzw. vernachlässigten Druckabhängigkeit der Viskosität entsteht trotz der endothermen Phasengrenze ein kleinskaliges Konvektionsmuster mit einer Vielzahl von sehr schwachen Aufströmen, die eine geringe Temperaturanomalie aufweisen. Das Konvektionsmuster ist durch die deutlich stärkeren Abströme geprägt. Die niedrigen Viskositäten in der Tiefe der Phasengrenze machen den Phasenübergang zu einer undurchdringlichen Barriere, die die Konvektion in zwei Schichten unterteilt. In der oberen Schicht kann die Phasengrenze das Konvektionsmuster nicht beeinflussen. Der verbleibende geringe Temperaturgradient in der unteren Grenzschicht dieser Schicht schwächt die Aufströme und führt zur Dominanz der Abströme. Damit die endotherme Phasengrenze die Anzahl der Aufströme reduzieren kann, darf eine Mindestviskosität in der Tiefe der Phasengrenze 
nicht unterschritten werden. Ein Verstärken der Druckabhängigkeit führt erneut zu einem Konvektionsmuster mit zwei Aufströmen, das sich durch die verstärkte Konvektion früher als im Referenzfall nach 1.6 - 2.1 Ga etabliert.

Ein tiefenabhängiger thermischer Ausdehnungskoeffizient unterdrückt Instabilitäten in der kernnahen thermischen Grenzschicht, auf die die endotherme Phasengrenze wirken kann. Gleichzeitig erhöht er die Bedeutung der Abströme. Der Einfluss der Phasengrenze auf das Konvektionsmuster nimmt somit ab. Für das Marsmodell mit endothermer Phasengrenze entsteht innerhalb von 1.3 - 1.9 Ga ein Konvektionsmuster mit drei Aufströmen. Ohne diesen Phasenübergang ist die Anzahl der Aufströme auf vier zurückgegangen.

Auch die Temperatur- und Druckabhängigkeit der thermischen Leitfähigkeit scheint, die kernnahe Grenzschicht zu stabilisieren und den Einfluss der Phasengrenze zu reduzieren. Allerdings ist der Einfluss in den Marsmodellen sehr gering. Ohne Phasengrenze verändert sich das Konvektionsmuster durch die Tiefenabhängigkeit der thermischen Leitfähigkeit nicht, mit Phasengrenze sind am Ende der Evolution drei (statt zwei) konzentrierte Aufströme beobachtbar.

Die Kombination von $\alpha(r)$ und $\lambda(r)$ führt zu einem sehr geringen Einfluss des endothermen Phasenübergangs auf das Konvektionsmuster. Mit und ohne Phasengrenze entsteht ein Konvektionsmuster mit drei konzentrierten Aufströmen. Lediglich die Zeit, die zum Erreichen dieses Musters benötigt wird, wird durch die endotherme Phasengrenze von $2.5 \mathrm{Ga}$ auf 1.0 - 1.5 Ga reduziert. Die zusätzlichen exothermen Phasengrenzen ermöglichen im Modell mit endothermer Phasengrenze wieder eine Reduzierung auf zwei Aufströme. Das Berücksichtigen der Zeit- bzw. Temperaturabhängigkeit der Positionen der Phasengrenzen führt zu keinen signifikanten Effekten. Das Konvektionsmuster mit zwei Aufströmen erweist sich auch dann als stabil, wenn nach Erreichen dieses Zustands die endotherme Phasengrenze entfernt wird.

Die Temperatureffekte der erweiterten Boussinesq-Näherung, insbesondere die latente Wärme der Phasenübergänge, haben keinen deutlichen Einfluss auf das Konvektionsmuster, wenn Viskositätseffekte durch den adiabatischen Temperaturanstieg geeignet kompensiert werden. Insgesamt sind die Unterschiede im Konvektionsmuster durch die Tiefenabhängigkeit der thermischen Parameter, wie sie im Mars vorliegen, insbesondere bei Berücksichtigung der endothermen Phasengrenze, gering. In diesem Fall entsteht ein Unterschied zwischen zwei bzw. drei Aufströmen, der eventuell von Details der Startkonfiguration beeinflusst werden kann, die in der Arbeit nicht weiter untersucht worden sind. Wichtig ist jedoch, dass der Einfluss der Phasengrenze, insbesondere bei der Kombination von $\alpha(r)$ und $\lambda(r)$ geringer wird und hier auch ohne endotherme Phasengrenze ein Konvektionsmuster mit drei konzentrierten Aufströmen erzeugt werden kann. Dieser Mechanismus ist nicht so effektiv wie die endotherme Phasengrenze, allerdings unabhängiger von der Kerngröße und könnte auch bei einem größeren Kern ohne endotherme Phasengrenze zu einer Reduzierung der Aufströme beitragen. Insbesondere die umfassenden Modelle mit Berücksichtigung der tiefenabhängigen thermischen Parameter und aller Phasengrenzen können sowohl in der Boussinesq-Näherung als auch in der erweiterten Boussinesq-Näherung den heute konzentrierten Vulkanismus auf dem Mars durch zwei konzentrierte Aufströme mit deutlicher Temperaturanomalie erklären. In der Entwicklung zu dieser Konfiguration sind die bis zum Ende verbleibenden Aufströme schon früh vorhanden und bleiben nahezu ortsfest, so dass auch die lange Geschichte des Vulkanismus in der Tharsis- und Elysium-Region verständlich wird. Die lange Lebensdauer der 
ortsfesten Aufströme, die fehlende Plattentektonik und die geringere Schwerebeschleunigung auf dem Mars erklären die gewaltige Größe der Marsvulkane. Den Modellen gelingt es allerdings nicht die Konzentration des Vulkanismus auf die beiden Zentren in nur 0.7 Ga zu erreichen, wie es die Datierungen der Vulkane in der alten Hemisphäre nahelegt. In der Temperaturentwicklung führt das Ausbilden einer niedrigviskosen Zone durch das Verstärken der Druckabhängigkeit der Viskosität bzw. der erhöhte thermische Ausdehnungskoeffizient im oberflächennahen Bereich zu einem Verstärken der Konvektion und einem effizienteren Abkühlen des Planeten. Das Ausbilden einer Zone mit niedriger thermischer Leitfähigkeit in der Nähe des Übergangs zur Lithosphäre hemmt das Abkühlen des Mantels. Insbesondere für den Marsmantel sind die Effekte eines tiefenabhängigen thermischen Ausdehnungskoeffizienten und einer tiefenabhängigen thermischen Leitfähigkeit auf die Temperaturentwicklung in halber Tiefe gering $(\sim 20 \mathrm{~K}$ nach $4.5 \mathrm{Ga})$ und kompensieren sich bei Kombination der Parameter. Die zusätzlichen exothermen Phasengrenzen ändern die Temperatur nicht signifikant. In halber Tiefe werden Temperaturen von $1605 \pm 5 \mathrm{~K}$ erreicht. Diese Temperatur liegt etwa im Bereich der Manteltemperaturen von ungefähr $1650 \mathrm{~K}$, die in parametrisierten Modellen ( $\alpha$ und $\lambda$ konstant) beobachtet werden, wenn das Lithosphärenwachstum berücksichtigt wird (Spohn et al. 2001a). Die Temperaturunterschiede durch die erweiterte Boussinesq-Näherung können durch die adiabatische Temperaturzunahme mit der Tiefe und die latente Wärme erklärt werden. Die viskose Reibung spielt im Marsmantel eine zu vernachlässigende Rolle.

An der KMG führen ein größeres Aktivierungsvolumen, ein tiefenabhängiger thermischer Ausdehnungskoeffizient und eine tiefenabhängige thermische Leitfähigkeit zu einem schnelleren Abkühlen, so dass sich dieser Effekt bei Kombination von $\alpha(r)$ und $\lambda(r)$ verstärkt. Insbesondere bei der Temperaturentwicklung an der KMG macht sich der verringerte Einfluss der endothermen Phasengrenze durch $\alpha(r)$ bzw. $\lambda(r)$ bemerkbar. Bei konstanten Materialparametern führt die endotherme Phasengrenze zu Kerntemperaturen, die nach $4.5 \mathrm{Ga}$ etwa $70 \mathrm{~K}$ über dem Fall ohne Phasengrenze liegen. Bei Kombination von $\alpha(r)$ und $\lambda(r)$ verringert sich dieser Unterschied auf lediglich $25 \mathrm{~K}$. Die Temperaturen liegen bei konstanten thermischen Parametern zwischen 1717 und 1790 K bzw. zwischen 1700 und $1735 \mathrm{~K}$ bei tiefenabhängigen thermischen Parametern, abhängig von den berücksichtigten Phasengrenzen. In der erweiterten Boussinesq-Näherung werden durch den Einfluss der Adiabate und die Beiträge der latente Wärme höhere Temperaturen zwischen 1975 K und 2090 K erreicht.

Die Temperaturentwicklung an der KMG ist eng mit der Frage nach der Existenz und Lebensdauer eines Dynamos im Mars verknüpft. Ein thermisch getriebener Dynamo ist möglich, wenn der Wärmefluss aus dem Kern über dem Wärmefluss entlang der Kernadiabate liegt. In allen Modellen liegt der Wärmefluss nach 4.5 Ga unter dem geschätzten Wertebereich bzw. an dessen unterer Grenze, so dass ein thermisch getriebener Dynamo im Mars heute nicht möglich ist. Gleichzeitig zeigt ein Vergleich zwischen den in den Kern interpolierten Temperaturen und den Schmelzkurven für Eisen mit einigen Prozent Schwefel, dass der Marskern bei einem Schwefelgehalt von mindestens $10 \%$ komplett flüssig ist und bisher keinen festen inneren Kern gebildet hat. Damit ist bis zum heutigen Zeitpunkt kein chemisch getriebener Dynamo im Mars möglich. Somit kann im Moment weder thermisch noch chemisch ein Dynamoprozess angetrieben werden, so dass das aktuelle Fehlen eines globalen Magnetfelds verständlich ist. In der Zukunft könnte durch das Wachsen eines inneren Kerns der Dynamoprozess neu gestartet werden. 
Die starken Krustenmagnetisierungen und ihr Fehlen in den großen Einschlagkratern legen jedoch nahe, dass Mars in den ersten 0.5 Ga seiner Entwicklung ein globales Magnetfeld und einen Dynamo besessen hat. Da der Kern flüssig ist, ist thermische Konvektion bei einem ausreichend überadiabatischen Wärmefluss möglich. Der Wärmefluss entlang der Adiabate ist nur unzureichend bekannt, so dass ein breites Intervall angeben wird, was eine genaue Bestimmung der Lebenszeit aus den Modellen nicht ermöglicht. Das Verstärken der Konvektion durch die stärker ausgeprägte niedrigviskose Zone (höheres $V^{*}$ ) erhöht den Wärmefluss aus dem Kern deutlich über die obere Grenze des Werteintervalls. Die Breite des Maximums im Wärmefluss nimmt gegenüber dem Referenzfall ab und ermöglicht kurze Lebenszeiten des Dynamos. Der Wärmefluss steigt in allen Simulationen durch den Initialisierungsprozess der Konvektion zunächst an, so dass der Dynamo unter Umständen erst verspätet aktiv wird. Durch diesen Effekt des Initialisierens ist es mit Hilfe der Simulationen schwierig, das genaue Einsetzen der Dynamoaktivität zu bestimmen. Ein für Mars realistisches Profil des thermischen Ausdehnungskoeffizienten hebt den Wärmefluss an der KMG gegenüber dem Referenzfall an. Es werden mittlere Werte des adiabatischen Wärmeflusses überschritten. Das Maximum im Wärmefluss bleibt allerdings bei Berücksichtigung der endothermen Phasengrenze sehr breit. Die tiefenabhängige thermische Leitfähigkeit erhöht den Wärmefluss nur geringfügig, so dass er auch bei Kombination von $\alpha(r)$ und $\lambda(r)$ vom Effekt des thermischen Ausdehnungskoeffizienten dominiert bleibt. Allerdings führen die exothermen Phasengrenzen zu einem deutlichen Anstieg des Wärmeflusses über den oberen Grenzwert hinaus, so dass ein thermisch getriebener Dynamo in der Anfangszeit wahrscheinlich ist. Wiederum ist das Maximum so breit, dass das Zeitfenster vermutlich nicht richtig getroffen werden kann. Akzeptiert man ein Konvektionsmuster mit drei Aufströmen und betrachtetet den Wärmefluss ohne endotherme Phasengrenze, so entsteht bei der Kombination von $\alpha(r)$ und $\lambda(r)$ unter Berücksichtigung der exothermen Phasengrenzen ein sehr schmales Maximum, dessen Amplitude einen thermisch getriebenen Dynamo sehr wahrscheinlich macht und dessen Breite auch eine kurze Lebensdauer zulässt. Ein sehr starkes Erhöhen der Startkerntemperatur erlaubt auch mit endothermer Phasengrenze eine kurze Periode der Aktivität.

Der Wärmefluss an der Oberfläche nach $4.5 \mathrm{Ga}$ ist von den Tiefenabhängigkeit der thermischen Parameter sowie von den Phasengrenzen nahezu unabhängig und liegt bei etwa $12.3 \pm 0.2 \mathrm{~mW} / \mathrm{m}^{2}$. Dieser Wert ist geringer als die Werte in parametrisierten Modellen zwischen 20 und $30 \mathrm{~mW} / \mathrm{m}^{2}$ (Spohn et al. 2001a). Zuber et al. (2000) schätzen den Wärmefluss aus der Dicke der elastischen Lithosphäre ab. Für die Tharsis-Erhebung wird ein Wert zwischen 10 und $20 \mathrm{~mW} / \mathrm{m}^{2}$ angegeben. Für das Hellas-Becken werden höhere Wärmeflüsse zwischen 38 und $56 \mathrm{~mW} / \mathrm{m}^{2}$ angenommen. Ein direkter Vergleich mit den Daten der Simulationen ist allerdings schwierig, da einerseits die Messdaten unterschiedliche Zeitpunkte wiedergeben und die Simulationsdaten einen gemittelten Wert für die Oberfläche angeben.

In den Modellen ist die Dicke der Lithosphäre aus den Profilen der Geschwindigkeit bestimmt worden. Die Lithosphäre ist durch sehr geringe Geschwindigkeiten charakterisiert, so dass der Wärmetransport durch Diffusion dominiert wird. Durch die vernachlässigbaren Geschwindigkeiten innerhalb der Lithosphäre formt sich eine kohärente Platte. Die Tiefenabhängigkeit der thermischen Parameter im Mars, sowie die unterschiedlichen Phasengrenzen beeinflussen die heutige Lithosphärendicke nur wenig. In den Modellen liegt ihr Wert zwischen 295 und 315 km. Der Vergleich der Simulationsdaten mit Be- 
obachtungsdaten ist schwierig. Es gibt Versuche, aus den gemessenen Topographie- und Schwerefelddaten auf die effektive elastische Lithosphärendicke zu schließen. Dabei wird die Lithosphäre als der Teil des Mantels aufgefasst, der über einen geologisch langen Zeitraum $\left(10^{8}\right.$ a) Spannungen aufrecht erhalten kann. Dies entspricht etwa der Lage der $650^{\circ}$ C Isotherme (Zuber et al. 2000). Mit diesem Verfahren ergeben sich Lithosphärendicken zwischen $5 \mathrm{~km}$ (Noachis Terra $\left(17.5^{\circ} \mathrm{S}-72.5^{\circ} \mathrm{S}, 40^{\circ} \mathrm{W}-40^{\circ} \mathrm{O}\right)$ ) und $250 \mathrm{~km}$ (Olympus Mons) (Zuber et al. 2000). Diese Daten gelten für den Zeitpunkt, als die Last, z.B. der Vulkankegel, aufgebracht worden ist. Die hier benutzte Beschreibung der Lithosphäre weicht von der in den Simulationen verwendeten Definition als kohärente Platte ab. Zudem geben die Modelle einen für den Planeten gemittelten Wert an, so dass zusammen mit den zeitlichen Unterschieden in den Daten ein Vergleich schwierig ist. Zur Bestimmung der rheologischen Lithosphäre liegen im Wesentlichen parametrisierte Modellrechnungen zur thermischen Evolution vor, die die heutige Dicke der Lithosphäre auf 100 - 400 km abschätzen. Die Dicke hängt in diesen Modellen stark vom angenommen Mechanismus des Wärmetransports und der Verteilung der radioaktiven Elemente ab (Spohn et al. 1998). Insgesamt können die hier präsentierten realistischeren Modelle den langlebigen, heute auf zwei Zentren konzentrierten Vulkanismus auf dem Mars erklären. Der heute geringe Wärmefluss aus dem Kern und das bisherige Fehlen eines inneren Kerns lassen weder thermische noch chemische Konvektion im Kern als Antriebsmechanismus für einen Dynamoprozess zu, so dass das Fehlen eines globalen Magnetfelds plausibel ist. In den umfassenden Modellen ist eine kurze Phase eines thermisch getriebenen Dynamos zu Beginn der Entwicklung möglich, die die starke Magnetisierung der alten Kruste erklären kann.

Im Konvektionsprogramm führen höhere effektive Rayleighzahlen (z.B. durch niedrigere Viskositäten bei hohen Temperaturen im Startprofil), hohe interne Heizraten und starke Viskositätskontraste sehr schnell zu numerischen Problemen, so dass die Wahl einiger Parameter, insbesondere der internen Heizrate und der Viskositätsparameter, durch die numerische Machbarkeit beschränkt werden (Kapitel (3.8) und (3.5)) und unterhalb der realistischen Werte für Mars oder zumindest an der unteren Grenze des angenommenen Bereichs liegen. Hier können Verbesserungen in der numerischen Umsetzung die Stabilität erhöhen, so dass Simulationen mit realistischeren Parametern z.B. im Viskositätsgesetz möglich werden. Ein Ansatz könnte sein, anstelle des Potentials für die Geschwindigkeit ein Potential für das Produkt aus Viskosität und Geschwindigkeit zu definieren. Diese Größe weist weniger starke Gradienten auf, da hohe Viskositäten mit geringen Geschwindigkeiten verbunden sind, so dass die numerische Stabilität eventuell erhöht werden kann. Die Veränderung der Parameter kann zukünftige Modelle realistischer gestalten. Zusätzlich könnte der Einfluss von geringen Mengen von Wasser in den oberen Schichten berücksichtigt werden, das das Fließverhalten des Gesteins verändert. Auch Schmelzen wirken sich auf die fluiddynamischen Eigenschaften aus. Die Berechnung der Schmelzen könnte zusätzlich Informationen über die genaue Entwicklung des Vulkanismus geben. Die Marsoberfläche ist von einer porösen Regolith- bzw. Megaregolithschicht bedeckt, die Wärme schlecht leitet. Ab einer gewissen Mächtigkeit könnte der isolierenden Charakter dieser Schicht die thermische Evolution beeinflussen.

Stein et al. (2004) stellen dreidimensionale kartesische Konvektionsmodelle mit einer temperatur- und spannungsabhängigen Viskosität vor, in denen es gelingt, wichtige Eigenschaften der Plattentektonik zu beschreiben. In ihren Modellen ist die Plattenbewe- 
gung allerdings nur auf kurze Intervalle beschränkt, die von langen Phasen einer stagnierenden Oberfläche gefolgt werden. Für Mars könnte die Berücksichtigung dieser NichtNewton'schen Rheologie eventuell konsistent ein kurze Phase der Plattentektonik simulieren und vielleicht zu einem Verständnis der Dichotomie beitragen.

In verschiedenen zukünftigen Marsmissionen ist u.a. der Einsatz von Seismometern auf dem Mars geplant. In der momentan gestoppten Netlander-Mission wird ein Netzwerk mit vier Seismometern angedacht. Das geophysikalische Paket der ESA-Mission ExoMars enthält ein Seismometer. Die Daten dieser zukünftigen Missionen können dazu beitragen, die innere Struktur des Mars und insbesondere die Größe des Kerns zu klären und so u.a. die Existenz des endothermen Phasenübergangs zu überprüfen.

\subsection{Evolutionsmodelle für Merkur}

In Kapitel 6 sind thermische Evolutionsmodelle für Merkur vorgestellt worden, die dazu beitragen sollen, die innere Struktur des großen Eisenkerns zu klären und mögliche Mechanismen zur Erzeugung des Magnetfeldes zu finden. Zunächst ist bei einer festen Rayleighzahl ( $R a=698)$ der Einfluss des Starttemperaturprofils auf die thermische Entwicklung des Planeten und das Ausbilden eines inneren Kerns analysiert worden. Es zeigt sich, dass bei höheren Mantelstarttemperaturen das Wachstum des inneren Kerns tendenziell später einsetzt, allerdings unterschiedliche Wachstumsraten dazu führen, dass sich die Größe des inneren Kerns annähert und nach $4.5 \mathrm{Ga}$ in einem engen Intervall von $r_{\mathrm{iK}}=1042 \mathrm{~km} \pm 58 \mathrm{~km}$ liegt. Höhere Anfangskerntemperaturen verzögern den Einsatz des Kernwachstums deutlicher und führen zu signifikant kleineren inneren Kernen $\left(r_{\mathrm{iK}}=818 \mathrm{~km}\left(T_{\mathrm{KMG}}(0)=2400 \mathrm{~K}\right)\right)$. Die prinzipielle Beobachtung bezüglich der Startkerntemperatur findet sich ebenfalls bei Conzelmann (1999).

Die heutige Temperatur an der KMG hängt nur schwach vom Startprofil ab und die Temperaturen liegen trotz anfänglicher Temperaturunterschiede von bis zu $300 \mathrm{~K}$ in einem engen Intervall: $T_{\mathrm{KMG}}=2014 \pm 11 \mathrm{~K}$. Das Temperaturprofil im Merkurmantel ist nach etwa 1.0 Ga durch eine dicke obere thermische Grenzschicht dominiert, die mehr als die Hälfte des Merkurmantels einnimmt. Die Konvektion ist dadurch auf eine dünne kernnahe Schicht mit einem geringen Temperaturkontrast beschränkt. Es werden nach $4.5 \mathrm{Ga}$ Lithosphärendicken von bis zu 294 km erreicht, wobei sowohl höhere Kernstarttemperaturen als auch höhere Mantelstarttemperaturen die Lithosphäre geringfügig verdünnen. Die dicke Lithosphäre und die nach 4.5 Ga schwache Konvektion lassen vermuten, dass rein konduktive Modelle die thermische Entwicklung und das Wachstum des inneren Kerns beschreiben könnten. Ein Vergleich der Modelle mit Simulationen, die nur die Wärmeleitung berücksichtigen, zeigt jedoch, dass dies nicht der Fall ist und unterstreicht die Bedeutung der Konvektion. In den konduktiven Modellen entstehen deutliche Unterschiede im Kernwachstum und der thermischen Entwicklung.

Das Ausbilden der dicken thermischen Grenzschicht unter der Oberfläche ist mit einem starken Abfall im Wärmefluss an der Oberfläche verbunden, der später in der Entwicklung einen nahezu vom Startprofil unabhängigen Wert annimmt.

Nach 4.5 Ga ist der Wärmefluss aus dem Kern für alle Startprofile zu gering, um einen thermisch getriebenen Dynamo zuzulassen. Der feste innere Kern nimmt allerdings höchstens $58 \%$ des Radius des gesamten Kerns ein, so dass ein ausreichender Anteil des Kerns 
flüssig bleibt und durch chemische Konvektion ein Dynamo angetrieben werden könnte, der eventuell die Existenz eines heutigen Magnetfeldes erklärt.

Die unterschiedlichen Starttemperaturbedingungen haben einen starken Einfluss auf die Volumenänderung des Planeten. Insbesondere das Abkühlen des zu Beginn sehr heißen Kerns führt zu Änderungen im Radius von bis zu 6 km, die über den beobachteten Werten liegen. Mit dem Referenzstartprofil ist die Änderung mit $2.8 \mathrm{~km}$ (für die letzten nach 3.8 $\mathrm{Ga})$ näher an den aus Messungen abgeschätzten Daten von $1 \mathrm{~km}$ bis $2 \mathrm{~km}$.

In einer zweiten Modellreihe ist der Einfluss des anfänglichen Schwefelgehaltes zwischen 1 und 4 Gew.-\% auf die Entwicklung des inneren Kerns untersucht worden. Da die Schmelzkurve von Eisen mit diesen geringen Schwefelzusätzen bisher noch nicht vermessen worden ist, werden dazu zwei verschiedene Parametrisierungen der Schmelzkurve der Fe-FeS-Legierung verwendet, die sich auf unterschiedlichen Messungen des Schmelzverhaltens (Boehler (1996a,c) und Fei et al. (1997, 2000b)) gründen. Ein Verringern des Schwefelgehalts erhöht die Schmelztemperaturen, so dass das Kernwachstum früher einsetzt und größere innere Kerne entstehen. Analog verzögert sich die Bildung des inneren Kerns bei einem höheren Schwefelanteil und es entstehen kleinere innere Kerne. Für die erste Parametrisierung der Schmelzkurve (vgl. Tabelle (6.1)) ist der innere Kern nach 4.5 Ga mit $1358 \mathrm{~km}$ bei 1 Gew.-\% Schwefel mehr als doppelt so groß wie im Fall mit 3 Gew.$\%$ Schwefel $(610 \mathrm{~km})$. Die höheren Schmelztemperaturen in der zweiten Parametrisierung liefern deutlich größere Radien für den inneren Kern (1123 km (3 Gew.-\% Schwefel)), so dass die genaue Kerngröße anhand der Modelle nicht festgelegt werden kann. Von der Parametrisierung der Schmelzkurve hängt es ab, ob bei einem noch höheren Schwefelanteil von z.B. 4 Gew.-\% überhaupt ein innerer Kern ausfriert. Ohne das Ausbilden eines inneren Kerns fehlt jedoch ein Antriebsmechanismus für einen hydrodynamischen Dynamo im Inneren, der das beobachtete Magnetfeld erklären kann. Aufgrund des geringen Wärmeflusses aus dem Kern in den Simulationen mit unterschiedlicher Anfangsschwefelkonzentration ist ein thermisch getriebener Dynamo nicht möglich. Die kleineren inneren Kerne bei höherem Schwefelgehalt verringern die Abnahme im Planetenradius, so dass bei einem Schwefelgehalt von 3 Gew.-\% ('Schmelzkurve I') bzw. 4 Gew.-\% ('Schmelzkurve II') Werte von 2.1 bzw. $2.3 \mathrm{~km}$ in den letzten $3.8 \mathrm{Ga}$ erreicht werden, die in die Nähe der Beobachtungen von 1 bis $2 \mathrm{~km}$ rücken. Um die Größe des inneren Kerns besser einschränken zu können, sind erweiterte Labormessungen für die Schmelzbedingungen von Eisen mit einigen Gewichtsprozent Schwefel bei Druck- und Temperaturbedingungen im Merkurkern erforderlich.

In einer dritten Simulationsreihe sind die bisher achsensymmetrischen Modelle für verschiedene Rayleighzahlen und damit unterschiedliche Mantelviskositäten zu vollständig dreidimensionalen Modellen erweitert worden. Diese Erweiterung des Modells ruft keine signifikanten Unterschiede in der Größe des inneren Kerns und der thermischen Situation nach 4.5 Ga hervor. Bei einer kleinen Rayleighzahl in der Nähe des Einsatzes der Konvektion gewinnen Details der anfänglich aufgebrachten Störung an Einfluss, so dass die anfängliche Entwicklung im achsensymmetrischen Fall geringfügig vom vollständig dreidimensionalen Fall abweicht. Das dreidimensionale Konvektionsmuster im Mantel ist kleinskalig und durch kalte Abströme bestimmt. Der geringe Temperaturkontrast zwischen Kern und Mantel unterstützt die Dominanz der Abströme, die auch in den achsensymmetrischen Modellen beobachtet werden konnte.

Mit einer erhöhten Rayleighzahl ( $R a=3490$ bzw. $R a=6980)$ kühlen sowohl Kern als 
auch Mantel schneller ab, so dass deutlich größere innere Kerne mit Radien von 1406 bzw. 1504 km möglich sind. Das starke Abkühlen des Systems bei hohen Rayleighzahlen führt aber darüber hinaus zu hohen, die Messdaten klar überschreitenden, Schrumpfungsraten für den Planetenradius. Auch beim Erhöhen der Rayleighzahl, die die Konvektion verstärkt und über weite Teile der Evolution den Wärmefluss aus dem Kern erhöht, liegt der heutige Wärmefluss aus dem Kern unter dem Wärmefluss entlang der Adiabate, so dass ein thermisch getriebener Dynamo ausgeschlossen werden kann.

Zusammenfassend haben sowohl die genaue Starttemperatursituation, die Schmelzbedingungen und der anfängliche Schwefelanteil als auch die angenommene Konvektionsstärke einen Einfluss auf die Entwicklung und die Größe des inneren Kerns. Die beobachtete Abnahme des Planetenradius von 1 bis $2 \mathrm{~km}$ lässt besonders warme Startmodelle mit sehr heißen Startkerntemperaturen unrealistischer erscheinen. Die Daten können bei der geeigneten Parametrisierung der Schmelzkurve mit einem Schwefelanteil im Kern von 2 - 4 Gew.- $\%$ bei einer niedrigen Rayleighzahl (hohe Referenzviskosität) erklärt werden. Allerdings sind die Beobachtung zur Abnahme des Planetenradius mit großen Unsicherheiten behaftet. In allen Modellen ist der heutige Wärmefluss aus dem Kern zu gering, um thermische Konvektion als Antriebsmechanismus für einen Dynamoprozess zuzulassen. Das Ausfrieren eines inneren Kerns und die dabei frei werdende leichte Komponente ermöglichen jedoch chemische Konvektion, die in einer ausreichend dicken, flüssigen Schicht einen Dynamo antreiben kann. Hier müssen weitere Analysen zu einer Abschätzung der Stärke des Magnetfeldes aus dem zur Verfügung stehenden Auftrieb beitragen. Die Abschätzung der Magnetfeldstärke könnte helfen, die Modellannahmen einzuschränken. Die Analyse der Topographie an der KMG und der auftretenden thermischen Anomalien in den dreidimensionalen Simulationen könnte Abschätzungen der Feldstärken und der Realisierbarkeit eines thermoelektrischen Dynamos (Stevenson 1987) ermöglichen.

Die Variation der Rayleighzahl und der Parametrisierung der Schmelzkurve zeigen einen weiten Bereich möglicher Radien des inneren Kerns auf. Um die innere Struktur besser einschränken zu können, ist eine verbesserte Abschätzung der Materialparameter im Merkurmantel (z.B. der Viskosität) erforderlich. Außerdem sind neue Erkenntnisse und Messungen des Schmelzverhaltens von Eisen mit einigen Prozent Schwefel bei Druckbedingungen des Kerns wichtig, die die Schmelzbedingungen besser festlegen.

Die Evolutionsmodelle können ähnlich wie die gezeigten Modelle für Mars durch tiefenabhängige Parameter $\alpha$ und $\lambda$ bzw. durch den Übergang zur erweiterten BoussinesqNäherung realitätsgetreuer gestaltet werden. Der Einfluss dieser tiefenabhängigen Parameter auf die thermische Entwicklung ist allerdings vermutlich noch geringer als im Mars, da im dünnen Mantel die Druckänderung kleiner ist. Eventuell kann aber die Veränderung des Konvektionsmusters im Zusammenhang mit dem thermoelektrischen Dynamo von Bedeutung sein.

Zunächst ist es wichtig, Möglichkeiten zu finden, die die Annahmen der Evolutionsmodelle einschränken. Viele der bisher vorliegenden Daten (Magnetfeld, Verringerung des Planetenradius) sind mit großen Unsicherheiten behaftet und andere wichtige Daten wie z.B. Angaben über das Trägheitsmoment fehlen völlig. Es ist zu hoffen, dass zukünftige Missionen wie BepiColombo die Datenlage deutlich verbessern und zusammen mit den Evolutionsmodellen ein stimmiges Bild der inneren Struktur und thermischen Entwicklung des Planeten Merkur und seines Magnetfeldes ergeben werden. 


\section{A Anhang}

\section{A.1 Abbildungsfunktion: radiales Gitter}

In Kapitel (4.1) wird eine nicht lineare Abbildungsfunktion zwischen den festen Tschebyscheff-Gitterpunkten $x_{k}$ und den radialen Leveln beschrieben:

$$
x_{k}=\sum_{i=0}^{8} a_{i} r_{k}^{i} \quad 0<k<N
$$

Die folgende Tabelle listet die Koeffizienten auf:

\begin{tabular}{|l|c|}
\hline$a_{i}$ & Wert \\
\hline$a_{0}$ & $3.660483748824042 \mathrm{e}+02$ \\
\hline$a_{1}$ & $-2.832787267928025 \mathrm{e}+03$ \\
\hline$a_{2}$ & $9.346631673220185 \mathrm{e}+03$ \\
\hline$a_{3}$ & $-1.726018714820188 \mathrm{e}+04$ \\
\hline$a_{4}$ & $1.954741055238378 \mathrm{e}+04$ \\
\hline$a_{5}$ & $-1.391874222895884 \mathrm{e}+04$ \\
\hline$a_{6}$ & $6.092002951922801 \mathrm{e}+03$ \\
\hline$a_{7}$ & $-1.499896621816303 \mathrm{e}+03$ \\
\hline$a_{8}$ & $1.591650212116777 \mathrm{e}+02$ \\
\hline
\end{tabular}

Tabelle A.1: Koeffizienten zur Darstellung der Abbildungsfunktion $x(r)$

Dieses Polynom wird durch die Addition einer linearen Funktion ggf. so korrigiert, dass sich für die beiden Randwerte $r_{i}$ und $r_{a}$, die durch die Bedingungen $r_{a}-r_{i}=1(2040 \mathrm{~km})$ und $r_{i} / r_{a}=0.4$ (für Mars) festliegen, die Werte -1 und +1 ergeben. Abbildung (A.1) zeigt die Zuordnung zwischen den Tschebyscheff-Gitterpunkten und den radialen Leveln, wie sie sich nach der Inversion von Gleichung (4.8) für den linearen Fall (blaue Kurve) bzw. von Gleichung (4.9) für das Polynom achter Ordnung (schwarze Kurve) ergibt. Mit dem Polynom achter Ordnung entstehen Unterschiede in der Tiefe der endothermen Phasengrenze $(r=1500 \mathrm{~km})$ und in der Nähe der Oberfläche. Das etwas schwächere Ansteigen der Funktion $r\left(x_{k}\right)$ liefert in der Tiefe der Phasengrenze eine geringfügig höhere Gitterpunktdichte. Der Einfluss der nicht linearen Abbildungsfunktion ist im oberflächennahen Bereich deutlicher. Der wesentlich stärkere Gradient von $r\left(x_{k}\right)$ führt dazu, dass weniger radiale Gitterpunkte in den oberen 200 km sind, die durch hohe Viskositäten und kleine Geschwindigkeiten in den Simulationen gekennzeichnet sind. Gleichzeitig nimmt die 


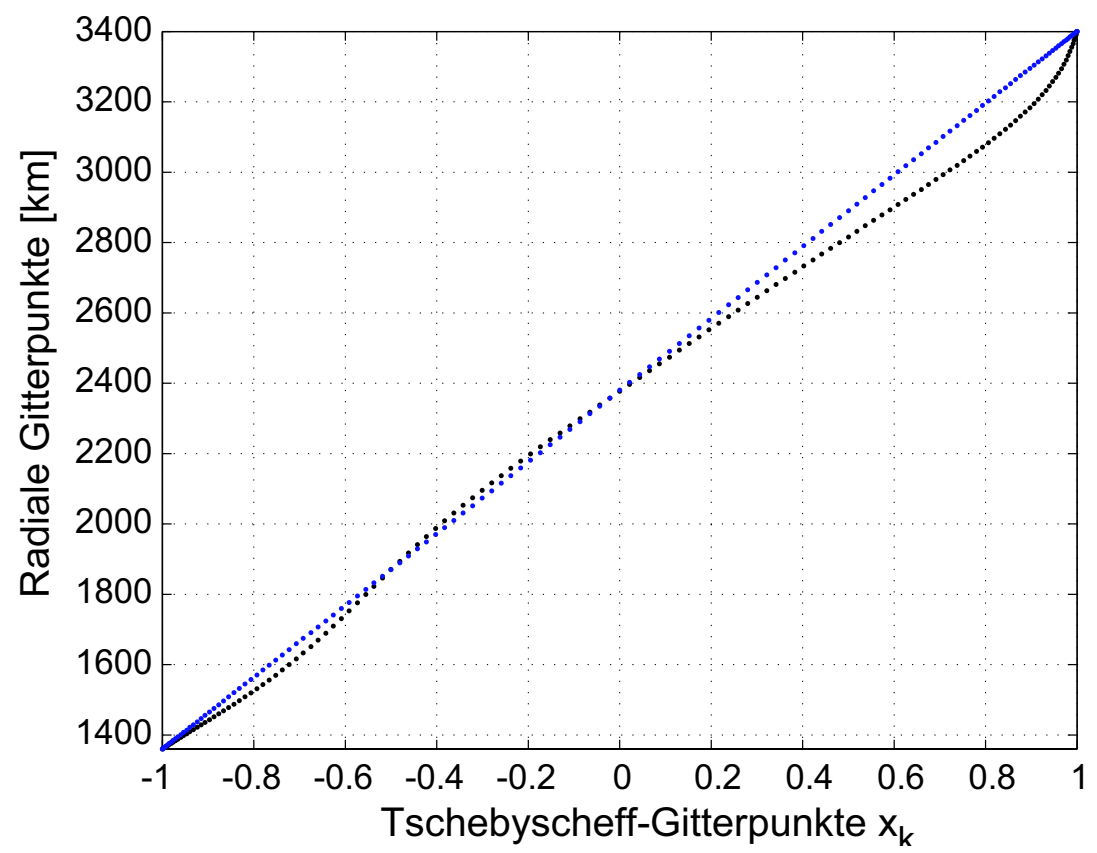

Abbildung A.1: Abbildungsfunktion zwischen den Tschebyscheff-Gitterpunkten und den radialen Leveln (blau: lineare Abbildungsfunktion nach Gleichung: (4.8), schwarz: Abbildungsfunktion nach Inversion von Polynom (4.9)).

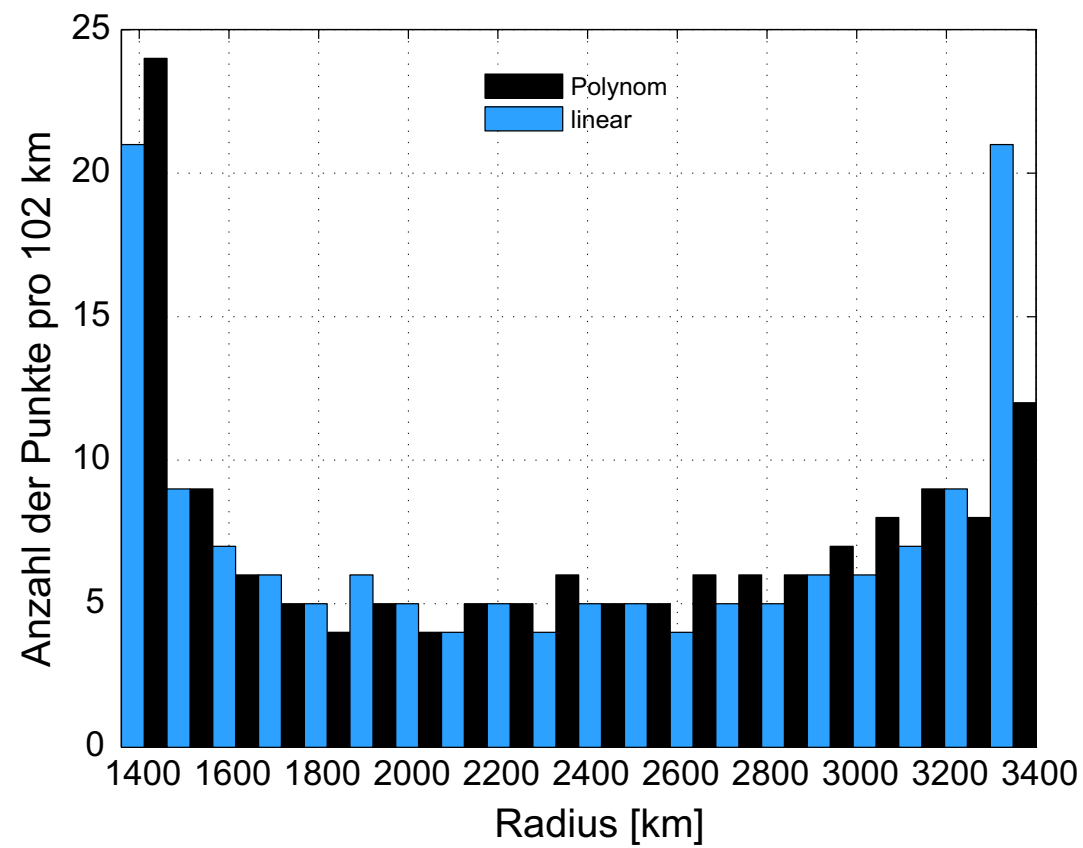

Abbildung A.2: Anzahl der Gitterpunkte in aufeinanderfolgenden Intervallen mit einer Länge von 102 km (blau: lineare Abbildungsfunktion, schwarz: Abbildungsfunktion mit einem Polynom achter Ordnung. 
Anzahl der Gitterpunkte zwischen $r=2600 \mathrm{~km}$ und $r=3200 \mathrm{~km} \mathrm{zu}$, in der starke Viskositätsgradienten zu erwarten sind. Die Punktdichte ist durch die Anzahl der radialen Gitterpunkte in aufeinanderfolgenden Intervallen der Länge $102 \mathrm{~km}$ in Abbildung (A.2) dargestellt.

\section{A.2 Bestimmung der Lithosphärendicke}

Die Lithosphärendicke wird aus den radialen Profilen der gemittelten Tangential- und Radialgeschwindigkeit ermittelt, indem der oberflächennahe Bereich bestimmt wird, in dem beide Geschwindigkeiten verschwinden bzw. einen Schwellenwert unterschreiten. Der rms-Wert der Radialgeschwindigkeit $v_{r}^{r m s}=\sqrt{\left\langle v_{r}(r)^{2}\right\rangle}$ wird als Wurzel des folgenden Ausdrucks berechnet:

$$
<v_{r}(r)^{2}>=\frac{1}{4 \pi r^{2}} \int_{0}^{\pi} \int_{0}^{2 \pi} v_{r}^{2}(\theta, \phi) r^{2} \sin \theta d \phi d \theta
$$

Die Radialgeschwindigkeit lässt sich durch das poloidale Potential in den Kugelflächenfunktionen folgendermaßen darstellen:

$$
v_{r}(r, \theta, \phi)=\frac{1}{r^{2}} \sum_{l, m} l(l+1) W_{l}^{m}(r) Y_{l}^{m}(\theta, \phi)
$$

Für das Integral folgt dann unter Ausnutzung der Normierung und Orthogonalität der Kugelflächenfunktionen:

$$
<v_{r}(r)^{2}>=\frac{1}{4 \pi r^{2}} \sum_{l, m} \frac{1}{r^{2}}\left[l(l+1) W_{l}^{m}(r)\right]^{2}
$$

Entsprechend gilt für den rms-Wert der Tangentialgeschwindigkeit $v_{\tan }^{r m s}=\sqrt{\left\langle v_{r}(r)^{2}\right\rangle}$ :

$$
<v_{\tan }(r)^{2}>=\frac{1}{4 \pi r^{2}} \int_{0}^{\pi} \int_{0}^{2 \pi}\left(v_{\phi}^{2}+v_{\theta}^{2}\right) r^{2} \sin \theta d \phi d \theta
$$

Dabei gilt folgende Darstellung durch Kugelflächenfunktion für $v_{\theta}$ und $v_{\phi}$ :

$$
\begin{gathered}
v_{\theta}=\frac{1}{r \sin \theta} \sum_{l, m} \frac{d W_{l}^{m}}{d r} \sin \theta \frac{\partial Y_{l}^{m}}{\partial \theta} \\
v_{\phi}=\frac{1}{r \sin \theta} \sum_{l, m} \frac{d W_{l}^{m}}{d r} \frac{\partial Y_{l}^{m}}{\partial \phi}
\end{gathered}
$$

Durch Einsetzen und Umformen ergibt sich dann:

$$
<v_{\tan }(r)^{2}>=\frac{1}{4 \pi r^{2}} \sum_{l, m} l(l+1)\left(\frac{d W_{l}^{m}(r)}{d r}\right)^{2}
$$

bzw. der gesuchte rms-Wert $v_{\tan }^{r m s}$ durch Wurzelziehen.

Der zu unterschreitende Schwellenwert wird durch die Peclet-Zahl $P e=v d / \kappa$ beschrieben, die den Beitrag der Advektion zum Wärmetransport ins Verhältnis zum Beitrag der 
Diffusion setzt und gleich der dimensionslosen Geschwindigkeit ist. Zur Bestimmung der Lithosphärendicke wird $P e=1.0$ verwendet, was bei den gewählten Parametern für Mars einer dimensionsbehafteten Geschwindigkeit von $0.0139 \mathrm{~mm} / \mathrm{a}$ entspricht. Der absolute Wert der Lithosphärendicke hängt aber vom verwendeten Schwellenwert ab. Dies ist in Abbildung (A.3) dargestellt. Die Lithosphärenmächtigkeit nimmt mit steigendem

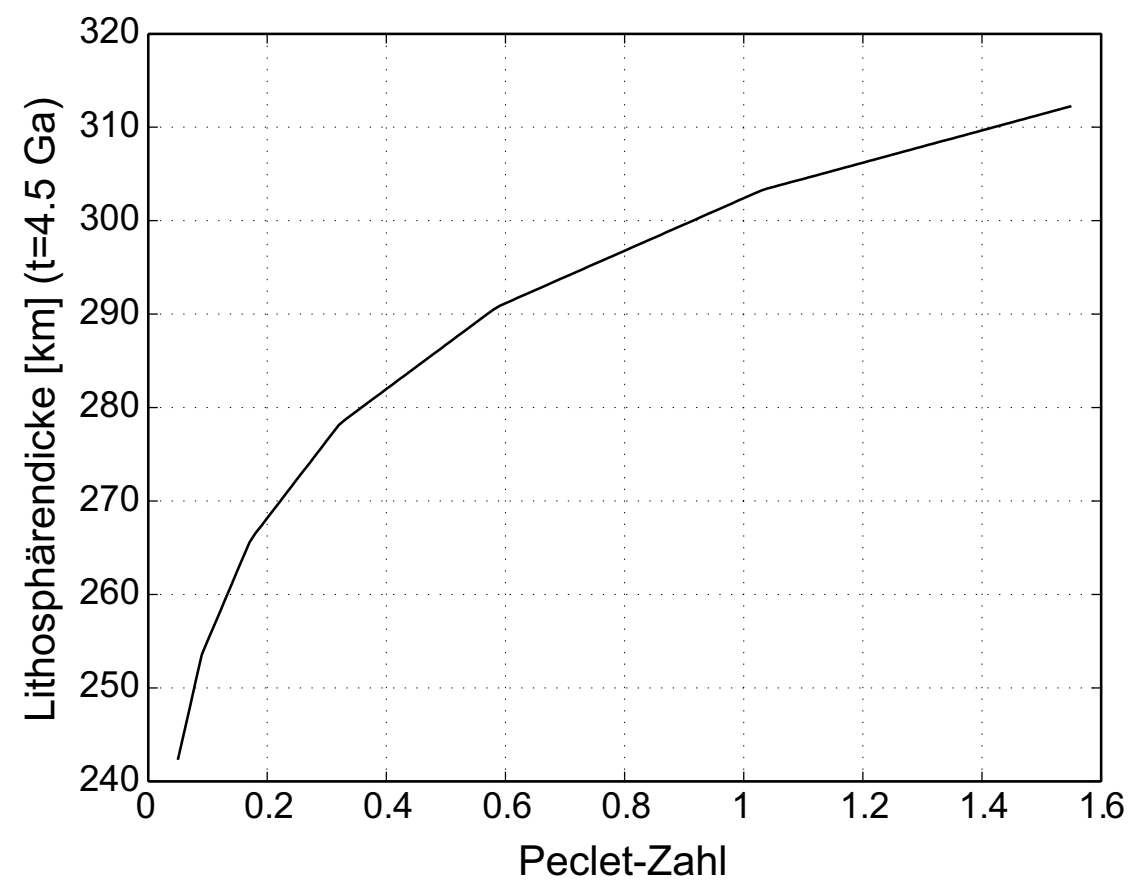

Abbildung A.3: Einfluss des Schwellenwertes bei der Bestimmung der Lithosphärendicke nach 4.5 Ga für das Marsmodell mit konstanten thermischen Parametern und endothermer Phasengrenze.

Schwellenwert zu. Dieser Anstieg ist jedoch nicht linear und für kleine Grenzgeschwindigkeiten deutlich stärker. Dies unterstützt die Wahl des Schwellenwertes bei $P e=1.0$, in dessen Bereich die Abhängigkeit deutlich geringer ist. In der Lithosphäre sind damit alle dimensionslosen Geschwindigkeiten kleiner als 1.0 ( $0.0139 \mathrm{~mm} / \mathrm{a})$, so dass hier der die Bedeutung des advektiven Wärmetransports gegenüber der Wärmeleitung abnimmt. 


\section{A.3 Modellparameter für Mars}

\begin{tabular}{|c|c|c|}
\hline & Symbol & Wert \\
\hline Planetenradius & $r_{a}$ & $3400 \cdot 10^{3} \mathrm{~m}$ \\
\hline Kernradius & $r_{i}$ & $1360 \cdot 10^{3} \mathrm{~m}$ \\
\hline Radienverhältnis & $\overline{r_{i} \backslash r_{a}}$ & 0.4 \\
\hline Gravitationsbeschleunigung & $g$ & $3.5 \mathrm{~m} / \mathrm{s}^{2}$ \\
\hline Manteldicke & $d$ & $2040 \cdot 10^{3} \mathrm{~m}$ \\
\hline Manteldichte & $\rho_{\text {Mantel }}$ & $3600 \mathrm{~kg} / \mathrm{m}^{3}$ \\
\hline Spezifische Wärmekapazität des Mantels & $C_{\text {Mantel }}$ & $1235 \mathrm{~J} / \mathrm{kg} \mathrm{K}$ \\
\hline Thermischer Ausdehnungskoeffizient & $\alpha_{\text {ref }}$ & $2.5 \cdot 10^{-5} \mathrm{~K}^{-1}$ \\
\hline Thermische Leitfähigkeit & $\lambda_{\text {ref }}$ & $4 \mathrm{~W} / \mathrm{mK}$ \\
\hline Thermische Diffusivität & $\kappa$ & $0.9 \cdot 10^{-6} \mathrm{~m}^{2} / \mathrm{s}$ \\
\hline Thermische Diffusionszeit & $d^{2} \backslash \kappa$ & $146.6 \mathrm{Ga}$ \\
\hline Kompressionsmodul & $K_{0}$ & $160 \mathrm{GPa}$ \\
\hline Viskosität & $\eta_{\text {ref }}$ & $3.3 \cdot 10^{21} \mathrm{~Pa} \mathrm{~s}$ \\
\hline Aktivierungsenergie & $E^{*}$ & $187.5 \mathrm{~kJ} / \mathrm{mol}$ \\
\hline Aktivierungsvolumen & $V^{*}$ & $3 \mathrm{~cm}^{3} / \mathrm{mol}$ \\
\hline 'cut-off'-Temperatur & $T_{\text {cutoff }}$ & $783 \mathrm{~K}$ \\
\hline Anfängliche Temperaturdifferenz & $\Delta T$ & $2000 \mathrm{~K}$ \\
\hline Temperatur an der Oberfläche & $T_{0}$ & $243 \mathrm{~K}$ \\
\hline Temperatur im Mantelinneren vor $4.5 \mathrm{Ga}$ & $T_{\mathrm{int}}$ & $1751 \mathrm{~K}$ \\
\hline Heizrate pro Volumen vor $4.5 \mathrm{Ga}$ & $H_{0}$ & $1.836 \cdot 10^{-8} \mathrm{~W} / \mathrm{m}^{3}$ \\
\hline Zerfallskonstante & $\lambda^{*}$ & $0.434(\mathrm{Ga})^{-1}$ \\
\hline Rayleighzahl bei Referenzviskosität & $\mathrm{Ra}$ & 1800000 \\
\hline Dissipationszahl & Di & 0.1445 \\
\hline Radius des Phasenübergangs: $\alpha$ - $\beta$-Spinell & $r_{\alpha \beta}$ & $2338 \mathrm{~km}$ \\
\hline Clapeyron-Steigung $(\alpha$ - $\beta$-Spinell) & $\gamma_{\alpha \beta}$ & $+2.5 \cdot 10^{6} \mathrm{~Pa} / \mathrm{K}$ \\
\hline Dichteänderung ( $\alpha$ - $\beta$-Spinell) & $(\Delta \rho)_{\alpha \beta}$ & $252 \mathrm{~kg} / \mathrm{m}^{3}$ \\
\hline Phasenparameter $(\alpha-\beta$-Spinell) & $P_{\alpha \beta}$ & +0.27230 \\
\hline Radius des Phasenübergangs: $\beta-\gamma$-Spinell & $r_{\beta \gamma}$ & $2053 \mathrm{~km}$ \\
\hline Clapeyron-Steigung $(\beta-\gamma-$ Spinell $)$ & $\gamma_{\beta \gamma}$ & $+5.0 \cdot 10^{6} \mathrm{~Pa} / \mathrm{K}$ \\
\hline Dichteänderung $(\beta-\gamma$-Spinell) & $(\Delta \rho)_{\beta \gamma}$ & $150 \mathrm{~kg} / \mathrm{m}^{3}$ \\
\hline Phasenparameter $(\beta-\gamma$-Spinell $)$ & $P_{\beta \gamma}$ & +0.32420 \\
\hline Radius des Phasenübergangs: $\gamma$-Spinell-Perowskit & $r_{\gamma p v}$ & $1497 \mathrm{~km}$ \\
\hline Clapeyron-Steigung $(\gamma$-Spinell-Perowskit) & $\gamma_{\gamma p v}$ & $-2.5 \cdot 10^{6} \mathrm{~Pa} / \mathrm{K}$ \\
\hline Dichteänderung $(\gamma$-Spinell-Perowskit $)$ & $(\Delta \rho)_{\gamma p v}$ & $252 \mathrm{~kg} / \mathrm{m}^{3}$ \\
\hline Phasenparameter ( $\gamma$-Spinell-Perowskit) & $P_{\gamma p v}$ & -0.27230 \\
\hline Kerndichte & $\rho_{\text {Kern }}$ & $8500 \mathrm{~kg} / \mathrm{m}^{3}$ \\
\hline Spezifische Wärmekapazität des Kerns & $C_{\text {Kern }}$ & $700 \mathrm{~J} / \mathrm{Kg} \mathrm{K}$ \\
\hline
\end{tabular}

Tabelle A.2: Parameter für die Marsmodelle (Referenzen, Diskussion siehe Kapitel (2)). 


\section{A.4 Modellparameter für Merkur}

\begin{tabular}{|l|c|c|}
\hline & Symbol & Wert \\
\hline Planetenradius & $r_{a}$ & $2440 \cdot 10^{3} \mathrm{~m}$ \\
\hline Kernradius & $r_{i}$ & $1900 \cdot 10^{3} \mathrm{~m}$ \\
\hline Radienverhältnis & $r_{i} \backslash r_{a}$ & 0.7787 \\
\hline Gravitationsbeschleunigung & $g$ & $3.8 \mathrm{~m} / \mathrm{s}^{2}$ \\
\hline Manteldicke & $d$ & $540 \cdot 10^{3} \mathrm{~m}$ \\
\hline Manteldichte & $\rho_{\text {Mantel }}$ & $2835 \mathrm{~kg} / \mathrm{m}^{3}$ \\
\hline Spezifische Wärmekapazität des Mantels & $C_{\text {Mantel }}$ & $1400 \mathrm{~J} / \mathrm{kg} \mathrm{K}$ \\
\hline Thermischer Ausdehnungskoeffizient & $\alpha$ & $2.0 \cdot 10^{-5} \mathrm{~K}^{-1}$ \\
\hline Thermische Leitfähigkeit & $\lambda$ & $4 \mathrm{~W} / \mathrm{mK}^{2}$ \\
\hline Thermische Diffusivität & $\kappa$ & $1.0 \cdot 10^{-6} \mathrm{~m}^{2} / \mathrm{s}$ \\
\hline Thermische Diffusionszeit & $d^{2} \backslash \kappa$ & $9.247 \mathrm{Ga}$ \\
\hline Viskosität in halber Tiefe (1573 K) & $\eta_{\mathrm{ref}}$ & $8.1 \cdot 10^{22} \mathrm{~Pa} \mathrm{~s}$ \\
\hline Aktivierungsenergie & $E^{*}$ & $466.07 \mathrm{~kJ} / \mathrm{mol}^{*}$ \\
\hline Aktivierungsvolumen & $V^{*}$ & $8.02 \mathrm{~cm} / \mathrm{mol}^{3}$ \\
\hline untere Viskositätsgrenze & $\eta_{1}$ & $4.2 \cdot 10^{16} \mathrm{~Pa} \mathrm{~s}$ \\
\hline obere Viskositätsgrenze & $\eta_{\infty}$ & $4.2 \cdot 10^{24} \mathrm{~Pa} \mathrm{~s}$ \\
\hline Anfängliche Temperaturdifferenz & $\Delta T$ & $1660 \mathrm{~K}$ \\
\hline Temperatur an der Oberfläche & $T_{0}$ & $440 \mathrm{~K}$ \\
\hline Temperatur im Mantelinneren vor $4.5 \mathrm{Ga}$ & $T_{\text {int }}$ & $1600 \mathrm{~K}$ \\
\hline Heizrate pro Volumen vor 4.5 Ga & $H_{0}$ & $5.2373 \cdot 10^{-8} \mathrm{~W} / \mathrm{m}^{3}$ \\
\hline Zerfallskonstante & $\lambda^{*}$ & $0.0495(\mathrm{Ga})^{-1}$ \\
\hline Rayleighzahl bei Referenzviskosität & Ra & 698 \\
\hline Kerndichte & $\rho_{\mathrm{Kern}}$ & $8352 \mathrm{~kg} / \mathrm{m}^{3}$ \\
\hline Dichte von Schwefel bei Kerndruck & $\rho_{\mathrm{S}}$ & $6077.78 \mathrm{~kg} / \mathrm{m}^{3}$ \\
\hline Dichte von Eisen bei Kerndruck & $\rho_{\mathrm{Fe}}$ & $8412.27 \mathrm{~kg} / \mathrm{m}^{3}$ \\
\hline Druck im Planetenzentrum & $P_{z}$ & $40 \mathrm{GPa}$ \\
\hline Druck an der KMG & $P_{\mathrm{KMG}}$ & $10 \mathrm{GPa}$ \\
\hline Spezifische Wärmekapazität des Kerns & $C_{\mathrm{Kern}}$ & $750 \mathrm{~J} / \mathrm{Kg} \mathrm{K}$ \\
\hline Latente Wärme & $L$ & $2.5 \cdot 10^{5} \mathrm{~J} / \mathrm{Kg}$ \\
\hline Konstanten der Kernadiabate & $T_{a} 1$ & $8 \cdot 10^{-12} \mathrm{~Pa}{ }^{-1}$ \\
\hline & $T_{a} 2$ & $-3.9 \cdot 10^{-23} \mathrm{~Pa}{ }^{-2}$ \\
\hline
\end{tabular}

Tabelle A.3: Verwendete Parameter für die Merkurmodelle (Conzelmann 1999). 


\section{A.5 Änderung des Planetenradius}

Das Abkühlen des Planeten ist mit einer Reduzierung des Volumens verbunden, die sich durch ein Abnahme des Radius bemerkbar macht. Dabei wird der Planet vereinfacht als ideale Kugel beschrieben, in der Masse und Energie erhalten bleiben. Die hier beschriebenen Berechnung der Radiusänderung entspricht dem Vorgehen von Conzelmann (1999) und Schubert et al. (1988).

Zur Volumenänderung trägt zum einen das Ausfrieren des inneren festen Kerns bei, da die Dichte des festen Eisens höher ist als die der Flüssigkeit. Der Dichteunterschied $\Delta \rho_{\mathrm{fl}}$ liegt nach Longhi et al. (1992) zwischen 200 und $300 \mathrm{~kg} / \mathrm{m}^{3}$. Für die vorliegenden Simulationen wird ein Wert von $200 \mathrm{~kg} / \mathrm{m}^{3}$ verwendet. Für die Radiusänderung durch das Ausbilden eines inneren Kerns $\Delta R_{\text {Planet }}\left(r_{i K}\right)$ gilt:

$$
\Delta R_{\text {Planet }}\left(r_{i K}\right)=-\frac{1}{3} \frac{\Delta \rho_{\mathrm{fl}}}{\rho_{\text {Kern }}} \frac{r_{i K}^{3}}{r_{a}^{2}}
$$

Der Kern liefert durch sein eigentliches Abkühlen einen zweiten Beitrag zur Volumenänderung. Stevenson et al. (1983) benutzen eine lineare Beziehung zwischen der Temperatur an der KMG $T_{\mathrm{KMG}}(t)$ und der über das Kernvolumen gemittelten Temperatur im Kern. Mit $\beta=1.11$ als Proportionalitätskonstante (Conzelmann 1999) lässt sich der Beitrag zur Änderung des Planetenradius wie folgt beschreiben:

$$
\Delta R_{\text {Planet }}\left(T_{\mathrm{KMG}}\right)=-\frac{r_{i}^{3}}{3 r_{a}^{2}} \alpha_{\mathrm{Fe}} \beta\left[T_{\mathrm{KMG}}(0)-T_{\mathrm{KMG}}(t)\right]
$$

Für den Ausdehungskoeffizient von Eisen $\alpha_{\mathrm{Fe}}$ wird ein Wert von $\alpha_{\mathrm{Fe}}=3 \cdot 10^{-5} \mathrm{~K}^{-1}$ benutzt (Boehler et al. 1990).

Den letzten Beitrag zur Verringerung des Planetenradius liefert der Mantel. Mit der über das Volumen gemittelten Temperatur $T_{\mathrm{V}}$ folgt:

$$
\Delta R_{\text {Planet }}\left(T_{\mathrm{V}}\right)=-\frac{r_{a}^{3}-r_{i}^{3}}{3 r_{a}^{2}} \alpha\left[T_{\mathrm{V}}(0)-T_{\mathrm{V}}(t)\right]
$$

Die volumen-gemittelte Temperatur bestimmt sich aus dem Integral:

$$
T_{\mathrm{V}}(t)=\frac{3}{4 \pi\left(r_{a}^{3}-r_{i}^{3}\right)} \int_{r_{i}}^{r_{a}} \int_{0}^{\pi} \int_{0}^{2 \pi} T(t, r, \phi, \theta) r^{2} \sin \theta d r d \theta d \phi
$$

Für die Gesamtänderung des Planetenradius gilt:

$$
\Delta R_{\text {Planet }}=\Delta R_{\text {Planet }}\left(r_{i K}\right)+\Delta R_{\text {Planet }}\left(T_{\text {KMG }}\right)+\Delta R_{\text {Planet }}\left(T_{\mathrm{V}}\right)
$$

Entsprechend der Temperaturänderung schrumpft der Planet bzw. verringert sich der Radius beim Abkühlen bzw. vergrößert sich der Radius beim Aufheizen des Planeten. 



\section{Literaturverzeichnis}

Acuña, M. H., Connerney, J. E. P., Ness, N. F., Lin, R. P., Mitchell, D., Carlson, C. W., McFadden, J., Anderson, K. A., Rème, H., Mazelle, C., Vigne, D., Wasilewski, P., Cloutier, P., 1999, Global distribution of crustal magnetization discovered by the Mars Global Surveyor MAG/ER experiment, Science, 284, 790-793

Akaogi, M., Ito, E., 1993, Refinement of enthalpy measurement of $\mathrm{MgSiO}_{3}$-perovskite and negative pressure-temperature slopes for perovskite-forming reactions, Geophysical Research Letters, 20, 1839-1842

Akaogi, M., Ito, E., Navrotsky, A., 1989, Olivine-modified-spinel transitions in the system $\mathrm{Mg}_{2} \mathrm{SiO}_{4}-\mathrm{Fe}_{2} \mathrm{SiO}_{4}$ : Calorimetric measurements, thermochemical calculations, and geophysical applications, Journal of Geophysical Research, 94, 15 671-15685

Anderson, J. D., Colombo, G., Esposito, P. B., Lau, E. L., Trager, G. P., 1987, The mass, gravity field, ephemeris of Mercury, Icarus, 71, 337-349

Anderson, O. L., 1967, Equation for thermal expansivity in planetary interiors, Journal of Geophysical Research, 72, 3661-3668

Anderson, O. L., 1995, Mineral physics of iron and of the core, Reviews of Geophysics, $33,429-441$

Anderson, O. L., 1998, The Grüneisen parameter for iron at outer core conditions and the resulting conductive heat and power in the core, Physics of the Earth and Planetary Interior, 109, 179-197

Anderson, O. L., Oda, H., Isaak, D., 1992, A model for the computation of thermal expansivity at high compression and high temperatures: $\mathrm{MgO}$ as an example, Geophysical Research Letters, 19, 1987-1990

Bertka, C. M., Fei, Y., 1996, Constraints on the mineralogy of an iron-rich martian mantle from high-pressure experiments, Planetary and Space Science, 44, 1269-1276

Bertka, C. M., Fei, Y., 1997, Mineralogy of the martian interior up to core-mantle boundary, Journal of Geophysical Research, 102, 5251-5264

Bertka, C. M., Fei, Y., 1998, Density profile of SNC model martian interior and the moment-of-inertia factor of Mars, Earth and Planetary Science Letters, 157, 79-88

Billich, H., 1995, Numerische Modellrechnungen zur Konvektion im Mond, Diplomarbeit, Institut für Geophysik, Universiät Göttingen 
Bina, C. R., Helffrich, G., 1994, Phase transitions Clapeyron slopes and transition zone seismic discontinuity topography, Journal of Geophysical Research, 99, 15 853-15860

Birch, F., 1968, Thermal expansion at high pressures, Journal of Geophysical Research, 73, 817-819

Boehler, R., 1986, The phase diagram of iron to 430 kbar, Geophysical Research Letters, $13,1153-1156$

Boehler, R., 1992, Melting of the Fe-FeO and the Fe-FeS systems at high pressure: Constraints on core temperatures, Earth and Planetary Science Letters, 111, 217-227

Boehler, R., 1993, Temperatures in the Earth's core from melting-point measurements of iron at high static pressures, Nature, 363, 534-536

Boehler, R., 1996a, Melting temperature of the Earth's mantle and core: Earth's thermal structure, Annual Review of Earth and Planetary Sciences, 24, 15-40

Boehler, R., 1996b, Fe-FeS eutectic temperatures to 620 kbar, Physics of the Earth and Planetary Interiors, 96, 181-186

Boehler, R., 1996c, Experimental constraints on melting conditions relevant to core formation, Geochimica et Cosmochimica Acta, 60, 1109-1112

Boehler, R., 2000, High-pressure experiments and the phase diagram of lower mantle and core materials, Reviews of Geophysics, 38, 221-245

Boehler, R., Chopelas, A., 1991, A new approch to laser heating in high pressure mineral physics, Geophysical Research Letters, 18, 1147-1150

Boehler, R., von Bargen, N., Chopelas, A., 1990, Melting, thermal expansion and phase transitions of iron at high pressures, Journal of Geophysical Research, 95, 21 73121736

Breuer, D., Yuen, D. A., Spohn, T., Zhang, S., 1998, Three dimensional models of martian mantle convection with phase transitions, Geophysical Research Letters, 25, 229-232

Buske, M., 2002, Dreidimensionale Evolutionsmodelle der Konvektion im Marsmantel, Diplomarbeit, Institut für Geophysik, Universität Göttingen

Butler, S. L., Peltier, W. R., Costin, S. O., 2005, Numerical models for the Earth's thermal history: Effects of inner-core solidification and core potassium, Physics of the Earth and Planetary Interiors, 152, 22-42

Cameron, A. G. W., Fegley, B., Benz, W., Slattery, W. L., 1988, The strange density of Mercury: Theoretical considerations, in Mercury, (Hg.) F. Vilas, C. R. Chapman, M. S. Matthews, S. 692-708, University of Arizona Press, Tucson

Carpozen, L., Gilder, S. A., Hart, R. J., 2005, Paleomagnetism of the Vredefort meteorite crater and implicatons for craters on Mars, Nature, 435, 198-201 
Chopelas, A., Boehler, R., 1989, Thermal expansion measurement at very high pressure, systematics, and a case for a chemically homogeneous mantle, Geophysical Research Letters, 16, 1347-1350

Chopelas, A., Boehler, R., 1992, Thermal expansivity in the lower mantle, Geophysical Research Letters, 19, 1983-1986

Chopelas, A., Boehler, R., Ko, T., 1994, Thermodynamics and behavior of $\gamma-\mathrm{Mg}_{2} \mathrm{SiO}_{4}$ at high pressure: Implications for $\mathrm{Mg}_{2} \mathrm{SiO}_{4}$ phase equilibrium, Physics and Chemistry of Minerals, 21, 351-359

Christensen, U., 1995, Effects of phase transitions on mantle convection, Annu. Rev. Earth Planet. Sci, 23, 65-87

Christensen, U., 2000, Vorlesungsskript: Einführung in die Geodynamik WS 00/01, Institut für Geophysik, Universität Göttingen

Christensen, U. R., 1984, Convection with pressure- and temperature-dependent nonNewtonian rheology, Geophys. J. R. Astron. Soc., 77, 343-384

Christensen, U. R., Yuen, D. A., 1985, Layered convection induced by phase transitions, Journal of Geophysical Research, 90, 10 291-10 300

Connerney, J. E. P., Ness, N. F., 1988, Mercury's magnetic field and interior, in Mercury, (Hg.) F. Vilas, C. R. Chapman, M. S. Matthews, S. 494-513, University of Arizona Press, Tucson

Connerney, J. E. P., Acuña, M. H., Wasilewski, P. J., Ness, N. F., Rème, H., Mazelle, C., Vignes, D., Lin, R. P., Mitchell, D. L., Cloutier, P. A., 1999, Magnetic lineation in the ancient crust of Mars, Science, 284, 794-798

Conzelmann, V., 1999, Thermische Evolution des Planeten Merkur berechnet unter Anwendung verschiedener Viskositätsgesetze, Dissertation, Westfälische Wilhelms Universität Münster

Dreibus, G., Wänke, H., 1985, Mars: A volatile rich planet, Meteoritics, 20, 367-382

Dreibus, G., Wänke, H., 1987, Volatiles on Earth and Mars: A comparison, Icarus, 71, 225-240

Dubuffet, F., Yuen, D. A., 2000, A thick pipe-like heat-transfer mechanism in the mantle: nonlinear coupling between 3-D convection and varible thermal conductivity., Geophysical Research Letters, 27, 17-20

Dubuffet, F., Yuen, D. A., Rainey, E. S. G., 2002, Controlling thermal chaos in the mantle by positive feedback from radiative thermal conductivity, Nonlinear Processes in Geophysics, 9, 311-323

Espostio, P. B., Banerdt, W. B., Lindal, G. F., Sjorgen, W. L., Slade, M. A., Bills, B. G., Smith, D. E., Blamino, G., 1992, Gravity and topography, in Mars, (Hg.) H. Kieffer, S. 209-248, University of Arizona Press, Tucson 
Fei, Y., Bertka, C. M., 1996, The state of the martian core, in Lunar and Planetary Institute Conference Abstracts, S. 351-352

Fei, Y., Prewitt, C. T., Mao, H., Bertka, C. M., 1995, Structure and density of FeS at high pressure and high temperature and the internal structure of Mars, Science, 268, 1892-1894

Fei, Y., Bertka, C. M., Finger, L. W., 1997, High-pressure iron-sulfur compound, $\mathrm{Fe}_{3} \mathrm{~S}_{2}$ and melting relations in the Fe-FeS system, Science, 275, 1621-1623

Fei, Y., Li, J., Bertka, C. M., 2000a, The equation of state of a new iron-sulfur compound $\mathrm{Fe}_{3} \mathrm{~S}$ and its application to modeling of the density of the martian core, in Lunar and Planetary Institute Conference Abstracts, S. 1722-1923

Fei, Y., Li, J., Bertka, C. M., Prewitt, C. T., 2000b, Structure type and bulk modulus of $\mathrm{Fe}_{3} \mathrm{~S}$, a new iron-sulfur compound, American Mineralogist, 85, 1830-1833

Folkner, W. M., Yoder, C. F., Yuan, D. N., Standish, E. M., Preston, R. A., 1997, Interior structure and seasonal mass redistribution of Mars from radio tracking of Mars Pathfinder, Science, 278, 1749-1752

Glatzmaier, G. A., 1984, Numerical simulations of stellar convective dynamos. I The model and method, Journal of Computational Physics, 55, 461-484

Goettel, K. A., 1988, Present bounds on the bulk composition of Mercury: Implications for planetary formation processes, in Mercury, (Hg.) F. Vilas, C. R. Chapman, M. S. Matthews, S. 613-621, University of Arizona Press, Tucson

Grasset, O., Parmentier, E. M., 1998, Thermal convection in volumetrically heated, infinite Prandtl number fluid with strongly temperature-dependent viscosity: Implications for planetary thermal evolution, Journal of Geophysical Research, 103, 18 171-18 181

Gudkova, T. V., Zharkov, V. N., 2004, Mars: interior structure and excitation of free oscillations, Physics of the Earth and Planetary Interiors, 142, 1-22

Hansen, U., Yuen, D. A., Kroening, S. E., 1991, Effects of depth-dependent thermal expansivity on mantle circulations and lateral thermal anomalies, Geophysical Research Letters, 18, 1261-1264

Hansen, U., Yuen, D. A., Kroening, S. E., Larsen, T. B., 1993, Dynamical consequences of depth-dependent thermal expansivity and viscosity on mantle circulations and thermal structure, Physics of the Earth and Planetary Interiors, 77, 205-223

Harder, H., 1998, Phase transitions and three-dimensional planform of thermal convection in the martian mantle, Journal of Geophysical Research, 103, 16775-16797

Harder, H., 2000, Mantle convection and the dynamic geoid of Mars, Geophysical Research Letters, 27, 301-304

Harder, H., Christensen, U. R., 1996, A one-plume model of martian mantle convection, Nature, 380, 507-509 
Harder, H., Schubert, G., 2001, Sulfur in Mercury's core, Icarus, 151, 118-122

Hauber, E., van Gasselt, S., Ivanov, B., Werner, S., Head, J. W., Neukum, G., Jaumann, R., Greeley, R., Mitchell, K. L., Muller, P., and the HRSC Co-Investigator Team, 2005, Discovery of a flank caldera and very young glacial acitvity at Hecates Tholus, Mars, Nature, 434, 356-360

Hauck, S. A., Dombard, A. J., Phillips, R. J., Solomon, S. C., 2004, Internal and tectonic evolution of Mercury, Earth and Planetary Science Letters, 222, 713-728

Hirose, K., 2002, Phase transitions in pyrolitic mantle around 670-km depth: Implications for upwelling of plumes from lower mantle, Journal of Geophysical Research, 107, $3.1-3.11$

Hofmeister, A. M., 1999, Mantle values of thermal conductivity and the geotherm from phonon lifetime, Science, 283, 1699-1706

Ita, J., Stixrude, L., 1992, Petrology, elasticity, and composition of the mantle transition zone, Journal of Geophysical Research, 97, 6849-6866

Ito, E., Takahashi, E., 1989, Postspinel transformation in the system $\mathrm{Mg}_{2} \mathrm{SiO}_{4}-\mathrm{Fe}_{2} \mathrm{SiO}_{4}$ and some geophysical implications, Journal of Geophysical Research, 94, $10637-$ 10646

Karato, S., Wu, P., 1993, Rheology of the upper mantle: A synthesis, Science, 260, 771778

Karato, S.-I., Li, P., 1992, Diffusion creep in perovskite: Implications for the rheology of the lower mantle, Science, 5049, 1238-1240

Katsura, T., Ito, E., 1989, The system $\mathrm{Mg}_{2} \mathrm{SiO}_{4}-\mathrm{Fe}_{2} \mathrm{SiO}_{4}$ at high pressures and temperatures: Precise determination of stabilities of olivine, modified spinel and spinel, Journal of Geophysical Research, 94, 15 663-15670

Kiefer, W. S., Bills, B. G., Nerem, R. S., 1996, An inversion of gravity and topography for mantle and crustal structure on Mars, Journal of Geophysical Research, 101, 92389252

Kieffer, H. H., Jakosky, B. M., Snyder, C. W., 1992, The planet Mars: from antiquity to the present, in Mars, (Hg.) H. Kieffer, S. 1-34, University of Arizona Press, Tucson

Kleine, T., Münker, C., Mezger, K., Palme, H., 2002, Rapid accretion and early core formation on asteroids and the terrestrial planets from Hf-W chronometry, Nature, 418, 952-954

Lee, D. C., Halliday, A. N., 1997, Core formation on Mars and differentiated asteroids, Nature, 388, 854-857

Leitch, A. M., Yuen, D. A., Sewell, G., 1991, Mantle convection with internal heating and pressure-dependent thermal expansivity, Earth and Planetary Science Letters, 102, 213-232 
Longhi, J., Knittle, E., Holloway, J. R., Wänke, H., 1992, The bulk composition, mineralogy and internal structure of Mars, in Mars, (Hg.) H. Kieffer, S. 184-208, University of Arizona Press, Tucson

Meade, C., Jeanloz, R., 1990, The strength of mantle silicates at high pressures and room temperature: implications for the viscosity of the mantle, Nature, 348, 533-535

Melosh, H. J., McKinnon, W. B., 1988, The tectonics of Mercury, in Mercury, (Hg.) F. Vilas, C. R. Chapman, M. S. Matthews, S. 374-400, University of Arizona Press, Tucson

Mohit, P. S., Arkani-Hamed, J., 2004, Impact demagnetizaton of the martian crust, Icarus, $168,305-317$

Neukum, G., Hiller, K., 1981, Martian ages, Journal of Geophysical Research, 86, 30973121

Neukum, G., Jaumann, R., Hoffmann, H., Hauber, E., Head, J. W., Basilevsky, A. T., Ivanov, B., Werner, S., van Gasselt, S., Murray, J., McCord, T., and the HRSC CoInvestigator Team, 2004, Recent and episodic volcanic and glacial activity on Mars revealed by the High Resolution Stereo Camera, Nature, 432, 971-979

Nimmo, F., Stevenson, D. J., 2000, Influence of early plate tectonics on the thermal evolution and magnetic field on Mars, Journal of Geophysical Research, 105, 11 969-11979

Purucker, M., Ravat, D., Frey, H., Voorhies, C., Sabaka, T., Acuña, M., 2000, An altitudenormalized magnetic map of Mars and its interpretation, Geophysical Research Letters, $27,2449-2452$

Reynard, B., Price, G. D., 1990, Thermal expansion of mantle minerals at high pressures - a theoretical study, Geophysical Research Letters, 17, 689-692

Sammis, C. G., Smith, J. C., Schubert, G., Yuen, D. A., 1977, Viscosity-depth profile of the Earth's mantle: Effects of polymorphic phase transitions, Journal of Geophysical Research, 82, 3747-3761

Schubert, G., Spohn, T., 1990, Thermal history of Mars and the sulfur content of its core, Journal of Geophysical Research, 95, 14 095-14 104

Schubert, G., Ross, M. N., Stevenson, D. J., Spohn, T., 1988, Mercury's thermal history and the generation of its magnetic field, in Mercury, (Hg.) F. Vilas, C. R. Chapman, M. S. Matthews, S. 429-460, University of Arizona Press, Tucson

Schubert, G., Russell, C. T., Moore, W. B., 2000, Timing of the martian dynamo, Nature, 408, 666-667

Schubert, G., Turcotte, D. L., Olson, P., 2001, Mantle Convection in the Earth and Planets, Cambridge University Press, Cambridge

Sleep, N. H., 1994, Martian plate tectonics, Journal of Geophysical Research, 99, 56395655 
Smith, D. E., Zuber, M. T., Solomon, S. C., Phillips, R. J., Head, J. W., Garvin, J. B., Banerdt, W. B., Muhleman, D. O., Pettengill, G. H., Neumann, G. A., Lemoine, F. G., Abshire, J. B., Aharonson, O., Brown, C. D., Hauck, S. A., Ivanov, A. B., McGovern, P. J., Zwally, H. J., Duxbury, T. C., 1999, The global topography of Mars and implications for surface evolution, Science, 284, 1495-1501

Sohl, F., Spohn, T., 1997, The interior structure of Mars: Implications from SNC meteorites, Journal of Geophysical Research, 102, 1613-1635

Spohn, T., 1991, Mantle differentian and thermal evolution of Mars, Mercury and Venus, Icarus, 90, 222-236

Spohn, T., Sohl, F., Breuer, D., 1998, Mars, The Astronomy and Astrophysics Review, 8, $181-236$

Spohn, T., Acuña, M. H., Breuer, D., Golombek, M., Greeley, R., Hallyday, A., Hauber, E., Jaumann, R., Sohl, F., 2001a, Geophysical constraints on the evolution of Mars, Space Science Reviews, 96, 1-34

Spohn, T., Sohl, F., Wieczerkowski, K., Conzelmann, V., 2001b, The interior structure of Mercury: what we know, what we expect from BepiColombo, Planetary and Space Science, 49, 1561-1570

Stein, C., Schmalzl, J., Hansen, U., 2004, The effect of rheological parameters on plate behaviour in a self-consistent model of mantle convection, Physics of the Earth and Planetary Interiors, 142, 225-255

Stevenson, D. J., 1987, Mercury's magnetic field: a thermoelectric dynamo?, Earth and Planetary Science Letters, 82, 114-120

Stevenson, D. J., 2001, Mars' core and magnetism, Nature - Insight, 412, 214-219

Stevenson, D. J., Spohn, T., Schubert, G., 1983, Magnetism and thermal evolution of the terrestrial planets, Icarus, 54, 466-489

Strom, R. G., 1997, Mercury: An overview, Advances in Space Research, 19, 1471-1485

Sunder-Plaßmann, T., 1996, Vergleich verschiedener Ansätze zur Untersuchung der thermischen Evolution konvektierender Systeme, Diplomarbeit, Institut für Geophysik, Universität Göttingen

Tilgner, A., 1999, Spectral methods for the simulation of incompressible flows in spherical shells, International Journal for numerical methods in fluids, 30, 713-724

Turcotte, D. L., Schubert, G., 2002, Geodynamics, Cambridge University Press, Cambridge, 2. Aufl.

Urakawa, S., Someya, K., Terasaki, H., Katsura, T., Yokoshi, S., Funakoshi, K., Utsumi, W., Katayama, Y., Sueda, Y., Irifune, T., 2004, Phase relationships and equation of state of $\mathrm{FeS}$ at high pressures and temperatures and implications for the internal structure of Mars, Physics of the Earth and Planetary Interiors, 143-144, 469-479 
van den Berg, A. P., Yuen, D. A., 2002, Delayed cooling of the Earth's mantle due to variable thermal conductivity and the formation of a low conductivity zone, Earth and Planetary Science Letters, 199, 403-413

van den Berg, A. P., Yuen, D. A., Allwardt, J. R., 2002, Non-linear effects from variable thermal conductivity and mantle internal heating: implications for massive melting and secular cooling of the mantle, Physics of the Earth and Planetary Interiors, 129, 359375

van den Berg, A. P., Yuen, D. A., Rainey, E. S. G., 2004, The influence of variable viscosity on delayed cooling due to variable thermal conductivity, Physics of the Earth and Planetary Interiors, 142, 283-295

Weinstein, S. A., 1995, The effects of a deep mantle endothermic phase change on the structure of thermal convection in silicate planets, Journal of Geophsysical Research, 100, 11719-11728

Weiss, B. P., Hojatollah, V., Baudenbacher, F. J., Kirschvink, J. L., Stewart, S. T., Shuster, D. L., 2002, Record of an ancient martian magnetic field in ALH84001, Earth and Planetary Science Letters, 201, 449-463

Whaler, K. A., Purucker, M. E., 2005, A spatially continuous magnetization model for Mars, Journal of Geophysical Research, 110, 1-11

Williams, J.-P., Nimmo, F., 2004, Thermal evolution of the martian core: Implications for an early dynamo, Geology, 32, 97-100

Wüllner, U., Harder, H., 1998, Convection underneath a crust inhomogeneously enriched in heat sources, application to martian mantle dynamics, Physics of the Earth and Planetary Interior, 109, 129-150

Yanagawa, T. K. B., Nakada, M., Yuen, D. A., 2004, A simplified mantle convection model for thermal conductivity stratification, Physics of the Earth and Planetary Interiors, $146,163-177$

Yoder, C. F., Konopliv, A. S., Yuan, D. N., 2003, Fluid core size of Mars from detection of the solar tide, Science, 300, 299-303

Yuen, D. A., Balachandar, S., Steinbach, V. C., Honda, S., Reuteler, D. M., Smedsmo, J. J., Lauer, G. S., 1995, Non-equilibrium effects of core-cooling and time-dependent internal heating on mantle flush events, Nonlinear Processes in Geophysics, 2, 206-221

Zharkov, V. N., Gudkova, T. V., 2000, Interior structure models, Fe/Si ratio and parameters of figure for Mars, Physics of the Earth and Planetary Interiors, 117, 407-420

Zhong, S., Zuber, M. T., 2001, Degree-1 mantle convection and the crustal dichotomy on Mars, Earth and Planetary Science Letters, 189, 75-84 
Zuber, M. T., Solomon, S. C., Phillips, R. J., Smith, D. E., Tyler, G. L., Aharonson, O., Balmino, G., Banerdt, W. B., Head, J. W., Johnson, C. L., Lemoine, F. G., McGovern, P. J., Neumann, G. A., Rowlands, D. D., Zhong, S., 2000, Internal structure and early thermal evolution of Mars from Mars Global Surveyor topography and gravity, Science, 287, 1788-1793 



\section{Danke}

An dieser Stelle möchte ich allen danken, die diese Arbeit ermöglicht haben und zur ihrer erfolgreichen Durchführung beigetragen haben.

Zunächst möchte ich mich bei Herrn Prof. Dr. Ulrich Christensen bedanken, der mich zu dieser Arbeit motiviert hat und der mir bei der Durchführung beratend zur Seite stand.

Herrn Prof. Dr. Andreas Tilgner möchte für die Übernahme des Korreferats danken.

Herr Dr. Johannes Wicht hat diese Arbeit Korrektur gelesen und dem Leser diverse Fehler und Sprachmonster erspart. Dafür ein herzliches Dankeschön. Außerdem war er immer ein zuverlässiger Ansprechpartner bei Problemen mit dem numerischen Code, der Physik oder dem Leben im Allgemeinen.

Herrn Dr. Dieter Schmitt, dem Koordinator der Research School, gilt mein Dank für die Organisation der vielen Vorträge, Vorlesungswochen und Seminare, die mir die Möglichkeit gegeben haben, über mein eigenes Forschungsgebiet heraus, andere Fachgebiete kennenzulernen.

Dann möchte ich mich bei meinen Kollegen und Freunden hier im Institut für die gute und herzliche Atmosphäre bedanken. Es klappt ja bekanntlich nicht immer alles auf Anhieb und insbesondere in diesen Phasen war es schön, im Institut viele offene Ohren und helfende Hände zu finden.

Diese Arbeit wurde am Max-Planck-Institut für Sonnensystemforschung in KatlenburgLindau im Rahmen der International Max Planck Research School angefertigt. Ich bedanke mich bei den Direktoren des Instituts und der MPG für diese Möglichkeit und die finanzielle Förderung. 



\section{Lebenslauf}

\section{Persönliche Daten}

Name

Geburtsdaten

Eltern

Familienstand

Staatsbürgerschaft

\section{Schulbildung}

08/1984-07/1988

08/1988 - 05/1997

$06 / 1997$

\section{Studium}

10/1997 - 09/1999

09/1999

$10 / 1999-11 / 2002$

$04 / 2001-09 / 2002$

$11 / 2002$

02/2003-03/2006
Monika Buske

14.07.1977 in Stadtlohn

Hanns-Volker Buske

Maria-Elisabeth Buske, geb. Theisen

ledig

deutsch
Von-Galen-Grundschule, Gescher

Gymnasium Nepomucenum, Coesfeld

Abitur

Grundstudium der Physik an der TU Clausthal

Vordiplom

Hauptstudium der Physik an der Georg-August-Universität, Göttingen

Diplomarbeit am Institut für Geophysik, Göttingen: "Dreidimensionale Evolutionsmodelle der Konvektion im Marsmantel"

Diplomprüfung

Doktorarbeit: "Dreidimensionale thermische Evolutionsmodelle für das Innere von Mars und Merkur"

Während dieser Zeit Tätigkeit als wissenschaftlicher Mitarbeiter am Max-Planck-Institut für Sonnensystemforschung in Katlenburg-Lindau 